The contents of this report are not to be used for advertising, publication, or promotional purposes. Citation of trade names does not constitute an official endorsement or approval of the use of such commercial products.

The findings of this report are not to be construed as an official Department of the Army position, unless so designated by other authorized documents. 


\section{Performance Characteristics of a Rapidly Installed Breakwater System}

by Michael J. Briggs

Coastal and Hydraulics Laboratory

U.S. Army Engineer Research and Development Center 3909 Halls Ferry Road

Vicksburg, MS 39180-6199

Final report

Approved for public release; distribution is unlimited 


\section{PREFACE}

This report discusses the dissertation research work performed during the period September 1997 to March 2001 by Dr. Michael J. Briggs (U.S. Engineer Research and Development Center (ERDC)). The work was sponsored by the ERDC Military Research, Development, Testing, and Evaluation (RDT\&E) Program in the Coastal and Hydraulics Laboratory (CHL).

The research was conducted under the general supervision of Mr. Thomas W. Richardson, Acting Director, CHL, and Mr. Dennis Markle, Chief, Harbors and Entrances Branch, CHL.

This report is a dissertation in partial fulfillment of the requirements for the degree of Doctor of Philosophy in Ocean Engineering from Texas A\&M University (TAMU), College Station, TX. The author would like to thank Professor Jun Zhang, TAMU, for serving as chair of his dissertation committee and for his helpful encouragement, guidance, and assistance. The author would also like to thank the other members of his committee including: Dr. Donald T. Resio and Dr. Zeki Demirbilek, CHL, Dr. Robert Randall and Mr. Robert Reid, TAMU, and Dr. Philip L.-F. Liu, Cornell University. Dr. Resio provided funding and freedom to work on this research as part of the overall RIBS project. Dr. Demirbilek was especially helpful in his support and assistance in the numerical modeling of the RIBS. The author gratefully acknowledges Mr. Ivano Melito, CHL, Dr. Leon Borgman, Borgman Inc, and Professor Nobuhisa Kobayashi, University of Delaware for their contributions.

At the time of publication of this report, Dr. James R. Houston was Director of ERDC, and Mr. Armando J. Roberto, Jr., was Acting Commander.

The contents of this report are not to be used for advertising, publication, or promotional purposes. Citation of trade names does not constitute an official endorsement or approval of the use of such commercial products. 


\section{TABLE OF CONTENTS}

CHAPTER Page

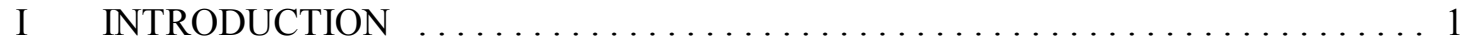

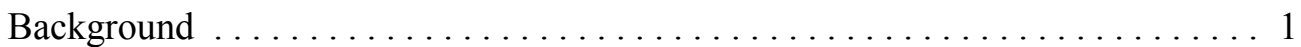

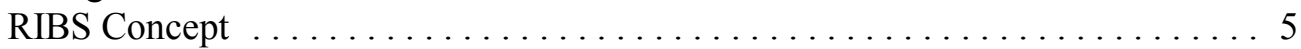

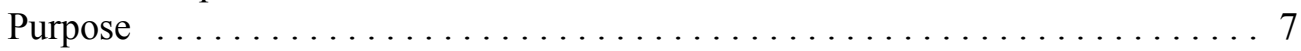

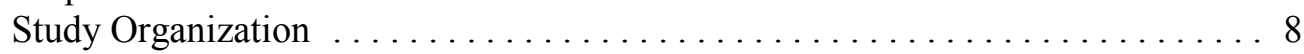

II EXPERIMENTAL MEASUREMENTS $\ldots \ldots \ldots \ldots \ldots \ldots \ldots \ldots \ldots \ldots \ldots \ldots$

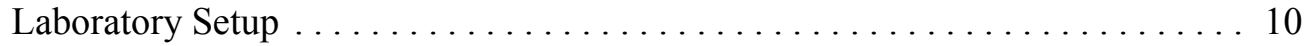

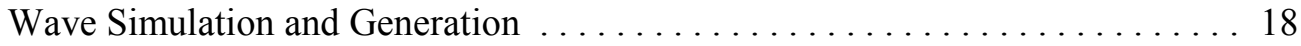

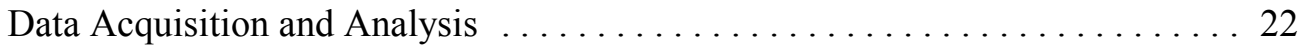

Measured Data . ................................. 27

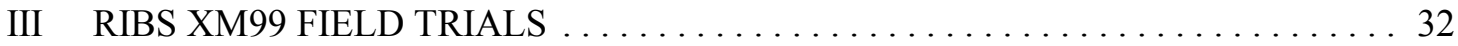

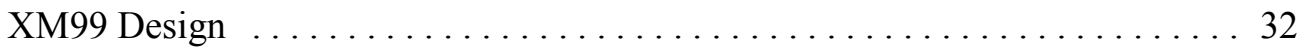

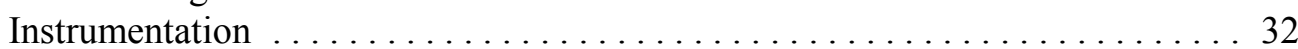

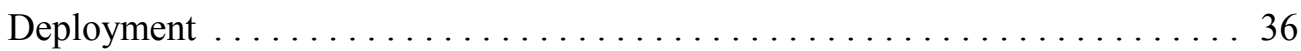

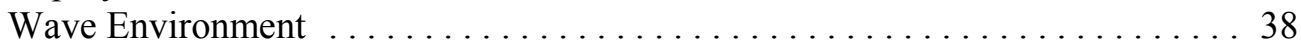

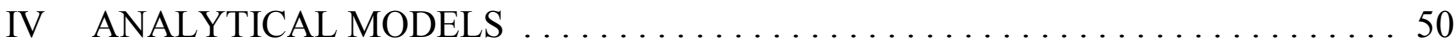

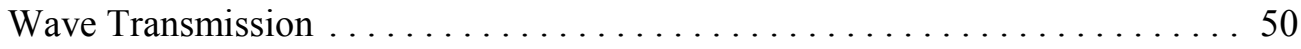

Hydrodynamic Pressure . . . . . . . . . . . . . . . . . . . . . . 58

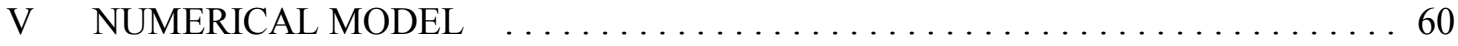

Theoretical Development ......................... 60

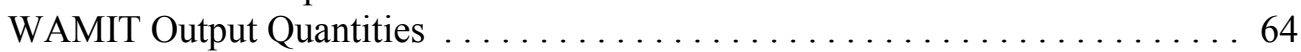

Model Generation Details ................................ 66

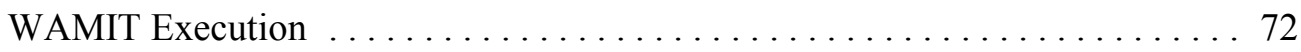

VI WAVE TRANSMISSION COEFFICIENTS $\ldots \ldots \ldots \ldots \ldots \ldots \ldots \ldots \ldots \ldots$

Fundamental Laboratory Experiments $\ldots \ldots \ldots \ldots \ldots \ldots \ldots \ldots \ldots \ldots$

XM99 Field Trials . . . . . . . . . . . . . . . . . . . . 87 
CHAPTER Page

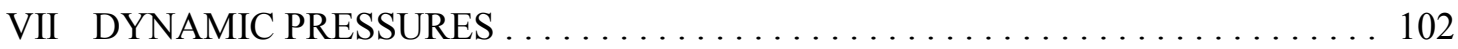

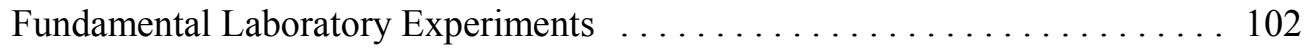

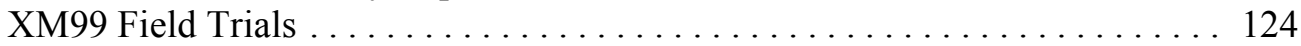

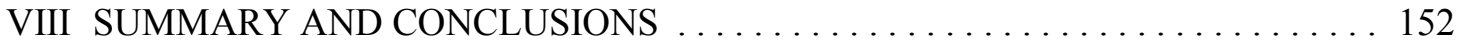

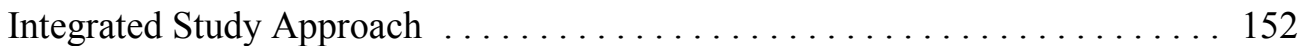

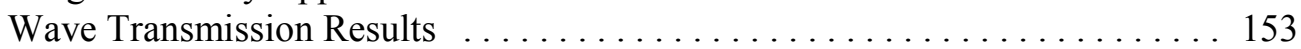

Dynamic Pressure Results . . . . . . . . . . . . . . . . . . . . . . 155

Areas for Future Research . . . . . . . . . . . . . . . . . . . . . . 158

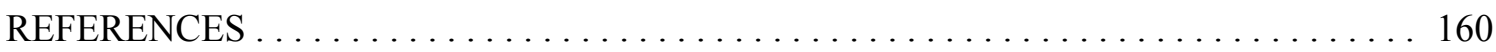

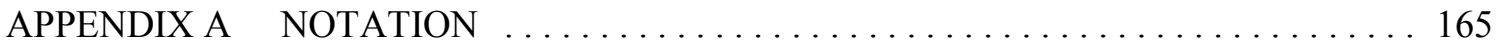

APPENDIX B FLab PRESSURE GAGE VOLTAGES $\ldots \ldots \ldots \ldots \ldots \ldots \ldots \ldots \ldots$

APPENDIX C FLab WAVE MEASUREMENTS $\ldots \ldots \ldots \ldots \ldots \ldots \ldots \ldots \ldots \ldots$

APPENDIX D MEASURED XM99 WAVE PARAMETERS $\ldots \ldots \ldots \ldots \ldots \ldots \ldots \ldots 1$

APPENDIX E $\quad$ XM99 MASS MOMENTS OF INERTIA . . . . . . . . . . . . . . . . 189

APPENDIX F FLab WAVE TRANSMISSION COEFFICIENTS $\ldots \ldots \ldots \ldots \ldots \ldots . . \ldots 3$

APPENDIX G XM99 WAVE TRANSMISSION COEFFICIENTS $\ldots \ldots \ldots \ldots \ldots .201$

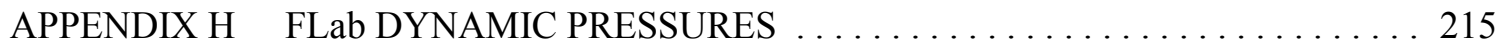

APPENDIX I XM99 ANALYTICAL DYNAMIC PRESSURES $\ldots \ldots \ldots \ldots \ldots \ldots . \ldots 235$

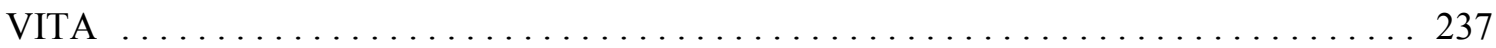




\section{LIST OF FIGURES}

FIGURE

Page

1 Rapidly Installed Breakwater System (RIBS) concept $\ldots \ldots \ldots \ldots \ldots \ldots \ldots$

2 Fundamental laboratory experiments in the DSWG Basin $\ldots \ldots \ldots \ldots \ldots \ldots$

3 Schematic of basin layout for $45 \mathrm{deg}$ interior angle configuration $\ldots \ldots \ldots \ldots \ldots$

4 Frequency spectra for operational series of the FLab (a) $T=5 \mathrm{sec}, \bar{\theta}=0 \mathrm{deg}$,

(b) $T=5 \mathrm{sec}, \bar{\theta}=20 \mathrm{deg}$, (c) $T=8 \mathrm{sec}, \bar{\theta}=0 \mathrm{deg}$, (d) $T=8 \mathrm{sec}, \bar{\theta}=20 \mathrm{deg}$,

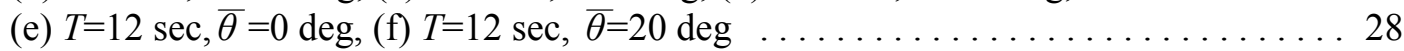

5 Directional spreading for operational series of the FLab (a) $T=5 \mathrm{sec}, \bar{\theta}=0 \mathrm{deg}$,

(b) $T=5 \mathrm{sec}, \bar{\theta}=20 \mathrm{deg}$, (c) $T=8 \mathrm{sec}, \bar{\theta}=0 \mathrm{deg}$, (d) $T=8 \mathrm{sec}, \bar{\theta}=20 \mathrm{deg}$,

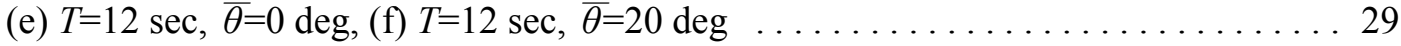

6 Frequency spectra and directional spreading for SS5 survival series ( $T=12 \mathrm{sec})$ of the FLab (a) $S(f), \bar{\theta}=0 \mathrm{deg}$, (b) $D(f, \theta), \bar{\theta}=20 \mathrm{deg}$, (c) $S(f), \bar{\theta}=0 \mathrm{deg}$, (d) $D(f, \theta), \bar{\theta}=20 \mathrm{deg}$. 30

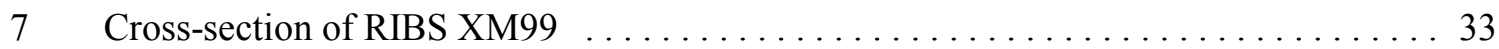

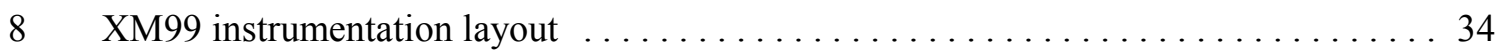

9 XM99 tow through Cape Canaveral Harbor, FL $\ldots \ldots \ldots \ldots \ldots \ldots \ldots \ldots \ldots \ldots \ldots \ldots \ldots$

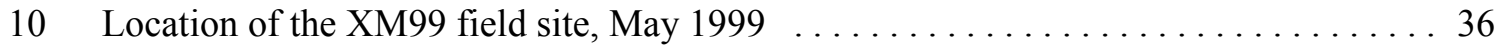

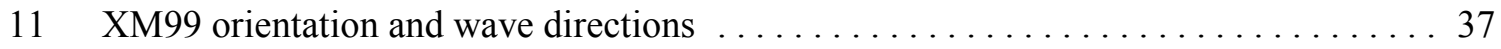

12 XM99 on station off Cape Canaveral, May $1999 \ldots \ldots \ldots \ldots \ldots \ldots \ldots \ldots \ldots$

13 Incident significant wave heights $\ldots \ldots \ldots \ldots \ldots \ldots \ldots \ldots \ldots \ldots \ldots \ldots \ldots \ldots \ldots \ldots \ldots \ldots \ldots \ldots$

14 GEDAP significant and maximum wave heights $\ldots \ldots \ldots \ldots \ldots \ldots \ldots \ldots$

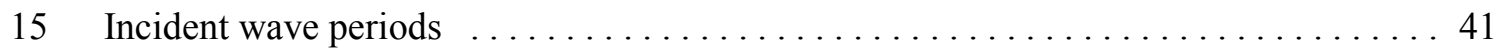

16 GEDAP significant and maximum wave periods $\ldots \ldots \ldots \ldots \ldots \ldots \ldots \ldots \ldots$

17 MLM incident wave direction and directional spread $\ldots \ldots \ldots \ldots \ldots \ldots \ldots \ldots$

18 Directional wave spectrum, May 25,1800 hours $\ldots \ldots \ldots \ldots \ldots \ldots \ldots \ldots$ 
19 Directional wave spectrum, unimodal swell, May 23, 0600 hours $\ldots \ldots \ldots \ldots \ldots 4$

20 Directional wave spectrum, bimodal with dominant sea mode, May 24, 1800 hours . . 46

21 Directional wave spectrum, bimodal with dominant swell mode, May 21, 1800 hours . 47

22 Joint distribution of wave period and height $\ldots \ldots \ldots \ldots \ldots \ldots \ldots \ldots \ldots$

23 Joint distribution of wave period and wave direction $\ldots \ldots \ldots \ldots \ldots \ldots \ldots$

24 Joint distribution of wave period and directional spread $\ldots \ldots \ldots \ldots \ldots \ldots \ldots \ldots$

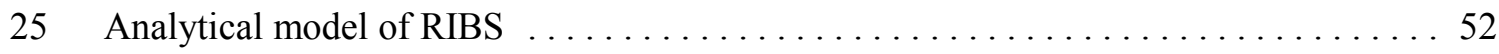

26 WAMIT panel layout for FLab - 2-D view $\ldots \ldots \ldots \ldots \ldots \ldots \ldots \ldots \ldots \ldots \ldots \ldots \ldots \ldots \ldots \ldots$

27 WAMIT panel layout for FLab $-3-\mathrm{D}$ view $\ldots \ldots \ldots \ldots \ldots \ldots \ldots \ldots \ldots \ldots \ldots \ldots \ldots \ldots \ldots \ldots$

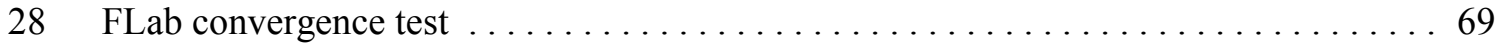

29 WAMIT panel layout for XM99 - 2-D view $\ldots \ldots \ldots \ldots \ldots \ldots \ldots \ldots \ldots \ldots \ldots \ldots$

30 WAMIT panel layout for XM99 - 3-D view $\ldots \ldots \ldots \ldots \ldots \ldots \ldots \ldots \ldots \ldots \ldots \ldots \ldots \ldots$

31 Measured $K_{T}$ for FLab, $\theta_{r}=45 \operatorname{deg}$ (a) $d / h=0.25$, (b) $d / h=0.50$, (c) $d / h=0.75$, (d) $d / h=1.00$, and $\theta_{r}=90 \mathrm{deg}$ (e) $d / h=0.50$, (f) $d / h=1.00 \ldots \ldots \ldots \ldots \ldots \ldots \ldots \ldots \ldots \ldots \ldots$

32 Comparison of monochromatic and spectral $K_{T}$ values for $d / h=0.50$ and $\theta_{r}=0 \mathrm{deg} \ldots 76$

33 Effect of wave height on $K_{T}$ for FLab with $T_{p}=12 \mathrm{sec}, \theta_{r}=45 \mathrm{deg}$ (a) $d / h=0.25$,

(b) $d / h=0.50$, (c) $d / h=0.75$, (d) $d / h=1.00$, and $\theta_{r}=90 \mathrm{deg}$ (e) $d / h=0.50$, (f) $d / h=1.00 \ldots 78$

34 WAMIT $K_{T}$ predictions for FLab, $\theta_{r}=45 \mathrm{deg}$ (a) $d / h=0.25$, (b) $d / h=0.50$, (c) $d / h=0.75$, (d) $d / h=1.00$, and $\theta_{r}=90 \mathrm{deg}$ (e) $d / h=0.50$, (f) $d / h=1.00 \ldots \ldots \ldots \ldots . .79$

35 Comparison of WAMIT vs. measured $K_{T}$ for FLab $\ldots \ldots \ldots \ldots \ldots \ldots \ldots \ldots$

36 Analytical model comparisons with measured $K_{T} \ldots \ldots \ldots \ldots \ldots \ldots \ldots \ldots$

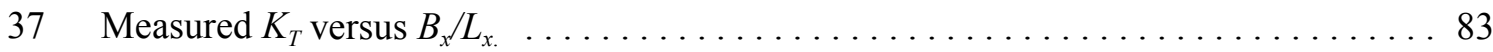

38 Effect of wave steepness on measured $K_{T}$ for FLab $\ldots \ldots \ldots \ldots \ldots \ldots \ldots \ldots \ldots$

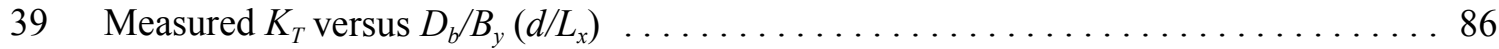


40 Wave transmission coefficient analysis by frequency $\ldots \ldots \ldots \ldots \ldots \ldots \ldots$

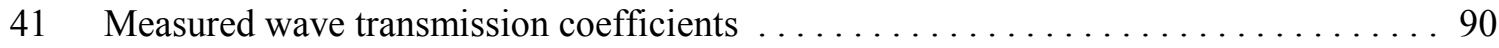

42 Joint distributions of wave transmission coefficient vs. (a) peak wave period $T_{p}$,

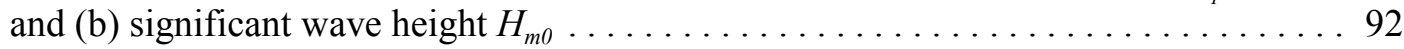

43 Joint distributions of wave transmission coefficient vs. (a) mean wave direction $\bar{\theta}$

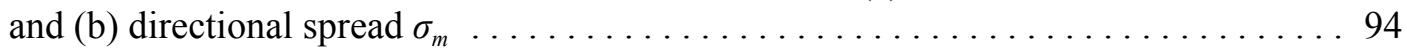

44 Comparison of measured wave transmission with WAMIT, PTT, and MPTT models . 96

45 Measured and analytical model wave transmission versus $T_{F}$ parameter (a) $K_{T}$ vs. $T_{F}$, (b) ratio of measured $K_{T}$ vs. predicted $K_{T} \ldots \ldots \ldots \ldots \ldots \ldots \ldots$

46 Measured $K_{T}$, least squares fit, and $95 \%$ confidence limits versus (a) $B / L$ and (b) $H / L$.

47 Measured $p_{1, S}$ vs. normalized distance from the bow for FLab $\theta_{r}=45 \mathrm{deg}$ (a) $d / h=0.25$,

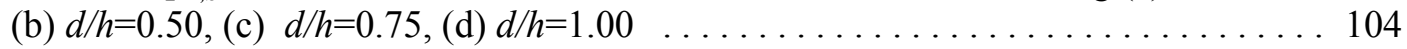

48 Measured $\bar{p}_{1, S}$ vs. normalized distance from the bow for FLab $\theta_{r}=45 \mathrm{deg}$ (a) $d / h=0.25$,

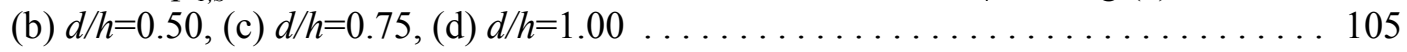

49 Comparison of average $p_{l, A v g}$ for regular and spectral waves for $\theta_{r}=45 \mathrm{deg}, d / h=1.00 \quad 106$

50 Effect of wave height on measured $p_{1, S}$ for FLab $T_{p}=12 \mathrm{sec}$ and $\theta_{r}=45 \mathrm{deg}$

(a) $d / h=0.25$, (b) $d / h=0.50$, (c) $d / h=0.75$, (d) $d / h=1.00 \ldots \ldots \ldots \ldots \ldots \ldots \ldots$

51 WAMIT predicted $\bar{p}_{W}$ for $d / h=1.00$ and $T=5 \mathrm{sec}$ (a) $\bar{\theta}=0 \mathrm{deg}$ and (b) $\bar{\theta}=20 \mathrm{deg} \ldots 108$

52 WAMIT predicted $\bar{p}_{W}$ for $d / h=1.00$ and $T=8 \mathrm{sec}$ (a) $\bar{\theta}=0 \mathrm{deg}$ and (b) $\bar{\theta}=20 \mathrm{deg} \ldots \ldots 109$

53 WAMIT predicted $\bar{p}_{W}$ for $d / h=1.00$ and $T=12 \sec$ (a) $\bar{\theta}=0 \mathrm{deg}$ and (b) $\bar{\theta}=20 \mathrm{deg} \ldots 110$

54 WAMIT $\bar{p}_{W}$ for FLab $\theta_{r}=45 \mathrm{deg}, T=5 \sec$ (a) $d / h=0.25$, (b) $d / h=0.50$, (c) $d / h=0.75$,

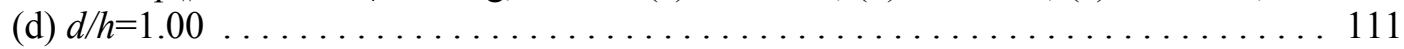

55 WAMIT predicted $\bar{p}_{W}$ for FLab $\theta_{r}=45 \mathrm{deg}, T=8 \mathrm{sec}, \bar{\theta}=0 \mathrm{deg}$ (solid line), $\bar{\theta}=20 \mathrm{deg}$ (dashed line) (a) $d / h=0.25$, (b) $d / h=0.50$, (c) $d / h=0.75$, (d) $d / h=1.00 \ldots \ldots 112$

56 WAMIT predicted $\bar{p}_{W}$ for FLab $\theta_{r}=45 \mathrm{deg}, T=12 \mathrm{sec}, \bar{\theta}=0 \mathrm{deg}$ (solid line), $\bar{\theta}=20 \mathrm{deg}$ (dashed line) (a) $d / h=0.25$, (b) $d / h=0.50$, (c) $d / h=0.75$, (d) $d / h=1.00 \ldots . .113$ 
57 WAMIT $\bar{p}_{1, W}$ for FLab $\theta_{r}=45 \operatorname{deg}$ (a) $d / h=0.25$, (b) $d / h=0.50$,

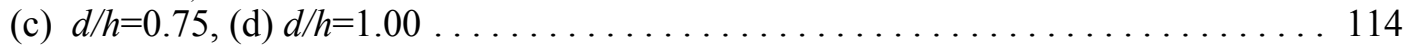

58 WAMIT $\bar{p}_{l, W}$ vs. measured $\bar{p}_{l, S}$ pressures for FLab (a) bow, (b) middle, (c) stern. . . 116

59 Analytical $\bar{p}_{1, A}$ for $\theta_{r}=45 \mathrm{deg}, d / h=0.50$ : (a) $T=5 \mathrm{sec}, \bar{\theta}=0 \mathrm{deg}$,

(b) $T=5 \mathrm{sec}, \bar{\theta}=20 \mathrm{deg}$, (c) $T=8 \mathrm{sec}, \bar{\theta}=0 \mathrm{deg}$, (d) $T=8 \mathrm{sec}, \bar{\theta}=20 \mathrm{deg}$,

(e) $T=12 \mathrm{sec}, \bar{\theta}=0 \mathrm{deg}$, (f) $T=12 \mathrm{sec}, \bar{\theta}=20 \mathrm{deg}$

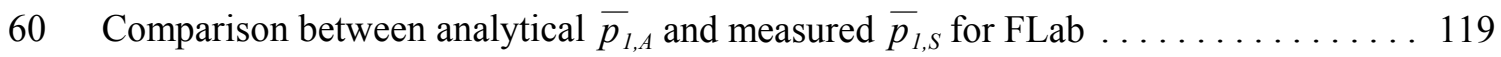

61 Measured dynamic pressure $\bar{p}_{1, S}$ vs. modified Reynold's Number $\operatorname{Re}_{x} \ldots \ldots \ldots \ldots$

62 Measured dynamic pressure $\bar{p}_{1, S}$ vs. Wiegel's transmission factor $T_{F} \ldots \ldots \ldots \ldots 121$

63 External pressure gage depths for XM99 (a) top level, (b) middle level,

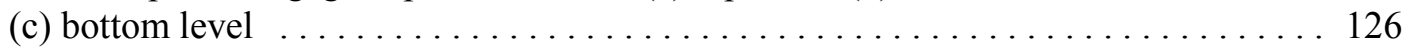

64 Measured dynamic pressures $p_{1, S}$ for XM99 (a) top level, (b) middle level,

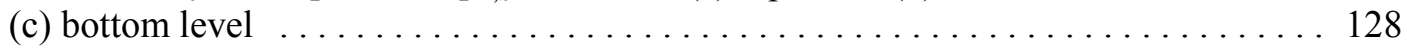

65 Maximum dynamic pressures $p_{1, \text { Max }}$ for XM99 (a) top level, (b) middle level,

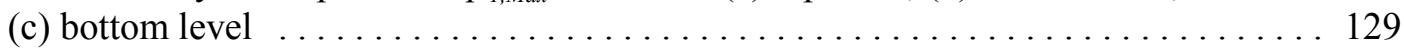

66 Measured $\bar{p}_{1, S}$ for XM99 "sea" waves: (a) $T=3 \mathrm{sec}, \bar{\theta}=0 \mathrm{deg}$,

(b) $T=3 \mathrm{sec}, \bar{\theta}=30 \mathrm{deg}$, (c) $T=3 \mathrm{sec}, \bar{\theta}=60 \mathrm{deg}$, (d) $T=5 \mathrm{sec}, \bar{\theta}=60 \mathrm{deg} \ldots \ldots \ldots \ldots 132$

67 Measured $\bar{p}_{1, S}$ for XM99 "swell" waves: (a) $T=8 \mathrm{sec}, \bar{\theta}=30 \mathrm{deg}$,

(b) $T=10 \mathrm{sec}, \bar{\theta}=30 \mathrm{deg}$, (c) $T=8 \mathrm{sec}, \bar{\theta}=60 \mathrm{deg}$, (d) $T=10 \mathrm{sec}, \bar{\theta}=60 \mathrm{deg} \ldots \ldots \ldots 133$

68 WAMIT predicted $\bar{p}_{W}$ for XM99 (a) $T=3 \mathrm{sec}, \bar{\theta}=0 \mathrm{deg}$, (b) $T=3 \mathrm{sec}, \bar{\theta}=30 \mathrm{deg}$,

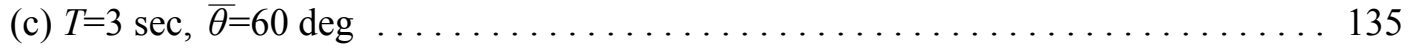

69 WAMIT predicted $\bar{p}_{W}$ for XM99 (a) $T=5 \mathrm{sec}, \bar{\theta}=0 \mathrm{deg}$, (b) $T=5 \mathrm{sec}, \bar{\theta}=30 \mathrm{deg}$,

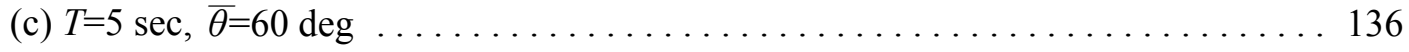

70 WAMIT predicted $\bar{p}_{W}$ for XM99 (a) $T=8 \mathrm{sec}, \bar{\theta}=0 \mathrm{deg}$, (b) $T=8 \mathrm{sec}, \bar{\theta}=30 \mathrm{deg}$,

(c) $T=8 \mathrm{sec}, \bar{\theta}=60 \mathrm{deg}$

71 WAMIT predicted $\bar{p}_{W}$ for XM99 (a) $T=10 \mathrm{sec}, \bar{\theta}=0 \mathrm{deg}$, (b) $T=10 \mathrm{sec}, \bar{\theta}=30 \mathrm{deg}$,

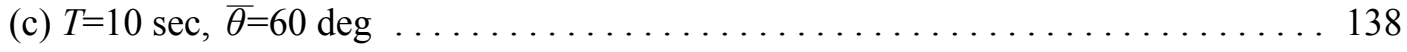


72 WAMIT $\bar{p}_{W}$ for XM99 "sea" waves (a) $T=3 \mathrm{sec}, \bar{\theta}=0 \mathrm{deg}$, (b) $T=5 \mathrm{sec}, \bar{\theta}=0 \mathrm{deg}$, (c) $T=3 \mathrm{sec}, \bar{\theta}=30 \mathrm{deg}$ (d) $T=5 \mathrm{sec}, \bar{\theta}=30 \mathrm{deg}$.

73 WAMIT $\bar{p}_{W}$ for XM99 “swell" waves (a) $T=8 \mathrm{sec}, \bar{\theta}=0 \mathrm{deg}$, (b) $T=10 \mathrm{sec}, \bar{\theta}=0 \mathrm{deg}$,

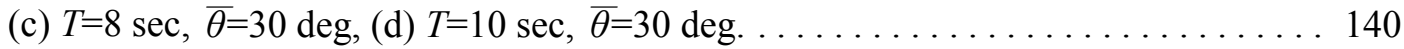

74 WAMIT $\bar{p}_{1, W}$ magnitude for XM99 “sea" waves (a) $T=3 \mathrm{sec}, \bar{\theta}=0 \mathrm{deg}$, (b) $T=5 \mathrm{sec}$,

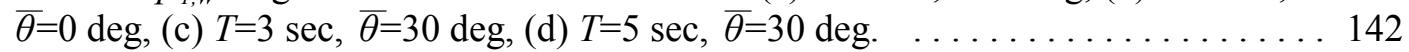

75 WAMIT $\bar{p}_{1, W}$ magnitude for XM99 "swell" waves (a) $T=8 \mathrm{sec}, \bar{\theta}=0 \mathrm{deg}$, (b) $T=10 \mathrm{sec}$, $\bar{\theta}=0 \mathrm{deg}$, (c) $T=8 \mathrm{sec}, \bar{\theta}=30 \mathrm{deg}$, (d) $T=10 \mathrm{sec}, \bar{\theta}=30 \mathrm{deg} . \ldots \ldots \ldots \ldots \ldots \ldots . \ldots \ldots$

76 Comparison of WAMIT $\bar{p}_{1, W}$ vs. measured $\bar{p}_{1, S}$ for XM99 (a) bow, (b) middle,

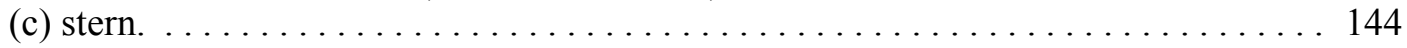

77 Analytical $\bar{p}_{l, A}$ for XM99 top level: (a) $T=3 \mathrm{sec}, \bar{\theta}=0 \mathrm{deg}$, (b) $T=5 \mathrm{sec}, \bar{\theta}=0 \mathrm{deg}$,

(c) $T=3 \mathrm{sec}, \bar{\theta}=30 \mathrm{deg}$, (d) $T=5 \mathrm{sec}, \bar{\theta}=30 \mathrm{deg}$, (e) $T=3 \mathrm{sec}, \bar{\theta}=60 \mathrm{deg}$,

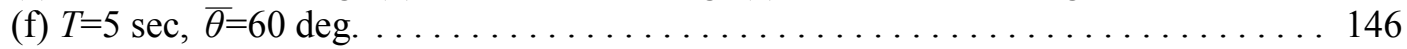

78 Analytical $\bar{p}_{1, A}$ for XM99 top level: (a) $T=8 \mathrm{sec}, \bar{\theta}=0 \mathrm{deg}$,

(b) $T=10 \mathrm{sec}, \bar{\theta}=0 \mathrm{deg}$, (c) $T=8 \mathrm{sec}, \bar{\theta}=30 \mathrm{deg}$, (d) $T=10 \mathrm{sec}, \bar{\theta}=30 \mathrm{deg}$,

(e) $T=8 \mathrm{sec}, \bar{\theta}=60 \mathrm{deg}$, (f) $T=10 \mathrm{sec}, \bar{\theta}=60 \mathrm{deg}$. 147

79 Analytical $\bar{p}_{l, A}$ for middle and bottom levels at $T=5 \mathrm{sec}$ (a) Middle, $\bar{\theta}=0 \mathrm{deg}$,

(b) Bottom, $\bar{\theta}=0 \mathrm{deg}$, (c) Middle, $\bar{\theta}=30 \mathrm{deg}$, (d) Bottom, $\bar{\theta}=30 \mathrm{deg}$,

(e) Middle, $\bar{\theta}=60 \mathrm{deg}$, (f) Bottom, $\bar{\theta}=60 \mathrm{deg}$.

80 Comparison of analytical $\bar{p}_{l, A}$ vs. measured $\bar{p}_{1, S}$ for XM99 (a) bow, (b) middle,

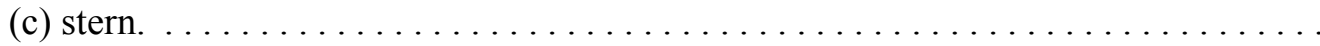




\section{LIST OF TABLES}

TABLE

Page

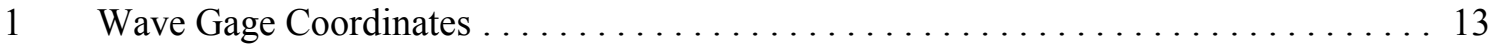

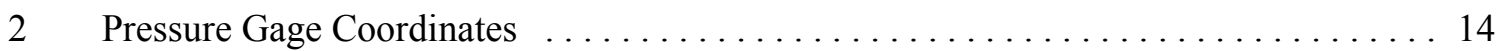

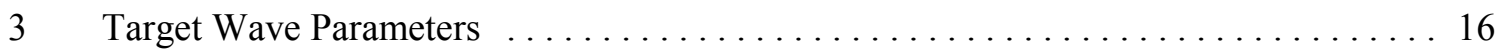

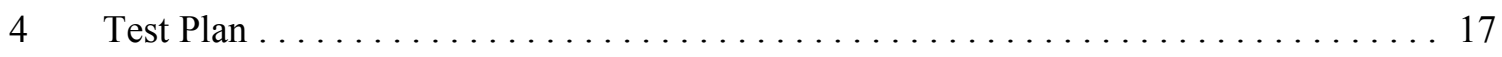

5 Simulation, Collection, and Analysis Parameters $\ldots \ldots \ldots \ldots \ldots \ldots \ldots \ldots \ldots \ldots \ldots \ldots$

6 Measured Wave Parameters . . . . . . . . . . . . . . . . . . . 31

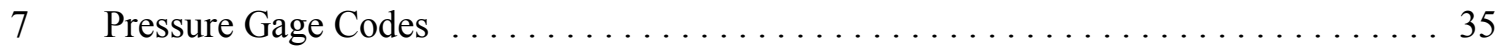

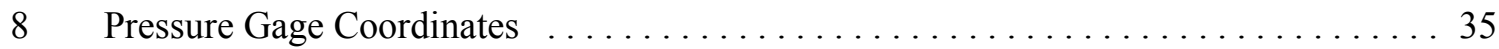

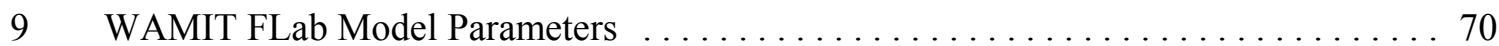

10 Incident Wave Angles: Global, Analytical, and Numerical $\ldots \ldots \ldots \ldots \ldots \ldots \ldots$

11 WAMIT and Measured $K_{T}$ Statistics $\ldots \ldots \ldots \ldots \ldots \ldots \ldots \ldots \ldots \ldots \ldots \ldots \ldots \ldots \ldots \ldots \ldots$

12 Summary of FLab Regression Analysis Parameters $\ldots \ldots \ldots \ldots \ldots \ldots \ldots \ldots$

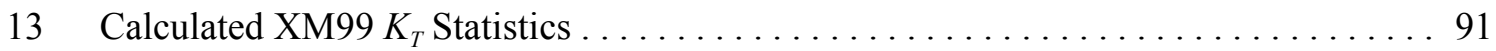

14 Summary of XM99 Wave Data Regression Analysis Parameters . . . . . . . . . . 93

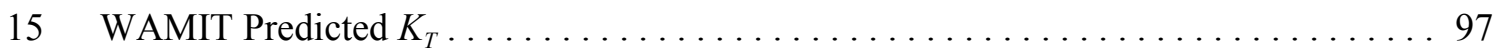

16 Summary of XM99 Regression Analysis Parameters $\ldots \ldots \ldots \ldots \ldots \ldots \ldots \ldots \ldots$

17 WAMIT and Measured Dynamic Pressure Statistics $\ldots \ldots \ldots \ldots \ldots \ldots \ldots \ldots$

18 Summary of Regression Analysis Parameters $\ldots \ldots \ldots \ldots \ldots \ldots \ldots \ldots \ldots \ldots \ldots$

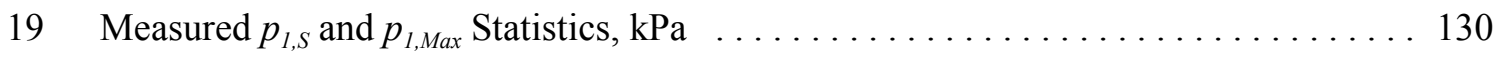

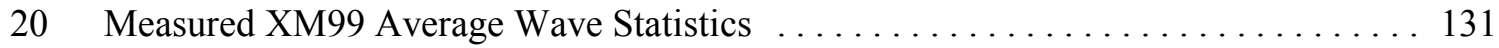




\section{CHAPTER I}

\section{INTRODUCTION}

\section{BACKGROUND}

\section{Historical Perspective}

The U.S. Army Corps of Engineers has the primary technical role in breakwater design and construction in the United States. Over the last 50 years, they have been actively engaged in a wide range of breakwater research, relating to civil and military missions. Breakwaters can be classified into two categories: gravity and floating. Gravity breakwaters rest on the bottom and usually extend above the surface. They maintain stability by incorporating sufficient mass (usually in rock or molded units) to resist wave-induced motions and provide a barrier to wave penetration. A new type of gravity breakwater is a berm breakwater that does not extend to the surface, but is slightly submerged. Gravity breakwaters are the types commonly seen in most ports and harbors around the world. Floating breakwaters are not as massive and must be anchored to the sea floor to maintain position and orientation. Usually, they occupy only the top section of the water column, but may also be submerged.

Floating wave breakwaters have historically been referred to as vertical wave barriers, curtain walls, wave screens, etc. For many years, the U.S. Army Engineer Research and Development Center (ERDC) has been involved with the design and deployment of floating breakwaters, primarily for application within bays or estuaries that are semiprotected from very large wave generation. Such structures typically are intended to attenuate waves with heights not exceeding $1.22 \mathrm{~m}$ and periods not exceeding $4 \mathrm{sec}$. Extrapolation to an open ocean environment is at least an order of magnitude greater in difficulty. In an oceanic environment, waves with heights up to $6.10 \mathrm{~m}$ and periods to $18 \mathrm{sec}$ are common during storm conditions.

Even though floating breakwaters have had little success outside of relatively protected waters, efforts have continued to design an effective breakwater for more robust wave climate

This dissertation follows the style and format of the ASCE Journal of Waterway, Port, Coastal, and Ocean Engineering. 
conditions. Designs have included floating, submerged, and suspended breakwaters, either solid or membrane. Mani and Jayakumar (1995) developed a suspended pipe breakwater, consisting of a row of closely spaced, vertical pipes, to protect a fishing harbor. Murali and Mani (1997) investigated a breakwater consisting of two rows of equally spaced vertical pipes with gaps, suspended from trapezoidal pontoons. A water-filled, flexible membrane attached to the sea floor was studied as an alternative to a rigid breakwater (Ohyama, et al. 1989; Tanaka et al. 1990; Tanaka et al. 1992). The flexible design utilizes interaction of incident and radiated waves generated by the membrane's motion to reduce the transmitted wave height.

\section{Military Significance}

Today's military must be capable of quickly and efficiently projecting its forces to any location around the globe. Furthermore, once these forces are on the ground, it is critical to adequately sustain these forces. Since 90 percent of the Army's equipment is moved via Sealift, there is a mandate for a strong chain of operations that can create robust, sea-based links between major depots within the U.S. and potential operational areas around the world. Recent

investments in infrastructure within the U.S. and in large Sealift ships have greatly increased our capacity to get necessary supplies to ports of debarkation in any theater. However, there are insufficient areas around the world that have large port facilities available that can directly load/offload modern deep-draft Sealift ships. Furthermore, given the importance of such facilities to U.S. force projection and sustainment, it is quite likely that such facilities would be high-priority targets for enemy actions. Given the limited number of ports around the world that meet Army needs for force projection and sustainment, and the likelihood that such ports may be damaged or denied to U.S. forces, it is critical that the Army maintains realistic forceprojection alternatives to large shore-based port facilities.

The only reasonable alternative available today is via Logistics Over the Shore (LOTS) or Joint Logistics Over the Shore (JLOTS, when two or more services are involved) operations. In these operations, supplies from Sealift ships are offloaded at offshore anchorages onto smaller vessels termed lighters. Lighters then transport the supplies to smaller ports and harbors, to causeways along the shore, or directly onto the beach. 


\section{The Problem}

Existing LOTS or JLOTS capabilities allow operations to be conducted in sea states 1 and 2 (SS1 and SS2), or significant wave heights up to $1 \mathrm{~m}$. Sea State 3 (SS3) conditions consist of waves with peak periods in the range 3 to $6 \mathrm{sec}$ and significant wave heights between 0.91 and $1.52 \mathrm{~m}$. Throughput rates documented during a LOTS exercise conducted at Ft. Story, VA, in 1984, show that SS3 conditions seriously diminish or halt system throughput completely. In 1991 and again in 1993, results from two major LOTS exercises provided evidence that ships and lighters were unable to perform their roles in SS3.

If global sea states remained primarily in SS1 and SS2 and only rarely attained SS3 and higher levels, the inability to continue operations into these conditions would not be a serious problem for U.S. forces. However, extensive studies have concluded that SS3 conditions can be expected more than 50 percent of the time in many areas of U.S. interest around the globe. As an example of the problems that this can cause, Operation Restore Hope in Somalia in 1993 was plagued by the inability to maintain an adequate throughput of critical supplies. Gen. Gordon R. Sullivan, then Army Chief of Staff, concluded that one of the primary contributors to these shortages was the inability of the ships offshore to discharge their cargo in SS3.

\section{Breakwater Performance}

According to Jones (1971), an ideal wave barrier will have the following performance characteristics: (a) good performance or attenuation of wave energy in the operating range of wave conditions, (b) high mobility or readily and rapidly transportable with existing land and sea equipment and constraints, (c) quick installation and removal without undue difficulty, (d) able to survive a "design" storm, (e) economic to build and use, and (f) reusable. Unfortunately, performance and mobility are conflicting requirements. As a result, we have never had a truly effective floating breakwater in energetic wave environments, with most being relegated to the relatively benign wave climates in sheltered marinas and aquiculture facilities.

Of the criteria above for rating a floating breakwater, the most important is performance or wave attenuation. Wave attenuation is accomplished by wave reflection, interference due to wave radiation, and energy extraction/dissipation due to breaking, absorption, turbulence, and 
friction. Based on his survey of performance data from 106 laboratory and field tests, Jones (1971) found that the velocity field is the most important parameter affecting floating breakwater performance. For water depth to wavelength ratios of $h / L>0.5$ (i.e., deepwater waves), the wave has circular orbits that decrease rapidly with distance below the surface. The velocity is only $1 / 5$ of its surface value at middepth. This condition corresponds to waves with wave periods smaller than about $4.4 \mathrm{sec}$ in $15.24 \mathrm{~m}$ water depth. Therefore, according to Jones, a fairly rigid barrier from the surface with depth of submergence (i.e., draft) less than the full depth blocks out a significant portion of the kinetic energy and is relatively effective. He suggested that a barrier blocking the upper half of the water column (i.e., from the surface to 50 percent of the depth) should be able to block 96 percent of the wave's energy (i.e., 4 percent transmitted, $K_{T}=0.2$ ) associated with $\mathrm{SS} 3$ waves.

For intermediate and shallow water waves (i.e., $h / L \leq 0.5$ ), however, the water particle motions are more in a horizontal line, moving back and forth. The amplitude and speed of the water particles become constant over the entire water column as the wave becomes a shallow water wave. A barrier occupying only 50 percent of the water column would transmit 49 percent of the wave energy. To achieve a reduction of 96 percent as before, the depth of submergence would have to be 90-95 percent of the water column.

Jones (1971) also found that wave steepness and relative breakwater length parallel to wave direction (i.e., width or beam) are important parameters affecting wave attenuation. Although less important, wave steepness becomes more significant if breaking and turbulence are present. Of course, larger wave heights require a larger structure (i.e., mass or inertia) for survivability and overtopping. For floating breakwaters aligned normal to the wave direction, the breakwater width or beam should be greater than the wavelength. In general for a given wavelength, if the draft is small, the breakwater width must be larger, and vice versa. Thus, if the draft is relatively shallow, the breakwater width needs to be longer to compensate.

Hales (1981) performed a literature review of the state-of-the-art in floating breakwaters. He noted that basic mechanisms of floating breakwaters include reflection, dissipation, interference, and energy conversion. Hales found that these breakwaters could be grouped according to concept as pontoon, sloping float, scrap tire, cylinder, and tethered float. His review of theoretical, field, and laboratory literature indicated that breakwater mass, radius of gyration (moment of inertia), and depth of submergence are the most important parameters. 
Two important nondimensional parameters are the breakwater width to wavelength ratio $(B / L)$ and water depth to wavelength $(h / L)$. Wave transmission and mooring forces decreased as the breakwater width increased to more than one-half the wavelength. Although reduced mooring forces seems intuitively wrong, the floating breakwater is long relative to the wave so that it experiences wave forces in opposite directions on different parts of the structure. He recommended floating breakwaters for use when wave periods are less than $4 \mathrm{sec}$ and wave heights do not exceed $1.22 \mathrm{~m}$.

Jones (1971) also reported that previous tests and studies have shown that to be effective, floating breakwaters must have widths on the order of one-fourth to one-third of the wavelength being attenuated and be very rigid and massive to prevent the breakwater from acting as a wavemaker. In addition, floating breakwaters have (a) a strong dependence of breakwater performance on wave period, (b) mooring difficulties, and (c) higher potential for structural failures during large wave events. These factors were the driving force behind recent floating breakwater developments at the ERDC.

\section{RIBS CONCEPT}

The new concept of the RIBS was designed with the breakwater performance criteria previously described. This concept promises to expand floating breakwater technology by allowing operations in SS3 conditions. The driving force behind the RIBS concept has been the fact that SS3 is a "war stopper"for present force projection plans and technology. The objective of the RIBS is to reduce the waves from SS3 to SS2 within the lee of the structure to facilitate military and civilian operations along exposed portions of the world's coastlines.

The RIBS is a floating breakwater with two legs in a "V" shape in plan view that provide a sheltered region from waves and currents. Figure 1 is an artist's rendition of what the full-scale RIBS will look like. According to the patent (Resio et al., 1997), the legs of the RIBS may be a continuous rigid structure, a hinged structure with one or more joints, a flexible water beam membrane structure, or a hybrid of rigid and flexible components. Based on previous experience, it appears that the RIBS legs will need to be of the order of 1.5 to 3 wavelengths in length and extend through the water column a depth sufficient to deflect most of the wave energy (i.e., order of 0.5 times the water depth). In the past, floating breakwaters were generally positioned 


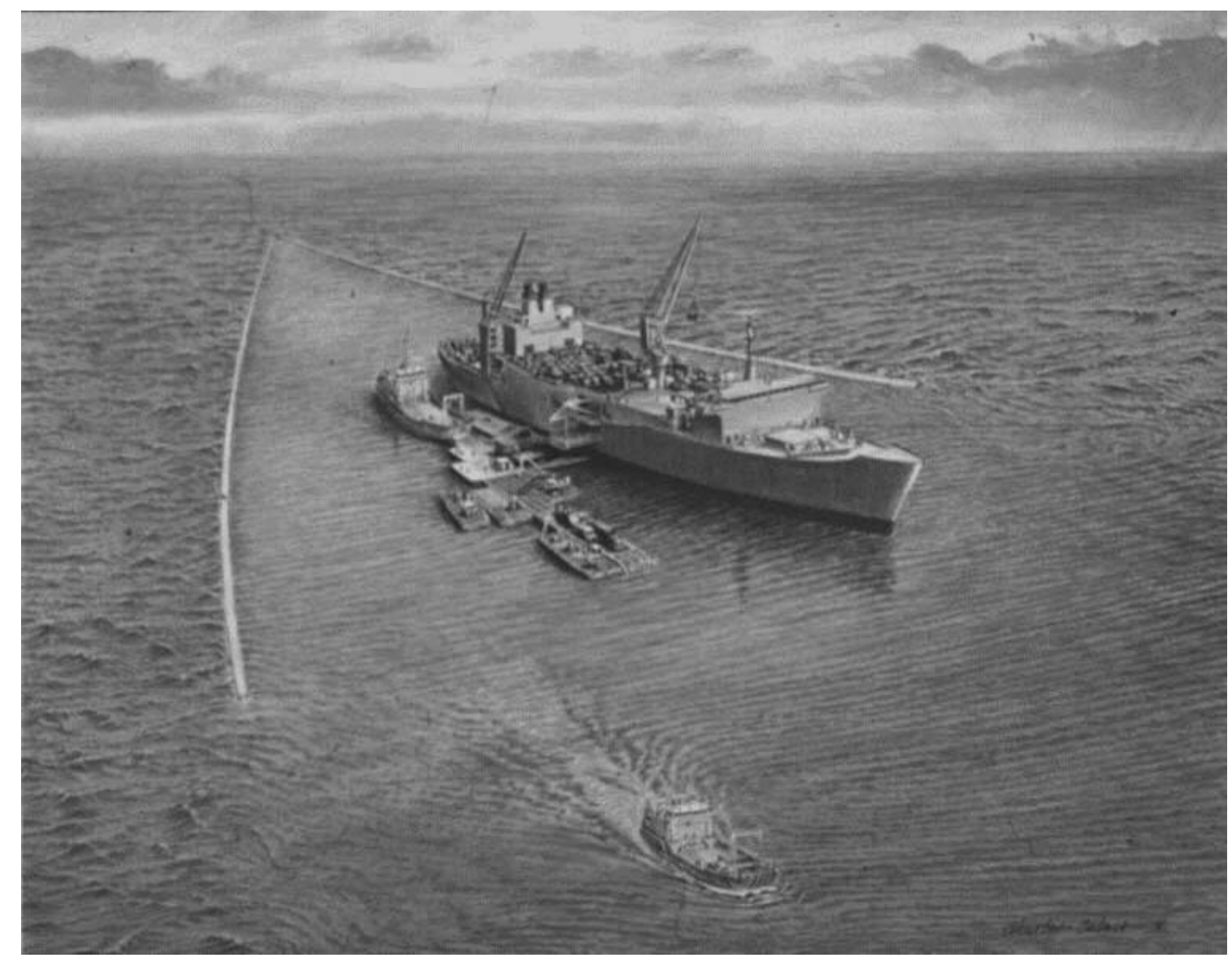

Figure 1. Rapidly Installed Breakwater System (RIBS) concept.

broadside to the incoming waves. The RIBS concept is based on the ability of the relatively thin leg structures to diffract and reflect waves because of the angle the legs make to the incoming waves (Resio et al., 1997). Each leg of the RIBS acts as a diffraction element for obliquely incident waves, leaving relatively calm water inside and behind the structure. Mooring loads are minimized because the RIBS shape is "streamlined" and should deflect most of the incoming waves rather than absorbing and reflecting them.

The goal of the RIBS program is to produce a floating breakwater that can meet the Department of Defenses's (DOD) needs for sea-state mitigation during JLOTS operations. To accomplish this, the final version of the RIBS must (a) reduce wave heights by at least 50 percent in the lee of the breakwater; (b) be rapidly deployable, even in SS3, and (c) be transportable with existing Army assets or a plan must exist to acquire necessary deployment assets. 
Since the beginning of the RIBS program in 1995, more than 100 laboratory and four field experiments have been conducted in support of the RIBS development. The RIBS XM99 is the latest of the field models tested in the RIBS program.

\section{PURPOSE}

The purpose of this research was to conduct an integrated study of analytical, numerical, laboratory, and field experiments to predict the performance and dynamic pressure of the RIBS. Various closed-form solutions for vertical barriers with simplified boundary conditions exist in the literature (Resio et al. 1995). These formulations are based on linear, asymptotic approximations for an infinitely long barrier. In real-world situations, however, complicated geometries and floatation elements give rise to complex, nonlinear systems. Consequently, although an understanding of the physics of these systems can provide guidance for the overall direction of these studies, mathematical and computer solutions are not yet capable of providing realistic solutions. Therefore, efforts have concentrated on laboratory-scale models and ocean-scale field trials of various RIBS concepts. In these experiments, V-shaped floating structures have been exposed to a wide range of incident wave conditions that have validated the RIBS concept. Thus, a key objective of this research is to document and compare the measured performance to predicted values from analytical, laboratory, and numerical models. A long-term goal is to improve these predictive capabilities with empirical relationships from measured data.

There are no analytical tools available for predicting wave transmission and dynamic pressures of the complicated RIBS floating structure in irregular seas. However, there are two simple, semi-empirical, analytical models for wave transmission of rigid, thin, vertical barriers in the presence of normally incident waves that will be used in this research (Ursell 1947, Wiegel 1960, Kriebel and Bollmann 1996). Dynamic pressures can be predicted using linear wave theory. Additional work in the analytical area for predicting wave transmission and dynamic pressure are an area of ongoing research.

Very little laboratory data exist for floating breakwaters. Giles and Eckert (1979) conducted a series of laboratory experiments to study wave transmission coefficients and mooring line loads for floating tire breakwaters. Harms et al. (1982) conducted a series of laboratory experiments of a floating breakwater composed of cylindrical members (i.e., steel or concrete 
pipes, telephone poles) in a matrix of scrap truck or automobile tires. Wiegel (1960) and Kriebel and Bollmann (1996) conducted experiments with regular waves. Broderick and Leonard (1995) investigated the nonlinear interactions of membranes using laboratory tests of a 1-m-diameter, fabric, cylindrical model.

Numerical solutions include various methods (Liu and Abbaspour 1982; Nakamura 1983; Losada et al.1992; Mandal and Dolai 1994). Porter and Evans (1995) developed an integral equation for the velocity in the gap using Chebychev polynomials. Kim and Kee (1996) investigated the performance of a vertical (extending to the sea floor), flexible-membrane, wave barrier with varying membrane tension, water depth, and mass density. Williams and Abul-Azm (1996) proposed a dual pontoon floating breakwater. Das et al. (1997) used one-term Galerkin approximations to evaluate reflection and transmission coefficients for three different types of barrier configurations. Lo (2000) investigated the performance of a flexible membrane using eigenfunction expansions for the velocity potential and membrane motions.

Since the RIBS is large relative to the wavelength of the design SS3 wave conditions, its presence will affect the wave field. Therefore, a three-dimensional, numerical radiation/diffraction code is required to perform the hydrodynamic analysis of the wavestructure interaction. The numerical model used in this study is the linear radiation/diffraction program WAMIT (Wave Analysis MIT) for the analysis of wave-structure interaction. Hydrodynamic analysis is used to estimate the response of a structure to waves in the six rigid body modes of surge, sway, heave, pitch, roll, and yaw. The focus in this study is the hydrodynamic parameters of wave transmission and dynamic pressure. Of the criteria for rating a floating breakwater, the most important is performance, as quantified by wave transmission. Dynamic pressures are an important quantity because they are used to calculate the wave forces and moments, operational stresses, and ultimate strength requirements for RIBS design and survivability.

\section{STUDY ORGANIZATION}

In Chapter II, the fundamental laboratory experiments conducted in 1997 are discussed. In the summer of 1999 the first ocean-scale field trials of the RIBS were conducted. A description of this field study, known as the XM99, is presented and discussed in Chapter III. In 
Chapter IV, the analytical models of wave transmission and dynamic pressure are described. In Chapter V, the WAMIT numerical model, boundary value problem, and parameter formulations are discussed. Comparisons between the analytical, numerical, laboratory, and field measurements of transmission coefficients are presented and discussed in Chapter VI. In Chapter VII a comparison of the dynamic pressure measurements and model predictions is presented and discussed. Finally, a summary and conclusions are presented with recommendations for future research in Chapter VIII. 


\section{CHAPTER II}

\section{EXPERIMENTAL MEASUREMENTS}

\section{LABORATORY SETUP}

These Fundamental Laboratory Experiments (hereafter referred to as FLab) were conducted in the directional spectral wave basin at the Coastal and Hydraulic Laboratory, U.S. Army Engineer Research and Development Center. Figure 2 shows the overall layout of the basin and the model RIBS. The purpose of this experiment was to gather some information relative to fundamental questions about the RIBS performance and to provide data for the preliminary design of the XM99 field version of the RIBS.

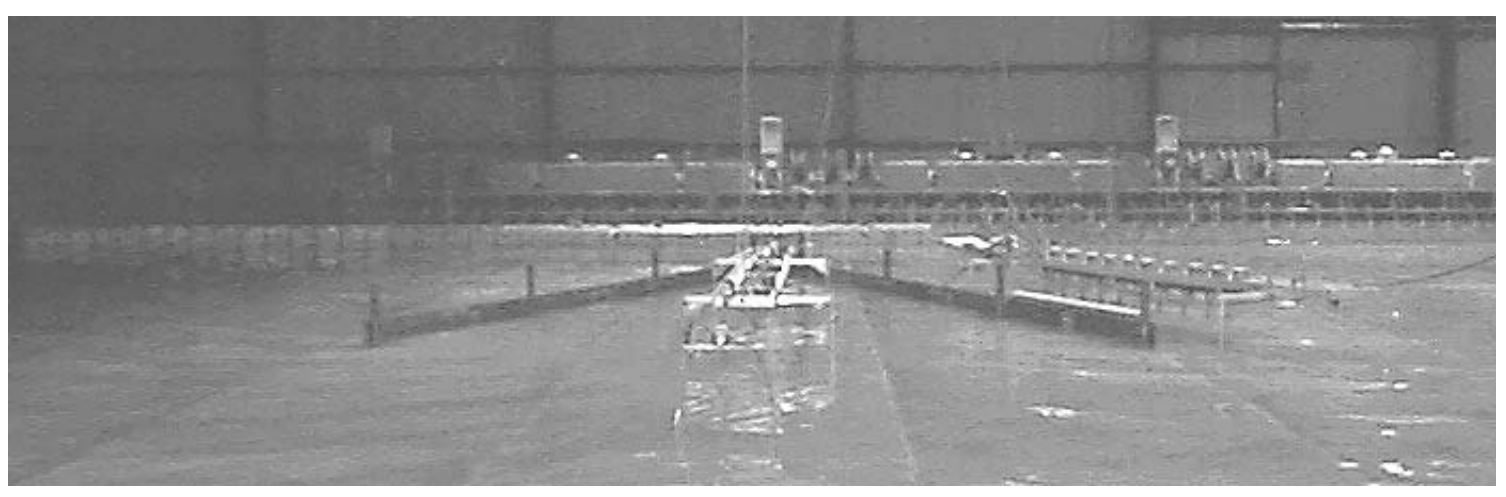

Figure 2. Fundamental laboratory experiments in the DSWG Basin.

\section{Directional Spectral Wave Basin}

Waves were generated in this flat-bottomed basin using the 27-m-long directional spectral wave generator (DSWG). The DSWG was designed and built by MTS Systems Corporation, Minneapolis, MN. It consists of 60 paddles in four modules that facilitate portability for relocation within the test facility for different research projects. Each paddle is $76 \mathrm{~cm}$ tall and 
$46 \mathrm{~cm}$ wide. The paddles are driven at the 61 joints in a piston (i.e., translational) mode (more efficient and representative of shallow-water environments) by $559 \mathrm{~W}$ electric direct current motors. This produces a cleaner, more continuous waveform with less cross-wave generation for wave periods greater than approximately $0.70 \mathrm{sec}$ than does driving individual paddles (Sand 1979). The "wetback" design has no bottom or end seals, but has flexible plastic plate seals which slide in guides between each paddle to provide continuity in the wave fronts.

Wave periods are typically above $0.6 \mathrm{sec}$. The range of strokes is $\pm 15 \mathrm{~cm}$, corresponding to $\mathrm{a} \pm 10 \mathrm{~V}$ input signal. Directional waves are generated using the "snake principle." Offset angles between paddles can be continuously varied within the range of 0 to $180 \mathrm{deg}$ to produce directional waves at angles approaching \pm 90 deg from the normal for most wave periods.

The perimeter of the 26-m-wide by 30-m-long basin was lined with synthetic horsehair to minimize wave reflections from the boundaries. The basin layout was referenced to a right-hand coordinate system with its origin at paddle 1 of the DSWG. Water depth was a constant $46 \mathrm{~cm}$, maintained within $\pm 9 \mathrm{~mm}$ of the desired level by an automatic water level float and solenoid control valve.

\section{RIBS Model Configurations}

Figure 3 is a schematic of the layout of the DSWG basin. The right-handed coordinate system was located at the edge of DSWG paddle 1 with positive $x$-axis pointing into the basin and positive $y$-axis parallel to the paddle face. The bow or tip of the model RIBS was located $5.73 \mathrm{~m}$ in front of the DSWG, along its centerline at $13.72 \mathrm{~m}$. The two legs $\left(L_{r}=9.1 \mathrm{~m}\right)$ were constructed of 15.9-mm-thick A36 steel plate and securely fastened at four locations along each leg to the concrete floor. This arrangement allowed vertical movement of the entire model to simulate four relative barrier drafts, $d / h=0.25,0.50,0.75$, and 1.00 ( $d=\mathrm{draft}, h=$ water depth $)$ of the water depth. Each set of three anchor points could swivel about the bow to accommodate two interior angles of 45 and $90 \mathrm{deg}$ to the centerline. These tests were conducted with a model scale of 1:32 (Froude Number) to simulate a breakwater of $292.61 \mathrm{~m}$ in a water depth of $14.63 \mathrm{~m}$ 


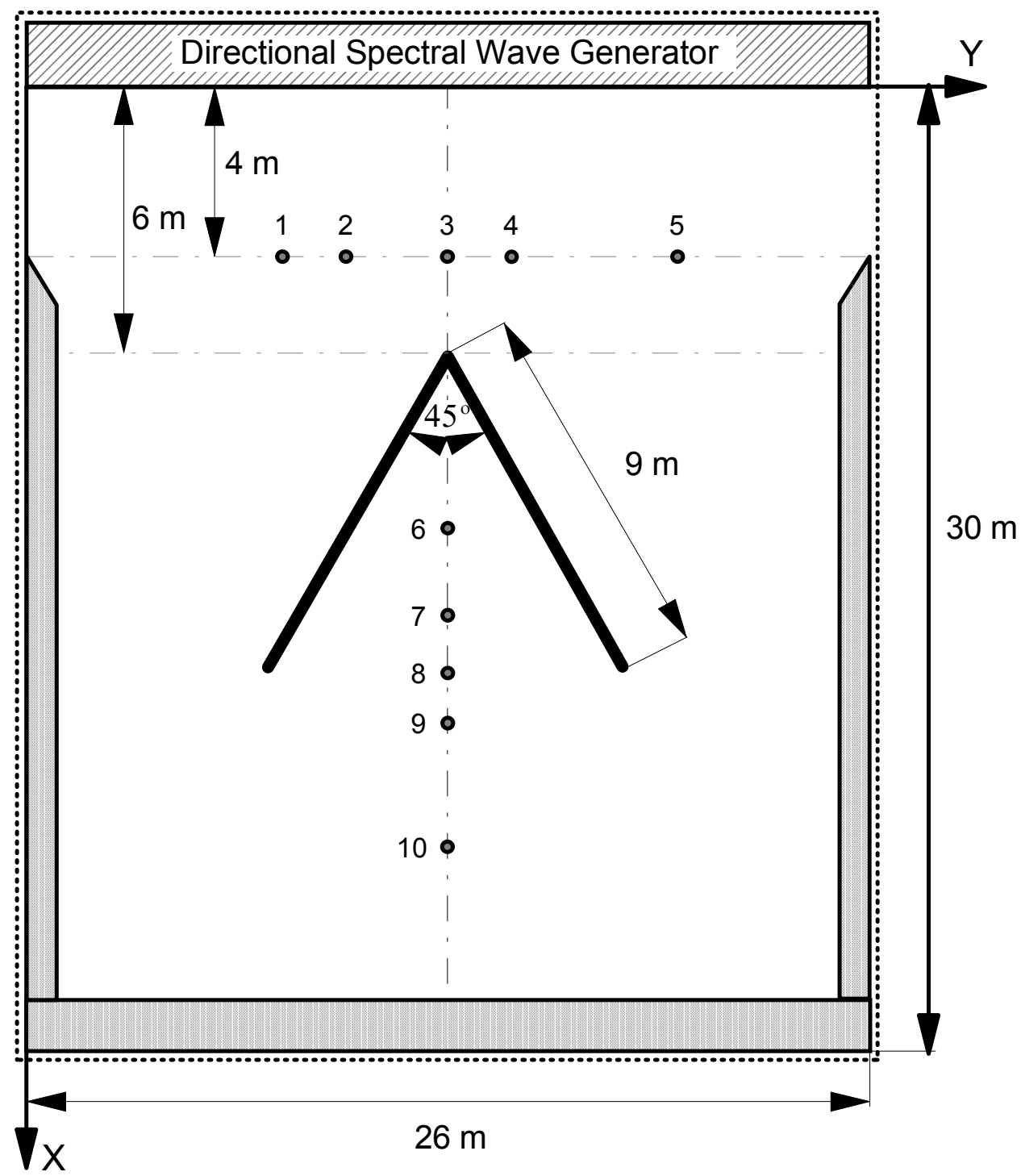

Figure 3. Schematic of basin layout for $45 \mathrm{deg}$ interior angle configuration.

\section{Instrumentation}

Wave gages. Ten capacitance wave gages were used to measure surface wave elevations. Five of the gages were positioned in a linear array $3.66 \mathrm{~m}$ in front of the model to record incident wave conditions. The linear array provides superior resolution capability for wave components at or near the same frequency and slightly different directions (Oltman-Shay 1987). A well-designed array must have a total length equal to the largest wavelength at the largest 
angle expected, and must also be short enough to avoid aliasing the higher frequency, smaller wavelength components. Thus, a lag spacing of $61 \mathrm{~cm}$ was used in the 2-3-1-7 linear array, so that wave periods with half wavelengths equal to $1,2,3,4,5,6,7,8,11$, and 13 lags were discernible.

The remaining five gages (i.e., gages 6 to 10) were located along the centerline in the lee of the model to measure transmitted wave parameters. These "transmission gages" were spaced to cover a range of normalized distances $D_{b} / L_{r x}$ in the lee of the structure for the two interior angles $\theta_{\mathrm{r}}$. This normalized distance was calculated as the ratio of the gage distance from the bow $D_{b}$ to the projected leg length $L_{r x}$, where $L_{r x}=L_{r} \cos \theta_{\mathrm{r}}$. These $D_{b} / L_{r x}$ ranged from 0.45 to 2.0 projected leg lengths along the centerline. All wave gages were mounted on long frames to minimize the amount of interference from the support legs. Table 1 lists the coordinates of these gages.

The wave gages were calibrated each day using an automated procedure of measurements at 11 vertical stops that covered the minimum and maximum surface elevations anticipated during the study. Calibration coefficients were obtained using a linear or quadratic least squares fit and averaging technique of the 21 voltage samples per gage to minimize the effects of slack in the gear drives and hysteresis in the sensors.

TABLE 1. Wave Gage Coordinates

\begin{tabular}{c|c|c}
\hline \hline Gage ID & $X, \mathrm{~m}$ & $Y, \mathrm{~m}$ \\
\hline \multicolumn{3}{|c|}{ (a) Incident Gage Array } \\
\hline 1 & 3.66 & 10.67 \\
\hline 2 & 3.66 & 11.89 \\
\hline 3 & 3.66 & 13.72 \\
\hline 4 & 3.66 & 14.33 \\
\hline 5 & 3.66 & 18.59 \\
\hline \multicolumn{3}{|c|}{ (b) Wave Transmission Gages } \\
\hline 6 & 9.54 & 13.72 \\
\hline 7 & 12.19 & 13.72 \\
\hline 8 & 14.17 & 13.72 \\
\hline 9 & 15.42 & 13.72 \\
\hline 10 & 18.53 & 13.72 \\
\hline \hline
\end{tabular}


Pressure gages. Three Druck, PDCR 80X, pressure gages were located on the starboard leg to measure external dynamic wave pressures. These gages were mounted at distances of $1.83 \mathrm{~m}$, $4.57 \mathrm{~m}$, and $7.32 \mathrm{~m}$ from the tip of the leg; corresponding to bow, middle, and stern locations, respectively. Table 2 lists coordinates from the local coordinate system with origin at DSWG paddle 1. The pressure sensor of each gage was mounted flush with the outside surface of the starboard leg. A set of twelve $9.53 \mathrm{~mm}$ diameter penetrations were drilled in the leg to position these pressure gages a distance of $8.56 \mathrm{~cm}$ from the surface for each of the four relative drafts. This distance was selected to fall sufficiently below the largest trough expected for the wave conditions being simulated.

TABLE 2. Pressure Gage Coordinates

\begin{tabular}{c|c|c|c|c}
\hline \hline Gage ID & Location & $X, \mathrm{~m}$ & $Y, \mathrm{~m}$ & $Z, \mathrm{~cm}$ \\
\hline
\end{tabular}

(a) 45 deg Interior Angle

\begin{tabular}{l|c|c|c|c}
\hline $11(21$ for 6,7 only) & Bow & 7.41 & 14.42 & 8.56 \\
\hline $12(22$ for 6,7 only) & Middle & 9.95 & 15.47 & 8.56 \\
\hline $13(23$ for 6,7 only) & Stern & 12.49 & 16.51 & 8.56 \\
\hline
\end{tabular}

(b) 90 deg Interior Angle

\begin{tabular}{l|c|c|c|c}
\hline $11(21$ for 6,7 only) & Bow & 7.02 & 15.00 & 8.56 \\
\hline $12(22$ for 6,7 only) & Middle & 8.96 & 16.95 & 8.56 \\
\hline 13 (23 for 6,7 only) & Stern & 10.90 & 18.89 & 8.56 \\
\hline \hline
\end{tabular}

A least squares linear fit was performed on the three pressure sensors to obtain calibration coefficients in volts per inch of water. These coefficients were subsequently converted to $\mathrm{N} / \mathrm{m}^{2}$ $\left(1 \mathrm{~N} / \mathrm{m}^{2}=1\right.$ Pascal) per inch of water. The voltage range varied between $\pm 8 \mathrm{~V}$ to $\pm 60 \mathrm{~V}$, based on the wave height and wave condition. Appendix B contains a listing of the relevant voltages and applicable run numbers for each model configuration and wave condition. 


\section{Wave Conditions}

The RIBS is designed to reduce Sea State 3 (SS3) waves to Sea State 2 (SS2) or less. These sea states are based on the Pierson-Moskowitz Sea Spectrum scale. Although defined by both wave period and height, the latter is the controlling parameter. The SS3 condition is defined for significant wave heights between $0.91 \mathrm{~m}$ to $1.52 \mathrm{~m}$ and range of wave periods from 3 to $6 \mathrm{sec}$. The RIBS is designed to survive a Sea State 5 (SS5) condition. Significant wave heights for SS5 range between $2.44 \mathrm{~m}$ to $3.66 \mathrm{~m}$. The range of wave periods is from 3.08 to $11.90 \mathrm{sec}$.

Thus, an operational series composed of waves primarily in the SS3 category and a survival series of SS5 waves were generated. Table 3 lists the target wave parameters for the fourteen wave conditions in these two test series. The operational series consisted of six monochromatic cases ("M") and six directional spectral ("D") wave cases. Peak wave periods were 5, 8, and 12 sec, scaled to the 1:32 Froude scale factor. The significant wave height was equivalent to 1.52 $\mathrm{m}$. The 12-s cases are not strictly in the SS3 window, but were included for completeness in testing the performance of the RIBS to larger wave periods with wave heights within the SS3 window. The survival series consisted of two directional spectral cases with a scaled wave period corresponding to $12 \mathrm{sec}$ and significant wave height of $3.66 \mathrm{~m}$. Wave directions for both series were $\bar{\theta}=0$ and $20 \mathrm{deg}$. The $\bar{\theta}=0 \mathrm{deg}$ cases were parallel to the RIBS center line and the $\bar{\theta}=20 \mathrm{deg}$ cases were simulated so that they impacted the starboard side of the RIBS model where the pressure gages were installed.

The wave height for the monochromatic wave cases was the energy-equivalent of the significant wave height defined as $\bar{H}=H_{m 0} / \sqrt{ } 2$. For the directional spectral cases, the Phillip's constant $\alpha$ was calculated based on the target $H_{m 0}$ wave height. The spectral peakedness factor $\gamma$ varied from 2.5 to 4.0 , as a function of wave period. The directional spreading $\sigma_{m}=10 \mathrm{deg}$ for all cases. This value is equivalent to a narrow directional spreading $S_{\max }=75$ for swell with long decay distance (Goda and Suzuki 1975). A detailed derivation of the directional spectrum and spreading function is contained in the next section entitled, "Wave Simulation and Generation." 
TABLE 3. Target Wave Parameters

\begin{tabular}{|c|c|c|c|c|c|c|}
\hline Test ID & Type & $\begin{array}{c}\text { Period } \\
(\mathrm{sec})\end{array}$ & $\begin{array}{c}\text { Height } \\
(\mathrm{cm})\end{array}$ & $\gamma$ & $\alpha$ & $\begin{array}{c}\bar{\theta} \\
(\operatorname{deg})\end{array}$ \\
\hline \multicolumn{7}{|c|}{ (a) Operational Series } \\
\hline M50 & Monochromatic & 0.88 & 3.3 & ---- & --- & 0 \\
\hline M52 & Monochromatic & 0.88 & 3.3 & --- & ---- & 20 \\
\hline M80 & Monochromatic & 1.41 & 3.3 & ---- & ---- & 0 \\
\hline M82 & Monochromatic & 1.41 & 3.3 & --- & --- & 20 \\
\hline M120 & Monochromatic & 2.12 & 3.3 & ---- & ---- & 0 \\
\hline M122 & Monochromatic & 2.12 & 3.3 & --- & ---- & 20 \\
\hline D50 & Spectral & 0.88 & 4.8 & 2.5 & 0.01522 & 0 \\
\hline D52 & Spectral & 0.88 & 4.8 & 2.5 & 0.01522 & 20 \\
\hline D80 & Spectral & 1.41 & 4.8 & 3.0 & 0.00348 & 0 \\
\hline D82 & Spectral & 1.41 & 4.8 & 3.0 & 0.00348 & 20 \\
\hline D120 & Spectral & 2.12 & 4.8 & 4.0 & 0.00117 & 0 \\
\hline D122 & Spectral & 2.12 & 4.8 & 4.0 & 0.00117 & 20 \\
\hline \multicolumn{7}{|c|}{ (b) Survival Series } \\
\hline D5120 & Spectral & 2.12 & 11.4 & 4.0 & 0.00677 & 0 \\
\hline D5122 & Spectral & 2.12 & 11.4 & 4.0 & 0.00677 & 20 \\
\hline
\end{tabular}




\section{Test Plan}

The test plan for the FLab consisted of a calibration phase to calibrate the wave conditions and a production phase for collecting the data in the different model configurations. Table 4 lists the run numbers, number of cases, monochromatic or directional spectral wave type, interior angle between RIBS legs, relative draft, and whether the RIBS model was in place. In all, 56 cases were run in the calibration phase and 83 cases in the production phase. Runs 23 and 24 were run after testing was completed to verify that the RIBS model did not significantly affect the wave calibrations.

TABLE 4. Test Plan

\begin{tabular}{l|l|l|l|l|l}
\hline \hline Run & No. Cases & Wave Type(s) & Interior Angle, deg & $d / h$ & Comments \\
\hline
\end{tabular}

(a) Calibration Phase

\begin{tabular}{c|c|c|c|c|c}
\hline 2 & 8 & M, D & 45 & 1.00 & RIBS model in place \\
\hline 3 & 3 & M, D & 45 & 1.00 & RIBS model in place \\
\hline 4 & 15 & M, D & 45 & 1.00 & RIBS model in place \\
\hline 5 & 14 & M, D & 45 & 1.00 & RIBS model in place \\
\hline 23 & 8 & D & --- & --- & Guide vanes only \\
\hline 24 & 8 & D & --- & --- & Empty basin \\
\hline
\end{tabular}

(b) Production Phase

\begin{tabular}{c|c|c|c|c|c}
\hline 6 & 14 & M, D & 45 & 1.00 & RIBS model in place \\
\hline 7 & 3 & M, D & 45 & 1.00 & RIBS model in place \\
\hline 8 & 14 & M, D & 45 & 0.75 & RIBS model in place \\
\hline 9 & 15 & M, D & 45 & 0.50 & RIBS model in place \\
\hline 10 & 16 & M, D & 45 & 0.25 & RIBS model in place \\
\hline 20 & 8 & D & 90 & 1.00 & RIBS model in place \\
\hline 21 & 3 & D & 90 & 0.50 & RIBS model in place \\
\hline 22 & 8 & D & 90 & 0.50 & RIBS model in place \\
\hline
\end{tabular}

Notes:

1. $\mathrm{M}=$ Monochromatic or regular wave $\mathrm{D}=$ Directional spectral wave. 


\section{WAVE SIMULATION AND GENERATION}

\section{Monochromatic Waves}

The DSWG utilizes the "snake principle" to generate waves with directions at an angle to the wavemaker. This terminology evolved because the wavemaker looks more or less like an undulating snake as the waveform progresses along its length. For monochromatic or regular waves, energy is generated at a discrete frequency and direction using a constant phase lag or offset phase angle between paddles. The wave direction or angle of wave propagation $\theta$ is

$$
\theta=\sin ^{-1} \frac{L}{Y}
$$

where $L=$ wavelength and $Y=$ distance along the wavemaker corresponding to one wave period or cycle of $360 \mathrm{deg}$. The distance $Y$ is related to the paddle width $B$ by

$$
Y=N_{l} B
$$

where $N_{l}=$ number of integer paddles required to make one cycle. It is a function of the phase angle $\varphi_{y}$

$$
N_{l}=\frac{360}{\varphi_{y}}
$$

The wave is propagated along the wavemaker at the proper angle by offsetting successive paddles by the phase $\varphi_{y}$. This phase is maintained for the entire time of wave generation and automatically recycles every $360 \mathrm{deg}$. There is a requirement to have a minimum of two paddles over a cycle distance $Y$. The smaller the paddle width $B$, the finer the resolution for making accurate directional waves at high frequencies.

The control signals $S_{c}(y, t)$ for the monochromatic wave cases were simulated for each of the 61 DSWG wave paddles as

$$
S_{c}(y, t)=\frac{H}{2} \cos \left(2 \pi f t+\varphi_{y}\right)
$$


where $H=$ target wave height, $y=$ location along DSWG, $t=$ time, and $f=$ frequency.

Once the wave elevation time series has been simulated for each of the 61 paddles, they are converted to corresponding stroke time series using a height-to-stroke transfer function. The three-dimensional form $F_{3}(f, \theta)$ was derived by Sand (1979) based on earlier work of Biesel (1954) and is valid for all nondimensional water depths $k h$. It is given by

$$
F_{3}(f, \theta)=\frac{H}{S}=\frac{2 \cosh (2 k h-1)}{(\sinh 2 k h+2 k h) \cos \theta}
$$

Finally, the stroke time history is converted to a voltage time series using the DSWG conversion factor. Since $20 \mathrm{~V}=30.5 \mathrm{~cm}$ for the maximum possible stroke and the analog-todigital (A/D) converter has a resolution of 4,096 digital units, the corresponding factor is 134.38 digital units per $\mathrm{cm}$ or a resolution of $0.074 \mathrm{~mm}$.

\section{Multidirectional Waves}

The directional wave spectrum $S(f, \theta)$ was parameterized as the product of the frequency spectrum $S(f)$ and the directional spreading function $D(f, \theta)$ as

$$
S(f, \theta)=S(f) D(f, \theta)
$$

where $f=$ frequency and $\theta=$ direction toward which the waves travel, measured clockwise from the $x$-axis. The directional spectrum must satisfy the constraints

$$
S(f)=\int_{0}^{2 \pi} S(f, \theta) d \theta ; \quad \int_{0}^{2 \pi} D(f, \theta) d \theta=1
$$

The TMA frequency spectrum $S_{T M A}(f)$ (Bouws et al. 1985), named for the Texel, MARSEN, and ARSLOE data sets, was used as the target spectrum. It is defined as

$$
S_{T M A}(f)=S_{J}(f) \Phi(f, h)
$$

where $S_{j}(f)=$ JONSWAP (Joint North Sea Wave Project) spectrum, $\Phi(f, h)=$ an empirical TMA parameter defined by Kitaigorodskii et al. (1975), and $h=$ water depth. The JONSWAP 
spectrum is defined by

$$
S_{J}(f)=\frac{\alpha g^{2}}{(2 \pi)^{4} f^{5}} e^{a} \gamma^{b}
$$

where $\alpha=$ Philip's constant, $g=$ gravitational acceleration, and $\gamma=$ peak enhancement factor.

The peak enhancement factor controls the peakedness of the spectrum and typically varies between 1 to 3.3 for sea conditions and 7 and higher for swell waves. Both $\alpha$ and $\gamma$ influence the energy contained in the wave spectrum. The functions $a$ and $b$ are given by

$$
a=-1.25\left(\frac{f_{p}^{4}}{f}\right) ; \quad b=e^{-\left[\frac{\left(f-f_{p}\right)^{2}}{2\left(\sigma f_{p}\right)^{2}}\right]}
$$

where $f_{p}=$ peak frequency and $\sigma=$ spectral width parameter. Typical values of the spectral width parameter on the left and right side of the peak frequency are $\sigma_{\mathrm{a}}=0.07$ and $\sigma_{\mathrm{b}}=0.09$, respectively. The function $\Phi(f, h)$ may be approximated as

$$
\Phi(f, h)= \begin{cases}\frac{\Omega^{2}}{2} & \text { for } \Omega<1 \\ 1-\frac{(2-\Omega)^{2}}{2} & \text { for } 1 \leq \Omega \leq 2 \\ 1 & \text { for } \Omega>2\end{cases}
$$

where $\Omega=2 \pi f \sqrt{h / g}$. The depth-limited TMA spectrum reduces to the JONSWAP spectrum in deep water.

The directional spreading function $D(f, \theta)$ used in this study was a wrapped normal spreading function (Borgman 1984). The Fourier representation of this function is

$$
D(f, \theta)=\frac{1}{2 \pi}+\frac{1}{\pi} \sum_{j=1}^{J} e^{\left[-\frac{\left(j \sigma_{m}\right)^{2}}{2}\right]} \cos j\left(\theta-\theta_{m}\right)
$$

where $\theta_{m}=$ the mean wave direction at frequency $f, \sigma_{m}=$ spreading standard deviation at frequency $f$ in radians, and $J$ is an arbitrary number of harmonics chosen to represent the Fourier series (typically 6 to 20 ). 
A deterministic amplitude, random phase model (Briggs et al. 1987; Borgman 1990) was used in the frequency domain to simulate realizations of the desired time series. The random surface elevation for each wave paddle at location $x, y$ and time $t$ is given by

$$
\eta(x, y, t)=2 \int_{0}^{\infty} \int_{0}^{2 \pi} A(f) e^{-i \Phi} e^{i \varphi}
$$

where $A(f)=$ deterministic amplitude function $=\sqrt{2 S(f, \theta) d f d \theta}, \Phi=$ independent random phase, uniformly distributed on $(0,2 \pi), \varphi=k x \cos \theta+k y \sin \theta-2 \pi f t, k=$ wavenumber $=2 \pi / L$, and $i=\sqrt{-1}$. This model is a double summation model since both frequency and direction are independent variables. The simulated spectrum always matches the target spectrum in this model. Although this method is the preferred model to represent a directional sea, it is phase locked (i.e., phase dependent) since different directions are summed at the same frequency. Phase locking gives a fluctuating RMS (i.e., root mean square) which tends to zero as the number of components becomes large (Pinkster 1984). Thus, a long time series (i.e., more than 1,000 components) was used to minimize this effect. An inverse Fourier transform was used to convert from the frequency domain to the time domain, where the length is constrained to be the product of integers 2,3 , and 5 raised to integer powers.

Once the wave elevation time series was estimated, it was converted to a voltage time series using the same procedure as previously described for the monochromatic waves using the height-to-stroke and stroke-to-voltage transfer functions.

\section{Simulation and Generation Parameters}

The digital to analog rate for the DSWG was $20 \mathrm{~Hz}$ (i.e., $\Delta \mathrm{t}=0.05 \mathrm{sec}$ ). Sixty-one time series of $1,000 \mathrm{sec}(20,000$ points) were generated with an even frequency increment $\Delta f=0.001$ $\mathrm{Hz}$. Lower cutoff frequency was $0.05 \mathrm{~Hz}$ for all peak wave periods. The TMA frequency spectrum was simulated with 30 frequencies unevenly spaced between the lower and upper (see Table 5) cutoff frequencies. The directional spreading function was likewise simulated with 30 directions unevenly spaced between $\pm 90 \mathrm{deg}$. The uneven spacing allows greater resolution near the peak frequency and the mean wave direction. Table 5 lists some additional wave simulation and generation parameters which varied with wave period. 


\section{DATA ACQUISITION AND ANALYSIS}

\section{Data Collection}

Data were collected in two phases: a calibration phase and a wave transmission phase. In the calibration phase, an iterative procedure was used to modify the control signals to match target parameters. In the wave transmission phase, the different model configurations were tested with the calibrated wave signals.

Table 5. Simulation, Collection, and Analysis Parameters

\begin{tabular}{l|c|c|c}
\hline \hline \multirow{2}{*}{ Description } & \multicolumn{3}{|c}{ Peak Wave Period, sec } \\
\cline { 2 - 4 } & $T_{p}=0.88$ & $T_{p}=1.41$ & $T_{p}=2.12$ \\
\hline
\end{tabular}

(a) Simulation and Generation

\begin{tabular}{l|c|c|c}
\hline Upper cutoff frequency, $f_{u}, \mathrm{~Hz}$ & 3.50 & 2.50 & 2.00 \\
\hline No. frequency components & 3,450 & 2,451 & 1,950 \\
\hline
\end{tabular}

(b) Data Collection

\begin{tabular}{l|c|c|c}
\hline Record length, $T_{r}$, sec & 200 & 300 & 500 \\
\hline Number of points, $N$ & 2000 & 3000 & 5000 \\
\hline Frequency increment, $\Delta f, \mathrm{~Hz}$ & 0.0050 & 0.0033 & 0.0020 \\
\hline
\end{tabular}

(c) Spectral Analysis

\begin{tabular}{l|c|c|c}
\hline Record length, $T_{r}, \mathrm{sec}$ & 200 & 240 & 300 \\
\hline No. of points, $N$ & 2,000 & 2,400 & 3,000 \\
\hline Frequency increment, $\Delta f, \mathrm{~Hz}$ & 0.0050 & 0.0042 & 0.0033 \\
\hline No. frequency components & 691 & 588 & 585 \\
\hline No. smoothed frequencies & 10 & 12 & 15 \\
\hline Degrees of freedom (DOF), $v$ & 20 & 24 & 30 \\
\hline
\end{tabular}

(d) Directional Spectral Analysis

\begin{tabular}{l|c|c|c}
\hline No. frequency components & 691 & 588 & 585 \\
\hline No. smoothed frequencies & 23 & 35 & 59 \\
\hline Effective DOF, $v$ & 46 & 70 & 118 \\
\hline \hline
\end{tabular}


Surface elevation data were sampled at $10 \mathrm{~Hz}$ (i.e., $\Delta t=0.10 \mathrm{sec}$ ) after waiting a sufficient time ( 35 to $50 \mathrm{sec}$ ) for slower traveling high-frequency waves to reach the back gages. For the monochromatic wave cases, data were collected for $100 \mathrm{sec}$. For the spectral cases, Goda (1985) recommends a minimum data collection of 200 waves at the peak period. Table 5 also summarizes the frequency-dependent data collection parameters.

\section{Data Analyses}

Data analyses consisted of (a) time domain zero-downcrossing analysis (ZCA), (b) single channel frequency spectra analysis (SCFA), and (c) a Maximum Likelihood Method directional wave spectra (MLM). The Coastal and Hydraulics Laboratory's TSAF (Time Series Analysis) software package was used for the ZCA and SCFA analyses on the transmitted wave gages and pressure sensors. The incident directional spectra were estimated from the 5-gage linear array using the MLM directional spectral analysis incorporated in the program NUSPEC (Briggs et al. 1987; Borgman 1990). Standard methods of time and frequency domain analysis, as specified by the IAHR $(1986,1997)$, were incorporated in the TSAF and NUSPEC software packages.

Zero-downcrossing analysis. The zero-downcrossing analysis was used to calculate average wave period $\bar{T}$ and height $\bar{H}$ for the monochromatic wave cases. In addition, the significant wave period $T_{1 / 3, d}$ and wave height $H_{1 / 3, d}$ were calculated for the directional spectral cases.

Single channel spectral analysis. For the SCFA, the data were zero-meaned, tapered by a 10-percent cosine bell window, Fourier transformed, and band averaged with a frequency resolution of $B_{e}=0.05 \mathrm{~Hz}$ between the lower $f_{l}=0.01 \mathrm{~Hz}$ and upper $f_{u}$ cutoff frequencies (which was frequency-dependent). Table 5 lists the frequency-dependent spectral analysis parameters, including record length $T_{r}$, number of points, frequency increment $\Delta f$, number of frequencies analyzed between $f_{l}$ and $f_{u}$, number of smoothed frequencies in each band, and degrees of freedom $v$.

Directional spectral analysis. The MLM directional spectral analysis is based on the relation that the autospectra and cross-spectra among all pairs of gages can be expressed as a linear combination of the directional components of $S(f, \theta)$ at that frequency. Details of this method can be found in Borgman (1984 and 1990) and Briggs et al. (1987). As in the simulation phase, 
the $S(f, \theta)$ is parameterized as the product of a frequency spectrum and a directional spreading function $D(f, \theta)$. The autospectral density $S_{i i}(f)$ for each of the $N$ wave gages is estimated, and a combined best estimate $\bar{S}(f)$ is obtained using a harmonic mean

$$
\bar{S}(f)=\left[\prod_{i=1}^{N} S_{i i}(f)\right]^{1 / N}
$$

The directional spreading function $D(f, \theta)$ is initially estimated by a truncated Fourier Series expansion of $L$ harmonics

$$
D(f, \theta)=\frac{1}{2 \pi}+\sum_{j=1}^{L} a_{j}(f) \cos (j \theta)+b_{j}(f) \sin (j \theta)
$$

where $a_{j}(f)=$ real Fourier coefficient of the spreading function and $b_{j}(f)=$ imaginary Fourier coefficient of the spreading function.

The autospectral and cross-spectral density estimates for each gage and each gage pair are calculated. The data records are zero-meaned, tapered by a 10-percent cosine bell window, and Fourier transformed using a "235" Fast Fourier Transform. A Gaussian smoothing function is used to smooth the estimates. Although it is similar to "band averaging" since raw spectral estimates are smoothed in the frequency domain, it gives a smoother transition because overlapping makes it more like a weighted moving average. The Gaussian smoothed line spectra $S_{m}$ for each frequency $m \Delta f$ is defined as

$$
\overline{S_{m}}=\frac{\sum_{j=-J}^{J} w_{j} S_{m-j}}{\sum_{j=-J}^{J} w_{j}}
$$

where $S_{m-j}=$ raw autospectral or cross-spectral estimate at frequency $(m-j) \Delta f$. The weights $w_{j}$ are defined over an integer number of spectral lines $J$ equivalent to $\pm 3 \sigma$ standard deviations of a Gaussian curve

$$
w_{j}=e^{-\left[\frac{(j \Delta t)^{2}}{2 \sigma}\right]}
$$

Since the area under a Gaussian curve $A_{G}=\sqrt{2 \pi} \sigma$, an equivalent rectangle having the same area has height of 1.0 (at $x=0$ ) and a bandwidth $b=A_{G}$. The number of bands averaged in each smoothed spectral estimate is considerably greater than a comparable frequency domain band 
averaging procedure. In band averaging, the resolution bandwidth $B_{e}$ is the product of the number of bands averaged and the basic frequency increment $\Delta f$ of the line spectra. In Gaussian smoothing, the number of bands $M$ in the smoothed average is given by

$$
M=\frac{6 \sigma}{\Delta f}
$$

Thus, for the same $f_{l}$ and $f_{u}$ cutoff frequencies and frequency resolution $B_{e}=0.05 \mathrm{~Hz}$, the effective degrees of freedom was increased using Gaussian smoothing (see Table 5).

The measured autospectral and cross-spectral estimates for each gage and pair of gages are substituted into the equations for the parameterized directional spectra for the autospectra $S_{i i}(f)$

$$
S_{i i}(f)=\int_{0}^{2 \pi} S(f, \theta) d \theta
$$

and the cross-spectra $S_{i j}(f)$

$$
S_{i j}(f)=\int_{0}^{2 \pi} S(f, \theta) e^{-i B_{i j}} d \theta
$$

where $B_{i j}=k X_{i j} \cos \theta+k Y_{i j} \sin \theta, X_{i j}=x_{i}-x_{j}, Y_{i j}=y_{i}-y_{j}, k=$ wavenumber, $x=x$-axis gage coordinate for gage at location $i$ or $j$, and $y=y$-axis gage coordinate at location $\mathrm{i}$ or $j$. The estimate for $\bar{S}(f)$ and $D(f, \theta)$ are substituted into the right-hand side of equations (19) and (20) for $S(f, \theta)$. Thus, for $N$ gages, a set of $N^{2}$ simultaneous linear equations (i.e., $N$ autospectral equations of the form of equation (19) and $N(N-1) / 2$ pairs of cross-spectral equations of the form of equation (20)) are solved for the Fourier coefficients $a_{l}(f)$ and $b_{l}(f)$ of the spreading function. A vector linear regression model is used to invert the matrix containing the spreading coefficients of $D(f, \theta)$.

Finally, a parameterized wrapped normal spreading function is fitted to the $D(f, \theta)$ to improve the initial distorted estimate due to truncation of the Fourier series. A resolution of 2 deg (i.e., 180 increments in $360 \mathrm{deg}$ ) was used for the directional spreading function estimates. Once the $a_{j}(f)$ and $b_{j}(f)$ are obtained, the mean wave direction $\bar{\theta}(f)$ is estimated as

$$
\bar{\theta}(f)=\tan ^{-1} \frac{b_{1}(f)}{a_{1}(f)}
$$

where $a_{1}$ and $b_{1}$ are the first harmonics. The mean of all $\theta(f)$ equals the overall mean wave direction or peak wave direction $\bar{\theta}$. 
An estimate of the directional spread was estimated from the width of the measured directional distribution. The measured directional spreading function was normalized by its peak value and the spread was estimated as the half-width at the 50 percent level of the spreading function.

\section{Control Signal Calibration}

During the calibration phase, the control signals were iteratively corrected until the desired target values were obtained. Because of (a) leakage around and under a "wetback" wavemaker, (b) electronic and mechanical losses of the DSWG, (c) use of linear wave theory, and (d) basin response characteristics; the desired wave spectrum is usually not faithfully reproduced. For the monochromatic cases, a linear gain factor was sufficient to correct the measured wave height over one iteration as the wave period and direction were accurately reproduced.

For the directional spectral cases, a response amplitude operator $(R A O)$ transfer function was calculated to compensate for observed variations in wave period, height, and spectral shape. The raw $R A O, H_{i}(f)$, for each wave gage in the incident gage array was calculated in the frequency domain as the ratio of the raw autospectral ordinates $S_{i i}(f)$ to the target autospectra $S_{p}(f)$ given by

$$
H_{i}(f)=\sqrt{\frac{S_{i i}(f)}{S_{p}(f)}}
$$

These raw values were then Gaussian smoothed with the same resolution bandwidth used in the directional spectral analysis and averaged to obtain a single frequency-dependent correction factor $\bar{H}(f)$. This value was set equal to 1.0 for values outside the lower and upper cutoff frequencies. Values greater than or less than threshold values of 100 or 0.01 were set to these respective lower and upper limits.

The original stroke time series $S_{c}(t)$ for each of the 61 paddles are Fourier transformed to the frequency domain for correction. The real and imaginary Fourier coefficients $U(f)$ and $V(f)$ are divided at each frequency by the appropriate value of the smoothed and averaged $\bar{H}(f)$. The corrected Fourier coefficients are then inverse Fourier transformed to the time domain to give the new control signal for the next iteration. The square root in equation (22) is because the 
spectral estimates are squared quantities of the Fourier coefficients since

$$
S_{i i}(f)=\left[U^{2}(f)+V^{2}(f)\right] \Delta f
$$

Finally, the digital control signals are low-pass filtered and converted to analog at run time.

\section{MEASURED DATA}

\section{Incident Waves}

At the end of the calibration phase, final incident wave parameters were calculated for the monochromatic and directional spectral wave cases. Figure 4 shows the measured frequency spectra from the MLM analysis for the six operational series cases D50, D52, D80, D82, D120, and D122. The measured directional spreading functions at the peak and overall for each of these six cases are shown in Figure 5. The frequency spectra and spreading functions for the two survival series cases D5120 and D5122 are illustrated in Figure 6. Table 6 lists measured wave parameters for the fourteen wave cases. Average wave period $\bar{T}$ and wave height $\bar{H}$ were calculated for the monochromatic cases. For the directional spectra cases, the peak wave period $T_{p}$, zero moment wave height $H_{m 0}$, mean wave direction $\bar{\theta}$, and directional spreading $\sigma_{h}$ were calculated.

In general, the agreement was very good, with most of the variation due to statistical uncertainty in the estimated parameters. The measured $\sigma_{h}$ values are slightly different from the target $\sigma_{m}$ because they are the half-width of the directional spread at half the maximum at the peak frequency.

\section{Wave Parameters}

Measured wave parameters for each of the 10 incident and transmitted wave gages are contained in Appendix C. Appendix Table $\mathrm{C} 1$ lists $H_{m 0}$ for each RIBS model configuration in the directional spectral cases. Appendix Table $\mathrm{C} 2$ contains a similar listing of the $T_{p}$ for these cases. Finally, Appendix Table $\mathrm{C} 3$ lists the $\bar{H}$ values for the monochromatic wave cases. 


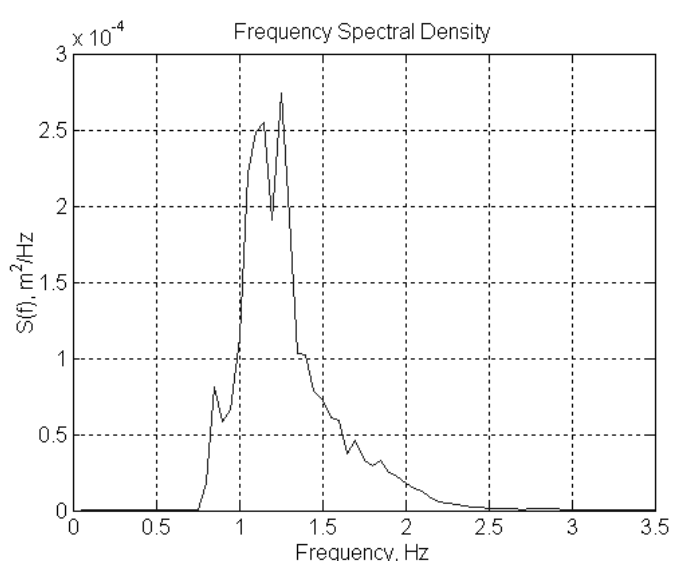

(a)

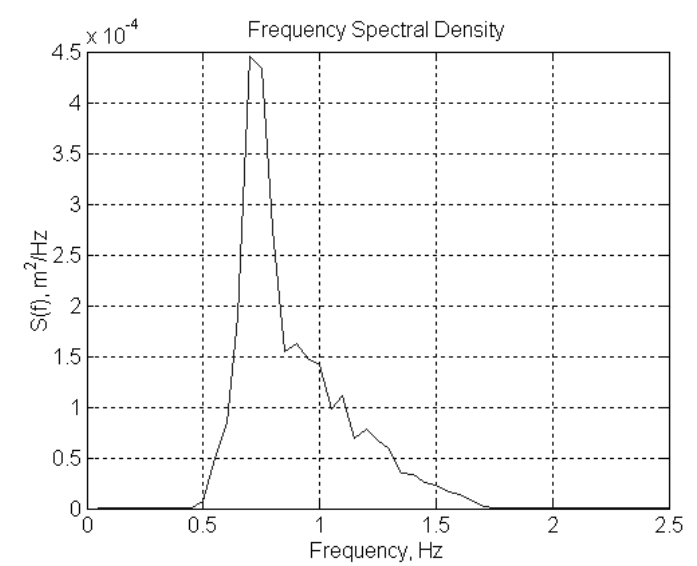

(c)

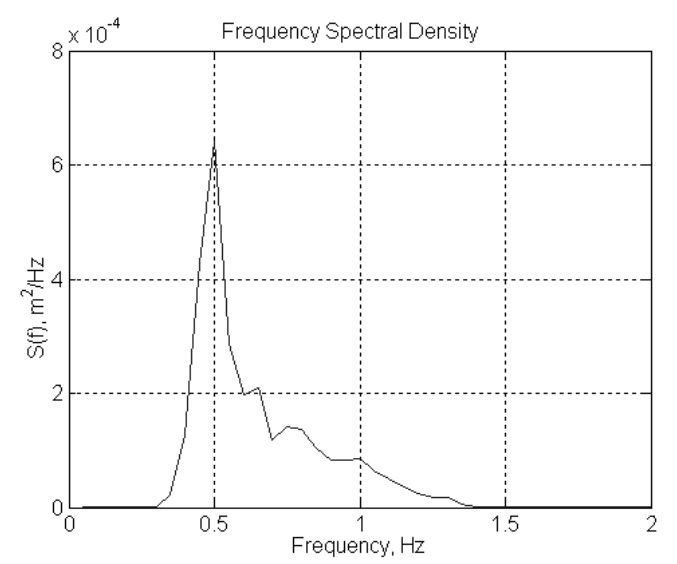

(e)

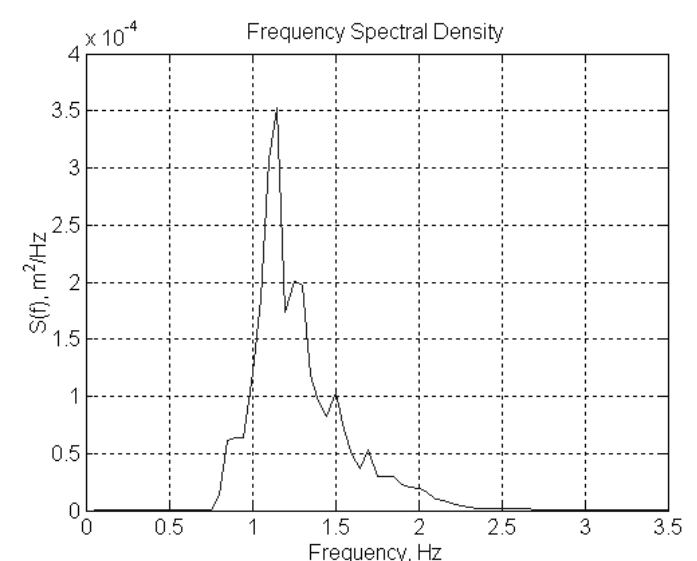

(b)

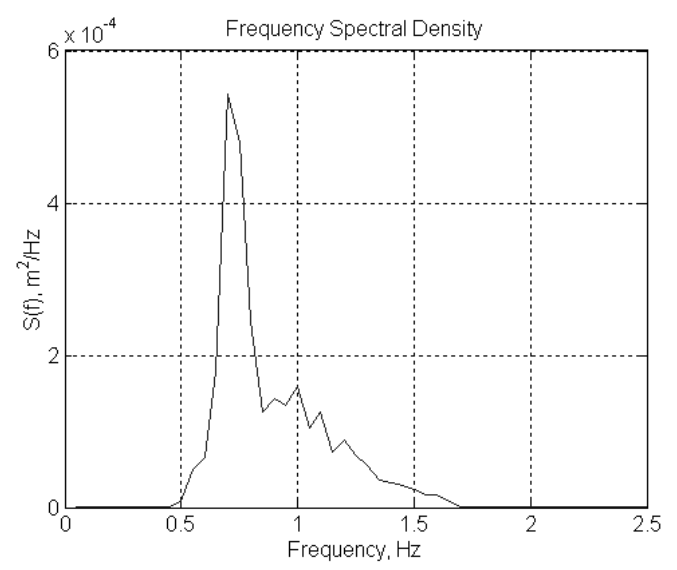

(d)

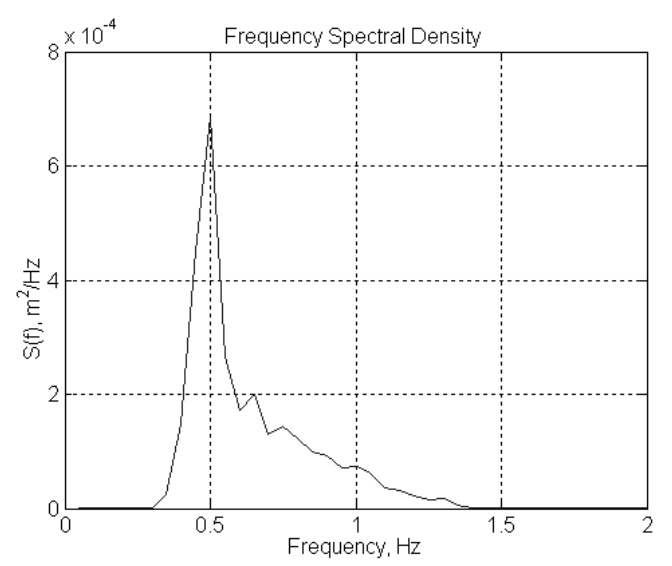

(f)

Figure 4. Frequency spectra for operational series of the FLab (a) $T=5 \mathrm{sec}, \bar{\theta}=0 \mathrm{deg}$, (b) $T=5 \mathrm{sec}, \bar{\theta}=20 \mathrm{deg}$, (c) $T=8 \mathrm{sec}, \bar{\theta}=0 \mathrm{deg}$, (d) $T=8 \mathrm{sec}, \bar{\theta}=20 \mathrm{deg}$, (e) $T=12 \mathrm{sec}, \bar{\theta}=0 \mathrm{deg}$, (f) $T=12 \mathrm{sec}, \bar{\theta}=20 \mathrm{deg}$. 


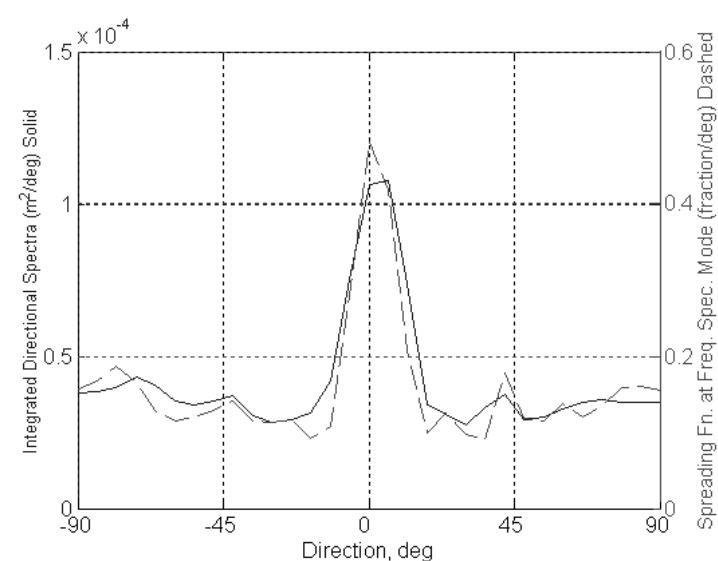

(a)

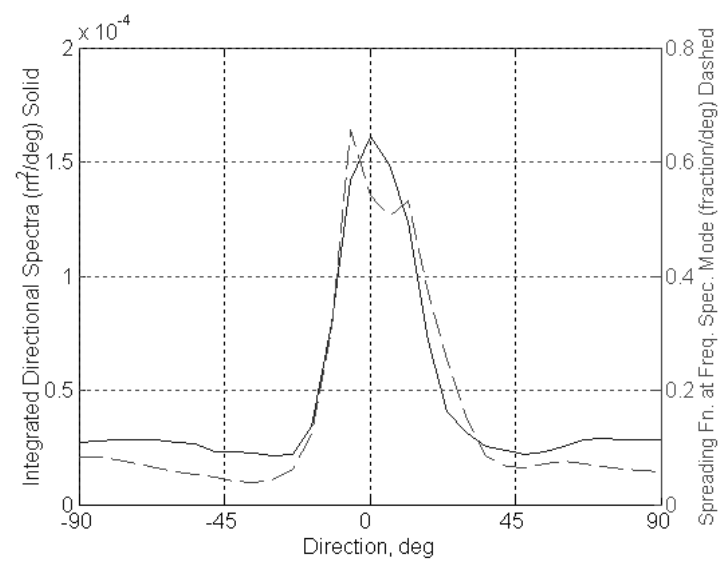

(c)

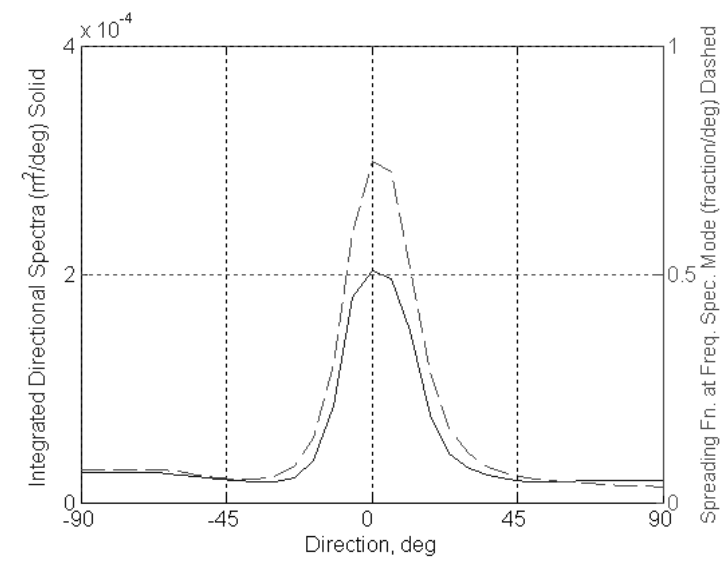

(e)

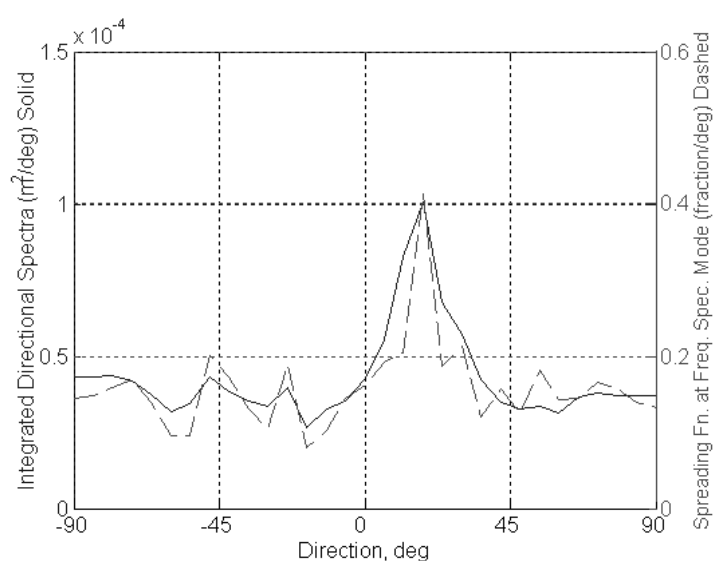

(b)

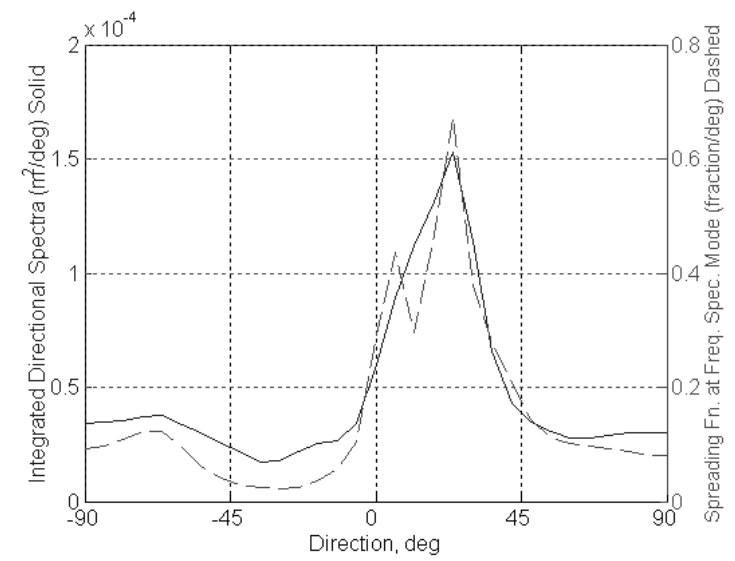

(d)

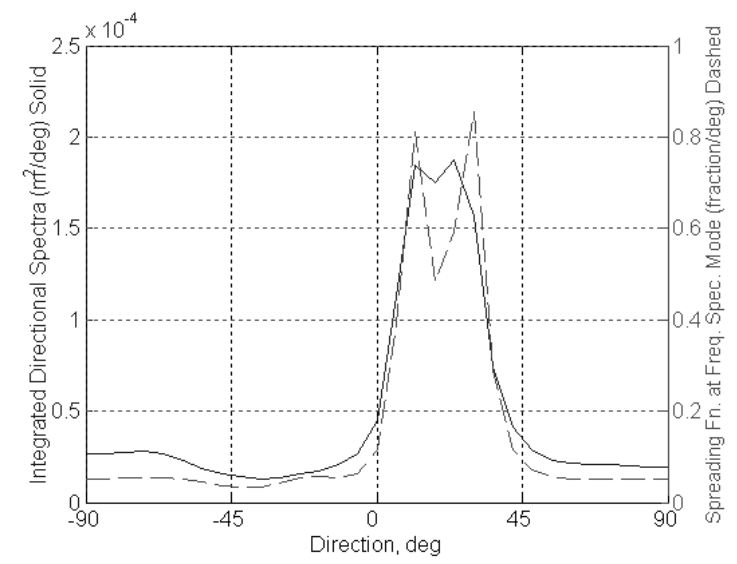

(f)

Figure 5. Directional spreading for operational series of the FLab (a) $T=5 \mathrm{sec}, \bar{\theta}=0 \mathrm{deg}$, (b) $T=5 \mathrm{sec}, \bar{\theta}=20 \mathrm{deg}$, (c) $T=8 \mathrm{sec}, \bar{\theta}=0 \mathrm{deg}$, (d) $T=8 \mathrm{sec}, \bar{\theta}=20 \mathrm{deg}$, (e) $T=12 \mathrm{sec}, \bar{\theta}=0 \mathrm{deg}$, (f) $T=12 \mathrm{sec}, \bar{\theta}=20 \mathrm{deg}$. 


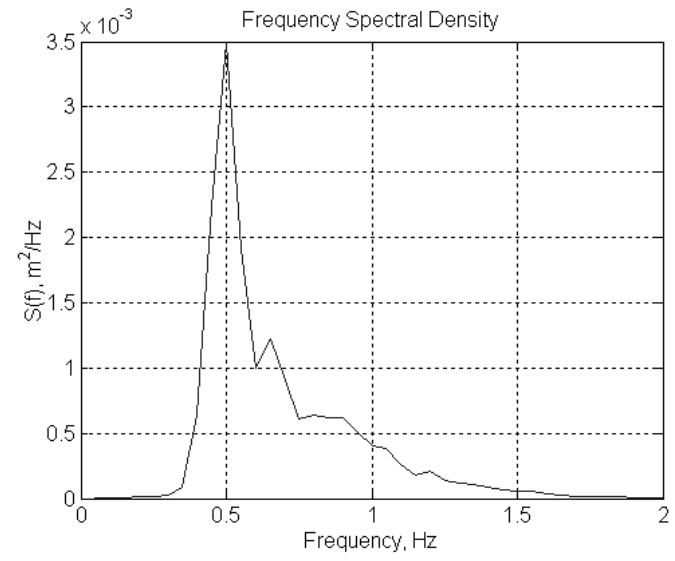

(a)

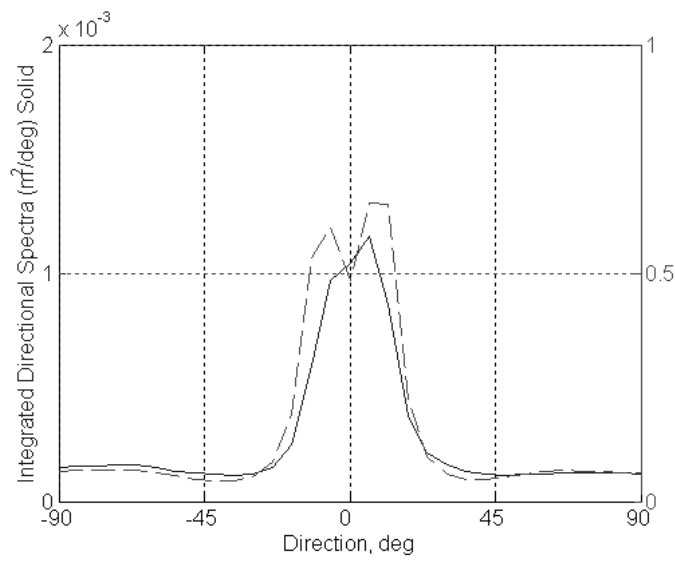

(c)

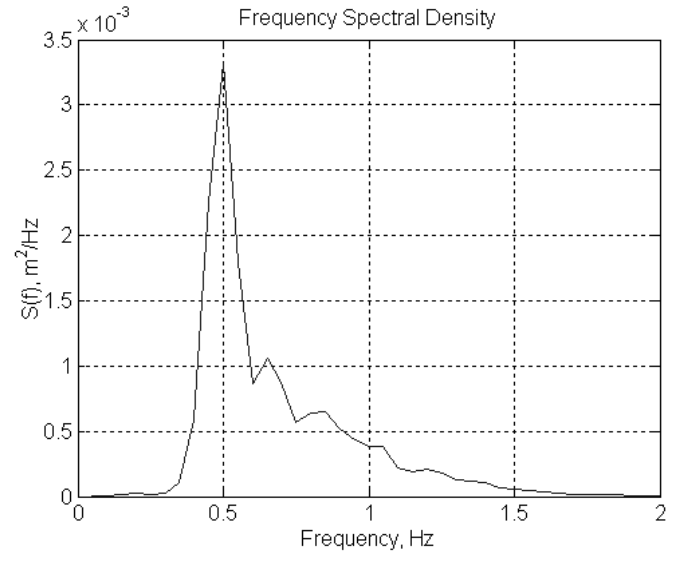

(b)

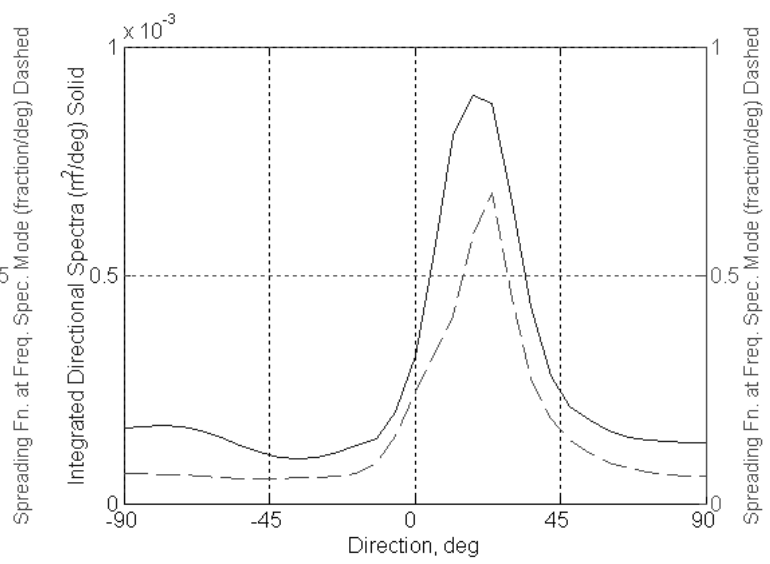

(d)

Figure 6. Frequency spectra and directional spreading for SS5 survival series ( $T=12 \mathrm{sec})$ of the FLab (a) $S(f), \bar{\theta}=0 \mathrm{deg}$, (b) $D(f, \theta), \bar{\theta}=20 \mathrm{deg}$, (c) $S(f), \bar{\theta}=0 \mathrm{deg}$, (d) $D(f, \theta), \bar{\theta}=20 \mathrm{deg}$. 
TABLE 6. Measured Wave Parameters

\begin{tabular}{|c|c|c|c|c|c|}
\hline Test ID & Type & $\begin{array}{c}\text { Period } \\
(\mathrm{sec})\end{array}$ & $\begin{array}{l}\text { Height } \\
(\mathrm{cm})\end{array}$ & $\begin{array}{c}\bar{\theta} \\
(\mathrm{deg})\end{array}$ & $\begin{array}{c}\sigma_{h} \\
(\operatorname{deg})\end{array}$ \\
\hline \multicolumn{6}{|c|}{ (a) Operational Series } \\
\hline M50 & Monochromatic & 0.88 & 3.3 & --- & ---- \\
\hline M52 & Monochromatic & 0.88 & 3.3 & ---- & ---- \\
\hline M80 & Monochromatic & 1.41 & 3.2 & ---- & ---- \\
\hline M82 & Monochromatic & 1.41 & 3.2 & --- & ---- \\
\hline M120 & Monochromatic & 1.41 & 3.0 & --- & ---- \\
\hline M122 & Monochromatic & 1.41 & 3.3 & --- & --- \\
\hline D50 & Spectral & 0.88 & 4.5 & 2 & 8 \\
\hline D52 & Spectral & 0.88 & 4.5 & 18 & 8 \\
\hline D80 & Spectral & 1.41 & 4.7 & -6 & 15 \\
\hline D82 & Spectral & 1.41 & 4.7 & 22 & 14 \\
\hline D120 & Spectral & 2.00 & 4.8 & 2 & 12 \\
\hline D122 & Spectral & 2.00 & 4.8 & 28 & 14 \\
\hline \multicolumn{6}{|c|}{ (b) Survival Series } \\
\hline D5120 & Spectral & 2.00 & 11.6 & 10 & 15 \\
\hline D5122 & Spectral & 2.00 & 11.3 & 22 & 13 \\
\hline
\end{tabular}




\section{CHAPTER III}

\section{RIBS XM99 FIELD TRIALS}

\section{XM99 DESIGN}

The first ocean-scaled version of the RIBS was deployed in Melbourne, FL, for 10 days from May 20 to 30, 1999. This ocean-scale version, henceforth referred to as XM99, had full scale dimensions, except that it was only about two thirds as long as the final design to minimize testing costs. The XM99 version explored a new construction concept that resembled a Venetian blind. It was also known as the "VE-RIBS," or vertically expandable RIBS because the legs tripled their draft once placed in the water. Each leg was $77.11 \mathrm{~m}$ long, $2.44 \mathrm{~m}$ wide, and 7.32 $\mathrm{m}$ deep. Based on daily diver measurements, water depth in the test site averaged $13.41 \mathrm{~m}$ during the deployment. The legs consisted of four horizontal levels, constructed from Mabey Truss frames, and attached to each other by a fabric material on all sides (Figure 7). The 1.95$\mathrm{m}$-tall top layer or top deck was the main structural element from which the other layers were supported. The vertical distance between each layer was variable: $0.91 \mathrm{~m}, 1.22 \mathrm{~m}$, and $1.83 \mathrm{~m}$ from the top down. To relieve large vertical and lateral bending stresses, it had four 60 -cm-long joints at the corners of the top deck, $45.72 \mathrm{~m}$ from the bow of each leg. The XM99 was moored at three points from buoys at the bow and stern of each leg.

\section{INSTRUMENTATION}

The XM99 instrumentation included sensors for measuring (a) incident wind, wave, and currents, (b) transmitted waves and pressures, and (c) structural motions, loads, and strains. Data for the entire system was monitored, collected, analyzed, and stored by an Integrated Motion Monitoring Information System (IMMIS) designed in cooperation with Coastal Leasing Corporation. These data were collected by "master-slave" data loggers located longitudinally at the bow, middle, and stern of each leg. Most of this instrumentation was concentrated on the top deck of the port leg, but some was also located on the starboard leg and the lower levels of the port leg. Sampling rates for most instrumentation was $4 \mathrm{~Hz}$, with analog inputs of 0-5 volts 


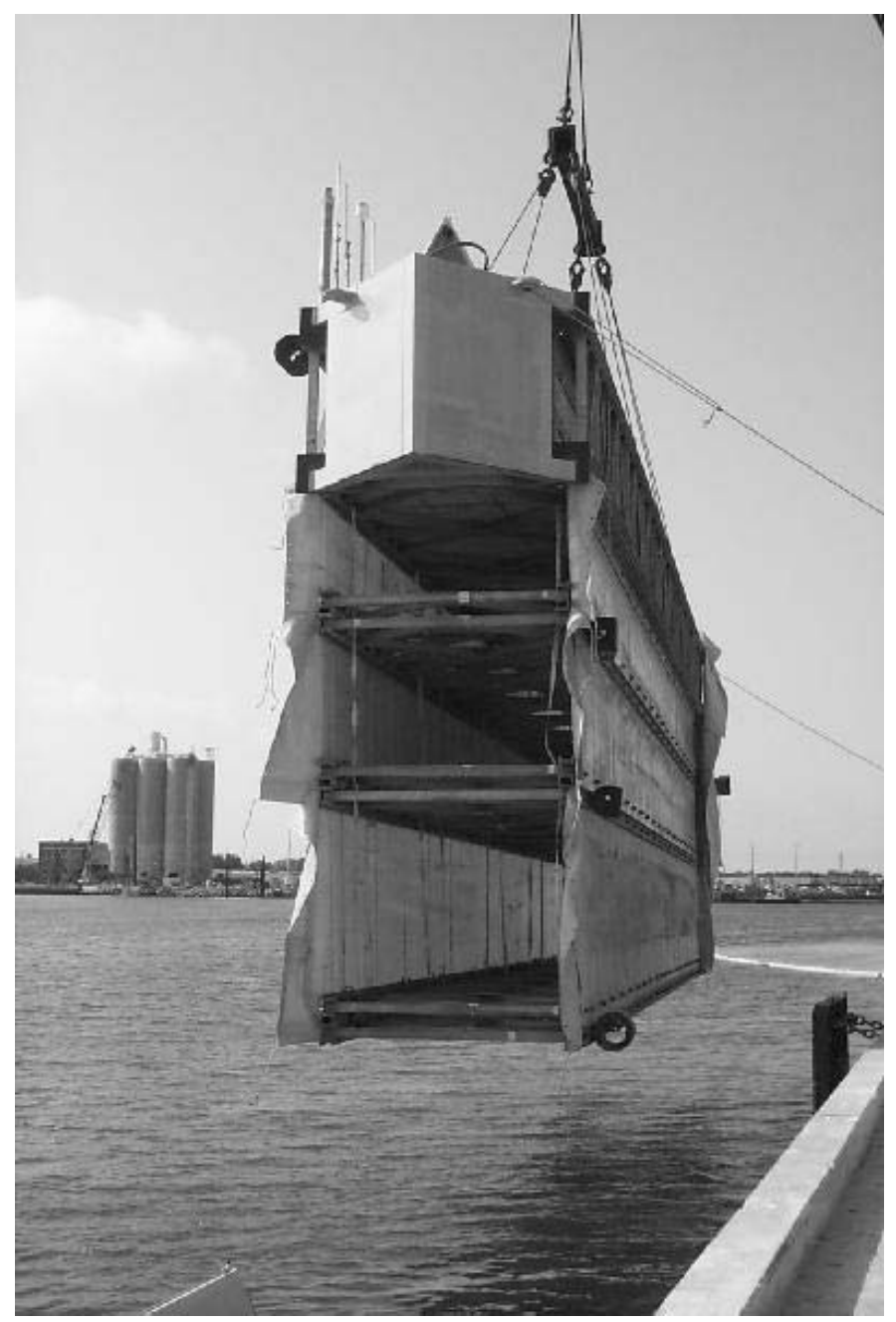

Figure 7. Cross-section of RIBS XM99.

direct current (VDC). A self-contained, omnidirectional Datasonics acoustic telemetry modem transferred data to a shore-based data acquisition and analysis system. Figure 8 shows some of the various types of instrumentation on the XM99.

A Datawell directional buoy, located about $457 \mathrm{~m}$ in front of the XM99, measured incident wave conditions. Transmitted wave heights were measured with a Datawell non-directional buoy, located $30.48 \mathrm{~m}$ from the nose or bow of the XM99 along the center line.

Pressure gages were installed on four levels of the port leg to collect wave-induced pressures for calibrating the hydrodynamic pressure estimates from the analytical and numerical models. On the external side, twelve pressure gages were located at bow, middle, and stern 


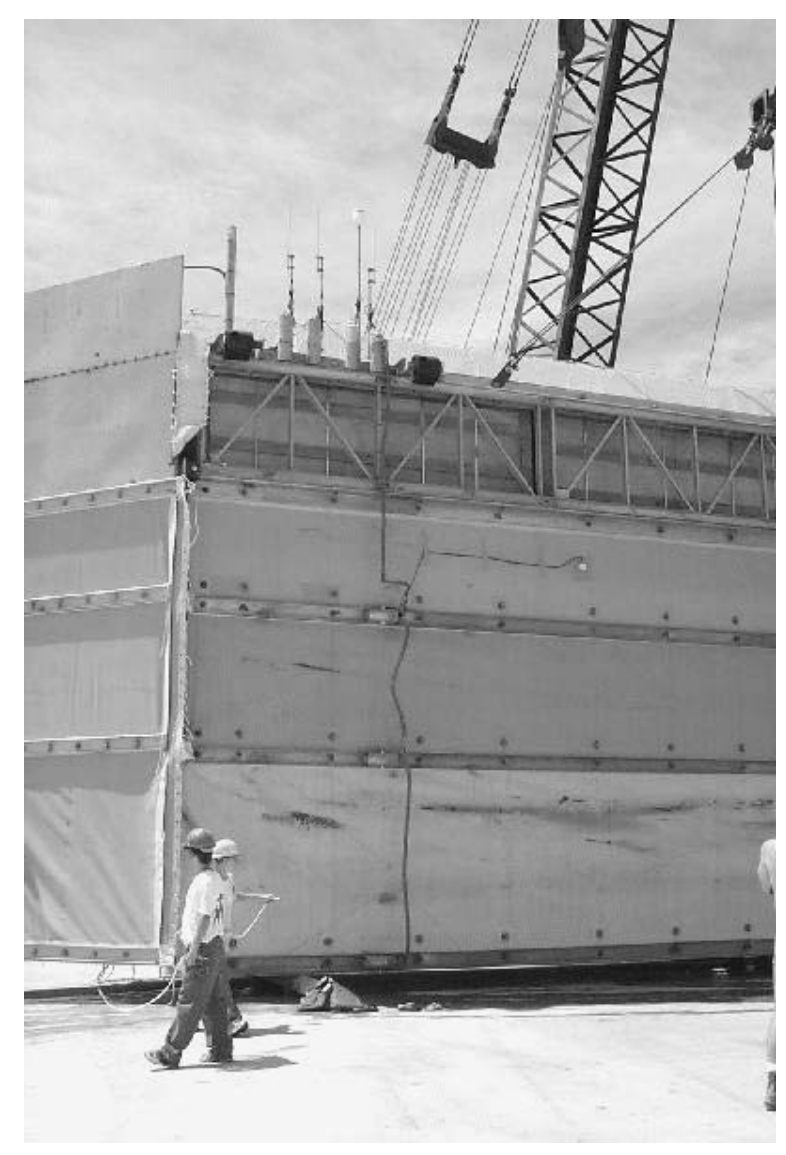

Figure 8. XM99 instrumentation layout.

stations on the surface, top deck, middle, and bottom levels. The three gages on the surface were intended to record atmospheric or reference pressures. On the internal side, five pressure gages were located at middle and stern stations on the top and middle levels and middle station on the bottom level. The internal gages were intended to measure the pressures on the interior of the XM99 for calculating net pressures and wave-induced loads on the structure. They are not discussed in this study. Table 7 is a schematic of the pressure gage locations and codes for the external and internal gages. Table 8 lists the coordinates for these gages in the local coordinate system with origin at the stern of the port leg.

Additional instrumentation included (1) an acoustic doppler current profiler, (2) an anemometer, (3) five Trimble Global Positioning System loggers on the top deck of each leg to monitor six degree of freedom (DOF) motions, (4) fourteen triaxial accelerometers were 
TABLE 7. Pressure Gage Codes

\begin{tabular}{c|c|c|c}
\hline \hline \multirow{2}{*}{ Level } & \multicolumn{3}{c}{ Station Location } \\
\cline { 2 - 4 } & Bow & Middle & Stern \\
\hline \multicolumn{5}{c}{ (a) Surface Reference Gages } \\
\hline Surface & 511 & 507 & 503 \\
\hline \multicolumn{4}{|c}{ (b) External Gages } \\
\hline Top & 512 & 508 & 504 \\
\hline Middle & 513 & 509 & 505 \\
\hline \hline Bottom & 514 & 510 & 506 \\
\hline
\end{tabular}

TABLE 8. Pressure Gage Coordinates

\begin{tabular}{|c|c|c|c|c|c|c|c|c|c|}
\hline \multirow[b]{2}{*}{ Level } & \multicolumn{3}{|c|}{ Bow, m } & \multicolumn{3}{|c|}{ Middle, $\mathrm{m}$} & \multicolumn{3}{|c|}{ Stern, $\mathrm{m}$} \\
\hline & $x$ & $y$ & $z$ & $x$ & $y$ & $z$ & $x$ & $y$ & $z$ \\
\hline \multicolumn{10}{|c|}{ (a) Surface Reference Gages } \\
\hline Surface & 67.71 & 0.00 & 0.38 & 35.16 & 0.00 & 0.38 & 1.20 & 0.00 & 0.38 \\
\hline \multicolumn{10}{|c|}{ (b) External Gages } \\
\hline Top & 67.71 & 0.00 & -3.11 & 35.16 & 0.00 & -3.11 & 1.20 & 0.00 & -3.11 \\
\hline Middle & 67.71 & 0.00 & -4.79 & 35.16 & 0.00 & -4.79 & 1.20 & 0.00 & -4.79 \\
\hline Bottom & 67.71 & 0.00 & -7.08 & 35.16 & 0.00 & -7.08 & 1.20 & 0.00 & -7.08 \\
\hline \multicolumn{10}{|l|}{ Notes: } \\
\hline \multicolumn{10}{|c|}{ 1. Local coordinate system origin on top deck at stern of port leg (see Figure 11). } \\
\hline \multicolumn{10}{|c|}{ 2. $x$-axis positive toward bow } \\
\hline \multicolumn{10}{|c|}{$y$-axis positive toward interior of port leg } \\
\hline$z$-ax & sitiv & from & deck. & & & & & & \\
\hline
\end{tabular}

co-located with the pressure gages on internal and external sides at the three levels (none with surface reference gages) and three on the external fabric of the port leg, (5) nine Hitec Products strain gages on the top deck on the corners and the diagonal at the bow, middle, and stern station cross sections, and (6) three National Scale load cells in the mooring lines. 


\section{DEPLOYMENT}

Deployment of the XM99 began at 6:00 a.m. on May 20. Wave forecasts were used to select a time when the wave environment was SS2 or less. Figure 9 shows the towout underway inside the harbor with the XM99 in its "folded" condition. The XM99 was on station north of the entrance channel by 11:00 a.m. (Figure 10). The site is located approximately four nautical miles offshore. By noon all mooring lines had been installed. Water depth at the test site was approximately $13.4 \mathrm{~m}$.

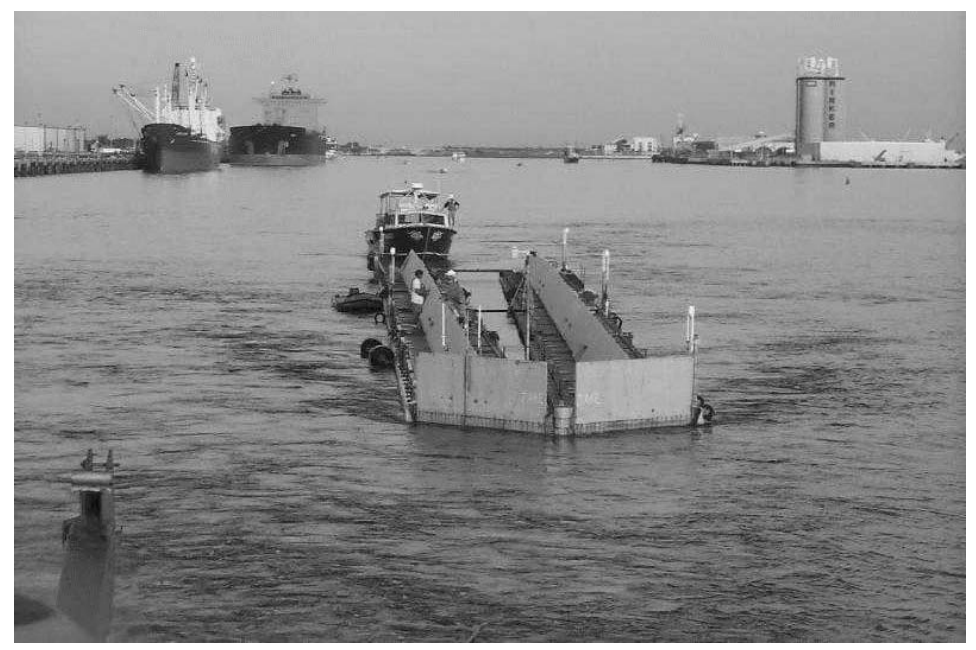

Figure 9. XM99 tow through Cape Canaveral Harbor, FL.

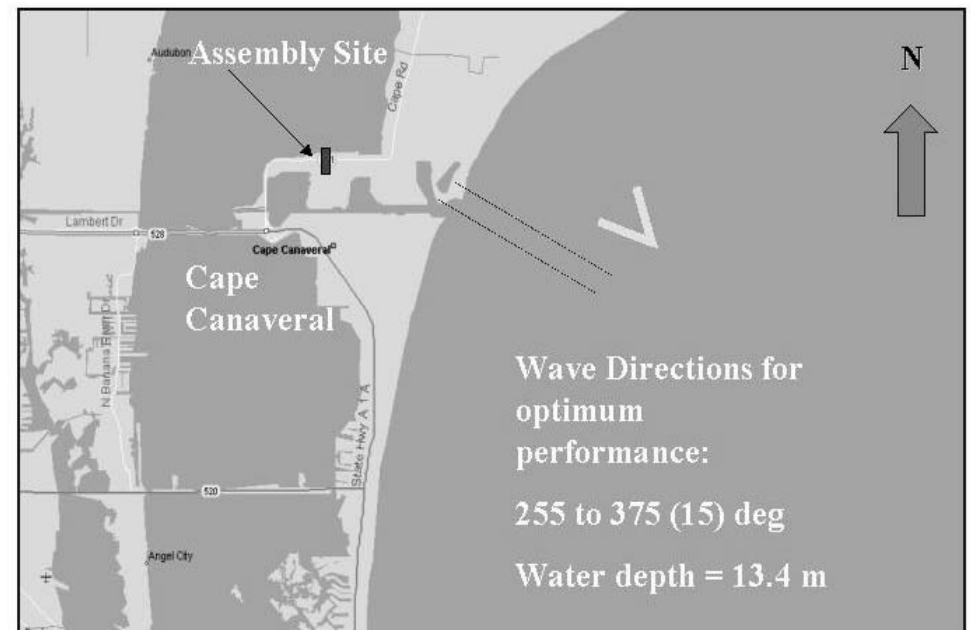

Figure 10. Location of the XM99 field site, May 1999. 
Figure 11 is a schematic of the global coordinate system. The centerline of the XM99 was aligned with the normal to the coast, approximately $135 \mathrm{deg}$. Thus, optimum performance for the XM99 occurred with waves traveling toward 315 deg (i.e., $135+180 \mathrm{deg}$ ), with an operating window of 120 deg between the normals to the port and starboard legs at 255 to 375 (15) deg. The local coordinate system for positioning the instrumentation is also shown in this figure. Figure 12 shows the XM99 when it was finally on station.

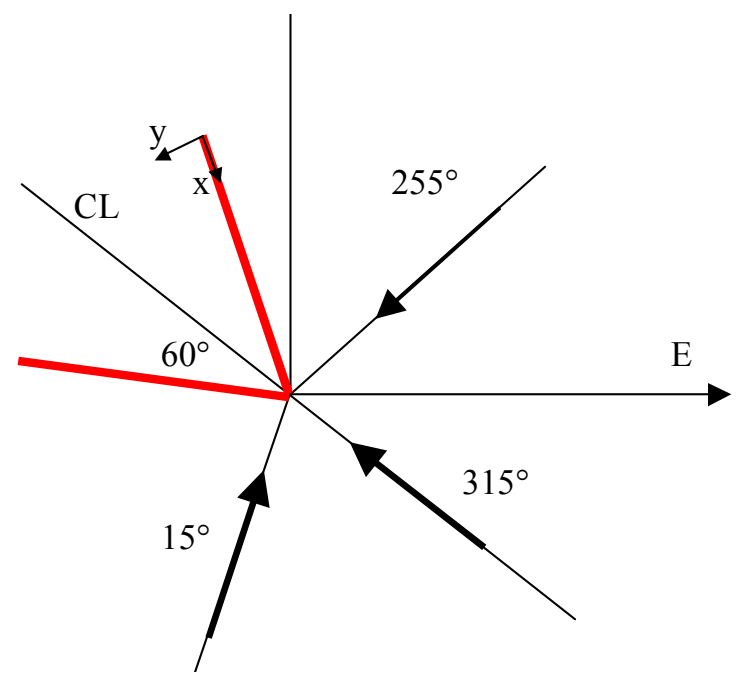

Figure 11. XM99 orientation and wave directions.

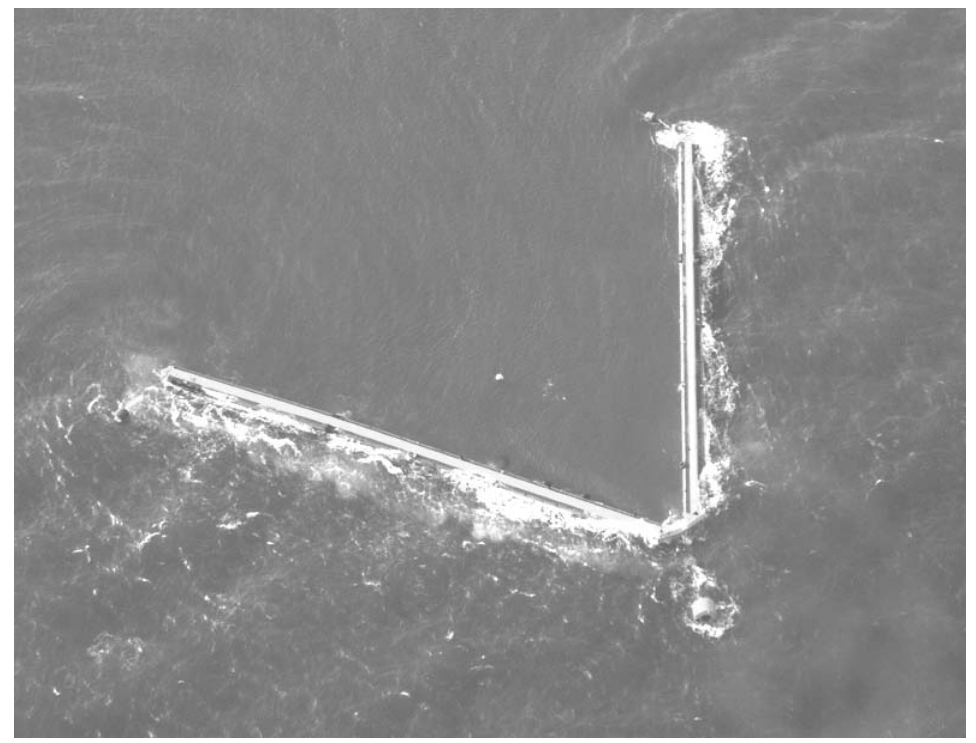

Figure 12. XM99 on station off Cape Canaveral, May 1999. 


\section{WAVE ENVIRONMENT}

\section{Processing Algorithms}

Incident waves were measured with the Datawell Directional Waverider buoy. These incident wave data were processed with three different algorithms. These included the Datawell onboard buoy processing, a GEDAP single channel frequency analysis, and a Maximum Likelihood Method (MLM) directional spectral analysis.

Datawell analysis. For the Datawell directional buoy, the directional wave spectra were estimated based on translational motions instead of wave slopes. The buoy contains a heavepitch-roll sensor, a three-axis compass, and two $x$ - and $y$-axis accelerometers. The three accelerations for heave and the two horizontal components were digitally integrated to get filtered displacements in the vertical, north, and west directions. Analog outputs of the accelerometers were low pass filtered with a cutoff frequency of $1.5 \mathrm{~Hz}$, resampled at $3.84 \mathrm{~Hz}$, and low-pass filtered again with a $10^{\text {th }}$ order filter with a cutoff of $0.6 \mathrm{~Hz}$. The Datawell algorithm calculates the Fast Fourier Transform (FFT) every half hour from eight overlapping segments of $200 \mathrm{sec}$ of data. The final record length is 1,535 points or 1,200 sec (i.e., 20 minutes). The number of degrees of freedom was 16, at a sampling frequency of $1.28 \mathrm{~Hz}$ (i.e., $\Delta \mathrm{t}=0.781 \mathrm{sec})$.

GEDAP analysis. GEDAP is a general purpose software package for control signal generation, time and frequency domain analyses, and data manipulation, archival, and presentation. It was originally developed by the National Research Council of Canada Hydraulics Laboratory (Miles 1997). It is a modular package that is fully integrated by a common file and support structure and an interactive graphics package.

The GEDAP program "VSD" was used to estimate the variance spectral density by smoothing the modified Periodogram of the total record. The modified Periodogram was obtained by multiplying the record by a 10 percent cosine bell window on the data to reduce leakage. Lower and upper cutoff frequencies of 0.0 and the Nyquist frequency of $0.64 \mathrm{~Hz}$ (i.e., half of the sampling frequency), respectively, were used. The filter or equivalent bandwidth was $0.01 \mathrm{~Hz}$ giving 20 degrees of freedom. 
MLM directional spectral analysis. For the MLM analysis, a Matlab version of the standard multidirectional spectral analysis software for the directional spectral wave generator was used. The data were zero-meaned, tapered by a 10-percent cosine bell window, and Fourier transformed to the frequency domain. The spectral and cross-spectral estimates were Gaussian smoothed with an effective bandwidth of $0.01 \mathrm{~Hz}$. A wrapped normal directional spreading function was used as an initial estimate and an MLM algorithm was used to improve this initial estimate. The overall mean wave direction and mean directional spreading were calculated according to the International Association for Hydraulic Research (IAHR) List of Sea State Parameters (1997) for multidirectional waves.

\section{Measured Wave Conditions}

Significant wave height, significant wave periods, mean wave direction and directional spreading were calculated with the analyses described above. Time series of each are described in the paragraphs below. Directional spectral plots and joint distribution plots are also presented. Appendix D contains a listing of the measured wave period, height, direction, and directional spreading for each hour during the field trials from May 20 to May 30.

Significant wave height. Significant wave heights from the GEDAP and Datawell analyses are compared in Figure 13. The MLM values are slightly smaller than these two and are not shown. Figure 14 shows the significant and maximum wave heights from the GEDAP analysis. The dashed lines represent the lower and upper SS3 limits. The blank spaces are due to breaks in the data. In general, the GEDAP values are higher than the Datawell, but have the same shapes and trends. During the deployment, significant wave heights were less than SS3, except for periods on May 22 and May 29. Maximum wave heights exceeded the SS3 lower limit on many occasions, however.

Significant wave periods. Significant wave periods from the GEDAP and Datawell analyses are shown in Figure 15. Figure 16 is the GEDAP analysis of the significant and maximum wave periods. This period corresponds to the period of the maximum wave height, and does not necessarily imply a larger value than the significant wave period.

The large fluctuations are due to the multi-modal character of the wave environment. The deployment period was characterized by unimodal sea only and swell only, and combined 


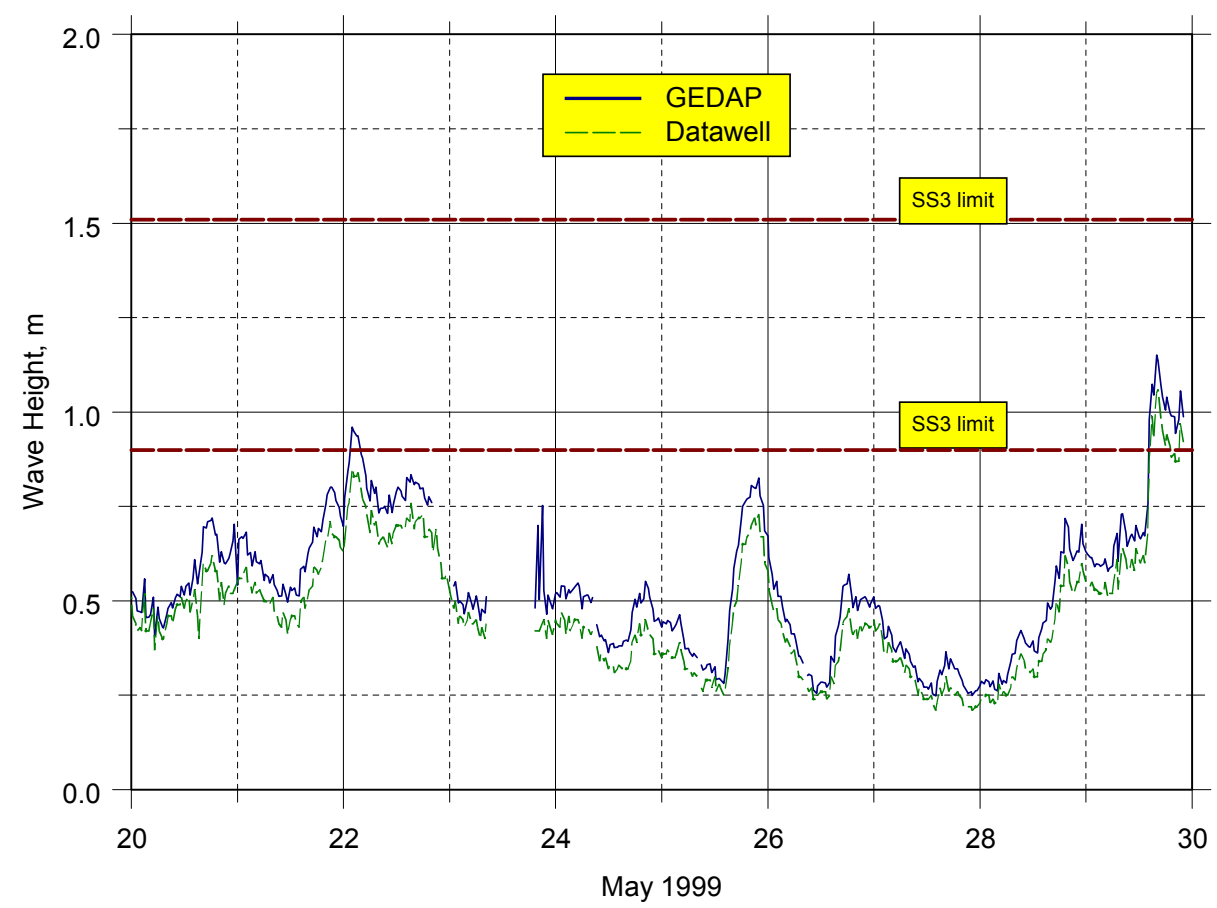

Figure 13. Incident significant wave heights.

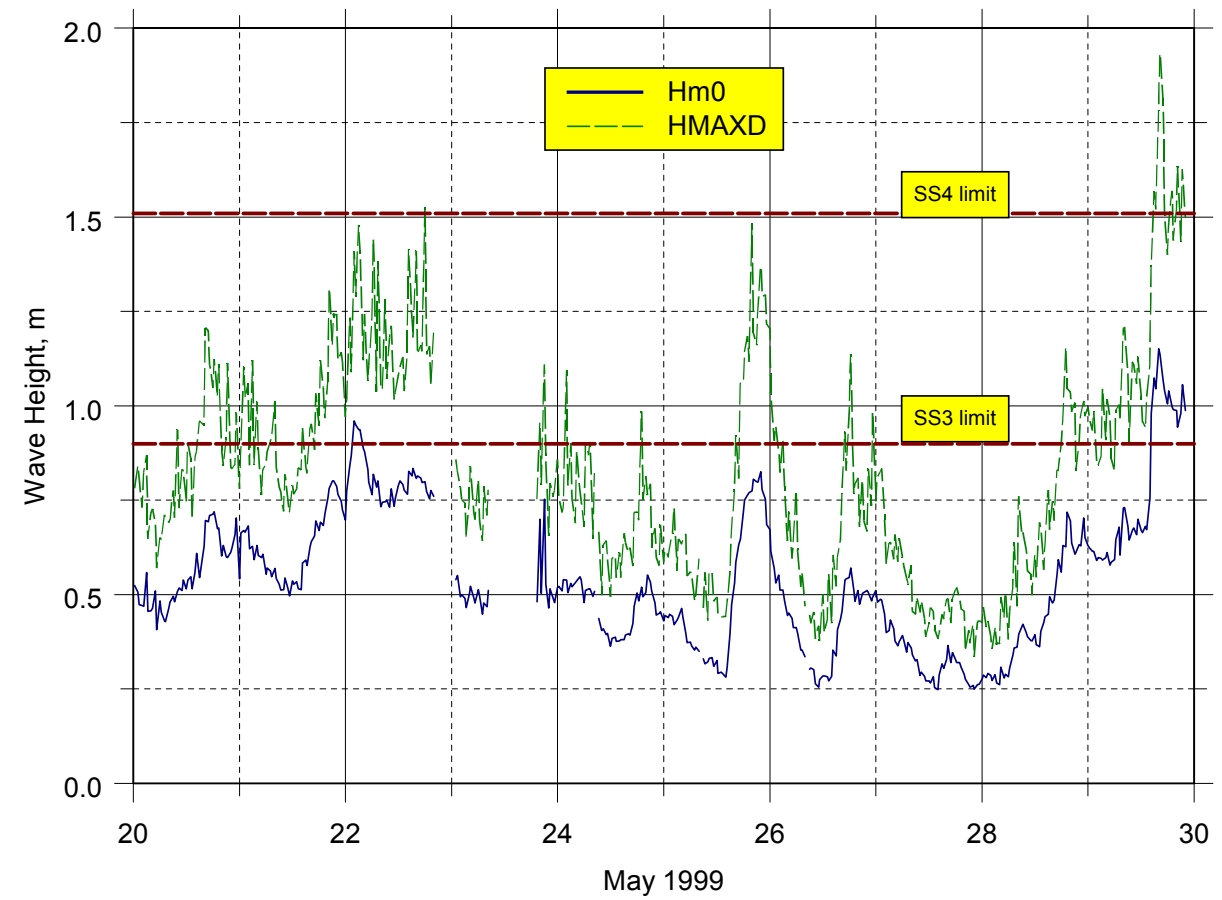

Figure 14. GEDAP significant and maximum wave heights. 


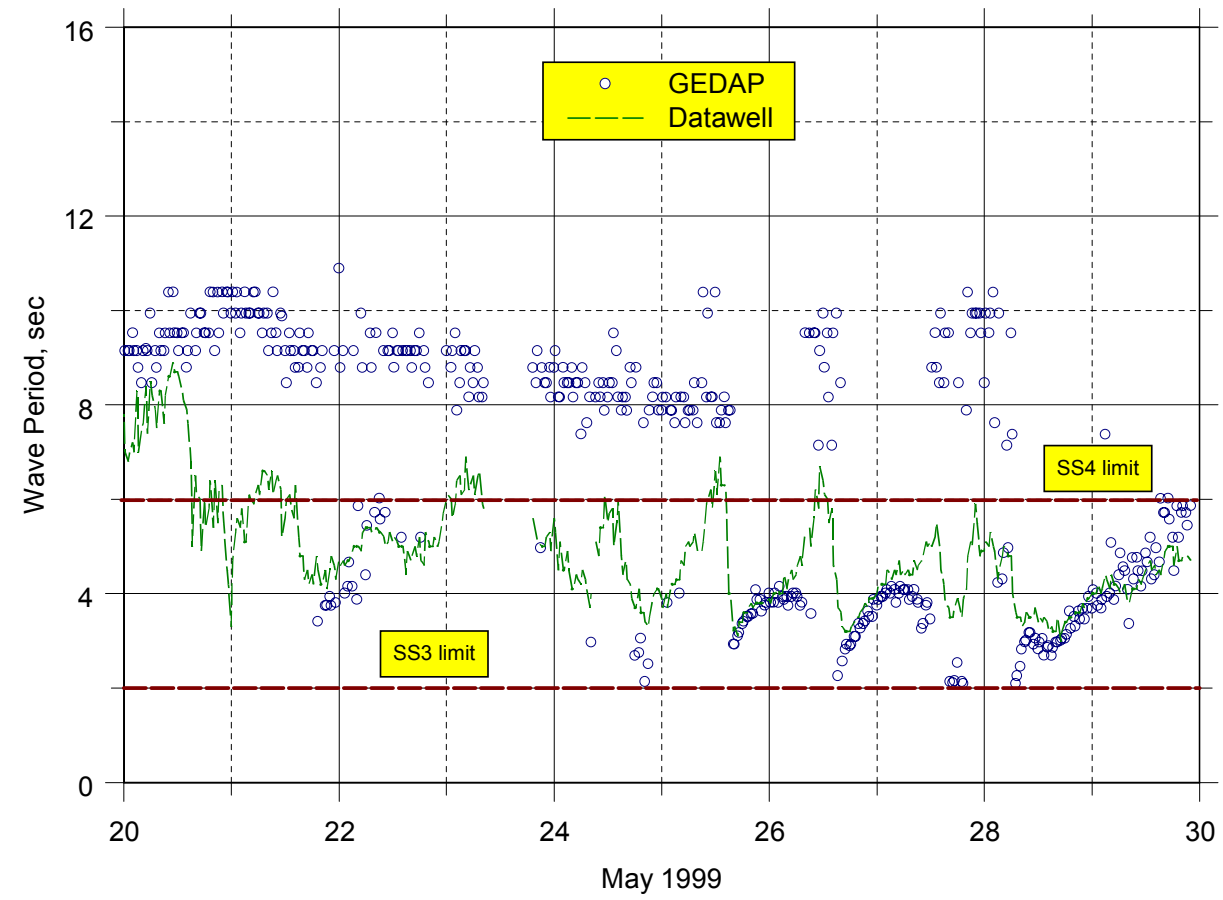

Figure 15. Incident wave periods.

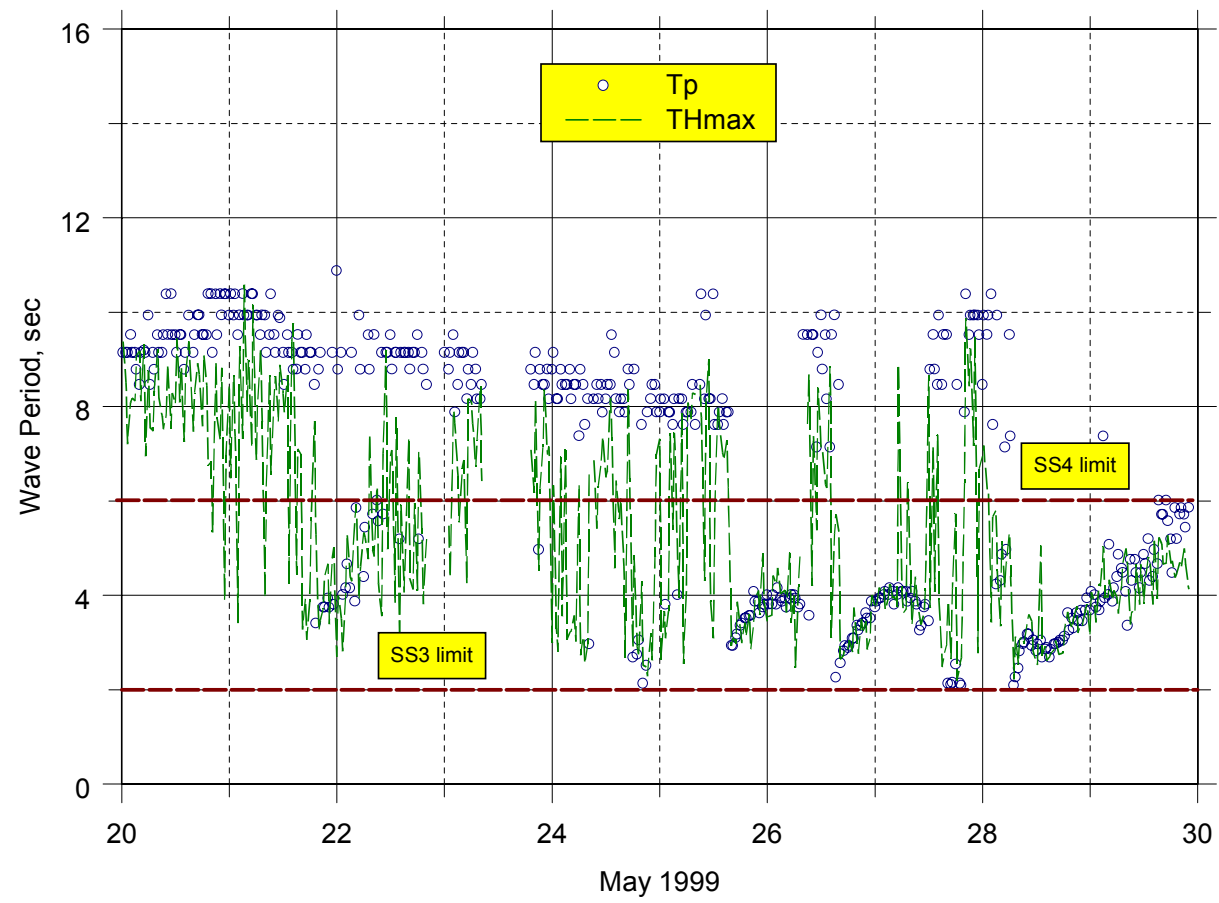

Figure 16. GEDAP significant and maximum wave periods. 
bimodal, and even trimodal, sea and swell wave spectra. Thus, the large variations in wave period are due to the shifting in wave energy between sea and swell peaks. In general, the GEDAP and MLM (not shown) values tend to track each other better, showing both sea and swell peaks. The Datawell values tend to track the sea peaks only.

Mean wave direction and spreading. Figure 17 is a time series of mean wave direction and directional spread at the peak frequency from the MLM analysis. The dashed line at 255 deg is the lower limit on the range of effective wave directions for the XM99. Most of the mean wave directions were within this window. The optimum "design" condition, with waves in the vicinity (i.e., $\pm 15 \mathrm{deg}$ ) of $315 \mathrm{deg}$, occurred for several hours between May 24 and May 27. Directional spreading ranged from 30 to $80 \mathrm{deg}$, the former value representative of a more narrow directional spreading.

Directional spectral plots. Figure 18 is a contour and three-dimensional plot of the directional wave spectra from the MLM directional spectral analysis. It shows a unimodal sea spectra for May 25, 1800 hours, with a peak period of $3.3 \mathrm{sec}$, significant wave height of $60 \mathrm{~cm}$, mean wave direction of $322 \mathrm{deg}$, and directional spreading of $39 \mathrm{deg}$.

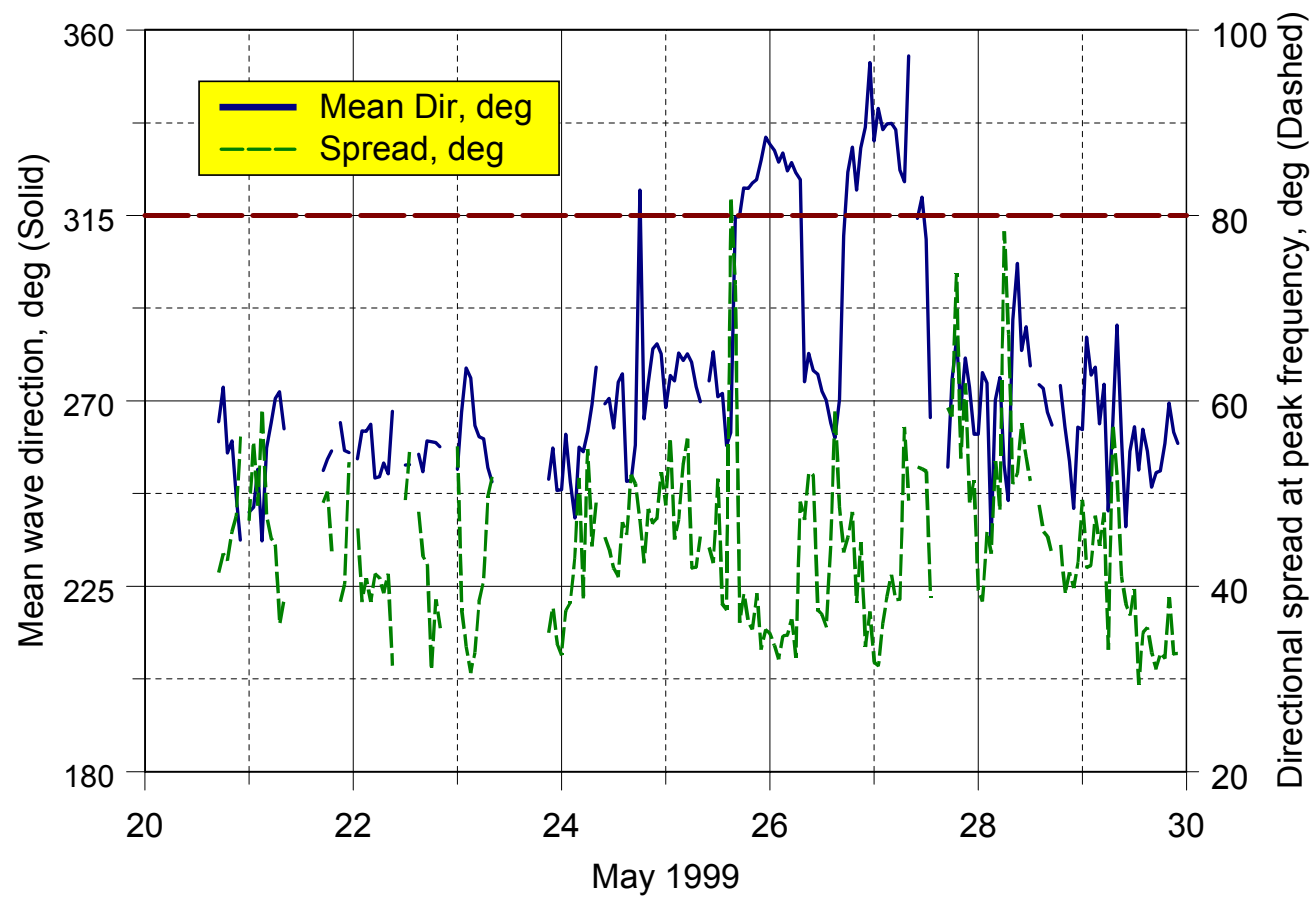

Figure 17. MLM incident wave direction and directional spread. 


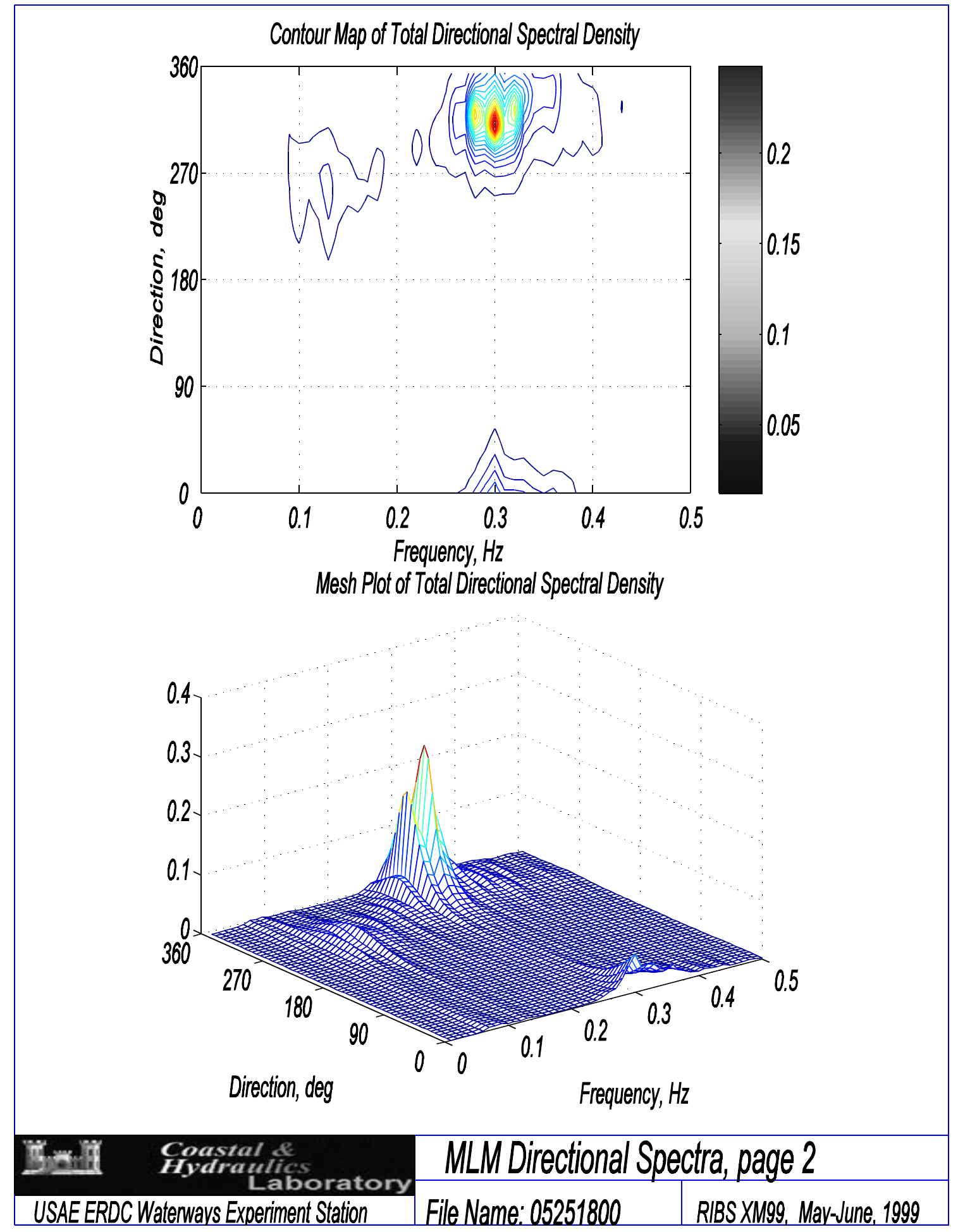

Figure 18. Directional wave spectrum, May 25, 1800 hours. 
Figures 19 to 21 are typical contour and three-dimensional plots of the directional wave spectra for various unimodal and bimodal spectra. Figure 19 is an example of a unimodal swell spectrum for May 23, 600 hours, with a peak period of $8.3 \mathrm{sec}$, wave height of $40 \mathrm{~cm}$, wave direction of $261 \mathrm{deg}$, and spread of $40 \mathrm{deg}$. A bimodal spectrum with a dominant sea mode for May 24, 1800 hours, is shown in Figure 20. The peak period is $2.7 \mathrm{sec}$, with a height of $41 \mathrm{~cm}$, direction of $321 \mathrm{deg}$, and spread of $47 \mathrm{deg}$. Finally, a bimodal spectrum with a dominant swell mode for May 21, 1800 hours, is shown in Figure 21. The peak period is $9.1 \mathrm{sec}$, with a height of $55 \mathrm{~cm}$, direction of $256 \mathrm{deg}$, and spread of $50 \mathrm{deg}$.

Joint distribution plots. Joint distribution or scatter plots of significant wave height, mean wave direction, and directional spread versus peak wave period from the GEDAP analysis are shown in Figures 22 to 24, respectively. These figures show the distributions for the entire 10day deployment. Figure 22 is the joint distribution for wave period and height. It shows that the wave data split into sea and swell categories. The sea conditions are also representative of the SS3 and smaller conditions with wave periods less than $6 \mathrm{sec}$. The largest recorded significant wave height was $1.15 \mathrm{~m}$ at a peak wave period of $4.71 \mathrm{sec}$. The largest peak period was $10.9 \mathrm{sec}$ at a significant wave height of $0.70 \mathrm{~m}$.

Figure 23 is the joint distribution of mean wave direction and peak wave period from the MLM analysis. As expected, wave directions are more tightly grouped in the swell waves (i.e., periods greater than $7 \mathrm{sec}$ ) than the sea waves. The lower limit at $255 \mathrm{deg}$ indicates that most of the waves encountered during the XM99 deployment were within the window of allowable wave directions for effective performance. The largest wave direction was at 354 deg with a wave period of $3.94 \mathrm{sec}$. The smallest wave direction of $236 \mathrm{deg}$ occurred at wave periods of both 4.23 and $10.39 \mathrm{sec}$. Waves with mean wave directions within \pm 15 deg of 315 deg occurred 22 times with wave periods ranging from 2.7 to $4.2 \mathrm{sec}$. These waves are probably all within the "design" direction window for the XM99 of 315 deg, parallel to its center line. Three occurrences were at $315 \mathrm{deg}$.

Figure 24 is the joint distribution for directional spread and peak wave period. Most of the spread values are between 30 and $60 \mathrm{deg}$. The maximum spread of $82 \mathrm{deg}$ occurs with a peak period of $7.9 \mathrm{sec}$. Some of the larger values above $60 \mathrm{deg}$ may be spurious points. The minimum value is $29.5 \mathrm{deg}$ at $4.2 \mathrm{sec}$. Values of 30 to $50 \mathrm{deg}$ are representative of fairly narrow spreading. In general, swell waves would be more narrow than sea conditions 


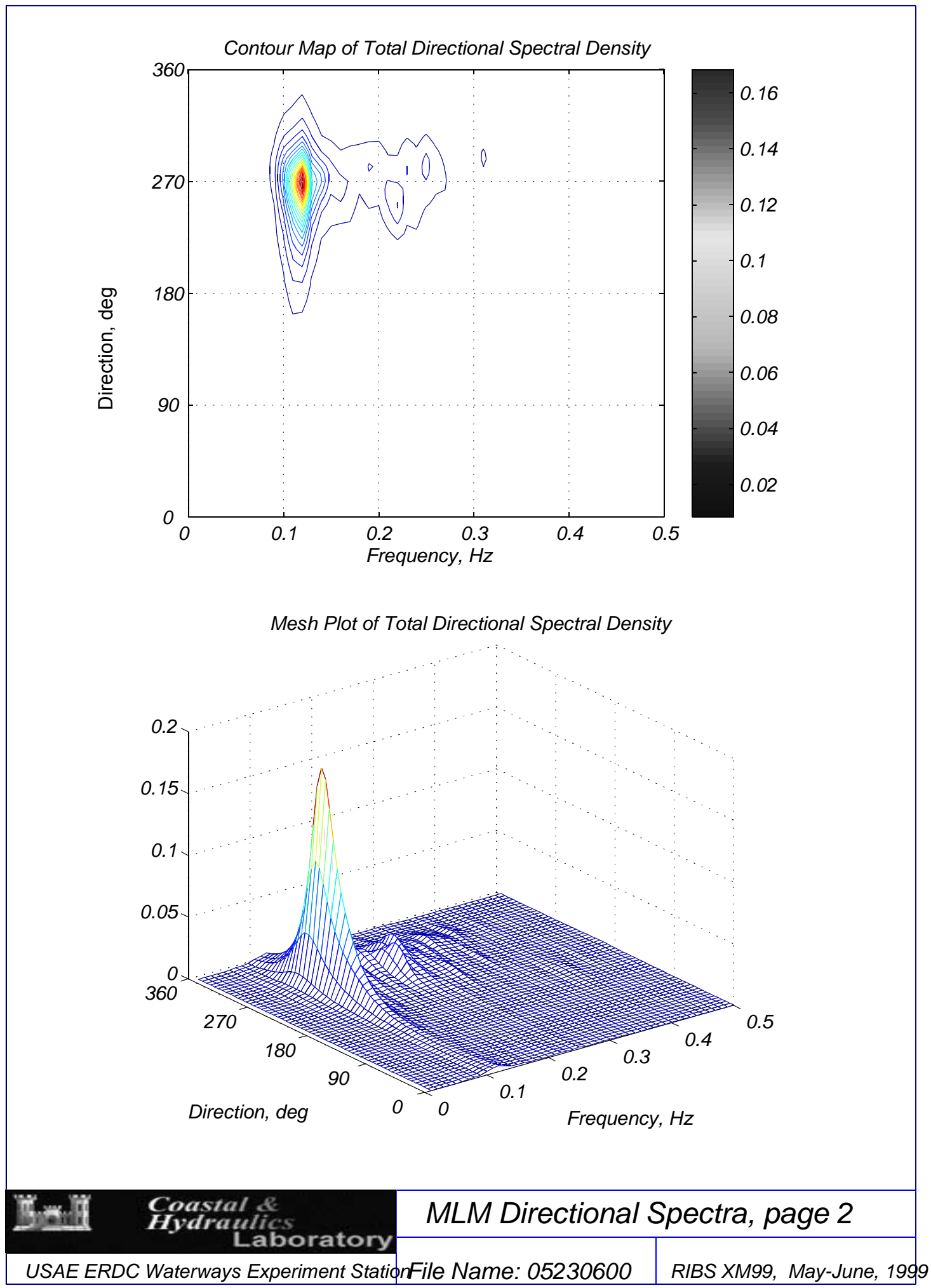

Figure 19. Directional wave spectrum, unimodal swell, May 23, 0600 hours. 


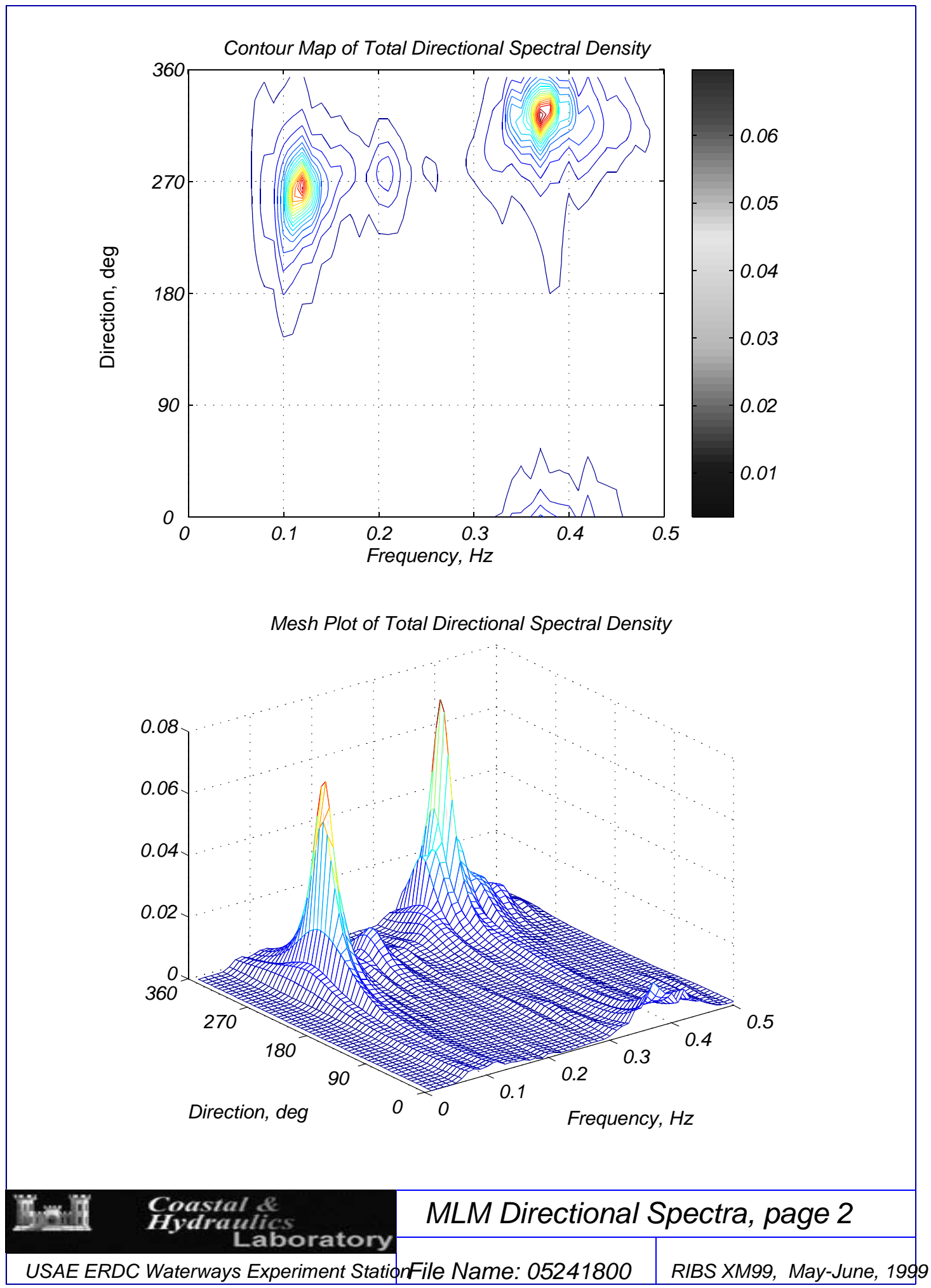

Figure 20. Directional wave spectrum, bimodal with dominant sea mode, May 24, 1800 hours. 


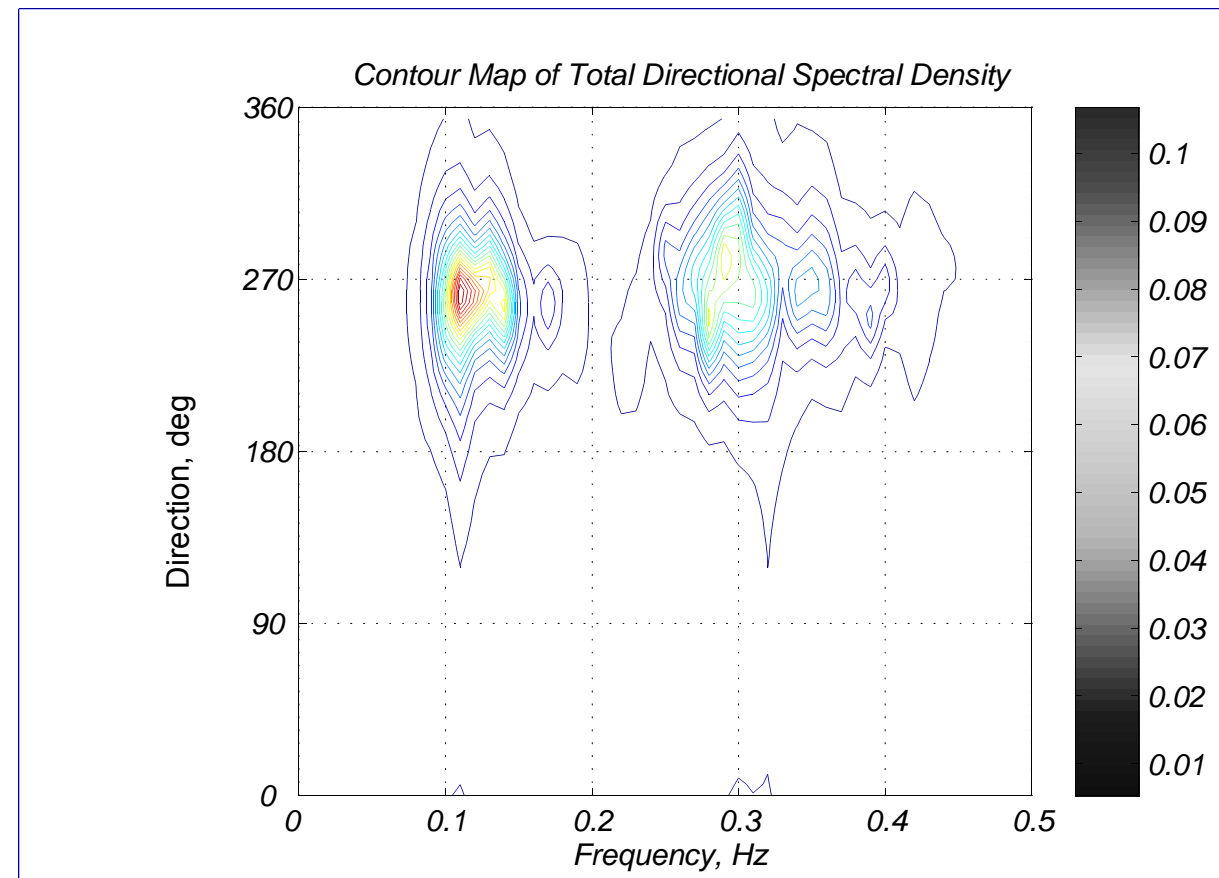

Mesh Plot of Total Directional Spectral Density

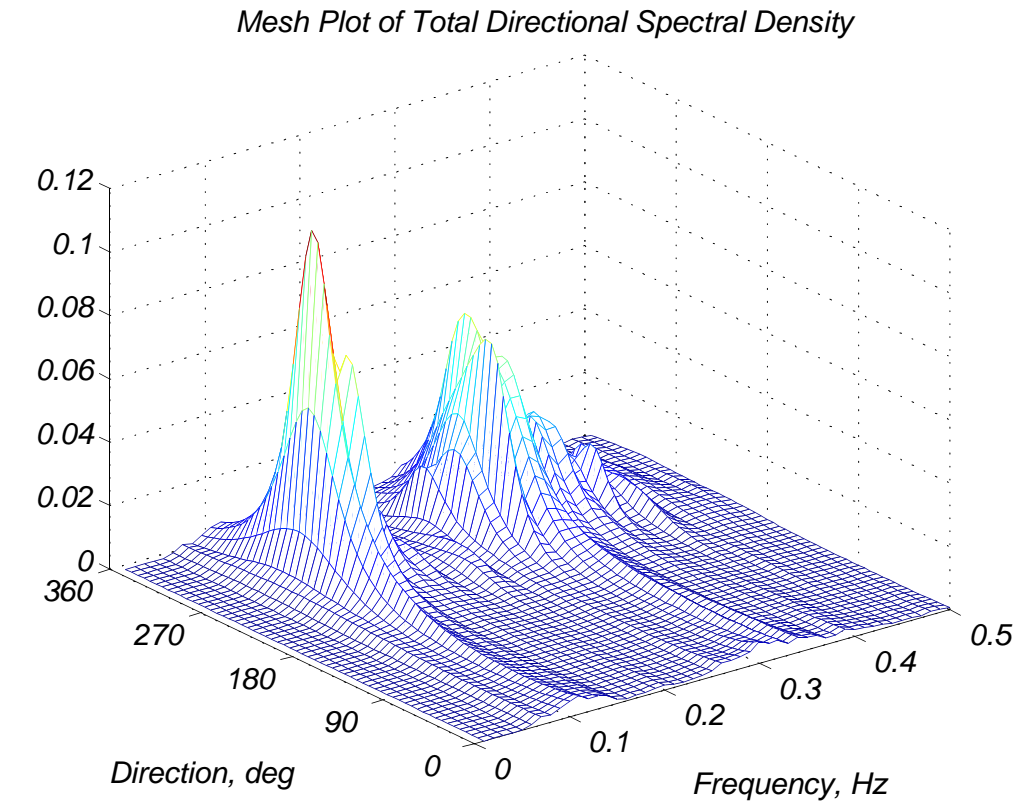

\section{Fant \\ Coastal if \\ Hydraulices \\ Laboratory}

MLM Directional Spectra, page 2

USAE ERDC Waterways Experiment StationFile Name: 05211800

RIBS XM99, May-June, 1999

Figure 21. Directional wave spectrum, bimodal with dominant swell mode, May 21, 1800 hours. 


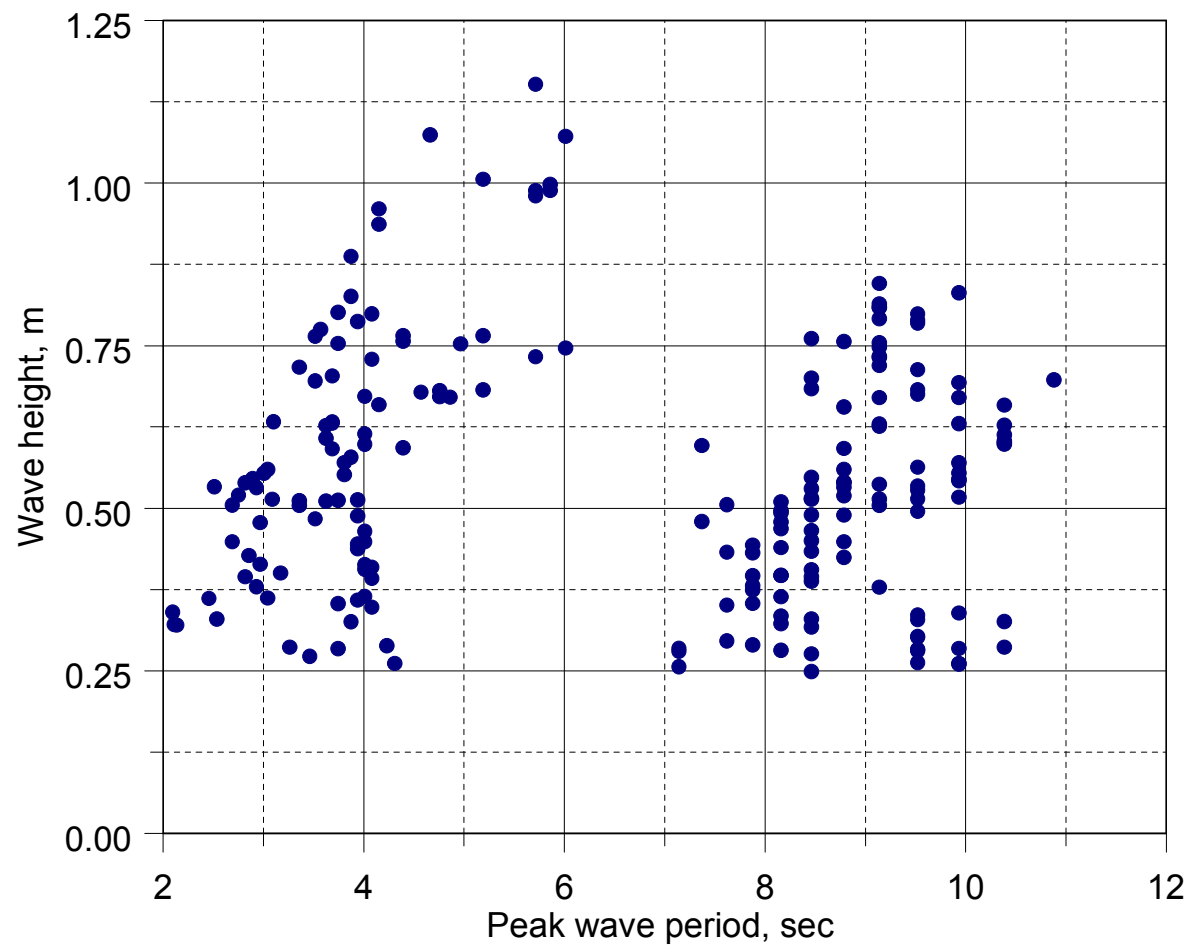

Figure 22. Joint distribution of wave period and height.

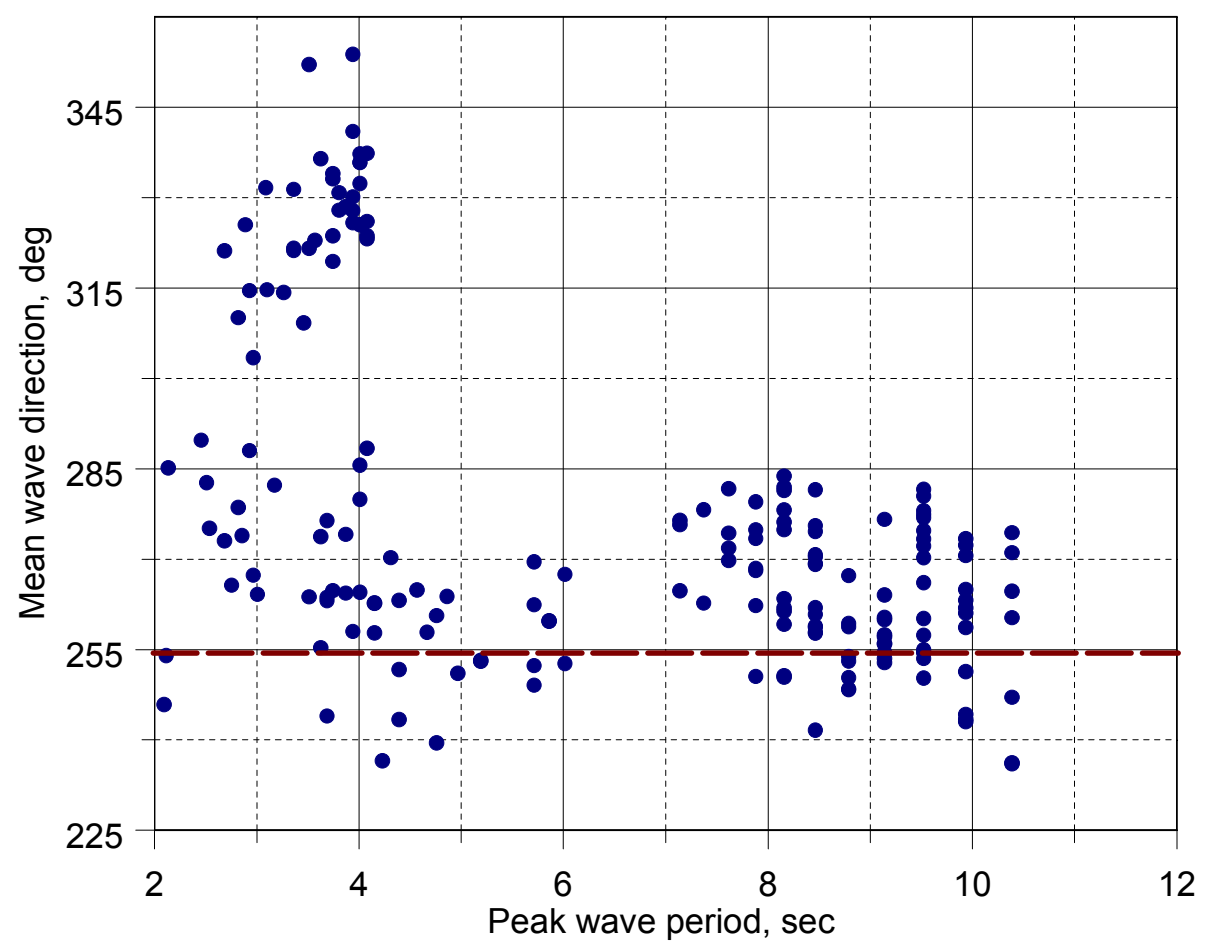

Figure 23. Joint distribution of wave period and wave direction. 


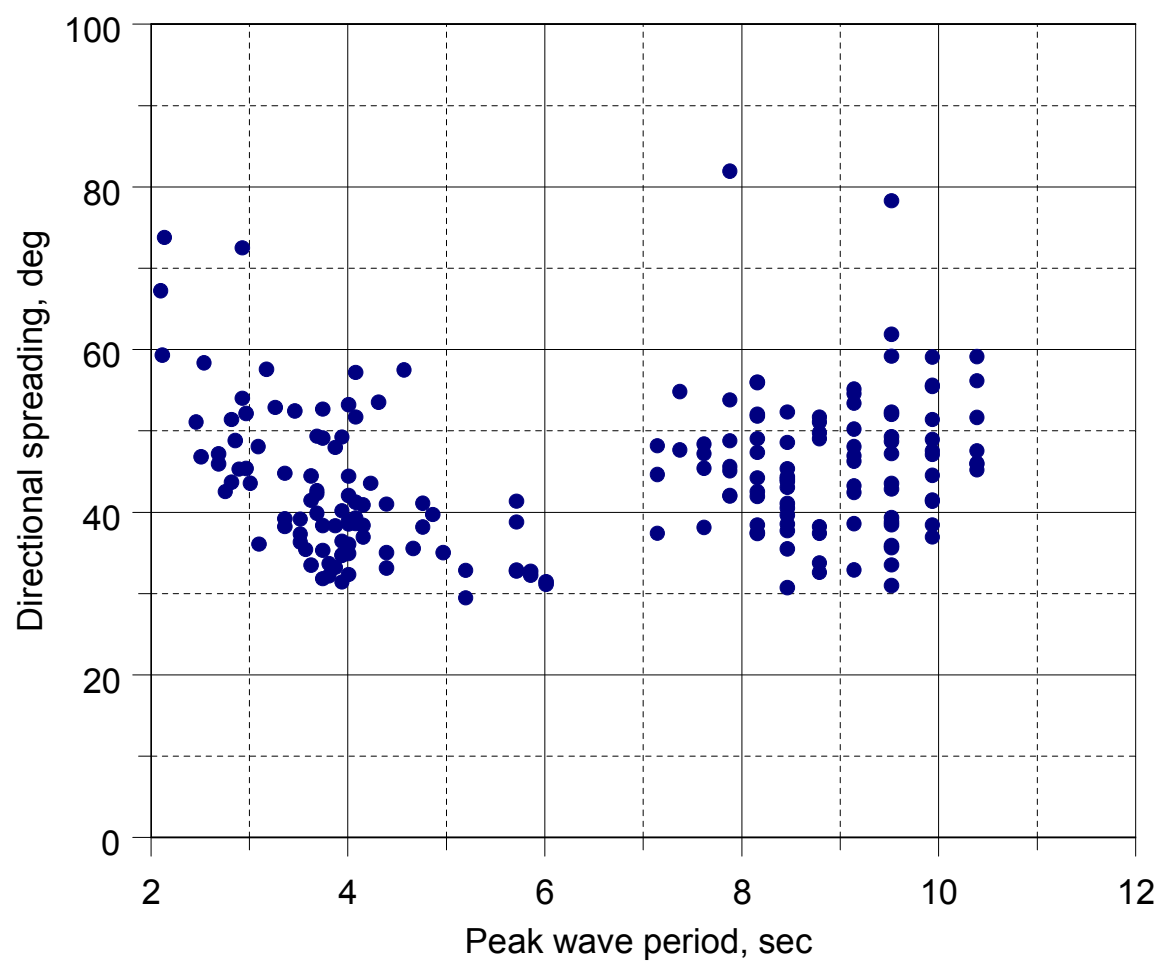

Figure 24. Joint distribution of wave period and directional spread. 


\section{CHAPTER IV}

\section{ANALYTICAL MODELS}

\section{WAVE TRANSMISSION}

\section{Background}

Breakwater performance or effectiveness is usually defined by the transmission coefficient $K_{T}$ that relates the size of the transmitted wave to the incident wave as

$$
K_{T}=\frac{H_{T}}{H_{I}}
$$

where $H_{T}$ is the transmitted wave height and $H_{I}$ is the incident wave height. A value of $K_{T}=0.50$ or less is indicative of very good performance as the transmitted wave height is reduced to one half of its incident value, equivalent to only 25 percent of its incident energy.

Wave transmission for a floating breakwater is a function of many wave and structural parameters. Harms et al. (1982) expressed $H_{T}$ as a function of the following wave and structure parameters

$$
H_{T}=f\left(H_{I}, L, d, B_{x}, B_{y}, m, k, \varepsilon, h, \gamma, v, g\right)
$$

where $H_{I}$ is incident wave height, $L$ is wavelength, $d$ is depth of submergence or draft, $B_{x}$ is width of the breakwater in the direction of wave travel, $B_{y}$ is breakwater length in the transverse direction, $m$ is breakwater mass, $k$ is spring constant or mooring line stiffness, $\varepsilon$ is horizontal excursion of the breakwater normal to its equilibrium position, $h$ is water depth, $\gamma$ is specific weight of water, $v$ is kinematic viscosity, and $g$ is gravitational acceleration. Of course, $L$ is a function of wave period $T$ or frequency $f$ and water depth $h$. Another parameter that Harms et al. (1982) did not mention is the flexural rigidity EI, defined as the product of the Modulus of Elasticity $E$ and the mass moment of inertia $I$. 
Harms et al. (1982) applied the Buckingham Pi Theorem to show that $K_{T}$ is a function of the following nondimensional parameters.

$$
K_{T}=f\left(\frac{L}{B_{x}}, \frac{H_{I}}{L}, \frac{d}{h}, \frac{B_{x}}{d}, \frac{B_{y}}{d}\right)
$$

where $L / B_{x}$ is a relative wavelength, $H_{I} / L$ is wave steepness, $d / h$ is a relative draft, $B_{x} / d$ is relative breakwater width, and $B_{y} / d$ is relative breakwater length. He also defined a breakwater Reynold's Number $R_{e}$ as

$$
R_{e}=\frac{V d}{v}=\frac{H_{I}}{L} \frac{d \sqrt{g L}}{v}
$$

The characteristic length is the breakwater draft $d$ and the velocity corresponds to the maximum wave-induced water particle velocity. The definition of the velocity is based on a deep water wave and is only approximately equal to this because it does not include a factor of $\sqrt{ }(\pi / 2)$.

Some important parameters for RIBS are the interior angle of the RIBS legs $\theta_{r}$ and the mean wave direction $\bar{\theta}$ or relative orientation to the waves. Directional spreading angles $\sigma_{m}$ can also be an important consideration in defining what wave directions the breakwater is actually exposed to.

Thus, most of these nondimensional parameters can be grouped into fixed quantities that are functions primarily of geometry and variable parameters that depend on the wave conditions. Relative breakwater width and length parameters can be defined in terms of water depth $h$ rather than draft $d$. Wave steepness can be expressed as $k a$ rather than $H / L$.

\section{Theory}

Two simple, semi-empirical analytical solutions are available (Wiegel 1960, Kriebel and Bollmann 1996) to predict wave transmission for rigid, thin, vertical barriers in the presence of waves. Both analytical models were based on normally incident waves on a straight breakwater (i.e., only one leg). Both are based on linear theory and have some shortcomings due to certain assumptions. The main shortcomings in their formulation are that they did not match dynamic pressures at the boundaries between regions and did not set the normal velocities on the face of 
the RIBS equal to zero because they did not include evanescent waves in the formulation. However, these two analytical formulations do give a quick estimate of wave transmission, especially for longer waves. In this study, they are compared to the measured data only to assess their range of applicability to the RIBS and as a first step in eventually improving these models in future research.

Kobayashi (1998) and Briggs et al. (1998) applied these models to the RIBS. Both analytical models can by represented by the definition sketch shown in Figure 25 for the RIBS. The fixed, impermeable, vertical legs form a "V" with an interior angle of $\theta_{r}$. The wave transmission of oblique waves problem is defined within two regions. Region 1 consists of the incident and reflected waves and Region 2 the transmitted waves. The origin of the analytical or body-fixed coordinate system is at the bow of the RIBS, with the positive $y$-axis along the starboard leg, the $x$-axis normal to this leg, and the z-axis positive up from the still water surface. This orientation simplifies the analysis as the $y$-axis is parallel to and positive along the starboard leg, where $x=0$. The water depth is defined by $h$ and the draft of the RIBS by $d$. The

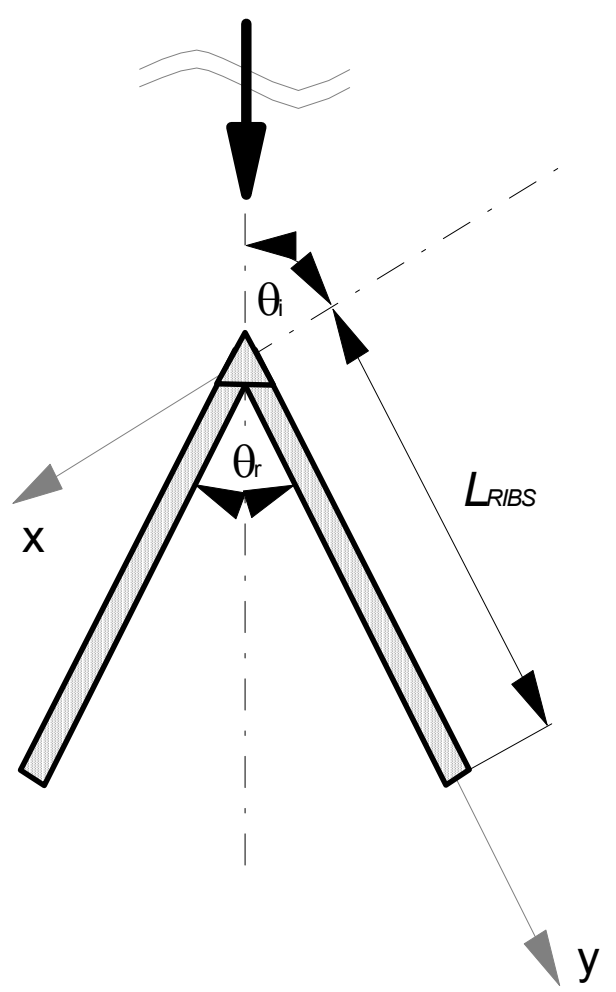

Figure 25. Analytical model of RIBS.

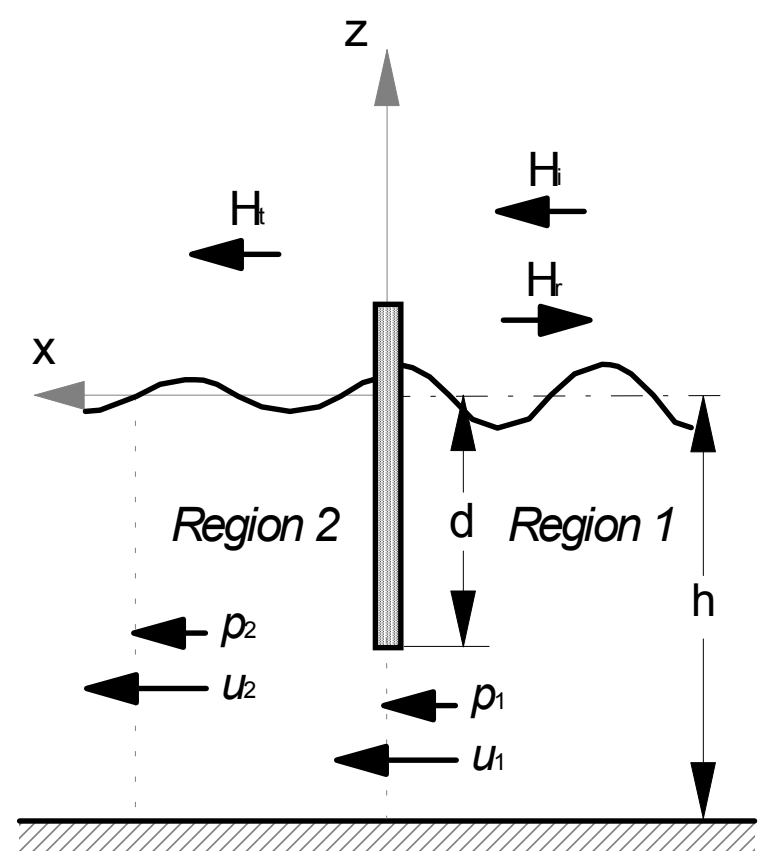


incident wave angle $\theta_{I}$ is measured counterclockwise from the positive $x$-axis. A wave direction of $0 \mathrm{deg}$ is normal or broadside to the starboard leg. Because of symmetry, wave directions on the port leg could equally be considered. Oblique waves in the range $0 \leq \theta_{I} \leq 90 \mathrm{deg}$ are considered in this analysis.

Regular (i.e., monochromatic) waves are used in the analysis for simplicity and are defined by the wave period $T$ and wave height $H_{I}$. Reflected and transmitted waves are characterized by wave heights $H_{R}=K_{R} H_{I}$ and $H_{T}=K_{T} H_{I}$, where $K_{R}$ is the reflection coefficient. Evanescent or spurious waves from the breakwater motions are higher-order phenomenon that are assumed to be small and decay rapidly with distance from the legs.

In the analytical model for wave transmission, the legs are assumed to be fixed, rigid, thin, vertical, and infinitely long. These assumptions are considered a reasonable first approximation. The fixed and rigid assumption is predicated on the fact that the bending rigidity of the RIBS legs is sufficient to prevent the RIBS from acting as a wavemaker, so that the displacements are reasonably small relative to the wave height. The beam of the RIBS is small relative to the wavelengths in SS3, so the thin assumption is justified. The vertical assumption is warranted as long as the inclination angle (i.e., angle of the RIBS measured from the vertical) of the RIBS leg does not exceed about 20 deg from the vertical (Liu and Abbaspour 1982). The infinitely long assumption is valid if the dimensions of the legs are sufficiently large relative to the projected wavelengths along the $x$ - and $y$-axes. One final assumption is that the transmitted waves from the starboard leg are not reflected from the port leg back into the interior of the RIBS. Of course, this does occur but it is assumed to be a small effect.

Free surface elevations for the incident $\eta_{I}$ and reflected $\eta_{R}$ waves in Region 1 are given by linear wave theory as

$$
\begin{gathered}
\eta_{I}=\frac{H_{I}}{2} \cos \left(k_{x} x+k_{y} y-\omega t\right) \\
\eta_{R}=\frac{H_{I}}{2} K_{R} \cos \left(-k_{x, 1} x+k_{y, 1} y-\omega t+\varphi_{R}\right)
\end{gathered}
$$

where 
$k_{x, 1}=k \cos \theta_{1}=$ wave number in the $x$-direction in Region 1 ,

$k_{y, 1}=k \sin \theta_{1}=$ wave number in the $y$-direction in Region 1,

$k=$ wave number given by $g k \tanh k h=\omega^{2}$,

$\theta_{1}=\theta_{I} \quad=$ wave angle in Region $1=$ incident wave angle,

$\omega=2 \pi / T=$ angular frequency,

$K_{R}=$ reflection coefficient, and

$\varphi_{\mathrm{R}}=$ reflection phase difference.

Units of $\eta_{\mathrm{I}}$ and $\eta_{\mathrm{R}}$ are the same as $H_{I}$ and $H_{R}$. In Region 2, the transmitted wave elevation $\eta_{\mathrm{T}}$ is

$$
\eta_{T}=\frac{H_{I}}{2} K_{T} \cos \left(k_{x, 2} x+k_{y, 2} y-\omega t+\varphi_{T}\right)
$$

where

$k_{x, 2}=k \cos \theta_{2}=$ wave number in the $x$-direction in Region 2,

$k_{y, 2}=k \sin \theta_{2}=$ wave number in the $y$-direction in Region 2, and

$\varphi_{\mathrm{T}}=$ transmission phase difference.

Although the two phase differences $\varphi_{\mathrm{R}}$ and $\varphi_{\mathrm{T}}$ should probably be retained, they were neglected to simplify the analysis.

The wave transmission beneath the RIBS leg is a function of the time-averaged energy flux $F$, or rate of work done by the dynamic pressure. This is known as the transmitted wave power and is equal to the incident wave power under the barrier. Wave power is the time average over one wave of the depth-integrated product of dynamic pressure and horizontal fluid velocity.

In Region 1 , the dynamic pressure $p_{l}$ and horizontal velocity $u_{l}$ by linear wave theory are

$$
\begin{gathered}
p_{1}=\rho g K_{p}\left(\eta_{I}+\eta_{R}\right) \\
u_{1}=\frac{g k}{\omega} K_{p}\left(\eta_{I}-\eta_{R}\right) \cos \theta_{1}
\end{gathered}
$$

where the pressure response factor $K_{p}$ is 


$$
K_{p}=\frac{\cosh [k(h+z)]}{\cosh k h}
$$

In Region 2 on the interior of the leg, the dynamic pressure $p_{2}$ and horizontal velocity $u_{2}$ are similarly defined by linear wave theory as

$$
\begin{gathered}
p_{2}=\rho g K_{p} \eta_{T} \\
u_{2}=\frac{g k}{\omega} K_{p} \eta_{T} \cos \theta_{2}
\end{gathered}
$$

Both Wiegel (1960) and Kriebel and Bollman (1996) assumed that the waves were normal to the breakwater leg (i.e., incident wave direction $\theta_{I}=0 \mathrm{deg}$ ). Therefore, their derivations did not include the $\cos \theta$ term in the previous equations. To simplify the analysis, an assumption is being made that the wave directions $\theta_{1}$ and $\theta_{2}$ and corresponding wavenumbers $k_{x}$ and $k_{y}$ are the same on both sides of the RIBS leg because the leg is assumed to be infinitely thin. This assumes that $\theta_{1}=\theta_{2}=\theta_{I}$. In reality, the RIBS leg has some thickness or beam so that $\theta_{1}$ and $\theta_{2}$ would probably be different due to turbulence and refraction as the wave goes under the leg. Also, if reflections within the two legs of the RIBS are included, then the $\theta_{2}$ direction might be better represented as a uniform distribution of angles from 0 to $360 \mathrm{deg}$.

According to Wiegel and Kriebel and Bollman, the time-averaged energy flux $F_{l}$ in Region 1 is

$$
F_{1}=\frac{1}{T} \int_{0}^{T} \int_{-h}^{-d} p_{1} u_{1} d z d t=\frac{\rho g^{2} k}{8 \omega} H_{I}^{2} \frac{\sinh [2 k(h-d)]+2 k(h-d)]}{4 k \cosh ^{2} k h}\left(1-K_{R}^{2}\right)
$$

Similarly in Region 2, the energy flux $F_{2}$ is

$$
F_{2}=\frac{1}{T} \int_{0}^{T} \int_{-h}^{0} p_{2} u_{2} d z d t=\frac{\rho g^{2} k}{8 \omega} H_{I}^{2} \frac{\sinh [2 k h]+2 k h]}{4 k \cosh ^{2} k h} K_{T}^{2}
$$

Setting the two energy fluxes equal to each other, both Wiegel and Kriebel and Bollman obtained an expression for the transmission coefficient. Wiegel's original Power Transmission 
Theory (PTT) for the wave transmission coefficient did not include the effects of reflected waves and was given by

$$
K_{T}=\sqrt{T_{F}}
$$

Kriebel and Bollman included the effects of the reflected waves and called their model the Modified Power Transmission Theory (MPTT) defined as

$$
K_{T}=\sqrt{\left(1-K_{R}^{2}\right) T_{F}}
$$

where the transmission factor $T_{F}$ is defined as

$$
T_{F}=\frac{\sinh [2 k(h-d)]+2 k(h-d)}{\sinh (2 k h)+2 k h}
$$

As a further step, Kriebel and Bollman obtained an expression relating the transmission and reflection coefficients by assuming continuity of the horizontal water particle velocities at the boundary along the starboard leg (i.e., $x=0$ ) between the two regions

$$
K_{T}=1-K_{R}
$$

Finally, they set the two expressions in Equations 39 and 41 equal to each other and solved for $K_{T}$ as a function of $T_{F}$

$$
K_{T}=\frac{2 T_{F}}{1+T_{F}}
$$

The Kriebel and Bollman (1996) formulation has some major weaknesses. First, they did not match the dynamic pressures at the boundaries between regions. Thus, their equation 41 does not satisfy wave energy conservation. Second, they did not include evanescent modes in their formulation. These "spurious" or "wave-making" modes are important in the "near field" close to the RIBS, but decay with distance.

Even though the Kriebel and Bollman formulation for $K_{T}$ in equation 41 is in error, an interesting observation is that if equation 41 is substituted into equation 39, then another definition of $T_{F}$ is given by 


$$
T_{F}=\frac{1-K_{R}}{1+K_{R}}
$$

This is the reflection coefficient used in many numerical models to simulate boundary reflections.

In summary, both the Wiegel and Kriebel and Bollman models were originally intended to describe wave transmission on normally incident waves on a single, rigid, thin breakwater. They derived the PTT and MPTT models for predicting the wave transmission coefficient $K_{T}$ as a function of a transmission factor $T_{F}$. Both Wiegel (1960) and Kriebel and Bollman (1996) made some assumptions relative to phase angles and did not include wave direction. The integration limits in their calculations of the energy flux are inconsistent. These models were never intended for a multi-legged structure such as the RIBS, but are being evaluated in this study to determine their range of applicability as semi-empirical tools.

\section{Influence of Wave Direction}

Other researchers have found a small variation in $K_{T}$ with incident wave direction. Losada et al. (1992) found that $K_{T}$ is almost constant in the range $0 \leq \theta_{I} \leq 60 \mathrm{deg}$ and approaches zero as $\theta_{I}$ $\Rightarrow 90$ deg. Sawargi (1995) noted in his book that Nakamura et al. (1981) found that $K_{T}$ was essentially unchanged for $\theta_{I}<40 \mathrm{deg}$ from the normal to the breakwater.

Since $K_{T}$ and $K_{R}$ are related, knowing $K_{R}$ should be useful in estimating $K_{T}$. Helm-Petersen (1998) investigated the reflection coefficients $K_{R}$ of three types of coastal structures with vertical fronts using multidirectional seas (up to 30 deg directional spreading) in a physical model. Coastal structures included a vertical porous structure (i.e., gabion baskets), and caissons with vertical fronts (fully reflecting), perforated vertical fronts with 25 percent porosity, and vertical fronts on top of impermeable mounds with and without berms. Of course, these structures extend to the bottom, so no wave transmission under the structure is included in these reflection coefficients. For the porous vertical structure, Helm-Petersen found that $K_{R}$ varied between 0.6 and 0.8 , decreased as the peak frequency increased, and remained constant for wave directions from 0 deg (i.e., normal) to $30 \mathrm{deg}$. For caissons with vertical fronts, $K_{R}$ was about 0.95 and showed little variation with incident wave angle ( 0 to $40 \mathrm{deg}$ ) or directional spreading. For the porous vertical front caissons, $K_{R}$ decreased from 0.5 for 0 deg incident wave 
angle to 0.3 at an angle of $60 \mathrm{deg}$. Finally, for vertical caissons on mounds, $K_{R}$ ranged from 0.6 for a mound with no berm to 0.35 with a berm. Thus, one might expect that the wave transmission coefficient is not too sensitive to wave directions up to about $40 \mathrm{deg}$ from the normal to the breakwater, although it might tend to increase since the reflection coefficient is decreasing.

In summary, wave transmission coefficients are mainly a function of the wave period $T$ or wavelength $L$, the water depth $h$, and the draft or depth of submergence $d$ of the RIBS. Typical nondimensional parameters used to describe the performance are the relative water depth $h / L$ and the relative draft of the breakwater $d / h$. There is probably some variation in the transmission coefficient with incident wave direction. What has not been considered in these derivations is wave energy dissipation. From an energy balance standpoint, the incident energy should equal the sum of the reflected, transmitted, and dissipated energy. Wave energy dissipation can occur due to wave breaking, absorption, turbulence, and flow separation under the legs due to the oscillatory nature of the waves.

\section{HYDRODYNAMIC PRESSURE}

Dynamic wave pressures are important quantities because they are used to calculate wave forces and moments on the RIBS. The approach used in this study is based on linear wave theory.

\section{Theory}

The total wave pressure consists of a hydrostatic term $p_{\text {static }}$ and a hydrodynamic $p_{1}$ term defined as

$$
p_{\text {tot }}=p_{\text {static }}+p_{1}=-\rho g z+\rho g \eta K_{p}
$$

where $\rho$ is the density of water, $g$ is gravitational acceleration, $z$ is the vertical coordinate measured positive upward from the water surface, $\eta$ is the free surface elevation, and $K_{p}$ is the pressure response factor. The hydrostatic term, a constant term based on position in the water column, controls the buoyancy force on the RIBS. 
The $p_{1}$ external (i.e., Region 1) dynamic pressure from linear wave theory was stated previously as equation 31 . Expanding this equation and retaining all terms

$$
p_{1}=\rho g A K_{p}\left[\left(1+K_{R}\right) \cos \left(k_{x, 1} x+k_{y, 1} y-\omega t\right)+2 K_{R} \sin \left(k_{x, 1} x\right) \sin \left(k_{y, 1} y-\omega t\right)\right]
$$

where the wave amplitude $A$ is assumed to be equal to one half the wave height $H$ and the bodyfixed coordinate system is used. On the external side of the RIBS leg, however, $x=0$. Equation 45 then can be reduced to

$$
p_{1}=\rho g A K_{p}\left[\left(1+K_{R}\right) \cos \left(k_{y, 1} y-\omega t\right)\right]
$$

One could consider using the Kriebel and Bollman formulation for the wave transmission coefficient derived in equation 41 in this equation for $K_{R}$. However, because of the many simplifying assumptions used in this formulation, a better approximation for $K_{R}$ might be to consider an energy balance. In this case, the wave heights are related as

$$
H_{I}^{2}=H_{R}^{2}+H_{T}^{2}
$$

Dividing through by the square of $H_{I}$ and rearranging, $K_{R}$ can be defined as

$$
K_{R}=\sqrt{1-K_{T}^{2}}
$$

Substituting in for $K_{R}$ into equation 46 and dividing by $\rho g A$, the normalized dynamic pressure $\bar{p}_{1}$ is given as

$$
\overline{p_{1, A}}=K_{p}\left[\left(1+\sqrt{1-K_{T}^{2}}\right) \cos \left(k_{y, 1} y-\omega t\right)\right]
$$

This is the analytical model that will be used in comparisons with the measured and numerical predictions of the dynamic pressure. 


\section{CHAPTER V}

\section{NUMERICAL MODEL}

\section{THEORETICAL DEVELOPMENT}

The numerical program WAMIT (Wave Analysis MIT), developed by the Massachusetts Institute of Technology, was used in this study (Newman 1994; Lee 1995a and 1995b; Newman 1997). It solves a boundary value problem consisting of finding the velocity potential that satisfies the Laplace equation and four linearized boundary conditions for the free surface, bottom, body surface, and the radiation condition at infinity. The numerical solution is the boundary element method based on a three-dimensional, constant panel method (CPM) that determines radiation and diffraction velocity potentials on the body wetted surface using Green's theorem by adjusting the strength of the distributed sources over the panels. The analysis is performed in the frequency domain, with the flow assumed to be ideal and harmonic in time.

In the solution, WAMIT provides the excitation forces and motion amplitudes and phases for a fixed or freely floating body resulting from diffraction and/or diffraction plus radiation potentials. For the laboratory model used in the fundamental tests, fluid loading is estimated for a rigid, fixed RIBS so that only the diffraction solution is provided. For the XM99 field experiment, however, fluid loading for both the diffraction and radiation solution are included because it was moored, somewhat flexible, and able to respond to wave forcing like a wavemaker.

Among the available general purpose boundary element codes for performing linear radiation/diffraction analysis of floating bodies, WAMIT is considered to be a computationally efficient program. The code has been thoroughly benchmarked for accuracy and is becoming an industry standard. It was developed by a consortium composed of industry and academic sponsors including Chevron, Det Norske Veritas, Exxon, Mobil, Naval Surface Warfare Center, Norsk Hydro, Offshore Technology Research Center, Petrobras, Saga, Shell, and Statoil. Krouse (1998) describes the feasibility studies being carried out by McDermott Technology Inc. and the Gulf Coast Regional Maritime Technology Center for the mobile offshore base using 
WAMIT to estimate Response Amplitude Operators (RAO) for the rigid body motions.

Fathi et al. (1994) used WAMIT to calculate the exciting forces and RAO's for a floating flexible container. Zhao and Triantafyllou (1994) used WAMIT to study the hydroelastic response of long flexible tubes in waves. Newman (1994) presented several examples illustrating the capabilities of the WAMIT numerical model for deformable bodies. Included among the examples were bending of a floating barge and motions of two barges connected by a hinge.

Lee (1997) used WAMIT to study the wave-interaction and structural deflections of a very large floating structure (VLFS). The structure was idealized as a barge, floating on shallow water, and approximated as a plate.

\section{Boundary Value Problem}

The fluid is assumed to be inviscid and incompressible and the flow irrotational. Small amplitude, time harmonic motions are assumed to justify linearization and superposition.

Potential theory permits the representation of the velocity field by the gradient of the velocity potential $\varphi$, which must satisfy Laplace's equation in the fluid domain

$$
\nabla^{2} \varphi=0
$$

Linearized boundary conditions on the free surface $z=0$ and bottom $z=-h$ are

$$
\begin{gathered}
\frac{\partial \varphi}{\partial z}=\frac{\omega^{2}}{g} \varphi \\
\frac{\partial \varphi}{\partial z}=0
\end{gathered}
$$

where $\omega$ is incident wave angular frequency and $g$ is gravitational acceleration. The velocity potential of the incident wave is therefore

$$
\varphi_{I}=\frac{i g A}{\omega} K_{p} e^{-i k\left(x \cos \theta_{i}+y \sin \theta_{i}-\omega t\right)}
$$

where $A$ is wave amplitude, $\theta_{i}$ is incident wave angle, $K_{p}$ is the pressure response factor which was defined in Chapter IV, $h$ is water depth, and $k$ is the wave number (positive, real root) which 
satisfies the dispersion relation

$$
\omega^{2}=g k \tanh k h
$$

Linearization permits the decomposition of the total velocity potential $\Phi$ into two components

$$
\Phi=\varphi_{R}+\varphi_{D}
$$

where $\varphi_{R}$ is the radiation potential and $\varphi_{D}$ is the diffraction potential. Similarly, the diffraction potential can be expressed as the combination of the incident wave potential $\varphi_{\mathrm{I}}$ and a scattered potential $\varphi_{\mathrm{S}}$

$$
\varphi_{D}=\varphi_{I}+\varphi_{S}
$$

The scattered potential is due to the disturbance of the incident wave field by the fixed body. Again, by superposition, we can define the radiation potential as

$$
\varphi_{R}=\sum_{j=1}^{6} \xi_{j} \varphi_{j}
$$

where $\xi_{\mathrm{j}}$ are the complex motion amplitudes corresponding to the six rigid-body degrees of freedom of surge, sway, heave, roll, pitch, and yaw in the increasing order of $j$. The $\varphi_{\mathrm{j}}$ are the unit amplitude radiation potentials in each mode in the absence of waves, and refer to the linear or angular velocity of the rigid body motion.

The appropriate boundary conditions for the diffraction problem are imposed on the undisturbed position of the body surface $S_{b}$ as

$$
\frac{\partial \varphi_{S}}{\partial n}=-\frac{\partial \varphi_{I}}{\partial n}
$$

where $\mathbf{n}=\left(n_{1}, n_{2}, n_{3}\right), \mathbf{x} \times \mathbf{n}=\left(\mathrm{n}_{4}, n_{5}, n_{6}\right)$, and $\mathbf{x}=(x, y, z)$. The unit vector $\mathbf{n}$ points out of the fluid domain and is normal to the body boundary and $\mathbf{x}$ is a position on the body boundary. However, since $\varphi_{D}=\varphi_{I}+\varphi_{S}$, this is equivalent to

$$
\frac{\partial \varphi_{D}}{\partial n}=0
$$


The radiation potential $\varphi_{\mathrm{j}}$ is subject to the conditions

$$
\frac{\partial \varphi_{j}}{\partial n}=n_{j}
$$

The final condition to make the boundary value problem unique is to prescribe a radiation condition at infinity. This condition states that any waves on the free surface, other than those due to the incident wave themselves, are due to the body and are radiating and decaying away from the body. The incident wave potential is excluded from this condition. The radiation boundary condition is also known as the Sommerfeld condition.

The boundary value problem is solved in WAMIT using Green's theorem to derive an integral equation for the diffraction and radiation velocity potentials on the body surface. The Green's function or wave source potential $G(\xi, x)$ is the velocity potential at the point $x$ due to a point source of strength $-4 \pi$ located at the point $\xi$. It satisfies the free surface and radiation boundary conditions. Singular components and logarithmic singularities of the Green's function may occur when the source and field points are close to each other and to the free surface. These types of problems are overcome by increasing the number of panels (i.e., decreasing the size of the panels) around the perimeter of the structure and along the free surface. A cosine spacing or finer regular spacing in these areas usually ensures convergence of the discretization scheme.

\section{Inertia Matrix}

For a body in free stable flotation without external constraints (i.e., mooring lines), the mass or inertia matrix can be input in two different ways. In the first method, WAMIT assumes that the body mass $m$ is equal to the mass of displaced water in free floatation and the inertia matrix is not directly input. This method is applicable for the fundamental laboratory experiments where the body is fixed (i.e., diffraction only). In the second method, the values for the inertia matrix are input directly to WAMIT. This method was used for the XM99 field trials. 
The inertia matrix consists of a translation $M_{11}$, rotation $M_{22}$, and interaction $M_{12}$ and $M_{21}$ partition sections. It is defined as

$$
\mathbf{M}=\left(\begin{array}{ll}
M_{11} & M_{12} \\
M_{21} & M_{22}
\end{array}\right)
$$

and the partitioned submatrices are given by

$$
\begin{gathered}
M_{11}=\left(\begin{array}{ccc}
m & 0 & 0 \\
0 & m & 0 \\
0 & 0 & m
\end{array}\right) \\
M_{12}=\left(\begin{array}{ccc}
0 & m z_{g} & -m y_{g} \\
-m z_{g} & 0 & m x_{g} \\
m y_{g} & -m x_{g} & 0
\end{array}\right) \\
M_{21}=\left(\begin{array}{ccc}
0 & -m z_{g} & m y_{g} \\
m z_{g} & 0 & -m x_{g} \\
-m y_{g} & m x_{g} & 0
\end{array}\right) \\
M_{22}=\left(\begin{array}{lll}
I_{11} & I_{12} & I_{13} \\
I_{21} & I_{22} & I_{23} \\
I_{31} & I_{32} & I_{33}
\end{array}\right)
\end{gathered}
$$

where $m$ is the body mass and $\left(x_{g}, y_{g}, z_{g}\right)$ are the coordinates of the center of gravity of the XM99. The moments of inertia $I_{j j}$ are rather complicated for the XM99 and are described in Appendix E.

\section{WAMIT OUTPUT QUANTITIES}

Once the first order solution for the total velocity potential is obtained, WAMIT estimates many different output quantities. Options may be selected for (1) wave transmission coefficients at selected locations in the wave field to evaluate RIBS performance, (2) dynamic pressure distributions on RIBS surfaces for calculating bending loads due to waves, (3) response 
amplitude operators (RAO's) in 6 DOF including surge, sway, heave, roll, pitch, and yaw, (4) excitation forces, and (5) mean drift surge and sway forces and yaw moment used to estimate pretension mooring line forces due to waves. In this study, however, only the wave transmission coefficient and dynamic pressure distributions are investigated.

\section{Wave Transmission Coefficient}

The free-surface elevation is obtained from the dynamic free surface boundary condition using the total velocity potential $\Phi$.

$$
\eta=-\frac{1}{g}\left\{\frac{\partial \Phi}{\partial t}\right\}_{z=0}
$$

The nondimensional form is equivalent to the transmission coefficient and is given by

$$
\bar{\eta}=\frac{\eta}{A}=K_{T}
$$

These values are calculated at each of the specified field points in the fluid domain.

\section{Dynamic Pressure}

The dynamic pressure on the body surface is related to the total velocity potential $\Phi$ by the linearized Bernoulli equation

$$
p=-\rho \frac{\partial \Phi}{\partial t}
$$

The normalized WAMIT pressure is

$$
\overline{p_{W}}=\frac{p}{\rho g A}
$$

where $\rho$ is water density.

The hydrodynamic pressure acting on the central point of each panel consists of components from the incident, scattered, (i.e., diffraction) and radiation potentials. The incident and 
scattered pressures are due to the pressure from the incident waves on the motionless structure. The radiated pressure component is due to the motion of the RIBS in still water.

It is interesting to note that both the wave transmission coefficient and the dynamic pressure are functions of the time derivative of $\Phi$. The only difference is the constant used and evaluation at the surface or $(x, y, z)$ coordinates.

\section{MODEL GENERATION DETAILS}

\section{FLab Model}

Model formulation. The fundamental laboratory model was created with the MultiSurf system for the design of geometric objects in three dimensions developed by AeroHydro, Inc. (1998). It maintains a relational database of points, curves, surfaces, and solids that preserves definitions and dependencies as underlying objects are altered.

The laboratory model was constructed by creating the points which define the starboard leg. Curves connecting these points were then added. The curves were combined to create surfaces, and surfaces to make solids. The starboard leg was then rotated by one-half of the interior angle and translated to place the right-hand WAMIT coordinate system midway between the leg. MultiSurf uses symmetry to create the port leg.

Two- and three-dimensional views of the panel layout for the FL4550 case are shown in Figures 26 and 27, respectively. The origin of the WAMIT coordinate system is halfway back

of the bow on the centerline at the water surface. The positive $x$-axis points to the stern, positive $y$-axis to the starboard leg, and positive $z$-axis upward from the water surface.

The dimensions of the WAMIT model were as close as possible to the actual laboratory model. The length was $9.14 \mathrm{~m}$ and the beam was $12.7 \mathrm{~mm}$. The draft was $11.4 \mathrm{~cm}, 22.9 \mathrm{~cm}$, $34.3 \mathrm{~cm}$, and $45.7 \mathrm{~cm}$ corresponding to the submergence levels of $d / h=0.25,0.50,0.75$, and 1.00. The interior angle for these four models was $45 \mathrm{deg}$. Two additional models were created for the $90 \mathrm{deg}$ interior angle configurations with $d / h=0.50$ and 1.00 .

Equal spacing was selected to simplify bookkeeping of the panel locations. Only one panel with a thickness of $12.7 \mathrm{~mm}$ was used in the transverse direction (i.e., along the beam). Eighty panels with a length of $11.43 \mathrm{~cm}$ were used in the axial direction of each leg. The panel height 


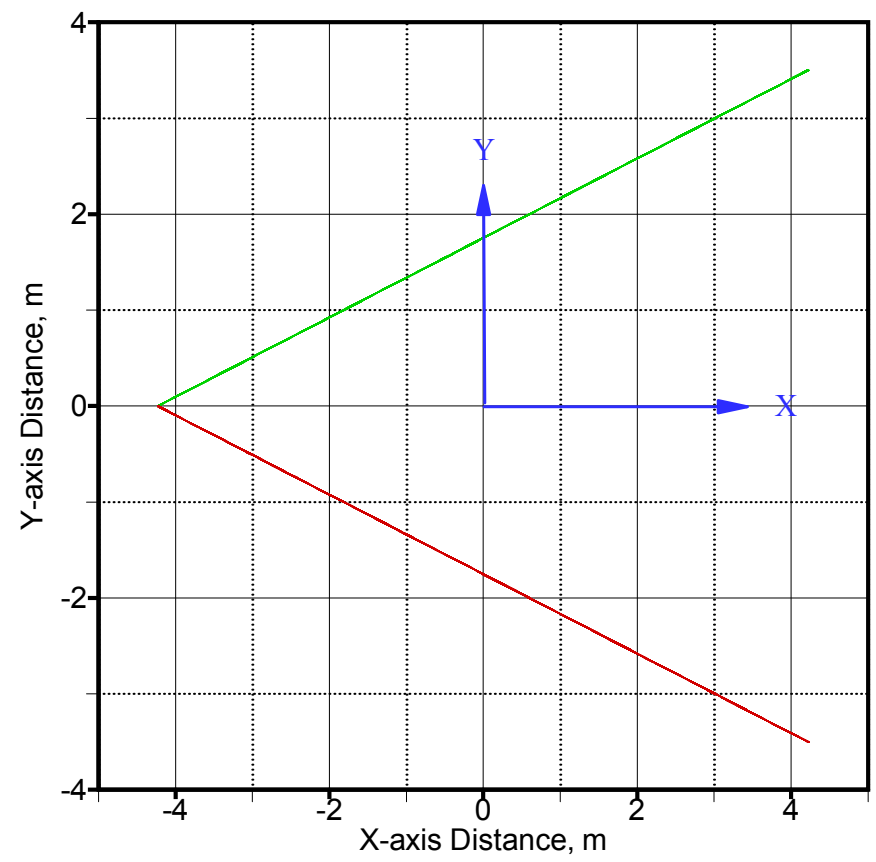

Figure 26. WAMIT panel layout for FLab - 2-D view.

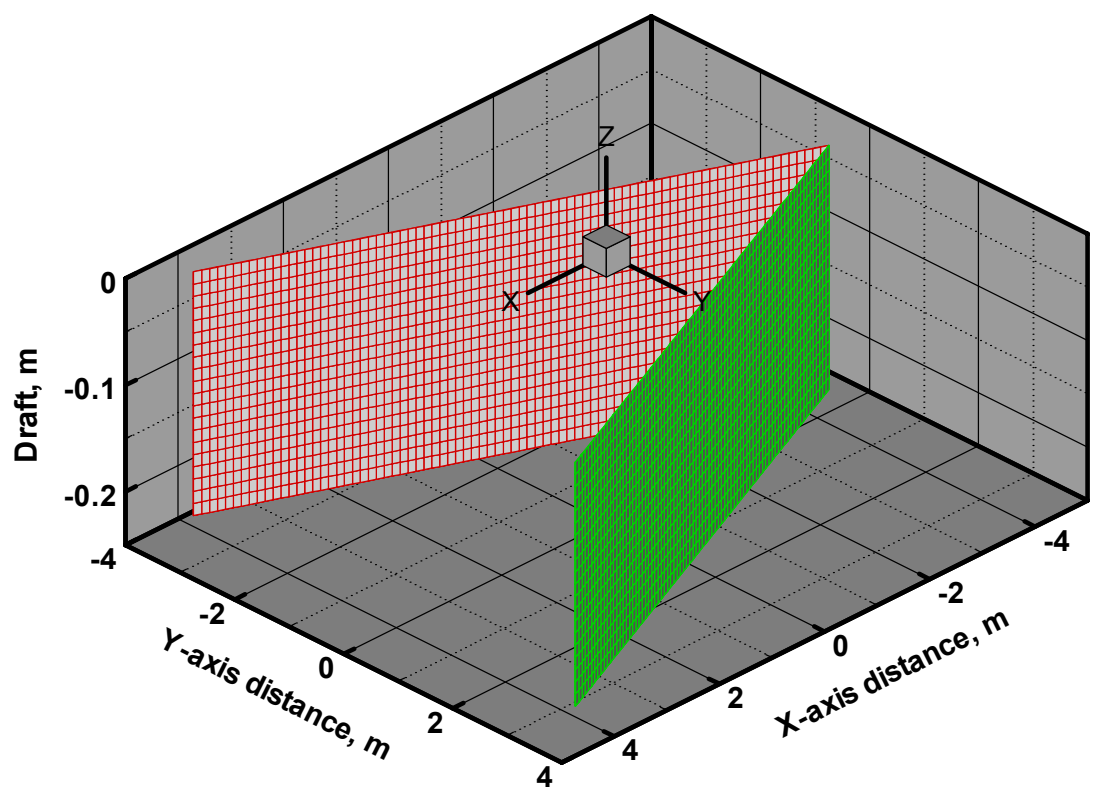

Figure 27. WAMIT panel layout for FLab - 3-D view. 
was fixed at $11.43 \mathrm{~mm}$ for all models and was selected to coincide with the placement of the pressure gages in the laboratory. The number of panels in the vertical direction was a function of the draft with $10,20,30$, and 40 panels for $d / h=0.25,0.50,0.75$, and 1.00 , respectively. Thus, the total number of panels per leg was 1,700, 3,320, 4,940, and 6,560, for the four models respectively.

Wave conditions. Six wave conditions from the laboratory experiment were simulated in the WAMIT runs. Waves were for the three wave periods of $0.88,1.41$, and $2.12 \mathrm{~s}$ (corresponding to 5,8 , and 12 s prototype) and two wave directions of 0 and $20 \mathrm{deg}$ from the center line of the model. In the WAMIT model, wave directions are measured counterclockwise from the $x$ axis.

Convergence tests. Numerical models approximate continuous flow problems with discrete approximations in both the fluid domain and the geometry of the structure. Continuous source and doublet distributions are approximated by constant distributions over panels and curved surfaces are approximated by flat panels. Both should converge to the true solution as the panel subdivision is uniformly refined. The secret here is "uniformly." The refinement should extend to all surface panels and in both directions to expect convergence.

Thus, one of the first steps in a numerical model formulation is to perform a convergence test (Newman and Lee 1992) to optimize the number of panels and the panel size by comparing a numerically predicted parameter against a target or "true" value. The convergence test ensures that an adequate number of panels have been used to properly resolve the boundary of the structure for computational accuracy. The wave transmission coefficient was used to optimize the Fundamental Laboratory models. Once the optimum number of panels was determined, these models were used for all subsequent analyses.

Newman and Lee (1992) recommend a minimum of 6 to 12 panels per wavelength for the smallest wavelength (i.e., highest frequency). The wavelength for the highest peak frequency of $1.14 \mathrm{~Hz}$ corresponding to $0.88 \mathrm{sec}$ was $1.22 \mathrm{~m}$. The panel length of $11.43 \mathrm{~cm}$ is equivalent to almost 11 panels per wavelength. Since the panel height and thickness were fixed at $11.43 \mathrm{~mm}$ and $12.7 \mathrm{~mm}$, respectively, this was thought to provide a good aspect ratio for the panels. Several different models were created with both fewer and greater panels to verify that this model was sufficiently discretized to achieve good resolution. Figure 28 shows the results from this convergence test series. 


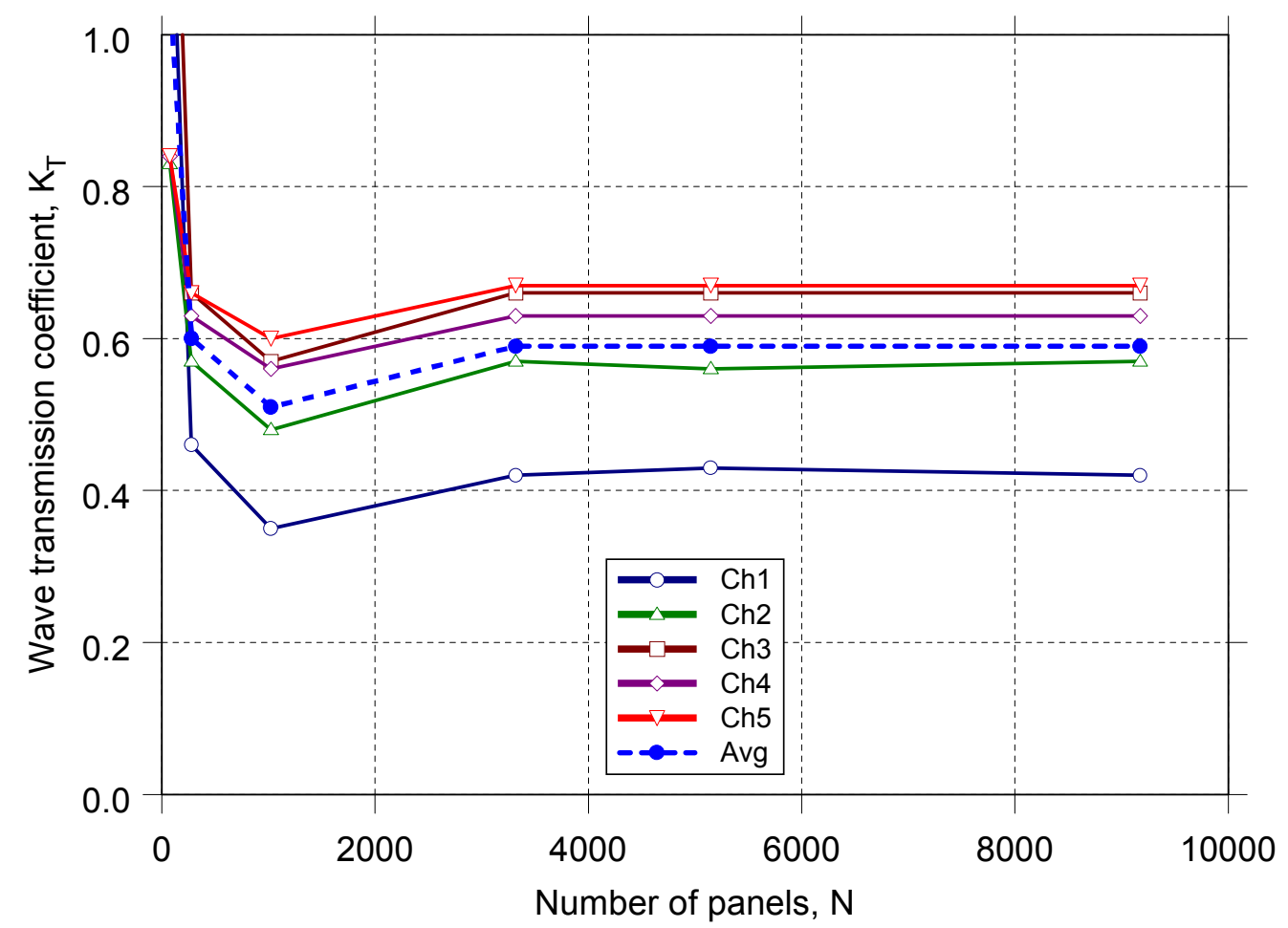

Figure 28. FLab convergence test.

Based on the results from this convergence test, the WAMIT models for all $d / h$ configurations were configured so that they had 80 panels in the longitudinal or axial directional, 1 panel across the beam, and 10 to 40 panels in the $z$-axis direction. Table 9 lists the WAMIT parameters for the different models including panel arrangement, total number $N$ of panels in each leg, panel length (axial direction), and panel height (z-direction).

\section{XM99 Model}

Model formulation. From a hydrodynamic point of view, the XM99 structure is relatively simple and can be modeled as a flat, rectangular barge using the program BARGEN (barge generator) pre-processing code supplied by MIT. Because of symmetry, BARGEN only requires input for the quarter model (i.e., quadrant) for each leg. Since only one leg of the RIBS is created, the program TRANSFORM rotates, shifts, and images the BARGEN single leg model to the full, two-legged RIBS model with the proper interior angle. 
TABLE 9. WAMIT FLab Model Parameters

\begin{tabular}{c|c|c|c|c|c|c}
\hline \hline \multirow{2}{*}{ Code } & $d / h$ & $\begin{array}{c}\text { Draft } d \\
(\mathrm{~cm})\end{array}$ & Panels & $N$ & $\begin{array}{c}x \text {-axis } \\
(\mathrm{cm})\end{array}$ & $\begin{array}{c}z \text {-axis } \\
(\mathrm{mm})\end{array}$ \\
\hline \multicolumn{7}{c}{$(\mathrm{a})$ 45 deg Interior Angle } \\
\hline 4525 & 0.25 & 11.43 & $80 \times 10$ & 1700 & 11.4 & 11.4 \\
\hline 4550 & 0.50 & 22.86 & $80 \times 20$ & 3320 & 11.4 & 22.9 \\
\hline 4575 & 0.75 & 34.29 & $80 \times 30$ & 4940 & 11.4 & 34.3 \\
\hline 45100 & 1.00 & 45.72 & $80 \times 40$ & 6560 & 11.4 & 45.7 \\
\hline & & $($ b) 90 deg Interior Angle & & \\
\hline 9050 & 0.50 & 22.86 & $80 \times 20$ & 3320 & 11.4 & 22.9 \\
\hline 90100 & 1.00 & 45.72 & $80 \times 40$ & 6560 & 11.4 & 45.7 \\
\hline \hline
\end{tabular}

Two- and three-dimensional views of the panel layout are shown in Figures 29 and 30, respectively. Analogous to the coordinate system for the FLab model, the WAMIT coordinate system for the XM99 model was located with the origin at the surface and centered along the longitudinal center line, halfway from the bow of the structure.

The dimensions of the WAMIT model of the XM99 were as close to the field experiment prototype as possible. The model had leg lengths of $76.2 \mathrm{~m}$, beam of $2.44 \mathrm{~m}$, and draft of 7.32 $\mathrm{m}$. The two legs were connected with an interior angle of $60 \mathrm{deg}$. Again, a criteria of a minimum of 6 to 12 panels per wavelength was used. Each leg (i.e., sides, ends, and bottom) was discretized with 2,596 panels using 50 longitudinal, 2 transverse, and 24 vertical panels with equal spacing. Thus, the panels were $1.52 \mathrm{~m}$ long, $1.22 \mathrm{~m}$ wide, and $30 \mathrm{~cm}$ high. The longitudinal panel length of $1.52 \mathrm{~m}$ corresponds to a minimum of 9.2 panels per wavelength for the shortest wavelength of $14.0 \mathrm{~m}$ at $T=3 \mathrm{sec}$. The leg length is equivalent to 5.4 wavelengths for this 3-sec wave period. The corresponding values for the longest wave at a 12-sec wave period (128.9 m wavelength) are 84.7 panels per wavelength and leg length of 0.6 times the wavelength. Equal spacing was selected to enhance bookkeeping since the panels were made small enough near the surface in the convergence tests (see below) to resolve high frequency (i.e., short wavelength) wave components. 


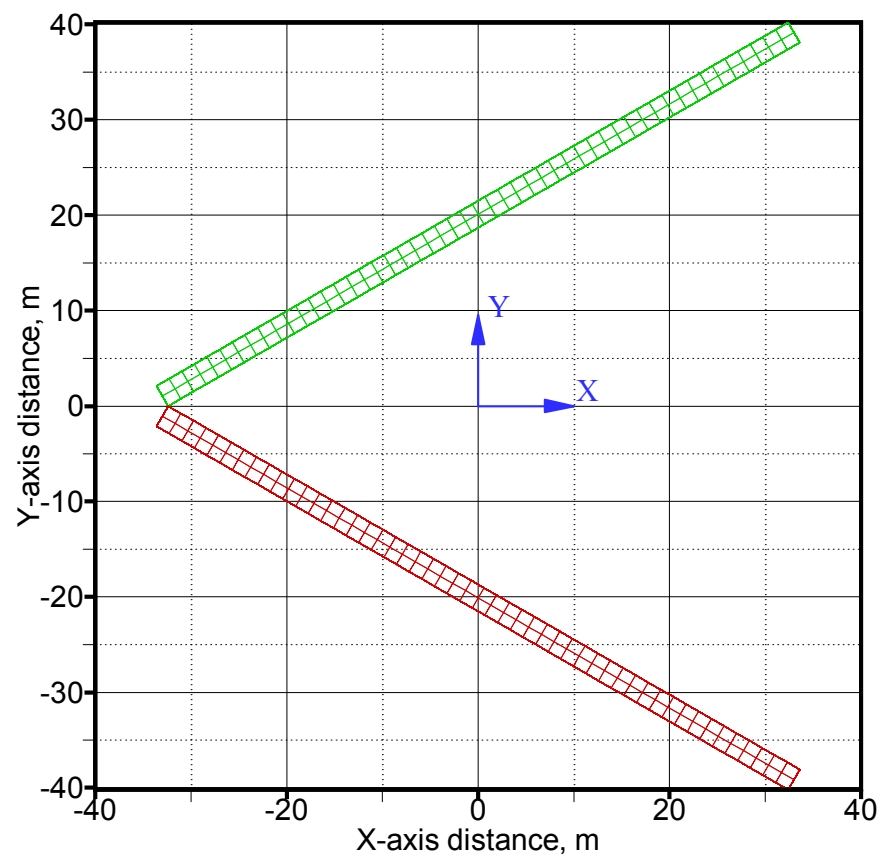

Figure 29. WAMIT panel layout for XM99 - 2-D view.

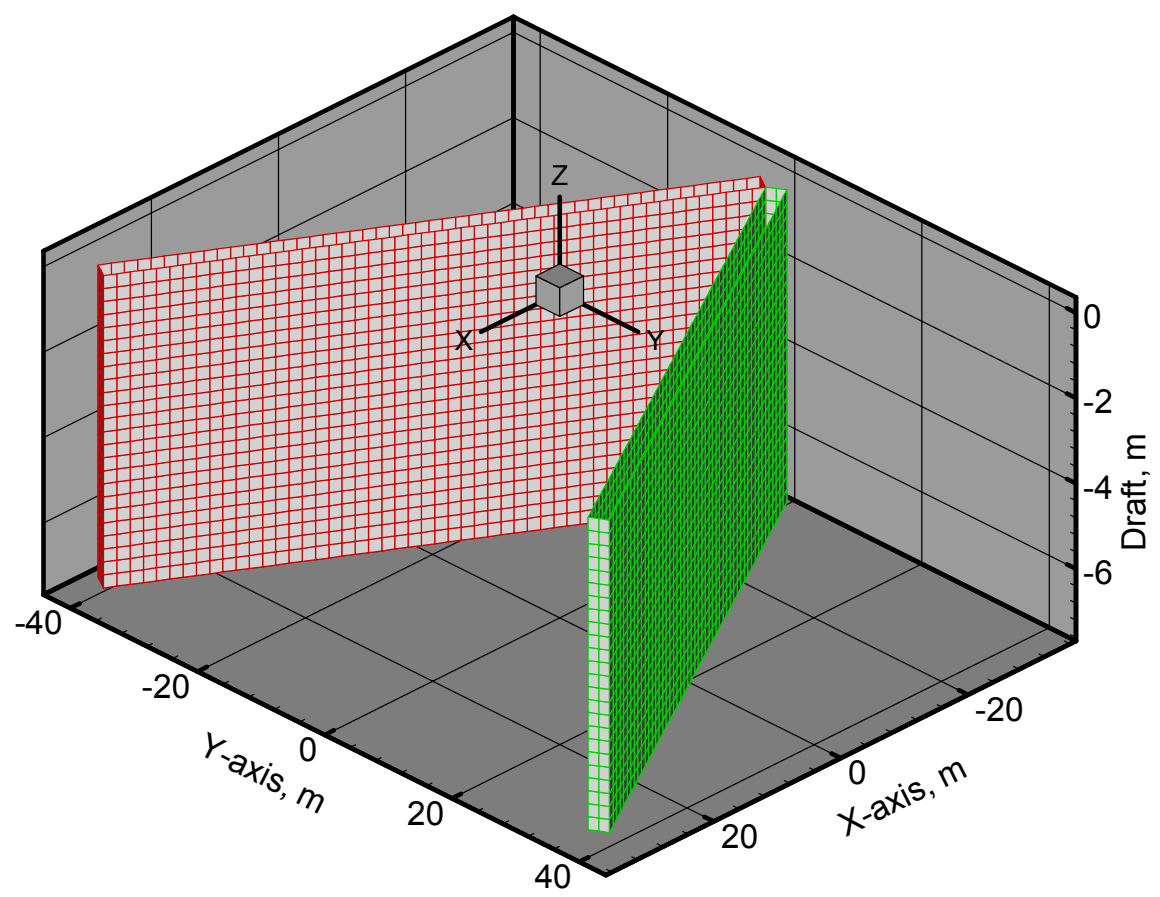

Figure 30. WAMIT panel layout for XM99 - 3-D view. 
Wave conditions. Wave conditions were selected to cover a range of wave periods and wave directions, representative of SS3 and those likely to occur during the deployment. Ten wave periods from 3 to $12 \mathrm{sec}$ were modeled. The "design" orientation of the XM99 was for the nose or bow to point to $135 \mathrm{deg}$ so that waves traveling towards $315 \mathrm{deg}$ (i.e., from $135 \mathrm{deg}$ ) were aligned with the center line or $x$-axis. Since wave directions are measured counterclockwise from the $x$-axis, waves with directions parallel to the center line of 0 deg (i.e., $315 \mathrm{deg}$ ), parallel to the starboard leg of $30 \mathrm{deg}$ (i.e., $285 \mathrm{deg}$ ), and normal to the port leg of $60 \mathrm{deg}$ (i.e., $255 \mathrm{deg}$ ) were simulated. Because of symmetry, only the half plane was modeled. Table 10 lists the correspondence between the global, the analytical body-fixed, and the WAMIT coordinate systems. The operating window of the XM99 was 120 deg, clockwise from 255 to 15 (i.e., 375) deg.

TABLE 10. Incident Wave Angles: Global, Analytical, and Numerical

\begin{tabular}{c|c|c|c}
\hline \hline Compass, deg & Analytical, deg & WAMIT, deg & Comment \\
\hline $15(375)$ & 0 & -60 & Normal to starboard leg \\
\hline 345 & 30 & -30 & \\
\hline 315 & 60 & 0 & Along center line \\
\hline 285 & 90 & 30 & Normal to port leg \\
\hline 255 & 120 & 60 &
\end{tabular}

\section{WAMIT EXECUTION}

WAMIT consists of two main modules, POTEN and FORCE. The POTEN module solves for the velocity potentials on each panel of the body for each component of the radiation and diffraction problem for all wave frequencies and headings. The FORCE module evaluates the hydrodynamic coefficients, motions, and first and second-order forces. Velocities and pressures on the body surface and at specified field points in the fluid domain can be estimated. 


\section{CHAPTER VI}

\section{WAVE TRANSMISSION COEFFICIENTS}

In this Chapter, wave transmission coefficients $K_{T}$ are calculated and presented for the fundamental laboratory experiments (FLab) and the XM99 field trials. These values are compared with the predictions of the two analytical models and the WAMIT numerical model. Empirical relationships between $K_{T}$ and several different nondimensional parameters are then calculated and discussed.

\section{FUNDAMENTAL LABORATORY EXPERIMENTS}

\section{Calculation Procedure}

As previously noted in Chapter IV, the wave transmission coefficient $K_{T}$ is given by

$$
K_{T}=\frac{H_{T}}{H_{I}}
$$

where $H_{I}$ is the incident wave height and $H_{T}$ is the transmitted wave height. In the case of the FLab, $H_{I}$ can be based on a zero moment wave height $H_{m 0}$ or a monochromatic (i.e., regular) wave height $\bar{H}$. The $H_{m 0}$ wave height is defined as

$$
H_{m 0}=4 \sqrt{m_{0}}
$$

where the zeroth moment $m_{0}$ is defined over all frequencies as

$$
m_{0}=\sum S(f) \Delta f
$$

The $\bar{H}$ wave height is related to $H_{m 0}$ by

$$
\bar{H}=\frac{H_{m 0}}{\sqrt{2}}
$$




\section{Measured Wave Transmission}

Spectral cases. Figure 31 shows the measured $K_{T}$ for the FLab. It is plotted versus the nondimensional parameter $D_{b} / L_{r}$, where $D_{b}$ is the distance of each gage from the bow of the RIBS and $L_{r}$ is the length of each RIBS leg. The first four frames (i.e., a to d) are for the four relative drafts $d / h=0.25,0.50,0.75$, and 1.00 of the model with the $\theta_{r}=45 \mathrm{deg}$ interior angle, respectively. The last two frames (i.e., e and f) are for the two $d / h=0.50$ and 1.00 of the model with the $\theta_{r}=90 \mathrm{deg}$ interior angle. Each frame contains six plots for the six wave conditions corresponding to prototype wave period $T=5$ (triangle), $T=8$ (square), and $T=12$ (circle) sec and mean wave direction $\bar{\theta}=0$ (solid symbol) and $\bar{\theta}=20$ (open symbol) deg. The solid lines represent the $\bar{\theta}=0 \mathrm{deg}$ and the dashed lines the $\bar{\theta}=20 \mathrm{deg}$ cases. A complete listing of the measured $K_{T}$ values from the FLab are contained in Appendix Table F1.

As expected, the $K_{T}$ decreases as wave period $T$ decreases and relative draft $d / h$ increases. The threshold level of $K_{T}=0.5$ is achieved for the SS3, $T=5 \mathrm{sec}$ cases for $d / h>0.25$. For the $T=8$ sec cases, this level is only achieved when $d / h>0.50$. For the $T=12 \mathrm{sec}$ cases, only when the RIBS is sitting on the bottom (i.e., $d / h=1.00$ ) is this threshold level attained. At this relative draft, all wave conditions are less than the threshold value.

In general, the $K_{T}$ are fairly uniform with distance in the lee of the RIBS. There is a tendency to increase slightly as the distance increases, especially as the distance exceeds the leg length (i.e., $D_{b} / L_{r}>1$ ).

Nakamura et al.(1985) found that there was very little difference in $K_{T}$ for $\bar{\theta}<40 \mathrm{deg}$ from the normal to a single leg floating breakwater. Both the $\bar{\theta}=0$ and $20 \mathrm{deg}$ cases are beyond this range, making a larger oblique angle to the RIBS legs. The $\bar{\theta}=0$ deg cases make an angle of $67.5 \mathrm{deg}$ to the normal of both legs and the $\bar{\theta}=20 \mathrm{deg}$ cases an angle of $47.5 \mathrm{deg}$ to the starboard leg. Thus, the $\bar{\theta}=20 \mathrm{deg}$ cases are almost parallel to the port leg and more normal to the starboard leg than the $\bar{\theta}=0$ deg cases. In the FLab experiments, the $\bar{\theta}=20 \mathrm{deg}$ cases (i.e., dashed lines) had up to a 10 percent variation when $T<12$ sec. For example, in Figure $31 \mathrm{~b}$ for the $T=5 \mathrm{sec}$ case at $\theta_{r}=45 \mathrm{deg}$ and $d / h=0.50$, the $K_{T}$ are 2 percent smaller on average. For the $T=8 \mathrm{sec}$ case, the $K_{T}$ is approximately 10 percent smaller. Some of this decrease in $K_{T}$ is probably due to an increase in the reflection coefficient $K_{R}$ because the wave is more normal to the RIBS leg. 


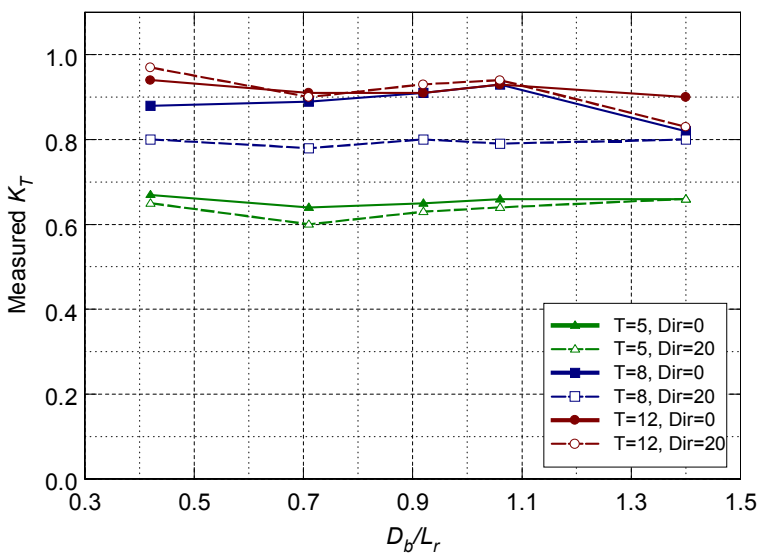

a) 45 deg interior angle, 25 percent draft.

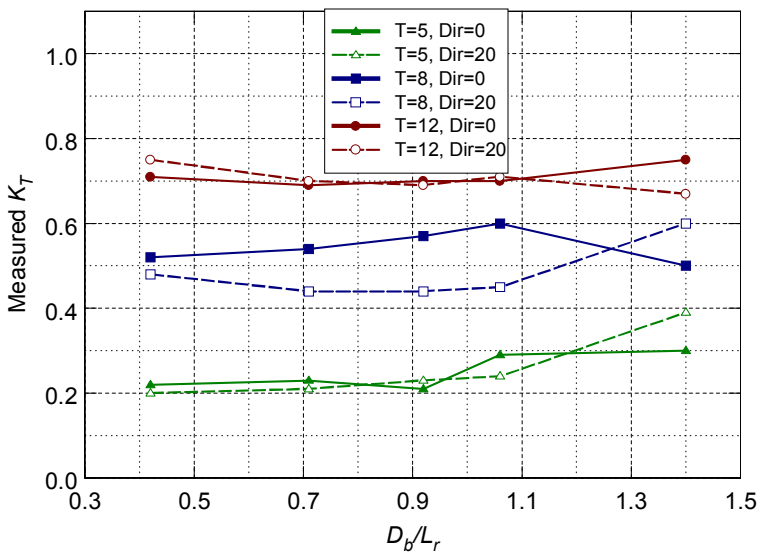

c) 45 deg interior angle, 75 percent draft.

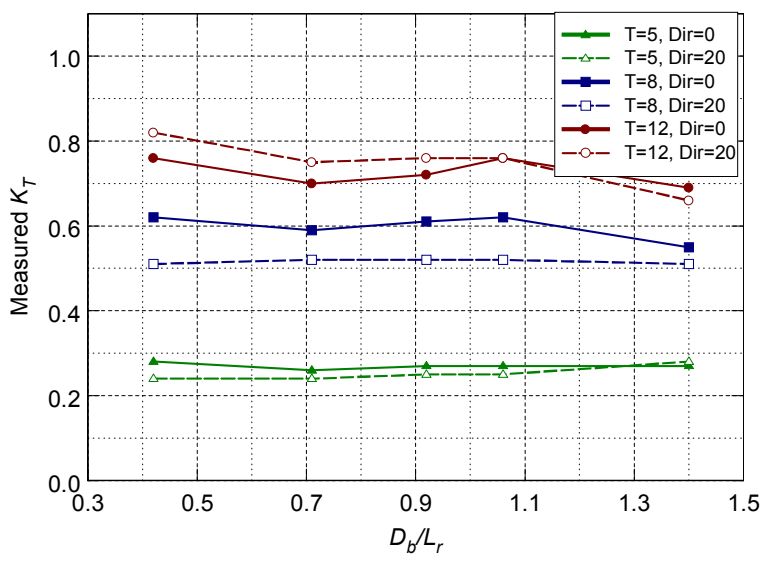

e) 90 deg interior angle, 50 percent draft.

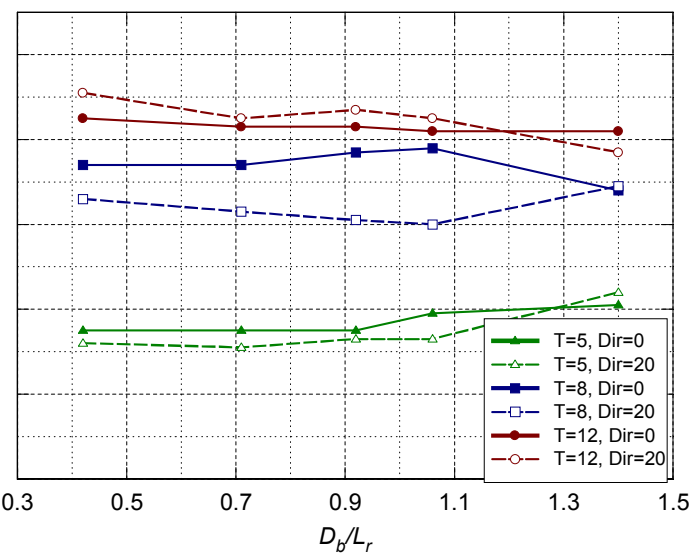

b) $45 \mathrm{deg}$ interior angle, 50 percent draft.

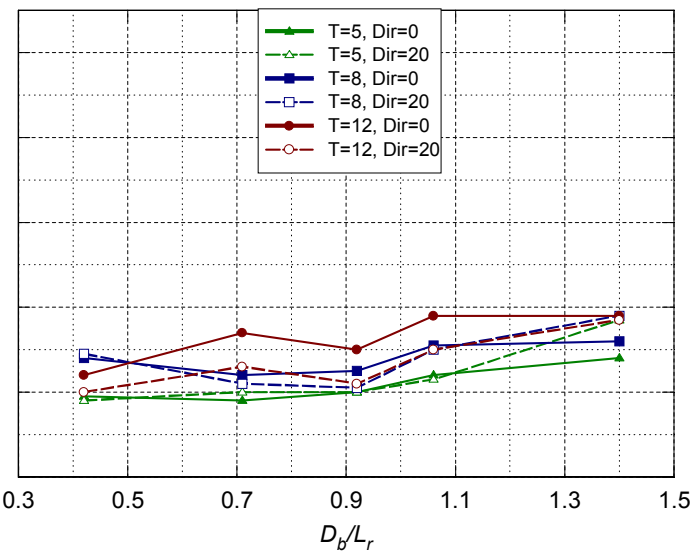

d) $45 \mathrm{deg}$ interior angle, 100 percent draft.

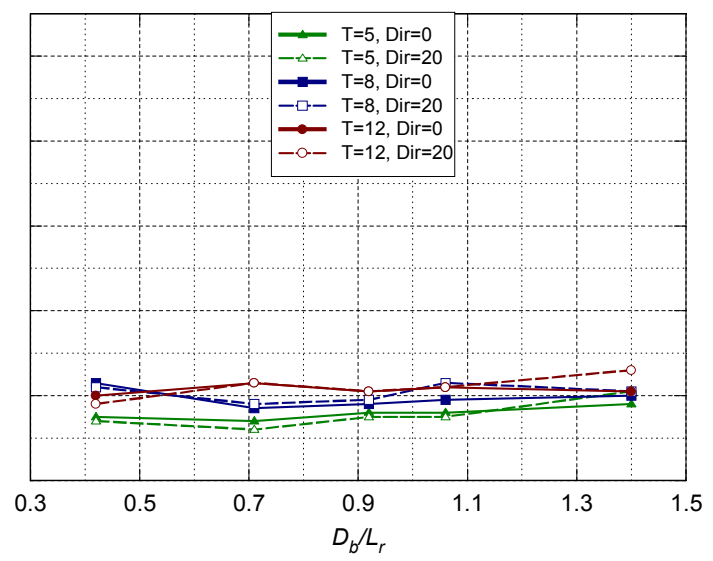

f) 90 deg interior angle, 100 percent draft.

Figure 31. Measured $K_{T}$ for FLab, $\theta_{r}=45 \mathrm{deg}$ (a) $d / h=0.25$, (b) $d / h=0.50$, (c) $d / h=0.75$, (d) $d / h=1.00$, and $\theta_{r}=90 \mathrm{deg}$ (e) $d / h=0.50$, (f) $d / h=1.00$. 
Finally, it was observed that the performance improves as the interior angle of the RIBS is increased from $\theta_{r}=45 \mathrm{deg}$ to $90 \mathrm{deg}$. An explanation is that as the RIBS interior angle increases, it becomes relatively longer in the transverse direction and more like a traditional breakwater. For the $d / h=0.50$ cases, the $K_{T}$ is 10 and 14 percent smaller for the $T=5$ and $8 \mathrm{sec}$ cases, respectively. The $K_{T}$ are even smaller and more tightly grouped together when the RIBS sits on the bottom.

Monochromatic cases. A limited number of monochromatic or regular wave cases were run for the interior angle of $\theta_{r}=45 \mathrm{deg}$ at the relative draft of $d / h=0.50$. Figure 32 compares the measured $K_{T}$ for the six monochromatic cases with their spectral counterparts. The spectral curves are the same as shown in the previous figure. The monochromatic values are shown as larger symbols to facilitate comparison.

Yamamoto (1981) found that regular waves could be used to approximate irregular waves in the estimation of transmission coefficients. In the FLab experiments, the monochromatic $K_{T}$ values were higher than the corresponding spectral values, except for the $T=5 \mathrm{sec}, \bar{\theta}=20 \mathrm{deg}$ case. For this case, the monochromatic $K_{T}$ follow the trends of the spectral $K_{T}$ very well. The

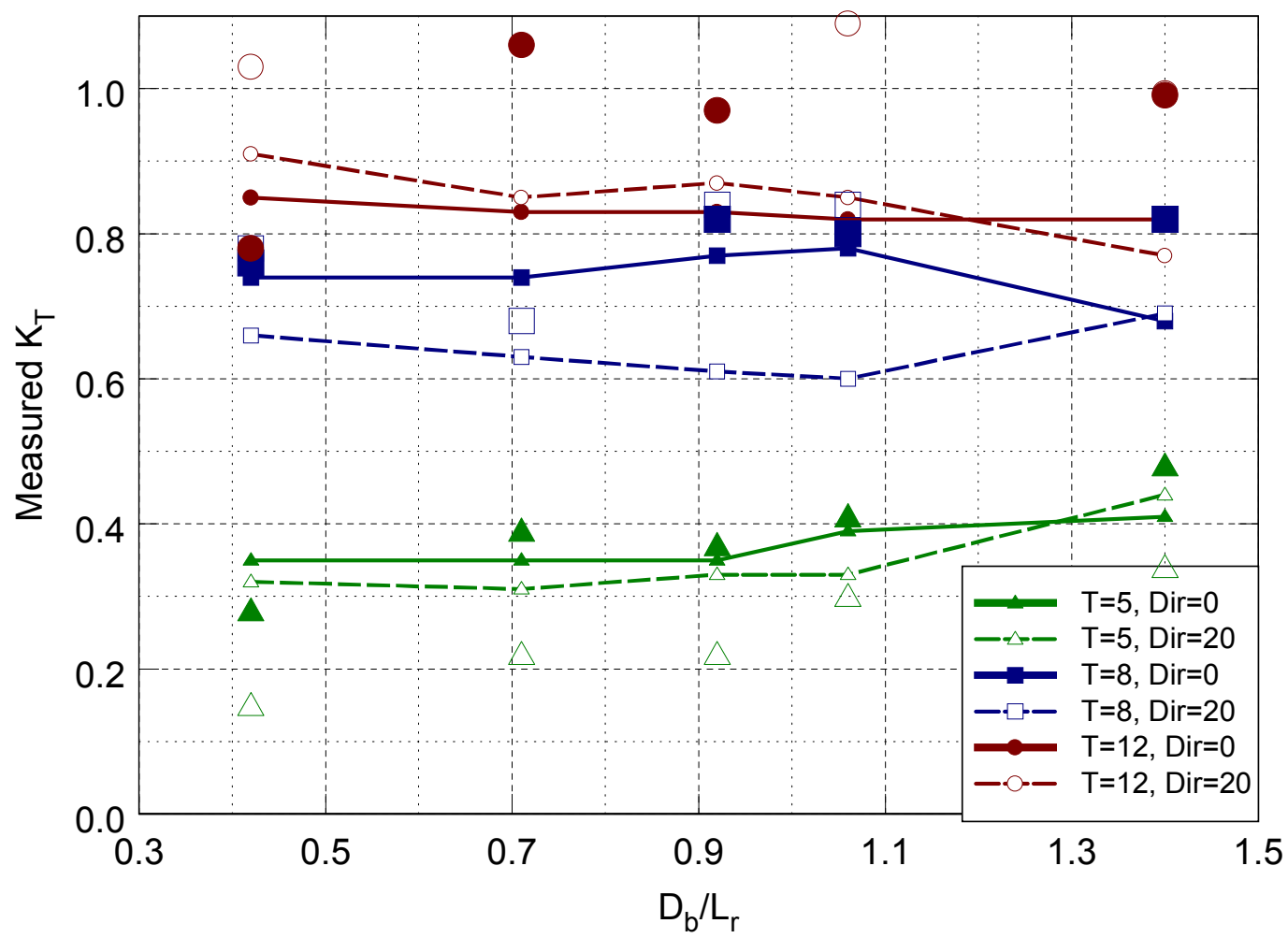

Figure 32. Comparison of monochromatic and spectral $K_{T}$ values for $d / h=0.50$ and $\theta_{r}=45 \mathrm{deg}$. 
variation in $K_{T}$ tended to increase as $T$ increased. However, the agreement between regular and irregular waves is good for the $T<6 \mathrm{sec}$ cases, which corresponds to the design limit for the RIBS.

Comparison of SS3 and SS5 cases. The $T=12 \mathrm{sec}$ cases are actually in the SS5 regime. This sea state is the survival "design" condition for the RIBS and has a wave height upper limit of $3.66 \mathrm{~m}$. Therefore, a limited number of cases were run to investigate the effect of this sea state on the RIBS performance. Since the wave height is larger, these conditions would also quantify the effect of increased wave height $H$ and wave steepness $H / L$ on the performance characteristics. Figure 33 illustrates these effects for the six combinations of interior angle $\theta_{r}$ and relative draft $d / h$. Each panel consists of six plots. The first two plots are for the SS3 wave conditions at the two wave directions and are the same as shown earlier. The next two plots are for the corresponding SS5 wave conditions. The last two plots (symbols only) show the difference in $K_{T}$ values between SS3 and SS5 wave conditions. The absolute value of these differences was taken, so all values are positive.

For all the plots, the differences in $K_{T}$ between SS3 and SS5 are very small. In general, the SS5 values are slightly smaller than the corresponding SS3 values. The minimum and maximum differences are -0.05 and 0.15 for all cases. The corresponding averages and the standard deviations are 0.02 and 0.04 . One would expect the SS5 cases to have slightly larger $K_{T}$ than the corresponding SS3 cases. The larger SS5 cases did experience some minor breaking, which explains some of this variation.

In summary, the RIBS should be positioned so that the relative draft is greater than 50 percent of the water depth. An interior angle greater than $45 \mathrm{deg}$ is desirable, given the tradeoff with mooring forces. Head seas will probably provide a conservative design as the RIBS tended to perform as well or better with a small angle in the mean wave direction.

\section{WAMIT Predicted Wave Transmission}

The predicted $K_{T}$ wave transmission coefficients from the WAMIT models are shown in Figure 34. The format of this figure is the same as the previous figures. In general, the WAMIT predictions were higher than the measured values. They also showed more variability as a 


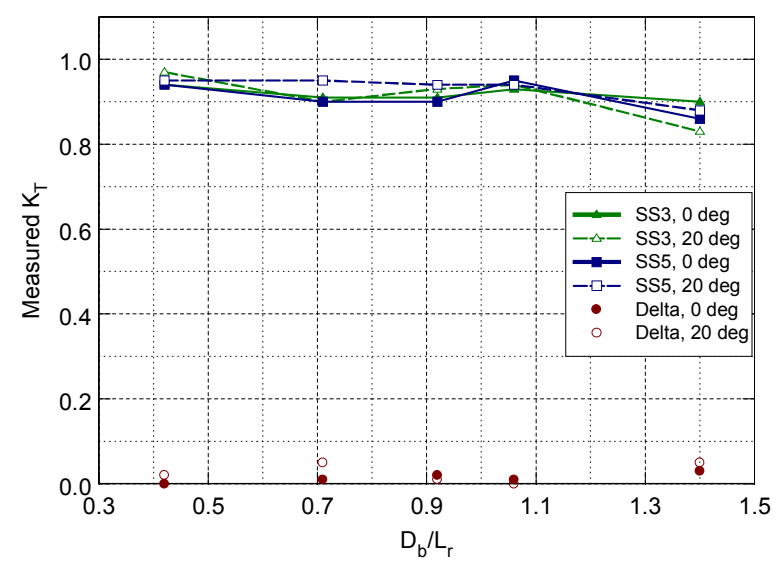

a) 45 deg interior angle, 25 percent draft.

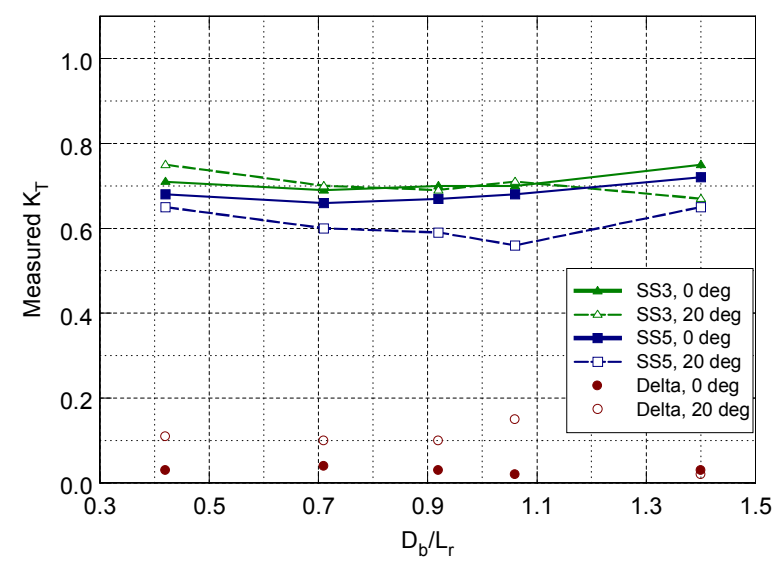

c) 45 deg interior angle, 75 percent draft.

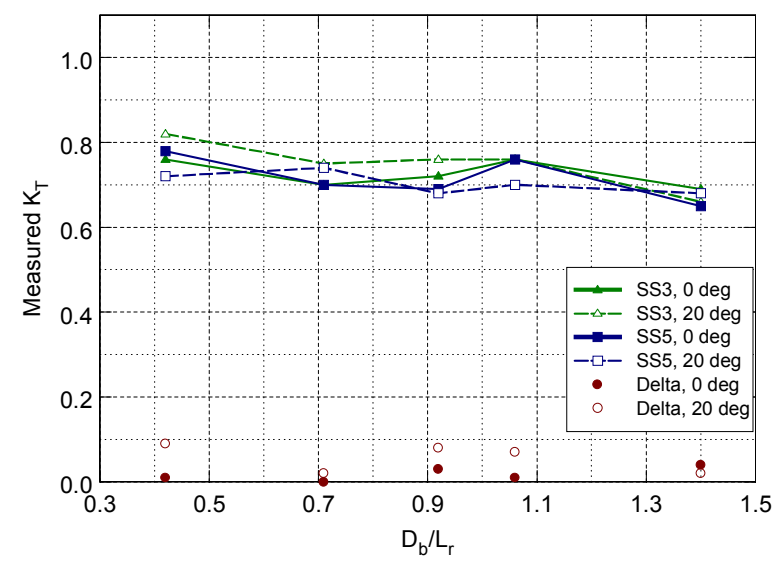

e) 90 deg interior angle, 50 percent draft.

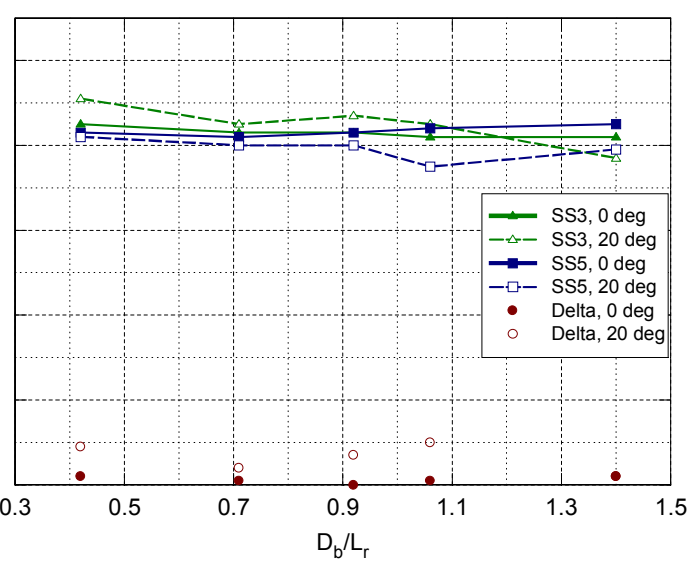

b) 45 deg interior angle, 50 percent draft.

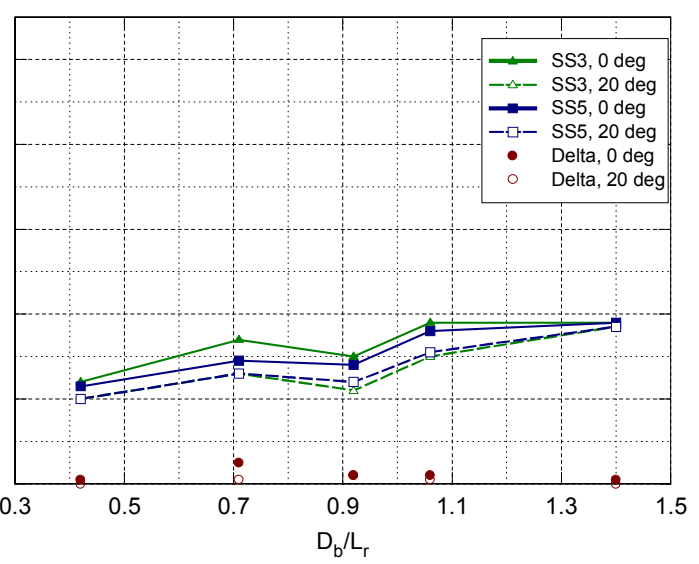

d) 45 deg interior angle, 100 percent draft.

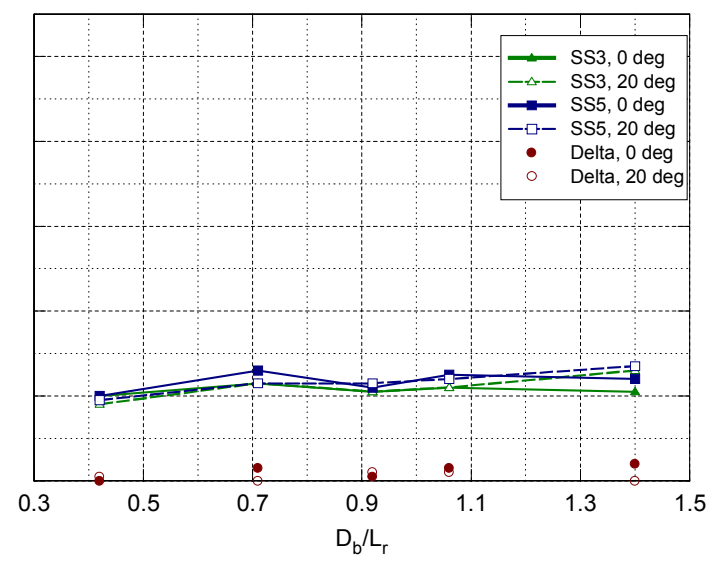

f) 90 deg interior angle, 100 percent draft.

Figure 33. Effect of wave height on $K_{T}$ for FLab with $T_{p}==12 \mathrm{sec}, \theta_{r}=45 \mathrm{deg}$ (a) $d / h=0.25$, (b) $d / h=0.50$, (c) $d / h=0.75$, (d) $d / h=1.00$, and $\theta_{r}=90 \mathrm{deg}$ (e) $d / h=0.50$, (f) $d / h=1.00$. 


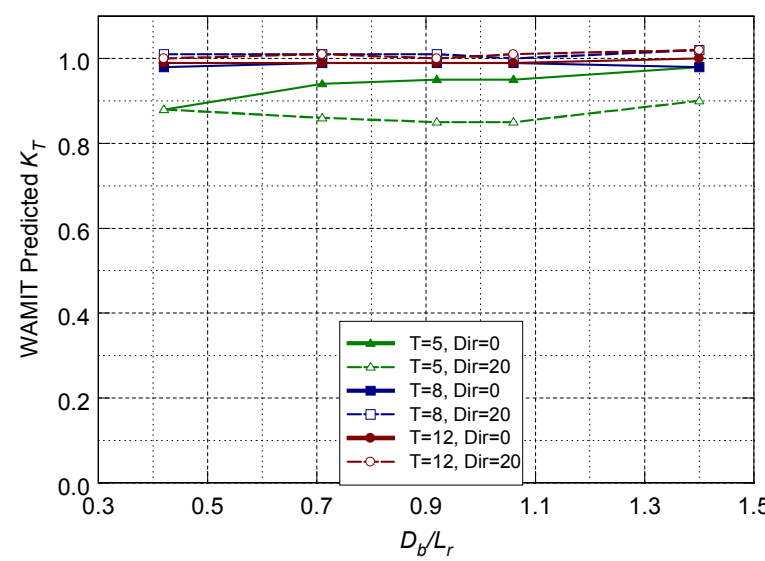

a) 45 deg interior angle, 25 percent draft.

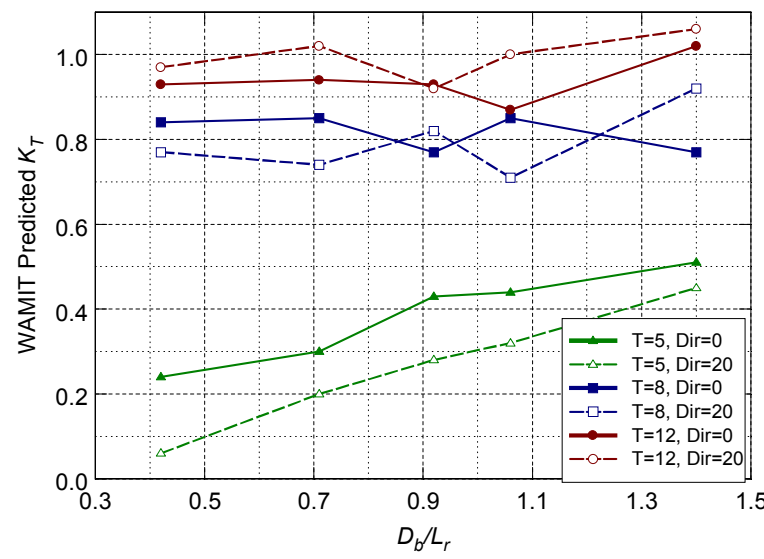

c) 45 deg interior angle, 75 percent draft.

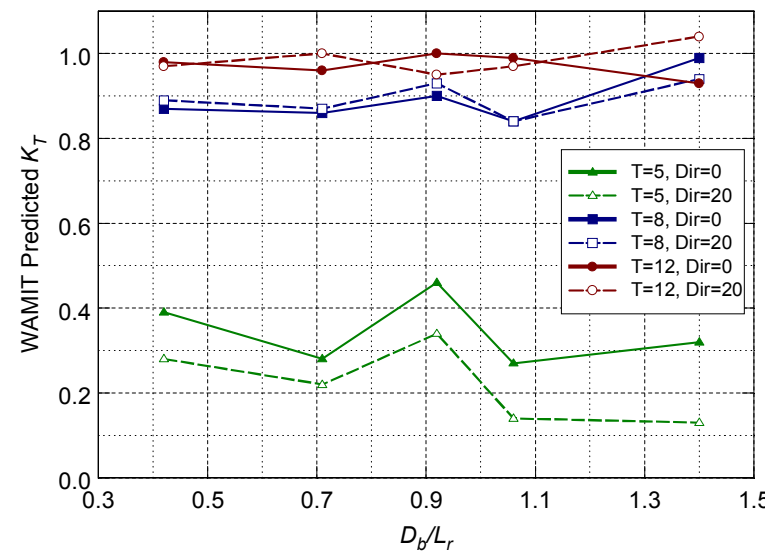

e) 90 deg interior angle, 50 percent draft.

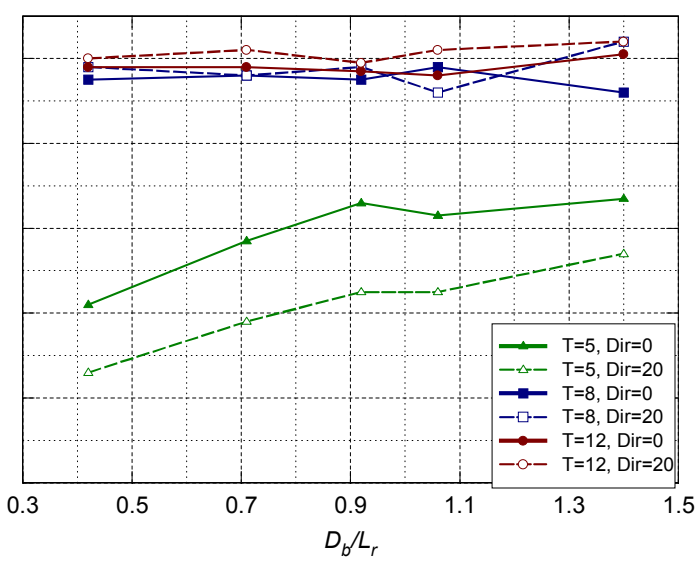

b) 45 deg interior angle, 50 percent draft.

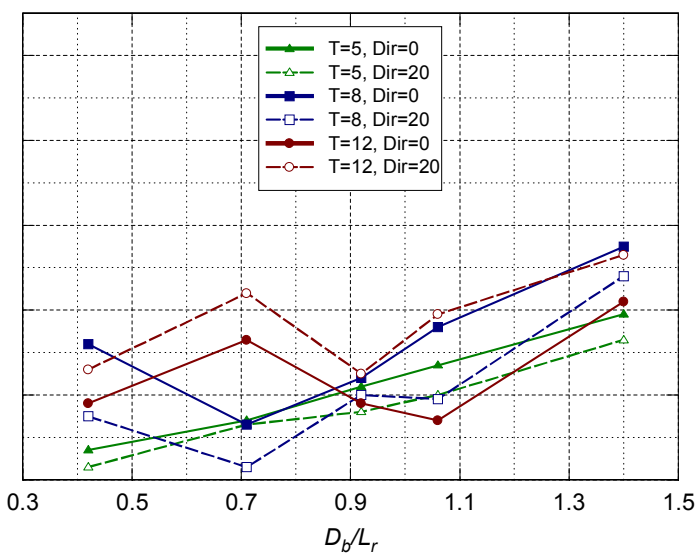

d) 45 deg interior angle, 100 percent draft.

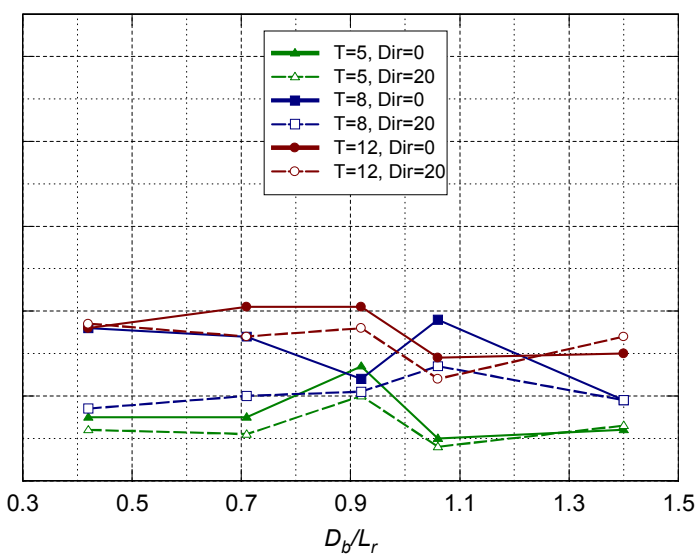

f) 90 deg interior angle, 100 percent draft.

Figure 34. WAMIT $K_{T}$ predictions for FLab, $\theta_{r}=45 \operatorname{deg}$ (a) $d / h=0.25$, (b) $d / h=0.50$, (c) $d / h=0.75$, (d) $d / h=1.00$, and $\theta_{r}=90 \mathrm{deg}$ (e) $d / h=0.50$, (f) $d / h=1.00$. 
function of $D_{b} / L_{r}$ (i.e., from gage to gage position). Numerical models tend to show more oscillation due to nodes and antinodes within the wave field. The WAMIT predicted $K_{T}$ values are listed in Appendix Table F2.

The averages over all gages of the measured and predicted $K_{T}$ values for each of the six $\theta_{r}$ and $d / h$ combinations are plotted against each other in Figure 35. A 45-deg line is also shown for easier observation of a 1:1 correlation between predicted and measured values. In general, the WAMIT predictions are slightly larger than the measured $K_{T}$, especially for the larger values.

Table 11 lists some statistics for the average WAMIT, average measured, and $\Delta K_{T}$ values. These statistics include the minimum, maximum, average, and standard deviation and are based on 36 values (i.e., 6 configurations and 6 wave conditions). The $\Delta K_{T}$ value was obtained by subtracting the measured $K_{T}$ from the WAMIT $K_{T}$ value. Thus, the average WAMIT $K_{T}$ was 0.13 higher than the corresponding measured $K_{T}$. An explanation is that WAMIT is based on potential flow and does not have the viscosity losses that the laboratory and prototype RIBS would experience.

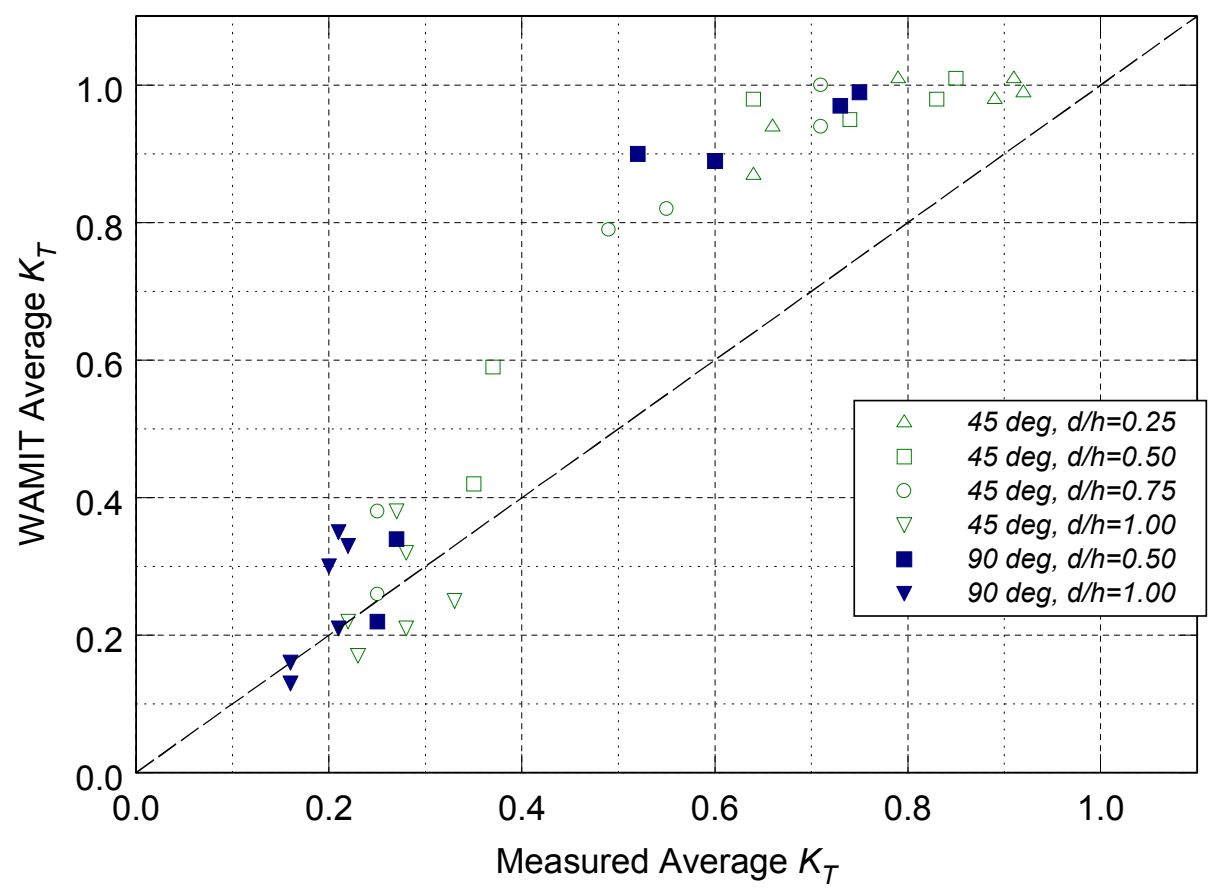

Figure 35. Comparison of WAMIT vs. measured $K_{T}$ for FLab. 
TABLE 11. WAMIT and Measured $K_{T}$ Statistics

\begin{tabular}{c|c|c|c}
\hline \hline Statistic & WAMIT $K_{T}$ & Measured $K_{T}$ & $\Delta K_{T}$ \\
\hline Minimum & 0.13 & 0.16 & -0.08 \\
\hline Maximum & 1.01 & 0.92 & 0.38 \\
\hline Average & 0.62 & 0.48 & 0.13 \\
\hline Standard Deviation & 0.34 & 0.26 & 0.13 \\
\hline \hline
\end{tabular}

\section{Analytical Model Comparisons}

Comparisons of the measured average $K_{T}$ versus the transmission factor $T_{F}$ in the PTT and MPTT analytical models are presented in Figure 36. The eight wave cases are plotted with unique symbols for each of the six $\theta_{r}$ and $d / h$ configurations. In general, measured $K_{T}$ is always greater than $T_{F}$. The second order polynomial trend line from the regression analysis for the entire data set is very good, with an $R^{2}=0.92$. The equation for this least squares fit line is given by

$$
K_{T}=a_{0}+a_{1} x+a_{2} x^{2}
$$

where $x$ is a dummy variable representing the independent nondimensional variable. Table 12 lists the square of the correlation coefficient $R^{2}$, and $a_{0}, a_{1}$, and $a_{2}$ coefficients of this trend line. The 95 percent confidence limits are also shown for this least squares regression analysis. The closeness of these confidence limits is an indication of the very good fit of the predicted line to the data.

Also plotted on this graph are the analytical PTT and MPTT model predictions corresponding to each laboratory data point. The PTT model predictions are larger than the MPTT model and a closer fit to the measured data. Both analytical models are smaller than the measured average $K_{T}$ values, but the PTT values are within the confidence limits. At $T_{F}=0$, both PTT and MPTT predict $K_{T}=0$ when the RIBS is on the bottom (i.e., $d / h=1.00$ ), whereas the measured $K_{T}$ have an intercept of 0.23. An explanation for this is that diffraction causes some transmission around the ends of the RIBS model even when it is on the bottom. The $T_{F}$ parameter is constant 


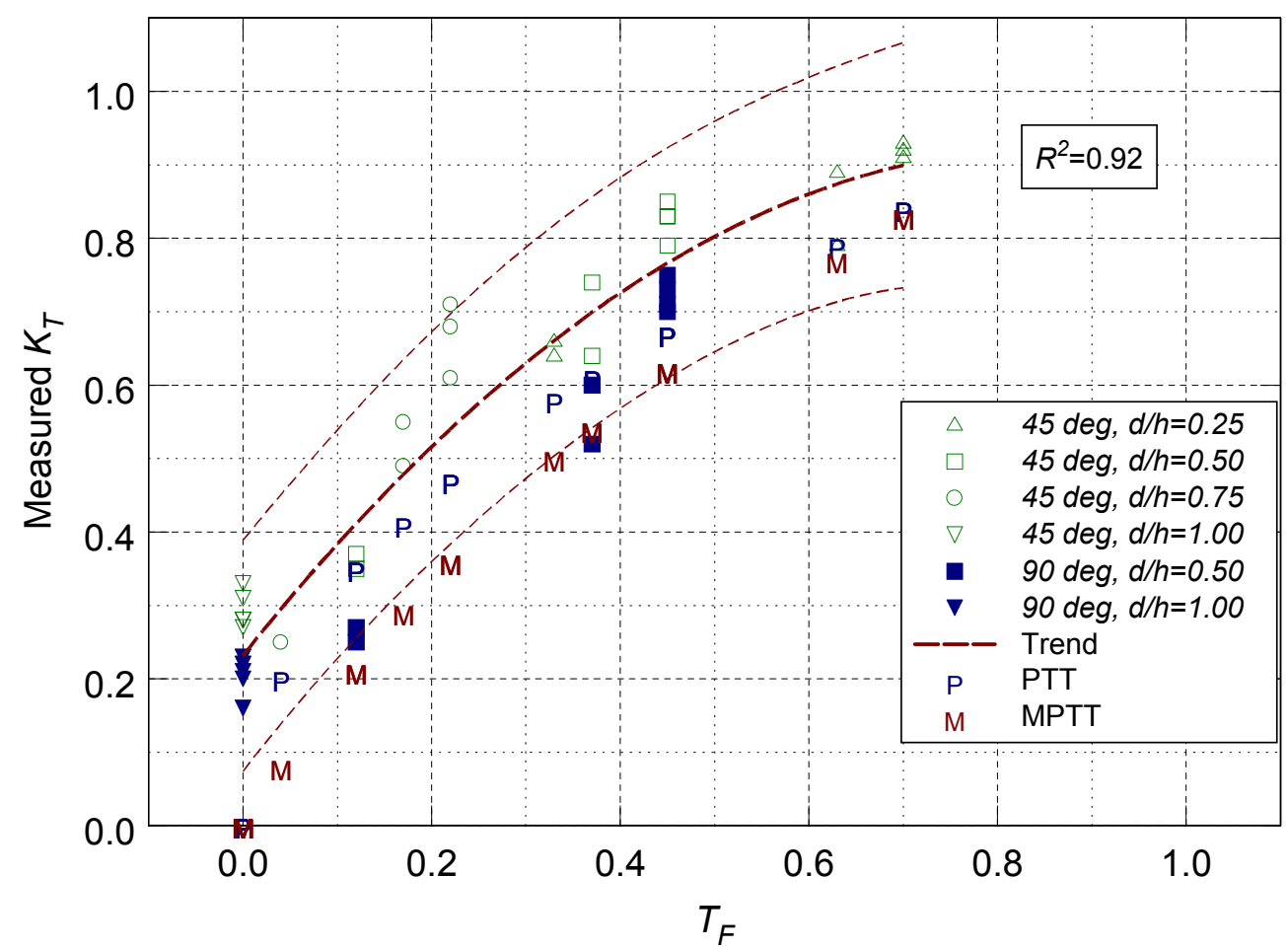

Figure 36. Analytical model comparisons with measured $K_{T}$.

TABLE 12. Summary of FLab Regression Analysis Parameters

\begin{tabular}{l|c|c|c|c|c}
\hline \hline \multicolumn{2}{c|}{ Independent Variable $x$} & \multirow{3}{*}{ Regression Coefficients } \\
\cline { 1 - 3 } Name & Symbol & $R^{2}$ & $a_{0}$ & $a_{1}$ & $a_{2}$ \\
\hline Transmission factor & $T_{F}$ & 0.92 & 0.23 & 1.61 & -0.92 \\
\hline Relative width & $B_{x} / L_{x}$ & 0.16 & 0.74 & -14.94 & 127.36 \\
\hline Wave steepness, $d / h<1$ & $H_{I} / L_{x}$ & 0.55 & 0.79 & 1.83 & -228.277 \\
\hline Wave steepness, $d / h=1$ & $H_{I} / L_{x}$ & 0.48 & 0.27 & 0.23 & -36.17 \\
\hline Composite & $D_{b} d / B_{r} L_{r}$ & 0.51 & 0.90 & -2.25 & 1.70 \\
\hline \hline
\end{tabular}

as a function of mean wave direction $\bar{\theta}$. In summary, even though the $T_{F}$ parameter does not include $\bar{\theta}$ in its formulation, it is a good predictor of the RIBS performance for the range of conditions tested in the FLab experiments. 


\section{FLab Empirical Relationships}

Several empirical relationships are investigated in this section. Included are the relative width $B_{x} / L_{x}$, wave steepness $H_{I} / L_{x}$, and a new composite parameter $D B$. For these comparisons, the global coordinate system used in the WAMIT model is used. In this system the $x$ direction is parallel to the RIBS center line.

Relative width. Relative width or beam is defined as the ratio of the projected RIBS beam in the x-direction $B_{x}$ to the projected wavelength in the $x$-direction $L_{x}$ (i.e., $L_{x}=L \cos \theta$ ). This $B_{x} / L_{x}$ ratio is the reciprocal of a definition of the relative wavelength parameter $L / B$. This parameter incorporates the RIBS geometric characteristics in the beam $B$ and the interior angle $\theta_{r}$ with the wave characteristics in the wave period $T$, water depth $h$, and mean wave direction $\bar{\theta}$.

Figure 37 shows the relationship between $B_{x} / L_{x}$ and measured $K_{T}$. The data is plotted the same as in the previous graph. The scatter in this data is very great and the regression analysis indicates a correspondingly low $R^{2}=0.15$. The regression coefficients are also listed in Table 12 . Thus, this parameter does not do a very good job predicting the RIBS performance.

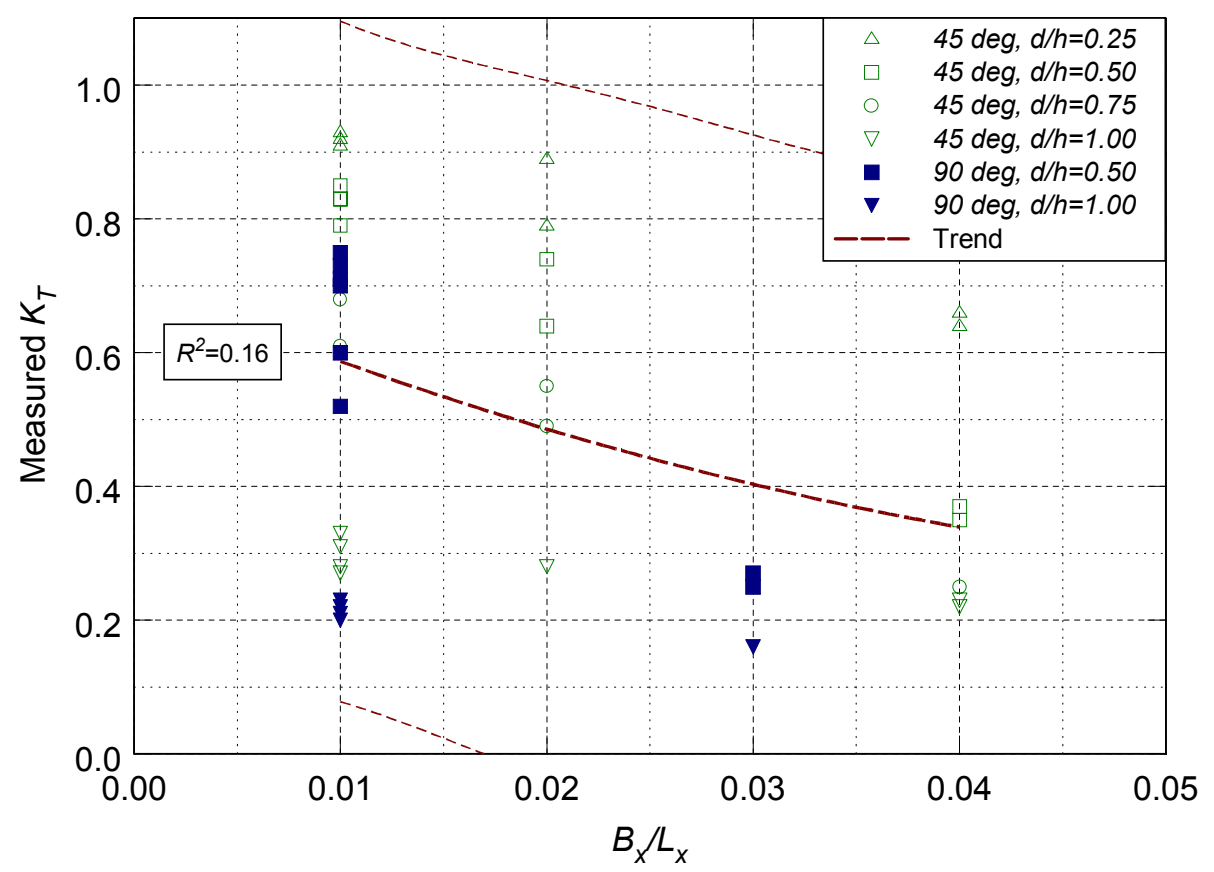

Figure 37. Measured $K_{T}$ versus $B_{x} / L_{x}$. 
Wave steepness. The comparisons between SS3 and SS5 wave heights in the previous section indicated that an increase in wave height did not have much effect on the measured $K_{T}$. These comparisons, however, were only based on wave periods of $T=12 \mathrm{sec}$ and did not cover a very broad range of wave steepness $H / L$. Figure 38 is a plot of measured average $K_{T}$ versus $H_{I} / L_{x}$ for the six RIBS model configurations and eight wave conditions. The eight SS5 wave conditions for the $\theta_{r}=45 \mathrm{deg}$ configuration are also included in this analysis. Wave steepness is calculated from the incident wave height $H_{I}$ and the projected wavelength $L_{x}$ in the direction of the $x$-axis or center line.

The data tended to align itself into two distinct groups: one based on the two configurations resting on the bottom (i.e., $d / h=1.00$ ) and a second group based on the four configurations above the bottom (i.e., $d / h<1.00$ ). The first group represents the cases where diffraction and flow under the RIBS are possible and the second group diffraction only. Therefore, two separate least square fit lines were evaluated and are shown in the figure. The 95 percent confidence

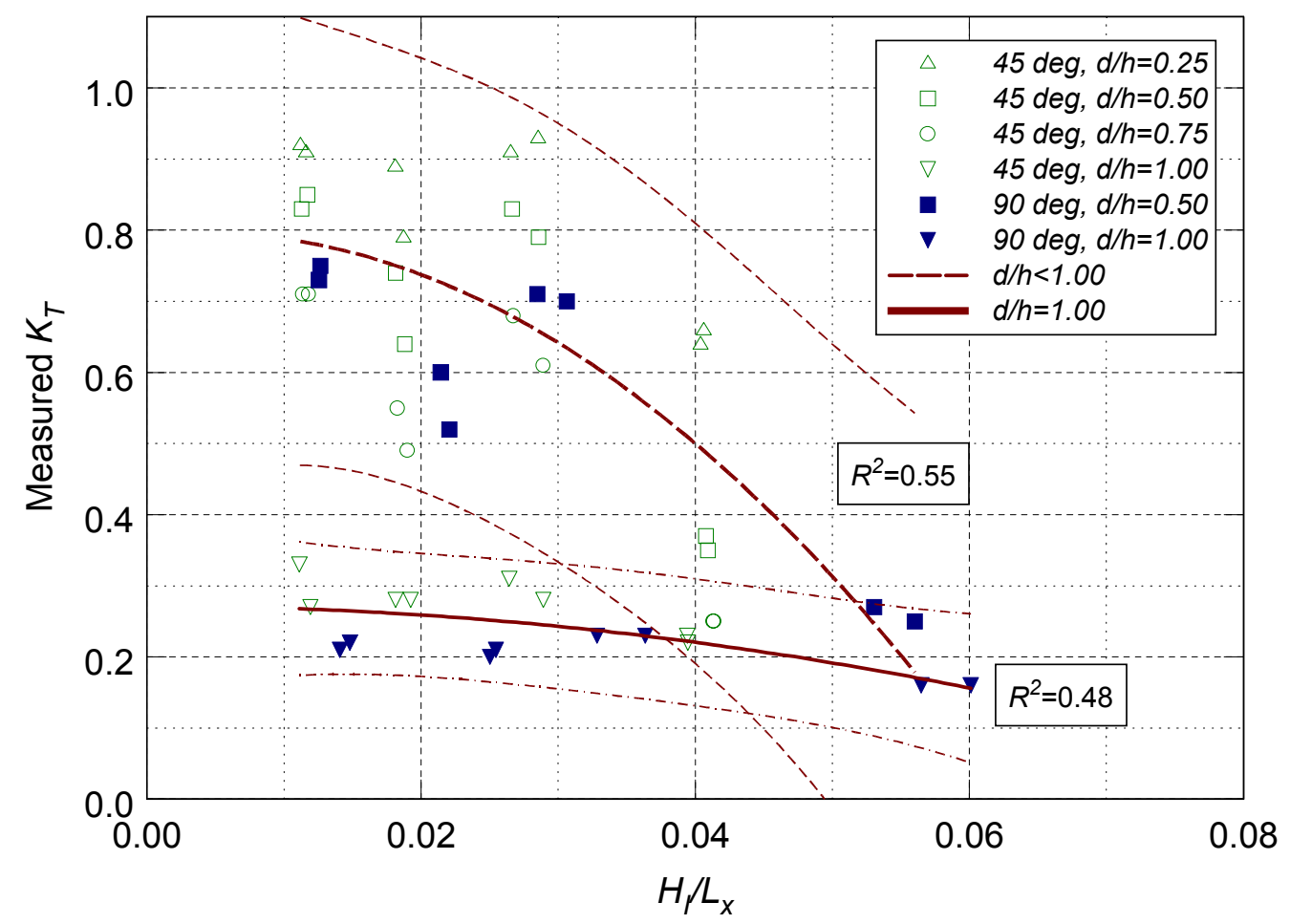

Figure 38. Effect of wave steepness on measured $K_{T}$ for FLab. 
limits for each curve are also shown. The $R^{2}=0.55$ and 0.48 for the two curves, respectively. These low values are due to the large scatter in the data, especially so for the first curve which has the wide 95 percent confidence intervals. In general, the $K_{T}$ decreases as $H_{I} / L_{x}$ increases, more so for the cases not resting on the bottom. For the second group of diffraction only cases, there appears to be a limit of $K_{T}=0.20$. The second order regression analysis coefficients are again listed in Table 12. In summary, this parameter has some validity in predicting RIBS performance. The scatter in the data and low correlation may be due to the limited data set of wave heights and wavelengths.

Composite parameter. The predictions of the relative width $B_{x} / L_{x}$ and wave steepness $H_{I} / L_{x}$ parameters showed a lot of scatter in the data. Therefore, a new composite parameter $D B$ was examined. It is a combination of three nondimensional parameters and is defined by

$$
D B=\frac{D_{b}}{B_{y}} \frac{d}{L_{x}}=\frac{D_{b}}{B_{y}} \frac{d}{h} \frac{h}{L_{x}}
$$

where $D_{b}$ is the distance from the bow to a gage in the lee of the RIBS measured parallel to the centerline, $B_{y}=L_{r} \sin \theta_{r}$ is the projected RIBS length in the y-direction (transverse to the centerline), $d / h$ is relative draft, and $h / L_{x}$ is a modified relative water depth. Thus, this new parameter incorporates many of the factors affecting a breakwater's performance.

Figure 39 shows the results of this parameter versus measured $K_{T}$. The regression analysis gave an $R^{2}=0.51$. Again, the regression analysis coefficients are listed in Table 12. Appendix Table F3 lists all of the empirical parameters investigated in this study.

\section{FLab Wave Transmission Summary}

For the FLab laboratory experiments, measured $K_{T}$ was calculated and presented for the five transmitted gage locations, six configurations, six operational and two survival wave cases. The $K_{T}$ decreased as wave period $T$ decreased and relative draft $d / h$ increased, and was fairly uniform with distance in the lee of the RIBS. The RIBS should be positioned so that the relative draft is greater than 50 percent of the water depth. An interior angle greater than $45 \mathrm{deg}$ is desirable, given the tradeoff with mooring forces. Head seas will probably provide a conservative design 


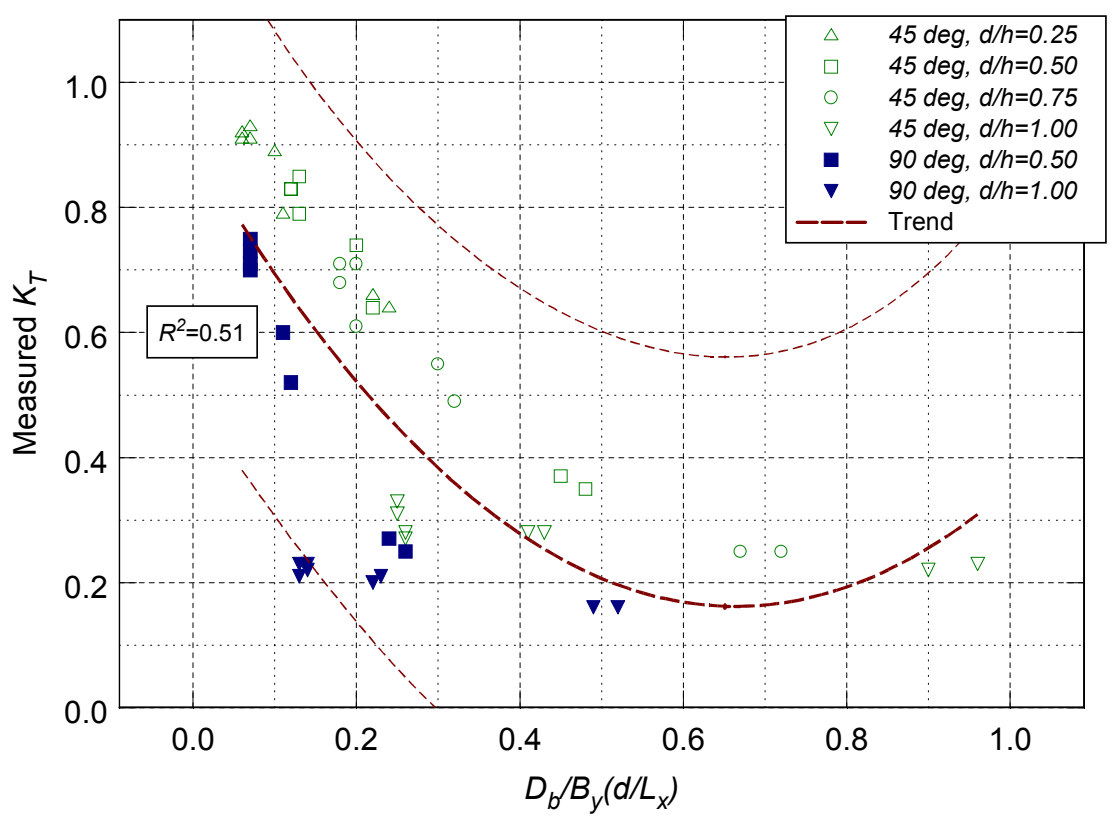

Figure 39. Measured $K_{T}$ versus $D_{b} / B_{y}\left(d / L_{x}\right)$.

as the RIBS tended to perform as well or better with a small angle in the mean wave direction $\bar{\theta}$.

In general, the WAMIT $K_{T}$ was higher than the measured values and showed more variability from gage to gage position. The correlation for the average over all five gages for each wave case and geometric configuration was excellent with an $R^{2}=0.95$. Since the average WAMIT $K_{T}$ was 0.13 higher than the corresponding measured value, it should provide some conservatism in the RIBS design.

The measured $K_{T}$ was compared to the analytical PTT and MPTT predictions using Wiegel's transmission factor $T_{F}$. The agreement was surprisingly good for the FLab data. The PTT model predictions were a closer fit to the measured data than the MPTT model. Although the $T_{F}$ parameter is not a function of mean wave direction $\bar{\theta}$, it was a reasonable predictor of the RIBS performance for the range of FLab conditions.

Several empirical relationships were investigated including a modified relative width $B_{x} / L_{x}$, modified wave steepness $H / L_{x}$, and a new composite parameter $D_{b} d / B_{y} L_{x}$. The last two gave the best correlation, but were not as good as Wiegel's transmission factor $T_{F}$. 


\section{XM99 FIELD TRIALS}

\section{Calculation Procedure}

The measured wave transmission coefficient $K_{T}$ for the XM99 field data is defined the same as previously discussed for the fundamental laboratory experiments. It is based on the zero moment wave height $H_{m 0}$ that is calculated from the entire frequency range of incident waves. Because the XM99 was shorter than a full-scale prototype RIBS (only about one-fourth as long), its performance would not be expected to be as good. A better way to evaluate the XM99 performance is to (1) restrict the range of frequencies used in the calculation of $K_{T}$ or (2) use an energy-based weighting function. In option 1 only wave periods less than $6 \mathrm{sec}$ (or frequencies greater than $0.17 \mathrm{~Hz}$ ) would be used in the estimation of $K_{\text {TSAve. }}$. This limited range of frequencies is more representative of the frequencies in SS3 of a scale model at 1 to 4 scale than a fullscale RIBS. In option 2 a new weighted transmission coefficient $K_{T, W g t}$ is calculated that is more influenced by the frequencies with more energy (i.e., peak periods) than those with little or no energy.

The steps in calculating these two versions of the wave transmission coefficient, $K_{T, S A v e}$ and $K_{T, W g t}$ are described below. First, a frequency-dependent transmission coefficient $K_{T}(f)$ is defined as the ratio of wave heights at individual wave frequencies as

$$
K_{T}(f)=\frac{H_{T}(f)}{H_{I}(f)}=\sqrt{\frac{S_{T}(f)}{S_{I}(f)}}
$$

where the $H_{m 0}$ wave height is used in the calculation of $H_{T}$ and $H_{I}$, and $S_{T}(f)$ and $S_{I}(f)$ are the transmitted and incident wave spectral estimates at frequency $f$, respectively. The incident values were measured from the Datawell directional buoy. Because of the distance from this buoy to the RIBS, it was assumed that these spectral estimates did not include any reflected energy from the RIBS.

For the energy-based option 2, a weighting function $W g t(f)$ is estimated as the ratio of the incident energy at each frequency to the total energy in the wave where $m_{0}$ is the zero moment previously defined.

$$
W g t(f)=\frac{S_{I}(f) \Delta f}{m_{0}}
$$


In the last step, the total wave transmission coefficient is estimated for the two options. In option 1, the short average wave transmission coefficient $K_{T, S A v e}$ is defined as

$$
K_{T, S A v e}=\frac{1}{N} \sum_{f_{l}}^{f_{u}} K_{T}(f)
$$

where $f_{l}$ and $f_{u}$ are lower and upper frequency limits, and $N$ is the number of frequencies between these limits. The value of $f_{l}$ is set to $0.17 \mathrm{~Hz}$, corresponding to $6 \mathrm{sec}$. In option 2 , the total transmission coefficient $K_{T, W e t}$ is defined as a weighted average of the frequency-dependent wave transmission coefficients.

$$
K_{T, W g t}=\sum_{f_{l}}^{f_{u}} W g t(f) K_{T}(f)
$$

This equation is identical to the previous equation, except that the weighting function is included. The cutoff limits $f_{l}$ and $f_{u}$ also cover the entire frequency range from zero to the Nyquist frequency.

\section{Measured Wave Transmission}

Frequency-dependent analysis. The first step in the analysis of the measured field data is to calculate the frequency-dependent wave transmission coefficient $K_{T}(f)$. The GEDAP analysis package (Miles 1997) was used in these calculations. Figure 40 is an example plot of the $K_{T}(f)$ analysis using the incident and transmitted wave spectra in the lower half and $K_{T}(f)$ in the upper half for the May 25, 1800-hr case. The horizontal dashed line is the desired cutoff for wave transmission at $K_{T}=0.5$. The vertical dashed line is at a frequency of $0.17 \mathrm{~Hz}$ (i.e., $6 \mathrm{sec}$ ), corresponding to an upper limit of SS3. The lower panel is a summary of the calculations for the transmission coefficient. Minimum and maximum values, the average, and 90-percent confidence limits are shown for the entire frequency band and the limited or short band of frequencies greater than $0.17 \mathrm{~Hz}$. The averages are simple averages over the values of $K_{T}$. For the short frequency band from 0.17 to $0.5 \mathrm{~Hz}$, the average $K_{T, S A v e}=0.37$. This compares to the value of $K_{T, S A v e}=0.51$ for the entire frequency band from 0 to $0.5 \mathrm{~Hz}$. 
07 February 2001 13:21

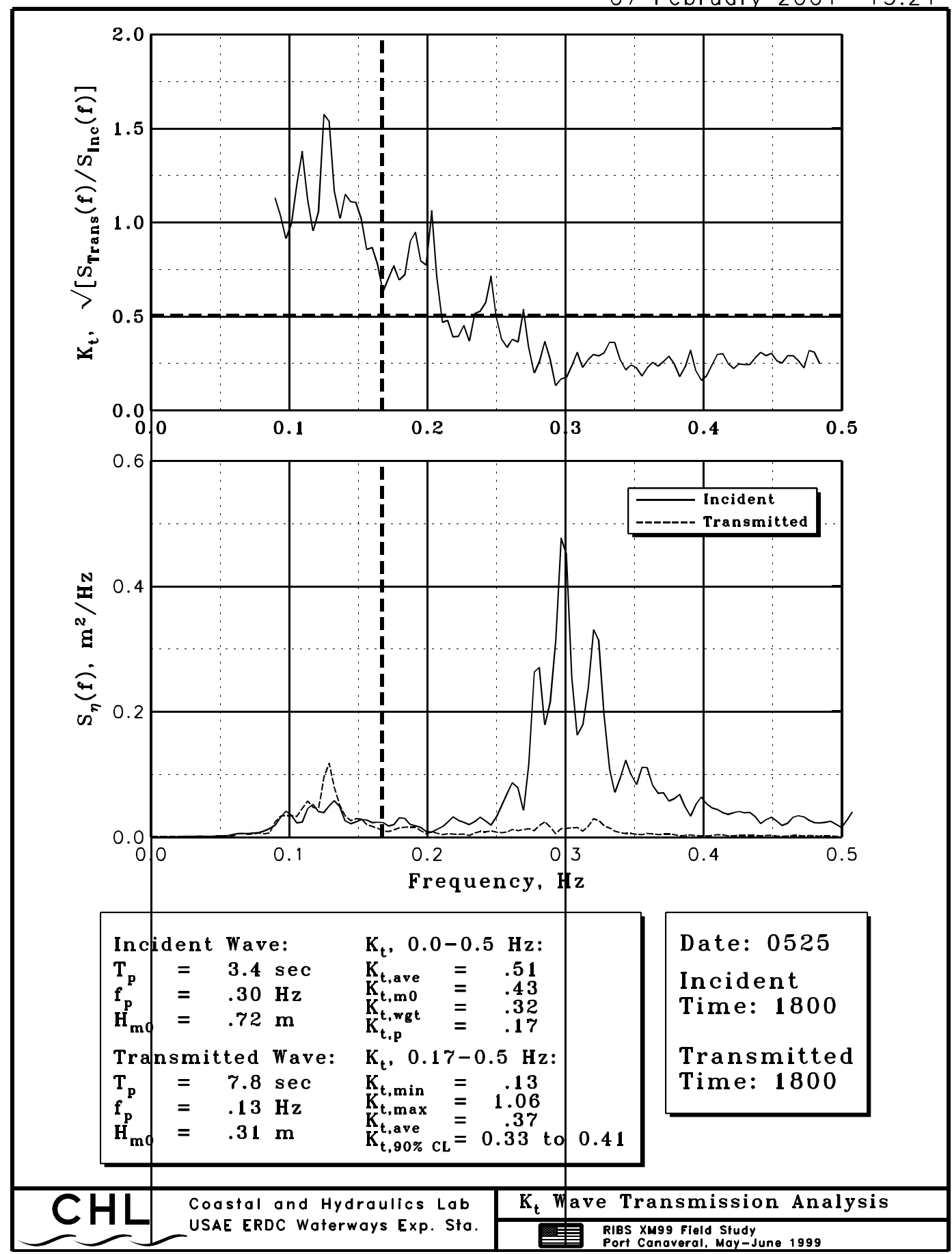

Figure 40. Wave transmission coefficient analysis by frequency. 
Some of the $K_{T}(f)$ are greater than 1.0 in the low frequencies. The relative amount of energy in these low frequencies is so small that the amount of wave amplification would not be noticed by an observer on a ship. Also, the energy values at these frequencies are so small that they are probably heavily influenced by signal noise and not actually as large as indicated. This is part of the justification for using an energy-based weighting function for the estimation of the wave transmission coefficient as these seemingly large amplifications will not be present when the $K_{T}$ are weighted. For the example shown in this figure, the transmitted wave has more low frequency energy than the incident wave. This is probably due to the XM99 making some waves due to its response to the incoming waves.

Time series plots. Figure 41 is a time series of wave transmission values during the 10 days of deployment. The dotted line is the traditional definition of the wave transmission coefficient $K_{T}$ (based on $H_{m 0}$ ), the dashed line is the short average $K_{T, S A v e}$, and the solid line is for the weighted $K_{T, W g t}$. The $K_{T, S A v e}$ and $K_{T, W g t}$ are always less than the $K_{T}$, with the amount of difference being a function of wave period and direction. The $K_{T, S A v e}$ is sometimes smaller than

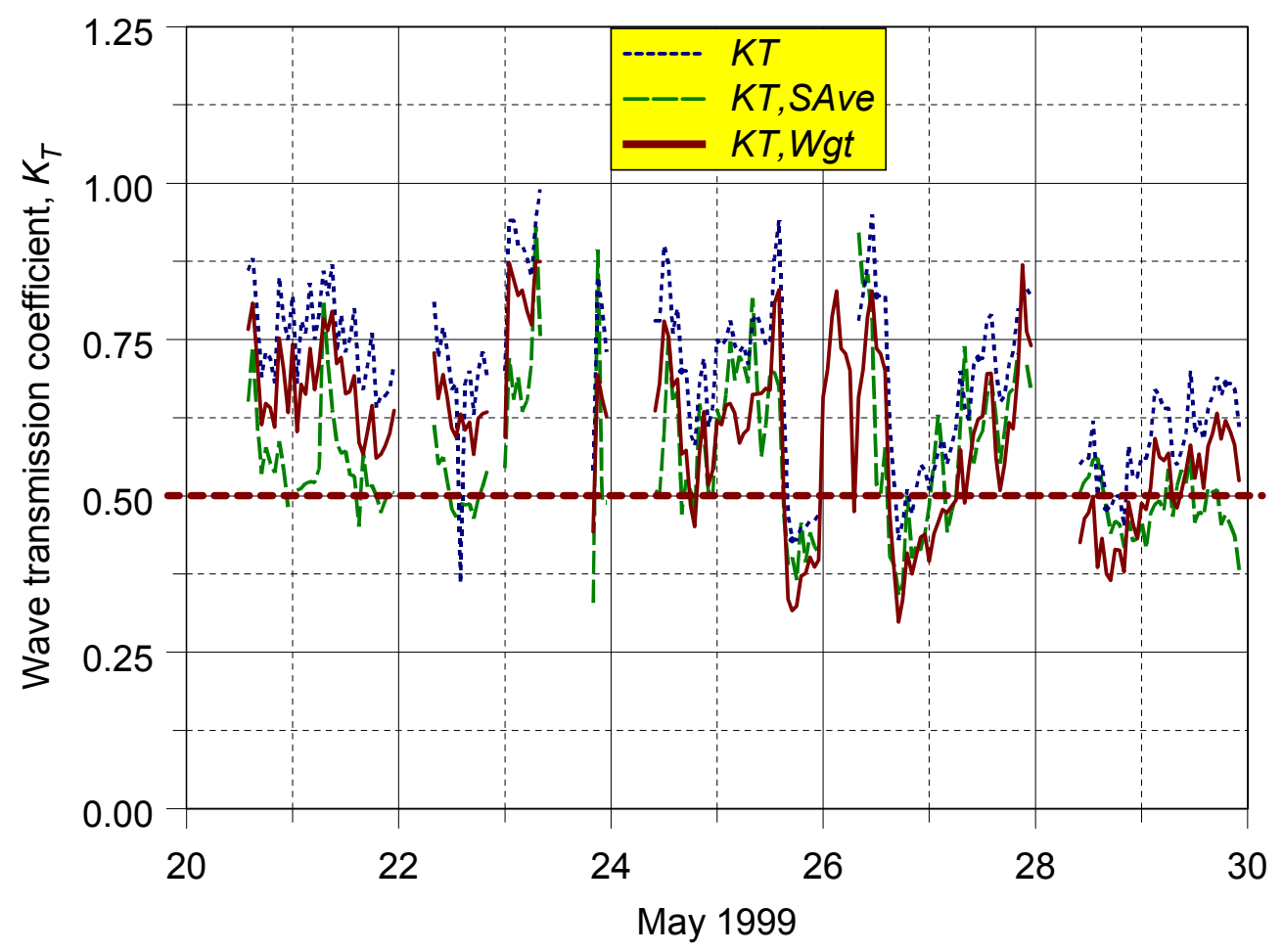

Figure 41. Measured wave transmission coefficients. 
the $K_{T, W g t}$, and vice versa, depending on the amount of energy in the spectrum and the mode shape. Table 13 lists the minimum, maximum, average, and standard deviation for each of the calculated wave transmission values. Both $K_{T, S A v e}$ and $K_{T, W g t}$ were less than the desired threshold level (i.e., 0.5) for several hours every day, with the best performances occurring between May 24 through 29. Threshold values were obtained for waves with peak periods as large as $10 \mathrm{sec}$. Appendix Table G1 contains listings of the measured $K_{T}$ for the different analysis methods for the entire XM99 deployment.

The XM99 performed well considering that it experienced some minor tearing in the curtains after the first two days, resulting in a reduced barrier to wave transmission. On the last day of the deployment, a storm caused some structural damages that resulted in reduced depth of submergence and increased wave transmission.

TABLE 13. Calculated XM99 $K_{T}$ Statistics

\begin{tabular}{c|c|c|c}
\hline \hline Statistic & $K_{T}$ & $K_{T, \text { Ave }}$ & $K_{T, W g t}$ \\
\hline Minimum & 0.36 & 0.33 & 0.30 \\
\hline Maximum & 0.99 & 0.93 & 0.87 \\
\hline Average & 0.69 & 0.55 & 0.60 \\
\hline Standard Deviation & 0.13 & 0.12 & 0.13 \\
\hline \hline
\end{tabular}

Joint distribution or scatter plots. In this section, joint distribution plots of $K_{T, W g t}$ versus measured values of $T_{p}, H_{m 0}, \bar{\theta}$, and $\sigma_{m}$ are presented and discussed. Although there are some slight differences between the distributions using $K_{T}$ and $K_{T, S A v e}$, the trends are the same so their plots will not be presented.

Figure $42 \mathrm{a}$ is the joint distribution between $K_{T, W g t}$ and $T_{p}$. These plots perform a dual function. First, they are scatter diagrams of the measured $K_{T, W g t}$ and the individual wave parameters. Second, they show the results of a quadratic least squares fit to the data and the 95 percent confidence limits. The square of the correlation coefficient $R^{2}$ is shown on each plot. 


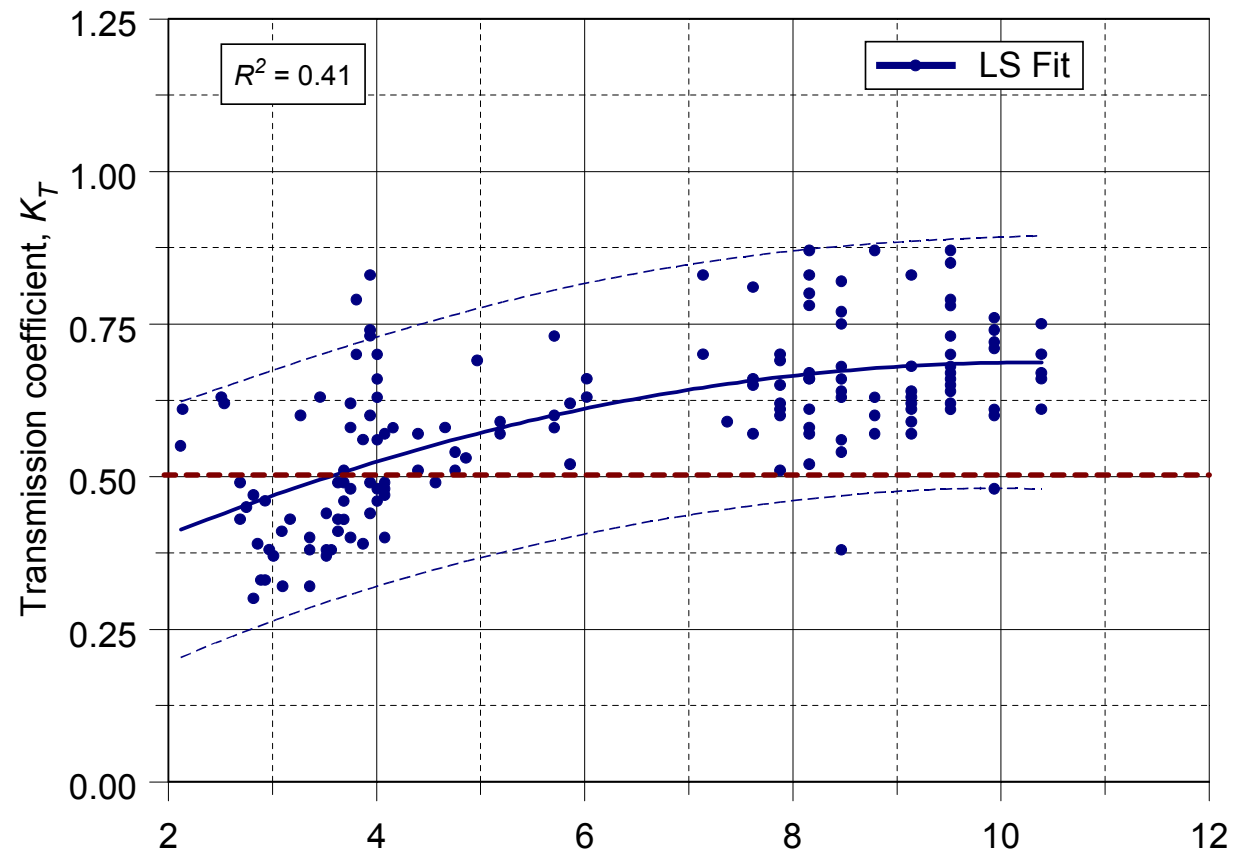

a)

Peak wave period $T_{P}$, sec

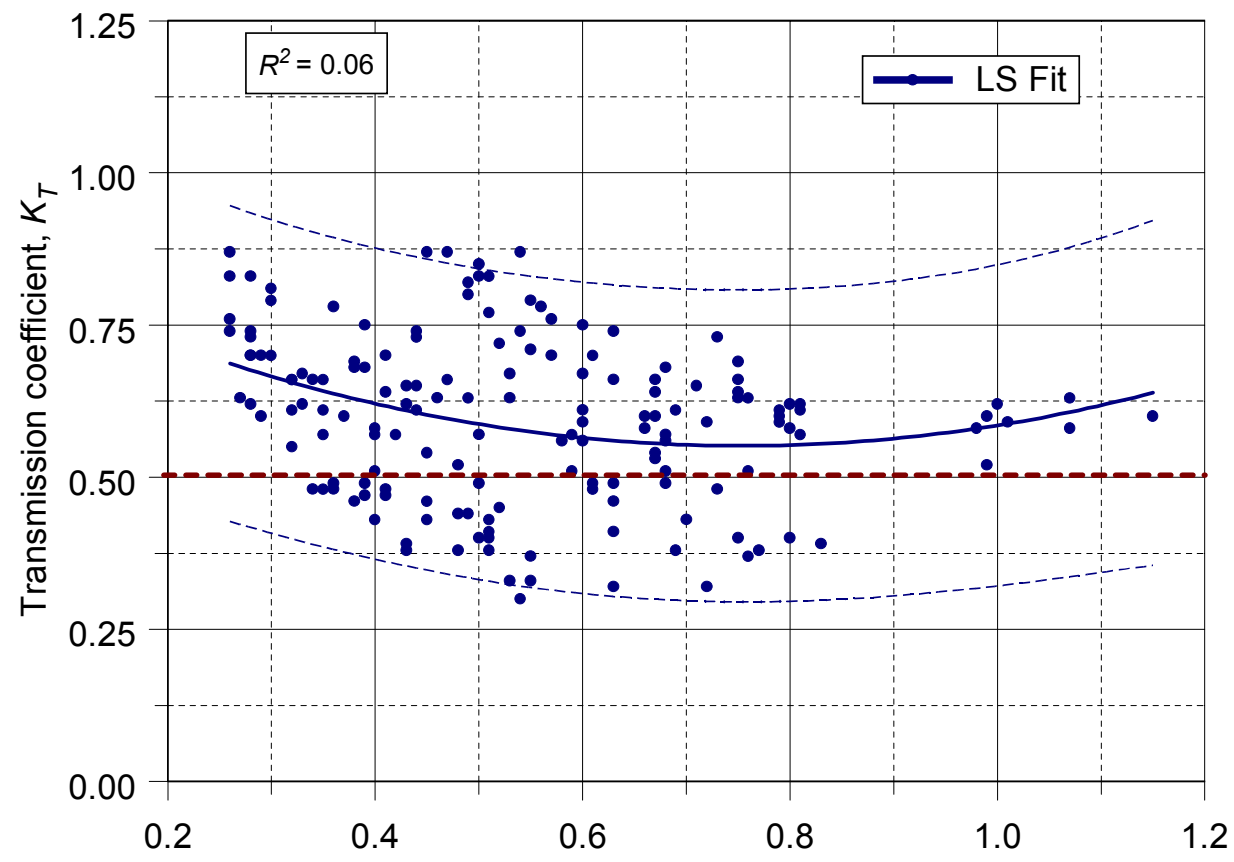

b)

Significant wave height $H_{m 0}, \mathrm{~m}$

Figure 42. Joint distributions of wave transmission coefficient vs. (a) peak wave period $T_{P}$, and (b) significant wave height $H_{m 0}$. 
The peak wave period in this figure can be for a unimodal sea or swell, or a dominant sea or swell mode of a bimodal or multimodal sea state. In general, the wave transmission values group into either sea or swell regimes depending on whether the dominant mode of the waves is in the sea or swell band of frequencies. Values larger than the threshold are most likely characterized by a significant amount of energy in the "swell" regime and/or wave direction and directional spreading exceeding the range of allowable directions for the XM99. The coefficients less than the threshold in the "swell" regime are due to a significant amount of energy at a "sea" peak in a bimodal or multi-modal wave condition.

The was $R^{2}=0.41$, a reasonable fit to the data. The other regression coefficients are listed in Table 14. This table has the same format as before.

TABLE 14. Summary of XM99 Wave Data Regression Analysis Parameters

\begin{tabular}{l|c|c|c|c|c}
\hline \hline \multicolumn{2}{c|}{ Independent Variable $x$} & \multicolumn{3}{|c}{ Regression Coefficients } \\
\cline { 1 - 1 } \cline { 5 - 6 } Name & Symbol & $R^{2}$ & $a_{0}$ & $a_{1}$ & $a_{2}$ \\
\hline Peak wave period & $T_{P}$ & 0.41 & 0.26 & 0.08 & -0.004 \\
\hline Wave height & $H_{m 0}$ & 0.06 & 0.81 & -0.65 & 0.42 \\
\hline Mean wave direction & $\bar{\theta}$ & 0.19 & 0.72 & -0.002 & $4 \mathrm{E}-06$ \\
\hline Directional spread & $\sigma_{m}$ & 0.003 & 0.57 & 0.002 & $-2 \mathrm{E}-05$ \\
\hline \hline
\end{tabular}

Figure $42 \mathrm{~b}$ is the joint distribution plot for $K_{T, W g t}$ versus $H_{m 0}$. Incident wave heights up to $0.83 \mathrm{~m}$ were efficiently reduced during this field trial. The eight largest wave heights occurred on the last day of the deployment and were not as effectively minimized (i.e., $K_{T, W g t} \leq 0.63$ ) due to the damage sustained by the XM99 during the storm. The $R^{2}=0.06$, a very small correlation. The least squares fit line coefficients for the second order equation are contained in Table 14.

The joint distribution of $K_{T, W g t}$ versus $\bar{\theta}$ is shown in Figure 43a. This figure has the same format as in the previous figures. The vertical line is the effective wave direction limit of 255 deg for the XM99. Surprisingly, there was even a value below the threshold for one case at 244 deg. The distribution is bimodal with the primary peak around 264 deg and a secondary peak 

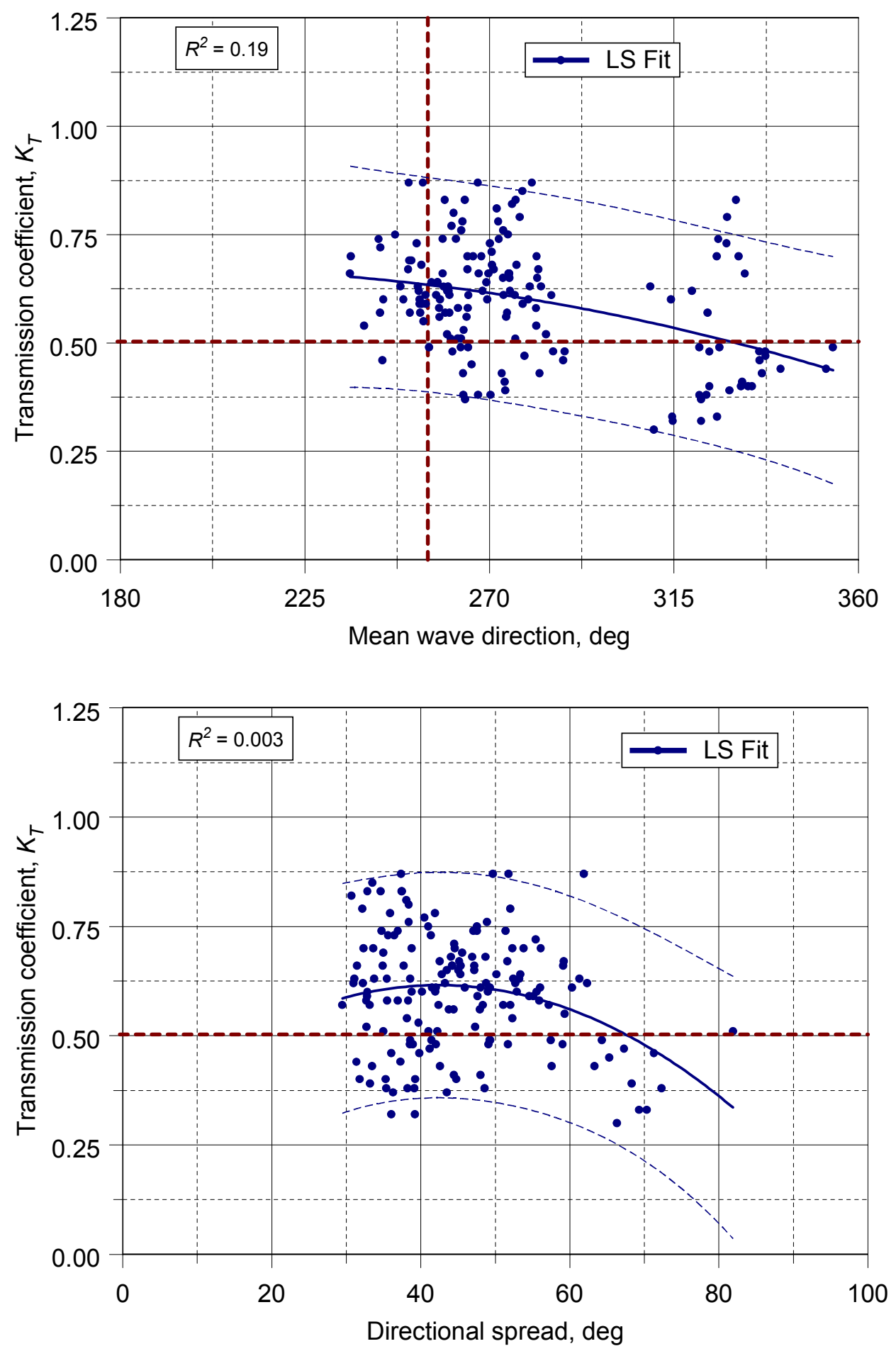

Figure 43. Joint distributions of wave transmission coefficient vs. (a) mean wave direction $\bar{\theta}$, and (b) directional spread $\sigma_{m}$. 
near $326 \mathrm{deg}$. The $R^{2}=0.19$ for the regression analysis with $\bar{\theta}$. This is a reasonable predictor and fit to the data.

The $\sigma$-distribution is narrower and unimodal with most of the values clustered around 40 deg (Figure 43b). This represents a fairly narrow directional spreading value. Values of $K_{T, W g t}$ were less than the threshold for mean wave directions in the range from 244 to $354 \mathrm{deg}$ and directional spreading from 31 to $72 \mathrm{deg}$. Even though the data is compact in direction-space, the correlation was almost nonexistent $\left(R^{2}=0.003\right)$ for $\sigma_{m}$. Regression coefficients are listed in Table 14 for the last two wave parameters.

\section{WAMIT Predicted Wave Transmission}

A comparison of field measurements and numerical and analytical predictions of wave transmission is shown in Figure 44. An example wave period of $3 \mathrm{sec}$ and wave direction parallel to the XM99 centerline (i.e., $315 \mathrm{deg}$ ) was selected for comparison. The measured value of $K_{T, W g t}$ is an average of all the waves with a wave period of $3 \pm 0.5 \mathrm{sec}$ and wave direction of $315 \pm 15 \mathrm{deg}$. A total of 9 cases were identified with these characteristics, including two cases with $\mathrm{K}_{\mathrm{T} \text {,Wgt }}$ 's greater than the threshold. These 9 cases had wave heights from $0.27 \mathrm{~m}$ to $0.72 \mathrm{~m}$, wave directions from 309 to $325 \mathrm{deg}$, and spreads from 36 to $72 \mathrm{deg}$. For this group, the average $K_{T, W g t}=0.41$, with 95 percent confidence limits of \pm 0.08 .

The curve is for the WAMIT prediction along the center line of the XM99. In general, the $K_{T}$ oscillates along the centerline, with values ranging from 0.02 to 0.4 . Based on analysis using a HOBEM (Higher Order Boundary Element Method) model, this oscillation appears to be characteristic of numerical models based on Green's Function. The XM99 legs may be contributing to interference which leads to the oscillation in the predictions. Also, the WAMIT predictions are based on regular waves without directional spreading, which would probably tend to smooth these oscillations. The agreement between numerical model and field measurements is reasonable. Of course, one should realize that the field measurement is for one location. Although it is assumed to be fixed, in reality the transmitted buoy experienced some movement about a watch circle of its mooring. So this value is an average for the range of positions within that watch circle during the data collection interval. Thus, the field value could 


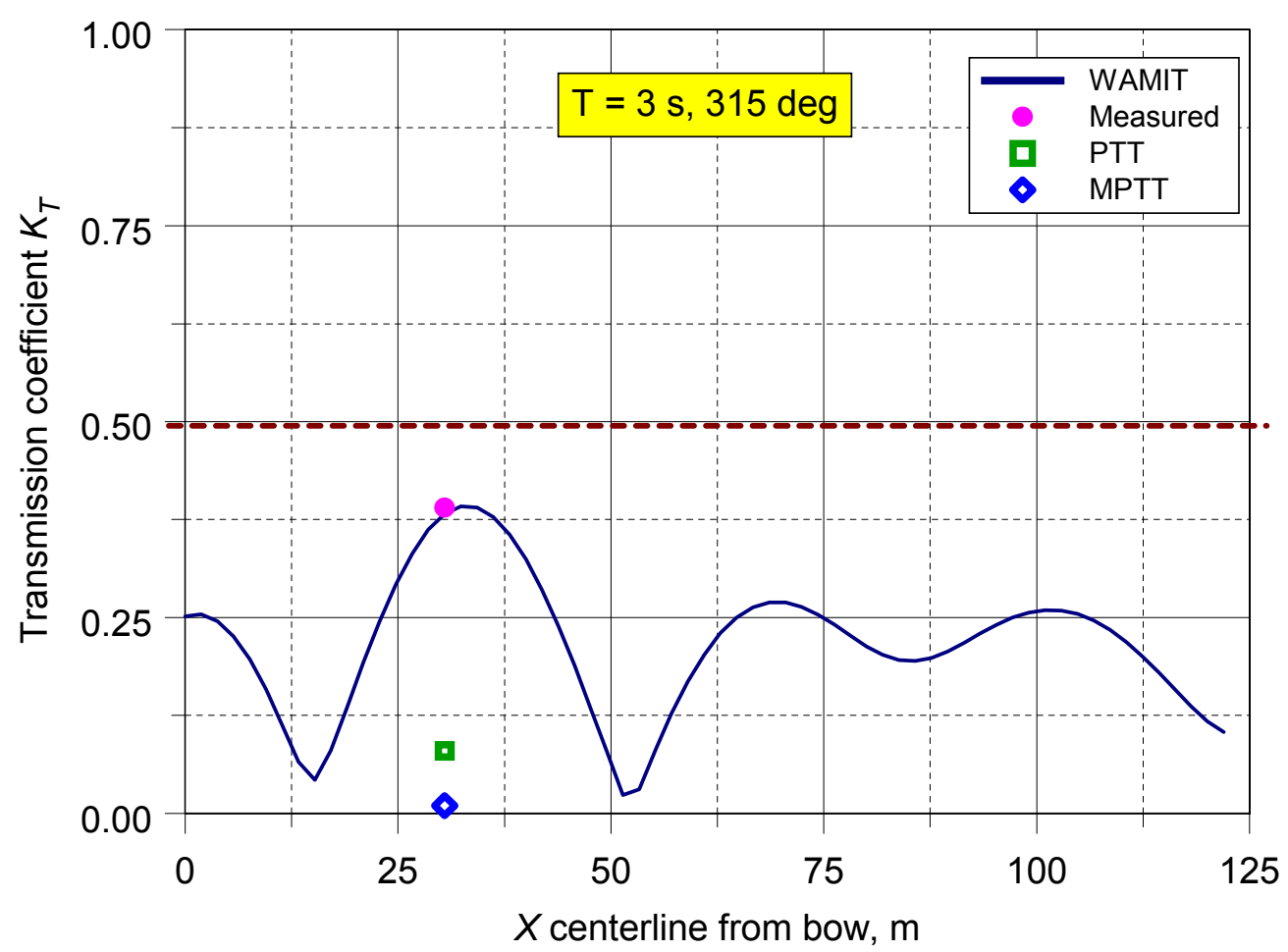

Figure 44. Comparison of measured wave transmission with WAMIT, PTT, and MPTT models.

be compared to other numerical model predictions within some reasonable watch circle of the center line position.

The predicted wave transmission for the PTT and MPTT models was calculated using the value of the transmission factor $T_{F}$ for a wave period $T=3 \mathrm{sec}$. Because these models are based on waves normal to a structure, the estimated $K_{T}$ is independent of location in Region 2. For this particular wave period, the two analytical models did not predict the wave transmission very well ( 0.08 for PTT and 0.01 for MPTT). However, the PTT analytical model prediction is within the WAMIT range of oscillations of $K_{T}$ in the lee of the XM99.

Table 15 lists the WAMIT-predicted $K_{T}$ for the 33 wave period and direction combinations at the location corresponding to the transmitted gage. The $K_{T}$ are averages of nine gages in two concentric circles (4 gages each) with 1.5 and 3.0 m radii about the transmitted gage. The $K_{T}$ in Figure 44 are based on values along the center line only. 
TABLE 15. WAMIT Predicted $K_{T}$

\begin{tabular}{c|c|c|c}
\hline \hline \multirow{2}{*}{ Period, sec } & \multicolumn{3}{|c}{ Direction, deg } \\
\cline { 2 - 4 } & $0(315)$ & $30(285)$ & $60(255)$ \\
\hline 2 & 0.07 & 0.06 & 0.12 \\
\hline 3 & 0.15 & 0.16 & 0.33 \\
\hline 4 & 0.51 & 0.26 & 0.30 \\
\hline 5 & 0.40 & 0.07 & 0.66 \\
\hline 6 & 0.34 & 1.28 & 0.31 \\
\hline 7 & 0.92 & 1.32 & 1.22 \\
\hline 8 & 1.15 & 1.24 & 1.19 \\
\hline 9 & 1.16 & 1.16 & 1.08 \\
\hline 10 & 1.13 & 1.11 & 1.07 \\
\hline 11 & 1.11 & 1.09 & 1.06 \\
\hline 12 & 1.09 & 1.07 & \\
\hline \hline
\end{tabular}

\section{Analytical Model Comparisons}

The top panel in Figure 45a shows the predicted $K_{T}$ values for the PTT and MPTT models plotted against the $T_{F}$ parameter defined in Chapter IV. The least squares fit lines, based on a second order polynomial, are shown for the PTT and MPTT models. In addition, the measured $K_{T, W g t}$ values, a least square fit, and 95 percent confidence limits are shown for comparison. The second order polynomial equation for this line is listed in Table 16 . The $R^{2}=0.42$ is not very good due to the large scatter in the data. The trend of the fitted line is similar to the trends of the two analytical models, however, increasing as $T_{F}$ increases.

Figure $45 \mathrm{~b}$ shows the ratio of the measured to the predicted $K_{T}$ values for the PTT and MPTT models. A spline fit curve is shown for each of the models rather than individual points. A ratio of 1.0 corresponds to a perfect fit. The two models under predict $K_{T}$ for small $T_{F}$, but improve as $T_{F}$ increases. Small $T_{F}$ values correspond to small wave periods. At approximately $T_{F}=0.15$, the PTT model predictions are within the 95 percent confidence limits. This corresponds to a wave period $T=5.2 \mathrm{sec}$. Similarly for the MPTT model, $T_{F}=0.30$ with a 


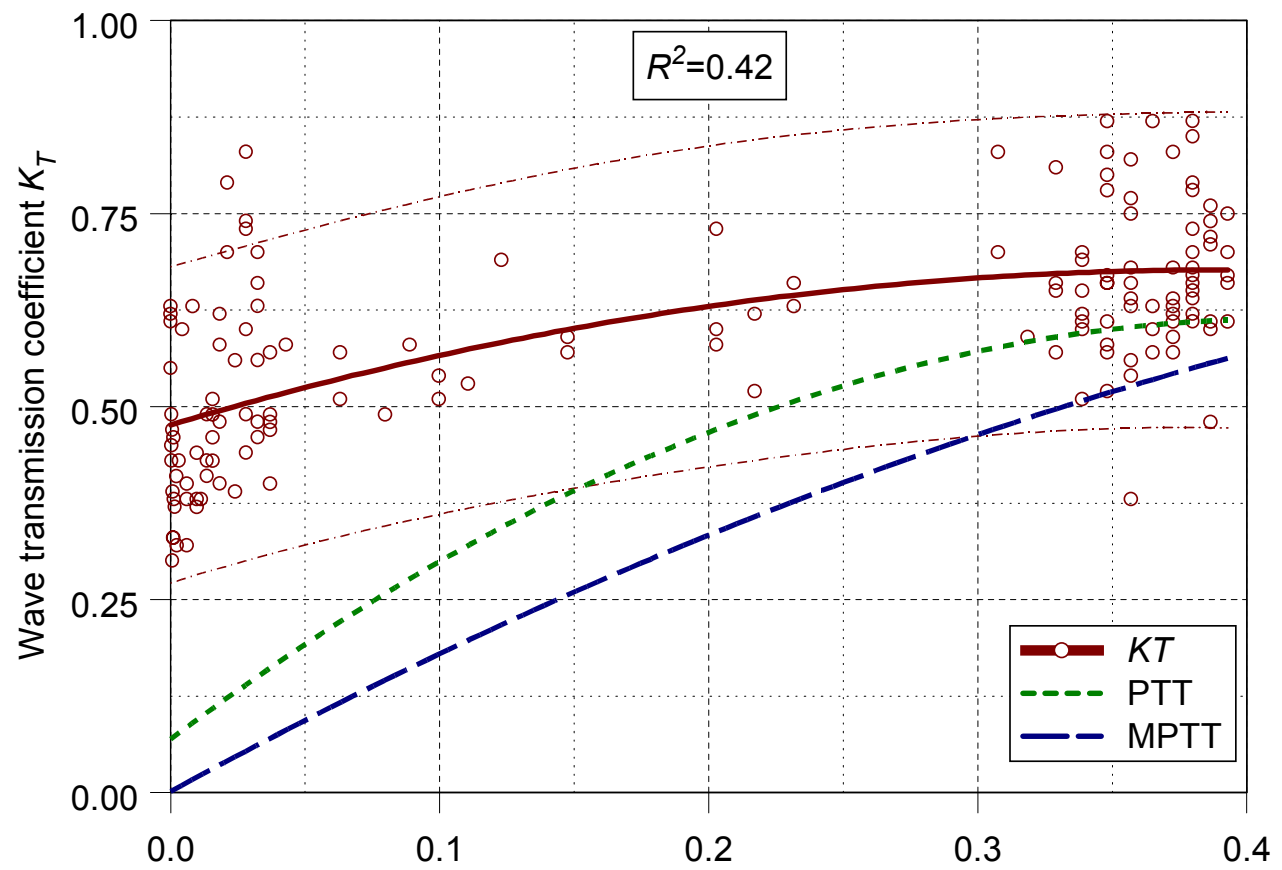

a)

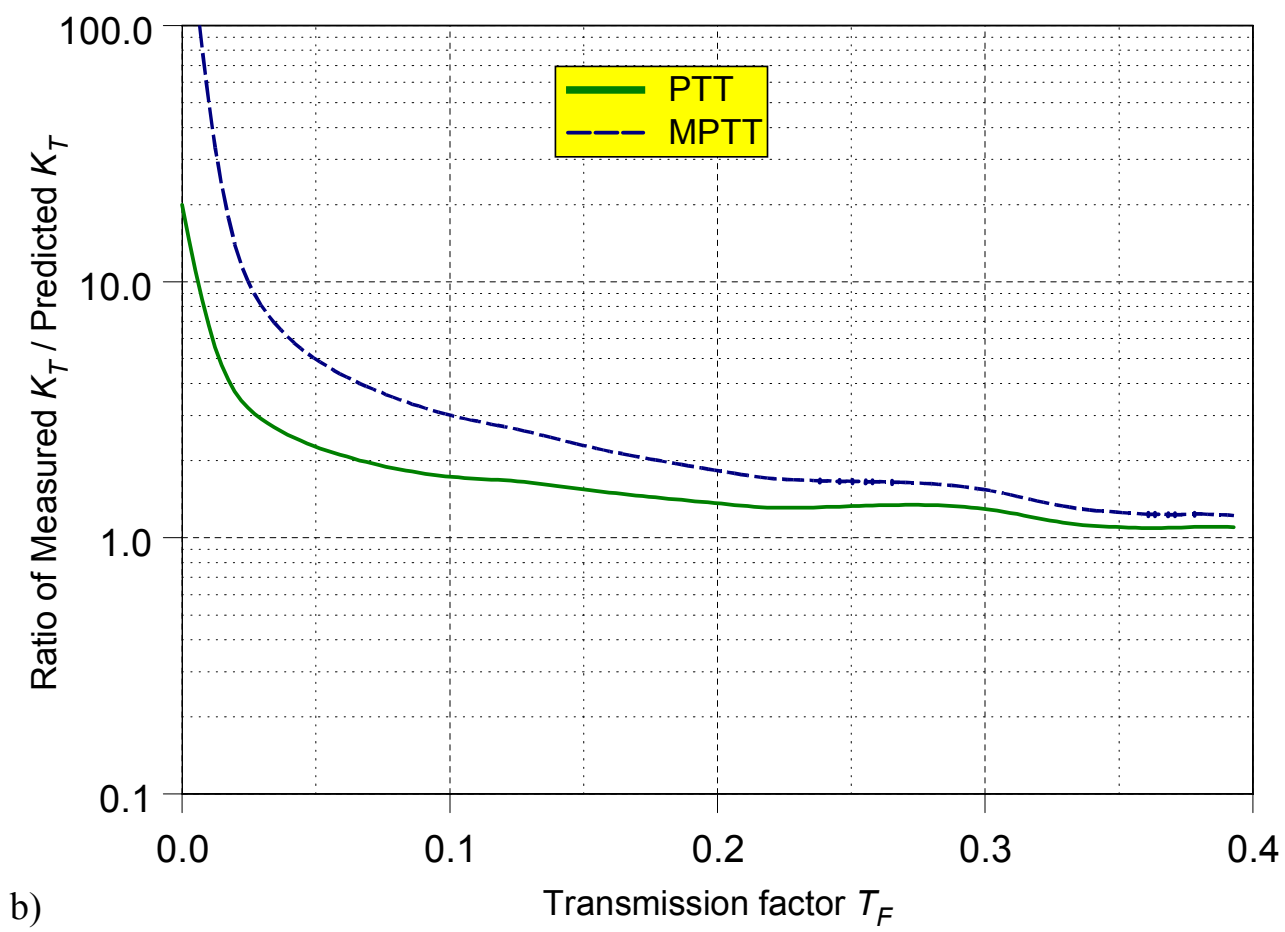

Figure 45. Measured and analytical model wave transmission versus $T_{F}$ parameter. (a) $K_{T}$ vs. $T_{F}$, (b) ratio of measured $K_{T}$ vs. predicted $K_{T}$. 
corresponding $T=7.0 \mathrm{sec}$ before its predictions are within the confidence limits. Thus, only the PTT model is applicable for the SS3 design limit of wave periods for the RIBS.

\section{XM99 Empirical Relationships}

The relative width and wave steepness empirical parameters are examined for the XM99. A complete listing of these parameters is contained in Appendix Table G2.

For traditional floating breakwaters, wave transmission is often a function of the relative breakwater width $B / L$ and wave steepness $H / L$, where $L$ is wavelength. Plots of $K_{T}$ versus these two parameters are shown in Figures 46a and 46b. In addition to the measured data, each plot includes the least squares second order polynomial fit line and the 95 percent confidence limits.

For the $B / L$ parameter, swell conditions correspond to small $B / L$ values and sea conditions to larger $B / L$ values because the XM99 width or beam $B$ is a constant. The equation for the least squares line for the parameter $B / L$ is contained in Table 16 . This empirical parameter has an $R^{2}=0.46$.

For the $H / L$ wave steepness parameter, swell waves also tend to correspond to smaller values of $H / L$ (for a constant wave height $H$ ). The second order polynomial equation for the wave steepness $H / L$ has an $R^{2}=0.48$. Table 16 lists the complete regression coefficients for this least squares fit.

Of the three empirical parameters, wave steepness was the best, but not significantly better than the others. In general, the existing analytical and empirical models do a reasonable job of predicting wave transmission for the XM99, but there is room for improvement.

\section{XM99 Wave Transmission Summary}

The measured $K_{T}$ during the 10-day deployment of the XM99 field trial was calculated using the traditional definition $K_{T}$, a simple average $K_{T, S A v e}$, and a weighted $K_{T, W g t}$. The measured $K_{T}$ were less than the $K_{T}=0.5$ desired level of efficiency for several hours every day, with the best performances on May 22, 23, 25, 26, and 28. The XM99 performed very well considering that it experienced some minor tearing in the curtains after the first two days, resulting in a reduced barrier to wave transmission. 


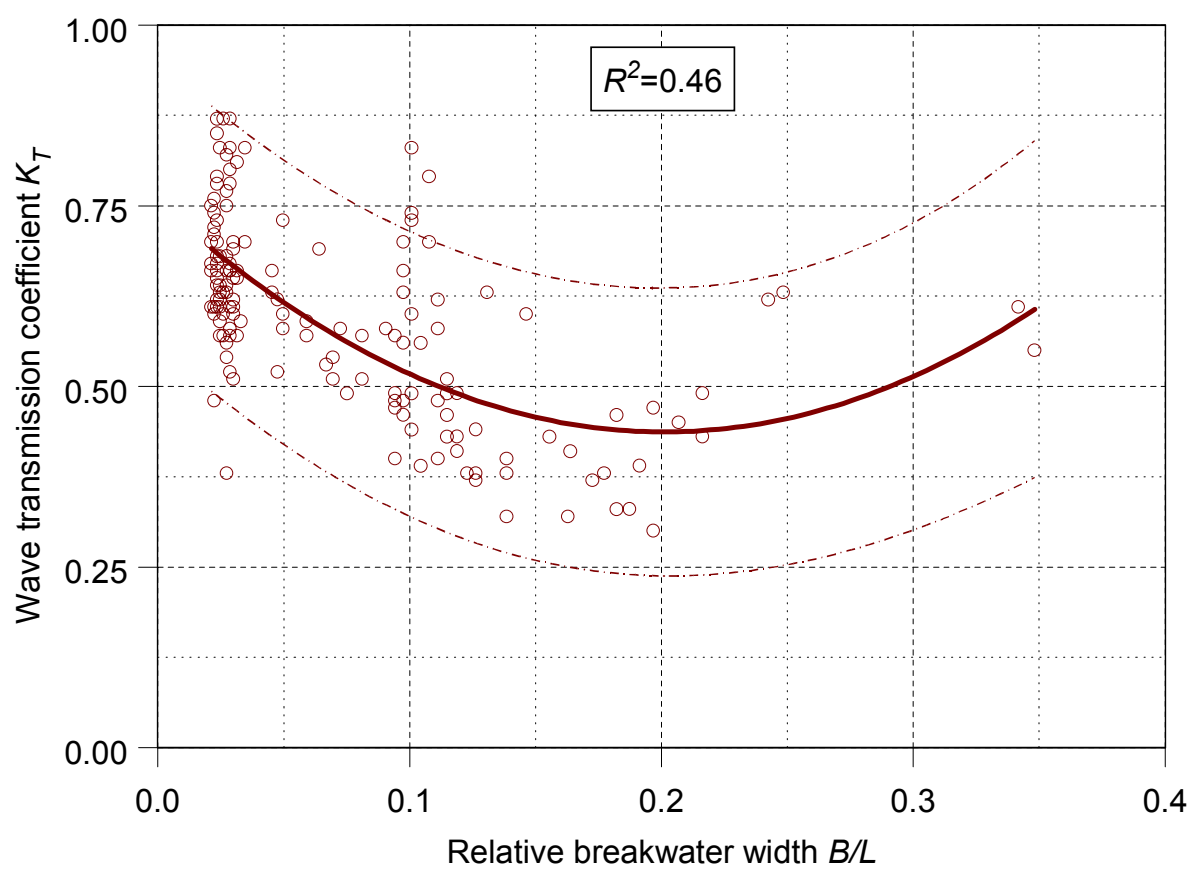

a) $B / L$

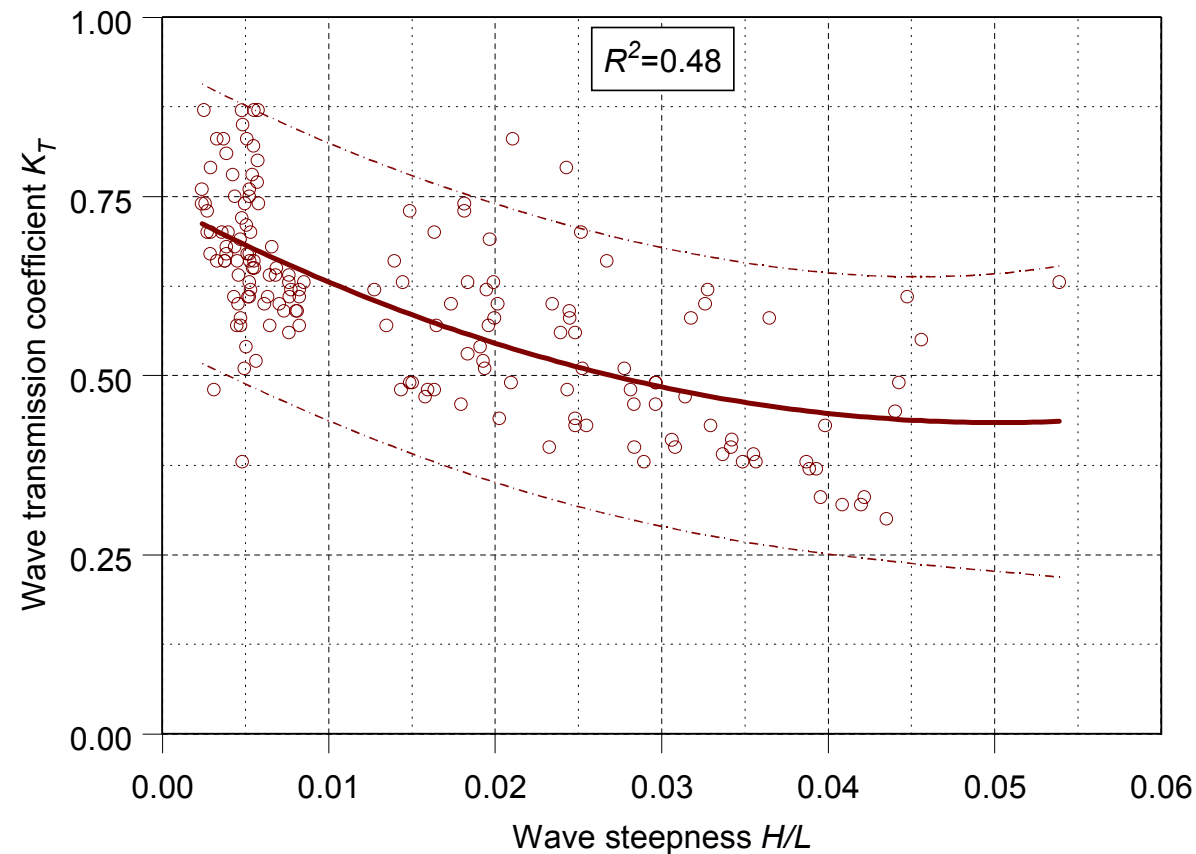

b) $H / L$

Figure 46. Measured $K_{T}$, least squares fit, and $95 \%$ confidence limits versus (a) $B / L$ and (b) $H / L$. 
TABLE 16. Summary of XM99 Regression Analysis Parameters

\begin{tabular}{l|c|c|c|c|c}
\hline \hline \multicolumn{2}{c|}{ Independent Variable $x$} & \multirow{3}{*}{ Regression Coefficients } \\
\cline { 1 - 3 } \cline { 5 - 6 } Name & Symbol & $R^{2}$ & $a_{0}$ & $a_{1}$ & $a_{2}$ \\
\hline Transmission factor & $T_{F}$ & 0.42 & 0.48 & 1.03 & -1.33 \\
\hline Relative width & $B / L$ & 0.46 & 0.75 & -3.16 & 7.84 \\
\hline Wave steepness & $H / L$ & 0.48 & 0.74 & -12.17 & 121.12 \\
\hline \hline
\end{tabular}

Joint distributions of the measured $K_{T}$ versus peak wave period $T_{p}$, significant wave height $H_{S}$, mean wave direction $\bar{\theta}$, and directional spread $\sigma_{m}$ were calculated. The measured $K_{T}$ were within the threshold mainly for the "sea" band of frequencies, although several bimodal cases with nearly equal sea and swell components were also successfully reduced. Incident wave heights up to 0.83 were efficiently reduced. Threshold $K_{T}$ values were obtained for wave directions almost broadside to the starboard leg and $10 \mathrm{deg}$ to the port side of the centerline, and the full range of observed directional spread from 30 to $60 \mathrm{deg}$.

The WAMIT-predicted $K_{T}$ for a wave period of $3 \mathrm{sec}$ and wave direction parallel to the XM99 centerline (i.e., $315 \mathrm{deg}$ ) was selected for comparison. The measured $K_{T, W g t}$ was an average of all the waves with a wave period of $3 \pm 0.5 \mathrm{sec}$ and wave direction of $315 \pm 15 \mathrm{deg}$. The agreement was very good.

The PTT and MPTT analytical models were compared with the measured $K_{T}$ versus Wiegel's transmission factor $T_{F}$. For the XM99 data, small values of $T_{F}$ correspond to small wave periods. The measured $K_{T}$ fell into two groups (for the "sea" and "swell" regimes) with a lot of scatter. Thus, the fit was not as good for this parameter as it had been for the FLab data. The XM99 data set includes more bimodal and multimodal waves and a wider range of wave directions and larger directional spreading than the FLab data. The PTT and MPTT models under predict $K_{T}$ for small $T_{F}$ values, but improve as $T_{F}$ increases. Only the PTT model would be useful for predicting $K_{T}$ within the design criteria of SS3 for the RIBS.

Again, several empirical parameters were investigated relative to measured $K_{T}$. Of the three parameters, wave steepness was the best, but not significantly better than the others. In general, the existing analytical and empirical models do not predict wave transmission for the XM99 very well. 


\section{CHAPTER VII}

\section{DYNAMIC PRESSURES}

In this Chapter dynamic pressures $p_{1}$ for the fundamental laboratory experiment (FLab) and XM99 field trials (XM99) are presented and discussed. Dynamic pressures are important because these are used to calculate wave forces and moments on the RIBS. First, the calculation procedures for calculating the dynamic pressures are described. Then, the measured dynamic pressures are presented and discussed. Next, the normalized pressures are compared with the predictions from the analytical and WAMIT numerical models. Finally, empirical relationships between the $p_{l}$ and several different nondimensional parameters are presented and discussed.

\section{FUNDAMENTAL LABORATORY EXPERIMENTS}

\section{Calculation Procedure}

The equations for the external (i.e., Region 1) dynamic pressure $p_{1}$ were presented in Chapter IV. The measured $p_{I, S}$ is calculated as an equivalent wave height (or double amplitude) using standard zero downcrossing and zero moment estimation techniques. The measured $p_{1, S}$ are presented in dimensional form, in units of $\mathrm{kPa}$. Details on these calculations are presented later in this section.

For comparisons between numerical and analytical models, normalized pressure values are required. The standard normalizing factor for dynamic pressure is $\rho g A$, where $A$ is the incident wave amplitude and is equal to one half the incident wave height $H$ by linear wave theory. Both WAMIT and analytical dynamic pressures are normalized in this manner. The analyticallypredicted $\bar{p}_{l, A}$ from linear wave theory was derived in Chapter IV and is repeated here

$$
\bar{p}_{1, A}=\frac{p_{1}}{\rho g A}=K_{p}\left(1+\sqrt{1-K_{T}^{2}}\right) \cos \left(k_{y} y-\omega t\right)
$$

This includes incident and reflected pressure. The reflection coefficient $K_{R}$ has been replaced by the $K_{T}$ determined from an energy balance approach and the term with $k_{x}$ has been set to zero 
because $x=0$ on the external side of the RIBS leg. Thus, for consistency, an equivalent normalized pressure $\bar{p}_{l, S}$ is obtained by dividing the measured $p_{l, S}$ by $\rho g H$.

\section{Measured Dynamic Pressure}

Spectral cases. As previously described in Chapter II, the FLab had three pressure gages installed at bow, middle, and stern locations on the starboard RIBS leg to measure external dynamic pressure. These gages were located on one level at $z=-8.5 \mathrm{~cm}$ from the surface for all $d / h$ drafts. Only the data for the four $\theta_{r}=45 \mathrm{deg}$ interior angle configurations are presented here.

For the spectral cases, four different dynamic pressure estimates $p_{1}$ were calculated. These include time domain estimates of the significant $p_{1, S}$, average $p_{1, A v e}$, and maximum $p_{1, \max }$ dynamic pressure, and the frequency domain zero-moment $p_{l, m 0}$. However, only the $p_{1, S}$ values are presented here because the other pressure estimates appeared to follow a Rayleigh distribution relative to magnitudes.

Figure 47 shows the measured external $p_{1, S}$ pressure for the FLab. The organization of these plots is similar as the $K_{T}$ wave transmission coefficients presented in the previous chapter. The measured $p_{1, S}$ are plotted versus normalized distance of the pressure gage from the bow of the RIBS. The gage distance is measured parallel to the starboard leg from the bow and is normalized by the RIBS leg length $L_{r}$. Thus, the three pressure gages were located at $0.2 L_{r}$, $0.5 L_{r}$, and $0.8 L_{r}$ from the bow. The four frames (i.e., a to d) correspond to the four relative drafts $d / h=0.25,0.50,0.75$, and 1.00 of the model with the $\theta_{r}=45$ deg interior angle, respectively. The six lines in each frame represent the six wave conditions, corresponding to prototype wave periods $T_{p}=5$ (triangle), 8 (square), and 12 (circle) sec and mean wave direction $\bar{\theta}=0$ (solid symbol) and 20 (open symbol) deg. The solid lines represent the $\bar{\theta}=0 \mathrm{deg}$ and the dashed lines the $\bar{\theta}=20 \mathrm{deg}$ cases. Appendix $\mathrm{H}$ contains a listing of $p_{1, S}, p_{1, A v e}$, and $p_{1, \text { Max }}$ pressures for the four $d / h$ configurations in Tables $\mathrm{H} 1$ to $\mathrm{H} 3$, respectively.

As expected, the dynamic pressure $p_{l, S}$ increases as $d / h$ increases, due to the increase in reflected energy on the external side of the RIBS leg. The $p_{1, S}$ are slightly larger for the waves at an angle $\bar{\theta}=20 \mathrm{deg}$ because the wave is more normal to the leg. In general, the $p_{1, S}$ are fairly constant for all three pressure gages for each wave case. There is a tendency to increase at the stern gage location, especially for the $\bar{\theta}=20$ deg cases. The largest variations occur at 


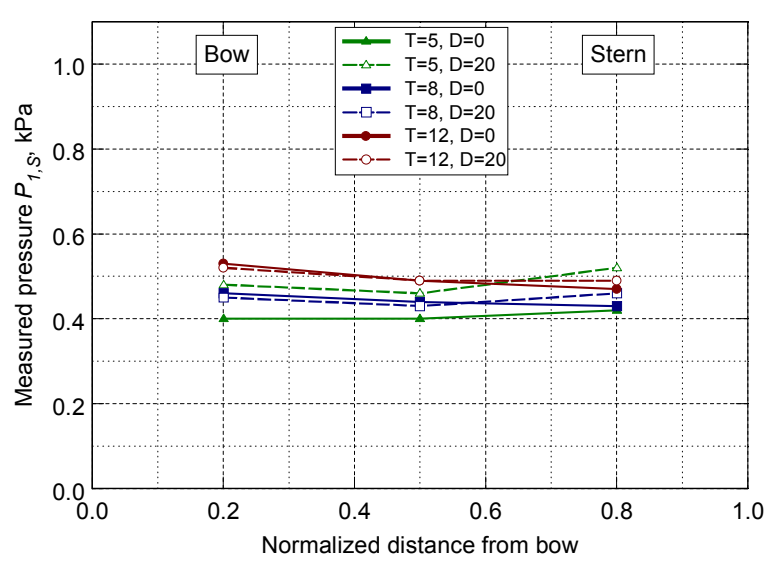

a) 45 deg interior angle, 25 percent draft.

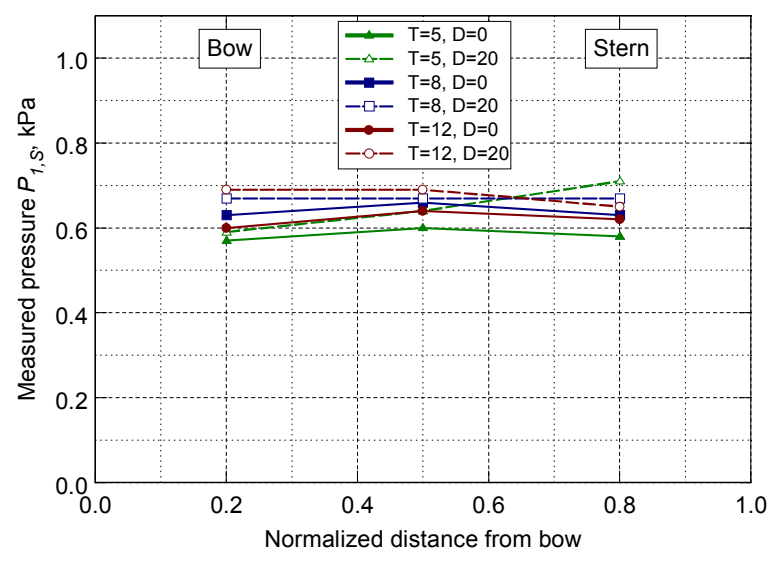

c) 45 deg interior angle, 75 percent draft.

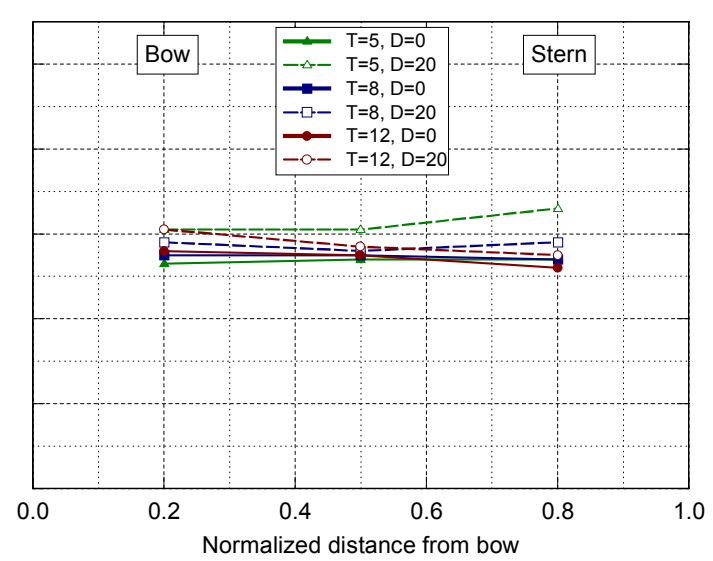

b) $45 \mathrm{deg}$ interior angle, 50 percent draft.

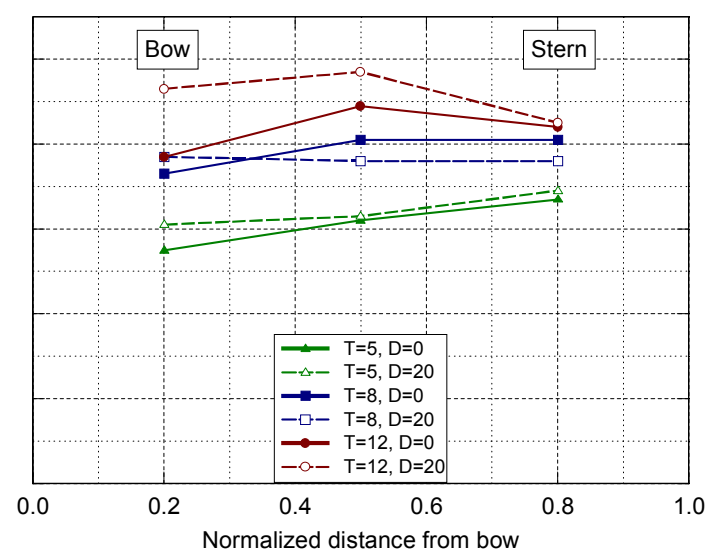

d) 45 deg interior angle, 100 percent draft.

Figure 47. Measured $p_{1, S}$ vs. normalized distance from the bow for FLab $\theta_{r}=45 \operatorname{deg}$ (a) $d / h=0.25$, (b) $d / h=0.50$, (c) $d / h=0.75$, (d) $d / h=1.00$.

$d / h=1.00$, when the RIBS is resting on the bottom. In this case, the $p_{I, S}$ is the largest because the wave can no longer go underneath the RIBS and the reflected energy is the greatest.

In general, the measured $p_{1, S}$ increases from the bow toward the stern of the RIBS. A possible explanation for this is the build-up and growth of stem-waves along the exterior of the RIBS legs. This Mach effect occurs when grazing waves ride along a solid boundary, and is strongly dependent on the incident wave period, and to a lesser extent, the wave height and direction.

As mentioned previously, normalized values are needed for comparing analytical and 
numerical predictions. The measured dynamic pressures $p_{1, S}$ were normalized by the factor $\rho g H$. Appendix Table H4 contains a listing of these normalized dynamic pressures $\bar{p}_{1, S}$ for each of the four configurations. The $\bar{p}_{I, S}$ are shown in Figure 48 . The format is identical to the plots of the dimensional $p_{l, S}$ values. In general, the trends are the same as the dimensional pressure values.

Monochromatic cases. Monochromatic waves were only run for the $d / h=1.00$ configuration. Figure 49 compares the average $p_{1, A v e}$ dynamic pressures among the six spectral and regular wave cases. The spectral cases are represented by the lines connecting the points. The data for the regular cases are shown with larger symbols and no lines. There is not much difference between the spectral and regular cases, although the regular wave values are slightly

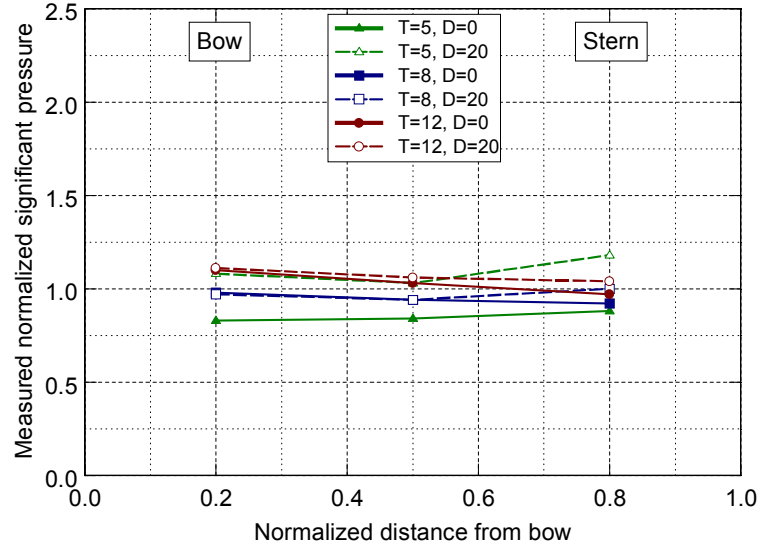

a) 45 deg interior angle, 25 percent draft.

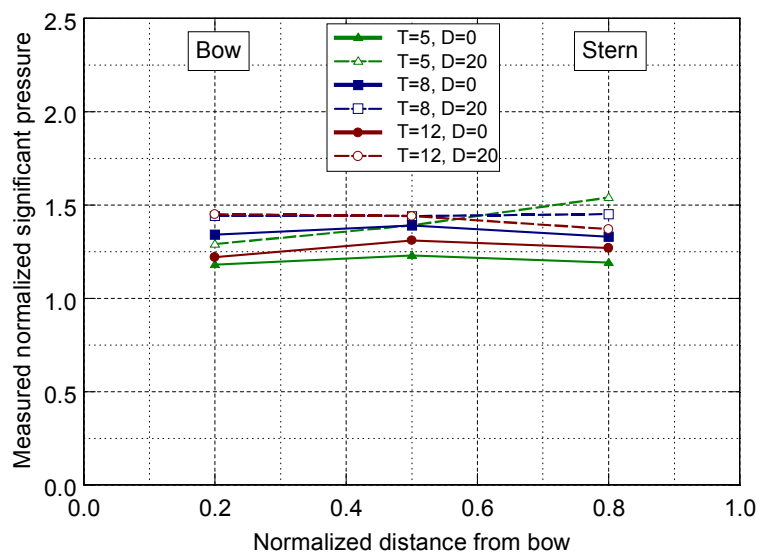

c) 45 deg interior angle, 75 percent draft.

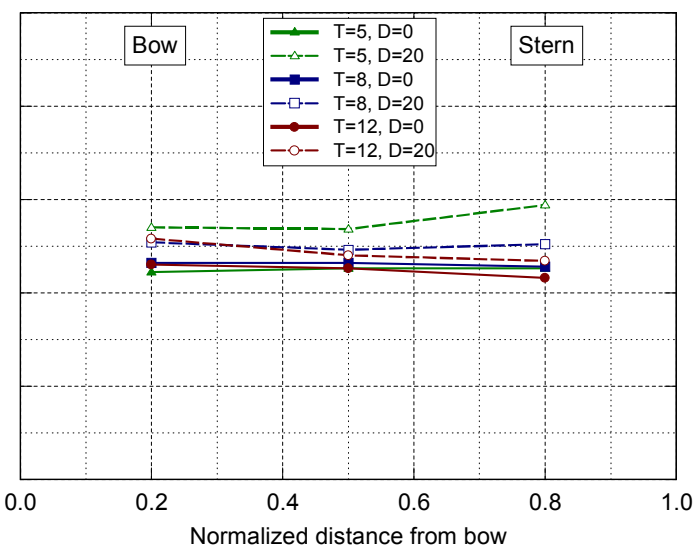

b) 45 deg interior angle, 50 percent draft.

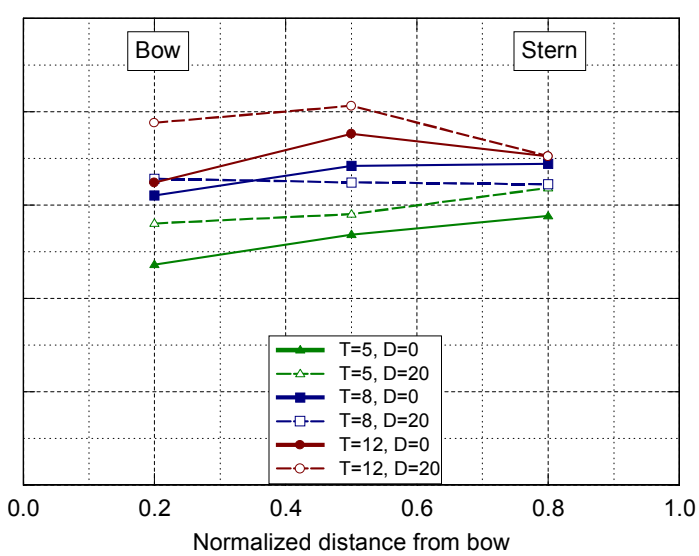

d) 45 deg interior angle, 100 percent draft.

Figure 48. Measured $\bar{p}_{1, S}$ vs. normalized distance from the bow for FLab $\theta_{r}=45 \mathrm{deg}$ (a) $d / h=0.25$, (b) $d / h=0.50$, (c) $d / h=0.75$, (d) $d / h=1.00$. 


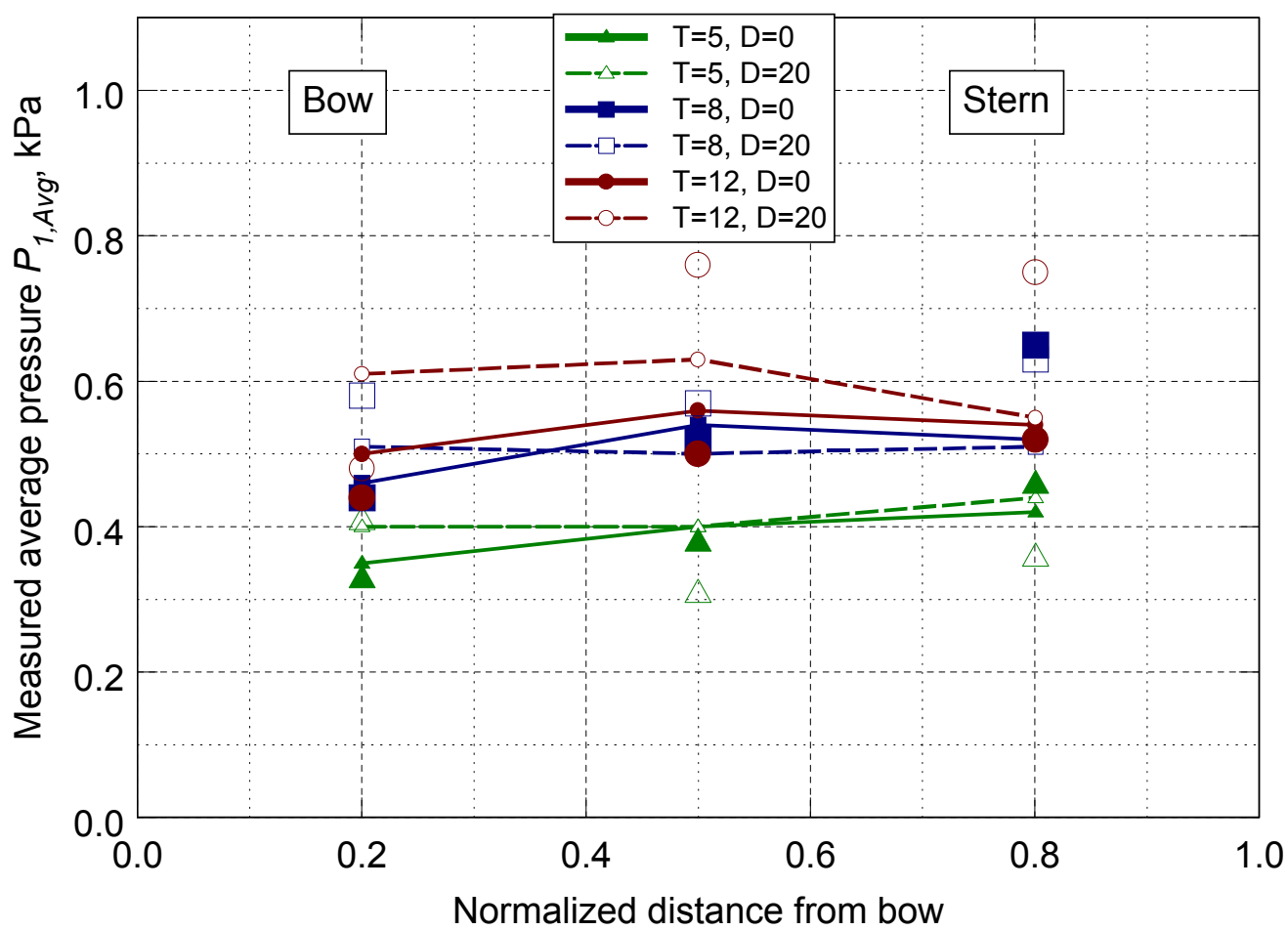

Figure 49. Comparison of average $p_{l, A v g}$ for regular and spectral waves for $\theta_{r}=45 \mathrm{deg}, d / h=1.00$. larger. The middle and stern gages show a significant difference for the $T_{p}=12 \mathrm{sec}$ and $\bar{\theta}=20$ deg case, however. Appendix Tables H5 and H6 contain a listing for the monochromatic cases of the $p_{1, A v e}$ and $p_{1, \text { Max }}$ pressures, respectively.

Comparison of SS3 and SS5 cases. As with the $\mathrm{K}_{\mathrm{T}}$ comparisons, dynamic pressures were recorded for the $T=12 \mathrm{sec}$ cases for two wave heights. The larger wave height corresponded to an SS5 wave condition with a height of $3.66 \mathrm{~m}$. Figure 50 compares the $p_{l, S}$ values of the SS3 and SS5 wave cases at the four $\theta_{r}=45 \mathrm{deg}$ interior angle configurations. In general, the $p_{1, S}$ increases as the $d / h$ increases. The $\bar{\theta}=20 \mathrm{deg}$ cases are larger than their corresponding $\bar{\theta}=0$ deg cases and the differences increase as $d / h$ increases. These trends are similar to the $p_{1, S}$ values.

The ratio of the SS5 to SS3 wave heights is about $2.4(=3.66 \mathrm{~m} / 1.52 \mathrm{~m})$, while the ratio of dynamic pressures ranges from a low of 6.4 to a high of 7.5. This pressure ratio increases as $d / h$ and $\bar{\theta}$ increase. The average for all cases is 6.9. Listings of the $p_{1, S}, p_{1, A v g}$, and $p_{1, M a x}$ pressures for the SS5 cases are also contained in Appendix Tables H1 to H3. 


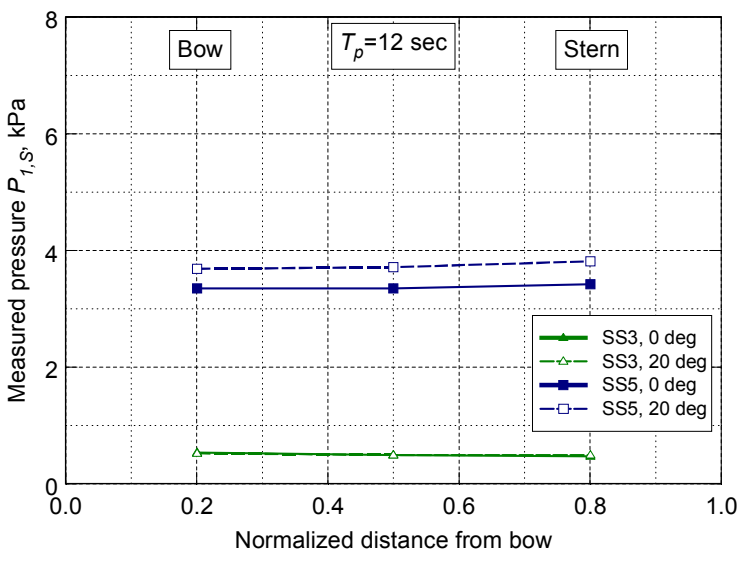

a) 45 deg interior angle, 25 percent draft.

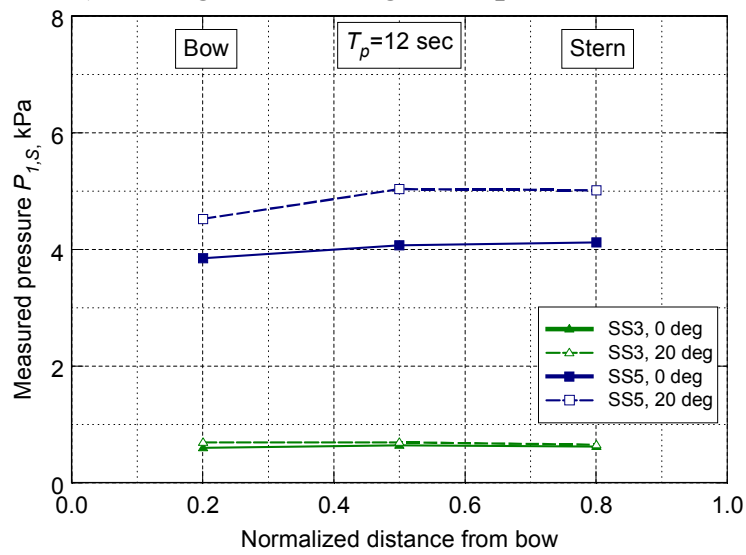

c) 45 deg interior angle, 75 percent draft.

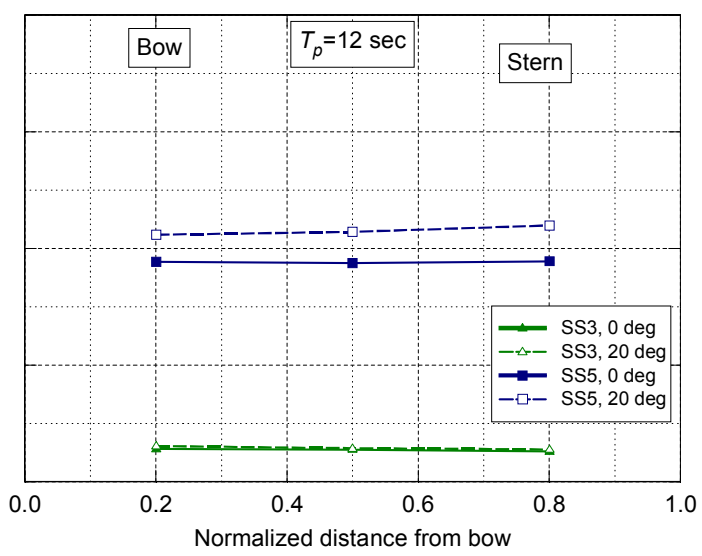

b) 45 deg interior angle, 50 percent draft.

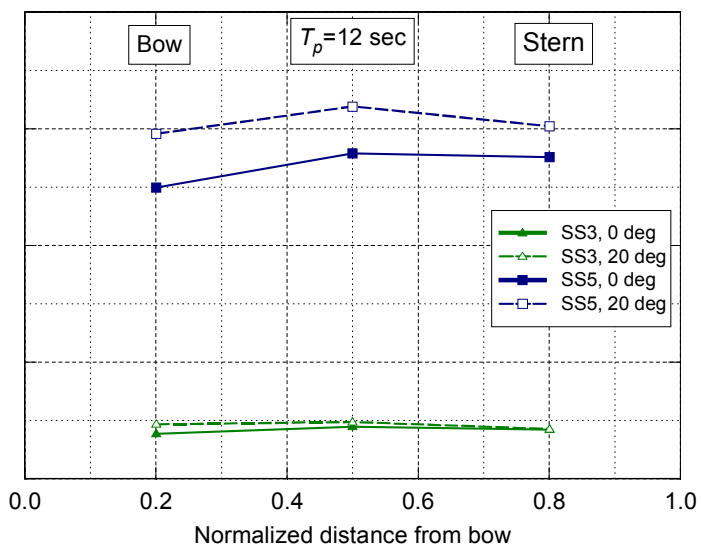

d) 45 deg interior angle, 100 percent draft.

Figure 50. Effect of wave height on measured $p_{1, S}$ for FLab $T_{p}=12 \mathrm{sec}$ and $\theta_{r}=45 \mathrm{deg}$ (a) $d / h=0.25$, (b) $d / h=0.50$, (c) $d / h=0.75$, (d) $d / h=1.00$.

\section{WAMIT Predicted Dynamic Pressures}

The predicted normalized dynamic pressure $\bar{p}_{W}$ from the WAMIT numerical models is estimated using the procedures described in Chapter V. Figures 51 to 53 illustrate the variation of dynamic pressure along the starboard leg for the six wave cases for $d / h=1.00$. These pressures are "snapshots" in time of the spatial variation of pressure along the external side of the leg. The forty curves in the figures correspond to the estimated pressure at the center of each 
a)

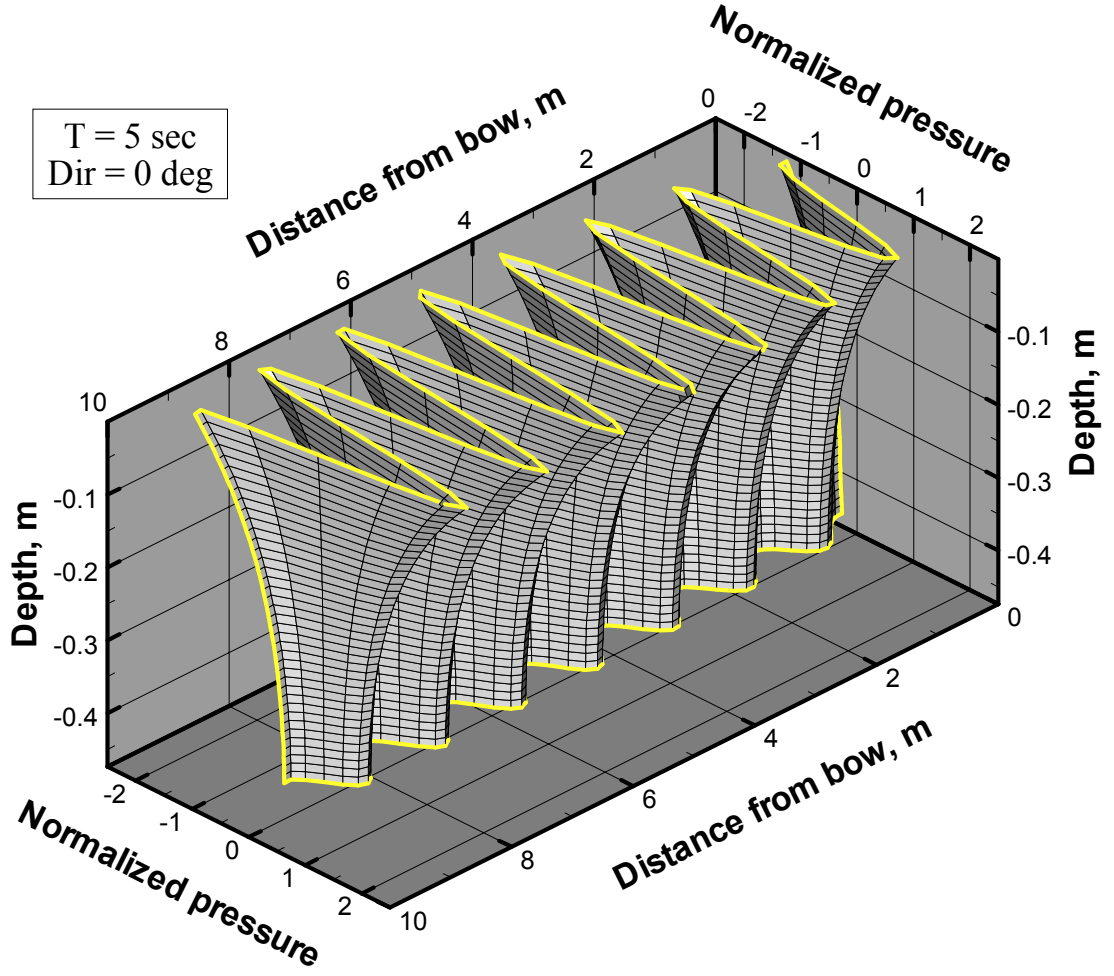

b)

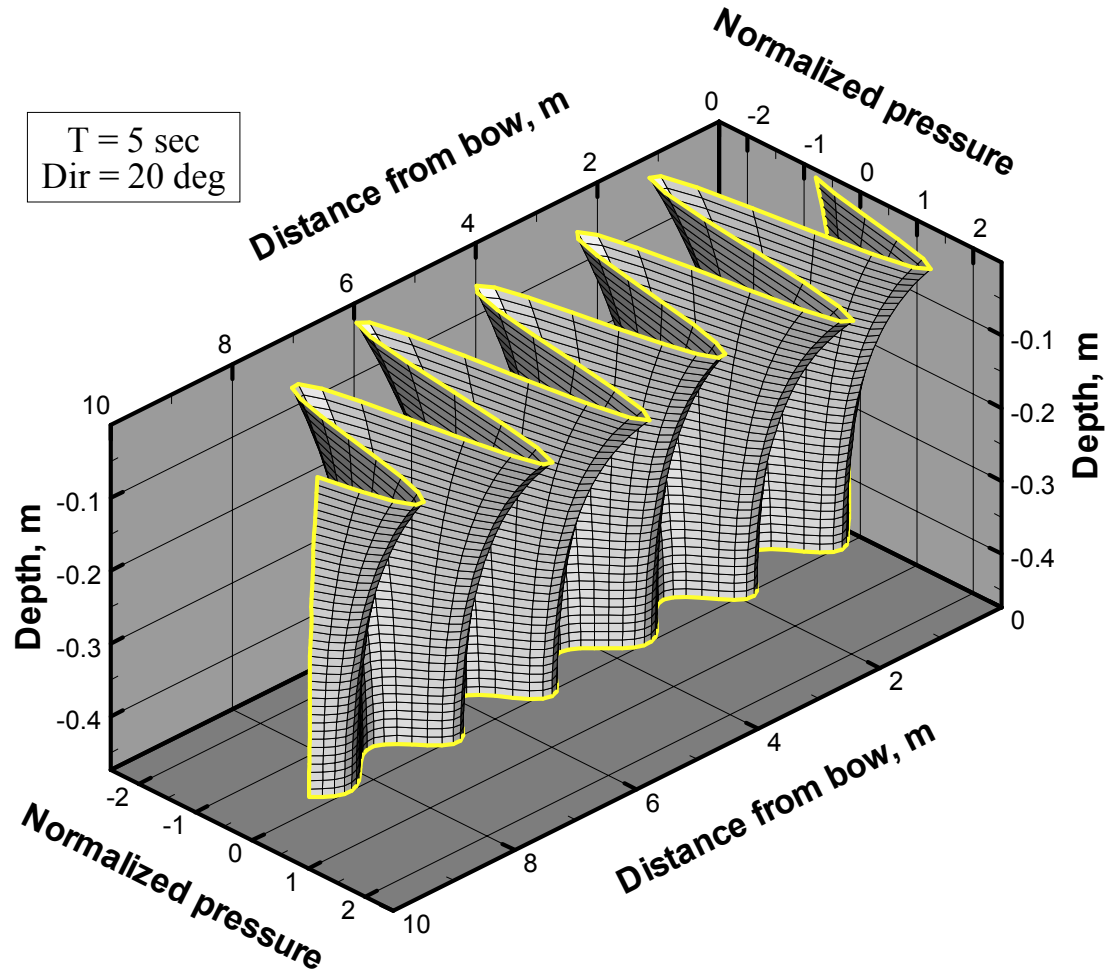

Figure 51. WAMIT predicted $\bar{p}_{W}$ for $d / h=1.00$ and $T=5 \mathrm{sec}$ (a) $\bar{\theta}=0 \mathrm{deg}$ and (b) $\bar{\theta}=20 \mathrm{deg}$. 
a)

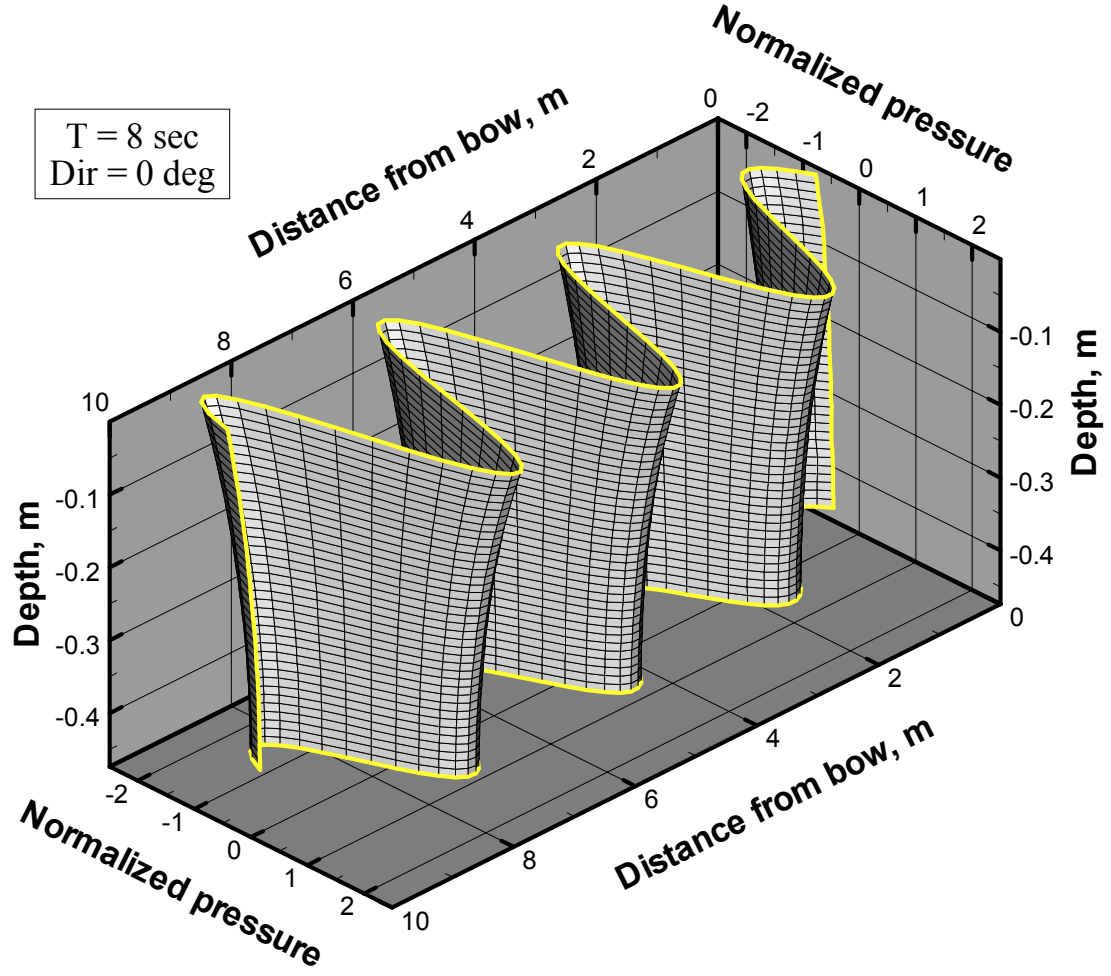

b)

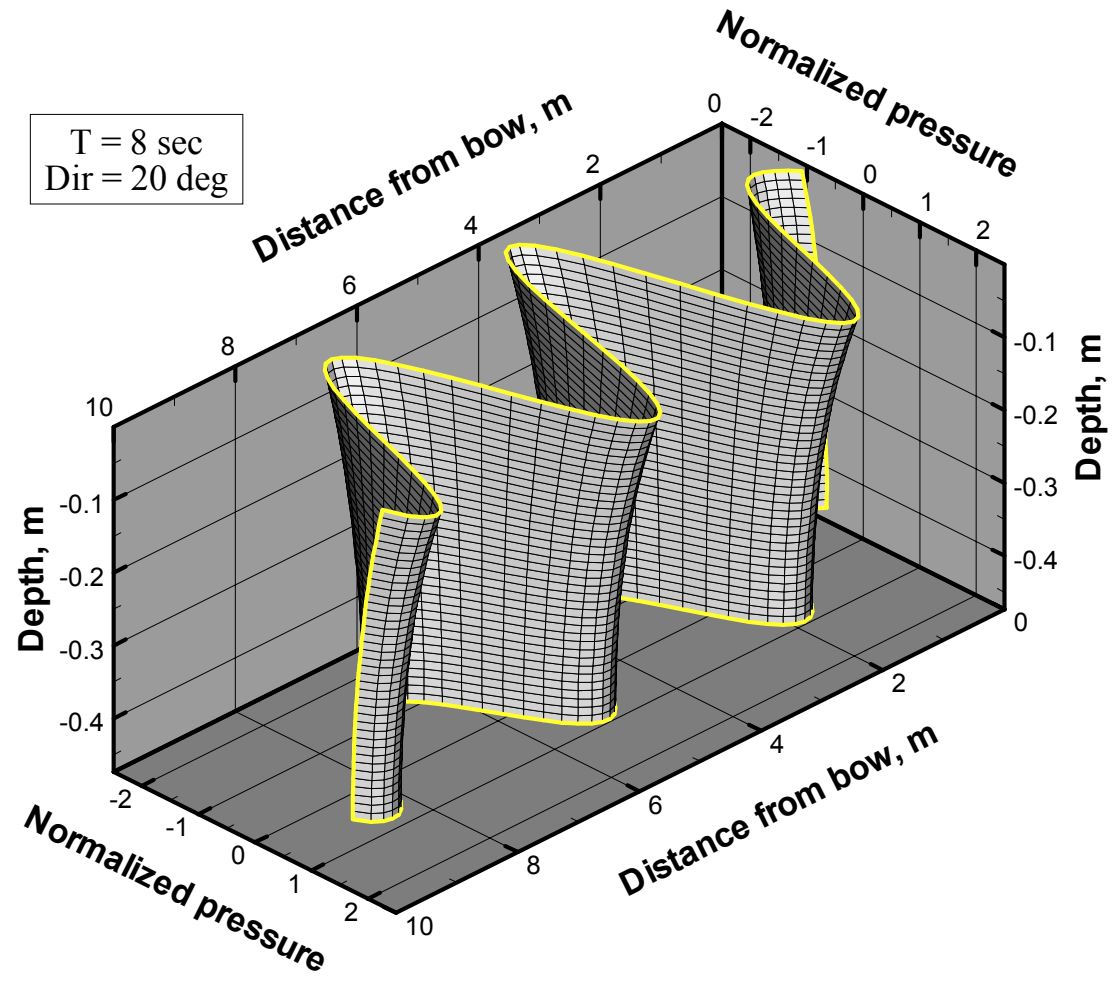

Figure 52. WAMIT predicted $\bar{p}_{W}$ for $d / h=1.00$ and $T=8 \mathrm{sec}$ (a) $\bar{\theta}=0 \mathrm{deg}$ and (b) $\bar{\theta}=20 \mathrm{deg}$. 
a)

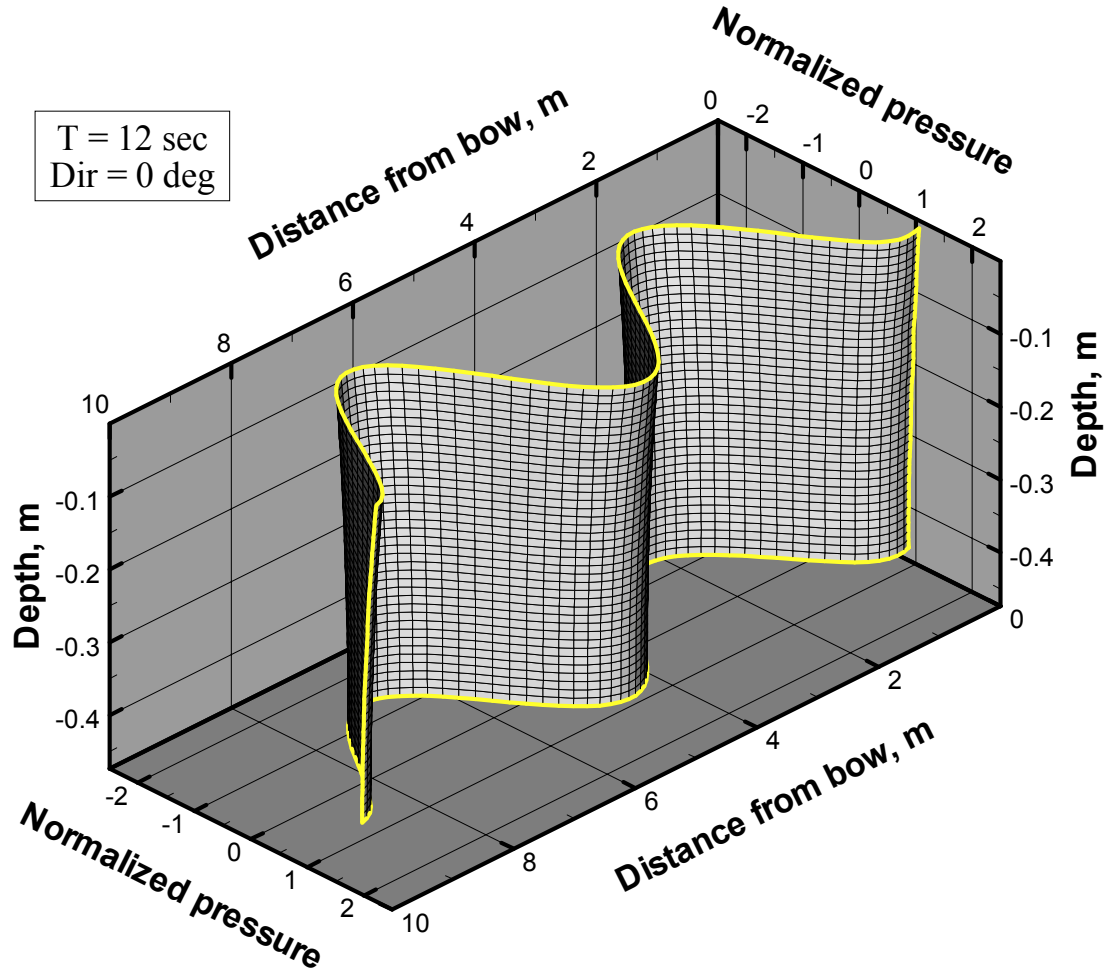

b)

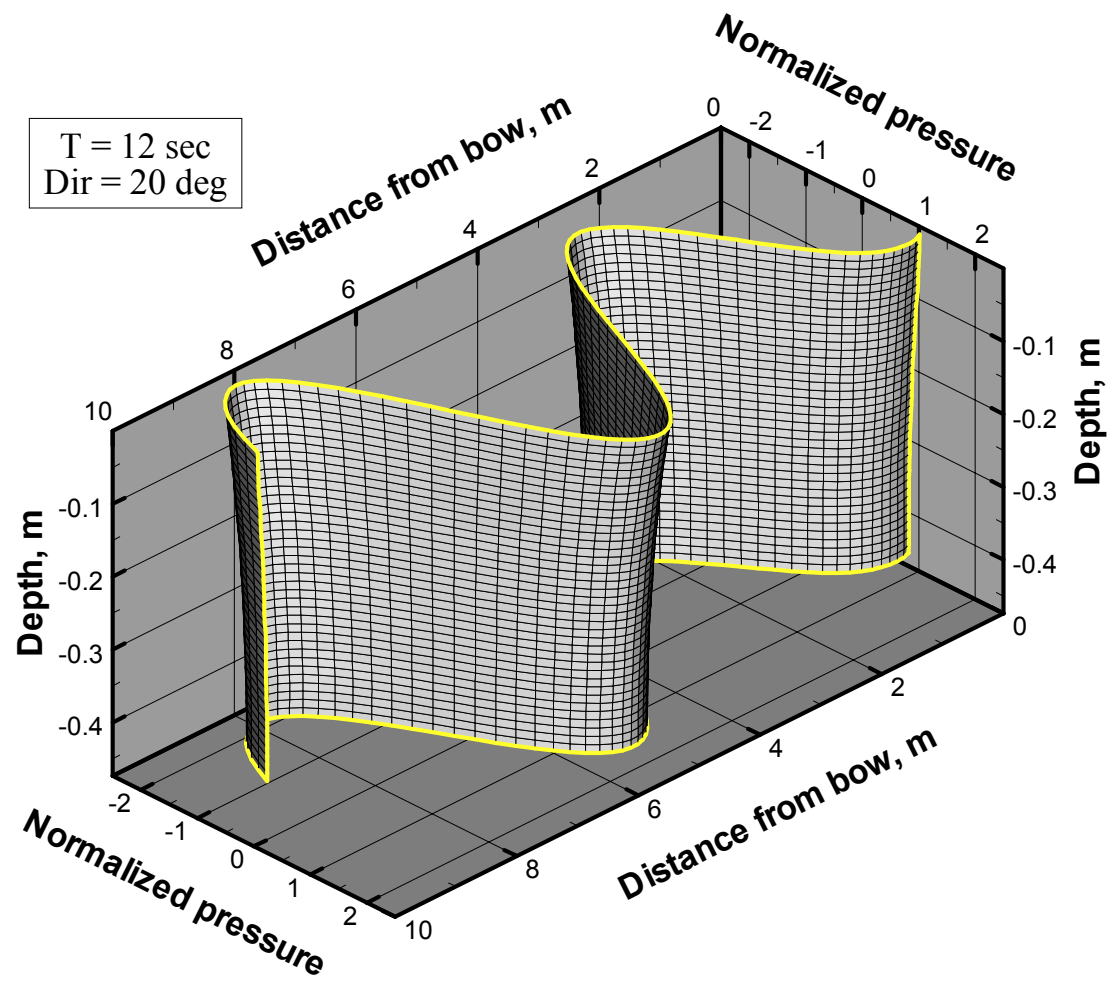

Figure 53. WAMIT predicted $\bar{p}_{W}$ for $d / h=1.00$ and $T=12 \mathrm{sec}$ (a) $\bar{\theta}=0 \mathrm{deg}$ and (b) $\bar{\theta}=20 \mathrm{deg}$. 
panel throughout the water column, spaced every $11.4 \mathrm{~mm}$ from the water surface. The pressure increases from the bottom to the surface. Positive pressures are due to the passage of the crests and negative pressures to the passage of the wave troughs. The number of waves along the RIBS leg varies as the wavelength changes according to the wave period. Although not shown, the variation of dynamic pressure with the other $d / h$ ratios is similar except that the drafts are shallower and do not go all the way to the bottom.

The three pressure gages are all located at the same vertical level at $z=-8.5 \mathrm{~cm}$ below the surface. The dynamic pressure variation at this level is shown in Figures 54 to 56 for each of

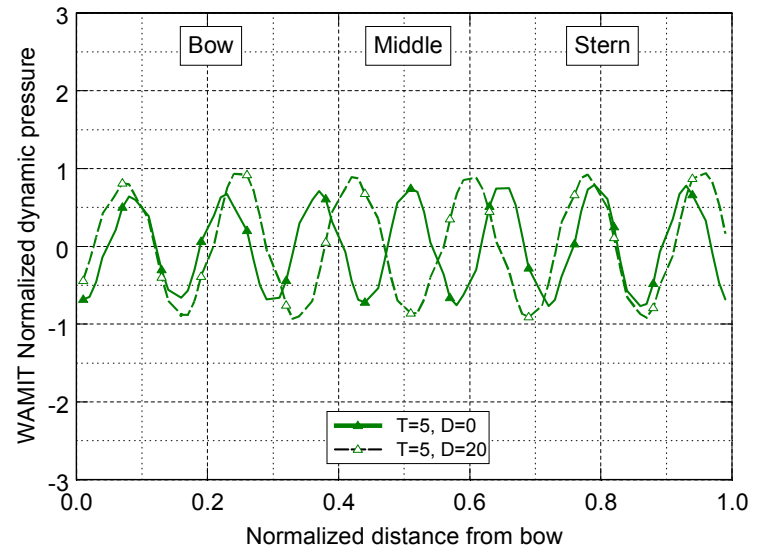

a) 45 deg interior angle, 25 percent draft.

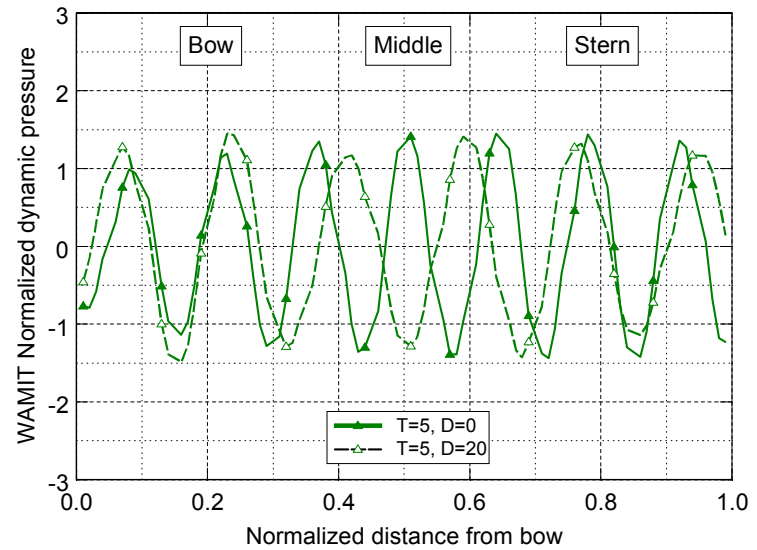

c) $45 \mathrm{deg}$ interior angle, 75 percent draft.

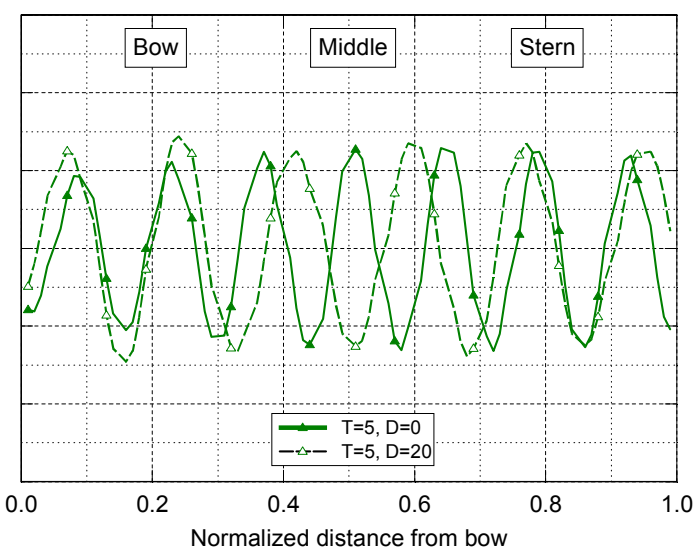

b) 45 deg interior angle, 50 percent draft.

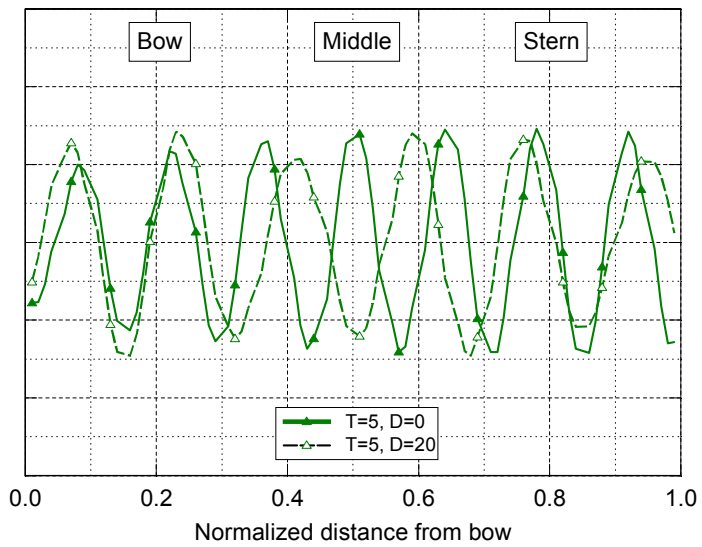

d) $45 \mathrm{deg}$ interior angle, 100 percent draft.

Figure 54. WAMIT $\bar{p}_{W}$ for FLab $\theta_{\mathrm{r}}=45 \mathrm{deg}$, T=5sec: (a) $d / h=0.25$, (b) $d / h=0.50$, (c) $d / h=0.75$, (d) $d / h=1.00$. 


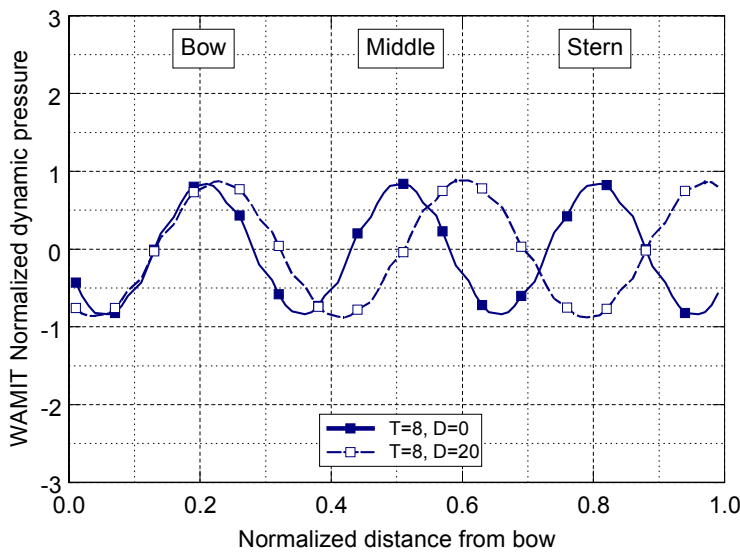

a) 45 deg interior angle, 25 percent draft.

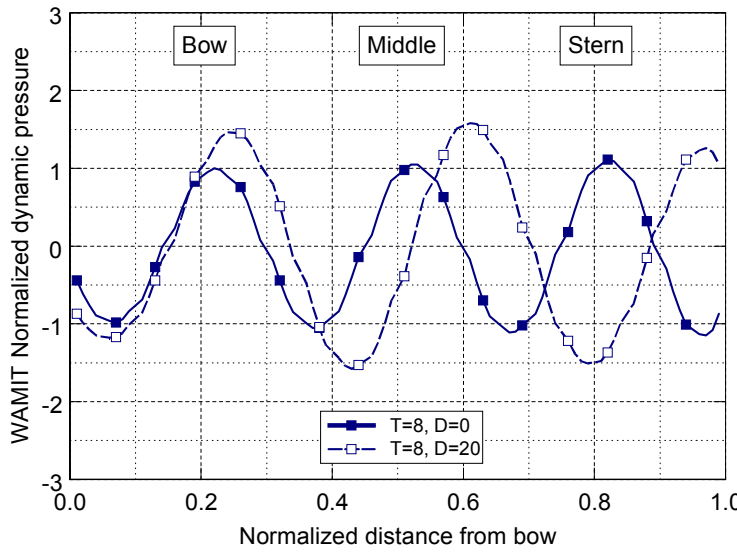

c) 45 deg interior angle, 75 percent draft.

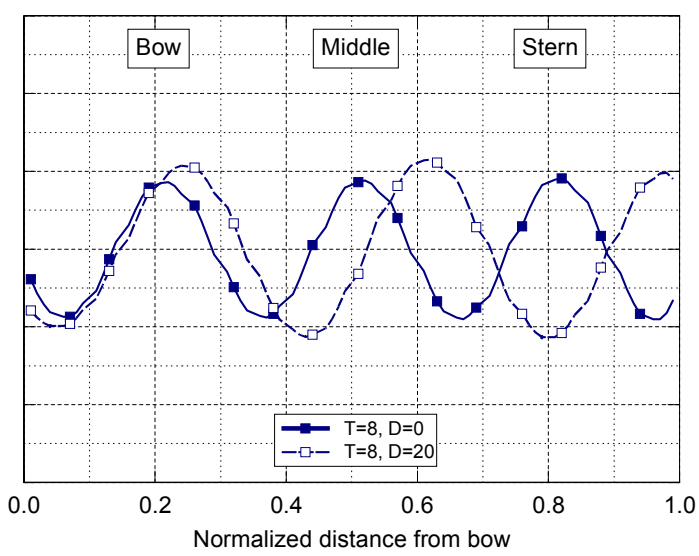

b) $45 \mathrm{deg}$ interior angle, 50 percent draft.

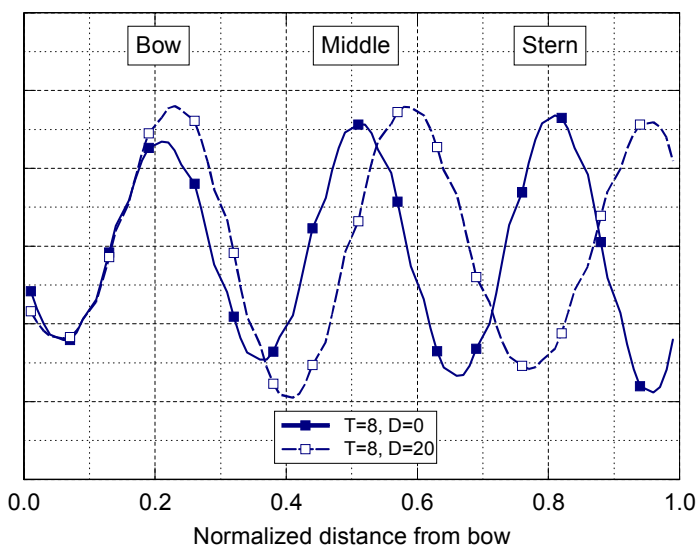

d) 45 deg interior angle, 100 percent draft.

Figure 55. WAMIT predicted $\bar{p}_{W}$ for FLab $\theta_{r}=45 \mathrm{deg}, T=8 \mathrm{sec}, \bar{\theta}=0 \mathrm{deg}$ (solid line), $\bar{\theta}=20 \mathrm{deg}$ (dashed line) (a) $d / h=0.25$, (b) $d / h=0.50$, (c) $d / h=0.75$, (d) $d / h=1.00$.

the three wave periods, respectively. The format is the same as previous figures with the four panels corresponding to the four $d / h$ ratios. The solid line corresponds to the $\bar{\theta}=0$ deg case and the dashed line to the $\bar{\theta}=20 \mathrm{deg}$ case for each wave period. The locations of the bow, middle, and stern pressure gages are identified at their normalized distances from the bow of $0.2 L_{r}, 0.5 L_{r}$, and $0.8 L_{r}$, respectively. These curves represent a "snapshot" in time of the relative pressure along the RIBS starboard leg. 


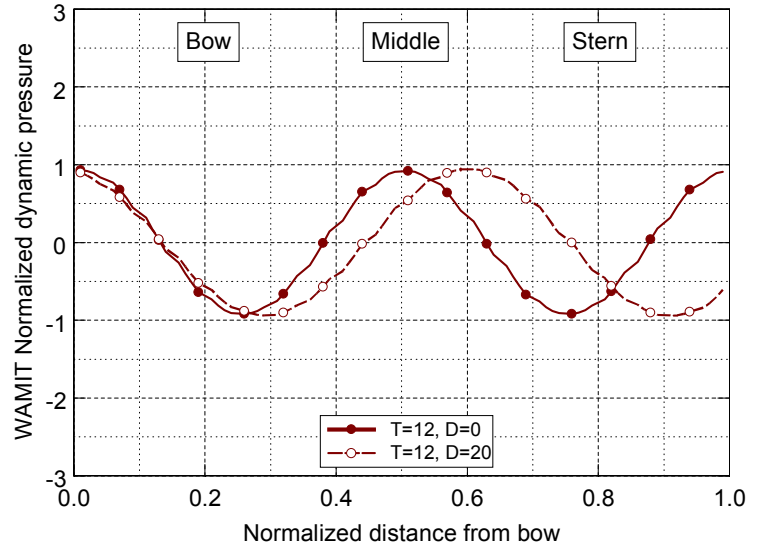

a) 45 deg interior angle, 25 percent draft.

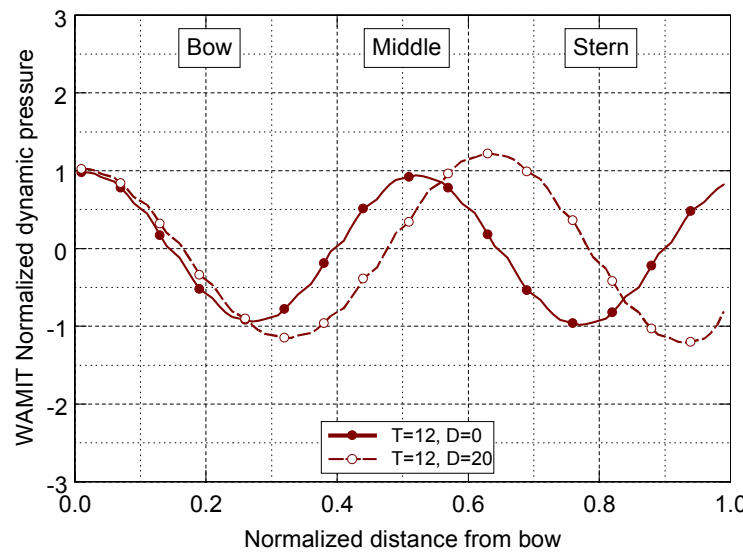

c) 45 deg interior angle, 75 percent draft.

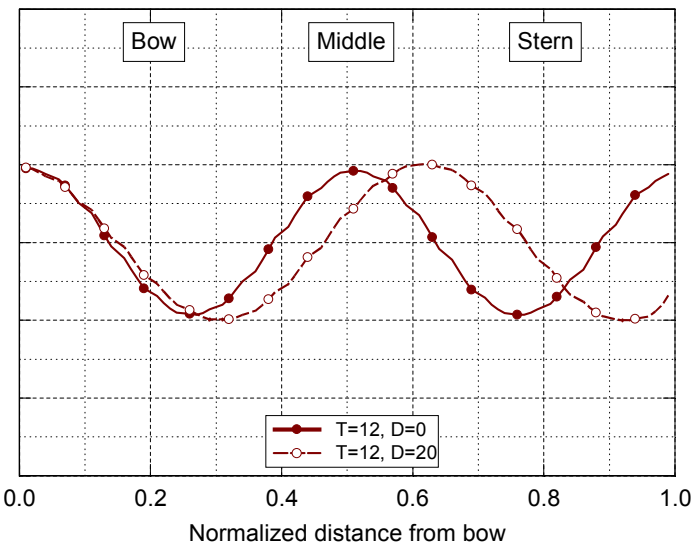

b) 45 deg interior angle, 50 percent draft.

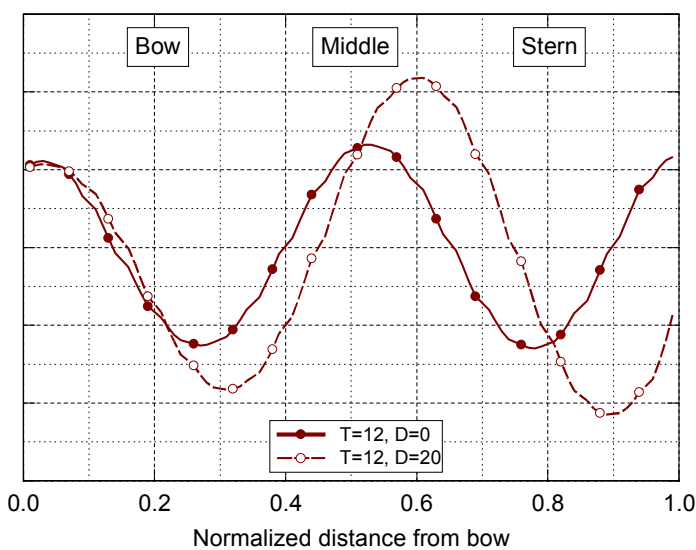

d) 45 deg interior angle, 100 percent draft.

Figure 56. WAMIT predicted $\bar{p}_{W}$ for FLab $\theta_{r}=45 \mathrm{deg}, T=12 \mathrm{sec}, \bar{\theta}=0 \mathrm{deg}$ (solid line), $\bar{\theta}=20 \mathrm{deg}$ (dashed line) (a) $d / h=0.25$, (b) $d / h=0.50$, (c) $d / h=0.75$, (d) $d / h=1.00$.

The relative phase between the two wave directions $\bar{\theta}$ can be observed on these plots. In general, the dynamic pressure magnitudes increase as $d / h$ increases and are nearly constant along the outer surface of the RIBS leg. As the $d / h$ increases, the dynamic pressures tend to increase slightly toward the stern, especially for the $T_{p}=12 \mathrm{sec}$ and $\bar{\theta}=20 \mathrm{deg}$ wave at $d / h=1.00$. For the $T_{p}=8 \mathrm{sec}$ cases, the $\bar{\theta}=20 \mathrm{deg}$ pressures are larger than the $\bar{\theta}=0$ deg cases. These cases also increase toward the middle gage and then decrease toward the stern. In summary, the WAMIT predicted $\bar{p}_{W}$ magnitude increases with $d / h$ and only slightly with $T_{p}$, especially for the $d / h=$ 1.00 cases. The $\bar{p}_{W}$ phase has a phase shift due to wave direction $\bar{\theta}$. Appendix Tables $\mathrm{H} 7$ to 
H10 contain listings of the WAMIT $\bar{p}_{W}$ for the six operational series wave cases and four $d / h=0.25,0.50,0.75,1.00$ configurations, respectively.

The WAMIT $\bar{p}_{1, W}$ at each gage location and $d / h$ configuration is shown in Figure 57 . The magnitude of this pressure was determined by taking one half of the crest to trough distance closest to the gage from the previous figures. The justification for this procedure is that this technique attempts to include some of the WAMIT variation in $\bar{p}_{W}$ along the length of the RIBS leg. This pressure is representative of what each gage would experience based on its relative position. At another instant in time, these pressures would change, but would still maintain their relative magnitudes to one another. This figure can be compared to the previously presented

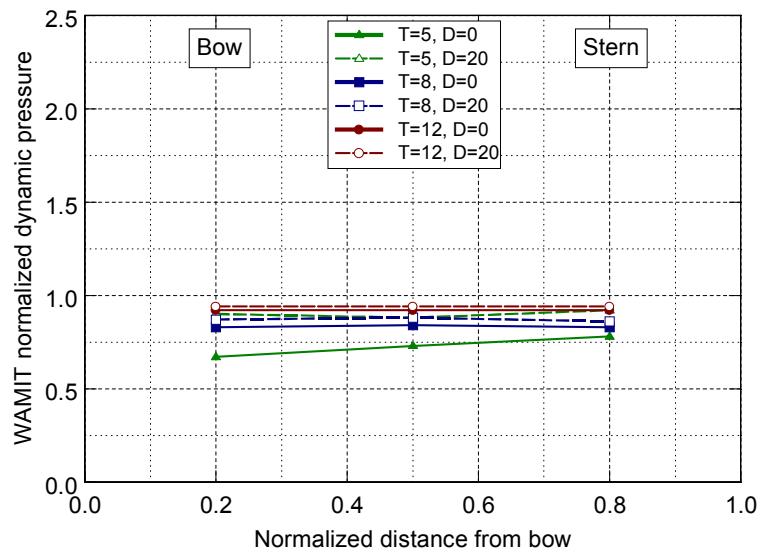

a) $45 \mathrm{deg}$ interior angle, 25 percent draft.

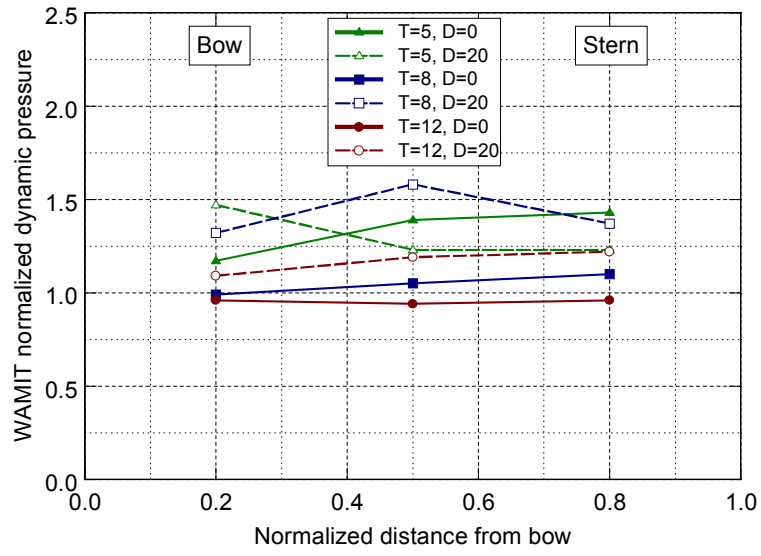

c) 45 deg interior angle, 75 percent draft.

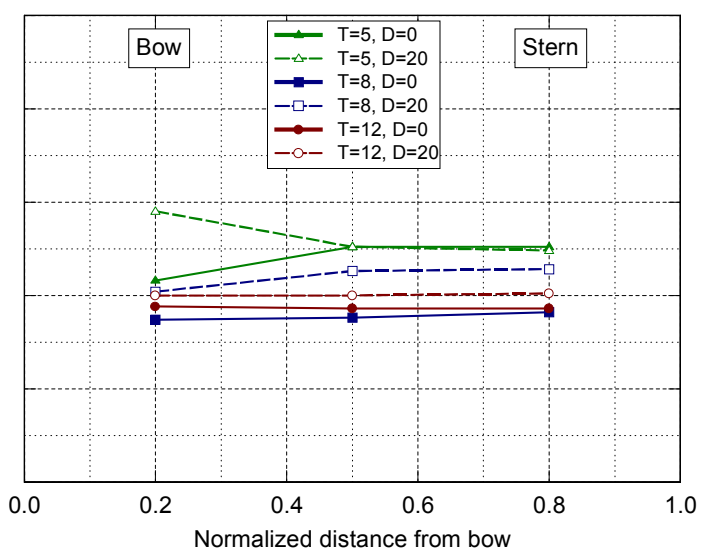

b) 45 deg interior angle, 50 percent draft.

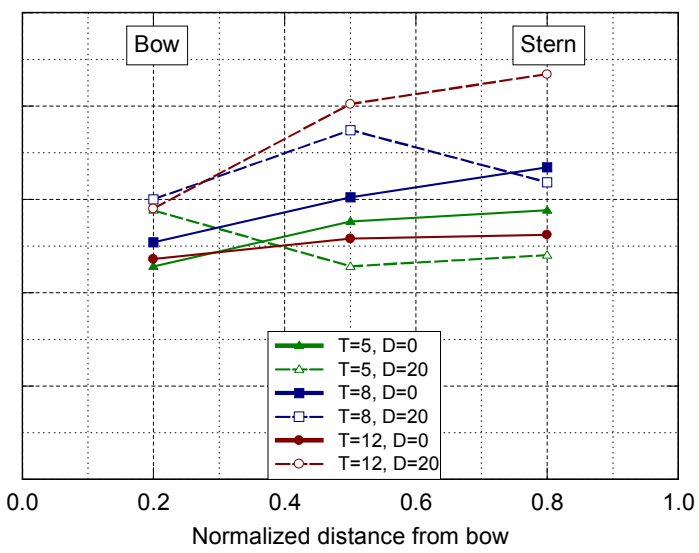

d) 45 deg interior angle, 100 percent draft.

Figure 57. WAMIT $\bar{p}_{1, W}$ for FLab $\theta_{r}=45 \operatorname{deg}$ (a) $d / h=25 \%$, (b) $d / h=0.50$, (c) $d / h=0.75$, (d) $d / h=1.00$. 
Figure 48 for the measured normalized significant pressures $\bar{p}_{l, S}$.

The agreement between measured $\bar{p}_{1, S}$ and WAMIT predicted $\bar{p}_{1, W}$ pressures is good. The three panels of Figure 58 show this relationship for the bow, middle, and stern gages, respectively. In each panel the individual pressure values at each $d / h$ ratio are shown. A 45-deg line is again included to improve readability. In general, the WAMIT predictions are slightly smaller than the measured pressures, but do not have a lot of scatter.

Table 17 lists the minimum, maximum, average, and standard deviation for the measured, WAMIT, and $\Delta \overline{\mathrm{p}}_{1}$ dynamic pressures. These statistics are based on the average of the three gages for six wave cases and four values of $d / h$, or a total of 72 values. The $\Delta \bar{p}_{1}$ was obtained by subtracting the WAMIT $\bar{p}_{I, W}$ from the measured $\bar{p}_{1, S}$ value at each of the 72 values. Thus, the average measured $\bar{p}_{l, S}$ was 0.28 higher than the corresponding WAMIT $\bar{p}_{1, W}$. Appendix Table H1 1 contains a listing of these WAMIT $\bar{p}_{1, W}$ for each $d / h$ configuration, including the SS5 conditions.

TABLE 17. WAMIT and Measured Dynamic Pressure Statistics

\begin{tabular}{c|c|c|c}
\hline \hline Statistic & WAMIT $\bar{p}_{1, W}$ & Measured $\bar{p}_{1, S}$ & $\Delta \bar{p}_{1}$ \\
\hline Minimum & 1.45 & 1.70 & -0.26 \\
\hline Maximum & 3.75 & 3.82 & 0.99 \\
\hline Average & 2.30 & 2.59 & 0.28 \\
\hline Standard Deviation & 0.56 & 0.53 & 0.28 \\
\hline \hline
\end{tabular}


a)

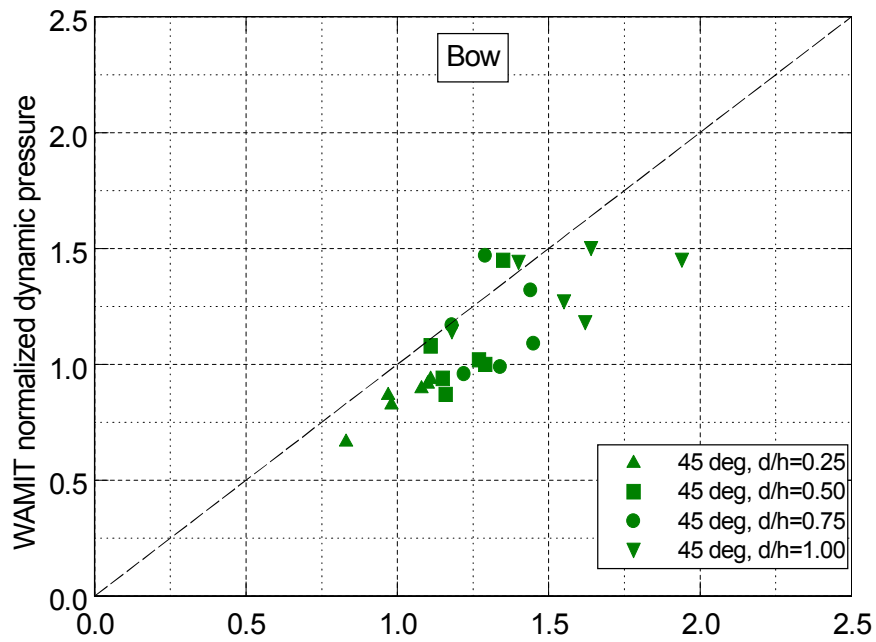

b)
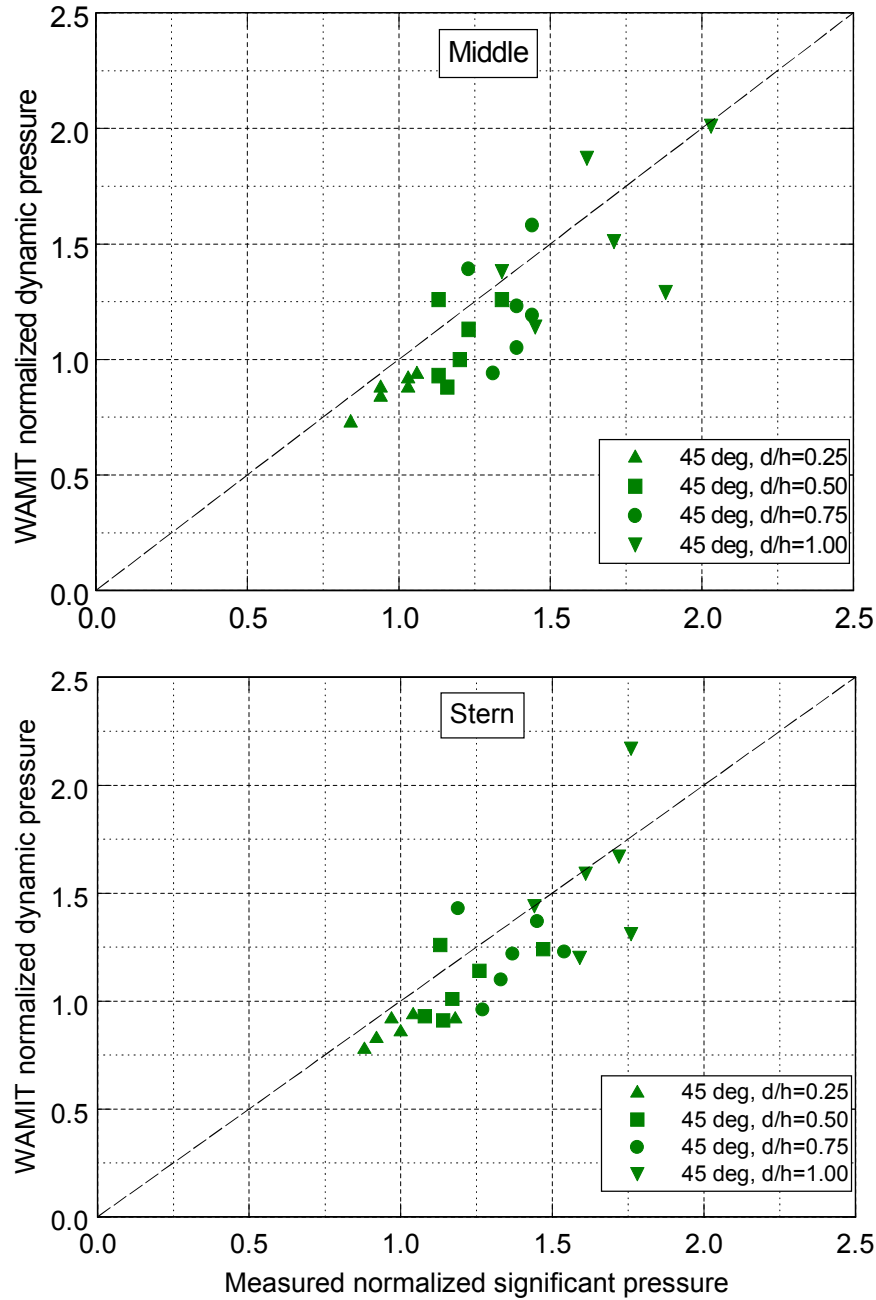

Figure 58. WAMIT $\bar{p}_{1, W}$ vs. measured $\bar{p}_{l, S}$ pressures for FLab (a) bow, (b) middle, (c) stern. 


\section{Comparisons of Measured and Analytical Pressures}

Analytical predictions of the normalized dynamic pressure $\bar{p}_{1, A}$ were estimated using equation 77 for each of the eight wave cases and four values of $d / h$ for $\theta_{r}=45$ deg interior angle. These predictions are based on the linear wave theory model defined earlier in Chapter IV. In the analytical coordinate system, the origin is at the bow and the positive $x$ - and $y$-axes are normal and parallel to the starboard leg, respectively. Therefore, the two wave directions of $\bar{\theta}=0$ and $20 \mathrm{deg}$ correspond to angles of 67.5 and $87.5 \mathrm{deg}$ in this coordinate system.

The predicted $\bar{p}_{l, A}$ magnitude is a function of the pressure response factor $K_{p}$ and reflection coefficient $K_{R}$. The measured $K_{T}$ was used as an estimate of the wave transmission coefficient and substituted for $K_{R}$ according to the energy-based derivation described in Chapter IV. In the analytical model, the $\bar{p}_{l, A}$ magnitude is constant for all pressure gages at each vertical level.

The phase $\bar{p}_{l, A}$ varies for each pressure gage and is a function of the mean wave direction $\bar{\theta}$ and $y$ coordinates. Both $\bar{p}_{l, A}$ magnitude and phase are functions of the wave period $T$, wavelength $L$, and wavenumber $k$, and wavenumber $y$-component $k_{y}$. Appendix Table H12 contains a listing of $K_{T}, k_{x}, k_{y}, K_{p}$, and $\bar{p}_{1, A}$ for each wave case and $d / h$ configuration.

Figure 59 shows the analytical normalized dynamic pressures $\bar{p}_{l, A}$ for each gage location for the six wave cases for $d / h=0.50$. Each of the six panels corresponds to a different wave period and direction combination. For each case, the wave period was discretized into ten even increments for a full cycle. Although the $\bar{p}_{l, A}$ magnitude is constant for each wave period $T$, it shows an increase as $T$ increases. This increase is due to the variation in the reflection coefficient with $T$. The estimated values of $K_{R}$ ranged from a low of 0.53 to a high of 0.94 . The phase of the $\bar{p}_{1, A}$ changes for each pressure gage. These plots can be compared to the corresponding plots from the WAMIT predictions shown in Figures 51 to 53. The difference between the two plots is that the WAMIT plots are "snapshots" in time (i.e., at a fixed time) and show spatial variation whereas the analytical figures are "snapshots" in space (i.e., at a fixed point) and show temporal variation. Each curve is for one wave cycle at a particular gage location. The magnitudes can be compared between the WAMIT and analytical plots. Although plots of the other three $d / h$ are not shown, the $\bar{p}_{l, A}$ magnitude increases slightly as $d / h$ increases.

The analytical $\bar{p}_{1, A}$ and measured $\bar{p}_{1, S}$ are compared in Figure 60 . The agreement is good, and in general, the analytical $\bar{p}_{l, A}$ are slightly larger than the measured $\bar{p}_{l, S}$. 


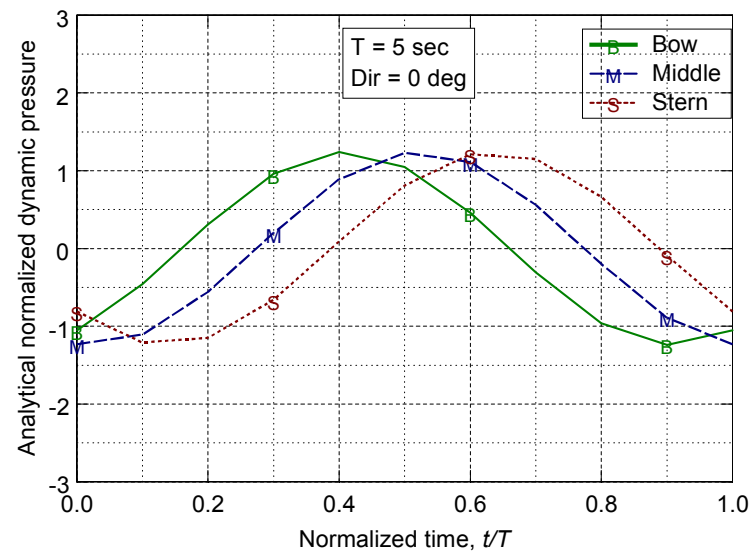

a) $T=5 \mathrm{sec}, \bar{\theta}=0 \mathrm{deg}$.

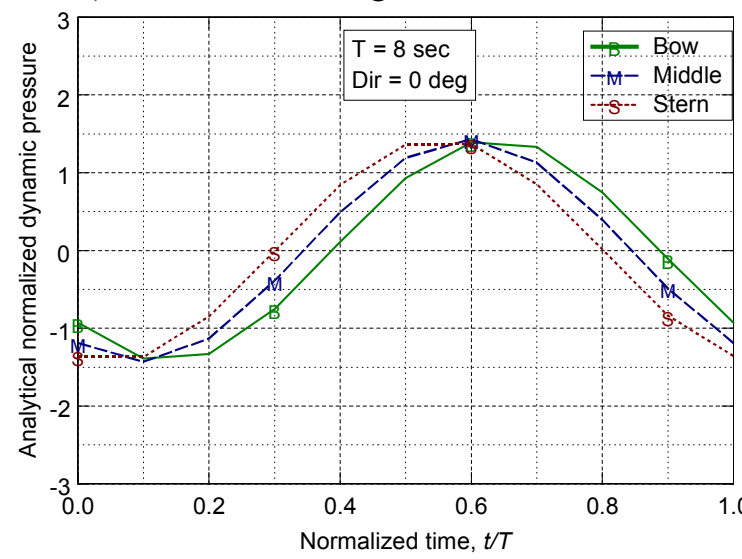

c) $T=8 \mathrm{sec}, \bar{\theta}=0 \mathrm{deg}$.

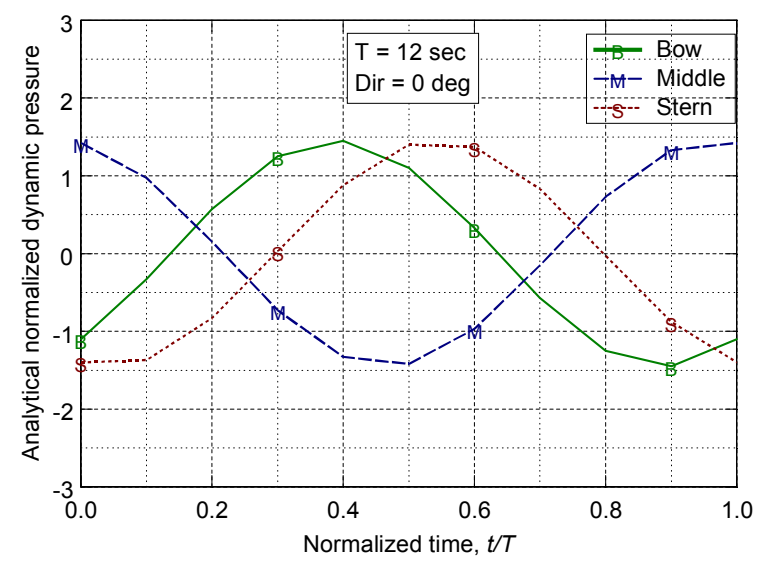

e) $T=12 \mathrm{sec}, \bar{\theta}=0 \mathrm{deg}$.

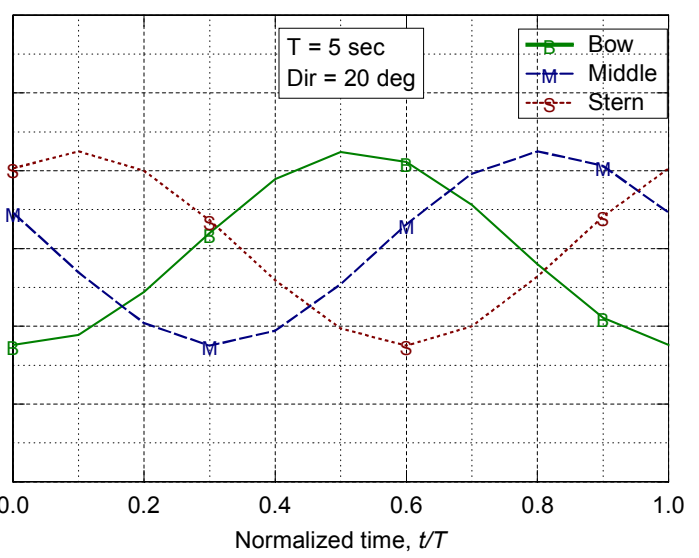

b) $T=5 \mathrm{sec}, \bar{\theta}=20 \mathrm{deg}$.

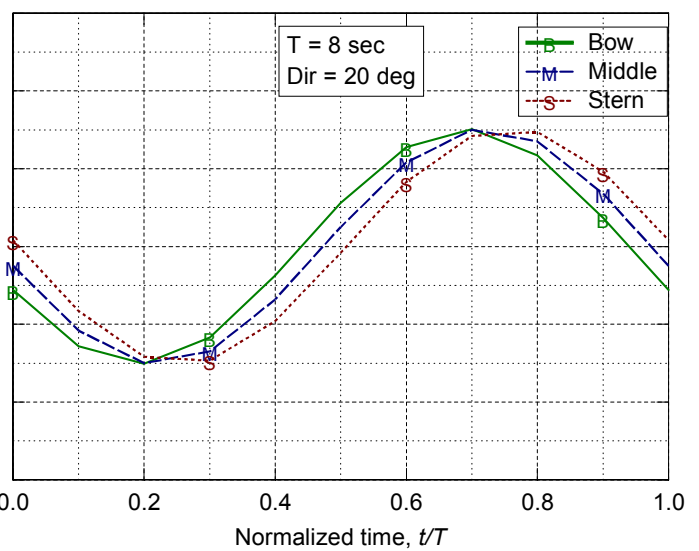

d) $T=8 \mathrm{sec}, \bar{\theta}=20 \mathrm{deg}$.

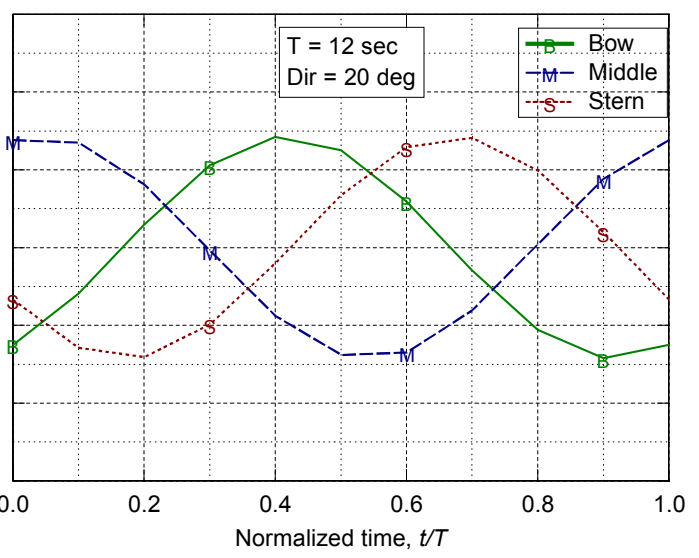

f) $T=12 \mathrm{sec}, \bar{\theta}=20 \mathrm{deg}$.

Figure 59. Analytical $\bar{p}_{1, A}$ for $\theta_{r}=45 \mathrm{deg}, d / h=0.50$ : (a) $T=5 \mathrm{sec}, \bar{\theta}=0 \mathrm{deg}$, (b) $T=5 \mathrm{sec}, \bar{\theta}=20 \mathrm{deg}$, (c) $T=8 \mathrm{sec}, \bar{\theta}=0 \mathrm{deg}$, (d) $T=8 \mathrm{sec}, \bar{\theta}=20 \mathrm{deg}$, (e) $T=12 \mathrm{sec}, \bar{\theta}=0 \mathrm{deg}$, (f) $T=12 \mathrm{sec}, \bar{\theta}=20 \mathrm{deg}$. 


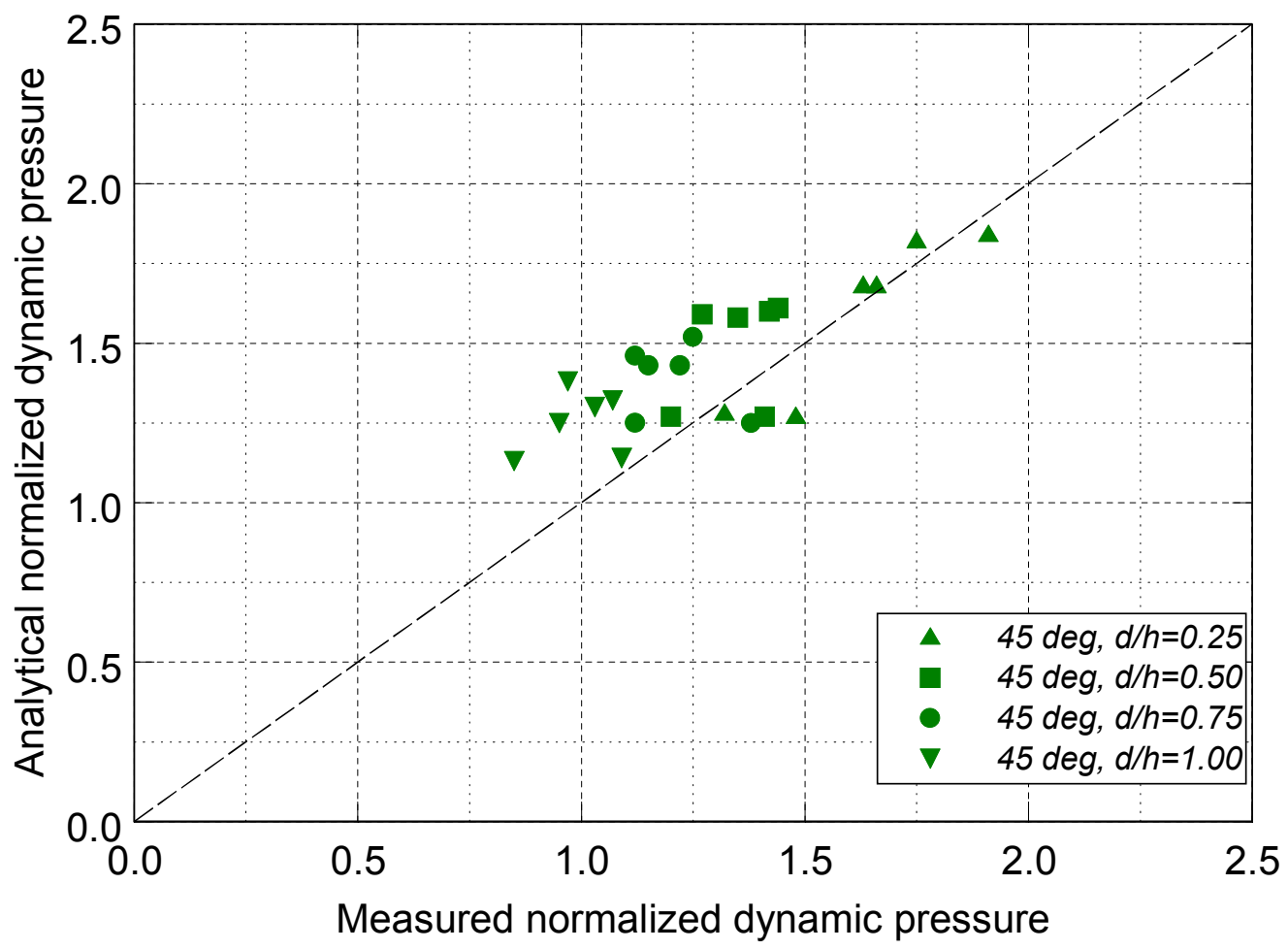

Figure 60. Comparison between analytical $\bar{p}_{l, A}$ and measured $\bar{p}_{l, S}$ for FLab.

\section{Empirical Normalized Pressure Relationships}

In this section, the measured $\bar{p}_{l, S}$ are compared with several nondimensional parameters. The $\bar{p}_{l, S}$ pressures are averages of the three pressure gages. Nondimensional parameters investigated include the relative width $B_{x} / L_{x}$, wave steepness $H_{I} / L_{x}$, pressure response factor $K_{P}$, modified Reynold's Number $R_{E}$, and Wiegel's transmission factor $T_{F}$. The WAMIT coordinate system with origin on the center line of the RIBS is used in the parameter definitions. These parameters were described previously in Chapter VI.

Relative width, wave steepness, and pressure response factor. For this fundamental laboratory data set, the $\bar{p}_{1, S}$ pressure does not show any relationship to relative width, wave steepness, or pressure response factor. The pressures align into three vertical groups according to wave period and wavelength. Thus, $\bar{p}_{I, S}$ depends mainly on wave period and the scatter is too extreme for a good fit to the data. 
Reynold's number. Next, a modified form of the Reynold's Number described in Chapter IV is investigated. The original formulation by Harms et al. (1982) was a function of wave height $H$, wavelength $L$, a characteristic breakwater length equal to the draft $d$, gravity $g$, and kinematic viscosity $v$. In this new formulation, mean wave direction $\bar{\theta}$ and the projected wavelength in the direction parallel to the RIBS center line (or the x-direction) are used. The new $R_{e x}$ is defined as

$$
R_{e_{x}}=\frac{H}{L \cos \theta} \frac{d \sqrt{g L \cos \theta}}{v}
$$

Figure 61 shows the relationship between the measured $\bar{p}_{l, S}$ and $R_{e x}$. The format is the same as the previous figure. The second order polynomial trend line from the regression analysis of all measured pressures is shown along with 95 percent confidence limits. The least squares fit is much better for this parameter with a square of the correlation coefficient $R^{2}=0.57$. Table 18 lists the square of the correlation coefficient $R^{2}$, and $a_{0}, a_{1}$, and $a_{2}$ coefficients of this trend line defined by

$$
\bar{p}_{1, S}=a_{0}+a_{1} x+a_{2} x^{2}
$$

Transmission factor $\mathbf{T}_{\mathbf{F}}$. The relationship between $\bar{p}_{l, S}$ and Wiegel's transmission factor $T_{F}$ (Chapter VI) is shown in Figure 62. The value of $T_{F}$ increases as the draft decreases. This parameter has the best fit of all the nondimensional parameters investigated. The square of the correlation coefficient $R^{2}=0.71$ for $T_{F}$.

TABLE 18. Summary of Regression Analysis Parameters

\begin{tabular}{l|c|c|c|c|c}
\hline \hline \multicolumn{2}{c|}{ Independent Variable $x$} & \multicolumn{3}{|c}{ Regression Coefficients } \\
\cline { 1 - 3 } Name & Symbol & $R^{2}$ & $a_{0}$ & $a_{1}$ & $a_{2}$ \\
\hline Relative width & $B_{x} / L_{x}$ & 0.03 & 1.54 & -61.33 & 3025 \\
\hline Wave steepness & $H_{I} / L_{x}$ & 0.03 & 1.38 & -3.26 & -9.21 \\
\hline Pressure response & $K_{P}$ & 0.04 & 1.58 & -1.19 & 1.01 \\
\hline Reynold's No. & $R_{e x}$ & 0.57 & 0.60 & $4 \mathrm{E}-05$ & $-5 \mathrm{E}-10$ \\
\hline Transmission factor & $T_{F}$ & 0.71 & 1.58 & -1.76 & 1.36 \\
\hline \hline
\end{tabular}




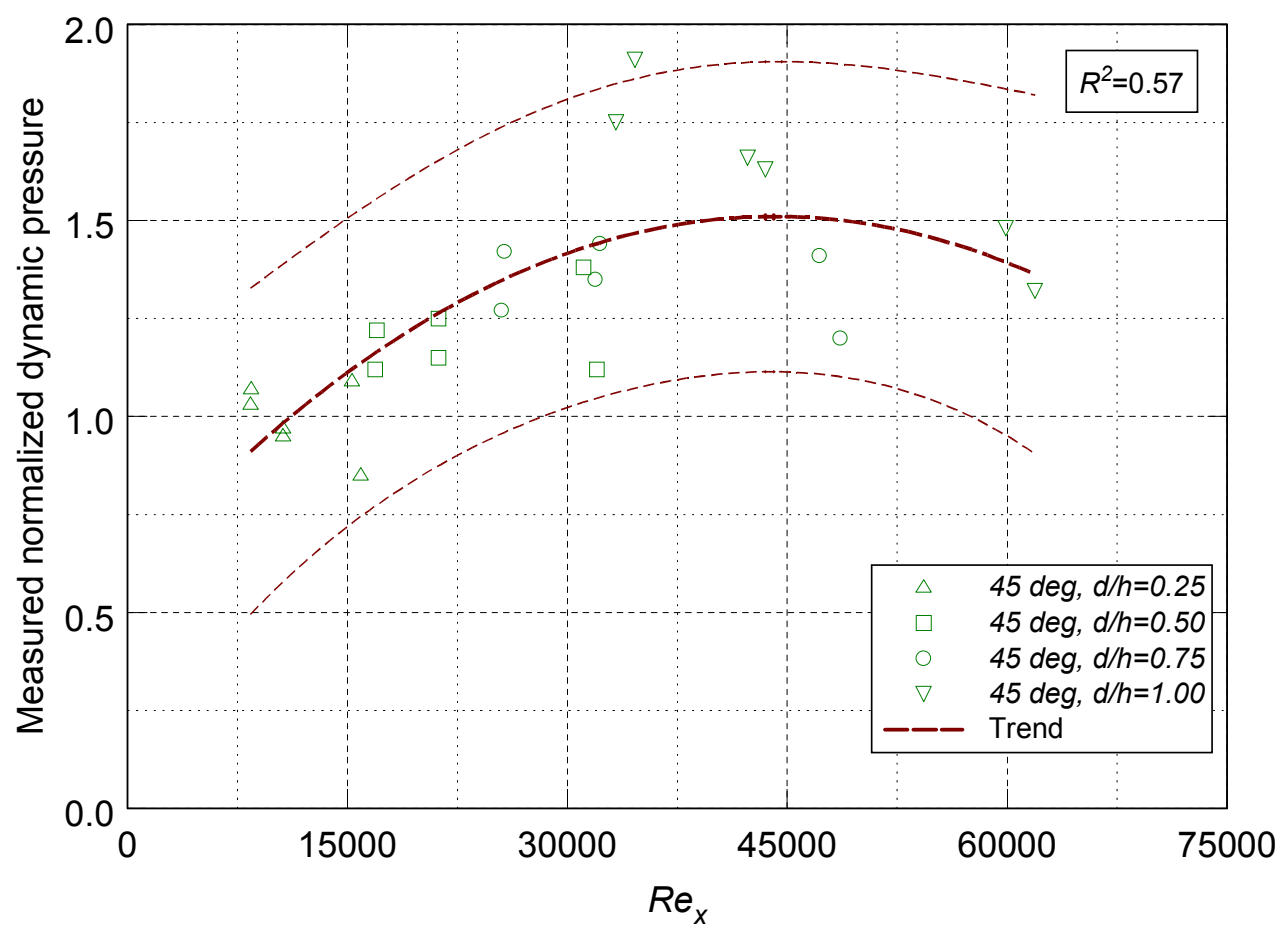

Figure 61. Measured dynamic pressure $\bar{p}_{1, S}$ vs. modified Reynold's Number $R e_{x}$.

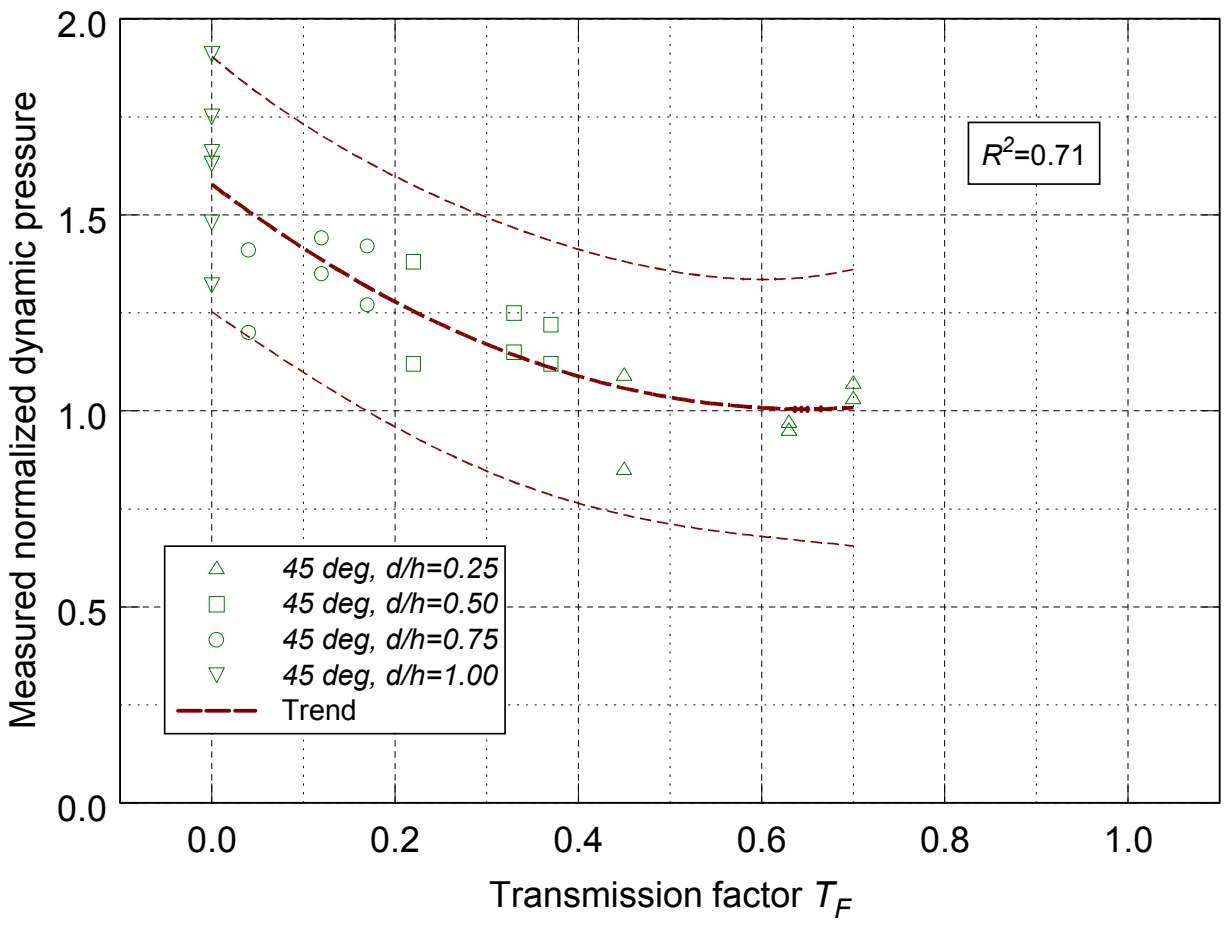

Figure 62. Measured dynamic pressure $\bar{p}_{1, S}$ vs. Wiegel's transmission factor $T_{F}$. 


\section{FLab Dynamic Pressure Summary}

The FLab had three pressure gages installed at bow, middle, and stern locations on the starboard RIBS leg. These gages were located on the same vertical level at $z=-8.5 \mathrm{~cm}$ from the surface for all relative drafts $d / h$.

The significant dynamic pressure $p_{l, S}$ increased as $d / h$ increased, due to the increase in reflected energy on the external side of the RIBS leg. The $p_{1, S}$ were slightly larger for the waves at an angle $\bar{\theta}=20 \mathrm{deg}$ because the wave was more normal to the leg. In general, the $p_{1, S}$ were fairly constant for all three pressure gages for each wave case. There was a tendency to increase at the stern gage location, especially for the $\bar{\theta}=20$ deg cases. The largest variations occurred when the RIBS was resting on the bottom. In this case, the $p_{1, S}$ was the largest because the wave could no longer go underneath the RIBS and the reflected energy was the greatest. Similar to the wave transmission coefficients, there was not much difference between the spectral and regular cases, although the regular wave values were slightly larger. The ratio of the SS5 to SS3 dynamic pressures ranged from a low of 6.4 to a high of 7.5 , while the wave height ratio was only about 2.4 . The average for all cases was 6.9 .

The measured $p_{1, S}$ were calculated as an equivalent wave height or double amplitude using standard zero downcrossing and zero moment estimation techniques. For comparison to the analytical and WAMIT models, the measured $p_{I, S}$ were divided by $\rho g H$ ( $H$ is the wave height) to obtain a normalized or nondimensional pressure $\bar{p}_{l, S}$. The normalized WAMIT $\bar{p}_{1, W}$ and analytical $\bar{p}_{l, A}$ dynamic pressures were divided by $\rho g A$ ( $A$ is the wave amplitude). The WAMIT $\bar{p}_{1, W}$ represents a "snapshot" in time of the relative pressure along the RIBS starboard leg. In general, the $\bar{p}_{l, W}$ increases as $d / h$ increases and is fairly constant along the RIBS leg. As $d / h$ increases, the $\bar{p}_{1, W}$ tends to increase slightly toward the stern.

For comparisons to the measured $\bar{p}_{I, S}$, the magnitude of the WAMIT $\bar{p}_{I, W}$ was determined by taking one half of the crest to trough distance closest to the gage. The justification for this procedure is that this technique attempts to include some of the WAMIT variation in $\bar{p}_{1, W}$ along the length of the RIBS. This $\bar{p}_{I, W}$ pressure is representative of what each pressure gage would experience based on its relative position. At another instant in time, these pressures would change, but would still maintain their relative magnitudes to each other. 
The agreement between measured $\bar{p}_{l, S}$ and WAMIT $\bar{p}_{1, W}$ was very good. Regression analysis correlations ranged from 0.64 to 0.67 for the individual pressure gages. In general, the WAMIT predictions are slightly smaller than the measured $\bar{p}_{l, S}$. The average measured $\bar{p}_{l, S}$ for all $d / h$ and $\theta_{r}$ combinations (i.e., 72 values) was 0.28 higher than the corresponding WAMIT $\bar{p}_{1, W}$.

The analytical model for dynamic pressure is based on linear wave theory and includes both incident and reflected waves. The predicted pressure magnitude is a function of the wave period $T$, wavelength $L$, water depth $h, y$-axis wavenumber $k_{y}$ ( $x=0$ on the RIBS leg, so no $k_{x}$ term), pressure response factor $K_{P}$, reflection coefficient $K_{R}$, and wave direction $\bar{\theta}$. The $K_{R}$ is based on an energy balance and is defined as $\sqrt{ }\left(1-K_{T}^{2}\right)$. The measured $K_{T}$ were used in the calculation of $K_{R}$. The analytical $\bar{p}_{l, A}$ are "snapshots" in space: they show an entire wave cycle for a specific location (i.e., a pressure gage). For each location, the $\bar{p}_{l, A}$ magnitude is constant, but the phase changes over a complete cycle. The $\bar{p}_{l, A}$ magnitude increased slightly as $d / h$ increased.

The analytical $\bar{p}_{I, A}$ were compared to the measured dynamic pressure $\bar{p}_{I, S}$. The agreement was excellent with an $R^{2}=0.93$. The analytical $\bar{p}_{l, A}$ were slightly smaller than the corresponding measured values. Thus, the analytical model did an excellent job of predicting dynamic pressure for this limited FLab data set.

Several empirical parameters were again investigated. As for the wave transmission coefficient analysis, the relative breakwater width $B / L$ and wave steepness $H / L$ parameters did not have sufficient variation in the number of wave periods or wave heights to be a good indicator. A modified Reynold's Number $R_{e x}$ parameter was investigated. It is a function of the wave height $H$, projected wavelength $L_{x}$, draft $d$, wave direction $\bar{\theta}$, gravity $g$, and kinematic viscosity $v$. The second order regression analysis gave an $R^{2}=0.57$. Again, the best predictor of the empirical parameters was the Wiegel's $T_{F}$ parameter. The least squares fit to the data produced an $R^{2}=0.71$.

In summary, the analytical models for dynamic pressure and the Wiegel $T_{F}$ were very good predictors for the FLab data set. The important parameter in the formulation is the inclusion of the reflection coefficient $K_{R}$. The accurate estimation of $K_{R}$ was possible because of the laboratory measurements of the transmission coefficient. This FLab data set represents an extensive collection of controlled laboratory experiments that should prove to be valuable to the coastal engineering community in verifying and improving analytical and numerical models. 


\section{XM99 FIELD TRIALS}

\section{Calculation Procedure}

General analysis procedure. Wave pressures were measured at twelve external locations on the port leg of the XM99. Gages were located at three stations on each of four vertical levels. The three stations are bow, middle, and stern. The four levels are the surface, top, middle, and bottom. Gage codes and coordinates were listed in Chapter III.

Data were collected at a sampling rate of $4 \mathrm{~Hz}$ for the first 30 minutes of every hour and transferred to the receiving computer during the second half hour. A total of 7,200 points were collected for each 30-minute record. These data were analyzed with the GEDAP software package (Miles 1997). The procedure is described in the paragraphs below. It is understood that each of the pressure quantities is a time series, but that the relationship as a function of time (i.e., "( $(t)$ ") is omitted for convenience.

The three surface pressure gages were reference gages to measure atmospheric pressure. The submerged pressure gages measured absolute pressure $p_{a b s}$ that included the local atmospheric pressure $p_{a t m}$. Therefore, the first step in the analysis procedure involved the estimation of the atmospheric pressure from the three surface gages. An average value for all three reference gages was calculated. This average value of the atmospheric pressure $p_{\text {atm }}$ was then subtracted from the absolute pressure to obtain the gage pressure $p_{\text {gage }}$ for each gage

$$
p_{\text {gage }}=p_{a b s}-\bar{p}_{a t m}
$$

These values remained stable during the course of the XM99 field trial, varying between a low of 101.35 to a high of about $102.46 \mathrm{kPa}$.

The second step in the procedure involved the estimation of the gage depths for the pressure gages. This depth is a function of the hydrostatic component of pressure $p_{\text {static }}$. Since this value is a constant for each pressure gage, it can be estimated by finding the mean or average of each gage. The gage depth is obtained by converting $p_{\text {static }}$ into an equivalent water depth (i.e., 50.9 $\mathrm{kPa} / \mathrm{m})$.

The third step in the analysis is to zero-mean the time series by subtracting $p_{\text {static }}$ to obtain the dynamic pressure $p_{1}$ 


$$
p_{1}=p_{\text {gage }}-p_{\text {static }}
$$

where the gage pressure $p_{\text {gage }}$ is the same as the total wave pressure $p_{\text {tot }}$ from Chapter IV. The time series records for each of the external and internal pressure gages now consisted of only the dynamic wave pressure component.

The final step in the procedure consisted of calculating time and frequency domain parameters from the time series for external pressure. In the time domain, a zero downcrossing analysis was performed. Calculated statistics included the minimum and maximum pressures, RMS pressure, significant pressure height and associated period, and maximum pressure height. In the frequency domain, a standard spectral analysis routine was used on the pressure data. This consisted of a 10 percent cosine bell window, variance restoration, and a frequency bandwidth of $0.01 \mathrm{~Hz}$ between zero and the Nyquist frequency at $2.0 \mathrm{~Hz}$ to obtain 16 DOF spectral estimates. Frequency domain parameters included a zero-moment pressure $p_{1, S}$ and peak period from the pressure spectrum.

Normalization procedure. The same procedure used for the FLab pressures will be used for the XM99 data. The measured $p_{1, S}$ is divided by $\rho g H$ to obtain the normalized $\bar{p}_{1, S}$. The WAMIT and analytical predicted pressures $\bar{p}_{l}$ are normalized by $\rho g A$ as before. The analytical $\bar{p}_{1}$ is calculated using equation 77 . These normalized pressures are then compared to each other and several empirical parameters.

\section{Measured Dynamic Pressure}

Gage depths. An important consideration in comparing measured dynamic pressures with the analytical and WAMIT predictions is the actual depth of the gages. The three panels of Figure 63 show the depth variation during the deployment for the top, middle, and bottom levels, respectively. Bow (solid line), middle (dashed line), and stern (dotted line) curves are shown for each level. For the top level, the variation in depth for all three gages was between approximately 2.70 and $3.08 \mathrm{~m}$. For the middle level gages, this variation was between 4.42 and $4.72 \mathrm{~m}$. The variation for the bottom level was between 6.77 and $7.86 \mathrm{~m}$. The jumps on May 22, 26, and 29 for some of the gages correspond to changes in the integrity of the fabric and straps connecting the different levels. These were especially significant for the bottom stern gage, 


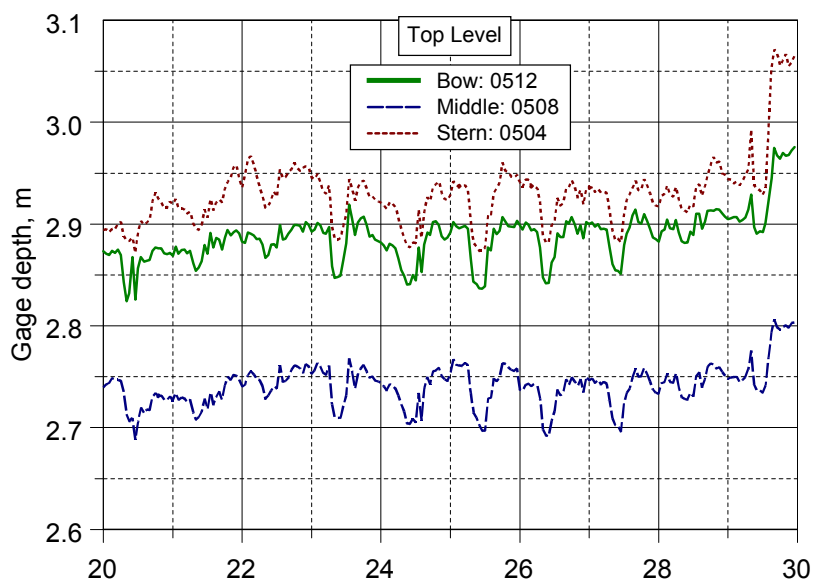

a)

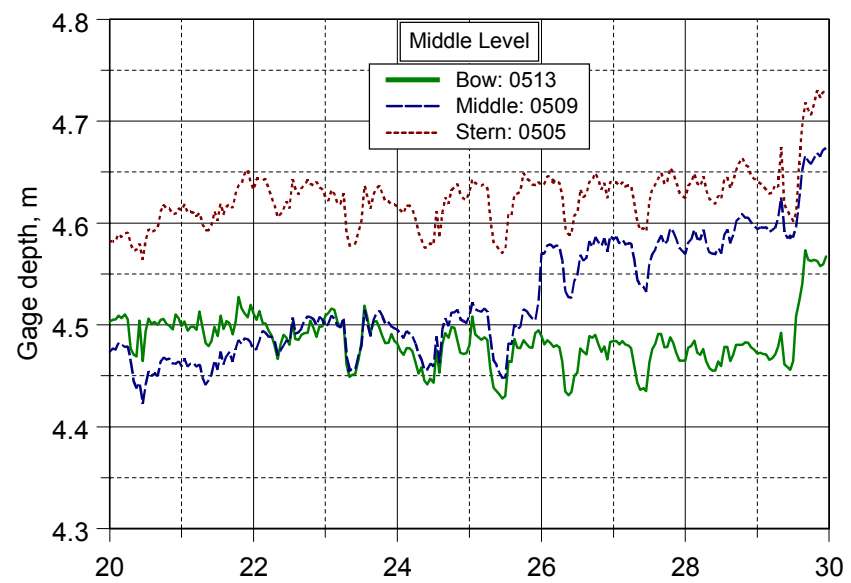

b)

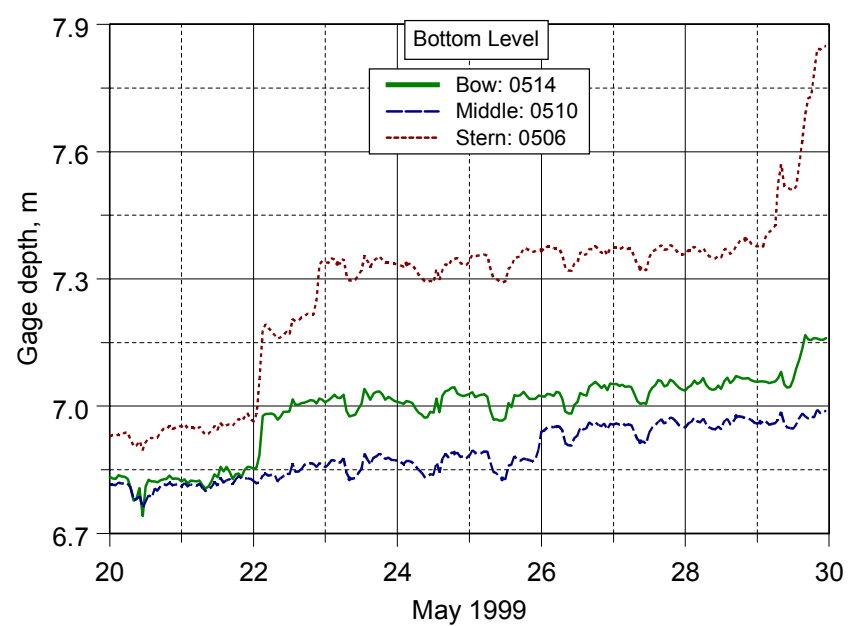

Figure 63. External pressure gage depths for XM99 (a) top level, (b) middle level, (c) bottom level. 
which reflected the cumulative displacements of the top two levels as well as some substantial deflection of its own on May 22 and 29.

For comparisons to the analytical and numerical models, an average depth of 2.90, 4.42, and $6.86 \mathrm{~m}$ was assumed for the three levels. A more thorough analysis would involve using actual gage depths rather than these averages in the comparisons with WAMIT and analytical models. Comparing these measurements of actual gage depths to the original values during the installation indicates that the XM99 had about 21 to $37 \mathrm{~cm}$ of freeboard relative to the top level.

External dynamic pressures. The significant dynamic pressures $p_{1, S}$ for the nine external pressure gages are shown in Figure 64 for each of the three levels. The format of this figure is similar to the previous figure. The bow gage on the middle level never worked correctly and will not be discussed further. These dynamic pressures followed the trends of incident significant wave height (Chapter III). The variability in dynamic pressure from bow to stern along a constant level is evident in these plots. On the top level, the bow gage usually had the largest magnitude. The stern gage usually had the largest pressure on the middle and bottom levels.

Figure 65 is a similar plot for the maximum dynamic pressure $p_{1, \operatorname{Max}}$. The $y$-axis scale is double the previous figure. The trends are identical to the significant dynamic pressures, but approximately 1.75 times larger. The largest significant pressure $p_{1, S}$ occurred on May 29 for the top level, bow gage and the largest maximum pressure $p_{l, M a x}$ for the middle level, stern gage. Table 19 lists the minimum, maximum, average, and standard deviation for each of the external gages for the significant and maximum dynamic pressures. The largest $p_{1, S}$ occurred on May 29 for the top level, bow gage and the largest $p_{l, M a x}$ for the middle level, stern gage. The range of average and maximum $p_{1, S}$ were 1.77 to $3.48 \mathrm{kPa}$ and 4.89 to $7.57 \mathrm{kPa}$, respectively. Similarly, average and maximum $p_{1, \text { Max }}$ were 3.11 to $5.94 \mathrm{kPa}$ and 9.24 to $15.03 \mathrm{kPa}$, respectively.

Normalized dynamic pressures. So far, the measured dynamic pressures have been organized as a time series according to the day and time collected. The first step in normalizing the measured dynamic pressure was to rearrange this data according to wave period and direction categories. Thus, the data was sorted according to wave period and then wave direction. The bins were selected such that the wave periods consisted of even wave periods from 2 to $12 \mathrm{sec}$. For the $3 \mathrm{sec}$ bin, all data greater than or equal to $2.5 \mathrm{sec}$ and less than $3.5 \mathrm{sec}$ was selected. Similar logic was used for all the other wave periods. 


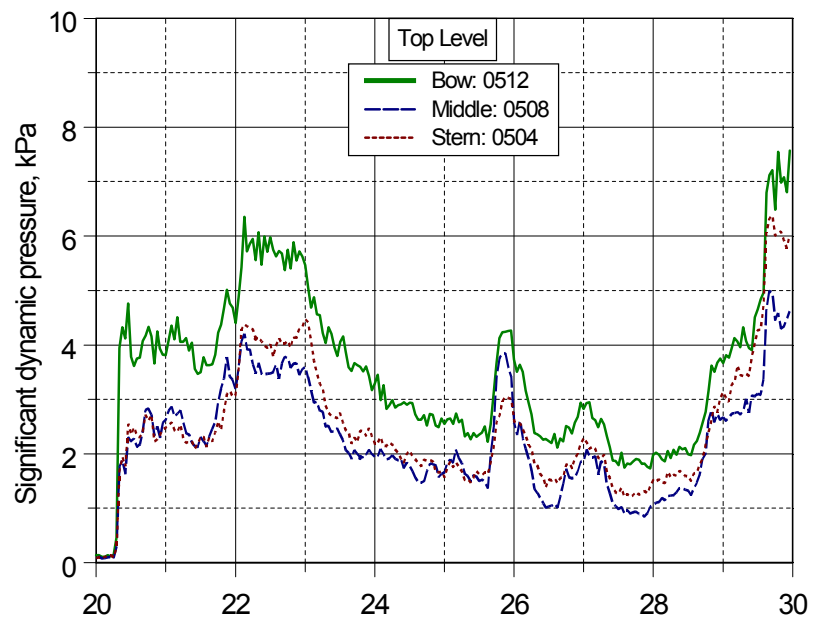

a)

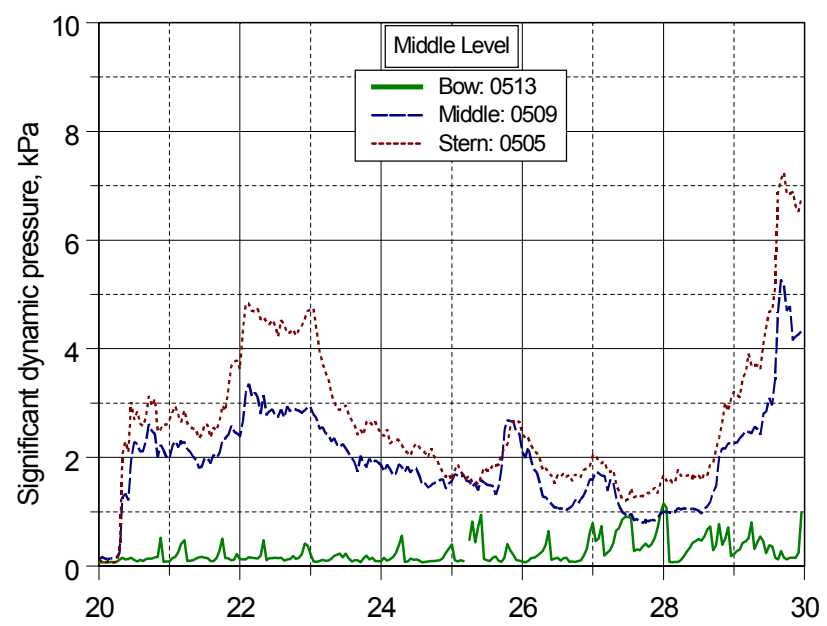

b)

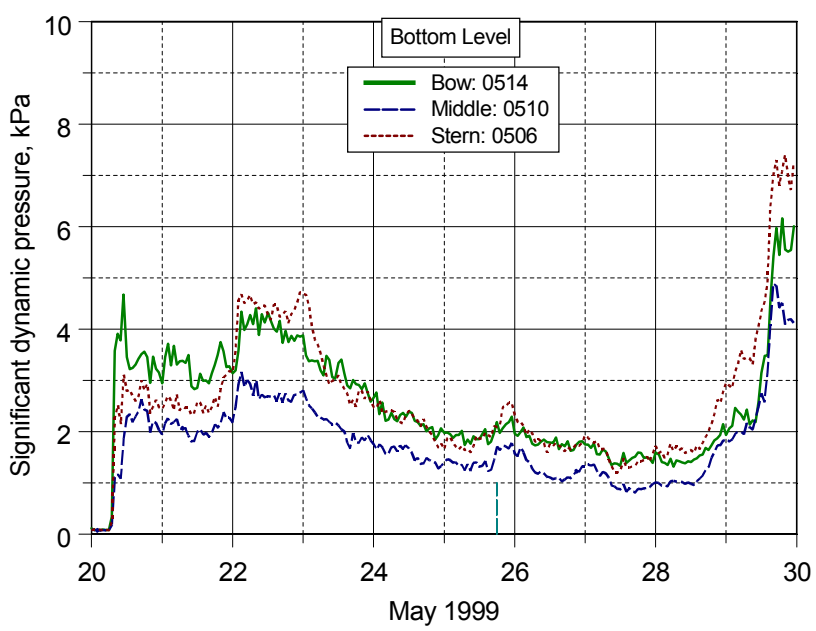

Figure 64. Measured dynamic pressures $p_{1, S}$ for XM99 (a) top level, (b) middle level, (c) bottom level. 
a)
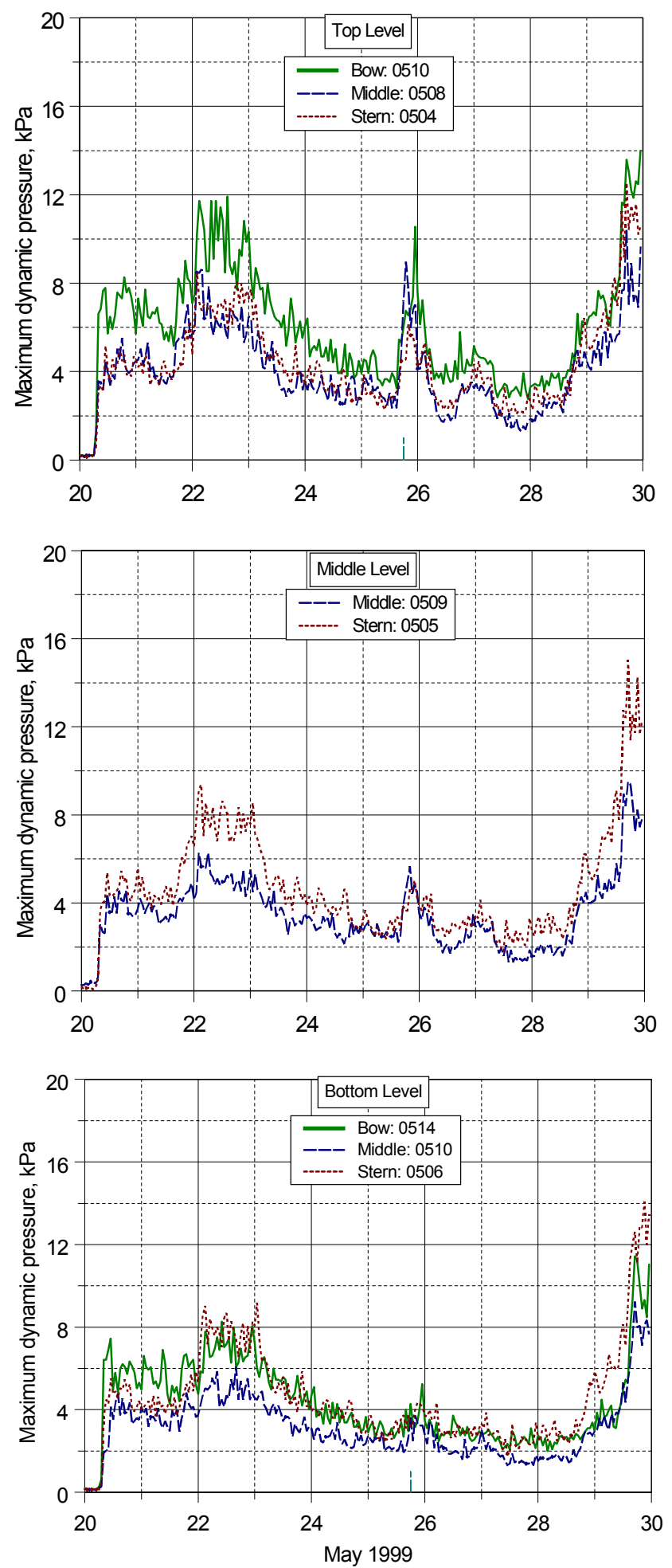

Figure 65. Maximum dynamic pressures $p_{1, \text { Max }}$ for XM99 (a) top level, (b) middle level, (c) bottom level. 
TABLE 19. Measured $p_{I S}$ and $p_{1 \mathrm{Mar}}$ Statistics, kPa

\begin{tabular}{|c|c|c|c|c|c|}
\hline Level & Gage & Minimum & Maximum & Average & Std Deviation \\
\hline \multicolumn{6}{|c|}{ (a) Significant Dynamic Pressures $p_{l, S}$} \\
\hline \multirow[t]{3}{*}{ Top } & Bow & 0.11 & 7.57 & 3.48 & 1.45 \\
\hline & Middle & 0.08 & 4.99 & 2.24 & 1.01 \\
\hline & Stern & 0.09 & 6.38 & 2.48 & 1.18 \\
\hline \multirow[t]{2}{*}{ Middle } & Middle & 0.12 & 5.27 & 1.94 & 0.87 \\
\hline & Stern & 0.07 & 7.24 & 2.65 & 1.35 \\
\hline \multirow[t]{3}{*}{ Bottom } & Bow & 0.08 & 6.16 & 2.55 & 1.12 \\
\hline & Middle & 0.07 & 4.89 & 1.77 & 0.82 \\
\hline & Stern & 0.07 & 7.40 & 2.62 & 1.33 \\
\hline \multicolumn{6}{|c|}{ (b) Maximum Dynamic Pressures $p_{1, \operatorname{Max}}$} \\
\hline \multirow[t]{3}{*}{ Top } & Bow & 0.14 & 14.00 & 5.94 & 2.62 \\
\hline & Middle & 0.14 & 10.41 & 3.99 & 1.86 \\
\hline & Stern & 0.14 & 12.55 & 4.36 & 2.12 \\
\hline \multirow[t]{2}{*}{ Middle } & Middle & 0.28 & 9.51 & 3.45 & 1.58 \\
\hline & Stern & 0.07 & 15.03 & 4.64 & 2.48 \\
\hline \multirow[t]{3}{*}{ Bottom } & Bow & 0.14 & 11.45 & 4.32 & 2.02 \\
\hline & Middle & 0.07 & 9.24 & 3.11 & 1.51 \\
\hline & Stern & 0.14 & 14.07 & 4.55 & 2.40 \\
\hline
\end{tabular}

Three wave direction bins were established. In the global coordinate system, these direction bins had centers at 315,285 , and $255 \mathrm{deg}$, with a 30 -deg range (15 deg on either side). These direction bins correspond to 0,30 , and $60 \mathrm{deg}$ in the WAMIT coordinate system. The first direction bin, representing waves traveling along the XM99 center line, included all wave directions from 330 to $300 \mathrm{deg}$. The second bin representing waves traveling parallel to the starboard leg, included all waves with directions between 300 and $270 \mathrm{deg}$. The last direction bin with waves traveling broadside to the port leg, included wave directions from 270 to 240 deg. Thus, for the first direction bin, all waves with directions greater than or equal to $330 \mathrm{deg}$ and less than $300 \mathrm{deg}$ were included. The same procedure was used for the other direction bins. There was some wave data that was not used because the mean wave directions were on the 
starboard side. This was done because the pressure gages were on the port side and would record different pressures for waves coming from the other side. These three direction bins were also designated as the 0,30 , and 60 deg bins, respectively.

According to this procedure there could have been 11 wave period bins and 3 direction bins, or a total of 33 bins. Only 17 bins were actually identified within these wave period and direction constraints. Table 20 lists the averages for the wave period, wave height, mean wave direction, and directional spread in each of the 17 bins. Also included are the number of waves, range of wave periods, and range of wave directions in each bin. Surprisingly, there were very few waves traveling along the XM99 center line, and most of those were in the smaller wave periods. There were a large number of waves in the swell category with wave periods greater than $7 \mathrm{sec}$.

After sorting the data, the measured $p_{l, S}$ dynamic pressures were normalized by dividing by $\rho g H$. The normalized dynamic pressure $\bar{p}_{1, S}$ is plotted in Figures 66 to 67 for the eight wave cases in the "sea" and "swell" regimes. The "sea" cases are represented by wave periods of 3

TABLE 20. Measured XM99 Average Wave Statistics

\begin{tabular}{l|c|c|c|c|c|c|c|}
\hline \hline Bin ID & $N$ & Periods, sec & Directions & $T_{p}, \mathrm{sec}$ & $H_{m 0}, \mathrm{~m}$ & $\bar{\theta}, \mathrm{deg}$ & $\sigma_{m,}$ deg \\
\hline 230 & 2 & $1.5 \leq T<2.5$ & $270 \leq \bar{\theta}<300$ & 2.30 & 0.34 & 287.43 & 62.40 \\
\hline 260 & 2 & $1.5 \leq T<2.5$ & $240 \leq \bar{\theta}<270$ & 2.11 & 0.33 & 249.89 & 63.25 \\
\hline 300 & 10 & $2.5 \leq T<3.5$ & $300 \leq \bar{\theta}<330$ & 3.08 & 0.50 & 315.58 & 47.95 \\
\hline 330. & 7 & $2.5 \leq T<3.5$ & $270 \leq \bar{\theta}<300$ & 2.79 & 0.42 & 279.07 & 51.83 \\
\hline 360 & 3 & $2.5 \leq T<3.5$ & $240 \leq \bar{\theta}<270$ & 2.91 & 0.52 & 265.71 & 43.79 \\
\hline 400 & 12 & $3.5 \leq T<4.5$ & $300 \leq \bar{\theta}<330$ & 3.87 & 0.53 & 324.67 & 39.77 \\
\hline 430 & 7 & $3.5 \leq T<4.5$ & $270 \leq \bar{\theta}<300$ & 3.94 & 0.55 & 278.31 & 47.87 \\
\hline 460 & 15 & $3.5 \leq T<4.5$ & $240 \leq \bar{\theta}<270$ & 3.96 & 0.73 & 258.83 & 39.92 \\
\hline 560 & 7 & $4.5 \leq T<5.5$ & $240 \leq \bar{\theta}<270$ & 4.89 & 0.79 & 257.75 & 38.71 \\
\hline 660 & 8 & $5.5 \leq T<6.5$ & $240 \leq \bar{\theta}<270$ & 5.82 & 0.96 & 259.09 & 34.15 \\
\hline 760 & 2 & $6.5 \leq T<7.5$ & $240 \leq \bar{\theta}<270$ & 7.26 & 0.38 & 263.70 & 49.69 \\
\hline 830 & 17 & $7.5 \leq T<8.5$ & $270 \leq \bar{\theta}<300$ & 8.11 & 0.41 & 276.73 & 45.55 \\
\hline 860 & 20 & $7.5 \leq T<8.5$ & $240 \leq \bar{\theta}<270$ & 8.22 & 0.45 & 260.08 & 45.13 \\
\hline 930 & 1 & $8.5 \leq T<9.5$ & $270 \leq \bar{\theta}<300$ & 9.14 & 0.38 & 276.61 & 46.90 \\
\hline 960 & 18 & $8.5 \leq T<9.5$ & $240 \leq \bar{\theta}<270$ & 8.98 & 0.64 & 256.14 & 44.89 \\
\hline 1030 & 14 & $9.5 \leq T<10.5$ & $270 \leq \bar{\theta}<300$ & 9.73 & 0.37 & 274.74 & 44.87 \\
\hline 1060 & 20 & $9.5 \leq T<10.5$ & $240 \leq \bar{\theta}<270$ & 9.84 & 0.58 & 255.74 & 48.16 \\
\hline \hline
\end{tabular}




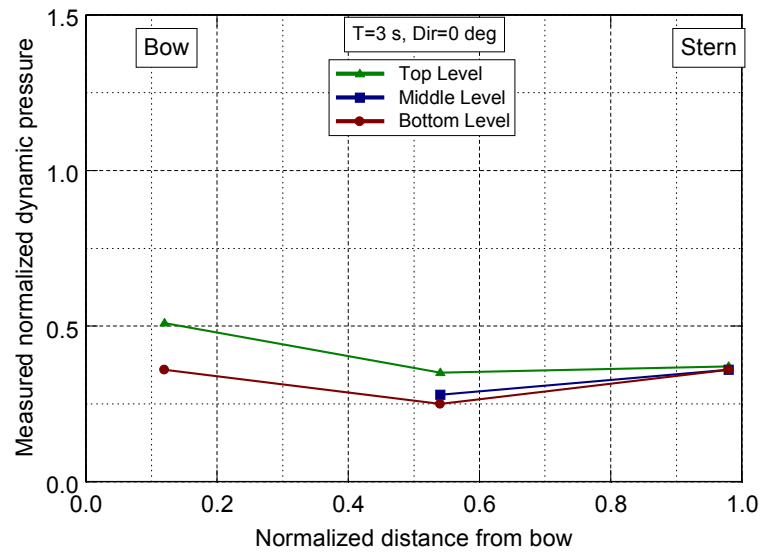

a) $T=3 \mathrm{sec}, \bar{\theta}=0 \mathrm{deg}$.

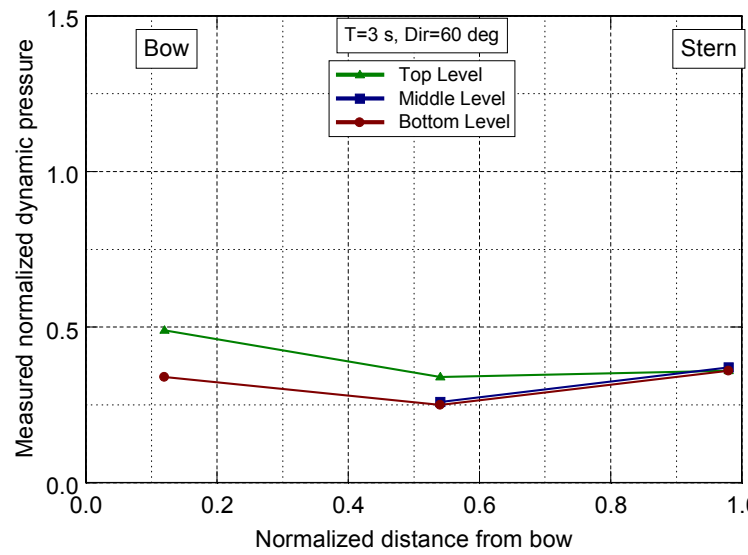

c) $T=3 \mathrm{sec}, \bar{\theta}=60 \mathrm{deg}$.

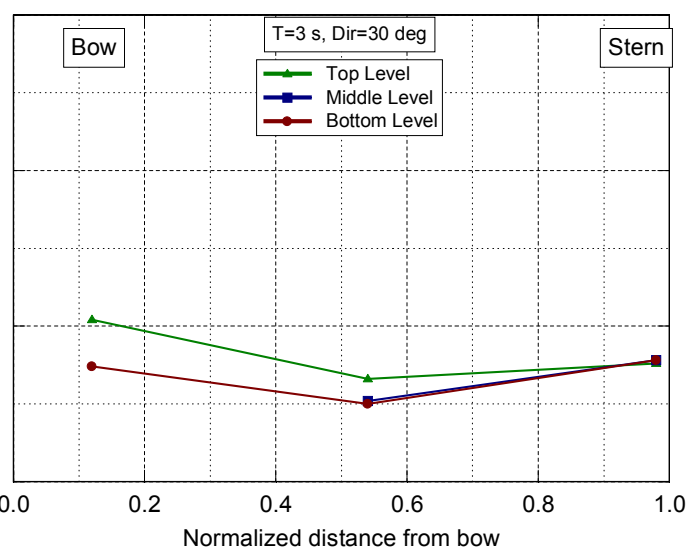

b) $T=3 \mathrm{sec}, \bar{\theta}=30 \mathrm{deg}$.

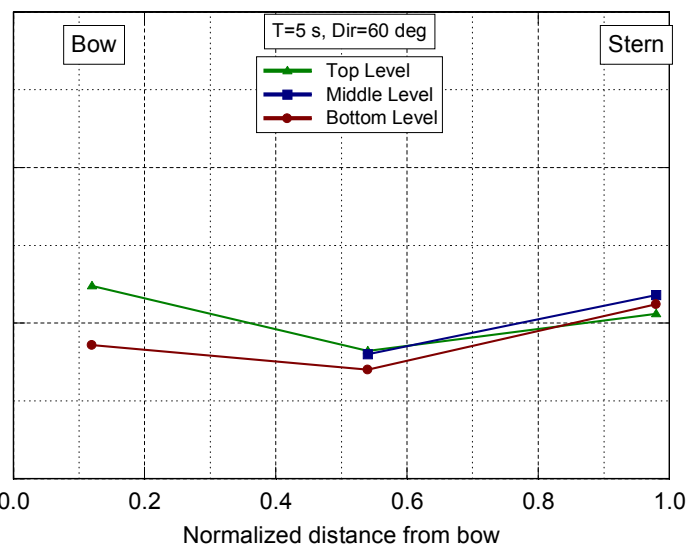

d) $T=5 \mathrm{sec}, \bar{\theta}=60 \mathrm{deg}$.

Figure 66. Measured $\bar{p}_{1, S}$ for XM99 "sea" waves: (a) $T=3 \mathrm{sec}, \bar{\theta}=0 \mathrm{deg}$, (b) $T=3 \mathrm{sec}, \bar{\theta}=30 \mathrm{deg}$, (c) $T=3 \mathrm{sec}, \bar{\theta}=60 \mathrm{deg}$, (d) $T=5 \mathrm{sec}, \bar{\theta}=60 \mathrm{deg}$.

and $5 \mathrm{sec}$ and wave directions from 0 to $60 \mathrm{deg}$ (i.e., 315 to $240 \mathrm{deg}$ ). The "swell" cases are similarly represented by wave periods of 8 and $10 \mathrm{sec}$ in the same wave directions. After sorting, it was discovered that there were no waves in four of the period/direction bins. These included $T=5 \mathrm{sec}, \bar{\theta}=0$ and $30 \mathrm{deg}$ bins and $T=8$ and $10 \mathrm{sec}, \bar{\theta}=0$ bins.

Although there is some variation among cases, the $\bar{p}_{1, S}$ are surprisingly similar in the "sea" and "swell" regimes and there is not much difference between gage pressures at different vertical levels. This is a surprising and unexpected result. The pressures were averaged for each wave 


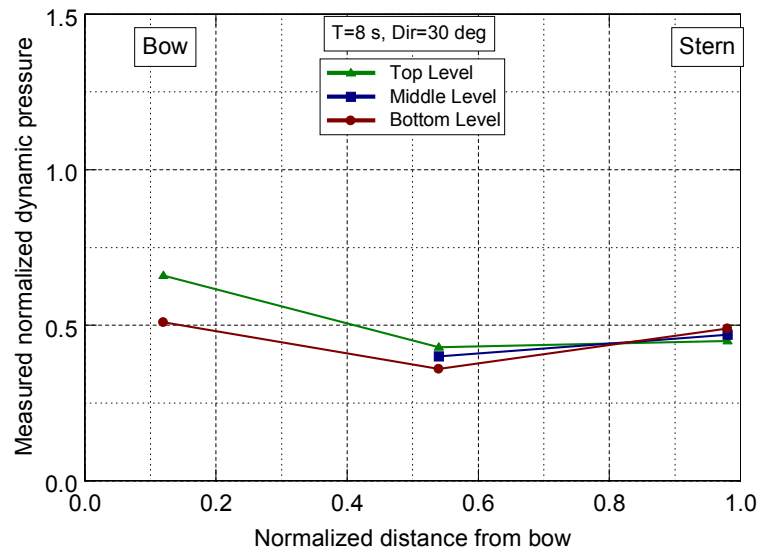

a) $T=8 \mathrm{sec}, \bar{\theta}=30 \mathrm{deg}$.

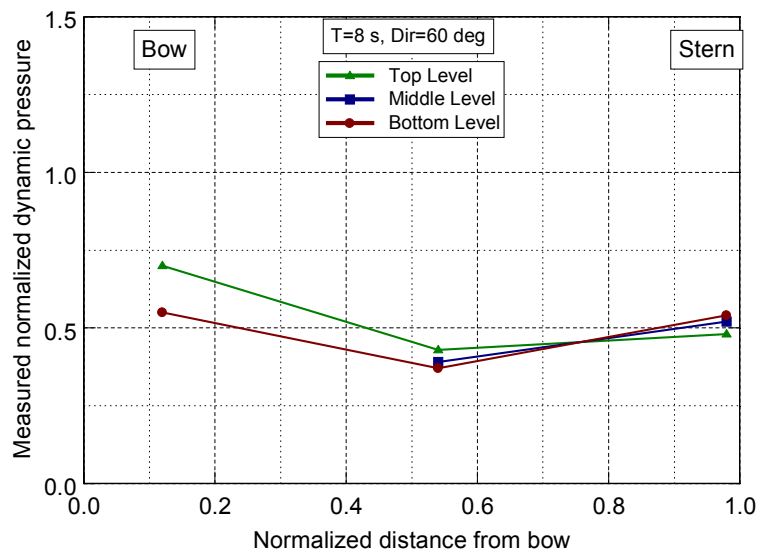

c) $T=8 \mathrm{sec}, \bar{\theta}=60 \mathrm{deg}$.

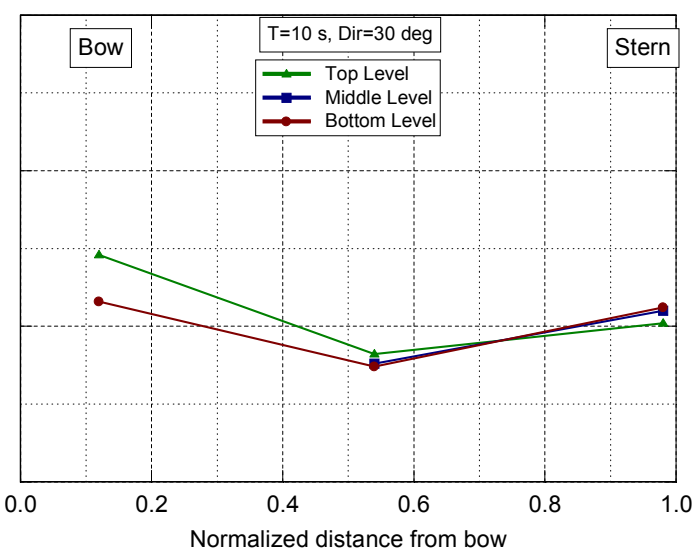

b) $T=10 \mathrm{sec}, \bar{\theta}=30 \mathrm{deg}$.

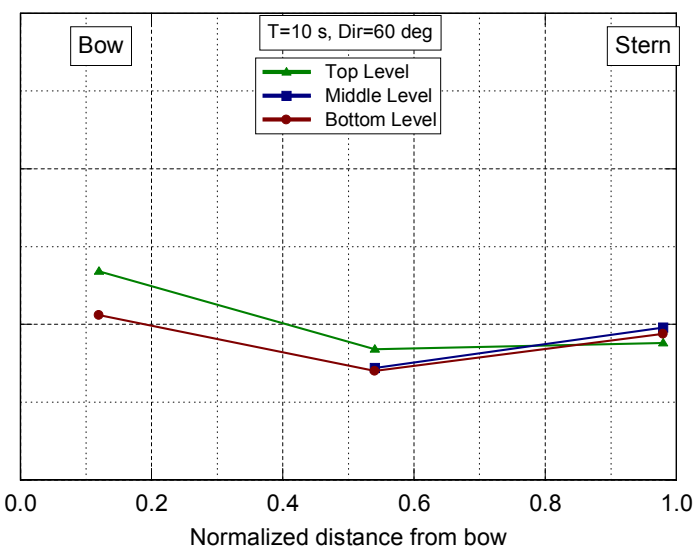

d) $T=10 \mathrm{sec}, \bar{\theta}=60 \mathrm{deg}$.

Figure 67. Measured $\bar{p}_{l, S}$ for XM99 "swell" waves: (a) $T=8 \mathrm{sec}, \bar{\theta}=30 \mathrm{deg}$, (b) $T=10 \mathrm{sec}$, $\bar{\theta}=30 \mathrm{deg}$, (c) $T=8 \mathrm{sec}, \bar{\theta}=60 \mathrm{deg}$, (d) $T=10 \mathrm{sec}, \bar{\theta}=60 \mathrm{deg}$.

period and direction bin, so there is some variation about each point that is not shown in the figures. Possible physical explanations for these discrepancies are nonlinear fluid motions and structural interactions (i.e., tail wagging, mach stem waves, oscillations of the fabric and levels, reflections between the two legs, etc.) that might add to the measured dynamic pressure at certain locations. Additional work is ongoing to understand the reasons for these unexpected results. 


\section{WAMIT Predicted Dynamic Pressures}

Pressure variation. The procedures described in Chapter V are used to predict the normalized dynamic pressures $\bar{p}_{W}$ from the WAMIT models. Figures 68 to 71 are threedimensional plots of the external $\bar{p}_{W}$ along the port leg of the XM99. The four figures bracket a range of wave periods that define sea (i.e., $3 \leq T<5 \mathrm{sec}$ ) and swell (i.e., $8 \leq T<10 \mathrm{sec}$ ) conditions and were observed during the field trials. The three panels in each figure contain the predicted $\bar{p}_{W}$ for the three wave direction bins, designated as 0,30 , and 60 deg (i.e., WAMIT coordinate system). These pressures are "snapshots" in time of the spatial variation of pressure along the external side of the port leg. The twenty-four curves corresponded to the estimated pressure at the center of each panel throughout the water column, spaced every $15 \mathrm{~cm}$ from the water surface.

As before, positive pressures are due to the passage of wave crests and forward horizontal velocities and negative pressures correspond to the passage of wave troughs and reversing horizontal velocities. The pressure oscillates along the length and decreases with depth along the XM99 port leg, except for the larger wave periods. As the wave period increases the wavelength increases and there are fewer waves along the XM99 length. For the longer period waves, the XM99 is much shorter than a wavelength and there is not a full wavelength of pressure within the "snapshot" of pressure along the entire leg length. For the larger periods in the swell category, the wave is considered an intermediate depth wave and the XM99 experiences almost the same dynamic pressure from the top to the bottom.

The $\bar{\theta}=60$ deg waves are normal to the port leg of the XM99. Most of the WAMIT predictions show a "flat" pressure distribution along the XM99 leg that has very little cyclical variation along the leg length. This is due to the relative phasing in the WAMIT model and the origin of the WAMIT coordinate system. A different phasing or origin location would produce a different "snapshot" of the pressure distribution. Since it is impossible to make any judgements about pressure amplitudes without a crest or trough present, the $\bar{\theta}=60$ deg wave cases are not included in any further WAMIT analysis.

These three-dimensional "snapshots" illustrate how $\bar{p}_{W}$ varies along and down the XM99 legs. For comparisons with the measured $\bar{p}_{l, S}$ and analytical $\bar{p}_{l, A}$, the spatial variation of $\bar{p}_{W}$ at each of the three levels is required. Figures 72 to 73 illustrate this spatial variation for the two sea and two swell category waves. Each panel shows the $\bar{p}_{W}$ variation for the top, middle, and 
(a)

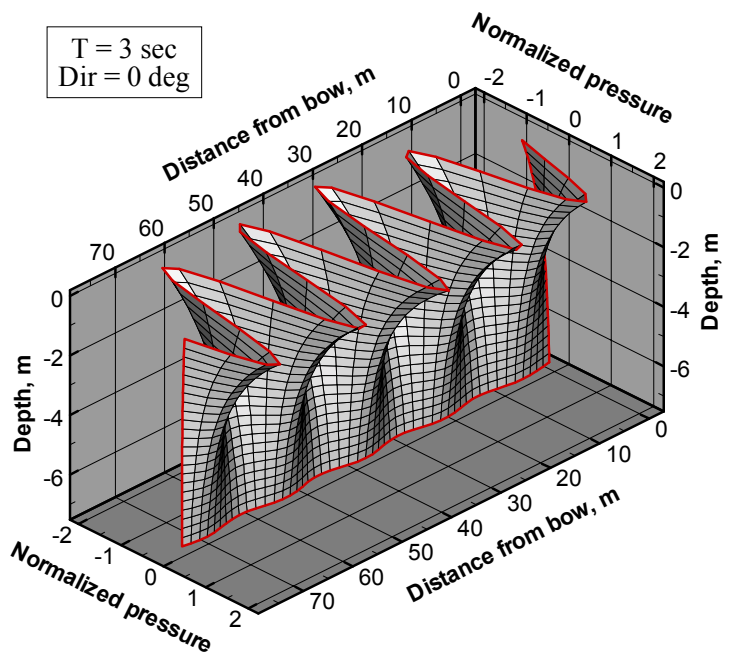

(b)

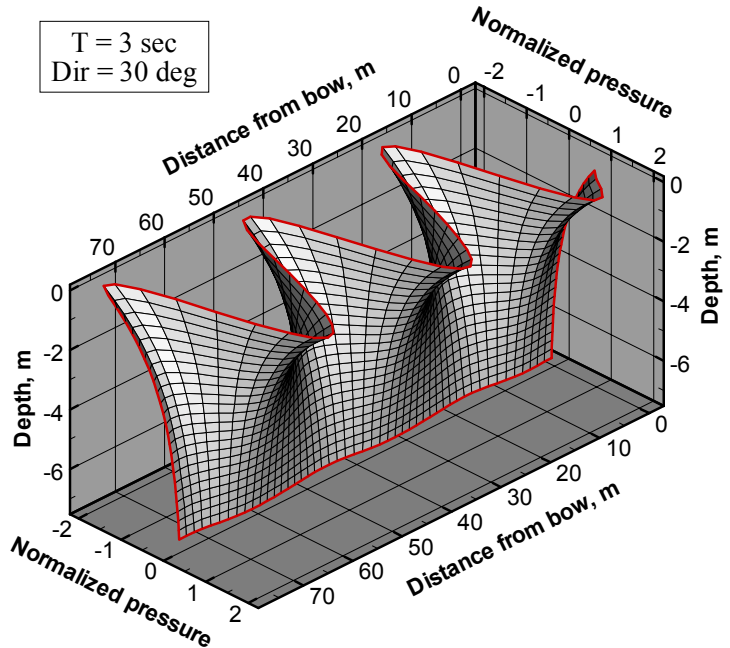

(c)

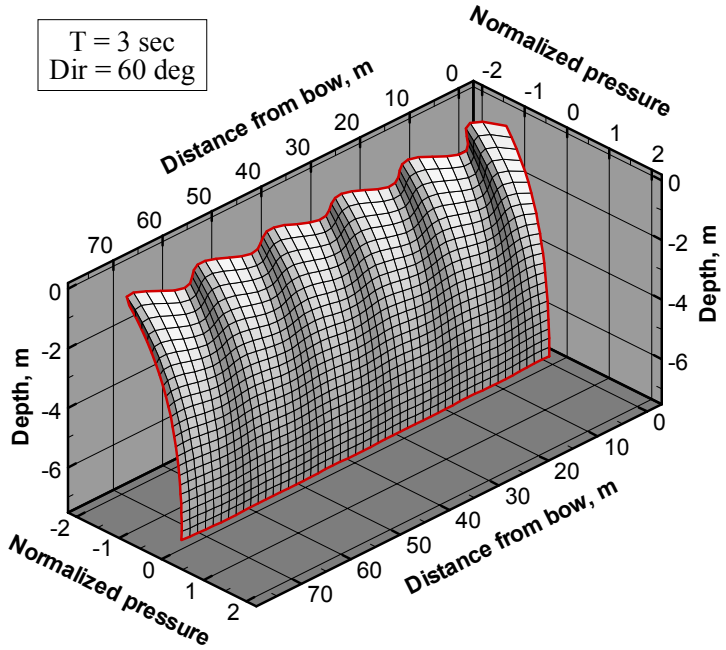

Figure 68. WAMIT predicted $\bar{p}_{W}$ for XM99 (a) $T=3 \mathrm{sec}, \bar{\theta}=0 \mathrm{deg}$, (b) $T=3 \mathrm{sec}, \bar{\theta}=30 \mathrm{deg}$, (c) $T=3 \mathrm{sec}, \bar{\theta}=60 \mathrm{deg}$. 
(a)

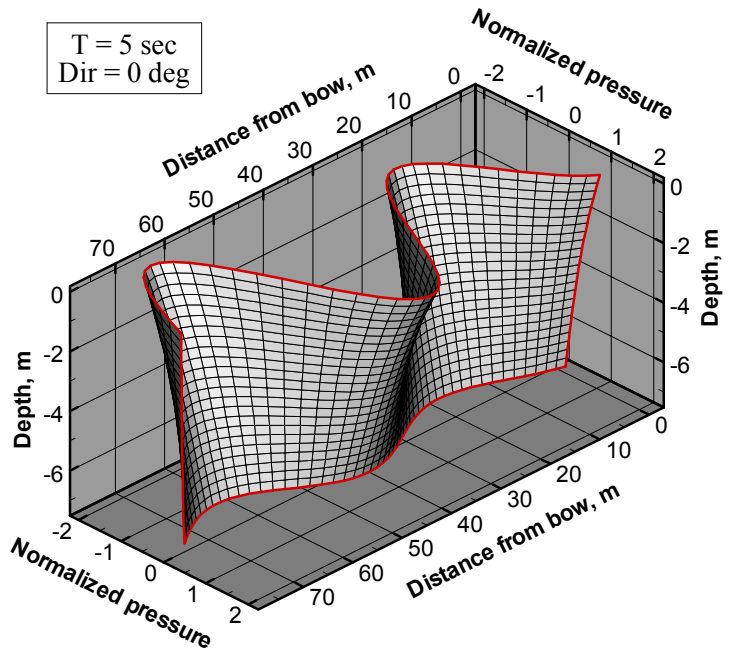

(b)

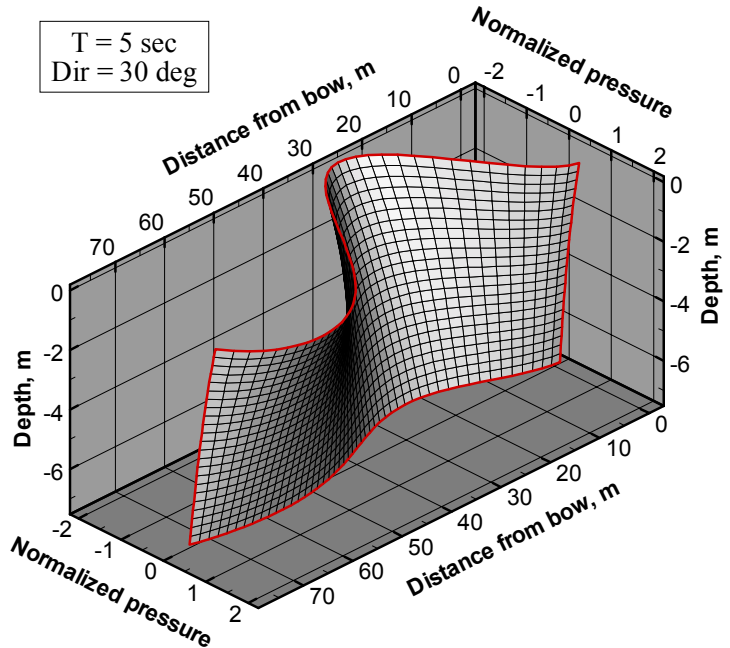

(c)

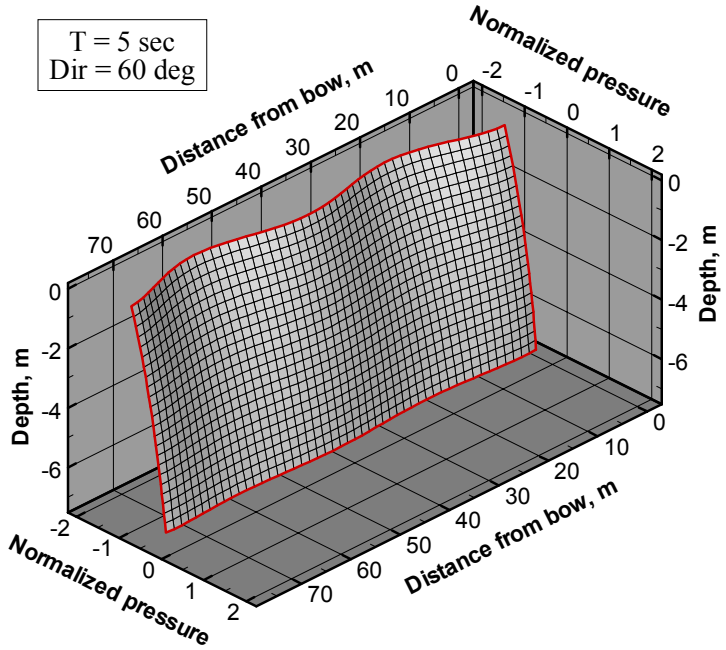

Figure 69. WAMIT predicted $\bar{p}_{W}$ for XM99 (a) $T=5 \mathrm{sec}, \bar{\theta}=0 \mathrm{deg}$, (b) $T=5 \mathrm{sec}, \bar{\theta}=30 \mathrm{deg}$, (c) $T=5 \mathrm{sec}, \bar{\theta}=60 \mathrm{deg}$. 
(a)

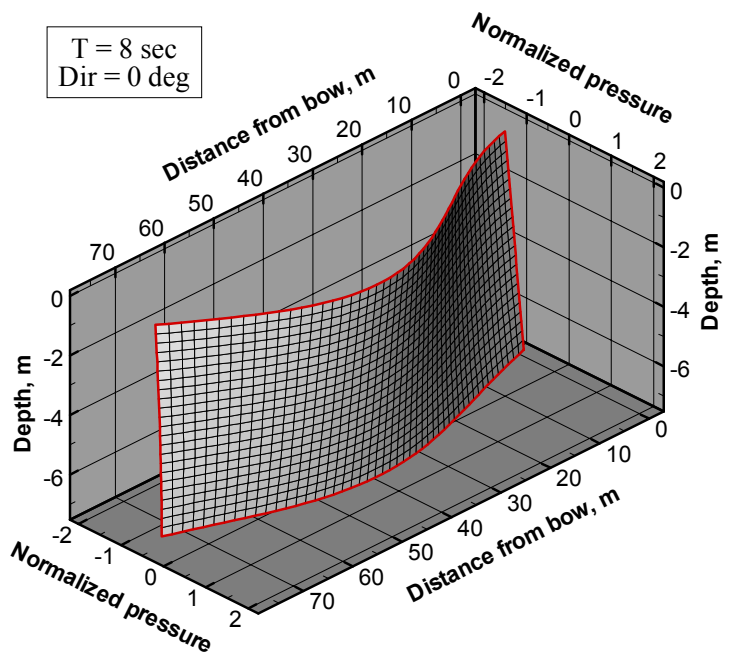

(b)

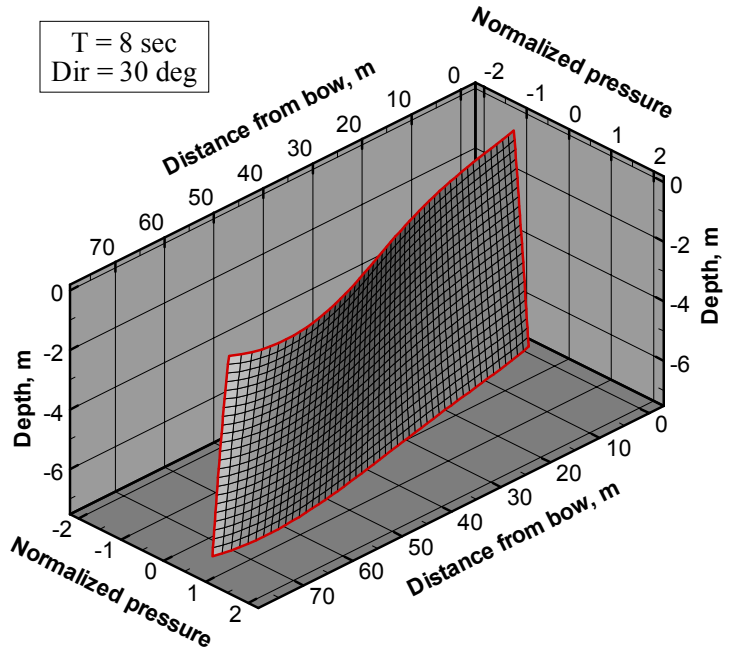

(c)

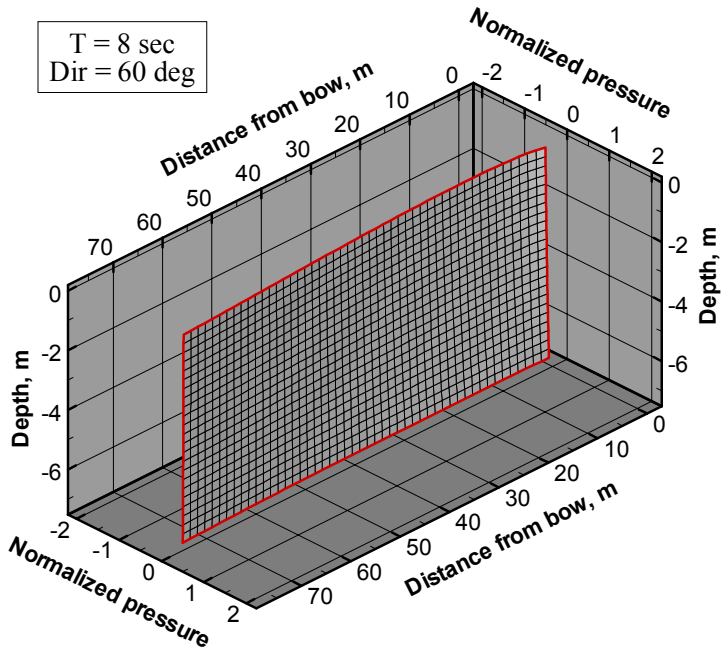

Figure 70. WAMIT predicted $\bar{p}_{W}$ for XM99 (a) $T=8 \mathrm{sec}, \bar{\theta}=0 \mathrm{deg}$, (b) $T=8 \mathrm{sec}, \bar{\theta}=30 \mathrm{deg}$, (c) $T=8 \mathrm{sec}, \bar{\theta}=60 \mathrm{deg}$. 
(a)

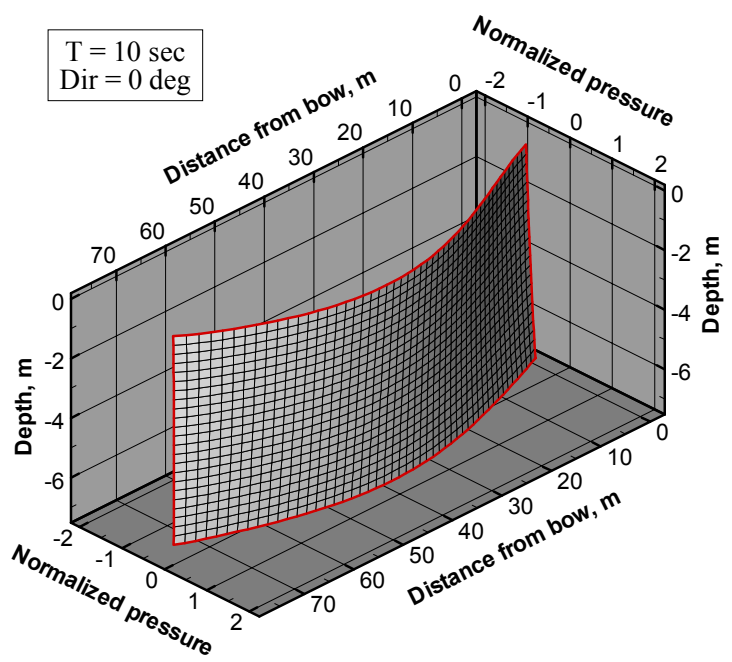

(b)

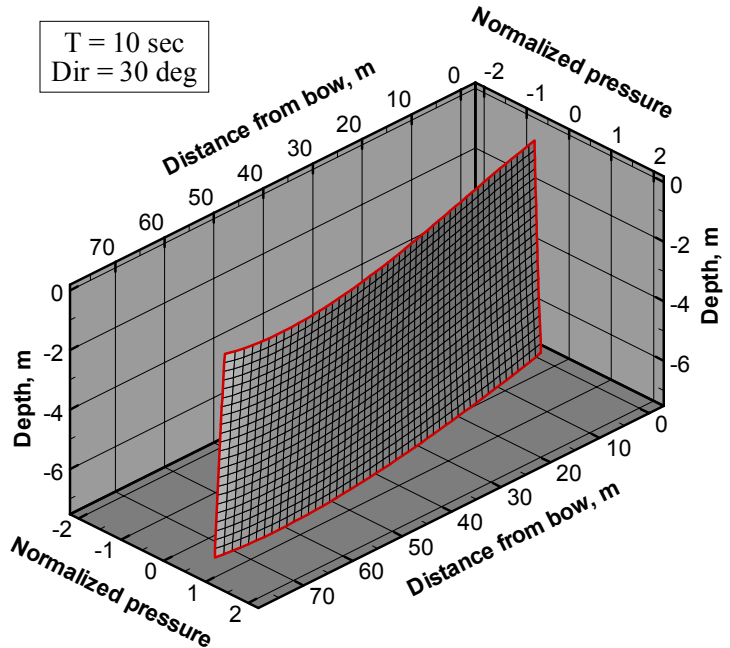

(c)

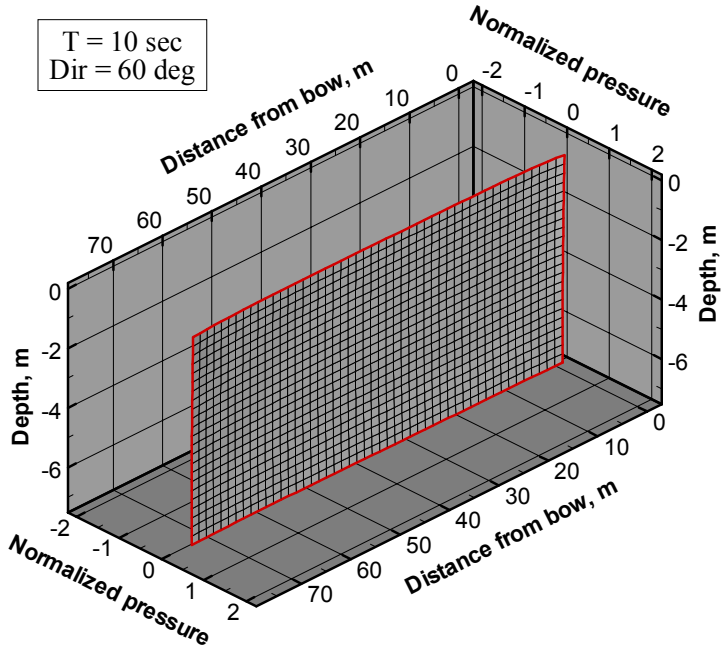

Figure 71. WAMIT predicted $\bar{p}_{W}$ for XM99 (a) $T=10 \mathrm{sec}, \bar{\theta}=0 \mathrm{deg}$, (b) $T=10 \mathrm{sec}, \bar{\theta}=30 \mathrm{deg}$, (c) $T=10 \mathrm{sec}, \bar{\theta}=60 \mathrm{deg}$. 


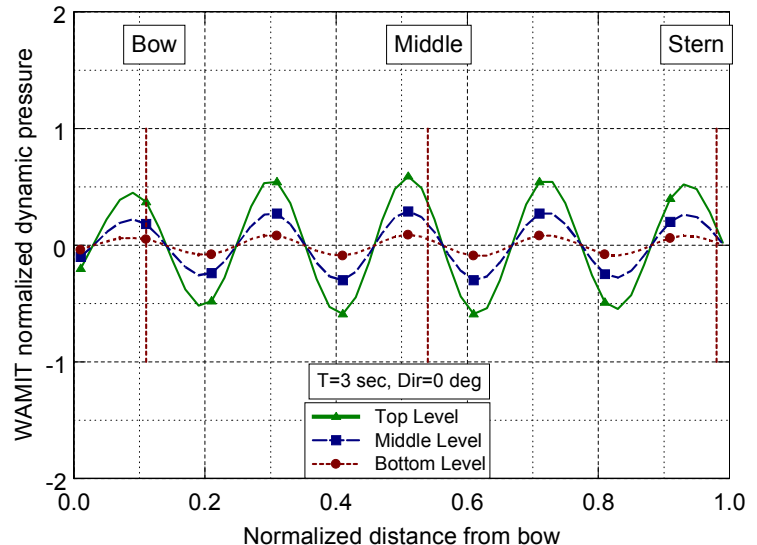

a) $T=3 \mathrm{sec}, \bar{\theta}=0 \mathrm{deg}$.

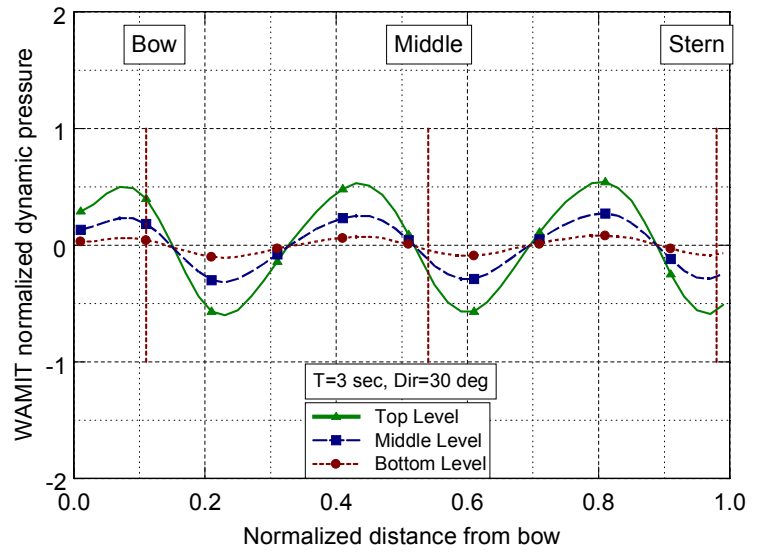

c) $T=3 \mathrm{sec}, \bar{\theta}=30 \mathrm{deg}$.

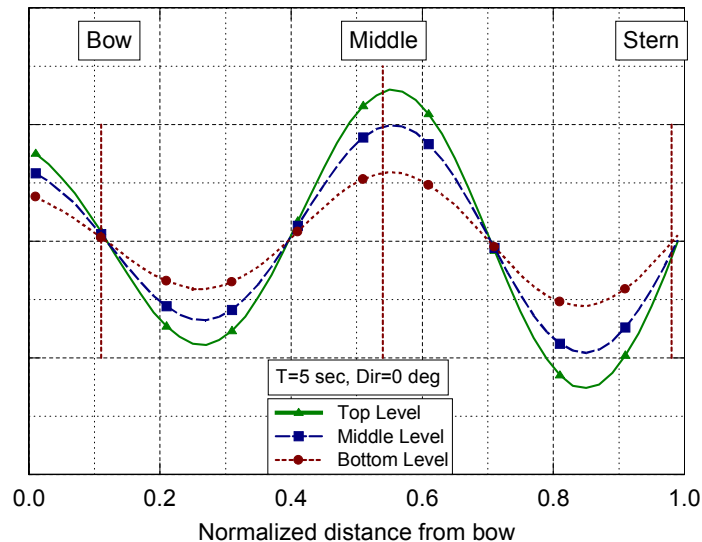

b) $T=5 \mathrm{sec}, \bar{\theta}=0 \mathrm{deg}$.

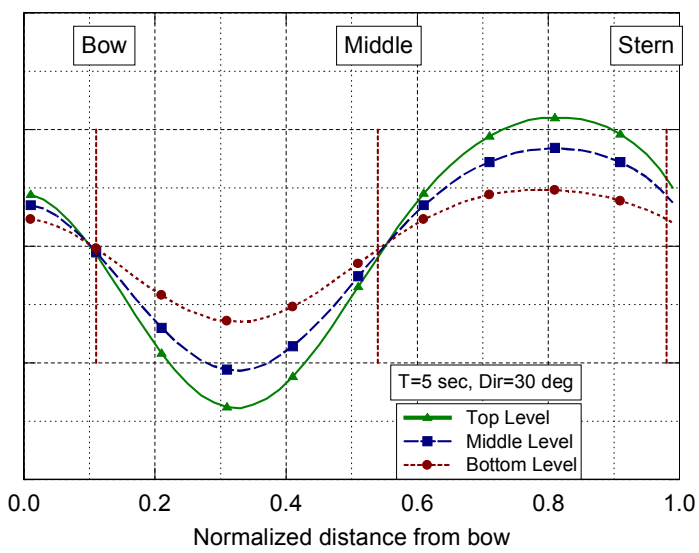

d) $T=5 \mathrm{sec}, \bar{\theta}=30 \mathrm{deg}$.

Figure 72. WAMIT $\bar{p}_{W}$ for XM99 "sea" waves (a) $T=3 \mathrm{sec}, \bar{\theta}=0 \mathrm{deg}$, (b) $T=5 \mathrm{sec}, \bar{\theta}=0 \mathrm{deg}$, (c) $T=3 \mathrm{sec}, \bar{\theta}=30 \mathrm{deg}$ (d) $T=5 \mathrm{sec}, \bar{\theta}=30 \mathrm{deg}$.

bottom levels at $z=-2.90$ (solid line), -4.42 (dashed line), and -6.86 (dotted line) $\mathrm{m}$. The dashed vertical lines are located at the horizontal positions of the bow, middle, and stern pressure gages.

For the $T=3 \mathrm{sec}, \bar{\theta}=0 \mathrm{deg}$ waves, the waves are parallel to the XM99 center line at $315 \mathrm{deg}$. There are several wavelengths along the XM99 leg length and they are fairly constant at each level and each location, although the middle gage is slightly larger than the bow and stern gages. The $\bar{p}_{W}$ decreases with depth, but follows similar trends as the top level. For the $T=3 \mathrm{sec}, \bar{\theta}=30$ deg waves, the waves are parallel to the starboard leg. There are fewer wavelengths along the 


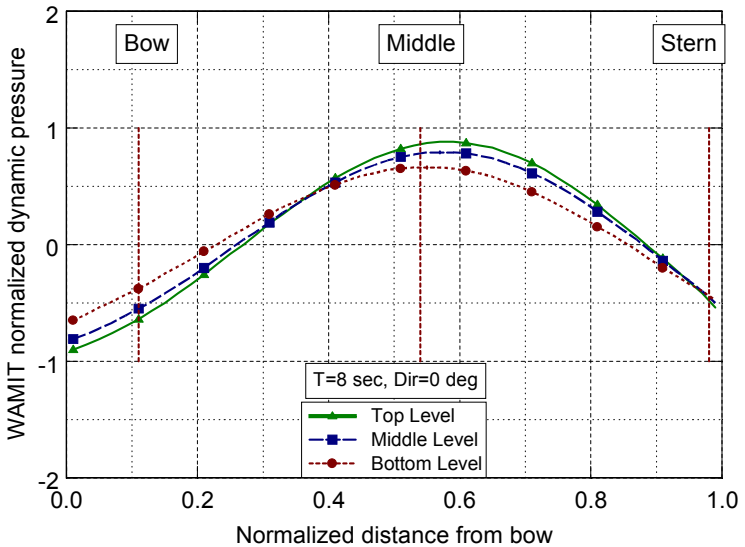

a) $T=8 \mathrm{sec}, \bar{\theta}=0 \mathrm{deg}$.

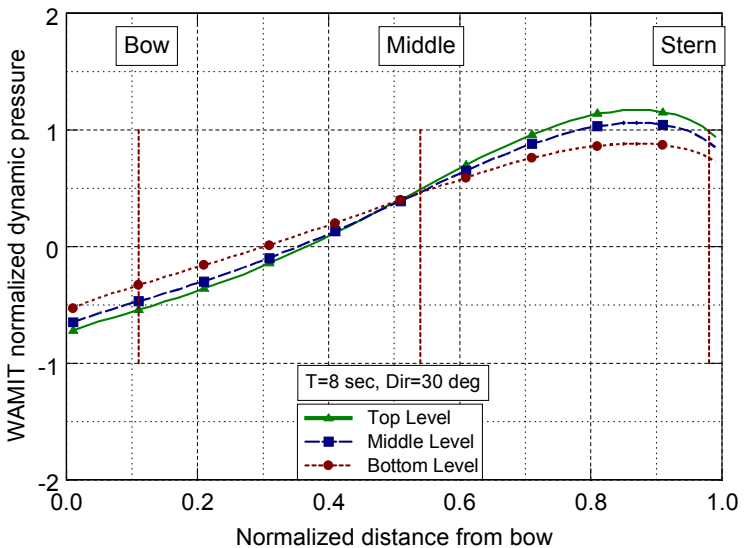

c) $T=8 \mathrm{sec}, \bar{\theta}=30 \mathrm{deg}$.

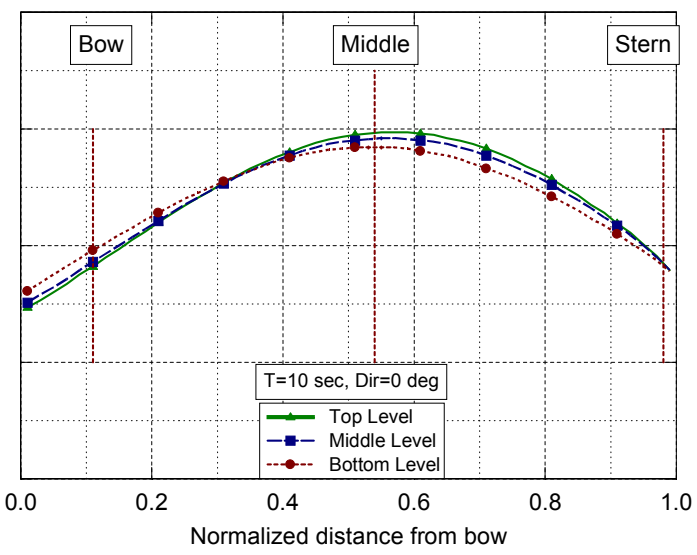

b) $T=10 \mathrm{sec}, \bar{\theta}=0 \mathrm{deg}$.

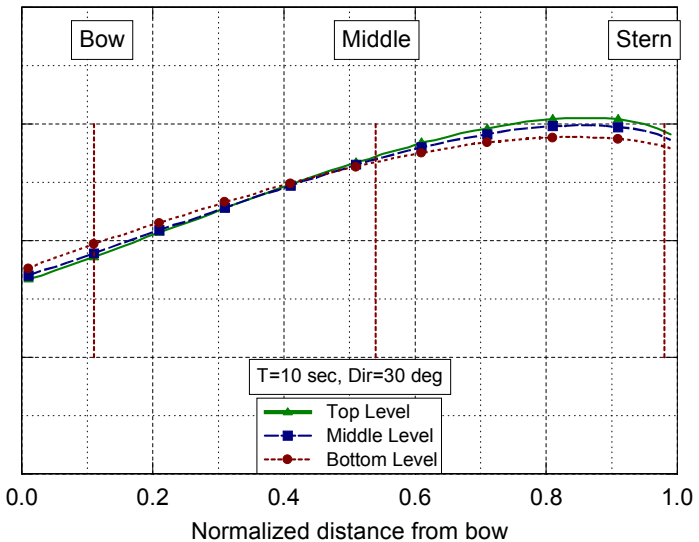

d) $T=10 \mathrm{sec}, \bar{\theta}=30 \mathrm{deg}$.

Figure 73. WAMIT $\bar{p}_{W}$ for XM99 "swell" waves (a) $T=8 \mathrm{sec}, \bar{\theta}=0 \mathrm{deg}$, (b) $T=10 \mathrm{sec}, \bar{\theta}=0 \mathrm{deg}$, (c) $T=8 \mathrm{sec}, \bar{\theta}=30 \mathrm{deg}$, (d) $T=10 \mathrm{sec}, \bar{\theta}=30 \mathrm{deg}$.

leg, and the $\bar{p}_{W}$ increases very slightly from the bow to the stern gages, especially on the top level.

For the $T=5 \mathrm{sec}, \bar{\theta}=0$ deg waves, there is only a little more than one wave cycle present on the XM99 leg at any instant in time. The $\bar{p}_{W}$ magnitude has increased slightly for this longer wave and increases from the bow to the stern. For the $T=5 \mathrm{sec}, \bar{\theta}=30 \mathrm{deg}$ waves, there is barely one wavelength along the leg. There is an increase in $\bar{p}_{W}$ from the bow to the middle, and then it remains constant. 
For the longer wave periods in the "swell" category, there is only one-half to one-fourth of a wavelength present along the XM99 leg. The $\bar{p}_{W}$ are almost constant at each level since the waves are intermediate depth waves. Because less than one wavelength is present, it is difficult to draw any conclusions relative to magnitude changes along the leg. Thus, it is assumed that the $\bar{p}_{W}$ is constant along the leg and with depth for the three levels.

Pressure magnitude. As done previously for the FLab data, a normalized $\bar{p}_{1, W}$ magnitude was calculated for each wave case. The magnitude of this pressure was determined by taking one half of the crest to trough distance closest to the gage from the previous figures. If the horizontal coordinate of the pressure gage did not fall within a WAMIT panel, the two adjacent panel $\bar{p}_{W}$ magnitudes were averaged along that level. This technique includes some of the WAMIT variation in $\bar{p}_{W}$ along the length of the RIBS leg. Thus, this pressure is representative of what each gage would experience based on its relative position. At another instant in time, these pressures would change, but would still maintain their relative magnitudes to one another.

Although these are just "snapshots" and the entire wave could be expected to travel along the leg over a wave cycle, it was assumed that the relative magnitudes among gages would be preserved. For the shorter waves in the "sea" category with one or more wavelengths present along the leg, this procedure seemed to work well. However, for the "swell" cases with less than one wavelength, it was assumed that the maximum $\bar{p}_{1, W}$ was present. This seemed justifiable since at least one crest or trough was usually observed.

Figures 74 to 75 show these $\bar{p}_{l, W}$ magnitudes for the "sea" and "swell" cases. The format is the same as in the previous figures. The trends for the variation in $\bar{p}_{1, W}$ are similar to those described earlier. The $T=5 \mathrm{sec}$ waves are larger than the $T=3 \mathrm{sec}$ waves. There is not much difference between the "swell" category waves, but the $\bar{\theta}=30$ deg waves are slightly larger than the $\bar{\theta}=0$ deg waves.

Comparison to measured pressures. A comparison of the WAMIT predicted $\bar{p}_{1, W}$ to the measured $\bar{p}_{1, S}$ is shown in Figure 76 . The three panels show the trends for the bow, middle, and stern pressure gages at all three levels. Only the data points for the eight cases are included (i.e., none of the $\bar{\theta}=60 \mathrm{deg}$ wave cases). In general, the WAMIT $\bar{p}_{1, W}$ are larger than the corresponding measured $\bar{p}_{1, S}$, especially as the $\bar{p}_{1, S}$ increase. 


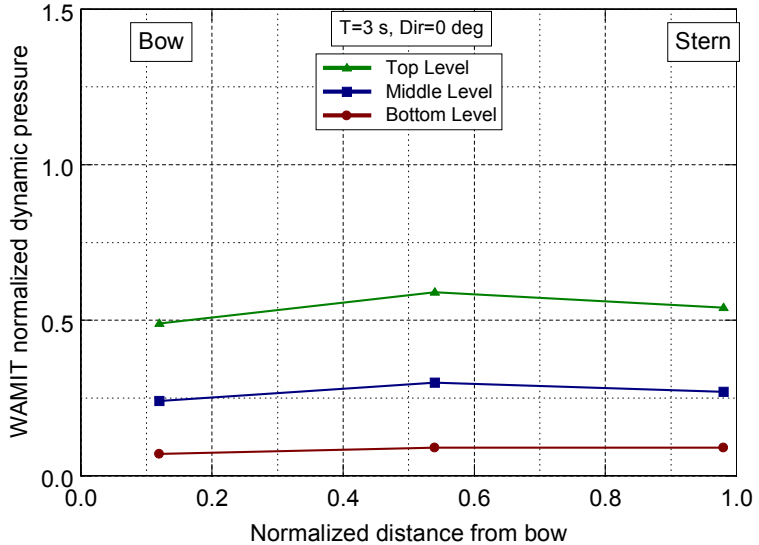

a) $T=3 \mathrm{sec}, \bar{\theta}=0 \mathrm{deg}$.

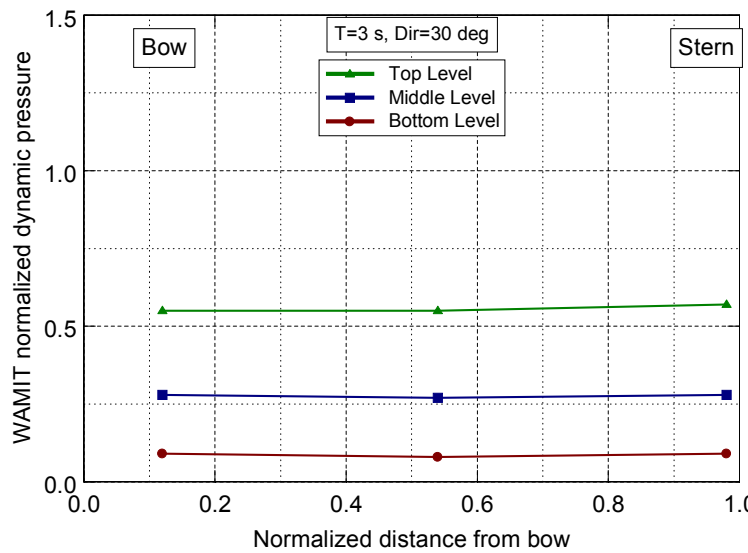

c) $T=3 \mathrm{sec}, \bar{\theta}=30 \mathrm{deg}$.

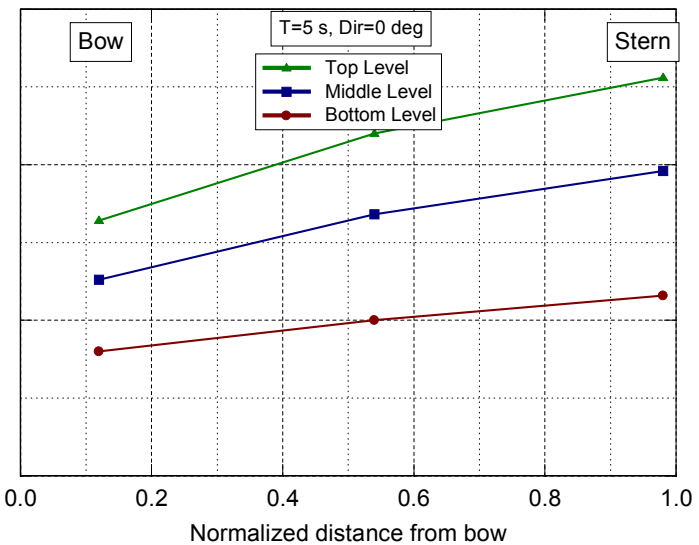

b) $T=5 \mathrm{sec}, \bar{\theta}=0 \mathrm{deg}$.

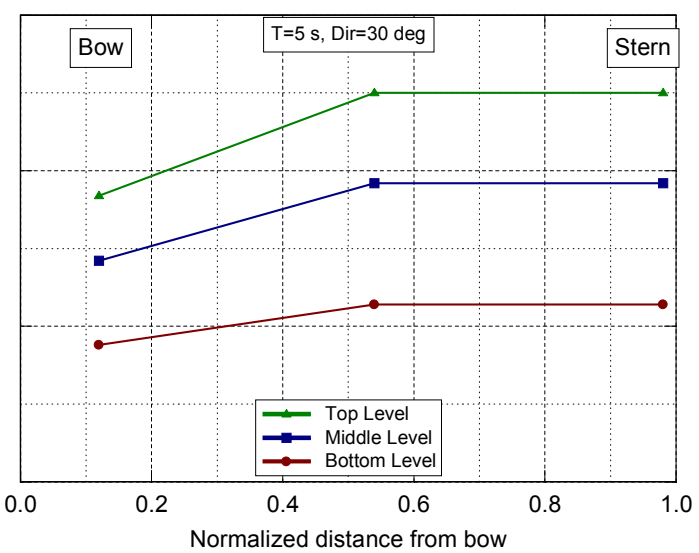

d) $T=5 \mathrm{sec}, \bar{\theta}=30 \mathrm{deg}$.

Figure 74. WAMIT $\bar{p}_{1, W}$ magnitude for XM99 "sea" waves (a) $T=3 \mathrm{sec}, \bar{\theta}=0 \mathrm{deg}$, (b) $T=5 \mathrm{sec}$, $\bar{\theta}=0 \mathrm{deg}$, (c) $T=3 \mathrm{sec}, \bar{\theta}=30 \mathrm{deg}$, (d) $T=5 \mathrm{sec}, \bar{\theta}=30 \mathrm{deg}$.

\section{Analytical Comparisons}

Pressure variation. Equation 77, from linear wave theory, was used to predict the normalized dynamic pressure $\bar{p}_{l, A}$ for each of the wave cases. The analytical coordinate system was the same as used previously for the FLab. Because of symmetry, the predicted $\bar{p}_{l, A}$ are the same as if the axes were on the port leg. The three wave bin directions of $\bar{\theta}=0,30$, and $60 \mathrm{deg}$ correspond to angles of 60,90 , and $120 \mathrm{deg}$, respectively, in the analytical coordinate system

As noted earlier, the predicted $\bar{p}_{l, A}$ magnitude is constant for all pressure gages at each 

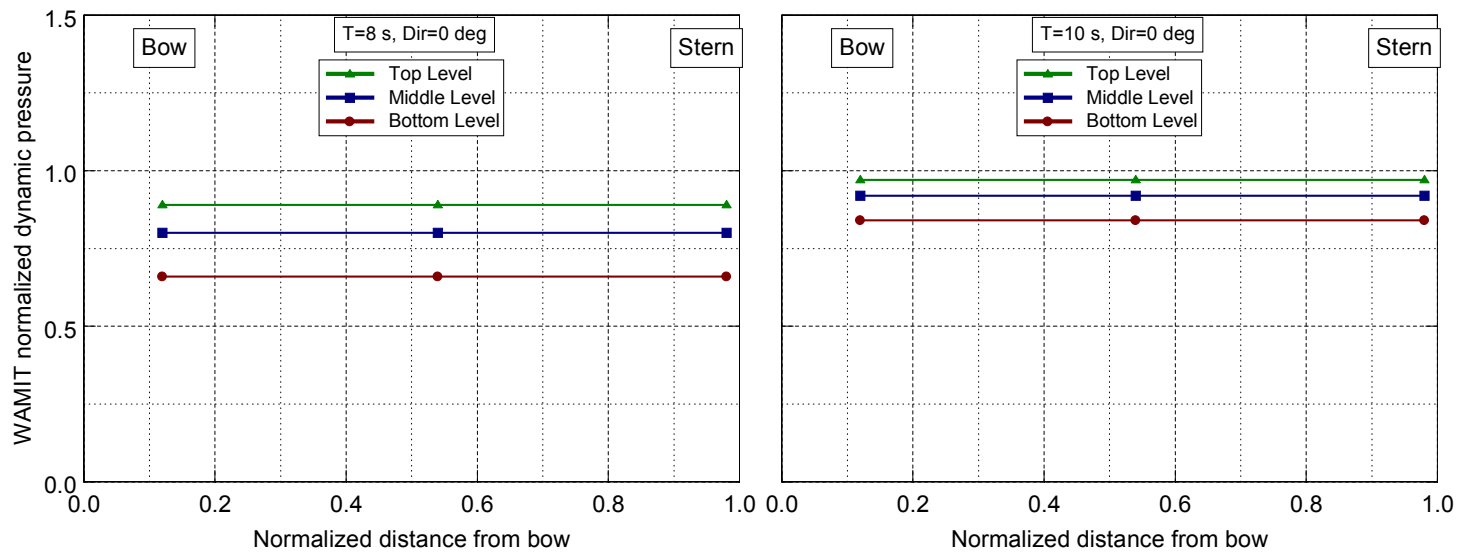

a) $T=8 \mathrm{sec}, \bar{\theta}=0 \mathrm{deg}$.

b) $T=10 \mathrm{sec}, \bar{\theta}=0 \mathrm{deg}$.
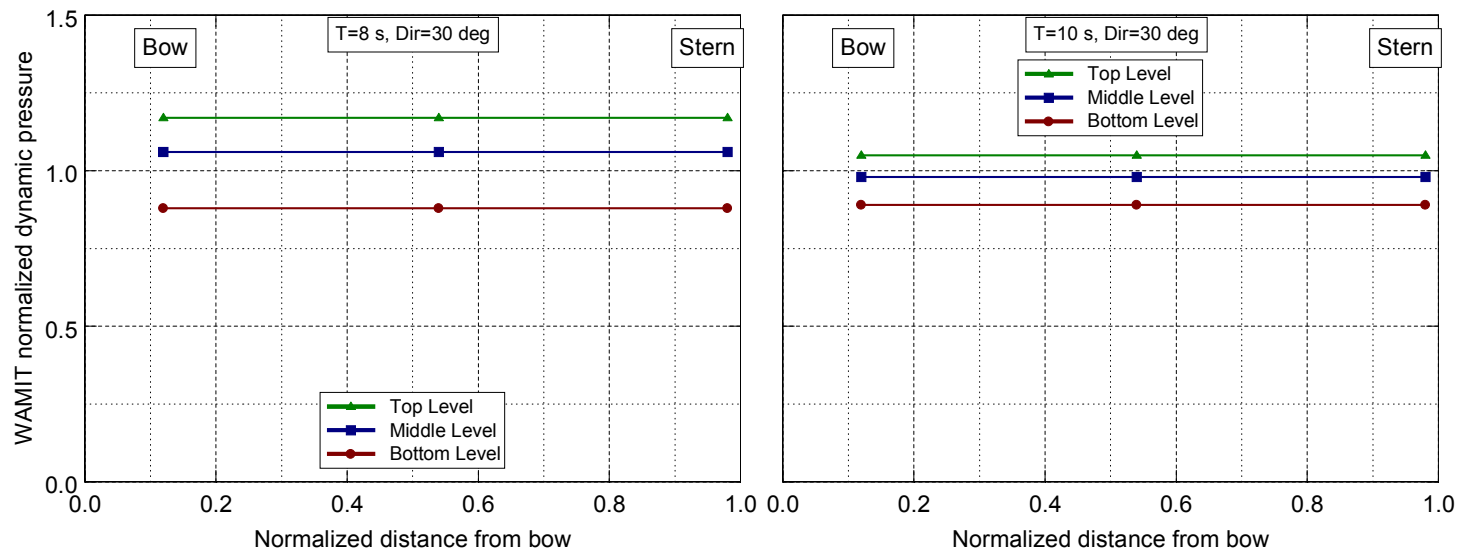

c) $T=8 \mathrm{sec}, \bar{\theta}=30 \mathrm{deg}$.

d) $T=10 \mathrm{sec}, \bar{\theta}=30 \mathrm{deg}$.

Figure 75. WAMIT $\bar{p}_{1, W}$ magnitude for XM99 “swell" waves (a) $T=8 \mathrm{sec}, \bar{\theta}=0 \mathrm{deg}$, (b) $T=10 \mathrm{sec}, \bar{\theta}=0 \mathrm{deg}$, (c) $T=8 \mathrm{sec}, \bar{\theta}=30 \mathrm{deg}$, (d) $T=10 \mathrm{sec}, \bar{\theta}=30 \mathrm{deg}$.

vertical level. The reflection coefficient $K_{R}$, based on an energy balance, was assumed to be equal to $\sqrt{ }\left(1-K_{T}^{2}\right)$. The WAMIT-estimated $K_{T}$ was used as an estimate of the wave transmission coefficient. A quick parameter study of the variation in $K_{T}$ within a "watch circle" of $3 \mathrm{~m}$ was performed. It was determined that the variation was insignificant relative to the predicted $K_{T}$ at the measurement location. Therefore, the single value predicted for this point was used rather than an average within the "watch circle." 
a)
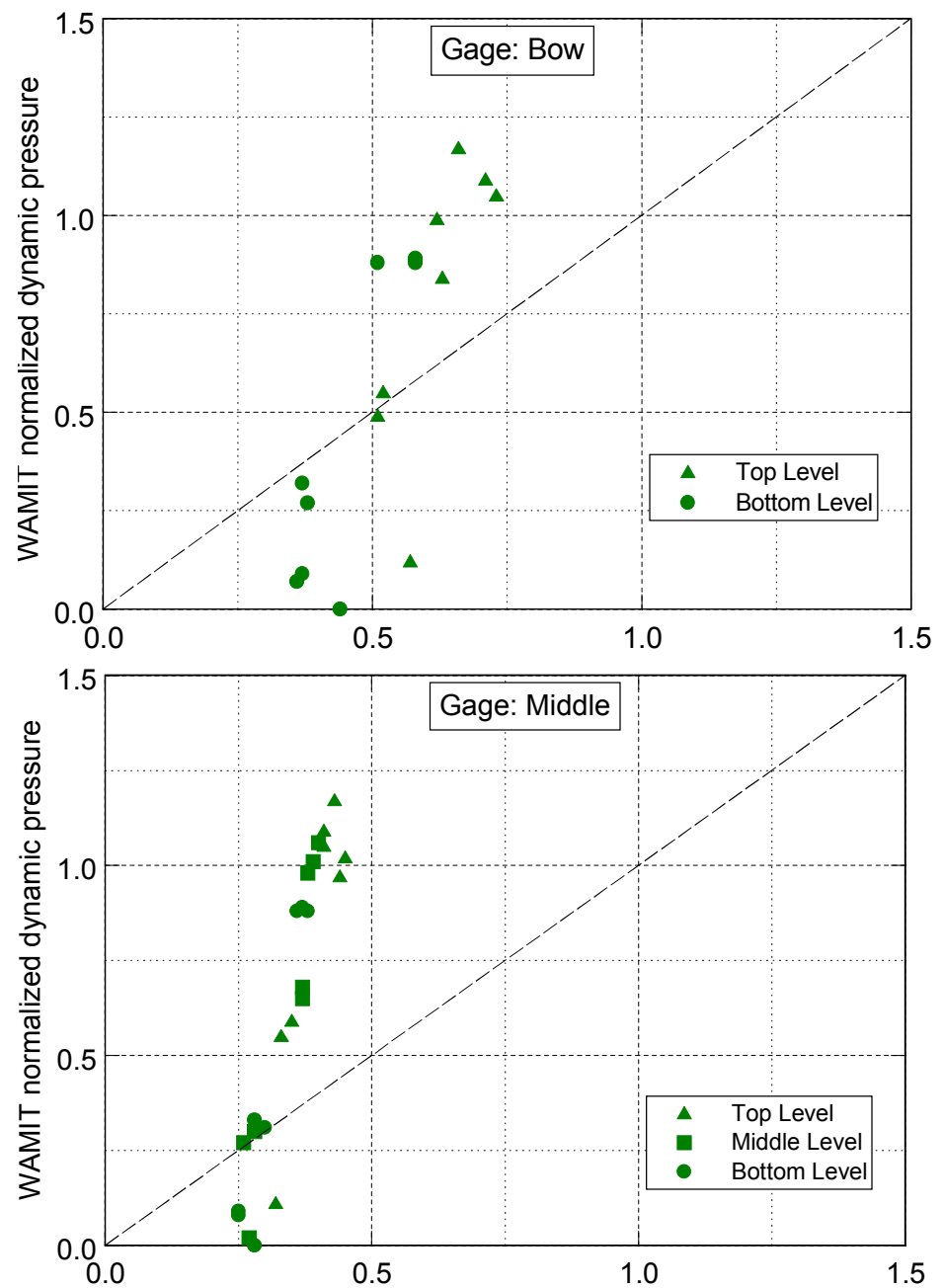

b)

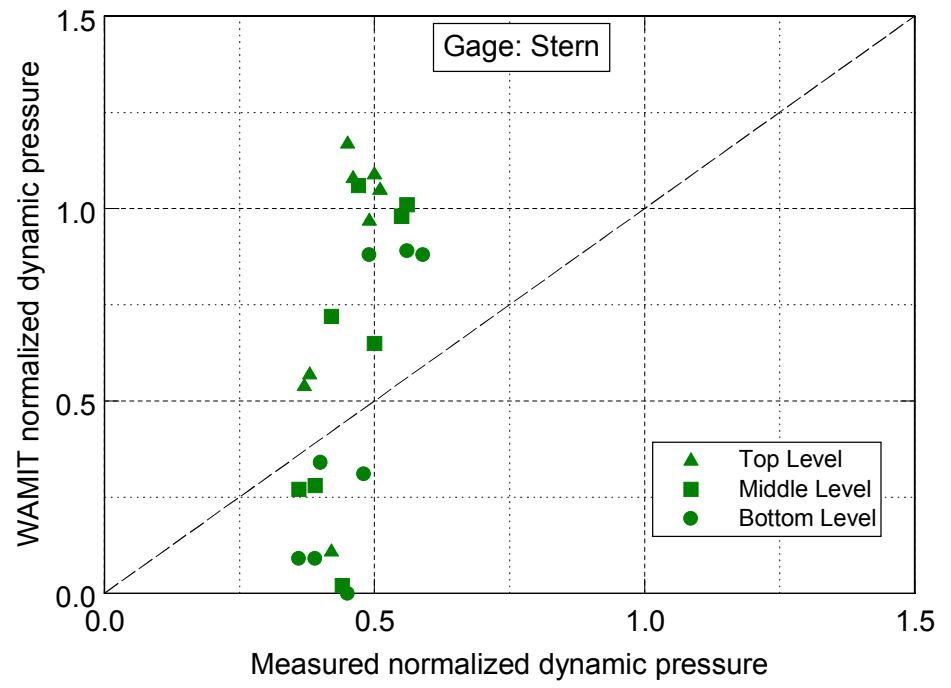

Figure 76. Comparison of WAMIT $\bar{p}_{1, W}$ vs. measured $\bar{p}_{1, S}$ for XM99 (a) bow, (b) middle, (c) stern. 
The phase $\bar{p}_{1, A}$ varies for each pressure gage and is a function of the mean wave direction $\bar{\theta}$ and $x$ and $y$ coordinates. Both $\bar{p}_{1, A}$ magnitude and phase are functions of the wave period $T$, wavelength $L$, and wavenumber $k$, and wavenumber $x$ - and $y$-components $k_{x}$ and $k_{y}$. Appendix I contains a listing of $K_{T}$ and $\bar{p}_{l, A}$ for each gage and wave case.

Figures 77 and 78 show the analytical normalized dynamic pressures $\bar{p}_{l, A}$ for each gage location at the top level for the "sea" and "swell" wave cases, respectively. As before, each of the six panels correspond to a different wave period and direction combination. The $\bar{p}_{1, A}$ magnitude is constant in space, but the phase changes for each pressure gage. It increases with wave period for the "sea" waves, but remains fairly constant for the "swell" wave cases. The magnitude is less for the "swell" cases than for the "sea" cases because the $K_{R}$ has decreased since most of the wave is passing through the XM99 for these longer $T$. Figure 79 illustrates the variation in $\bar{p}_{l, A}$ magnitude for the middle and bottom levels with $T=5 \mathrm{sec}$ waves. Comparing this figure with the previous figure for the top level, the magnitude decreases as the gage depth increases and the phasing remains constant.

These plots can be compared to the corresponding plots from the WAMIT predictions shown in Figures 72 to 73 . The analytical $\bar{p}_{1, A}$ magnitudes are very similar to the WAMIT predictions. These values also tend to support the argument that the magnitudes for the $\bar{\theta}=60$ deg cases are very similar to the other wave direction cases that the WAMIT predictions did not show.

Finally, the analytical $\bar{p}_{l, A}$ and measured $\bar{p}_{l, S}$ are compared in Figure 80 for bow, middle, and stern gage locations. Each panel includes top, middle, and bottom level gages. As previously discussed, the measured $\bar{p}_{1, S}$ seemed to be smaller than expected in several cases.

\section{XM99 Dynamic Pressure Summary}

Wave dynamic pressures were measured at twelve external locations on the port leg of the XM99. Gages were located at three stations on each of four vertical levels. The three stations were bow, middle, and stern. The four levels were the surface, top, middle, and bottom. The bow gage on the middle level never worked properly, however.

An important consideration in comparing measured dynamic pressures with the analytical and WAMIT predictions is the actual depth of the gages. For comparison purposes, the depths 


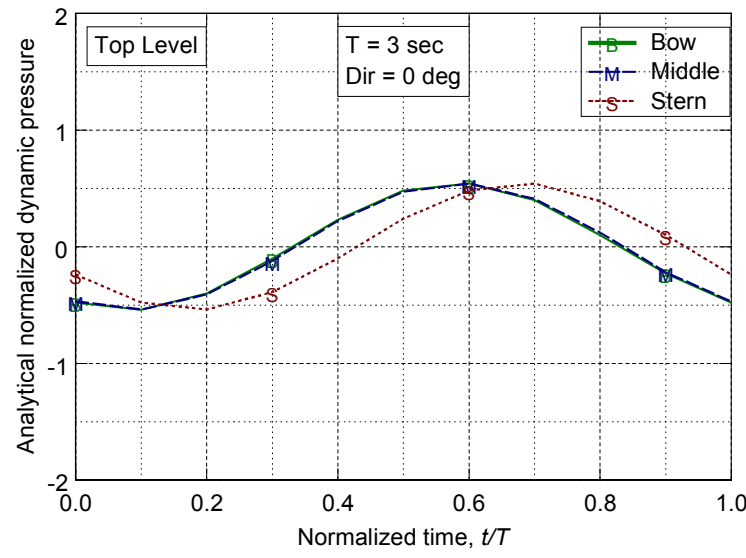

a) $T=3 \mathrm{sec}, \bar{\theta}=0 \mathrm{deg}$.

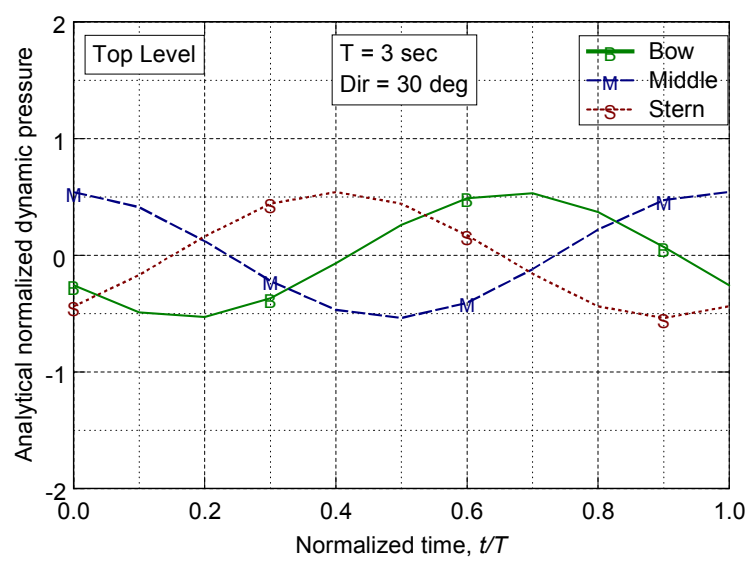

c) $T=3 \mathrm{sec}, \bar{\theta}=30 \mathrm{deg}$.

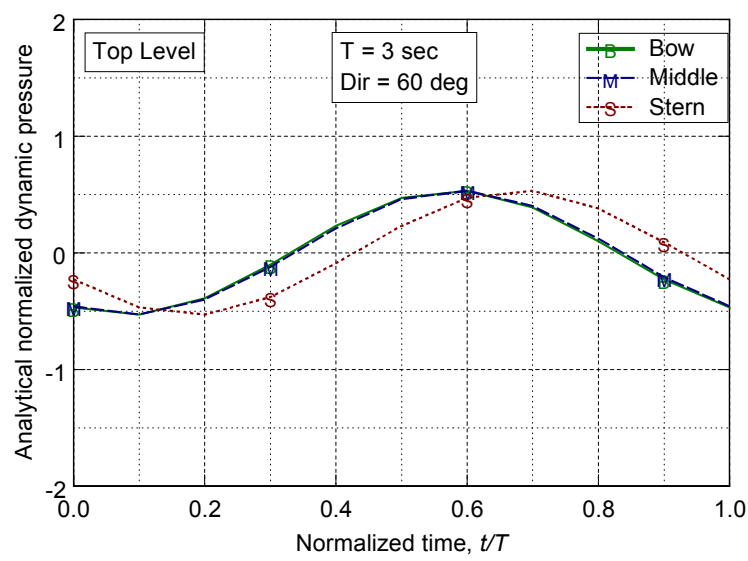

e) $T=3 \mathrm{sec}, \bar{\theta}=60 \mathrm{deg}$.

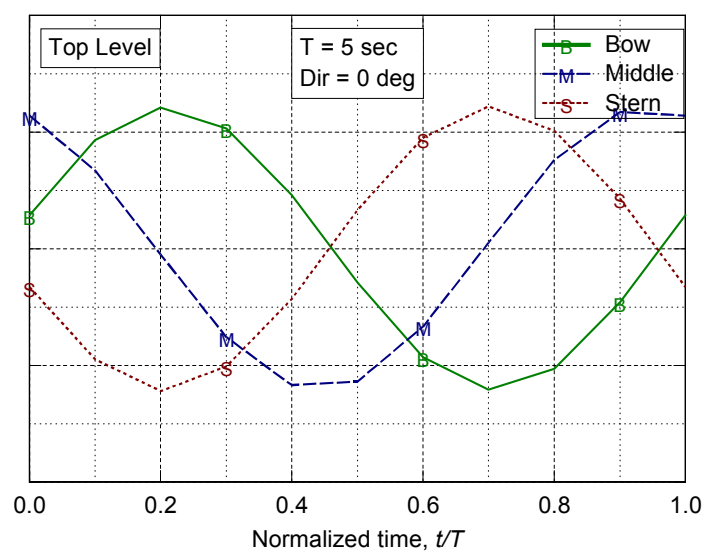

b) $T=5 \mathrm{sec}, \bar{\theta}=0 \mathrm{deg}$.

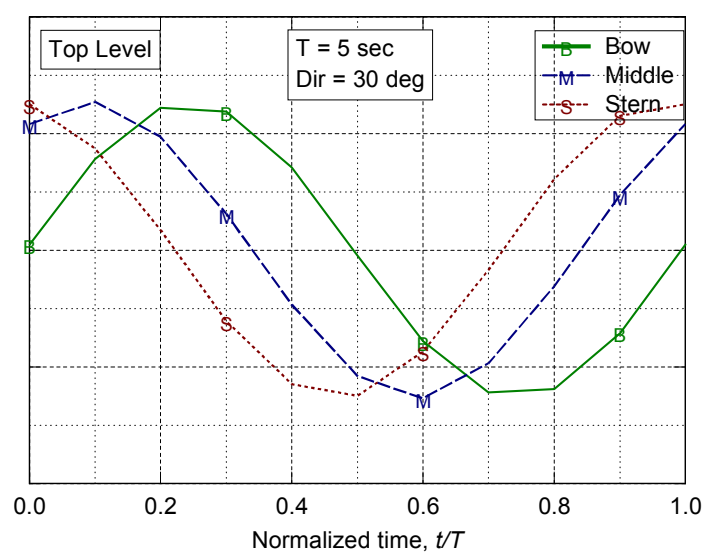

d) $T=5 \mathrm{sec}, \bar{\theta}=30 \mathrm{deg}$.

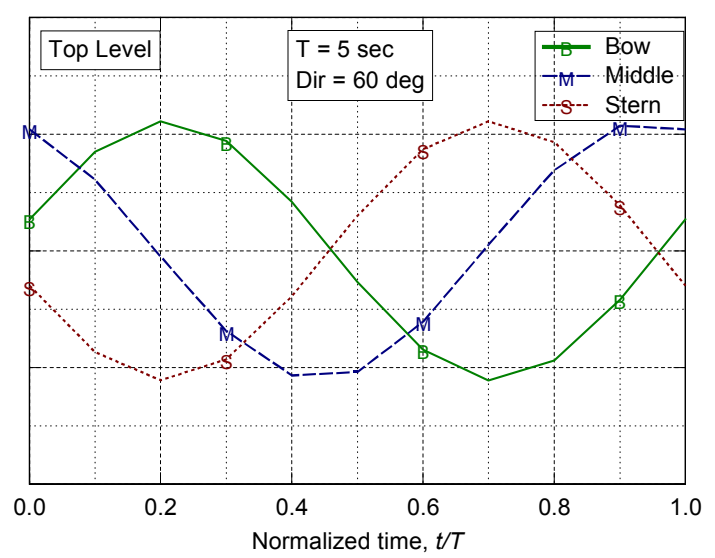

f) $T=5 \mathrm{sec}, \bar{\theta}=60 \mathrm{deg}$.

Figure 77. Analytical $\bar{p}_{1, A}$ for XM99 top level: (a) $T=3 \mathrm{sec}, \bar{\theta}=0 \mathrm{deg}$, (b) $T=5 \mathrm{sec}, \bar{\theta}=0 \mathrm{deg}$, (c) $T=3 \mathrm{sec}, \bar{\theta}=30 \mathrm{deg}$, (d) $T=5 \mathrm{sec}, \bar{\theta}=30 \mathrm{deg}$, (e) $T=3 \mathrm{sec}, \bar{\theta}=60 \mathrm{deg}$, (f) $T=5 \mathrm{sec}, \bar{\theta}=60 \mathrm{deg}$. 


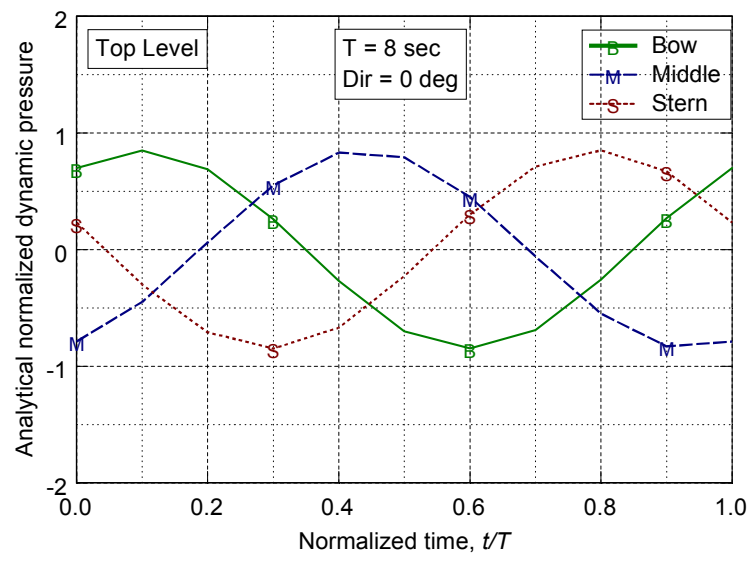

a) $T=8 \mathrm{sec}, \bar{\theta}=0 \mathrm{deg}$.

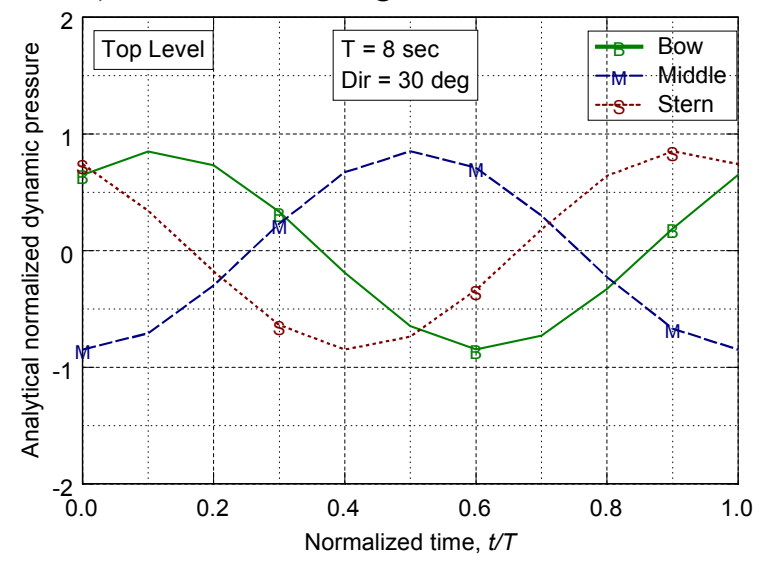

c) $T=8 \mathrm{sec}, \bar{\theta}=30 \mathrm{deg}$.

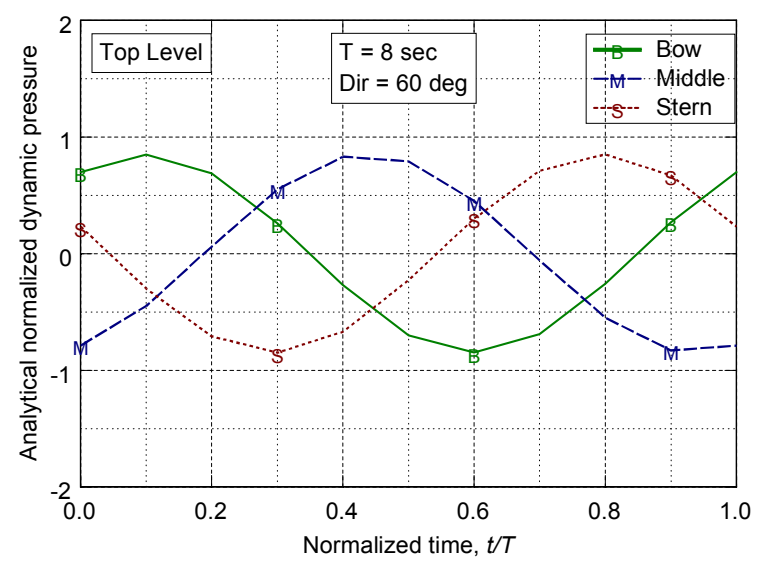

e) $T=8 \mathrm{sec}, \bar{\theta}=60 \mathrm{deg}$.

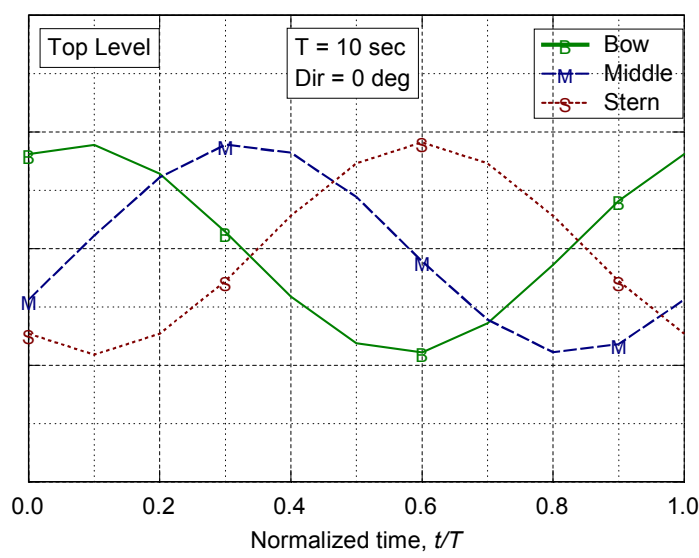

b) $T=10 \mathrm{sec}, \bar{\theta}=0 \mathrm{deg}$.

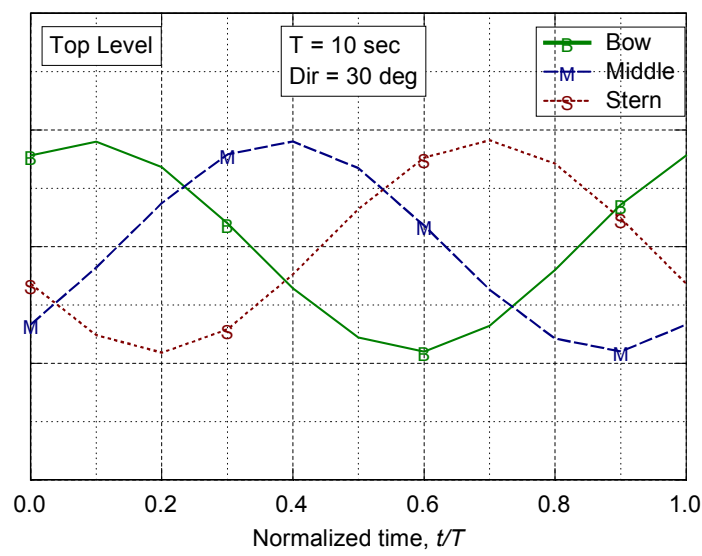

d) $T=10 \mathrm{sec}, \bar{\theta}=30 \mathrm{deg}$.

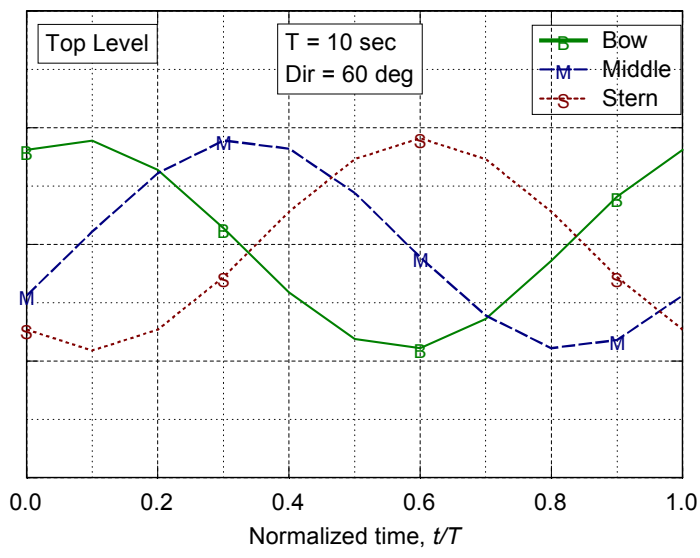

f) $T=10 \mathrm{sec}, \bar{\theta}=60 \mathrm{deg}$.

Figure 78. Analytical $\bar{p}_{1, A}$ for XM99 top level: (a) $T=8 \mathrm{sec}, \bar{\theta}=0 \mathrm{deg}$, (b) $T=10 \mathrm{sec}, \bar{\theta}=0 \mathrm{deg}$, (c) $T=8 \mathrm{sec}, \bar{\theta}=30 \mathrm{deg}$, (d) $T=10 \mathrm{sec}, \bar{\theta}=30 \mathrm{deg}$, (e) $T=8 \mathrm{sec}, \bar{\theta}=60 \mathrm{deg}$, (f) $T=10 \mathrm{sec}, \bar{\theta}=60 \mathrm{deg}$. 


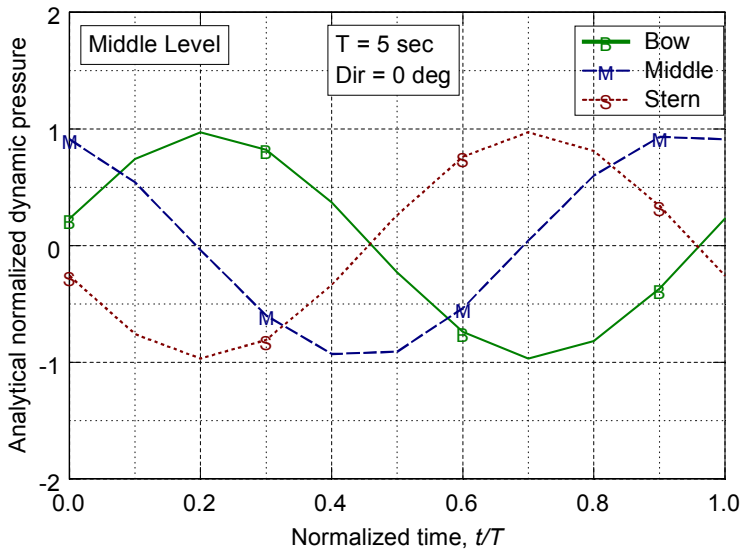

a) Middle level, $T=5 \mathrm{sec}, \bar{\theta}=0 \mathrm{deg}$.

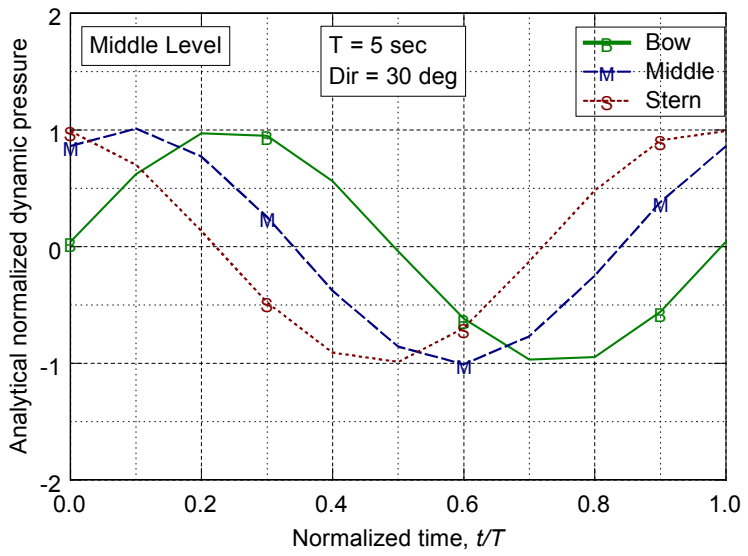

c) Middle level, $T=5 \mathrm{sec}, \bar{\theta}=30 \mathrm{deg}$.

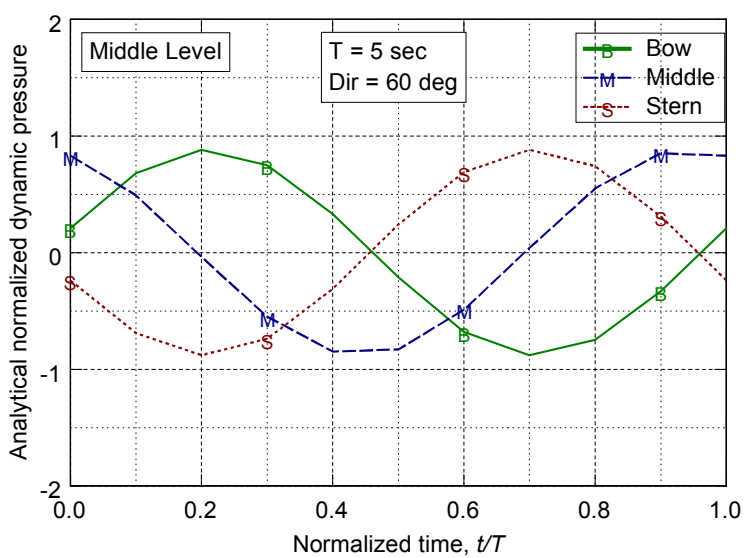

e) Middle level, $T=5 \mathrm{sec}, \bar{\theta}=60 \mathrm{deg}$.

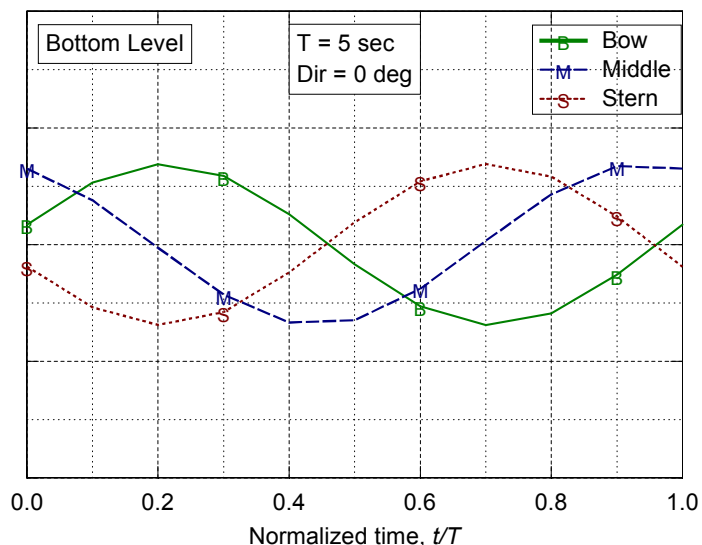

b) Bottom level, $T=5 \mathrm{sec}, \bar{\theta}=0 \mathrm{deg}$.

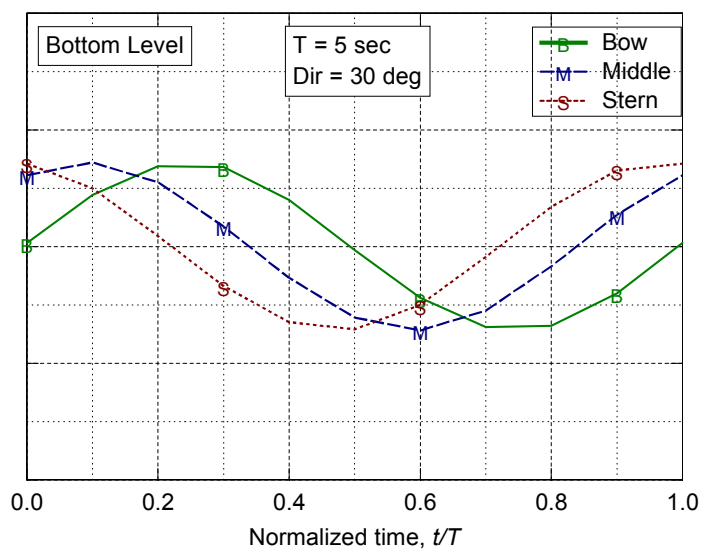

d) Bottom level, $T=5 \mathrm{sec}, \bar{\theta}=30 \mathrm{deg}$.

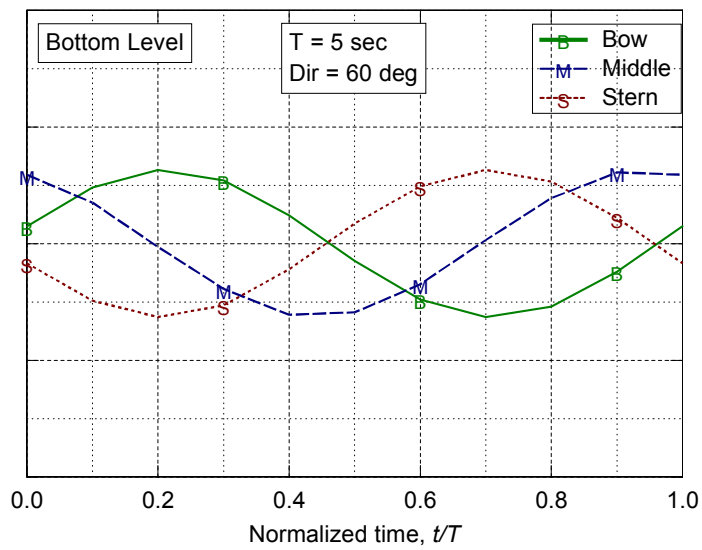

f) Bottom level, $T=5 \mathrm{sec}, \bar{\theta}=60 \mathrm{deg}$.

Figure 79. Analytical $\bar{p}_{l, A}$ for middle and bottom levels at $T=5 \mathrm{sec}$ (a) Middle, $\bar{\theta}=0 \mathrm{deg}$, (b) Bottom, $\bar{\theta}=0 \mathrm{deg}$, (c) Middle, $\bar{\theta}=30 \mathrm{deg}$, (d) Bottom, $\bar{\theta}=30 \mathrm{deg}$, (e) Middle, $\bar{\theta}=60 \mathrm{deg}$, (f) Bottom, $\bar{\theta}=60 \mathrm{deg}$. 
a)
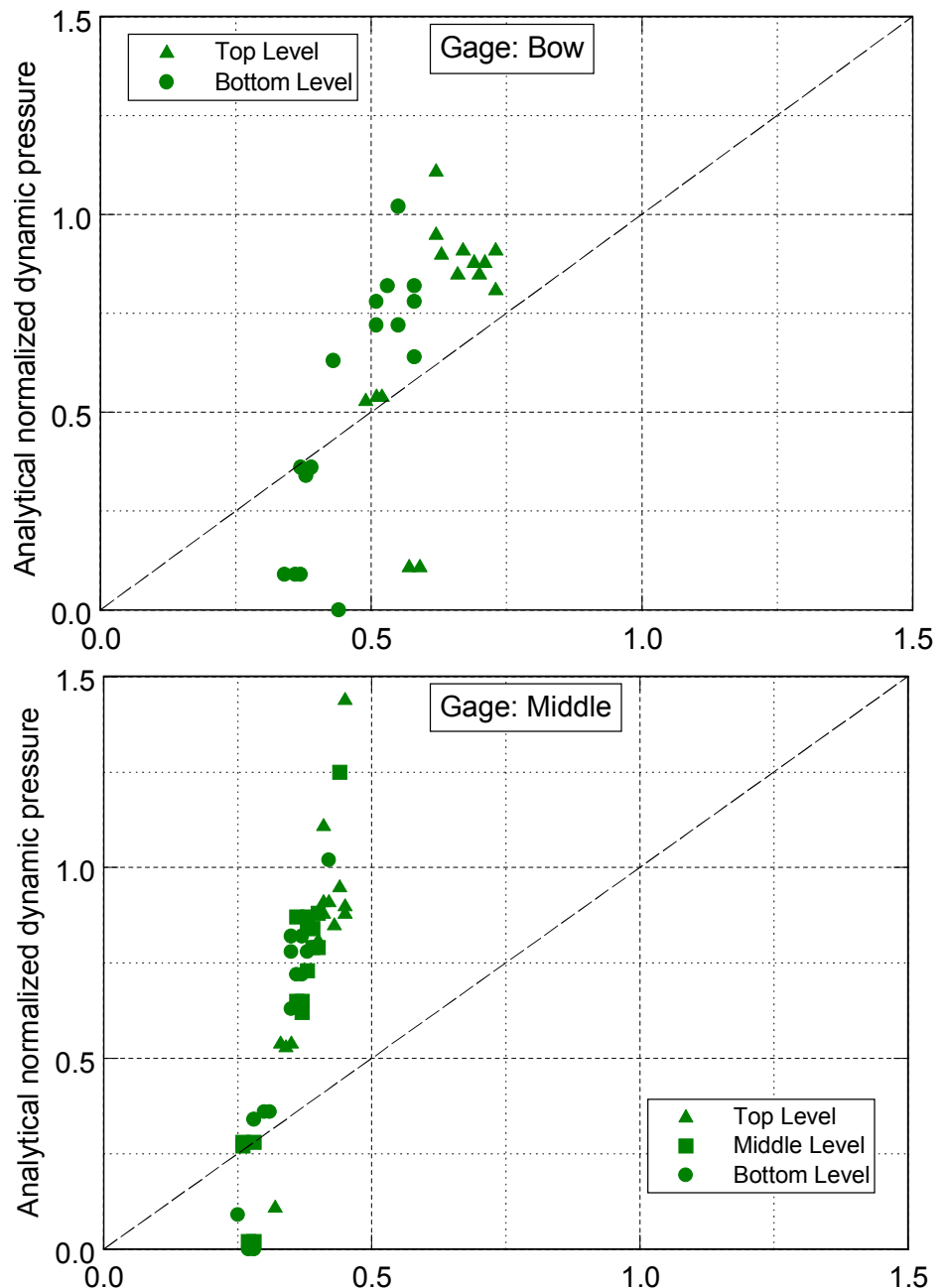

b)

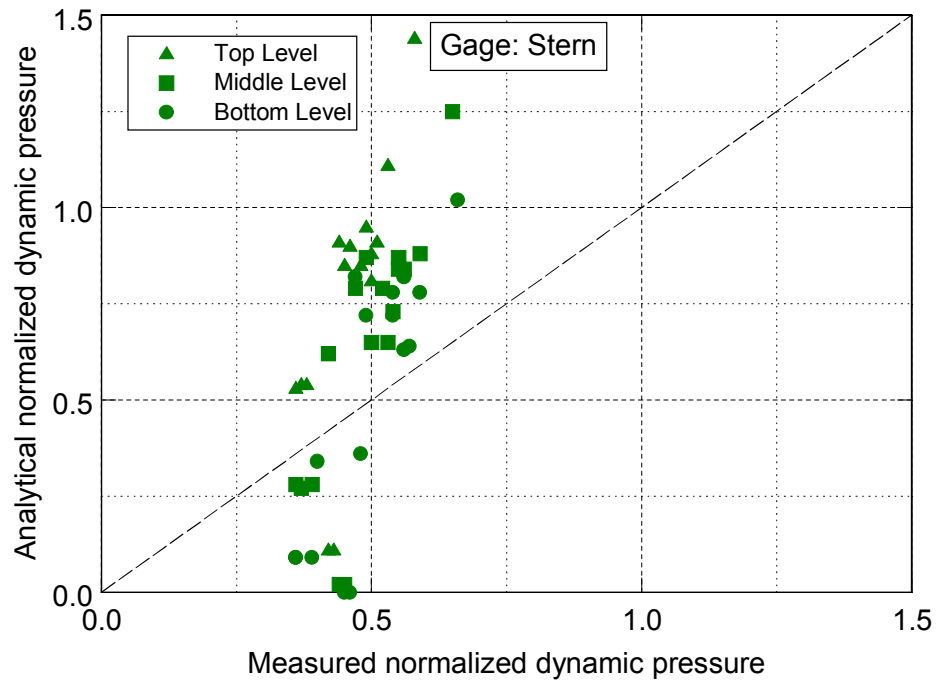

Figure 80. Comparison of analytical $\bar{p}_{1, A}$ vs. measured $\bar{p}_{l, S}$ for XM99 (a) bow, (b) middle, (c) stern. 
were assumed to remain constant and equal to the values used in the models. However, the pressure gages did show some variation over the course of the deployment due to tears in the membrane fabric between levels. For the top level, the variation in depth for all three gages was between approximately 2.70 and $3.08 \mathrm{~m}$ (i.e., $38 \mathrm{~cm}$ variation). For the middle level gages, this variation was between 4.42 and $4.72 \mathrm{~m}$ (i.e., $30 \mathrm{~cm}$ variation). The variation for the bottom level was the largest, varying between 6.77 and $7.86 \mathrm{~m}$ (i.e., $1.09 \mathrm{~m}$ variation). In the comparisons to WAMIT and the analytical models, an average depth of 2.90, 4.42, and $6.86 \mathrm{~m}$ was assumed for the three levels.

The measured significant dynamic pressures $p_{1, S}$ showed the same trends as the measured wave heights. On the top level, the bow gage usually had the largest magnitude. The stern gage usually had the largest pressure on the middle and bottom levels. The maximum dynamic pressures $p_{1, \text { Max }}$ were 1.75 times larger than the $p_{1, S}$, in line with expected Rayleigh statistics. The largest $p_{1, S}$ occurred on May 29 for the top level, bow gage and the largest $p_{1, \operatorname{Max}}$ for the middle level, stern gage. The range of average and maximum $p_{1, S}$ were 1.77 to $3.48 \mathrm{kPa}$ and 4.89 to $7.57 \mathrm{kPa}$, respectively. Similarly, average and maximum $p_{1, \text { Max }}$ were 3.11 to $5.94 \mathrm{kPa}$ and 9.24 to $15.03 \mathrm{kPa}$, respectively.

For comparisons with the analytical and WAMIT predictions, the measured $p_{1, S}$ were sorted into bins according to wave period and direction categories. These bins were selected such that the wave periods consisted of integer wave periods from 2 to $12 \mathrm{sec}$. Three wave direction bins were established with centers at 315, 285, and $255 \mathrm{deg}$, and a 30-deg range (15 deg on either side). Only data with wave directions within the port leg window were included. Of the 33 possible bins (i.e., 11 wave period bins and 3 direction bins), only 17 bins were actually identified within these wave period and direction constraints.

After sorting the data, the measured $p_{l, S}$ were divided by $\rho g H$ as before to obtain the normalized pressure $\bar{p}_{l, S}$. The magnitude of the WAMIT $\bar{p}_{1, W}$ and analytical $\bar{p}_{l, A}$ were determined as before for the FLab pressures by taking one half of the crest to trough distance closest to each pressure gage. The measured $\bar{p}_{1, S}$ pressures were somewhat smaller and less variable between levels than expected, especially for the stern gages. The analytical and WAMIT predictions, however, exhibited a more reasonable variation with vertical elevation of the pressure gages and had good agreement with each other. Possible explanations for these discrepancies are nonlinear fluid motions and structural interactions (i.e., tail wagging, mach 
stem waves, oscillations of the fabric and levels, reflections between the two legs, etc.) that might add to the measured dynamic pressure at certain locations and are not included in the analytical and numerical models. An investigation using the maximum measured pressures in lieu of the significant pressures might prove useful. Because of this lack of variation in the normalized measured pressures between vertical levels, empirical parameters were not investigated for the XM99 pressure gages. Additional work is ongoing to understand the reasons for these unexpected results. 


\section{CHAPTER VIII}

\section{SUMMARY AND CONCLUSIONS}

\section{INTEGRATED STUDY APPROACH}

The purpose of this research was to conduct an integrated study of analytical, numerical, laboratory, and field experiments to predict and evaluate the performance and structural response of the Army's new Rapidly Installed Breakwater System (RIBS). The RIBS is a floating breakwater with two legs in a "V" shape in plan view that provides a sheltered region from waves and currents. The hydrodynamic performance of a floating breakwater can be quantified by its ability to reduce waves in the sheltered region and this may be quantified by the wave transmission coefficient $K_{T}$. The structural response can be estimated using the waveinduced dynamic pressures along the structure. These pressures can be used to calculate the wave forces and moments.

The objective of RIBS is to reduce Sea State 3 (SS3) wave conditions to Sea State 2 for vessel loading/offloading during military amphibious operations. The SS3 conditions consist of waves with periods in the range 3 to $6 \mathrm{sec}$ and wave heights between 0.9 and $1.5 \mathrm{~m}$. The RIBS must also satisfy other constraints relative to transportation, assembly, deployment, and demobilization. A patent has been issued to U.S. Army Engineer Research and Development Center (ERDC) inventors (including the author) by the U. S. Patent Office.

Several RIBS geometries and configurations were tested in the directional spectral wave basin at the ERDC in 1997 to investigate the effects of draft, interior angle, and shape on performance. In these Fundamental Laboratory experiments (FLab), the RIBS was idealized as a thin, rigid, fixed, vertical barrier corresponding to a 293-m-long RIBS in 15-m-deep water. The physical model scale was 1:32. Because the model was fixed and rigid, no RIBS motions and interactions with the mooring lines were modeled. Four relative drafts of 0.50, 0.75, 1.00, and two interior angles of 45 and 90 deg were studied. Both wave transmission and dynamic pressures were measured and evaluated. More than 170 test cases were run and evaluated.

The ERDC deployed the first ocean-scaled version of the RIBS off Port Canaveral, FL, during May 20-30, 1999. This XM99 prototype was approximately $77 \mathrm{~m}$ long, $2.4 \mathrm{~m}$ wide, and $7.3 \mathrm{~m}$ deep. Water depth was $13.41 \mathrm{~m}$. The novel construction technique consisted of rigid 
steel truss frames and flexible membrane panels in a "Venetian Blind" arrangement. This arrangement allowed the XM99 to vertically expand during deployment and compress for shipping and storage. The interior was filled with sea water to increase its inertia and resistance to motion. This was one of the most heavily instrumented floating breakwater studies ever conducted. Incident directional and transmitted waves were measured with buoys and dynamic pressures were measured with twelve external gages on the port leg.

The analytical models used in this study are based on linear wave theory and idealize the RIBS as a fixed, rigid, vertical barrier. These models include contributions to the wave transmission and dynamic pressure from incident, reflected, and transmitted waves. They are designated the power transmission (PTT) and modified power transmission (MPTT) models. Because of some shortcomings in their formulation, they are compared to the measured data only to assess their range of applicability to the RIBS.

The linear, radiation/diffraction program WAMIT (Wave Analysis MIT) was the numerical model used in this study. WAMIT solves a boundary value problem by calculating the velocity potential that satisfies the Laplace equation and four linearized boundary conditions for the free surface, bottom, body surface, and the radiation condition at infinity. In the solution for the diffraction-only problem for the laboratory model, WAMIT provides the wave transmission coefficients and dynamic pressures on a fixed, rigid structure. For the diffraction-plus-radiation potential problem of the XM99 field trial, WAMIT calculates the wave transmission and pressures on a rigid, floating model.

\section{WAVE TRANSMISSION RESULTS}

The performance of a floating breakwater is quantified by the wave transmission coefficient $K_{T}$. This coefficient is defined as the ratio of the transmitted wave height $H_{T}$ to the incident wave height $H_{I}$. Both are calculated over the entire record duration and include energy contributions from all frequencies. For the FLab experiments, $K_{T}$ was defined this way because its scaled length was prototype scale. The XM99, however, was smaller in length and shallower in draft than a full-scale prototype. Thus, it was not expected to perform as well in full-scale SS3 conditions and several new analysis methods were proposed and investigated to evaluate the XM99 performance in terms of waves that corresponded to its ocean-scale size. A weighted 
average of frequency-dependent wave transmission coefficients was calculated using two different weighting functions. One was a simple average $K_{T, S A v e}$ from a lower cutoff frequency corresponding to the SS3 frequency and the second method involved an energy-based weight $K_{T, W g t}$ determined from the amount of energy in each frequency band.

For the FLab laboratory experiments, measured $K_{T}$ was calculated and presented for the five transmitted gage locations, six configurations, six operational and two survival wave cases. The $K_{T}$ decreased as wave period $T$ decreased and relative draft $d / h$ increased, and was fairly uniform with distance in the lee of the RIBS. From these results it was determined that the RIBS should be positioned so that the relative draft is greater than 50 percent of the water depth and an interior angle greater than $45 \mathrm{deg}$ should be used. Head seas will probably provide a conservative design as the RIBS tended to perform as well or better with a small angle in the mean wave direction $\bar{\theta}$.

The WAMIT-predicted $K_{T}$ was higher than the measured values and showed more variability from gage to gage position. The correlation for the average over all five gages for each wave case and geometric configuration was excellent. Since the average WAMIT $K_{T}$ was 0.13 higher than the corresponding measured value, it should provide some conservatism in the RIBS design.

The measured $K_{T}$ was compared to the analytical PTT and MPTT predictions using Wiegel's transmission factor $T_{F}$. The agreement was surprisingly good for the FLab data. The PTT model predictions were a closer fit to the measured data than the MPTT model. Although the $T_{F}$ parameter is not a function of mean wave direction $\bar{\theta}$, it was a reasonable predictor of the RIBS performance for the range of FLab conditions tested.

Several empirical relationships were investigated including a modified relative width $B_{x} / L_{x}$, modified wave steepness $H / L_{x}$, and a new composite parameter $D_{b} d / B_{y} L_{x}$. The last two gave the best correlation, but were not as good as Wiegel's transmission factor $T_{F}$.

The measured $K_{T}$ during the 10-day deployment of the XM99 field trials was calculated using the traditional definition $K_{T}$, a simple average $K_{T, S A v e}$, and a weighted $K_{T, W g t}$. The measured $K_{T}$ were less than the $K_{T}=0.5$ desired level of efficiency for several hours every day, with the best performances on May 22, 23, 25, 26, and 28. Incident wave heights up to 0.83 were efficiently reduced. Threshold $K_{T}$ values were obtained for wave directions almost broadside to the starboard leg and $10 \mathrm{deg}$ to the port side of the centerline, and the full range of 
observed directional spread from 30 to $60 \mathrm{deg}$. The XM99 performed very well considering that it experienced some minor tearing in the curtains after the first two days, resulting in a reduced barrier to wave transmission.

The WAMIT-predicted $K_{T}$ for a wave period of $3 \mathrm{sec}$ and wave direction parallel to the XM99 centerline (i.e., $315 \mathrm{deg}$ ) showed very good agreement with measured values that were an average of all the waves with a wave period of $3 \pm 0.5 \mathrm{sec}$ and wave direction of $315 \pm 15 \mathrm{deg}$.

The PTT and MPTT analytical models were compared with the measured $K_{T}$. The agreement was not as good for the XM99 as it had been for the FLab data. The PTT and MPTT models under predict $K_{T}$ for small $T_{F}$ values, but improve as $T_{F}$ increases. Only the PTT model would be useful for predicting $K_{T}$ within the design criteria of SS3 for the RIBS, however. A possible explanation is that the XM99 data set includes more bimodal and multimodal waves and a wider range of wave directions and larger directional spreading than the FLab data.

Again, several empirical parameters were investigated relative to measured $K_{T}$. Of the three parameters, wave steepness was the best, but not significantly better than the others. In general, the existing analytical and empirical models do not predict wave transmission for the XM99 very well.

\section{DYNAMIC PRESSURE RESULTS}

The Flab had three pressure gages installed at bow, middle, and stern locations on the starboard RIBS leg. These gages were located on the same vertical level at $z=-8.5 \mathrm{~cm}$ from the surface for all relative drafts $d / h$. The significant dynamic pressure $p_{l, S}$ increased as $d / h$ increased, due to the increase in reflected energy on the external side of the RIBS leg. In general, the $p_{l, S}$ were fairly constant for all three pressure gages for each wave case. There was a tendency to increase at the stern gage location, especially for the $\bar{\theta}=20 \mathrm{deg}$ cases. The largest variations occurred when the RIBS was resting on the bottom. For this case, the $p_{l, S}$ was the largest because the wave could no longer go underneath the RIBS and the reflected energy was the greatest. Similar to the wave transmission coefficients, there was not much difference between the spectral and regular cases, although the regular wave values were slightly larger. The ratio of the SS5 to SS3 dynamic pressures ranged from a low of 6.4 to a high of 7.5, while the wave height ratio was only about 2.4. The average for all cases was 6.9. 
The measured $p_{1, S}$ were calculated as an equivalent wave height or double amplitude using standard zero downcrossing and zero moment estimation techniques. For comparison to the analytical and WAMIT models, the measured $p_{1, S}$ were divided by $\rho g H$ ( $H$ is the wave height) to obtain a normalized or nondimensional pressure $\bar{p}_{l, S}$. The normalized WAMIT $\bar{p}_{l, W}$ and analytical $\bar{p}_{l, A}$ dynamic pressures were divided by $\rho g A$.

For comparisons to the measured $\bar{p}_{1, S}$, the magnitude of the WAMIT $\bar{p}_{1, W}$ was determined by taking one half of the crest to trough distance closest to the gage. In general, the $\bar{p}_{1, W}$ increased as $d / h$ increased and was fairly constant along the RIBS leg. As $d / h$ increased, the $\bar{p}_{1, W}$ tended to increase slightly toward the stern. The agreement between measured $\bar{p}_{I, S}$ and WAMIT $\bar{p}_{1, W}$ was very good with the WAMIT predictions being slightly smaller than the measured $\bar{p}_{l, S}$. The average measured $\bar{p}_{l, S}$ for all $d / h$ and $\theta_{r}$ combinations (i.e., 72 values) was 0.28 higher than the corresponding WAMIT $\bar{p}_{1, W}$.

The analytical model for dynamic pressure is based on linear wave theory and includes both incident and reflected waves. The predicted pressure magnitude is a function of the wave period $T$, wavelength $L$, water depth $h, y$-axis wavenumber $k_{y}$ ( $x=0$ on the RIBS leg, so no $k_{x}$ term), pressure response factor $K_{P}$, reflection coefficient $K_{R}$, and wave direction $\bar{\theta}$. The $K_{R}$ is based on an energy balance and is defined as $\sqrt{ }\left(1-K_{T}^{2}\right)$. The measured $K_{T}$ were used in the calculation of $K_{R}$. For each location, the $\bar{p}_{l, A}$ magnitude is constant, but the phase changes over a complete cycle. The $\bar{p}_{l, A}$ magnitude increased slightly as $d / h$ increased.

The agreement between the analytical $\bar{p}_{l, A}$ and measured dynamic pressure $\bar{p}_{l, S}$ was excellent. The analytical $\bar{p}_{l, A}$ were slightly smaller than the corresponding measured values.

Several empirical parameters were again investigated. Again, the best predictor of the empirical parameters was the Wiegel's $T_{F}$ parameter.

For the XM99 field trials, wave dynamic pressures were measured at twelve external locations on the port leg of the XM99. Gages were located at three stations on each of four vertical levels. The three stations were bow, middle, and stern. The four levels were the surface, top, middle, and bottom.

An important consideration in comparing measured dynamic pressures with the analytical and WAMIT predictions is the actual depth of the gages. For comparison purposes, the depths were assumed to remain constant and equal to the values used in the models. However, the pressure gages did show some variation over the course of the deployment due to tears in the 
membrane fabric between levels. In the comparisons to WAMIT and the analytical models, an average depth of 2.90, 4.42, and $6.86 \mathrm{~m}$ was assumed for the three levels. A more exacting comparison is planned in the future to look at the individual variations in the pressure gages.

The measured significant dynamic pressures $p_{1, S}$ showed the same trends as the measured wave heights. On the top level, the bow gage usually had the largest magnitude. The stern gage usually had the largest pressure on the middle and bottom levels. The maximum dynamic pressures $p_{l, M a x}$ were 1.75 times larger than the $p_{l, S}$, in line with expected Rayleigh statistics.

For comparisons with the analytical and WAMIT predictions, the measured $p_{l, S}$ were sorted into bins according to wave period and direction categories. Only data with wave directions within the port leg window were included. Of the 33 possible bins (i.e., 11 wave period bins and 3 direction bins), only 17 bins were actually identified within these wave period and direction constraints.

After sorting the data, the measured $p_{1, S}$ were divided by $\rho g H$ as before to obtain the normalized pressure $\bar{p}_{l, S}$. The magnitude of the WAMIT $\bar{p}_{1, W}$ and analytical $\bar{p}_{l, A}$ were determined as before for the FLab pressures by taking one half of the crest to trough distance closest to each pressure gage. The measured $\bar{p}_{l, S}$ pressures were somewhat smaller and less variable between levels than expected, especially for the stern gages. The analytical and WAMIT predictions, however, exhibited a more reasonable variation with vertical elevation of the pressure gages and had good agreement with each other. Possible explanations for these discrepancies are nonlinear fluid motions and structural interactions (i.e., tail wagging, mach stem waves, oscillations of the fabric and levels, reflections between the two legs, etc.) that might add to the measured dynamic pressure at certain locations and are not included in the analytical and numerical models. An investigation using the maximum measured pressures in lieu of the significant pressures might prove useful. Because of this lack of variation in the normalized measured pressures between vertical levels, empirical parameters were not investigated for the XM99 pressure gages. Additional work is ongoing to understand the reasons for these unexpected results. 


\section{AREAS FOR FUTURE RESEARCH}

Promising data were obtained from both the FLab laboratory and XM99 field data sets. Although most of the data has been analyzed, much remains to be done with this data. Because these were relatively large data sets, it was impossible to investigate every potential relationship within the scope of this dissertation. Additional research should be done with these data sets in comparison to work of other researchers, especially in the area of the reflection coefficient and its relationship to the transmission coefficient. These invaluable data sets could shed more light on the performance characteristics of RIBS.

For the Fundamental Laboratory Experiments, much of the data for the 90 deg interior angle configuration was not analyzed in detail. This data should be further analyzed to investigate the performance characteristics and structural responses to a RIBS design with wider interior angles.

Similarly, for the XM99 field trials, most of the interior pressure gages were not analyzed and discussed. These gages provide useful information for estimating net pressures that determine wave force loadings on the RIBS. This data could prove to be very useful.

The measured normalized pressures were somewhat smaller and less variable between levels than expected, especially for the stern gages. Possible explanations for these discrepancies are nonlinear fluid motions and structural interactions (i.e., tail wagging, mach stem waves, oscillations of the fabric and levels, reflections between the two legs, etc.) that might add to the measured dynamic pressure at certain locations and are not included in the analytical and numerical models. Additional work is ongoing to understand the reasons for these unexpected results.

Two simple, semi-empirical analytical models were used to predict wave transmission for the RIBS. They are based on a rigid, thin, vertical barrier in the presence of normally-incident waves. Although they were fair predictors of wave transmission for the fundamental laboratory experiments, they did not do a very good job for the XM99 field trials. The main shortcomings are that they do not match dynamic pressure at the boundaries between incident and transmitted waves. Additional research is ongoing to improve these models by including evanescent waves and proper matching of hydrodynamic parameters at the boundaries. 
The WAMIT model was used to model a very limited RIBS structure. This numerical model has many more capabilities including hydroelastic response, modeling of structural joints, and mooring line interactions. An interesting feature of WAMIT is its ability to perform hydroelastic analysis by incorporating structural deformations and flexibility into the analysis. Structural deformations can be described by using a finite set of mode shapes that are added to the standard six degrees of freedom (DOF) of a floating rigid body. The normal velocities on the body are specified using orthogonal shape functions corresponding to these generalized modes. Vertical and lateral bending deformations are included by idealizing the RIBS legs as simple beams with appropriate boundary conditions. Flexibility in the form of multiple bodies connected by hinges or joints can be evaluated using generalized modes. Joints are idealized as separate degrees of freedom between modules and added as another generalized mode of body motion. Finally, bodies which are not freely floating in waves can be analyzed. This situation arises in those cases in which the bodies are constrained by arbitrary linear external forces (e.g., by an elastic mooring cable).

The WAMIT model uses the constant panel method (CPM), while higher order boundary element methods (HOBEM) can also be used to solve the velocity potentials. The HOBEM has some advantages relative to CPM in that they (1) can use any higher order element to model the body surface (usually quadratic or cubic isoparametric elements), (2) can use the same interpolating or shape function for velocity potential, its derivatives, and elements, (3) do not have any discontinuities and "leaks"due to curved elements, (4) provide higher accuracy because the source strength is exact, and (5) require less computer time. Future research might investigate the additional advantages of using an "HOBEM" type numerical model on the RIBS structure. 


\section{REFERENCES}

Abul-Azm, A.G. (1993). "Wave diffraction through submerged breakwaters," J. Waterway,

Port, Coastal, and Ocean Engrg., ASCE, 119(6), 587-605.

AeroHydro, Inc. (1998). “MultiSurf 3D Surface Design Software,” Users Manual, Southwest Harbor, ME.

American Institute of Steel Construction, Inc. (1990). Manual of Steel Construction. New York.

Biesel, F. (1954). "Wave machines." Proc. 1st Conf. Ships and Waves, Hoboken, NJ, 288-304.

Borgman, L.E. (1984). "Directional spectrum estimation for the Sxy gauges," Report to US Army Waterways Experiment Station, Vicksburg, MS, 1-104.

Borgman, L.E. (1990). "Irregular ocean waves: kinematics and forces," The Sea, Vol 9, Part A, ed. Bernard LeMehaute, John Wiley and Sons, New York, 121-168.

Borgman, L.E. and Earle, M. (1999). "Harvest experiment and intercomparison: MATLAB programs and instructions, technical papers, and supporting information," Version 1.1, Report to US Army Waterways Experiment Station, Vicksburg, MS.

Bouws, E., Gunther, H., Rosenthal, W., and Vincent, C. (1985). "Similarity of the wind wave spectrum in finite depth water." J. Geophys. Res., 90(C1), 975-986.

Briggs, M.J., Borgman, L.E., and Outlaw, D.G. (1987). "Generation and analysis of directional spectral waves in a laboratory basin." Proc. Offshore Tech. Conf., Houston, TX, 495-502.

Briggs, M.J., Fowler, J.E., Bratos, S.M., Resio, D.T., and Pollock, C.B. (1996). "RIBS: A potential solution for logistics-over-the-shore," $20^{\text {th }}$ Army Science Conference, Norfolk, VA, 827-831.

Briggs, M.J., Demirbilek, Z., and Matheu, E.E. (1998). "An integrated study of wave phenomena affecting floating breakwater design for JLOTS," $21^{\text {st }}$ Army Science Conference, Norfolk, VA, 575-580.

Broderick, L.L. and Leonard, J.W. (1995). "Nonlinear response of membranes to ocean waves using boundary and finite elements," Ocean Engng., 22(7), 731-745. 
Das, P., Dolai, D.P., and Mandal, B.N. (1997). "Oblique wave diffraction by parallel thin vertical barriers with gaps," J. Waterway, Port, Coastal, and Ocean Engrg., ASCE, 163-171.

Fathi, D.E., Lee, C.-H. and Newman, J.N. (1994). "Computation of wave induced motions on a flexible container," Hydroelasticity in Marine Technology, Rotterdam, The Netherlands, 301308.

Fowler, J.E., Resio, D.T., Briggs, M.J., and Pollock, C.B. (1996). "Potential uses for the rapidly installed breakwater system," International Conference on Coastal Engrg., Chapter 127, Orlando, FL, 1631-1639.

Fowler, J.E., Pollock, C.B., Resio, D.T., and Turk, G.F. (1997). “Development of a rapidly installed breakwater (RIB) system," CERCular, U.S. Army Engineer Waterways Experiment Station, Vicksburg, MS.

Giles, M.L. and Eckert J.W. (1979). "Determination of mooring load and transmitted wave height for a floating tire breakwater," Coastal Engineering Technical Report AID No. 79-4, Coastal Engineering Research Center, Fort Belvoir, VA.

Goda, Y. (1985). Random Seas and Design of Maritime Structures. Univ. Tokyo Press, Tokyo, Japan.

Goda, Y. and Suzuki, Y. (1975). "Computation of refraction and diffraction of sea waves with Mitsuyasu's directional spectrum." Tech. Note No. 230, Port Harbour Res. Inst., Yokosuka, Japan.

Hales, Lyndell Z. (1981). "Floating breakwaters: state-of-the-art literature review," Technical Report No. 81-1, Coastal Engineering Research Center, Fort Belvoir, VA.

Harms, V.W., Westerink, J.J., Sorensen, R.M., and McTamany, J.E. (1982). "Wave transmission and mooring force characteristics of pipe-tire floating breakwaters," Technical Paper No. 82-4, Coastal Engineering Research Center, Fort Belvoir, VA.

Helm-Petersen, Jacob. (1998). "Estimation of wave disturbance in harbors," Ph.D. dissertation, Hydraulics \& Coastal Engineering Laboratory, Aalborg University, Aalborg, Denmark.

IAHR. (1986). "List of sea state parameters," Supplement to Bulletin No. 52, IAHR Working Group on Wave Generation and Analysis, 1-24.

IAHR. (1997). "IAHR list of sea state parameters," IAHR Seminar on Interaction of Ocean Structures with Multidirectional Waves, San Francisco, 15-24. 
Jones, J.B. (1971). “Transportable breakwaters - a survey of concepts," NTIS Technical Report $A D-887$ 841, Naval Facilities Engineering Command, Port Hueneme, CA.

Kim, M.H. and Kee, S.T. (1996). "Flexible-membrane wave barrier I: Analytic and numerical solutions," J. Waterway, Port, Coastal, and Ocean Engrg., ASCE, 122(1), 46-53.

Kitaigordskii, S.A., Krasitskii, V.P., and Zaslavskii, M.M. (1975). "On Phillips theory of equilibrium range in the spectra of wind generated gravity waves," J. Physical Oceanography, $5(3), 410-420$.

Kobayashi, N. (1998). "RIB wave field model development," Contract Report DACA39-98-M0279, Center for Applied Coastal Research, University of Delaware, Newark, DE.

Kriebel, D.L. and Bollman, C.A. (1996). "Wave transmission past vertical wave barriers," $25^{\text {th }}$ International Conference on Coastal Engrg., Orlando, FL, Chapter 191, 2470-2483.

Krouse, J. (1998). "Virtual ocean computer simulation," Sea Technology, 39(11), 64-65.

Lee, C.-H. (1995a). “WAMIT Theory Manual,” Report 95-2, MIT, Cambridge, MA.

Lee, C.-H. (1995b). “WAMIT User's Manual,” Version 5.3, MIT, Cambridge, MA.

Lee, C.-H. (1997). “Wave interaction with huge floating structure,” BOSS 97, Delft, The Netherlands, 2, 253-268.

Lee, S.-W. and Webster, W.C. (1994). "A preliminary to the design of a hydroelastic model of a floating airport." Hydroelasticity in Marine Technology, Rotterdam, The Netherlands, 351-362.

Liu, P.L.-F. and Abbaspour, M. (1982). "Wave scattering by a rigid thin barrier," J. Waterway, Port, Coastal, and Ocean Division, ASCE, 108(WW4), 479-491.

Lo, E.Y.M. (1998). "Flexible dual membrane wave barrier," J. Waterway, Port, Coastal, and Ocean Engrg., ASCE, 124(5), 264-271.

Lo, E.Y.M. (2000). "Performance of a flexible membrane wave barrier of a finite vertical extent," Coastal Engineering Journal, 42(2), 237-251.

Losada, I.J., Losada, M.A., and Roldan, A.J. (1992). "Propagation of oblique incident waves past rigid vertical thin barriers," Applied Ocean Research, 14, 191-199. 
Mandal, B.N. and Dolai, D.P. (1994). "Oblique water wave diffraction by thin vertical barriers in water of uniform finite depth," Applied Ocean Research, 195-203.

Mani, J.S. and Jayakumar, S. (1995). "Wave transmission by suspended pipe breakwater," $J$. Waterway, Port, Coastal, and Ocean Engrg., ASCE, 121(6), 335-338.

Mei, C.C. (1983). Applied Dynamics of Ocean Surface Waves, Wiley-Interscience, New York.

Miles, M.D. (1997). “GEDAP user's guide for windows NT," Technical Report HYD-TR-021, Canadian Hydraulics Center, Ottawa, Ontario, Canada, 1-96.

Murali, K. and Mani, J.S. (1997). "Performance of cage floating breakwater," J. Waterway, Port, Coastal, and Ocean Engrg., ASCE, 123(4), 172-179.

Nakamura, T. (1983). "Numerical analysis on wave boundary-value problem around a thinwalled body of arbitrary section," $30^{\text {th }}$ Japanese Conference on Coastal Engrg., JSCE, Japan, 410-414.

Nakamura, T. and Morita, S. (1985). "Wave attenuation method by the use of wave resonance between double-row of curtain walls," $32^{\text {nd }}$ Japanese Conference on Coastal Engrg., JSCE, Japan, 525-529.

Newman, J.N. (1994). "Wave effects on deformable bodies," Applied Ocean Research, 16, 4759.

Newman, J.N. (1997). "Recent results from numerical model tests," OTRC '97 International Conference, Nonlinear Design Aspects of Physical Model Tests, College Station, TX, 1-27.

Newman, J.N. and Lee, C.-H. (1992). "Sensitivity of wave loads to the discretization of bodies," BOSS '92, BPP Technicall Services, Ltd., London, United Kingdom, 50-66.

Ohyama, T., Tanaka, M., Kiyokawa, T., Uda, T., and Murai, Y. (1989). "Transmission and reflection characteristics of waves over a submerged flexible mound," Coastal Engineering in Japan, 32(1), 53-68.

Oltman-Shay, J.M. (1987). "Linear arrays: wind wave directional measurement systems," Seminar, US Army Waterways Experiment Station, Vicksburg, MS.

Peratrovich, Nottingham, and Drage, Inc. (1992). "Wave barrier development," Final Report, Alaska Science and Technology Foundation, Anchorage, AK. 
Pinkster, J.A. (1984). "Numerical modeling of directional seas," Symposium on Description and Modeling of Directional Seas, Technical University of Denmark, Copenhagen, C11-C19.

Porter, R. and Evans, D.V. (1995). "Complimentary approximations to wave scattering by vertical barriers," J. Fluid Mech., 294, 155-180.

Resio, D.T., Fowler, J.E., and Briggs, M.J. (1995). "RIBS: A Potential solution to the lots seastate problem," Army Operations Research Symposium (AORS), Ft. Lee, VA.

Resio, D.T., Briggs, M.J., Fowler, J.E., and Markle, D. (1997). "Floating V-shaped breakwater," U.S. Patent No. 5,702,203.

Sand, S. E. (1979). "Three-dimensional structure of ocean waves." Series Paper No. 24, Inst. of Hydrodynamics and Hydraulic Engrg., Tech. Univ. of Denmark, Copenhagen, Denmark.

Sawaragi, T. (1995), Coastal Engineering - Waves, Beaches, Wave-Structure Interactions, Elsevier, New York.

Tanaka, M., Ohyama, T., Kiyokawa, T., Uda, T., and Omata, A. (1990). "Wave control by flexible underwater mound," Offshore Technology Conference, Paper 6405, Houston, TX, 551-558.

Tanaka, M., Ohyama, T., Kiyokawa, T., Uda, T., and Omata, A. (1992). "Characteristics of wave dissipation by flexible submerged breakwater and utility of the device," International Conference on Coastal Engrg., Chapter 123, Venice, Italy, 1613-1624.

Ursell, F. (1947). "The effect of a fixed barrier on surface waves in deep water," Proc. Camb. Phil. Soc., 43, 374-82.

Wiegel, R.L. (1960). "Transmission of waves past a rigid vertical thin barrier," J. Waterway, Port, Coastal, and Ocean Division, ASCE, 86(WW1), Mar 1-12.

Williams, A.N. and Abul-Azm, A.G. (1996). "Hydrodynamic analysis of dual pontoon floating breakwater," $6^{\text {th }}$ ISOPE, Los Angeles, CA, 560-565.

Yamamoto, T. (1981). "Moored floating breakwater response to regular and irregular waves," Applied Ocean Research, 3(1), 27-36.

Zhao, R. and Triantafyllou, M. (1994). "Hydroelastic analyses of a long flexible tube in waves," Hydroelasticity in Marine Technology, Rotterdam, The Netherlands, 287-300. 
APPENDIX A

NOTATION 


\begin{tabular}{|c|c|}
\hline$a$ & parameter in TMA spectrum \\
\hline$a_{j}(f)$ & real Fourier coefficient of the spreading function \\
\hline$a_{m}$ & Fourier coefficients of the even terms \\
\hline$a_{0}$ & intercept in quadratic least squares fit equation \\
\hline$a_{1}$ & slope in quadratic least squares fit equation \\
\hline$a_{1}(f)$ & first harmonic real Fourier coefficient \\
\hline$a_{2}$ & second order coefficient in quadratic least squares fit equation \\
\hline$A$ & wave amplitude \\
\hline$A(f)$ & deterministic spectral amplitude function \\
\hline$A_{G}$ & area under a Gaussian curve \\
\hline$b$ & parameter in TMA spectrum \\
\hline$b_{1}(f)$ & first harmonic imaginary Fourier coefficient \\
\hline$b_{j}(f)$ & imaginary Fourier coefficient of spreading function \\
\hline$b_{m}$ & imaginary Fourier coefficient of spreading function \\
\hline$B$ & DSWG paddle width \\
\hline$B$ & XM99 beam or width \\
\hline$B_{e}$ & resolution bandwidth, $\mathrm{Hz}$ \\
\hline$B_{x}$ & width of the breakwater in the direction of wave travel \\
\hline$B_{y}$ & projected RIBS length in the y-direction (transverse to the center line) \\
\hline$B_{x} / d$ & relative breakwater width \\
\hline$B_{x} / L_{x}$ & modified relative breakwater width \\
\hline$B_{y} / d$ & relative breakwater length \\
\hline C & wave celerity for shallow water \\
\hline$C_{g}$ & wave group velocity \\
\hline$C_{i j}$ & co-spectra, the real part of the cross-spectrum \\
\hline$d$ & RIBS draft or depth of submergence \\
\hline$d / h$ & relative depth or draft \\
\hline$D(f, \theta)$ & directional spreading function \\
\hline$D_{b}$ & distance from bow to transmitted gage measured parallel to center line \\
\hline$D_{b} / L_{r}$ & normalized gage distance from bow of RIBS \\
\hline$e$ & 2.71828 \\
\hline
\end{tabular}




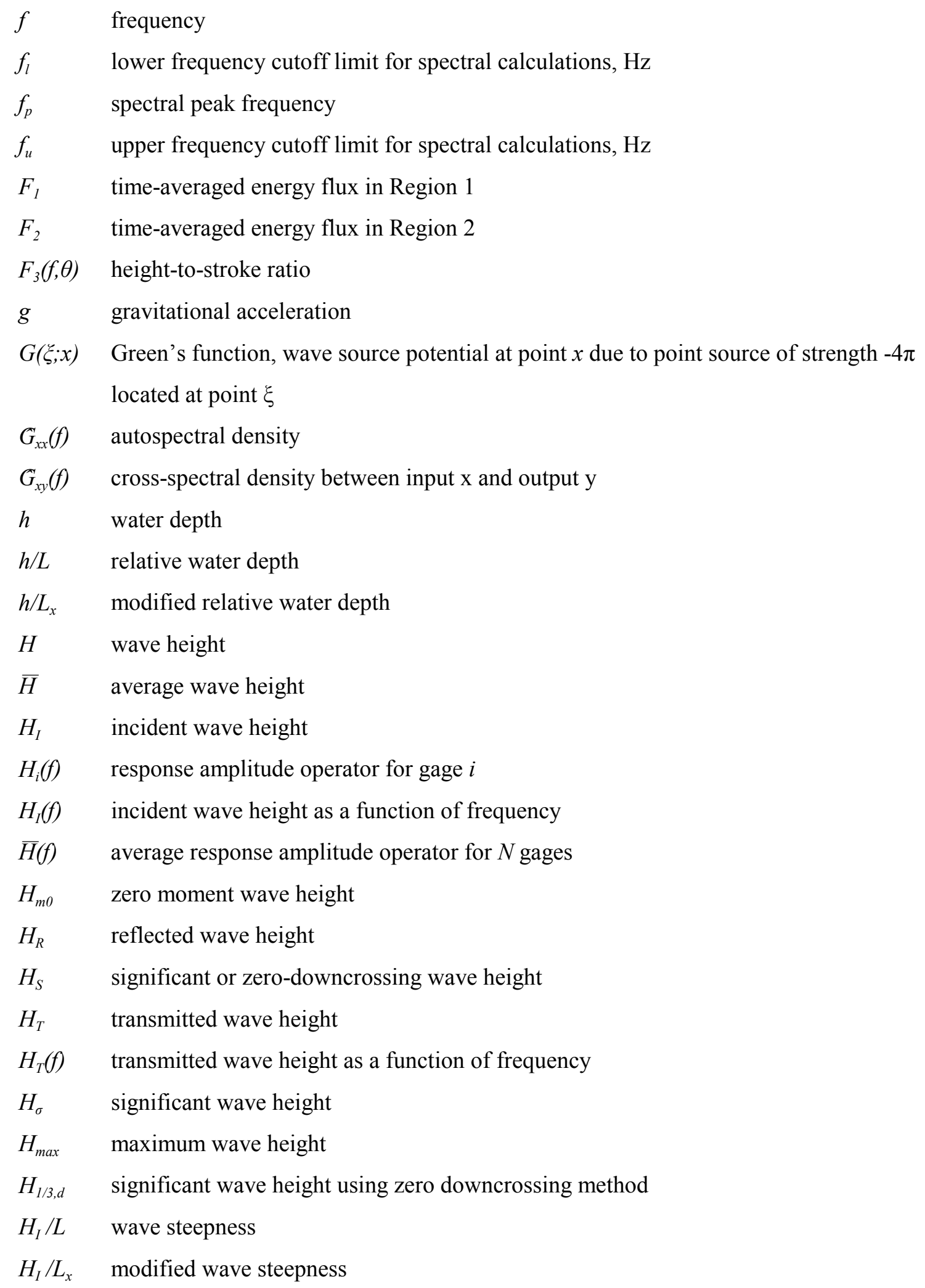


$H / S \quad$ height-to-stroke ratio

$i \quad \sqrt{ }-1$

I incident

$I_{j j} \quad$ rotational moments of inertia for RIBS

$j \quad$ summation index for direction

$J \quad$ number of direction bands

$J$ number of harmonics chosen to represent the Fourier series (typically 6 to 20)

$k \quad$ wavenumber

$k \quad$ spring constant or mooring line stiffness

$\mathrm{ka} \quad$ wave steepness

$k_{x} \quad$ component of the wavenumber in the $\mathrm{x}$-direction

$k_{x, 1} \quad$ wavenumber in the $x$-direction in Region 1

$k_{x, 2} \quad$ wavenumber in the $x$-direction in Region 2

$k_{y} \quad$ component of the wavenumber in the y-direction

$k_{y, 1} \quad$ wavenumber in the $y$-direction in Region 1

$k_{y, 2} \quad$ wavenumber in the $y$-direction in Region 2

$K_{p} \quad$ pressure response factor

$K_{R} \quad$ reflection coefficient

$K_{T} \quad$ wave transmission coefficient

$K_{T}(f) \quad$ frequency-dependent transmission coefficient

$K_{T, S A v e} \quad$ average wave transmission coefficient between lower and upper cutoff frequencies

$K_{T, W g t} \quad$ weighted wave transmission coefficient

$L \quad$ wavelength

$L \quad$ number of harmonics in a truncated Fourier series expansion used to calculate an initial directional spreading function

$L_{p} \quad$ wavelength corresponding to $f_{p}$

$L_{r} \quad$ scale ratio between model and prototype

$L_{r} \quad$ RIBS leg length

$L_{x} \quad$ component of wavelength in the $x$-direction

$L_{y} \quad$ component of wavelength in the $y$-direction

$L / B_{x} \quad$ relative wavelength 


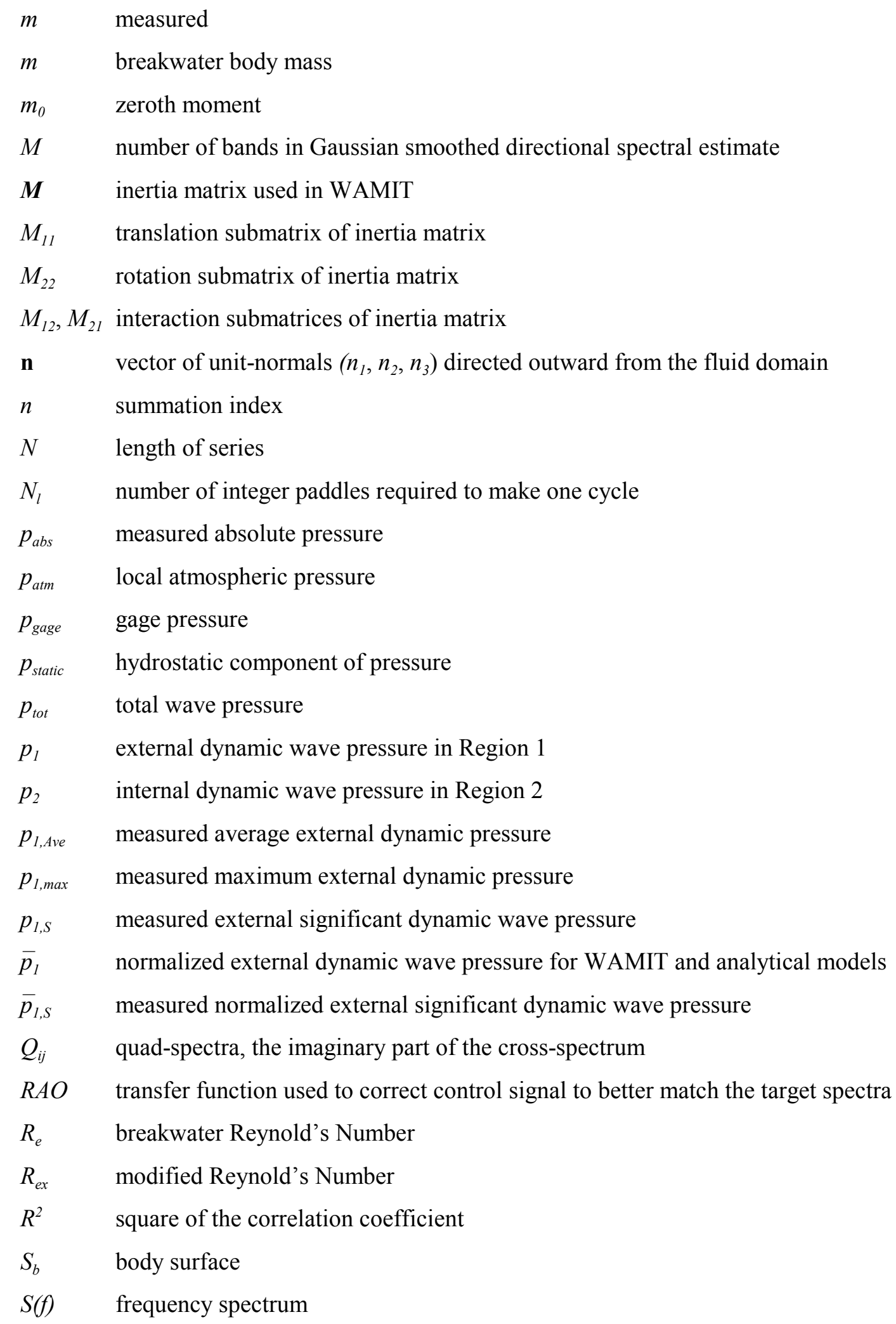


$\bar{S}(f) \quad$ average frequency spectrum for $N$ gages

$\bar{S}(f) \quad$ average spectral ordinate at frequency $f$

$S(f, \theta) \quad$ directional wave spectrum

$S_{c}(y, t) \quad$ wavemaker stroke control signal

$S_{i i}(f) \quad$ autospectral density for gage $i$

$S_{I}(f) \quad$ incident wave spectral estimate at frequency $f$

$S_{j}(f) \quad$ JONSWAP (Joint North Sea Wave Project) spectrum

$S_{m} \quad$ Gaussian smoothed line spectra

$S_{m-j} \quad$ raw autospectral or cross-spectral estimate at frequency $(m-j) \Delta f$

$S_{T}(f) \quad$ transmitted wave spectrum

$S_{\max } \quad$ Goda directional spreading parameter

$S_{T}(f) \quad$ transmitted wave spectral estimate at frequency $f$

$S_{\text {TMA }}(f) \quad$ TMA frequency spectra

SS3 Sea state 3

SS5 Sea state 5

$t$ time

$T \quad$ wave period

$\bar{T} \quad$ average wave period

$T_{F} \quad$ Wiegel's transmission factor

$T_{p} \quad$ spectral wave peak period

$T_{r} \quad$ time series duration or record length, sec

$T_{1 / 3, d} \quad$ significant wave period using zero downcrossing method

$u_{l} \quad$ horizontal velocity in Region 1

$u_{2} \quad$ horizontal velocity in Region 2

$U(f) \quad$ real Fourier coefficients in directional wave spectrum

$V(f) \quad$ imaginary Fourier coefficients in directional wave spectrum

$w_{j} \quad$ weights for directional wave spectrum

$W g t(f) \quad$ weighting function, ratio of incident energy at frequency $f$ to total energy in wave

$x \quad x$-axis coordinate

$x \quad$ direction perpendicular to wavemaker

$\mathbf{x} \quad$ vector of $(x, y, z)$ coordinates 


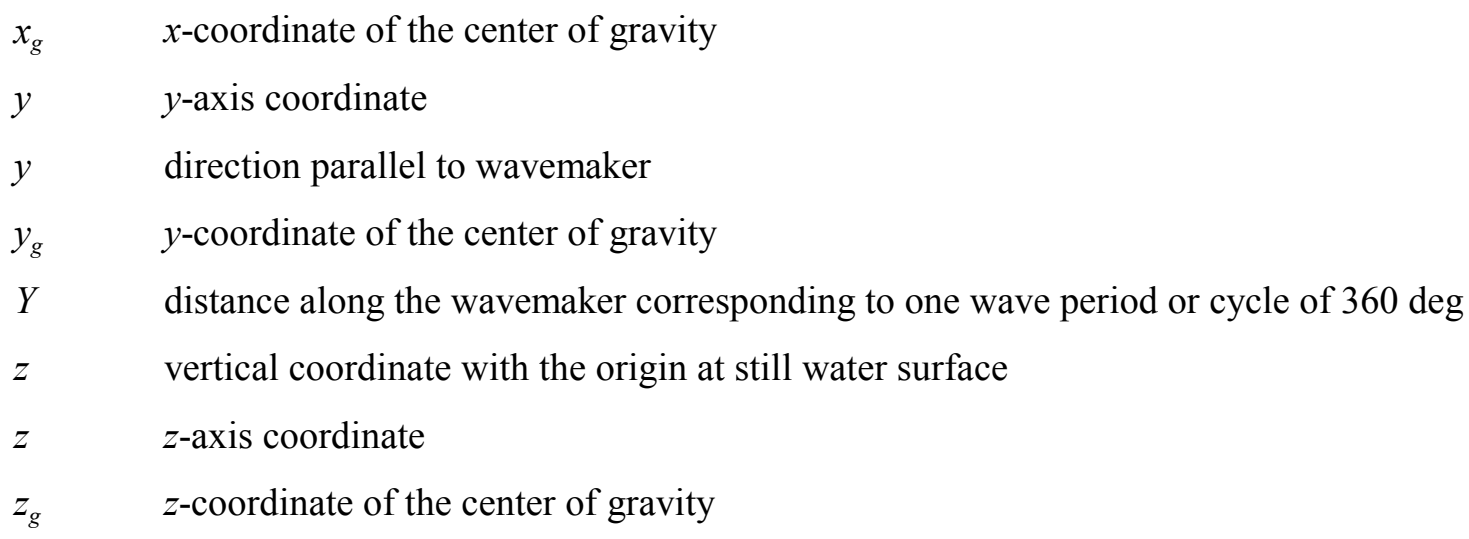

Greek Symbols

\begin{tabular}{ll}
$\alpha$ & Philip's constant \\
$\gamma$ & peak enhancement factor \\
$\gamma$ & specific weight of water \\
$\Delta f$ & frequency increment, Hz \\
$\Delta t$ & time interval, sec \\
$\varepsilon$ & horizontal excursion of the breakwater normal to its equilibrium position \\
$\eta$ & water surface elevation \\
$\bar{\eta}$ & normalized water surface elevation \\
$\eta_{I}$ & incident wave surface elevation \\
$\eta_{R}$ & reflected wave surface elevation \\
$\theta$ & direction of wave propagation, waves travel toward this direction \\
$\theta_{1}$ & incident wave angle \\
$\theta_{m}(f)$ & mean wave direction as a function of frequency \\
$\theta_{r}$ & interior angle of RIBS, deg \\
$\theta_{1}$ & wave direction in Region 1 \\
$\theta_{2}$ & wave direction in Region 2 \\
$\bar{\theta}$ & overall mean wave direction for all frequencies \\
$v$ & degrees of freedom \\
$v$ & kinematic viscosity \\
$\xi$ & six rigid-body degree of freedom body motions \\
$\xi_{j}$ & normalized body motions \\
\hline
\end{tabular}




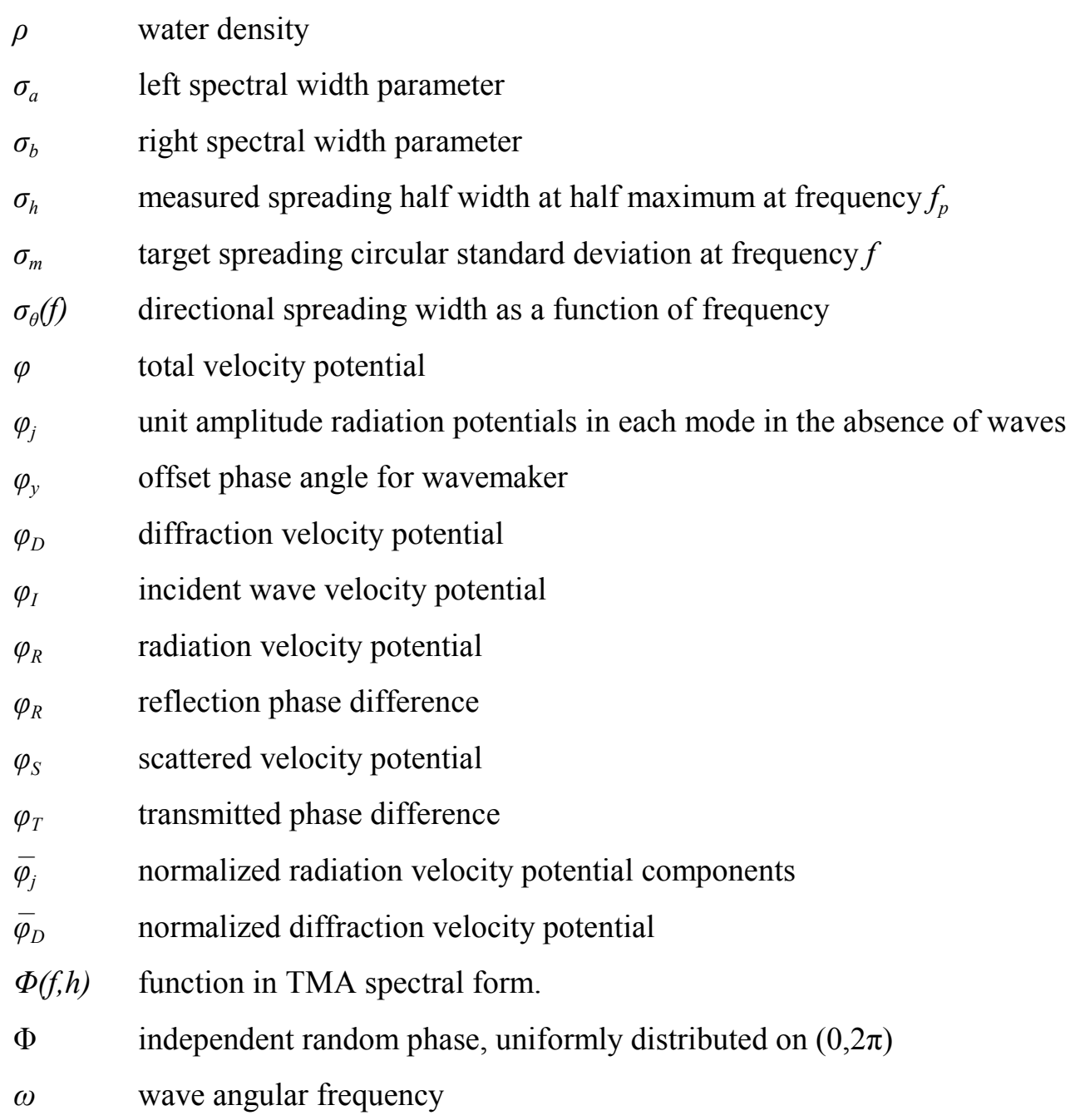


APPENDIX B

FLab PRESSURE GAGE VOLTAGES 


\begin{tabular}{|c|c|c|c|c|}
\hline \multicolumn{5}{|c|}{$\begin{array}{l}\text { Appendix B1 } \\
\text { Pressure Gage Voltages } \\
\text { DSWG Basin Fundamental Laborato }\end{array}$} \\
\hline Test ID & $\begin{array}{c}\text { Run } \\
\text { No. }\end{array}$ & $\begin{array}{l}d / h \\
(\%) \\
\end{array}$ & $\begin{array}{c}\text { Range } \\
\pm \mathbf{V}\end{array}$ & Comments \\
\hline \multicolumn{5}{|c|}{ Operational Series, 45 deg Interior Angle } \\
\hline M50 & $6-10$ & $100-25$ & 8 & \\
\hline M52 & $6-10$ & $100-25$ & 8 & \\
\hline M80 & $6-10$ & $100-25$ & 10 & $6 \&$ 7: Stern gage neg offset \\
\hline M82 & $6-10$ & $100-25$ & 10 & \\
\hline M120 & $6-10$ & $100-25$ & 10 & \\
\hline M122 & $6-10$ & $100-25$ & 10 & \\
\hline D50 & $6-10$ & $100-25$ & 10 & \\
\hline D52 & $6-10$ & $100-25$ & 10 & \\
\hline D80 & $6-10$ & $100-25$ & 10 & \\
\hline D82 & $6-10$ & $100-25$ & 10 & \\
\hline D120 & $6-10$ & $100-25$ & 10 & 6\& 7: Bad bow gage \\
\hline D122 & $6-10$ & $100-25$ & 10 & \\
\hline \multicolumn{5}{|c|}{ Survival Series, 45 deg Interior Angle } \\
\hline D5120 & $6-10$ & $100-25$ & 30 & \\
\hline D5122 & $6-10$ & $100-25$ & 30 & \\
\hline \multicolumn{5}{|c|}{ Operational Series, 90 deg Interior Angle } \\
\hline D50 & $20-22$ & 100,50 & 20 & $20 \&$ 21: Stern gage pos offset \\
\hline D52 & $20-22$ & 100,50 & 20 & $20: \pm 10$ \\
\hline D80 & $20-22$ & 100,50 & 20 & $20: \pm 10$ \\
\hline D82 & $20-22$ & 100,50 & 20 & $20 \& 21: \pm 10$ \\
\hline D120 & $20-22$ & 100,50 & 20 & \\
\hline D122 & $20-22$ & 100,50 & 20 & \\
\hline \multicolumn{5}{|c|}{ Survival Series, $90 \mathrm{deg}$ Interior Angle } \\
\hline D5120 & $20-22$ & 100,50 & 60 & \\
\hline D5122 & $20-22$ & 100,50 & 60 & \\
\hline \multicolumn{5}{|c|}{$\begin{array}{l}\text { Notes: } \\
\text { 1. Runs } 6 \text { and } 7 \text { had } 23 \text { gages: } 20 \text { wave gages, } 3 \text { pressure gages, command and feedback. } \\
\text { 2. All other runs had } 13 \text { gages: } 10 \text { wave gages } 3 \text { pressure gages, command and feedback. } \\
\text { 3. Doubled coefficient from } 3.6 \text { to } 7.2 \mathrm{E}-02,1 / 26 / 98 \text {, middle of run } 20, T=12 \mathrm{sec} \text { runs. }\end{array}$} \\
\hline
\end{tabular}


APPENDIX C

FLab WAVE MEASUREMENTS 


\begin{tabular}{|c|c|c|c|c|c|c|c|c|c|c|c|}
\hline \multicolumn{12}{|c|}{$\begin{array}{l}\text { Appendix } \mathrm{C} 1 \\
\text { Measured } H_{m 0} \text { Wave Heights in Centimeters } \\
\text { DSWG Basin Fundamental Laboratory Experiments (FLab) }\end{array}$} \\
\hline \multirow{2}{*}{$\begin{array}{l}\text { Case } \\
\text { Name }\end{array}$} & \multirow{2}{*}{$\begin{array}{l}\text { Run } \\
\text { No. }\end{array}$} & \multicolumn{10}{|c|}{ Gages } \\
\hline & & 1 & 2 & 3 & 4 & 5 & 6 & 7 & 8 & 9 & 10 \\
\hline \multicolumn{12}{|c|}{45 deg Interior Angle, $100 \%$ Penetration } \\
\hline R3_50D & 7 & 4.61 & 5.14 & 4.28 & 4.63 & 4.98 & 0.89 & 0.86 & 0.92 & 1.14 & 1.32 \\
\hline R3 52D & 7 & 4.64 & 4.52 & 4.03 & 4.13 & 4.87 & 0.79 & 0.88 & 0.88 & 1.01 & 1.65 \\
\hline R3 80D & 6 & 4.32 & 4.88 & 5.13 & 5.12 & 4.62 & 1.34 & 1.14 & 1.19 & 1.51 & 1.52 \\
\hline R3 $82 \mathrm{D}$ & 6 & 4.60 & 5.15 & 5.06 & 4.87 & 4.26 & 1.38 & 1.05 & 0.99 & 1.43 & 1.83 \\
\hline R3120D & 6 & 4.36 & 4.43 & 5.32 & 5.34 & 4.86 & 1.18 & 1.65 & 1.47 & 1.86 & 1.84 \\
\hline R3122D & 6 & 4.53 & 5.00 & 5.24 & 5.16 & 4.55 & 0.98 & 1.26 & 1.09 & 1.45 & 1.80 \\
\hline R5120D & 6 & 11.35 & 10.38 & 12.38 & 12.32 & 11.33 & 2.71 & 3.31 & 3.28 & 4.14 & 4.43 \\
\hline R5122D & 6 & 11.19 & 12.30 & 12.67 & 12.06 & 11.18 & 2.40 & 3.13 & 2.90 & 3.68 & 4.37 \\
\hline \multicolumn{12}{|c|}{$45 \mathrm{deg}$ Interior Angle, $75 \%$ Penetration } \\
\hline R3 50D & 8 & 4.44 & 5.18 & 4.85 & 5.18 & 5.09 & 1.09 & 1.11 & 1.06 & 1.42 & 1.49 \\
\hline R3 52D & 8 & 4.57 & 4.73 & 4.41 & 4.62 & 4.99 & 0.91 & 0.98 & 1.06 & 1.11 & 1.80 \\
\hline R3_80D & 8 & 4.32 & 4.93 & 5.15 & 5.16 & 4.62 & 2.51 & 2.60 & 2.76 & 2.90 & 2.43 \\
\hline R3 $82 \mathrm{D}$ & 8 & 4.63 & 5.13 & 4.83 & 4.72 & 4.35 & 2.29 & 2.10 & 2.11 & 2.12 & 2.86 \\
\hline R3120D & 8 & 4.45 & 4.52 & 5.41 & 5.46 & 5.02 & 3.52 & 3.43 & 3.50 & 3.48 & 3.74 \\
\hline R3122D & 8 & 4.50 & 4.98 & 5.10 & 5.08 & 4.57 & 3.65 & 3.40 & 3.36 & 3.43 & 3.26 \\
\hline R5120D & 8 & 11.12 & 10.56 & 12.53 & 12.66 & 11.60 & 7.92 & 7.67 & 7.88 & 7.91 & 8.41 \\
\hline R5122D & 8 & 10.93 & 12.43 & 12.68 & 12.16 & 11.21 & 7.68 & 7.11 & 7.01 & 6.63 & 7.72 \\
\hline \multicolumn{12}{|c|}{$45 \mathrm{deg}$ Interior Angle, $50 \%$ Penetration } \\
\hline R3 50D & 9 & 4.64 & 4.97 & 4.75 & 5.08 & 4.99 & 1.71 & 1.70 & 1.72 & 1.92 & 1.99 \\
\hline R3 52D & 9 & 4.48 & 4.89 & 4.45 & 4.43 & 4.79 & 1.50 & 1.44 & 1.54 & 1.52 & 2.03 \\
\hline R3 $80 \mathrm{D}$ & 9 & 4.39 & 4.87 & 5.08 & 5.13 & 4.57 & 3.55 & 3.56 & 3.70 & 3.76 & 3.25 \\
\hline R3 $82 \mathrm{D}$ & 9 & 4.57 & 5.10 & 4.82 & 4.69 & 4.25 & 3.09 & 2.96 & 2.85 & 2.83 & 3.25 \\
\hline R3120D & 9 & 4.45 & 4.44 & 5.34 & 5.46 & 4.96 & 4.19 & 4.09 & 4.10 & 4.07 & 4.06 \\
\hline R3122D & 9 & 4.50 & 4.93 & 5.05 & 5.04 & 4.52 & 4.38 & 4.07 & 4.17 & 4.08 & 3.68 \\
\hline R5120D & 9 & 11.14 & 10.51 & 12.42 & 12.58 & 11.55 & 9.67 & 9.50 & 9.65 & 9.76 & 9.85 \\
\hline R5122D & 9 & 11.15 & 12.22 & 12.45 & 11.85 & 10.98 & 9.63 & 9.43 & 9.39 & 8.76 & 9.26 \\
\hline
\end{tabular}




\begin{tabular}{|c|c|c|c|c|c|c|c|c|c|c|c|}
\hline \multicolumn{12}{|c|}{$\begin{array}{l}\text { Appendix C1 } \\
\text { Measured } H_{m 0} \text { Wave Heights in Centimeters }\end{array}$} \\
\hline \multirow{2}{*}{$\begin{array}{l}\text { Case } \\
\text { Name }\end{array}$} & \multirow{2}{*}{$\begin{array}{c}\text { Run } \\
\text { No. }\end{array}$} & \multicolumn{10}{|c|}{ Gages } \\
\hline & & 1 & 2 & 3 & 4 & 5 & 6 & 7 & 8 & 9 & 10 \\
\hline \multicolumn{12}{|c|}{$45 \mathrm{deg}$ Interior Angle, 25\% Penetration } \\
\hline R3_50D & 10 & 4.47 & 4.96 & 4.72 & 5.07 & 5.11 & 3.26 & 3.10 & 3.17 & 3.22 & 3.20 \\
\hline R3 52D & 10 & 4.39 & 4.65 & 4.35 & 4.47 & 4.87 & 2.97 & 2.74 & 2.87 & 2.93 & 2.98 \\
\hline R3 80D & 10 & 4.35 & 4.83 & 5.18 & 5.15 & 4.47 & 4.24 & 4.26 & 4.38 & 4.47 & 3.94 \\
\hline R3_82D & 10 & 4.53 & 5.16 & 4.89 & 4.63 & 4.07 & 3.70 & 3.65 & 3.71 & 3.66 & 3.74 \\
\hline R3120D & 10 & 4.44 & 4.44 & 5.26 & 5.42 & 4.98 & 4.61 & 4.48 & 4.49 & 4.58 & 4.41 \\
\hline R3122D & 10 & 4.47 & 4.88 & 4.99 & 4.95 & 4.53 & 4.62 & 4.30 & 4.45 & 4.47 & 3.95 \\
\hline R5120D & 10 & 10.87 & 10.50 & 12.51 & 12.74 & 11.39 & 10.87 & 10.44 & 10.44 & 10.97 & 10.02 \\
\hline R5122D & 10 & 11.21 & 12.32 & 12.35 & 11.86 & 10.84 & 11.10 & 11.11 & 11.00 & 11.00 & 10.31 \\
\hline \multicolumn{12}{|c|}{90 deg Interior Angle, $100 \%$ Penetration } \\
\hline R3 50D & 20 & 6.99 & 6.80 & 6.08 & 7.30 & 6.68 & 1.03 & 0.93 & 1.06 & 1.06 & 1.20 \\
\hline R3_52D & 20 & 6.56 & 6.35 & 6.31 & 7.56 & 7.08 & 0.95 & 0.84 & 1.03 & 1.04 & 1.40 \\
\hline R3 80D & 20 & 6.07 & 6.64 & 7.24 & 6.80 & 6.40 & 1.53 & 1.11 & 1.23 & 1.29 & 1.32 \\
\hline R3 82D & 20 & 5.90 & 6.25 & 6.49 & 6.36 & 6.70 & 1.42 & 1.14 & 1.23 & 1.45 & 1.34 \\
\hline R3120D & 20 & 5.76 & 6.14 & 6.55 & 6.25 & 6.12 & 1.23 & 1.41 & 1.29 & 1.36 & 1.27 \\
\hline R3122D & 20 & 5.58 & 5.93 & 6.30 & 6.35 & 6.22 & 1.09 & 1.42 & 1.25 & 1.33 & 1.60 \\
\hline R5120D & 20 & 13.44 & 14.55 & 15.03 & 14.46 & 14.27 & 2.89 & 3.71 & 3.16 & 3.53 & 3.51 \\
\hline R5122D & 20 & 13.79 & 15.10 & 15.82 & 14.86 & 15.08 & 2.79 & 3.44 & 3.36 & 3.59 & 3.97 \\
\hline \multicolumn{12}{|c|}{90 deg Interior Angle, 50\% Penetration } \\
\hline R3_50D & 22 & 6.50 & 6.37 & 6.08 & 6.43 & 6.43 & 1.80 & 1.68 & 1.71 & 1.75 & 1.71 \\
\hline R3_52D & 22 & 6.26 & 6.21 & 5.91 & 6.14 & 7.02 & 1.48 & 1.50 & 1.56 & 1.57 & 1.74 \\
\hline R3_80D & 22 & 5.48 & 5.82 & 5.88 & 6.04 & 5.21 & 3.54 & 3.38 & 3.45 & 3.53 & 3.10 \\
\hline R3_82D & 22 & 5.15 & 5.70 & 5.82 & 5.33 & 5.48 & 2.82 & 2.83 & 2.85 & 2.88 & 2.78 \\
\hline R3120D & 22 & 5.11 & 5.35 & 5.70 & 5.86 & 5.42 & 4.20 & 3.84 & 3.95 & 4.18 & 3.80 \\
\hline R3122D & 22 & 4.86 & 5.29 & 5.32 & 5.38 & 5.14 & 4.24 & 3.91 & 3.93 & 3.97 & 3.43 \\
\hline R5120D & 22 & 11.90 & 11.91 & 12.85 & 12.91 & 12.66 & 9.70 & 8.66 & 8.58 & 9.40 & 8.08 \\
\hline R5122D & 22 & 12.17 & 13.22 & 12.67 & 12.42 & 12.41 & 9.10 & 9.27 & 8.54 & 8.76 & 8.56 \\
\hline
\end{tabular}




\begin{tabular}{|c|c|c|c|c|c|c|c|c|c|c|c|}
\hline \multicolumn{12}{|c|}{$\begin{array}{l}\text { Appendix } \mathrm{C} 2 \\
\text { Measured } T_{p c} \text { Wave Periods }\end{array}$} \\
\hline \multirow{2}{*}{$\begin{array}{l}\text { Case } \\
\text { Name } \\
\end{array}$} & \multirow{2}{*}{$\begin{array}{c}\text { Run } \\
\text { No. }\end{array}$} & \multicolumn{10}{|c|}{ Gages } \\
\hline & & 1 & 2 & 3 & 4 & 5 & 6 & 7 & 8 & 9 & 10 \\
\hline \multicolumn{12}{|c|}{$45 \mathrm{deg}$ Interior Angle, $100 \%$ Penetration } \\
\hline R3_50D & 7 & 0.90 & 0.81 & 0.92 & 0.78 & 0.88 & 0.94 & 0.86 & 0.89 & 0.94 & 0.88 \\
\hline R3 52D & 7 & 0.88 & 0.81 & 0.90 & 0.87 & 0.89 & 0.81 & 0.90 & 0.90 & 0.92 & 0.81 \\
\hline R3_80D & 6 & 1.39 & 1.40 & 1.30 & 1.30 & 1.39 & 1.40 & 1.59 & 1.30 & 1.39 & 1.40 \\
\hline R3 $82 \mathrm{D}$ & 6 & 1.39 & 1.39 & 1.30 & 1.30 & 1.40 & 1.39 & 1.39 & 1.40 & 1.39 & 1.39 \\
\hline R3120D & 6 & 1.95 & 1.96 & 2.03 & 1.96 & 1.94 & 1.41 & 1.94 & 1.94 & 2.03 & 2.08 \\
\hline R3122D & 6 & 1.96 & 2.03 & 1.96 & 1.96 & 1.96 & 1.96 & 2.29 & 1.96 & 1.96 & 2.04 \\
\hline R5120D & 6 & 1.94 & 2.01 & 2.03 & 2.03 & 2.16 & 1.94 & 2.27 & 1.90 & 1.94 & 1.90 \\
\hline R5122D & 6 & 2.03 & 2.03 & 1.94 & 1.94 & 2.01 & 1.99 & 2.16 & 1.94 & 1.99 & 1.99 \\
\hline \multicolumn{12}{|c|}{$45 \mathrm{deg}$ Interior Angle, $75 \%$ Penetration } \\
\hline R3 50D & 8 & 0.82 & 0.88 & 0.88 & 0.78 & 0.88 & 1.18 & 1.18 & 0.89 & 1.18 & 0.88 \\
\hline R3_52D & 8 & 0.89 & 0.93 & 0.93 & 0.89 & 0.88 & 1.18 & 0.90 & 0.91 & 0.90 & 0.88 \\
\hline R3_80D & 8 & 1.39 & 1.29 & 1.29 & 1.30 & 1.39 & 1.28 & 1.45 & 1.33 & 1.33 & 1.45 \\
\hline R3 82D & 8 & 1.39 & 1.34 & 1.34 & 1.31 & 1.37 & 1.44 & 1.30 & 1.30 & 1.42 & 1.40 \\
\hline R3120D & 8 & 2.03 & 2.03 & 2.03 & 2.03 & 1.94 & 1.96 & 2.03 & 2.16 & 1.94 & 2.03 \\
\hline R3122D & 8 & 2.03 & 1.96 & 1.96 & 1.96 & 1.94 & 1.96 & 2.00 & 2.03 & 2.00 & 2.19 \\
\hline R5120D & 8 & 1.90 & 2.01 & 2.01 & 2.03 & 2.16 & 1.94 & 2.03 & 2.03 & 1.92 & 2.01 \\
\hline R5122D & 8 & 1.96 & 1.94 & 1.94 & 1.94 & 2.01 & 1.94 & 2.01 & 2.05 & 2.10 & 1.94 \\
\hline \multicolumn{12}{|c|}{$45 \mathrm{deg}$ Interior Angle, $50 \%$ Penetration } \\
\hline R3_50D & 9 & 0.90 & 0.88 & 0.88 & 0.78 & 0.89 & 1.18 & 1.13 & 0.89 & 1.18 & 1.18 \\
\hline R3 52D & 9 & 0.89 & 0.90 & 0.90 & 0.89 & 0.89 & 0.96 & 0.97 & 0.96 & 0.90 & 0.87 \\
\hline R3 80D & 9 & 1.39 & 1.32 & 1.32 & 1.28 & 1.39 & 1.29 & 1.29 & 1.33 & 1.33 & 1.45 \\
\hline R3_82D & 9 & 1.39 & 1.39 & 1.39 & 1.31 & 1.37 & 1.30 & 1.30 & 1.30 & 1.42 & 1.40 \\
\hline R3120D & 9 & 2.03 & 1.96 & 1.96 & 2.03 & 1.94 & 1.96 & 2.03 & 2.16 & 1.94 & 2.03 \\
\hline R3122D & 9 & 2.03 & 1.96 & 1.96 & 1.96 & 2.00 & 1.96 & 2.00 & 2.03 & 2.00 & 2.00 \\
\hline R5120D & 9 & 1.90 & 2.01 & 2.01 & 2.03 & 1.91 & 1.94 & 2.03 & 2.03 & 1.92 & 2.01 \\
\hline R5122D & 9 & 1.96 & 1.94 & 1.94 & 1.94 & 2.01 & 1.94 & 2.05 & 2.05 & 2.10 & 1.94 \\
\hline
\end{tabular}




\begin{tabular}{|c|c|c|c|c|c|c|c|c|c|c|c|}
\hline \multicolumn{12}{|c|}{$\begin{array}{l}\text { Appendix } \mathrm{C} 2 \\
\text { Measured } T_{p c} \text { Wave Periods }\end{array}$} \\
\hline \multirow{2}{*}{$\begin{array}{l}\text { Case } \\
\text { Name }\end{array}$} & \multirow{2}{*}{$\begin{array}{l}\text { Run } \\
\text { No. }\end{array}$} & \multicolumn{10}{|c|}{ Gages } \\
\hline & & 1 & 2 & 3 & 4 & 5 & 6 & 7 & 8 & 9 & 10 \\
\hline \multicolumn{12}{|c|}{$45 \mathrm{deg}$ Interior Angle, $25 \%$ Penetration } \\
\hline R3 50D & 10 & 0.82 & 0.94 & 0.94 & 0.79 & 0.88 & 0.81 & 0.88 & 0.90 & 0.89 & 0.89 \\
\hline R3 52D & 10 & 0.89 & 0.90 & 0.90 & 0.89 & 0.89 & 0.89 & 0.86 & 0.89 & 0.89 & 0.87 \\
\hline R3_80D & 10 & 1.39 & 1.32 & 1.32 & 1.28 & 1.37 & 1.29 & 1.29 & 1.33 & 1.33 & 1.29 \\
\hline R3_82D & 10 & 1.39 & 1.40 & 1.40 & 1.40 & 1.39 & 1.30 & 1.31 & 1.38 & 1.42 & 1.34 \\
\hline R3120D & 10 & 2.03 & 1.96 & 1.96 & 2.03 & 1.94 & 1.96 & 2.17 & 2.16 & 1.94 & 2.03 \\
\hline R3122D & 10 & 2.03 & 1.96 & 1.96 & 1.96 & 1.94 & 1.96 & 2.00 & 2.00 & 1.94 & 2.19 \\
\hline R5120D & 10 & 1.90 & 2.01 & 2.01 & 2.01 & 1.91 & 1.92 & 2.03 & 2.03 & 2.01 & 2.01 \\
\hline R5122D & 10 & 2.00 & 1.94 & 1.94 & 2.01 & 2.01 & 2.01 & 2.05 & 2.10 & 1.94 & 2.01 \\
\hline \multicolumn{12}{|c|}{$90 \mathrm{deg}$ Interior Angle, 100\% Penetration } \\
\hline R3 50D & 20 & 0.89 & 0.88 & 0.78 & 0.88 & 0.88 & 0.93 & 0.92 & 0.89 & 0.85 & 0.89 \\
\hline R3 52D & 20 & 0.88 & 0.80 & 0.90 & 0.89 & 0.88 & 0.90 & 0.87 & 0.89 & 0.87 & 0.88 \\
\hline R3 $80 \mathrm{D}$ & 20 & 1.39 & 1.33 & 1.33 & 1.30 & 1.30 & 1.40 & 1.41 & 1.36 & 1.36 & 1.34 \\
\hline \begin{tabular}{|l} 
R3 82D \\
\end{tabular} & 20 & 1.40 & 1.30 & 1.30 & 1.30 & 1.35 & 1.39 & 1.40 & 1.37 & 1.39 & 1.36 \\
\hline R3120D & 20 & 1.95 & 2.03 & 2.03 & 1.96 & 1.92 & 2.17 & 2.04 & 2.08 & 1.96 & 1.97 \\
\hline R3122D & 20 & 2.03 & 1.96 & 1.96 & 1.96 & 2.31 & 2.03 & 2.04 & 2.03 & 1.97 & 1.99 \\
\hline R5120D & 20 & 1.94 & 2.01 & 2.01 & 1.94 & 2.11 & 2.03 & 2.03 & 2.17 & 1.95 & 1.99 \\
\hline R5122D & 20 & 1.99 & 2.01 & 2.01 & 2.01 & 2.11 & 2.16 & 2.01 & 2.01 & 2.01 & 2.01 \\
\hline \multicolumn{12}{|c|}{$90 \mathrm{deg}$ Interior Angle, $50 \%$ Penetration } \\
\hline R3_50D & 22 & 0.88 & 0.90 & 0.90 & 0.88 & 0.89 & 0.92 & 0.90 & 0.92 & 0.97 & 0.97 \\
\hline R3 52D & 22 & 0.90 & 0.78 & 0.78 & 0.89 & 0.90 & 0.89 & 0.87 & 0.91 & 0.89 & 0.87 \\
\hline R3 80D & 22 & 1.39 & 1.33 & 1.33 & 1.29 & 1.28 & 1.30 & 1.30 & 1.29 & 1.39 & 1.29 \\
\hline R3_82D & 22 & 1.39 & 1.39 & 1.39 & 1.30 & 1.37 & 1.38 & 1.32 & 1.38 & 1.42 & 1.40 \\
\hline R3120D & 22 & 2.03 & 2.03 & 2.03 & 2.03 & 1.94 & 1.96 & 2.17 & 2.17 & 1.96 & 2.03 \\
\hline R3122D & 22 & 2.03 & 1.96 & 1.96 & 1.96 & 2.00 & 2.00 & 2.00 & 2.00 & 1.94 & 2.19 \\
\hline R5120D & 22 & 1.90 & 2.01 & 2.01 & 2.03 & 1.94 & 1.94 & 2.03 & 1.90 & 1.92 & 2.14 \\
\hline R5122D & 22 & 1.99 & 2.01 & 2.01 & 2.01 & 2.07 & 2.01 & 2.05 & 2.10 & 1.94 & 2.01 \\
\hline
\end{tabular}




\begin{tabular}{|c|c|c|c|c|c|c|c|c|c|c|c|}
\hline \multicolumn{12}{|c|}{\begin{tabular}{|l} 
Appendix $C 3$ \\
Measured $\bar{H}$ Wave Heights in Centimeters \\
DSWG Basin Fundamental Laboratory Experiments (FLab) \\
\end{tabular}} \\
\hline \multirow{2}{*}{$\begin{array}{l}\text { Case } \\
\text { Name } \\
\end{array}$} & \multirow{2}{*}{$\begin{array}{c}\text { Run } \\
\text { No. }\end{array}$} & \multicolumn{10}{|c|}{ Gages } \\
\hline & & 1 & 2 & 3 & 4 & 5 & 6 & 7 & 8 & 9 & 10 \\
\hline \multicolumn{12}{|c|}{$45 \mathrm{deg}$ Interior Angle, $50 \%$ Penetration } \\
\hline R3_50M & 9 & 2.89 & 3.99 & 2.68 & 3.51 & 3.04 & 0.91 & 1.25 & 1.18 & 1.33 & 1.56 \\
\hline R3 52M & 9 & 4.34 & 2.48 & 3.50 & 3.39 & 3.55 & 0.52 & 0.76 & 0.77 & 1.03 & 1.16 \\
\hline R3_80M & 9 & 3.28 & 2.34 & 3.35 & 3.21 & 2.84 & 2.27 & 3.16 & 2.45 & 2.40 & 2.47 \\
\hline R3_82M & 9 & 3.01 & 3.35 & 3.01 & 3.93 & 3.78 & 2.68 & 2.34 & 2.88 & 2.87 & 3.98 \\
\hline R3120M & 9 & 3.06 & 2.71 & 4.55 & 4.48 & 3.93 & 2.92 & 3.99 & 3.63 & 1.54 & 3.72 \\
\hline R3122M & 9 & 3.16 & 2.96 & 4.65 & 4.24 & 2.60 & 3.64 & 4.12 & 5.08 & 3.84 & 3.50 \\
\hline
\end{tabular}


APPENDIX D

MEASURED XM99 WAVE PARAMETERS 


\begin{tabular}{|c|c|c|c|c|c|c|}
\hline \multicolumn{7}{|c|}{$\begin{array}{l}\text { Appendix D1 } \\
\text { Measured XM99 Wave Parameters }\end{array}$} \\
\hline Date & $\begin{array}{c}\text { Time } \\
\text { (Hour) }\end{array}$ & $\begin{array}{c}T_{p} \\
\text { (sec) }\end{array}$ & $\begin{array}{l}H_{m 0} \\
(\mathrm{~m})\end{array}$ & $\begin{array}{c}\text { Mean } \\
\text { Direction } \bar{\theta} \\
(\text { deg) }\end{array}$ & $\begin{array}{l}\text { Spread } \sigma_{m} \\
\quad(\text { deg })\end{array}$ & Mode \\
\hline 20-May & 1400 & 8.79 & 0.56 & $\mathrm{NA}$ & NA & $\mathrm{NA}$ \\
\hline 20-May & 1500 & 9.94 & 0.54 & NA & NA & NA \\
\hline 20-May & 1600 & 9.14 & 0.63 & NA & NA & NA \\
\hline 20-May & 1700 & 9.94 & 0.69 & 264.95 & 41.49 & 1 \\
\hline 20-May & 1800 & 9.52 & 0.71 & 273.33 & 43.50 & 1 \\
\hline 20-May & 1900 & 9.52 & 0.67 & 257.35 & 42.82 & 1 \\
\hline 20-May & 2000 & 10.39 & 0.60 & 260.26 & 45.91 & 1 \\
\hline 20-May & 2100 & 10.39 & 0.60 & 247.07 & 47.53 & 1 \\
\hline 20-May & 2200 & 10.39 & 0.61 & 236.20 & 56.13 & 1 \\
\hline 20-May & 2300 & 10.39 & 0.66 & NA & NA & NA \\
\hline 21-May & 0 & 9.94 & 0.54 & 243.01 & 47.07 & 1 \\
\hline 21-May & 100 & 9.94 & 0.67 & 244.21 & 55.57 & 1 \\
\hline 21-May & 200 & 9.52 & 0.68 & 253.46 & 48.69 & 1 \\
\hline 21-May & 300 & 10.39 & 0.63 & 236.01 & 59.10 & 3 \\
\hline 21-May & 400 & 9.94 & 0.63 & 258.63 & 47.54 & 1 \\
\hline 21-May & 500 & 10.39 & 0.60 & 264.69 & 45.19 & 1 \\
\hline 21-May & 600 & 9.94 & 0.55 & 270.61 & 44.48 & 1 \\
\hline 21-May & 700 & 9.52 & 0.56 & 272.20 & 35.89 & 1 \\
\hline 21-May & 800 & 9.94 & 0.57 & 263.18 & 38.42 & 1 \\
\hline 21-May & 900 & 9.52 & 0.53 & NA & NA & NA \\
\hline 21-May & 1000 & 9.52 & 0.51 & NA & NA & NA \\
\hline 21-May & 1100 & 9.94 & 0.52 & 243.41 & 55.43 & 1 \\
\hline 21-May & 1200 & 9.14 & 0.54 & NA & NA & NA \\
\hline 21-May & 1300 & 9.52 & 0.53 & 250.22 & 59.19 & 1 \\
\hline 21-May & 1400 & 9.14 & 0.51 & NA & NA & NA \\
\hline 21-May & 1500 & 8.79 & 0.59 & $\overline{\mathrm{NA}}$ & $\overline{\mathrm{NA}}$ & NA \\
\hline 21-May & 1600 & 9.14 & 0.63 & NA & NA & NA \\
\hline 21-May & 1700 & 8.79 & 0.66 & 253.04 & 49.00 & 3 \\
\hline 21-May & 1800 & 9.14 & 0.67 & 255.89 & 50.17 & 3 \\
\hline 21-May & 1900 & 8.47 & 0.68 & 257.87 & 43.78 & 3 \\
\hline 21-May & 2000 & 8.79 & 0.76 & NA & NA & $\mathrm{NA}$ \\
\hline 21-May & 2100 & 3.75 & 0.80 & 264.76 & 38.34 & 4 \\
\hline 21-May & 2200 & 3.94 & 0.79 & 257.99 & 40.16 & 4 \\
\hline
\end{tabular}




\begin{tabular}{|c|c|c|c|c|c|c|}
\hline \multicolumn{7}{|c|}{\begin{tabular}{|l} 
Appendix D1 \\
Measured XM99 Wave Parameters
\end{tabular}} \\
\hline Date & $\begin{array}{c}\text { Time } \\
\text { (Hour) }\end{array}$ & $\begin{array}{c}T_{p} \\
(\text { sec) }\end{array}$ & $\begin{array}{l}H_{m 0} \\
(\mathrm{~m})\end{array}$ & $\begin{array}{c}\text { Mean } \\
\text { Direction } \\
\text { (deg) }\end{array}$ & $\begin{array}{l}\text { Spread } \sigma_{m} \\
\quad(\text { deg })\end{array}$ & Mode \\
\hline 21-May & 2300 & 9.14 & 0.75 & 257.44 & 53.38 & 4 \\
\hline 22-May & 0 & 10.88 & 0.70 & $\mathrm{NA}$ & $\mathrm{NA}$ & NA \\
\hline 22-May & 100 & 9.14 & 0.85 & 255.97 & 46.25 & 3 \\
\hline 22-May & 200 & 4.16 & 0.96 & 262.73 & 38.35 & 4 \\
\hline 22-May & 300 & 4.16 & 0.94 & 262.63 & 40.84 & 4 \\
\hline 22-May & 400 & 3.87 & 0.89 & 264.34 & 38.31 & 4 \\
\hline 22-May & 500 & 9.94 & 0.83 & 251.30 & 41.33 & 4 \\
\hline 22-May & 600 & 4.40 & 0.76 & 251.65 & 40.97 & 3 \\
\hline 22-May & 700 & 9.52 & 0.78 & 254.90 & 39.31 & 5 \\
\hline 22-May & 800 & 5.71 & 0.73 & 252.29 & 41.34 & 5 \\
\hline 22-May & 900 & 6.02 & 0.75 & 267.43 & 31.44 & 4 \\
\hline 22-May & 1000 & 9.14 & 0.73 & $\mathrm{NA}$ & $\mathrm{NA}$ & $\mathrm{NA}$ \\
\hline 22-May & 1100 & 9.14 & 0.73 & $\mathrm{NA}$ & NA & $\mathrm{NA}$ \\
\hline 22-May & 1200 & 9.52 & 0.79 & 254.47 & 49.28 & 3 \\
\hline 22-May & 1300 & 9.14 & 0.79 & 254.49 & 54.55 & 3 \\
\hline 22-May & 1400 & 5.19 & 0.77 & $\overline{\mathrm{NA}}$ & $\mathrm{NA}$ & NA \\
\hline 22-May & 1500 & 9.14 & 0.81 & 257.07 & 48.03 & 3 \\
\hline 22-May & 1600 & 9.14 & 0.81 & 252.78 & 43.25 & 3 \\
\hline 22-May & 1700 & 9.14 & 0.81 & 260.32 & 42.39 & 3 \\
\hline 22-May & 1800 & 9.52 & 0.80 & 260.08 & 30.97 & 3 \\
\hline 22-May & 1900 & 9.14 & 0.75 & 259.95 & 38.57 & 3 \\
\hline 22-May & 2000 & 8.47 & 0.76 & 258.87 & 35.47 & 3 \\
\hline 23-May & 0 & 9.14 & 0.72 & 253.48 & 55.11 & 3 \\
\hline 23-May & 100 & 8.79 & 0.54 & 267.26 & 37.37 & 1 \\
\hline 23-May & 200 & 9.52 & 0.50 & 278.03 & 33.50 & 1 \\
\hline 23-May & 300 & 8.47 & 0.49 & 275.56 & 30.69 & 1 \\
\hline 23-May & 400 & 9.14 & 0.50 & 264.04 & 32.86 & 1 \\
\hline 23-May & 500 & 8.16 & 0.49 & 261.33 & 38.42 & 1 \\
\hline 23-May & 600 & 8.47 & 0.51 & 260.79 & 40.49 & 1 \\
\hline 23-May & 700 & 8.79 & 0.45 & 253.85 & 49.69 & 1 \\
\hline 23-May & 800 & 8.16 & 0.47 & 250.39 & 51.79 & 1 \\
\hline 23-May & 2000 & 8.47 & 0.70 & $\mathrm{NA}$ & NA & $\mathrm{NA}$ \\
\hline
\end{tabular}




\begin{tabular}{|c|c|c|c|c|c|c|}
\hline \multicolumn{7}{|c|}{\begin{tabular}{|l} 
Appendix D1 \\
Measured XM99 Wave Parameters
\end{tabular}} \\
\hline Date & $\begin{array}{c}\text { Time } \\
\text { (Hour) }\end{array}$ & $\begin{array}{c}T_{p} \\
(\text { sec) }\end{array}$ & $\begin{array}{l}H_{m 0} \\
(\mathrm{~m})\end{array}$ & $\begin{array}{c}\text { Mean } \\
\text { Direction } \\
\text { (deg) }\end{array}$ & $\begin{array}{l}\text { Spread } \sigma_{m} \\
\quad(\text { deg })\end{array}$ & Mode \\
\hline 23-May & 2100 & 4.97 & 0.75 & 251.00 & 35.00 & 4 \\
\hline 23-May & 2200 & 8.47 & 0.47 & 258.63 & 37.72 & 1 \\
\hline 23-May & 2300 & 8.79 & 0.49 & 248.31 & 33.77 & 1 \\
\hline 24-May & 0 & 8.79 & 0.52 & 248.44 & 32.60 & 1 \\
\hline 24-May & 100 & 8.16 & 0.51 & 261.87 & 37.35 & 1 \\
\hline 24-May & 200 & 8.79 & 0.54 & 250.27 & 38.23 & 1 \\
\hline 24-May & 300 & 8.47 & 0.53 & 241.57 & 43.02 & 1 \\
\hline 24-May & 400 & 8.79 & 0.53 & 258.75 & 51.70 & 1 \\
\hline 24-May & 500 & 8.47 & 0.55 & 257.66 & 38.52 & 1 \\
\hline 24-May & 600 & 7.37 & 0.48 & 262.69 & 54.80 & 3 \\
\hline 24-May & 700 & 8.47 & 0.52 & 269.10 & 44.34 & 3 \\
\hline 24-May & 800 & 8.16 & 0.50 & 278.14 & 49.02 & 3 \\
\hline 24-May & 900 & NA & NA & $\mathrm{NA}$ & $\mathrm{NA}$ & NA \\
\hline 24-May & 1000 & 8.47 & 0.41 & 269.26 & 45.32 & 3 \\
\hline 24-May & 1100 & 8.47 & 0.39 & 270.65 & 44.04 & 3 \\
\hline 24-May & 1200 & 8.16 & 0.36 & 263.45 & 41.92 & 1 \\
\hline 24-May & 1300 & 8.47 & 0.39 & 274.54 & 41.03 & 1 \\
\hline 24-May & 1400 & 9.14 & 0.38 & 276.61 & 46.90 & 1 \\
\hline 24-May & 1500 & 7.88 & 0.38 & 250.50 & 45.56 & 1 \\
\hline 24-May & 1600 & 8.16 & 0.40 & 250.66 & 52.01 & 1 \\
\hline 24-May & 1700 & 8.79 & 0.42 & 259.25 & 51.06 & 3 \\
\hline 24-May & 1800 & 2.69 & 0.50 & 321.19 & 47.16 & 4 \\
\hline 24-May & 1900 & 2.75 & 0.52 & 265.68 & 42.52 & 3 \\
\hline 24-May & 2000 & 7.62 & 0.50 & 274.34 & 48.32 & 3 \\
\hline 24-May & 2100 & 2.51 & 0.53 & 282.63 & 46.81 & 1 \\
\hline 24-May & 2200 & 8.16 & 0.48 & 283.80 & 47.33 & 3 \\
\hline 24-May & 2300 & 8.47 & 0.45 & 281.45 & 52.30 & 3 \\
\hline
\end{tabular}




\begin{tabular}{|c|c|c|c|c|c|c|}
\hline \multicolumn{7}{|c|}{\begin{tabular}{|l} 
Appendix D1 \\
Measured XM99 Wave Parameters
\end{tabular}} \\
\hline Date & $\begin{array}{c}\text { Time } \\
\text { (Hour) }\end{array}$ & $\begin{array}{c}T_{p} \\
(\text { sec) }\end{array}$ & $\begin{array}{l}H_{m 0} \\
(\mathrm{~m})\end{array}$ & $\begin{array}{c}\text { Mean } \\
\text { Direction } \\
\text { (deg) }\end{array}$ & $\begin{array}{l}\text { Spread } \sigma_{m} \\
\quad(\text { deg })\end{array}$ & Mode \\
\hline 25-May & 0 & 7.88 & 0.43 & 268.39 & 48.75 & 3 \\
\hline 25-May & 100 & 8.16 & 0.44 & 276.16 & 56.01 & 3 \\
\hline 25-May & 200 & 7.88 & 0.44 & 274.86 & 45.05 & 3 \\
\hline 25-May & 300 & 7.62 & 0.43 & 281.70 & 47.19 & 3 \\
\hline 25-May & 400 & 4.01 & 0.46 & 279.87 & 53.21 & 3 \\
\hline 25-May & 500 & 8.16 & 0.40 & 281.40 & 55.92 & 3 \\
\hline 25-May & 600 & 7.88 & 0.37 & 279.47 & 41.96 & 3 \\
\hline 25-May & 700 & 7.88 & 0.35 & 273.42 & 42.04 & 3 \\
\hline 25-May & 800 & 7.62 & 0.35 & 269.78 & 45.37 & 3 \\
\hline 25-May & 900 & 8.47 & 0.33 & NA & NA & $\mathrm{NA}$ \\
\hline 25-May & 1000 & 8.16 & 0.32 & 274.84 & 44.21 & 3 \\
\hline 25-May & 1100 & 8.16 & 0.33 & 281.95 & 42.54 & 3 \\
\hline 25-May & 1200 & 10.39 & 0.33 & 271.06 & 51.62 & 3 \\
\hline 25-May & 1300 & 7.62 & 0.30 & 271.80 & 38.09 & 1 \\
\hline 25-May & 1400 & 8.16 & 0.28 & 259.15 & 37.47 & 1 \\
\hline 25-May & 1500 & 7.88 & 0.40 & 262.27 & 81.90 & NA \\
\hline 25-May & 1600 & 2.93 & 0.53 & 314.58 & 72.49 & NA \\
\hline 25-May & 1700 & 3.10 & 0.63 & 314.73 & 36.07 & 2 \\
\hline 25-May & 1800 & 3.36 & 0.72 & 321.64 & 39.20 & 2 \\
\hline 25-May & 1900 & 3.52 & 0.76 & 321.58 & 36.28 & 2 \\
\hline 25-May & 2000 & 3.57 & 0.77 & 322.89 & 35.37 & 2 \\
\hline 25-May & 2100 & 4.08 & 0.80 & 323.65 & 39.25 & 2 \\
\hline 25-May & 2200 & 3.87 & 0.83 & 328.51 & 33.19 & 2 \\
\hline 25-May & 2300 & 3.75 & 0.75 & 334.01 & 35.28 & 2 \\
\hline 26-May & 0 & 4.01 & 0.67 & 332.33 & 34.92 & 2 \\
\hline 26-May & 100 & 3.81 & 0.57 & 330.80 & 33.63 & 2 \\
\hline 26-May & 200 & 3.81 & 0.55 & 327.92 & 32.16 & 2 \\
\hline 26-May & 300 & 3.94 & 0.51 & 330.13 & 34.63 & 2 \\
\hline 26-May & 400 & 3.94 & 0.44 & 325.82 & 34.72 & 2 \\
\hline 26-May & 500 & 3.94 & 0.44 & 327.83 & 36.43 & 4 \\
\hline 26-May & 600 & 4.01 & 0.41 & 325.44 & 32.33 & 2 \\
\hline 26-May & 700 & 3.75 & 0.35 & 323.69 & 49.11 & 4 \\
\hline 26-May & 800 & 9.52 & 0.34 & 274.68 & 47.19 & 3 \\
\hline
\end{tabular}




\begin{tabular}{|c|c|c|c|c|c|c|}
\hline \multicolumn{7}{|c|}{\begin{tabular}{|l} 
Appendix D1 \\
Measured XM99 Wave Parameters
\end{tabular}} \\
\hline Date & $\begin{array}{c}\text { Time } \\
\text { (Hour) }\end{array}$ & $\begin{array}{c}T_{p} \\
(\text { sec) }\end{array}$ & $\begin{array}{l}H_{m 0} \\
(\mathrm{~m})\end{array}$ & $\begin{array}{c}\text { Mean } \\
\text { Direction } \\
\text { (deg) }\end{array}$ & $\begin{array}{l}\text { Spread } \sigma_{m} \\
\quad(\text { deg })\end{array}$ & Mode \\
\hline 26-May & 900 & 9.52 & 0.30 & 281.54 & 52.29 & 3 \\
\hline 26-May & 1000 & 9.52 & 0.30 & 277.42 & 52.02 & 3 \\
\hline 26-May & 1100 & 7.14 & 0.26 & 276.42 & 37.42 & 3 \\
\hline 26-May & 1200 & 9.94 & 0.28 & 272.32 & 36.94 & 1 \\
\hline 26-May & 1300 & 9.52 & 0.28 & 270.17 & 35.63 & 1 \\
\hline 26-May & 1400 & 7.14 & 0.28 & 264.71 & 44.58 & 1 \\
\hline 26-May & 1500 & 9.94 & 0.34 & 261.05 & 59.05 & 1 \\
\hline 26-May & 1600 & 8.47 & 0.43 & 270.31 & 48.57 & 3 \\
\hline 26-May & 1700 & 2.82 & 0.54 & 310.09 & 43.67 & 4 \\
\hline 26-May & 1800 & 2.89 & 0.55 & 325.49 & 45.28 & 4 \\
\hline 26-May & 1900 & 3.09 & 0.51 & 331.64 & 48.01 & 2 \\
\hline 26-May & 2000 & 3.36 & 0.51 & 321.20 & 38.23 & 2 \\
\hline 26-May & 2100 & 3.36 & 0.50 & 331.35 & 44.77 & 4 \\
\hline 26-May & 2200 & 3.63 & 0.51 & 336.44 & 33.46 & 2 \\
\hline 26-May & 2300 & 3.52 & 0.48 & 352.12 & 37.27 & 4 \\
\hline 27-May & 0 & 3.75 & 0.51 & 333.07 & 31.80 & 2 \\
\hline 27-May & 100 & 3.94 & 0.49 & 340.99 & 31.40 & 2 \\
\hline 27-May & 200 & 4.01 & 0.45 & 335.88 & 36.02 & 2 \\
\hline 27-May & 300 & 4.01 & 0.41 & 337.25 & 38.93 & 2 \\
\hline 27-May & 400 & 4.08 & 0.41 & 337.35 & 41.21 & 2 \\
\hline 27-May & 500 & 4.01 & 0.36 & 335.81 & 38.54 & 2 \\
\hline 27-May & 600 & 4.08 & 0.39 & 326.06 & 38.60 & 2 \\
\hline 27-May & 700 & 4.08 & 0.35 & 323.18 & 57.17 & 2 \\
\hline 27-May & 800 & 3.94 & 0.36 & 353.73 & 49.24 & $\mathrm{NA}$ \\
\hline 27-May & 900 & 3.87 & 0.32 & NA & NA & $\mathrm{NA}$ \\
\hline 27-May & 1000 & 3.27 & 0.29 & 314.24 & 52.89 & 4 \\
\hline 27-May & 1100 & 3.75 & 0.28 & 319.41 & 52.67 & 4 \\
\hline 27-May & 1200 & 3.46 & 0.27 & 309.22 & 52.42 & 4 \\
\hline 27-May & 1300 & 9.52 & 0.28 & 266.03 & 38.79 & 3 \\
\hline 27-May & 1400 & 8.47 & 0.25 & NA & NA & NA \\
\hline 27-May & 1500 & 8.47 & 0.32 & NA & NA & NA \\
\hline 27-May & 1600 & 9.52 & 0.33 & $\mathrm{NA}$ & $\mathrm{NA}$ & NA \\
\hline 27-May & 1700 & 2.12 & 0.32 & 253.92 & 59.31 & 4 \\
\hline
\end{tabular}




\begin{tabular}{|c|c|c|c|c|c|c|}
\hline \multicolumn{7}{|c|}{$\begin{array}{l}\text { Appendix D1 } \\
\text { Measured XM99 Wave Parameters }\end{array}$} \\
\hline Date & $\begin{array}{c}\text { Time } \\
\text { (Hour) }\end{array}$ & $\begin{array}{c}T_{p} \\
\text { (sec) }\end{array}$ & $\begin{array}{l}H_{m 0} \\
(\mathbf{m})\end{array}$ & $\begin{array}{c}\text { Mean } \\
\text { Direction } \\
\text { (deg) }\end{array}$ & $\begin{array}{l}\text { Spread } \sigma_{m} \\
\quad(\text { deg })\end{array}$ & Mode \\
\hline 27-May & 1800 & 2.54 & 0.33 & 275.09 & 58.33 & 3 \\
\hline 27-May & 1900 & 2.14 & 0.32 & 285.14 & 73.76 & 3 \\
\hline 27-May & 2000 & 7.88 & 0.29 & 268.09 & 53.79 & 3 \\
\hline 27-May & 2100 & 9.52 & 0.26 & 280.42 & 61.88 & 1 \\
\hline 27-May & 2200 & 9.94 & 0.26 & 273.38 & 48.90 & 1 \\
\hline 27-May & 2300 & 9.94 & 0.26 & 261.88 & 51.40 & 1 \\
\hline 28-May & 0 & 8.47 & 0.28 & 261.88 & 39.64 & 1 \\
\hline 28-May & 100 & 9.52 & 0.28 & 276.81 & 38.41 & 1 \\
\hline 28-May & 200 & 10.39 & 0.29 & 274.37 & 45.95 & 3 \\
\hline 28-May & 300 & 4.23 & 0.29 & 236.53 & 43.55 & 3 \\
\hline 28-May & 400 & 4.31 & 0.26 & 270.24 & 53.50 & 3 \\
\hline 28-May & 500 & 7.14 & 0.28 & 275.66 & 48.15 & 3 \\
\hline 28-May & 600 & 9.52 & 0.28 & 254.55 & 78.27 & 3 \\
\hline 28-May & 700 & 2.10 & 0.34 & 245.85 & 67.19 & 5 \\
\hline 28-May & 800 & 2.46 & 0.36 & 289.72 & 51.04 & 4 \\
\hline 28-May & 900 & 2.97 & 0.41 & 303.37 & 52.13 & 4 \\
\hline 28-May & 1000 & 3.17 & 0.40 & 282.25 & 57.57 & 2 \\
\hline 28-May & 1100 & 2.93 & 0.38 & 287.99 & 53.97 & 2 \\
\hline 28-May & 1200 & 2.82 & 0.39 & 278.56 & 51.38 & 4 \\
\hline 28-May & 1300 & 3.05 & 0.36 & NA & NA & NA \\
\hline 28-May & 1400 & 2.86 & 0.43 & 273.88 & 48.80 & 4 \\
\hline 28-May & 1500 & 2.69 & 0.45 & 273.06 & 45.94 & 2 \\
\hline 28-May & 1600 & 2.97 & 0.48 & 267.31 & 45.35 & 2 \\
\hline 28-May & 1700 & 3.01 & 0.55 & 264.14 & 43.50 & 2 \\
\hline 28-May & 1800 & 3.05 & 0.56 & NA & NA & NA \\
\hline 28-May & 1900 & 3.63 & 0.63 & 273.71 & 44.46 & 2 \\
\hline 28-May & 2000 & 3.52 & 0.69 & 263.70 & 39.13 & 2 \\
\hline 28-May & 2100 & 3.63 & 0.61 & 255.31 & 41.46 & 2 \\
\hline 28-May & 2200 & 3.69 & 0.63 & 243.93 & 39.83 & 2 \\
\hline 28-May & 2300 & 3.69 & 0.70 & 263.65 & 42.60 & 2 \\
\hline 29-May & 0 & 3.69 & 0.63 & 263.06 & 49.32 & 2 \\
\hline 29-May & 100 & 4.01 & 0.61 & 285.53 & 42.03 & 2 \\
\hline 29-May & 200 & 3.69 & 0.59 & 276.35 & 42.26 & 2 \\
\hline
\end{tabular}




\begin{tabular}{|c|c|c|c|c|c|c|}
\hline \multicolumn{7}{|c|}{\begin{tabular}{|l} 
Appendix D1 \\
Measured XM99 Wave Parameters
\end{tabular}} \\
\hline Date & $\begin{array}{c}\text { Time } \\
\text { (Hour) }\end{array}$ & $\begin{array}{c}T_{p} \\
(\mathbf{s e c})\end{array}$ & $\begin{array}{l}H_{m 0} \\
(\mathbf{m})\end{array}$ & $\begin{array}{c}\text { Mean } \\
\text { Direction } \bar{\theta} \\
(\mathrm{deg})\end{array}$ & $\begin{array}{l}\text { Spread } \sigma_{m} \\
\quad(\text { deg })\end{array}$ & Mode \\
\hline 29-May & 300 & 7.37 & 0.60 & 278.13 & 47.61 & 4 \\
\hline 29-May & 400 & 4.01 & 0.60 & 264.43 & 44.40 & 2 \\
\hline 29-May & 500 & 3.87 & 0.58 & 274.09 & 47.94 & 2 \\
\hline 29-May & 600 & 4.40 & 0.59 & 243.35 & 33.14 & 2 \\
\hline 29-May & 700 & 4.57 & 0.68 & 264.85 & 57.45 & 2 \\
\hline 29-May & 800 & 4.08 & 0.73 & 288.38 & 51.71 & 2 \\
\hline 29-May & 900 & 4.76 & 0.68 & 260.63 & 41.04 & 2 \\
\hline 29-May & 1000 & 4.76 & 0.67 & 239.46 & 38.14 & 2 \\
\hline 29-May & 1100 & 4.16 & 0.66 & 257.78 & 36.90 & 2 \\
\hline 29-May & 1200 & 4.86 & 0.67 & 263.78 & 39.71 & 2 \\
\hline 29-May & 1300 & 5.19 & 0.68 & 253.17 & 29.47 & 2 \\
\hline 29-May & 1400 & 4.40 & 0.76 & 263.11 & 35.00 & 2 \\
\hline 29-May & 1500 & 4.66 & 1.07 & 257.79 & 35.51 & 2 \\
\hline 29-May & 1600 & 5.71 & 1.15 & 249.07 & 32.86 & 2 \\
\hline 29-May & 1700 & 6.02 & 1.07 & 252.59 & 31.10 & 2 \\
\hline 29-May & 1800 & 5.19 & 1.01 & 253.01 & 32.81 & 2 \\
\hline 29-May & 1900 & 5.86 & 1.00 & 259.75 & 32.24 & 2 \\
\hline 29-May & 2000 & 5.71 & 0.99 & 269.50 & 38.78 & 2 \\
\hline 29-May & 2100 & 5.71 & 0.98 & 262.38 & 32.71 & 2 \\
\hline 29-May & 2200 & 5.86 & 0.99 & 259.69 & 32.73 & 2 \\
\hline \multicolumn{7}{|l|}{ Notes: } \\
\hline \multicolumn{7}{|c|}{ 1. Mode signifies type of spectral shape } \\
\hline \multicolumn{7}{|c|}{$1=$ swell only } \\
\hline \multicolumn{7}{|c|}{$2=$ sea only } \\
\hline \multicolumn{7}{|c|}{$3=$ bimodal swell dominant } \\
\hline \multicolumn{7}{|c|}{$4=$ bimodal sea dominant } \\
\hline 2. $\mathrm{NA}=$ & vailable & & & & & \\
\hline
\end{tabular}


APPENDIX E

XM99 MASS MOMENTS OF INERTIA 


\section{Theory}

This Appendix descrixbes the procedure for calculating the mass moments of inertia for the XM99 as used in the WAMIT numerical model. Details are given for the pitch, roll, and yaw moments. The RIBS XM99 is idealized as a beam or channel (American Institute of Steel Construction Handbook 1990) in these calculations.

\section{Global coordinate system}

The global coordinate system and layout for the RIBS is the same as used in the main report for the WAMIT layout.

\section{Pitch mass moment of inertia}

Figure E.1 is a schematic of the RIBS XM99 layout for one leg relative to pitch motions. A local coordinate system is defined relative to the global system. The y' axis is parallel to the longitudinal axis of the leg, with origin at the center of gravity C. The XM99 leg has length $\mathrm{L}=76.2 \mathrm{~m}$ and beam $\mathrm{B}=2.44 \mathrm{~m}$. The angle $\varphi$ between the local $\mathrm{x}$ '-axis and the global $\mathrm{x}$-axis is

$$
\varphi=90-\theta_{r}
$$

The first step is to calculate the area moment of inertia $\mathrm{I}_{\mathrm{y}}$ about the global $\mathrm{y}$-axis for the one leg. It is defined as

$$
I_{y}=I_{x} \sin ^{2} \varphi+I_{y} \cos ^{2} \varphi
$$

where,

$\mathrm{I}_{\mathrm{x}},=\frac{B L^{3}}{12}=$ area moment of inertia about the local $\mathrm{x}$ '-axis and

$\mathrm{I}_{\mathrm{y}^{\prime}}=\frac{L B^{3}}{12}=$ area moment of inertia about the local y'-axis.

After substitution and rearranging, $\mathrm{I}_{\mathrm{y}}$ becomes

$$
I_{y}=\frac{B L}{12}\left[\left(L \cos \theta_{r}\right)^{2}+\left(B \sin \theta_{r}\right)^{2}\right]
$$

The next step is to calculate the pitch mass moment of inertia $\mathrm{I}_{\mathrm{yy}}$ given by

$$
I_{y y}=\frac{\rho B L T}{12}\left[\left(L \cos \theta_{r}\right)^{2}+\left(B \sin \theta_{r}\right)^{2}\right]
$$


where $\rho$ is water density and T is the RIBS draft. Finally, the total pitch moment for both RIBS legs is

$$
I_{y y_{t o t}}=2 I_{y y}=\frac{\rho B L T}{6}\left[\left(L \cos \theta_{r}\right)^{2}+\left(B \sin \theta_{r}\right)^{2}\right]
$$

\section{Roll mass moment of inertia}

The procedure is similar as before for the pitch moment. Because of the offset $y_{c}$ of the center of gravity with the global $\mathrm{x}$-axis, the parallel axis theorem is required to calculate the area moment of inertia for one leg about the $\mathrm{x}$-axis. It is given by

$$
I_{x}=\bar{I}_{x}+A y_{c}^{2}
$$

where

$$
\bar{I}_{x}=I_{\dot{x}} \cos ^{2} \varphi+I_{\dot{y}} \sin ^{2} \varphi=\frac{B L}{12}\left[\left(L \sin \theta_{r}\right)^{2}+\left(B \cos \theta_{r}\right)^{2}\right]
$$

and

$$
A y_{c}^{2}=B L\left(\frac{L}{2} \cos \varphi_{r}\right)^{2}=\frac{B L^{3}}{4} \sin ^{2} \theta_{r}
$$

Combining Equation E.6 and Equation E.7 and rearranging, the area moment of inertia about the $\mathrm{x}$-axis is

$$
I_{x}=\frac{B L}{12}\left[4\left(L \sin \theta_{r}\right)^{2}+\left(B \cos \theta_{r}\right)^{2}\right]
$$

The roll mass moment of inertia $I_{x x}$ for one RIBS leg is

$$
I_{x x}=\rho T I_{x}=\frac{\rho B L T}{12}\left[4\left(L \sin \theta_{r}\right)^{2}+\left(B \cos \theta_{r}\right)^{2}\right]
$$

Finally, the mass moment of inertia in roll for both RIBS legs is

$$
I_{x x_{t o t}}=2 I_{x x}=\frac{\rho B L T}{6}\left[4\left(L \sin \theta_{r}\right)^{2}+\left(B \cos \theta_{r}\right)^{2}\right]
$$

\section{Yaw mass moment of inertia}

The yaw mass moment of inertia $\mathrm{I}_{z z t o t}$ is just the sum of the pitch and roll moments given as

$$
I_{z z_{t o t}}=I_{x x_{t o t}}+I_{y y_{t o t}}
$$




\section{Example Problem}

For the XM99, the input parameters are

$$
\begin{gathered}
\mathrm{L}=76.2 \mathrm{~m} \\
\mathrm{~B}=2.44 \mathrm{~m} \\
\mathrm{~T}=7.32 \mathrm{~m} \\
\theta_{\mathrm{r}}=30 \mathrm{deg} \\
\rho=1025 \mathrm{~kg} / \mathrm{m}^{3}
\end{gathered}
$$

The roll mass moment of inertia is given by Equation E.10 as

$$
I_{x x_{\text {tot }}}=\frac{1025(2.44)(76.2)(7.32)}{6}\left[4(76.2 \sin 30)^{2}+(2.44 \cos 30)^{2}\right]=1.351 x 10^{9} \mathrm{~kg}-\mathrm{m}^{2}
$$

The pitch mass moment of inertia is given by Equation E.4 as

$$
I_{y y_{\text {tot }}}=\frac{1025(2.44)(76.2)(7.32)}{6}\left[(76.2 \cos 30)^{2}+(2.44 \sin 30)^{2}\right]=1.013 \times 10^{9} \mathrm{~kg}-\mathrm{m}^{2}
$$

Finally, the yaw mass moment of inertia is given by Equation E.11

$$
I_{z z_{t o t}}=2.364 \times 10^{9} \mathrm{~kg}-\mathrm{m}^{2}
$$
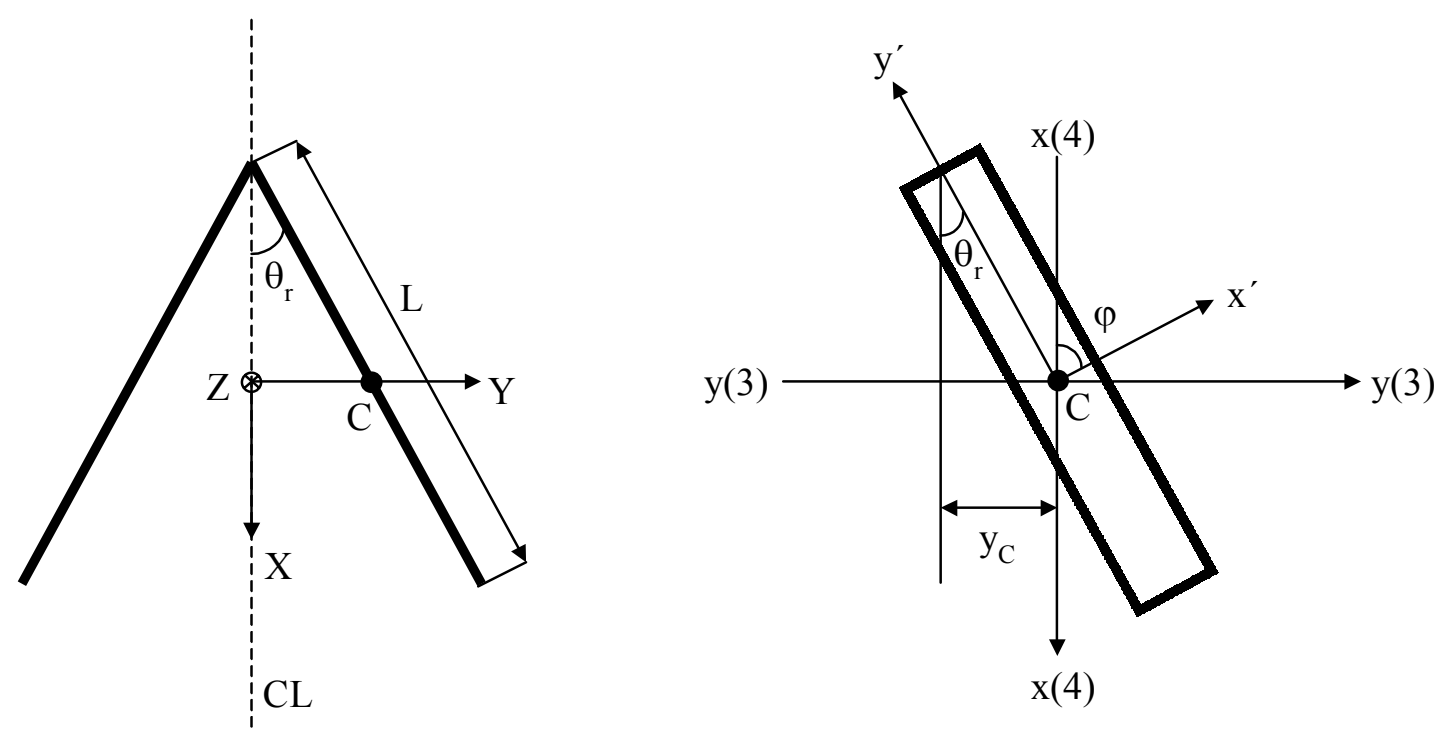

Figure E1. Schematic of RIBS XM99 for moment of inertia calculations. (a) plan view with global coordinate system, (b) expanded view of one leg with local coordinate systems. 
APPENDIX F

FLab WAVE TRANSMISSION COEFFICIENTS 


\begin{tabular}{|c|c|c|c|c|c|c|c|c|}
\hline $\begin{array}{l}\text { Appendix } \\
\text { Measurec } \\
\text { DSWG B }\end{array}$ & $\begin{array}{l}\mathrm{F} 1 \\
\text { Spect } \\
\sin \mathrm{Fu} \\
\end{array}$ & $\begin{array}{l}K_{T} W a \\
\text { dament }\end{array}$ & $\begin{array}{l}\text { Trans } \\
\text { abora }\end{array}$ & $\begin{array}{l}\text { on Co } \\
\text { Exper }\end{array}$ & $\begin{array}{l}\text { ients } \\
\text { it (FL }\end{array}$ & & & \\
\hline Case & Run & $\operatorname{Avg} H$ & & & $\boldsymbol{K}_{T}$ & & & $\operatorname{Avg} K_{T}$ \\
\hline Name & No. & $1-5$ & 6 & 7 & 8 & 9 & 10 & 6-10 \\
\hline $45 \mathrm{deg}$ Int & rior $\mathrm{A}$ & gle, $100^{\circ}$ & netrat & & & & & \\
\hline R3_50D & 7 & 4.73 & 0.19 & 0.18 & 0.20 & 0.24 & 0.28 & 0.22 \\
\hline R3 52D & 7 & 4.44 & 0.18 & 0.20 & 0.20 & 0.23 & 0.37 & 0.23 \\
\hline R3_80D & 6 & 4.81 & 0.28 & 0.24 & 0.25 & 0.31 & 0.32 & 0.28 \\
\hline R3_82D & 6 & 4.79 & 0.29 & 0.22 & 0.21 & 0.30 & 0.38 & 0.28 \\
\hline R3120D & 6 & 4.86 & 0.24 & 0.34 & 0.30 & 0.38 & 0.38 & 0.33 \\
\hline R3122D & 6 & 4.90 & 0.20 & 0.26 & 0.22 & 0.30 & 0.37 & 0.27 \\
\hline R5120D & 6 & 11.55 & 0.23 & 0.29 & 0.28 & 0.36 & 0.38 & 0.31 \\
\hline R5122D & 6 & 11.88 & 0.20 & 0.26 & 0.24 & 0.31 & 0.37 & 0.28 \\
\hline $45 \mathrm{deg}$ Int & rior $\mathrm{A}$ & gle, $75 \%$ & netratic & & & & & \\
\hline R3 50D & 8 & 4.95 & 0.22 & 0.23 & 0.21 & 0.29 & 0.30 & 0.25 \\
\hline R3 52D & 8 & 4.66 & 0.20 & 0.21 & 0.23 & 0.24 & 0.39 & 0.25 \\
\hline R3 80D & 8 & 4.84 & 0.52 & 0.54 & 0.57 & 0.60 & 0.50 & 0.55 \\
\hline R3 $82 \mathrm{D}$ & 8 & 4.73 & 0.48 & 0.44 & 0.44 & 0.45 & 0.60 & 0.49 \\
\hline R3120D & 8 & 4.97 & 0.71 & 0.69 & 0.70 & 0.70 & 0.75 & 0.71 \\
\hline R3122D & 8 & 4.85 & 0.75 & 0.70 & 0.69 & 0.71 & 0.67 & 0.71 \\
\hline R5120D & 8 & 11.69 & 0.68 & 0.66 & 0.67 & 0.68 & 0.72 & 0.68 \\
\hline R5122D & 8 & 11.88 & 0.65 & 0.60 & 0.59 & 0.56 & 0.65 & 0.61 \\
\hline $45 \mathrm{deg}$ In & $01 \mathrm{~A}$ & gle, $50 \%$ & netratic & & & & & \\
\hline R3 50D & 9 & 4.89 & 0.35 & 0.35 & 0.35 & 0.39 & 0.41 & 0.37 \\
\hline R3 52D & 9 & 4.61 & 0.32 & 0.31 & 0.33 & 0.33 & 0.44 & 0.35 \\
\hline R3 80D & 9 & 4.81 & 0.74 & 0.74 & 0.77 & 0.78 & 0.68 & 0.74 \\
\hline R3_82D & 9 & 4.68 & 0.66 & 0.63 & 0.61 & 0.60 & 0.69 & 0.64 \\
\hline R3120D & 9 & 4.93 & 0.85 & 0.83 & 0.83 & 0.82 & 0.82 & 0.83 \\
\hline R3122D & 9 & 4.81 & 0.91 & 0.85 & 0.87 & 0.85 & 0.77 & 0.85 \\
\hline R5120D & 9 & 11.64 & 0.83 & 0.82 & 0.83 & 0.84 & 0.85 & 0.83 \\
\hline R5122D & 9 & 11.73 & 0.82 & 0.80 & 0.80 & 0.75 & 0.79 & 0.79 \\
\hline
\end{tabular}




\begin{tabular}{|c|c|c|c|c|c|c|c|c|}
\hline $\begin{array}{l}\text { Appendi } \\
\text { Measure } \\
\text { DSWG B }\end{array}$ & $\begin{array}{l}\text { F1 } \\
\text { Spect } \\
\sin \text { Fu }\end{array}$ & $\begin{array}{l}K_{T} \mathrm{Wa} \\
\text { dament }\end{array}$ & $\begin{array}{l}\text { Trans } \\
\text { abora }\end{array}$ & $\begin{array}{l}\text { on Co } \\
\text { Exper }\end{array}$ & $\begin{array}{l}\text { ients } \\
\text { it (FL }\end{array}$ & & & \\
\hline Case & Run & $\operatorname{Avg} H$ & & & $\boldsymbol{K}_{T}$ & & & $\operatorname{Avg} K_{T}$ \\
\hline Name & & $1-5$ & 6 & 7 & 8 & 9 & 10 & 6-10 \\
\hline $45 \mathrm{deg}$ In & rior $\mathrm{A}$ & gle, $25 \%$ & netrati & & & & & \\
\hline R3_50D & 10 & 4.87 & 0.67 & 0.64 & 0.65 & 0.66 & 0.66 & 0.66 \\
\hline R3 52D & 10 & 4.55 & 0.65 & 0.60 & 0.63 & 0.64 & 0.66 & 0.64 \\
\hline R3 80D & 10 & 4.80 & 0.88 & 0.89 & 0.91 & 0.93 & 0.82 & 0.89 \\
\hline R3_82D & 10 & 4.66 & 0.80 & 0.78 & 0.80 & 0.79 & 0.80 & 0.79 \\
\hline R3120D & 10 & 4.90 & 0.94 & 0.91 & 0.91 & 0.93 & 0.90 & 0.92 \\
\hline R3122D & 10 & 4.77 & 0.97 & 0.90 & 0.93 & 0.94 & 0.83 & 0.91 \\
\hline R5120D & 10 & 11.60 & 0.94 & 0.90 & 0.90 & 0.95 & 0.86 & 0.91 \\
\hline R5122D & 10 & 11.72 & 0.95 & 0.95 & 0.94 & 0.94 & 0.88 & 0.93 \\
\hline $90 \mathrm{deg}$ In & rior $\mathrm{A}$ & gle, $100^{\circ}$ & tidat & & & & & \\
\hline R3_50D & 20 & 6.77 & 0.15 & 0.14 & 0.16 & 0.16 & 0.18 & 0.16 \\
\hline R3_52D & 20 & 6.77 & 0.14 & 0.12 & 0.15 & 0.15 & 0.21 & 0.16 \\
\hline R3 $80 \mathrm{D}$ & 20 & 6.63 & 0.23 & 0.17 & 0.18 & 0.19 & 0.20 & 0.20 \\
\hline R3_82D & 20 & 6.34 & 0.22 & 0.18 & 0.19 & 0.23 & 0.21 & 0.21 \\
\hline R3120D & 20 & 6.16 & 0.20 & 0.23 & 0.21 & 0.22 & 0.21 & 0.21 \\
\hline R3122D & 20 & 6.08 & 0.18 & 0.23 & 0.21 & 0.22 & 0.26 & 0.22 \\
\hline R5120D & 20 & 14.35 & 0.20 & 0.26 & 0.22 & 0.25 & 0.24 & 0.23 \\
\hline R5122D & 20 & 14.93 & 0.19 & 0.23 & 0.23 & 0.24 & 0.27 & 0.23 \\
\hline $90 \mathrm{deg}$ In & rior $\mathrm{A}$ & gle, $50 \%$ & eetratic & & & & & \\
\hline R3_50D & 22 & 6.36 & 0.28 & 0.26 & 0.27 & 0.27 & 0.27 & 0.27 \\
\hline R3_52D & 22 & 6.31 & 0.24 & 0.24 & 0.25 & 0.25 & 0.28 & 0.25 \\
\hline R3 $80 \mathrm{D}$ & 22 & 5.68 & 0.62 & 0.59 & 0.61 & 0.62 & 0.55 & 0.60 \\
\hline R3_82D & 22 & 5.49 & 0.51 & 0.52 & 0.52 & 0.52 & 0.51 & 0.52 \\
\hline R3120D & 22 & 5.49 & 0.76 & 0.70 & 0.72 & 0.76 & 0.69 & 0.73 \\
\hline R3122D & 22 & 5.20 & 0.82 & 0.75 & 0.76 & 0.76 & 0.66 & 0.75 \\
\hline R5120D & 22 & 12.45 & 0.78 & 0.70 & 0.69 & 0.76 & 0.65 & 0.71 \\
\hline R5122D & 22 & 12.58 & 0.72 & 0.74 & 0.68 & 0.70 & 0.68 & 0.70 \\
\hline
\end{tabular}




\begin{tabular}{|c|c|c|c|c|c|c|c|c|}
\hline \multicolumn{9}{|c|}{$\begin{array}{l}\text { Appendix F2 } \\
\text { Measured Monochromatic } K_{T} \text { Wave Transmission Coefficients } \\
\text { DSWG Basin Fundamental Laboratory Experiment (FLab) }\end{array}$} \\
\hline \multirow{2}{*}{$\begin{array}{l}\text { Case } \\
\text { Name } \\
\end{array}$} & \multirow{2}{*}{$\begin{array}{l}\text { Run } \\
\text { No. }\end{array}$} & \multirow{2}{*}{$\begin{array}{c}\operatorname{Avg} H \\
1-5 \\
\end{array}$} & \multicolumn{5}{|c|}{$K_{T}$} & \multirow{2}{*}{$\begin{array}{c}\operatorname{Avg} K_{T} \\
6-10\end{array}$} \\
\hline & & & 6 & 7 & 8 & 9 & 10 & \\
\hline \multicolumn{9}{|c|}{$45 \mathrm{deg}$ Interior Angle, $50 \%$ Penetration } \\
\hline R3 $50 \mathrm{M}$ & 9 & 3.22 & 0.28 & 0.39 & 0.37 & 0.41 & 0.48 & 0.39 \\
\hline R3 52M & 9 & 3.45 & 0.15 & 0.22 & 0.22 & 0.30 & 0.34 & 0.25 \\
\hline R3 $80 \mathrm{M}$ & 9 & 3.00 & 0.76 & 1.05 & 0.82 & 0.80 & 0.82 & 0.85 \\
\hline R3_82M & 9 & 3.42 & 0.78 & 0.68 & 0.84 & 0.84 & 1.16 & 0.86 \\
\hline R3120M & 9 & 3.75 & 0.78 & 1.06 & 0.97 & 0.41 & 0.99 & 0.84 \\
\hline R3122M & 9 & 3.52 & 1.03 & 1.17 & 1.44 & 1.09 & 0.99 & 1.15 \\
\hline
\end{tabular}




\begin{tabular}{|c|c|c|c|c|c|c|c|c|}
\hline $\begin{array}{l}\text { Appendix } \\
\text { WAMIT } \\
\text { DSWG B }\end{array}$ & $\begin{array}{l}\text { F3 } \\
\text { Predicte } \\
\text { asin Fun } \\
\end{array}$ & $\begin{array}{c}K_{T} \text { Wa } \\
\text { lamenta }\end{array}$ & $\begin{array}{l}\text { Trans } \\
\text { abora }\end{array}$ & $\begin{array}{l}\text { on Co } \\
\text { Exper }\end{array}$ & $\begin{array}{l}\text { ients } \\
\text { it (FL }\end{array}$ & & & \\
\hline Case & Period & $\operatorname{Dir} \bar{\theta}$ & & & $\boldsymbol{K}_{T}$ & & & $\operatorname{Avg} K_{T}$ \\
\hline Name & (sec) & (deg) & 6 & 7 & 8 & 9 & 10 & $6-10$ \\
\hline $45 \mathrm{deg}$ Int & erior Ang & e, $100 \%$ & netrat & & & & & \\
\hline FL45100 & 0.88 & 0 & 0.07 & 0.14 & 0.22 & 0.27 & 0.39 & 0.22 \\
\hline FL45100 & 0.88 & 20 & 0.03 & 0.13 & 0.16 & 0.20 & 0.33 & 0.17 \\
\hline FL45100 & 1.41 & 0 & 0.32 & 0.13 & 0.24 & 0.36 & 0.55 & 0.32 \\
\hline FL45100 & 1.41 & 20 & 0.15 & 0.03 & 0.20 & 0.19 & 0.48 & 0.21 \\
\hline FL45100 & 2.12 & 0 & 0.18 & 0.33 & 0.18 & 0.14 & 0.42 & 0.25 \\
\hline FL45100 & 2.12 & 20 & 0.26 & 0.44 & 0.25 & 0.39 & 0.53 & 0.38 \\
\hline $45 \mathrm{deg}$ Int & erior Ang & e, $75 \%$ & etratic & & & & & \\
\hline FL4575 & 0.88 & 0 & 0.24 & 0.30 & 0.43 & 0.44 & 0.51 & 0.38 \\
\hline FL4575 & 0.88 & 20 & 0.06 & 0.20 & 0.28 & 0.32 & 0.45 & 0.26 \\
\hline FL4575 & 1.41 & 0 & 0.84 & 0.85 & 0.77 & 0.85 & 0.77 & 0.82 \\
\hline FL4575 & 1.41 & 20 & 0.77 & 0.74 & 0.82 & 0.71 & 0.92 & 0.79 \\
\hline FL4575 & 2.12 & 0 & 0.93 & 0.94 & 0.93 & 0.87 & 1.02 & 0.94 \\
\hline FL4575 & 2.12 & 20 & 0.97 & 1.02 & 0.92 & 1.00 & 1.06 & 1.00 \\
\hline $45 \mathrm{deg}$ Int & erior Ang &, $50 \%$ & etratic & & & & & \\
\hline FL4550 & 0.88 & 0 & 0.42 & 0.57 & 0.66 & 0.63 & 0.67 & 0.59 \\
\hline FL4550 & 0.88 & 20 & 0.26 & 0.38 & 0.45 & 0.45 & 0.54 & 0.42 \\
\hline FL4550 & 1.41 & 0 & 0.95 & 0.96 & 0.95 & 0.98 & 0.92 & 0.95 \\
\hline FL4550 & 1.41 & 20 & 0.98 & 0.96 & 0.98 & 0.92 & 1.04 & 0.98 \\
\hline FL4550 & 2.12 & 0 & 0.98 & 0.98 & 0.97 & 0.96 & 1.01 & 0.98 \\
\hline FL4550 & 2.12 & 20 & 1.00 & 1.02 & 0.99 & 1.02 & 1.04 & 1.01 \\
\hline $45 \mathrm{deg}$ In & erior Ang & e, $25 \%$ & netratic & & & & & \\
\hline FL4525 & 0.88 & 0 & 0.88 & 0.94 & 0.95 & 0.95 & 0.98 & 0.94 \\
\hline FL4525 & 0.88 & 20 & 0.88 & 0.86 & 0.85 & 0.85 & 0.90 & 0.87 \\
\hline FL4525 & 1.41 & 0 & 0.98 & 0.99 & 0.99 & 0.99 & 0.98 & 0.98 \\
\hline FL4525 & 1.41 & 20 & 1.01 & 1.01 & 1.01 & 1.00 & 1.02 & 1.01 \\
\hline FL4525 & 2.12 & 0 & 0.99 & 0.99 & 0.99 & 0.99 & 1.00 & 0.99 \\
\hline FL4525 & 2.12 & 20 & 1 & 1.01 & 1.001 & 1.01 & 1.02 & 1.01 \\
\hline
\end{tabular}




\begin{tabular}{|c|c|c|c|c|c|c|c|c|}
\hline $\begin{array}{l}\text { Appendix } \\
\text { WAMIT } \\
\text { DSWG B }\end{array}$ & $\begin{array}{l}\text { F3 } \\
\text { Predicte } \\
\text { asin Fun } \\
\end{array}$ & $\begin{array}{l}K_{T} \mathrm{Wa} \\
\text { lament }\end{array}$ & $\begin{array}{l}\text { Trans } \\
\text { abora } \\
\end{array}$ & $\begin{array}{l}\text { on Co } \\
\text { Exper } \\
\end{array}$ & $\begin{array}{l}\text { ients } \\
\text { it (FL }\end{array}$ & & & \\
\hline Case & Period & $\operatorname{Dir} \bar{\theta}$ & & & $\boldsymbol{K}_{T}$ & & & $\operatorname{Avg} K_{T}$ \\
\hline Name & $(\mathrm{sec})$ & (deg) & 6 & 7 & 8 & 9 & 10 & $6-10$ \\
\hline $90 \mathrm{deg}$ Int & erior Ang & e, $100^{\circ}$ & enetrat & & & & & \\
\hline FL90100 & 0.88 & 0 & 0.15 & 0.15 & 0.27 & 0.1 & 0.12 & 0.16 \\
\hline FL90100 & 0.88 & 20 & 0.12 & 0.11 & 0.20 & 0.08 & 0.13 & 0.13 \\
\hline FL90100 & 1.41 & 0 & 0.36 & 0.34 & 0.24 & 0.38 & 0.19 & 0.30 \\
\hline FL90100 & 1.41 & 20 & 0.17 & 0.20 & 0.21 & 0.27 & 0.19 & 0.21 \\
\hline FL90100 & 2.12 & 0 & 0.36 & 0.41 & 0.41 & 0.29 & 0.3 & 0.35 \\
\hline FL90100 & 2.12 & 20 & 0.37 & 0.34 & 0.36 & 0.24 & 0.34 & 0.33 \\
\hline $90 \mathrm{deg}$ Int & erior Ang &, $50 \%$ & netrati & & & & & \\
\hline FL9050 & 0.88 & 0 & 0.39 & 0.28 & 0.46 & 0.27 & 0.32 & 0.34 \\
\hline FL9050 & 0.88 & 20 & 0.28 & 0.22 & 0.34 & 0.14 & 0.13 & 0.22 \\
\hline FL9050 & 1.41 & 0 & 0.87 & 0.86 & 0.90 & 0.84 & 0.99 & 0.89 \\
\hline FL9050 & 1.41 & 20 & 0.89 & 0.87 & 0.93 & 0.84 & 0.94 & 0.9 \\
\hline FL9050 & 2.12 & 0 & 0.98 & 0.96 & 1.00 & 0.99 & 0.93 & 0.97 \\
\hline FL9050 & 2.12 & 20 & 0.97 & 1.00 & 0.95 & 0.97 & 1.04 & 0.99 \\
\hline
\end{tabular}




\begin{tabular}{|c|c|c|c|c|c|c|c|c|c|}
\hline \multicolumn{10}{|c|}{$\begin{array}{l}\text { Appendix F4 } \\
\text { Average } K_{T} \text { Wave Transmission Parameters } \\
\text { DSWG Basin Fundamental Laboratory Experiment (FLab) }\end{array}$} \\
\hline \multirow{2}{*}{$\begin{array}{l}\text { Period } \\
\text { (sec) }\end{array}$} & \multirow{2}{*}{$\begin{array}{l}\text { Dir } \bar{\theta} \\
\text { (deg) }\end{array}$} & \multirow[b]{2}{*}{$T_{F}$} & \multirow[b]{2}{*}{$B_{x} / L_{x}$} & \multirow[b]{2}{*}{$H / L_{x}$} & \multirow{2}{*}{$\begin{array}{c}D_{b} / B_{y}{ }^{*} \\
\left(d / L_{x}\right)\end{array}$} & \multicolumn{4}{|c|}{$K_{T}$} \\
\hline & & & & & & PTT & MPTT & WAMIT & Meas \\
\hline \multicolumn{10}{|c|}{45 deg Interior Angle, $100 \%$ Penetration } \\
\hline 0.88 & 0.00 & 0.00 & 0.040 & 0.039 & 0.90 & 0.00 & 0.00 & 0.22 & 0.22 \\
\hline 0.88 & 20.00 & 0.00 & 0.043 & 0.039 & 0.96 & 0.00 & 0.00 & 0.17 & 0.23 \\
\hline 1.41 & 0.00 & 0.00 & 0.018 & 0.018 & 0.41 & 0.00 & 0.00 & 0.32 & 0.28 \\
\hline 1.41 & 20.00 & 0.00 & 0.019 & 0.019 & 0.43 & 0.00 & 0.00 & 0.21 & 0.28 \\
\hline 2.12 & 0.00 & 0.00 & 0.011 & 0.011 & 0.25 & 0.00 & 0.00 & 0.25 & 0.33 \\
\hline 2.12 & 20.00 & 0.00 & 0.012 & 0.012 & 0.26 & 0.00 & 0.00 & 0.38 & 0.27 \\
\hline 2.12 & 0.00 & 0.00 & 0.011 & 0.026 & 0.25 & 0.00 & 0.00 & 0.25 & 0.31 \\
\hline 2.12 & 20.00 & 0.00 & 0.012 & 0.029 & 0.26 & 0.00 & 0.00 & 0.38 & 0.28 \\
\hline \multicolumn{10}{|c|}{45 deg Interior Angle, $75 \%$ Penetration } \\
\hline 0.88 & 0.00 & 0.04 & 0.040 & 0.041 & 0.67 & 0.20 & 0.08 & 0.38 & 0.25 \\
\hline 0.88 & 20.00 & 0.04 & 0.043 & 0.041 & 0.72 & 0.20 & 0.08 & 0.26 & 0.25 \\
\hline 1.41 & 0.00 & 0.17 & 0.018 & 0.018 & 0.30 & 0.41 & 0.29 & 0.82 & 0.55 \\
\hline 1.41 & 20.00 & 0.17 & 0.019 & 0.019 & 0.32 & 0.41 & 0.29 & 0.79 & 0.49 \\
\hline 2.12 & 0.00 & 0.22 & 0.011 & 0.011 & 0.18 & 0.47 & 0.36 & 0.94 & 0.71 \\
\hline 2.12 & 20.00 & 0.22 & 0.012 & 0.012 & 0.20 & 0.47 & 0.36 & 1.00 & 0.71 \\
\hline 2.12 & 0.00 & 0.22 & 0.011 & 0.027 & 0.18 & 0.47 & 0.36 & 0.94 & 0.68 \\
\hline 2.12 & 20.00 & 0.22 & 0.012 & 0.029 & 0.20 & 0.47 & 0.36 & 1.00 & 0.61 \\
\hline \multicolumn{10}{|c|}{45 deg Interior Angle, $50 \%$ Penetration } \\
\hline 0.88 & 0.00 & 0.12 & 0.040 & 0.041 & 0.45 & 0.35 & 0.21 & 0.59 & 0.37 \\
\hline 0.88 & 20.00 & 0.12 & 0.043 & 0.041 & 0.48 & 0.35 & 0.21 & 0.42 & 0.35 \\
\hline 1.41 & 0.00 & 0.37 & 0.018 & 0.018 & 0.20 & 0.61 & 0.54 & 0.95 & 0.74 \\
\hline 1.41 & 20.00 & 0.37 & 0.019 & 0.019 & 0.22 & 0.61 & 0.54 & 0.98 & 0.64 \\
\hline 2.12 & 0.00 & 0.45 & 0.011 & 0.011 & 0.12 & 0.67 & 0.62 & 0.98 & 0.83 \\
\hline 2.12 & 20.00 & 0.45 & 0.012 & 0.012 & 0.13 & 0.67 & 0.62 & 1.01 & 0.85 \\
\hline 2.12 & 0.00 & 0.45 & 0.011 & 0.027 & 0.12 & 0.67 & 0.62 & 0.98 & 0.83 \\
\hline 2.12 & 20.00 & 0.45 & 0.012 & 0.029 & 0.13 & 0.67 & 0.62 & 1.01 & 0.79 \\
\hline
\end{tabular}




\begin{tabular}{|c|c|c|c|c|c|c|c|c|c|}
\hline \multicolumn{10}{|c|}{$\begin{array}{l}\text { Appendix F4 } \\
\text { Average } K_{T} \text { Wave Transmission Parameters } \\
\text { DSWG Basin Fundamental Laboratory Experiment (FLab) }\end{array}$} \\
\hline \multirow{2}{*}{$\begin{array}{l}\text { Period } \\
\text { (sec) }\end{array}$} & \multirow{2}{*}{$\begin{array}{c}\text { Dir } \bar{\theta} \\
\text { (deg) }\end{array}$} & \multirow[b]{2}{*}{$\boldsymbol{T}_{F}$} & \multirow[b]{2}{*}{$B_{x} / L_{x}$} & \multirow[b]{2}{*}{$H / L_{x}$} & \multirow{2}{*}{$\begin{array}{c}D_{b} / B_{y}{ }^{*} \\
\left(d / L_{x}\right)\end{array}$} & \multicolumn{4}{|c|}{$\boldsymbol{K}_{T}$} \\
\hline & & & & & & PTT & MPTT & WAMIT & Meas \\
\hline \multicolumn{10}{|c|}{$45 \mathrm{deg}$ Interior Angle, $25 \%$ Penetration } \\
\hline 0.88 & 0.00 & 0.33 & 0.040 & 0.041 & 0.22 & 0.58 & 0.50 & 0.94 & 0.66 \\
\hline 0.88 & 20.00 & 0.33 & 0.043 & 0.040 & 0.24 & 0.58 & 0.50 & 0.87 & 0.64 \\
\hline 1.41 & 0.00 & 0.63 & 0.018 & 0.018 & 0.10 & 0.79 & 0.77 & 0.98 & 0.89 \\
\hline 1.41 & 20.00 & 0.63 & 0.019 & 0.019 & 0.11 & 0.79 & 0.77 & 1.01 & 0.79 \\
\hline 2.12 & 0.00 & 0.70 & 0.011 & 0.011 & 0.06 & 0.84 & 0.83 & 0.99 & 0.92 \\
\hline 2.12 & 20.00 & 0.70 & 0.012 & 0.012 & 0.07 & 0.84 & 0.83 & 1.01 & 0.91 \\
\hline 2.12 & 0.00 & 0.70 & 0.011 & 0.027 & 0.06 & 0.84 & 0.83 & 0.99 & 0.91 \\
\hline 2.12 & 20.00 & 0.70 & 0.012 & 0.029 & 0.07 & 0.84 & 0.83 & 1.01 & 0.93 \\
\hline \multicolumn{10}{|c|}{$90 \mathrm{deg}$ Interior Angle, $100 \%$ Penetration } \\
\hline 0.88 & 0.00 & 0.00 & 0.031 & 0.056 & 0.49 & 0.00 & 0.00 & 0.16 & 0.16 \\
\hline 0.88 & 20.00 & 0.00 & 0.033 & 0.060 & 0.52 & 0.00 & 0.00 & 0.13 & 0.16 \\
\hline 1.41 & 0.00 & 0.00 & 0.014 & 0.025 & 0.22 & 0.00 & 0.00 & 0.30 & 0.20 \\
\hline 1.41 & 20.00 & 0.00 & 0.015 & 0.025 & 0.23 & 0.00 & 0.00 & 0.21 & 0.21 \\
\hline 2.12 & 0.00 & 0.00 & 0.008 & 0.014 & 0.13 & 0.00 & 0.00 & 0.35 & 0.21 \\
\hline 2.12 & 20.00 & 0.00 & 0.009 & 0.015 & 0.14 & 0.00 & 0.00 & 0.33 & 0.22 \\
\hline 2.12 & 0.00 & 0.00 & 0.008 & 0.033 & 0.13 & 0.00 & 0.00 & 0.35 & 0.23 \\
\hline 2.12 & 20.00 & 0.00 & 0.009 & 0.036 & 0.14 & 0.00 & 0.00 & 0.33 & 0.23 \\
\hline \multicolumn{10}{|c|}{$90 \mathrm{deg}$ Interior Angle, $50 \%$ Penetration } \\
\hline 0.88 & 0.00 & 0.12 & 0.031 & 0.053 & 0.24 & 0.35 & 0.21 & 0.34 & 0.27 \\
\hline 0.88 & 20.00 & 0.12 & 0.033 & 0.056 & 0.26 & 0.35 & 0.21 & 0.22 & 0.25 \\
\hline 1.41 & 0.00 & 0.37 & 0.014 & 0.021 & 0.11 & 0.61 & 0.54 & 0.89 & 0.60 \\
\hline 1.41 & 20.00 & 0.37 & 0.015 & 0.022 & 0.12 & 0.61 & 0.54 & 0.90 & 0.52 \\
\hline 2.12 & 0.00 & 0.45 & 0.008 & 0.013 & 0.07 & 0.67 & 0.62 & 0.97 & 0.73 \\
\hline 2.12 & 20.00 & 0.45 & 0.009 & 0.013 & 0.07 & 0.67 & 0.62 & 0.99 & 0.75 \\
\hline 2.12 & 0.00 & 0.45 & 0.008 & 0.028 & 0.07 & 0.67 & 0.62 & 0.97 & 0.71 \\
\hline 2.12 & 20.00 & 0.45 & 0.009 & 0.031 & 0.07 & 0.67 & 0.62 & 0.99 & 0.70 \\
\hline
\end{tabular}


APPENDIX G

XM99 WAVE TRANSMISSION COEFFICIENTS 


\begin{tabular}{|c|c|c|c|c|c|c|}
\hline \multicolumn{7}{|c|}{$\begin{array}{l}\text { Appendix G1 } \\
\text { Measured Wave Transmission Coefficients } \\
\text { XM99 Field Trials }\end{array}$} \\
\hline \multirow[b]{2}{*}{ Date } & \multirow{2}{*}{$\begin{array}{c}\text { Time } \\
\text { (Hour) }\end{array}$} & \multicolumn{5}{|c|}{ Wave Transmission Coefficients, $K_{T}$} \\
\hline & & $H_{m 0}$ & Wgt & Ave & Save & $T_{p}$ \\
\hline 20-May & 1400 & 0.86 & 0.77 & 0.71 & 0.65 & 0.69 \\
\hline 20-May & 1500 & 0.88 & 0.81 & 0.78 & 0.74 & 0.97 \\
\hline 20-May & 1600 & 0.79 & 0.72 & 0.67 & 0.62 & 1.16 \\
\hline 20-May & 1700 & 0.69 & 0.61 & 0.60 & 0.54 & 0.68 \\
\hline 20-May & 1800 & 0.73 & 0.65 & 0.65 & 0.58 & 0.89 \\
\hline 20-May & 1900 & 0.72 & 0.64 & 0.62 & 0.55 & 0.97 \\
\hline 20-May & 2000 & 0.68 & 0.61 & 0.62 & 0.54 & 0.75 \\
\hline 20-May & 2100 & 0.85 & 0.75 & 0.68 & 0.59 & 1.23 \\
\hline 20-May & 2200 & 0.78 & 0.70 & 0.62 & 0.54 & 1.24 \\
\hline 20-May & 2300 & 0.75 & 0.63 & 0.60 & 0.48 & 1.29 \\
\hline 21-May & 0 & 0.82 & 0.74 & NA & $\mathrm{NA}$ & 0.97 \\
\hline 21-May & 100 & 0.68 & 0.60 & 0.60 & 0.51 & 0.75 \\
\hline 21-May & 200 & 0.78 & 0.68 & 0.61 & 0.51 & 0.98 \\
\hline 21-May & 300 & 0.76 & 0.66 & 0.60 & 0.52 & 0.82 \\
\hline 21-May & 400 & 0.84 & 0.74 & 0.62 & 0.52 & 1.36 \\
\hline 21-May & 500 & 0.75 & 0.67 & 0.61 & 0.52 & 0.88 \\
\hline 21-May & 600 & 0.79 & 0.71 & 0.65 & 0.54 & 0.95 \\
\hline 21-May & 700 & 0.86 & 0.78 & 0.85 & 0.81 & 0.65 \\
\hline 21-May & 800 & 0.82 & 0.76 & 0.75 & 0.71 & 0.95 \\
\hline 21-May & 900 & 0.87 & 0.80 & 0.71 & 0.64 & 0.87 \\
\hline 21-May & 1000 & 0.73 & 0.71 & 0.66 & 0.58 & 0.94 \\
\hline 21-May & 1100 & 0.79 & 0.72 & 0.65 & 0.57 & 0.80 \\
\hline 21-May & 1200 & 0.73 & 0.66 & 0.63 & 0.57 & 0.86 \\
\hline 21-May & 1300 & 0.75 & 0.67 & 0.61 & 0.53 & 0.77 \\
\hline 21-May & 1400 & 0.80 & 0.69 & 0.64 & 0.53 & 0.72 \\
\hline 21-May & 1500 & 0.68 & 0.59 & 0.54 & 0.45 & 0.75 \\
\hline 21-May & 1600 & 0.67 & 0.57 & 0.63 & 0.57 & 0.51 \\
\hline 21-May & 1700 & 0.71 & 0.60 & 0.62 & 0.51 & 1.12 \\
\hline 21-May & 1800 & 0.76 & 0.64 & 0.63 & 0.52 & 1.45 \\
\hline 21-May & 1900 & 0.64 & 0.56 & 0.58 & 0.50 & 0.73 \\
\hline 21-May & 2000 & 0.66 & 0.57 & 0.57 & 0.47 & 0.90 \\
\hline 21-May & 2100 & 0.66 & 0.58 & 0.60 & 0.49 & 0.49 \\
\hline
\end{tabular}




\begin{tabular}{|c|c|c|c|c|c|c|}
\hline \multicolumn{7}{|c|}{$\begin{array}{l}\text { Appendix G1 } \\
\text { Measured Wave Transmission Coefficients } \\
\text { XM99 Field Trials } \\
\end{array}$} \\
\hline \multirow[b]{2}{*}{ Date } & \multirow{2}{*}{$\begin{array}{c}\text { Time } \\
\text { (Hour) }\end{array}$} & \multicolumn{5}{|c|}{ Wave Transmission Coefficients, $K_{T}$} \\
\hline & & $H_{m 0}$ & Wgt & Ave & Save & $T_{p}$ \\
\hline 21-May & 2200 & 0.67 & 0.60 & 0.58 & 0.51 & 0.59 \\
\hline 21-May & 2300 & 0.71 & 0.64 & 0.60 & 0.50 & 0.89 \\
\hline 22-May & 800 & 0.81 & 0.73 & 0.71 & 0.61 & 0.95 \\
\hline 22-May & 900 & 0.72 & 0.66 & 0.63 & 0.55 & 0.59 \\
\hline 22-May & 1000 & 0.77 & 0.69 & 0.67 & 0.56 & 0.91 \\
\hline 22-May & 1100 & 0.73 & 0.66 & 0.62 & 0.52 & 0.96 \\
\hline 22-May & 1200 & 0.67 & 0.61 & 0.57 & 0.48 & 0.90 \\
\hline 22-May & 1300 & 0.68 & 0.59 & 0.59 & 0.47 & 0.86 \\
\hline 22-May & 1400 & 0.36 & 0.63 & 0.60 & 0.49 & 0.69 \\
\hline 22-May & 1500 & 0.68 & 0.61 & 0.59 & 0.49 & 0.91 \\
\hline 22-May & 1600 & 0.70 & 0.62 & 0.60 & 0.49 & 0.86 \\
\hline 22-May & 1700 & 0.63 & 0.57 & 0.57 & 0.46 & 0.58 \\
\hline 22-May & 1800 & 0.70 & 0.62 & 0.61 & 0.49 & 0.66 \\
\hline 22-May & 1900 & 0.73 & 0.63 & 0.61 & 0.51 & 0.95 \\
\hline 22-May & 2000 & 0.69 & 0.63 & 0.62 & 0.54 & 0.63 \\
\hline 23-May & 0 & 0.70 & 0.59 & 0.62 & 0.54 & 0.86 \\
\hline 23-May & 100 & 0.94 & 0.87 & 0.79 & 0.72 & 0.96 \\
\hline 23-May & 200 & 0.94 & 0.85 & 0.78 & 0.65 & 0.68 \\
\hline 23-May & 300 & 0.90 & 0.82 & 0.76 & 0.69 & 0.85 \\
\hline 23-May & 400 & 0.90 & 0.83 & 0.71 & 0.64 & 0.94 \\
\hline 23-May & 500 & 0.88 & 0.80 & 0.74 & 0.65 & 0.52 \\
\hline 23-May & 600 & 0.85 & 0.77 & 0.77 & 0.71 & 0.70 \\
\hline 23-May & 700 & 0.94 & 0.87 & 0.95 & 0.93 & 0.87 \\
\hline 23-May & 800 & 0.99 & 0.87 & 0.82 & 0.76 & 1.14 \\
\hline 23-May & 2000 & 0.54 & 0.44 & 0.44 & 0.33 & 0.71 \\
\hline 23-May & 2100 & 0.85 & 0.69 & 0.93 & 0.89 & 0.34 \\
\hline 23-May & 2200 & 0.80 & 0.66 & 0.62 & 0.49 & 1.44 \\
\hline 23-May & 2300 & 0.73 & 0.63 & 0.60 & 0.49 & 0.77 \\
\hline
\end{tabular}




\begin{tabular}{|c|c|c|c|c|c|c|}
\hline \multicolumn{7}{|c|}{$\begin{array}{l}\text { Appendix G1 } \\
\text { Measured Wave Transmission Coefficients } \\
\text { XM99 Field Trials }\end{array}$} \\
\hline \multirow[b]{2}{*}{ Date } & \multirow{2}{*}{$\begin{array}{c}\text { Time } \\
\text { (Hour) }\end{array}$} & \multicolumn{5}{|c|}{ Wave Transmission Coefficients, $K_{T}$} \\
\hline & & $\boldsymbol{H}_{m \theta}$ & Wgt & Ave & Save & $T_{n}$ \\
\hline 24-May & 1100 & 0.78 & 0.68 & 0.62 & 0.50 & 0.98 \\
\hline 24-May & 1200 & 0.90 & 0.78 & 0.72 & 0.60 & 1.38 \\
\hline 24-May & 1300 & 0.87 & 0.75 & 0.84 & 0.75 & 1.19 \\
\hline 24-May & 1400 & 0.76 & 0.68 & 0.70 & 0.65 & 0.88 \\
\hline 24-May & 1500 & 0.80 & 0.69 & 0.75 & 0.67 & 1.14 \\
\hline 24-May & 1600 & 0.70 & 0.57 & 0.61 & 0.47 & 0.92 \\
\hline 24-May & 1700 & 0.70 & 0.57 & 0.64 & 0.53 & 0.82 \\
\hline 24-May & 1800 & 0.60 & 0.49 & 0.60 & 0.51 & 0.27 \\
\hline 24-May & 1900 & 0.58 & 0.45 & 0.58 & 0.47 & 0.18 \\
\hline 24-May & 2000 & 0.68 & 0.57 & 0.71 & 0.65 & 0.97 \\
\hline 24-May & 2100 & 0.72 & 0.63 & 0.67 & 0.60 & 0.57 \\
\hline 24-May & 2200 & 0.61 & 0.52 & 0.62 & 0.51 & 0.79 \\
\hline 24-May & 2300 & 0.64 & 0.54 & 0.60 & 0.49 & 0.75 \\
\hline 25-May & 0 & 0.75 & 0.62 & 0.73 & 0.64 & 0.93 \\
\hline 25-May & 100 & 0.74 & 0.61 & 0.73 & 0.62 & 0.88 \\
\hline 25-May & 200 & 0.75 & 0.65 & 0.74 & 0.66 & 0.97 \\
\hline 25-May & 300 & 0.78 & 0.65 & 0.79 & 0.75 & 1.08 \\
\hline 25-May & 400 & 0.74 & 0.63 & 0.75 & 0.69 & 0.42 \\
\hline 25-May & 500 & 0.73 & 0.58 & 0.80 & 0.72 & 1.20 \\
\hline 25-May & 600 & 0.74 & 0.60 & 0.80 & 0.71 & 0.99 \\
\hline 25-May & 700 & 0.71 & 0.61 & 0.74 & 0.68 & 0.73 \\
\hline 25-May & 800 & 0.78 & 0.66 & 0.88 & 0.82 & 0.78 \\
\hline 25-May & 900 & 0.79 & 0.66 & 0.78 & 0.68 & 0.81 \\
\hline 25-May & 1000 & 0.77 & 0.66 & 0.69 & 0.56 & 0.89 \\
\hline 25-May & 1100 & 0.74 & 0.67 & 0.68 & 0.61 & 0.93 \\
\hline 25-May & 1200 & 0.75 & 0.67 & 0.75 & 0.70 & 0.75 \\
\hline 25-May & 1300 & 0.88 & 0.81 & 0.77 & 0.70 & 1.01 \\
\hline 25-May & 1400 & 0.94 & 0.83 & 0.80 & 0.68 & 0.75 \\
\hline 25-May & 1500 & 0.65 & 0.51 & 0.59 & 0.48 & 0.88 \\
\hline 25-May & 1600 & 0.47 & 0.33 & 0.51 & 0.39 & 0.16 \\
\hline 25-May & 1700 & 0.43 & 0.32 & 0.54 & 0.40 & 0.28 \\
\hline 25-May & 1800 & 0.43 & 0.32 & 0.51 & 0.37 & 0.17 \\
\hline
\end{tabular}




\begin{tabular}{|c|c|c|c|c|c|c|}
\hline \multicolumn{7}{|c|}{$\begin{array}{l}\text { Appendix G1 } \\
\text { Measured Wave Transmission Coefficients } \\
\text { XM99 Field Trials } \\
\end{array}$} \\
\hline \multirow[b]{2}{*}{ Date } & \multirow{2}{*}{$\begin{array}{c}\text { Time } \\
\text { (Hour) }\end{array}$} & \multicolumn{5}{|c|}{ Wave Transmission Coefficients, $K_{T}$} \\
\hline & & $\boldsymbol{H}_{m 0}$ & Wgt & Ave & Save & $T_{p}$ \\
\hline 25-May & 1900 & 0.45 & 0.37 & 0.55 & 0.46 & 0.30 \\
\hline 25-May & 2000 & 0.45 & 0.38 & 0.51 & 0.39 & 0.22 \\
\hline 25-May & 2100 & 0.46 & 0.40 & 0.54 & 0.44 & 0.40 \\
\hline 25-May & 2200 & 0.46 & 0.39 & 0.55 & 0.42 & 0.29 \\
\hline 25-May & 2300 & 0.47 & 0.40 & 0.52 & 0.41 & 0.43 \\
\hline 26-May & 0 & NA & 0.66 & NA & NA & 0.93 \\
\hline 26-May & 100 & NA & 0.70 & NA & NA & 1.00 \\
\hline 26-May & 200 & NA & 0.79 & NA & NA & 0.90 \\
\hline 26-May & 300 & NA & 0.83 & NA & NA & 1.17 \\
\hline 26-May & 400 & NA & 0.74 & NA & NA & 0.81 \\
\hline 26-May & 500 & NA & 0.73 & NA & NA & 1.01 \\
\hline 26-May & 600 & NA & 0.70 & NA & NA & 0.87 \\
\hline 26-May & 700 & NA & 0.48 & NA & $\mathrm{NA}$ & 0.65 \\
\hline 26-May & 800 & 0.78 & 0.66 & 0.90 & 0.92 & 0.93 \\
\hline 26-May & 900 & 0.82 & 0.70 & 0.88 & 0.83 & 1.00 \\
\hline 26-May & 1000 & 0.87 & 0.79 & 0.89 & 0.85 & 0.90 \\
\hline 26-May & 1100 & 0.95 & 0.83 & 0.89 & 0.78 & 1.17 \\
\hline 26-May & 1200 & 0.82 & 0.74 & 0.66 & 0.52 & 0.81 \\
\hline 26-May & 1300 & 0.82 & 0.73 & 0.65 & 0.50 & 1.01 \\
\hline 26-May & 1400 & 0.82 & 0.70 & 0.71 & 0.58 & 0.87 \\
\hline 26-May & 1500 & 0.61 & 0.48 & 0.54 & 0.40 & 0.65 \\
\hline 26-May & 1600 & 0.52 & 0.38 & 0.51 & 0.39 & 0.76 \\
\hline 26-May & 1700 & 0.43 & 0.30 & 0.47 & 0.34 & 0.17 \\
\hline 26-May & 1800 & 0.46 & 0.33 & 0.51 & 0.36 & 0.19 \\
\hline 26-May & 1900 & 0.51 & 0.41 & 0.60 & 0.50 & 0.36 \\
\hline 26-May & 2000 & 0.47 & 0.38 & 0.52 & 0.40 & 0.23 \\
\hline 26-May & 2100 & 0.51 & 0.40 & 0.56 & 0.42 & 0.21 \\
\hline 26-May & 2200 & 0.55 & 0.43 & 0.57 & 0.41 & 0.29 \\
\hline 26-May & 2300 & 0.54 & 0.44 & 0.58 & 0.44 & 0.34 \\
\hline
\end{tabular}




\begin{tabular}{|c|c|c|c|c|c|c|}
\hline \multicolumn{7}{|c|}{$\begin{array}{l}\text { Appendix G1 } \\
\text { Measured Wave Transmission Coefficients } \\
\text { XM99 Field Trials }\end{array}$} \\
\hline \multirow[b]{2}{*}{ Date } & \multirow{2}{*}{$\begin{array}{c}\text { Time } \\
\text { (Hour) }\end{array}$} & \multicolumn{5}{|c|}{ Wave Transmission Coefficients, $K_{T}$} \\
\hline & & $\boldsymbol{H}_{m \theta}$ & Wgt & Ave & Save & $T_{n}$ \\
\hline 27-May & 0 & 0.51 & 0.40 & 0.60 & 0.49 & 0.27 \\
\hline 27-May & 100 & 0.54 & 0.44 & 0.64 & 0.55 & 0.28 \\
\hline 27-May & 200 & 0.57 & 0.46 & 0.71 & 0.63 & 0.38 \\
\hline 27-May & 300 & 0.59 & 0.48 & 0.68 & 0.55 & 0.29 \\
\hline 27-May & 400 & 0.55 & 0.47 & 0.57 & 0.44 & 0.47 \\
\hline 27-May & 500 & 0.58 & 0.48 & 0.60 & 0.48 & 0.22 \\
\hline 27-May & 600 & 0.61 & 0.49 & 0.66 & 0.50 & 0.32 \\
\hline 27-May & 700 & 0.70 & 0.57 & 0.78 & 0.61 & 0.45 \\
\hline 27-May & 800 & 0.64 & 0.49 & 0.84 & 0.74 & 0.27 \\
\hline 27-May & 900 & 0.62 & 0.55 & 0.70 & 0.62 & 0.44 \\
\hline 27-May & 1000 & 0.71 & 0.60 & 0.69 & 0.55 & 0.37 \\
\hline 27-May & 1100 & 0.72 & 0.62 & 0.69 & 0.59 & 0.40 \\
\hline 27-May & 1200 & 0.72 & 0.63 & 0.69 & 0.60 & 0.31 \\
\hline 27-May & 1300 & 0.78 & 0.70 & 0.75 & 0.66 & 1.06 \\
\hline 27-May & 1400 & 0.79 & 0.70 & 0.77 & 0.69 & 1.07 \\
\hline 27-May & 1500 & 0.70 & 0.56 & 0.72 & 0.62 & 0.76 \\
\hline 27-May & 1600 & 0.65 & 0.51 & 0.67 & 0.55 & 0.79 \\
\hline 27-May & 1700 & 0.66 & 0.55 & 0.70 & 0.60 & 0.52 \\
\hline 27-May & 1800 & 0.71 & 0.62 & 0.73 & 0.66 & 0.32 \\
\hline 27-May & 1900 & 0.73 & 0.61 & 0.77 & 0.67 & 0.42 \\
\hline 27-May & 2000 & 0.80 & 0.70 & 0.80 & 0.73 & 0.92 \\
\hline 27-May & 2100 & NA & 0.87 & NA & NA & 0.84 \\
\hline 27-May & 2200 & 0.83 & 0.76 & 0.77 & 0.71 & 0.93 \\
\hline 27-May & 2300 & 0.82 & 0.74 & 0.77 & 0.67 & 1.04 \\
\hline 28-May & 1000 & 0.55 & 0.43 & 0.64 & 0.50 & 0.19 \\
\hline 28-May & 1100 & 0.56 & 0.46 & 0.62 & 0.52 & 0.23 \\
\hline 28-May & 1200 & 0.56 & 0.47 & 0.63 & 0.53 & 0.33 \\
\hline 28-May & 1300 & 0.62 & 0.50 & 0.70 & 0.56 & 0.27 \\
\hline 28-May & 1400 & 0.50 & 0.39 & 0.67 & 0.56 & 0.26 \\
\hline 28-May & 1500 & 0.55 & 0.43 & 0.66 & 0.52 & 0.14 \\
\hline 28-May & 1600 & 0.48 & 0.38 & 0.58 & 0.48 & 0.21 \\
\hline 28-May & 1700 & 0.48 & 0.37 & 0.60 & 0.44 & 0.26 \\
\hline
\end{tabular}




\begin{tabular}{|c|c|c|c|c|c|c|}
\hline \multicolumn{7}{|c|}{$\begin{array}{l}\text { Appendix G1 } \\
\text { Measured Wave Transmission Coefficients } \\
\text { XM99 Field Trials } \\
\end{array}$} \\
\hline \multirow[b]{2}{*}{ Date } & \multirow{2}{*}{$\begin{array}{c}\text { Time } \\
\text { (Hour) }\end{array}$} & \multicolumn{5}{|c|}{ Wave Transmission Coefficients, $K_{T}$} \\
\hline & & $\boldsymbol{H}_{m \theta}$ & Wgt & Ave & Save & $T_{p}$ \\
\hline 28-May & 1800 & 0.50 & 0.41 & 0.61 & 0.46 & 0.31 \\
\hline 28-May & 1900 & 0.50 & 0.41 & 0.57 & 0.45 & 0.27 \\
\hline 28-May & 2000 & 0.45 & 0.38 & 0.54 & 0.42 & 0.38 \\
\hline 28-May & 2100 & 0.58 & 0.49 & 0.60 & 0.46 & 0.52 \\
\hline 28-May & 2200 & 0.53 & 0.46 & 0.56 & 0.43 & 0.66 \\
\hline 28-May & 2300 & 0.53 & 0.43 & 0.52 & 0.43 & 0.29 \\
\hline 29-May & 0 & 0.56 & 0.49 & 0.57 & 0.46 & 0.42 \\
\hline 29-May & 100 & 0.56 & 0.48 & 0.53 & 0.42 & 0.62 \\
\hline 29-May & 200 & 0.59 & 0.51 & 0.59 & 0.47 & 0.34 \\
\hline 29-May & 300 & 0.67 & 0.59 & 0.58 & 0.49 & 1.10 \\
\hline 29-May & 400 & 0.66 & 0.56 & 0.59 & 0.49 & 0.42 \\
\hline 29-May & 500 & 0.64 & 0.56 & 0.59 & 0.48 & 0.53 \\
\hline 29-May & 600 & 0.64 & 0.57 & 0.64 & 0.54 & 0.56 \\
\hline 29-May & 700 & 0.56 & 0.49 & 0.60 & 0.47 & 0.64 \\
\hline 29-May & 800 & 0.55 & 0.48 & 0.61 & 0.52 & 0.38 \\
\hline 29-May & 900 & 0.57 & 0.51 & 0.62 & 0.54 & 0.55 \\
\hline 29-May & 1000 & 0.60 & 0.54 & 0.63 & 0.52 & 0.57 \\
\hline 29-May & 1100 & 0.70 & 0.58 & 0.72 & 0.56 & 0.31 \\
\hline 29-May & 1200 & 0.60 & 0.53 & 0.56 & 0.46 & 0.47 \\
\hline 29-May & 1300 & 0.62 & 0.57 & 0.58 & 0.47 & 0.55 \\
\hline 29-May & 1400 & 0.59 & 0.51 & 0.56 & 0.47 & 0.31 \\
\hline 29-May & 1500 & 0.64 & 0.58 & 0.58 & 0.51 & 0.71 \\
\hline 29-May & 1600 & 0.66 & 0.60 & 0.55 & 0.51 & 0.67 \\
\hline 29-May & 1700 & 0.69 & 0.63 & 0.58 & 0.51 & 0.57 \\
\hline 29-May & 1800 & 0.66 & 0.59 & 0.54 & 0.45 & 0.56 \\
\hline 29-May & 1900 & 0.68 & 0.62 & 0.55 & 0.47 & 0.73 \\
\hline 29-May & 2000 & 0.68 & 0.60 & 0.57 & 0.45 & 0.91 \\
\hline 29-May & 2100 & 0.67 & 0.58 & 0.53 & 0.44 & 1.02 \\
\hline 29-May & 2200 & 0.61 & 0.52 & 0.48 & 0.38 & 0.73 \\
\hline
\end{tabular}




\begin{tabular}{|c|c|c|c|c|c|c|}
\hline \multicolumn{6}{|c|}{$\begin{array}{l}\text { Appendix G1 } \\
\text { Measured Wave Transmission Coefficients } \\
\text { XM99 Field Trials }\end{array}$} & \\
\hline \multirow[b]{2}{*}{ Date } & \multirow[b]{2}{*}{$\begin{array}{c}\text { Time } \\
\text { (Hour) }\end{array}$} & \multicolumn{5}{|c|}{ Wave Transmission Coefficients, $K_{T}$} \\
\hline & & $H_{m 0}$ & Wgt & Ave & Save & $T_{p}$ \\
\hline \multicolumn{7}{|c|}{$\begin{array}{l}\text { Notes: } \\
\text { 1. Five different methods of calculating } \mathrm{K}_{\mathrm{T}} \\
\mathrm{H}_{\mathrm{m} 0}=\text { Based on significant wave height } \mathrm{H}_{\mathrm{m} 0} \\
\mathrm{Wgt}=\text { Energy-based weighted average } \\
\text { Ave }=\text { Average of } \mathrm{K}_{\mathrm{T}}(\mathrm{f}) \text { over all frequencies } \\
\text { Save }=\text { Average of } \mathrm{K}_{\mathrm{T}}(\mathrm{f}) \text { over range } \leq 6 \mathrm{sec} \\
\mathrm{T}_{\mathrm{p}}=\mathrm{K}_{\mathrm{T}} \text { at peak period } \mathrm{T}_{\mathrm{p}} \\
\text { 2. } \mathrm{NA}=\text { Not available }\end{array}$} \\
\hline
\end{tabular}




\begin{tabular}{|c|c|c|c|c|c|c|}
\hline \multicolumn{7}{|c|}{$\begin{array}{l}\text { Appendix G2 } \\
\text { Calculated XM99 Wave Transmission Parameters } \\
\text { XM99 Field Trials }\end{array}$} \\
\hline Date & $\begin{array}{c}\text { Time } \\
\text { (Hour) }\end{array}$ & $\begin{array}{c}L \\
(\mathbf{m})\end{array}$ & $k h$ & $T_{F}$ & $B / L$ & $H / L$ \\
\hline 20-May & 1700 & 108.83 & 0.77 & 0.387 & 0.022 & 0.006 \\
\hline 20-May & 1800 & 103.39 & 0.82 & 0.380 & 0.024 & 0.007 \\
\hline 20-May & 1900 & 103.39 & 0.82 & 0.380 & 0.024 & 0.006 \\
\hline 20-May & 2000 & 114.57 & 0.74 & 0.393 & 0.021 & 0.005 \\
\hline 20-May & 2100 & 114.57 & 0.74 & 0.393 & 0.021 & 0.005 \\
\hline 20-May & 2200 & 114.57 & 0.74 & 0.393 & 0.021 & 0.005 \\
\hline 21-May & 0 & 108.83 & 0.77 & 0.387 & 0.022 & 0.005 \\
\hline 21-May & 100 & 108.83 & 0.77 & 0.387 & 0.022 & 0.006 \\
\hline 21-May & 200 & 103.39 & 0.82 & 0.380 & 0.024 & 0.007 \\
\hline 21-May & 300 & 114.57 & 0.74 & 0.393 & 0.021 & 0.005 \\
\hline 21-May & 400 & 108.83 & 0.77 & 0.387 & 0.022 & 0.006 \\
\hline 21-May & 500 & 114.57 & 0.74 & 0.393 & 0.021 & 0.005 \\
\hline 21-May & 600 & 108.83 & 0.77 & 0.387 & 0.022 & 0.005 \\
\hline 21-May & 700 & 103.39 & 0.82 & 0.380 & 0.024 & 0.005 \\
\hline 21-May & 800 & 108.83 & 0.77 & 0.387 & 0.022 & 0.005 \\
\hline 21-May & 1100 & 108.83 & 0.77 & 0.387 & 0.022 & 0.005 \\
\hline 21-May & 1300 & 103.39 & 0.82 & 0.380 & 0.024 & 0.005 \\
\hline 21-May & 1700 & 93.71 & 0.90 & 0.365 & 0.026 & 0.007 \\
\hline 21-May & 1800 & 98.39 & 0.86 & 0.373 & 0.025 & 0.007 \\
\hline 21-May & 1900 & 89.36 & 0.94 & 0.357 & 0.027 & 0.008 \\
\hline 21-May & 2100 & 21.95 & 3.84 & 0.018 & 0.111 & 0.036 \\
\hline 21-May & 2200 & 24.21 & 3.48 & 0.028 & 0.101 & 0.033 \\
\hline 21-May & 2300 & 98.39 & 0.86 & 0.373 & 0.025 & 0.008 \\
\hline 22-May & 800 & 49.08 & 1.72 & 0.203 & 0.050 & 0.015 \\
\hline 22-May & 900 & 53.77 & 1.57 & 0.232 & 0.045 & 0.014 \\
\hline 22-May & 1200 & 103.39 & 0.82 & 0.380 & 0.024 & 0.008 \\
\hline 22-May & 1300 & 98.39 & 0.86 & 0.373 & 0.025 & 0.008 \\
\hline 22-May & 1500 & 98.39 & 0.86 & 0.373 & 0.025 & 0.008 \\
\hline 22-May & 1600 & 98.39 & 0.86 & 0.373 & 0.025 & 0.008 \\
\hline 22-May & 1700 & 98.39 & 0.86 & 0.373 & 0.025 & 0.008 \\
\hline 22-May & 1800 & 103.39 & 0.82 & 0.380 & 0.024 & 0.008 \\
\hline 22-May & 1900 & 98.39 & 0.86 & 0.373 & 0.025 & 0.008 \\
\hline
\end{tabular}




\begin{tabular}{|c|c|c|c|c|c|c|}
\hline \multicolumn{7}{|c|}{$\begin{array}{l}\text { Appendix G2 } \\
\text { Calculated XM99 Wave Transmission Parameters } \\
\text { XM99 Field Trials } \\
\end{array}$} \\
\hline Date & $\begin{array}{c}\text { Time } \\
\text { (Hour) }\end{array}$ & $\begin{array}{c}L \\
(\mathbf{m}) \\
\end{array}$ & $k \boldsymbol{h}$ & $\boldsymbol{T}_{F}$ & $B / L$ & $H / L$ \\
\hline 22-May & 2000 & 89.36 & 0.94 & 0.357 & 0.027 & 0.009 \\
\hline 23-May & 0 & 98.39 & 0.86 & 0.373 & 0.025 & 0.007 \\
\hline 23-May & 100 & 93.71 & 0.90 & 0.365 & 0.026 & 0.006 \\
\hline 23-May & 200 & 103.39 & 0.82 & 0.380 & 0.024 & 0.005 \\
\hline 23-May & 300 & 89.36 & 0.94 & 0.357 & 0.027 & 0.005 \\
\hline 23-May & 400 & 98.39 & 0.86 & 0.373 & 0.025 & 0.005 \\
\hline 23-May & 500 & 85.09 & 0.99 & 0.348 & 0.029 & 0.006 \\
\hline 23-May & 600 & 89.36 & 0.94 & 0.357 & 0.027 & 0.006 \\
\hline 23-May & 700 & 93.71 & 0.90 & 0.365 & 0.026 & 0.005 \\
\hline 23-May & 800 & 85.09 & 0.99 & 0.348 & 0.029 & 0.006 \\
\hline 23-May & 2100 & 38.08 & 2.21 & 0.123 & 0.064 & 0.020 \\
\hline 23-May & 2200 & 89.36 & 0.94 & 0.357 & 0.027 & 0.005 \\
\hline 23-May & 2300 & 93.71 & 0.90 & 0.365 & 0.026 & 0.005 \\
\hline 24-May & 1000 & 89.36 & 0.94 & 0.357 & 0.027 & 0.005 \\
\hline 24-May & 1100 & 89.36 & 0.94 & 0.357 & 0.027 & 0.004 \\
\hline 24-May & 1200 & 85.09 & 0.99 & 0.348 & 0.029 & 0.004 \\
\hline 24-May & 1300 & 89.36 & 0.94 & 0.357 & 0.027 & 0.004 \\
\hline 24-May & 1400 & 98.39 & 0.86 & 0.373 & 0.025 & 0.004 \\
\hline 24-May & 1500 & 81.17 & 1.04 & 0.339 & 0.030 & 0.005 \\
\hline 24-May & 1600 & 85.09 & 0.99 & 0.348 & 0.029 & 0.005 \\
\hline 24-May & 1700 & 93.71 & 0.90 & 0.365 & 0.026 & 0.004 \\
\hline 24-May & 1800 & 11.30 & 7.46 & 0.000 & 0.216 & 0.044 \\
\hline 24-May & 1900 & 11.81 & 7.14 & 0.000 & 0.207 & 0.044 \\
\hline 24-May & 2000 & 77.48 & 1.09 & 0.329 & 0.032 & 0.006 \\
\hline 24-May & 2100 & 9.84 & 8.57 & 0.000 & 0.248 & 0.054 \\
\hline 24-May & 2200 & 85.09 & 0.99 & 0.348 & 0.029 & 0.006 \\
\hline 24-May & 2300 & 89.36 & 0.94 & 0.357 & 0.027 & 0.005 \\
\hline
\end{tabular}




\begin{tabular}{|c|c|c|c|c|c|c|}
\hline \multicolumn{7}{|c|}{$\begin{array}{l}\text { Appendix G2 } \\
\text { Calculated XM99 Wave Transmission Parameters } \\
\text { XM99 Field Trials }\end{array}$} \\
\hline Date & $\begin{array}{c}\text { Time } \\
\text { (Hour) }\end{array}$ & $\begin{array}{c}L \\
(\mathbf{m})\end{array}$ & $k h$ & $T_{F}$ & $B / L$ & $H / L$ \\
\hline 25-May & 0 & 81.17 & 1.04 & 0.339 & 0.030 & 0.005 \\
\hline 25-May & 100 & 85.09 & 0.99 & 0.348 & 0.029 & 0.005 \\
\hline 25-May & 200 & 81.17 & 1.04 & 0.339 & 0.030 & 0.005 \\
\hline 25-May & 300 & 77.48 & 1.09 & 0.329 & 0.032 & 0.006 \\
\hline 25-May & 400 & 25.08 & 3.36 & 0.032 & 0.097 & 0.018 \\
\hline 25-May & 500 & 85.09 & 0.99 & 0.348 & 0.029 & 0.005 \\
\hline 25-May & 600 & 81.17 & 1.04 & 0.339 & 0.030 & 0.005 \\
\hline 25-May & 700 & 81.17 & 1.04 & 0.339 & 0.030 & 0.004 \\
\hline 25-May & 800 & 77.48 & 1.09 & 0.329 & 0.032 & 0.005 \\
\hline 25-May & 1000 & 85.09 & 0.99 & 0.348 & 0.029 & 0.004 \\
\hline 25-May & 1100 & 85.09 & 0.99 & 0.348 & 0.029 & 0.004 \\
\hline 25-May & 1200 & 114.57 & 0.74 & 0.393 & 0.021 & 0.003 \\
\hline 25-May & 1300 & 77.48 & 1.09 & 0.329 & 0.032 & 0.004 \\
\hline 25-May & 1400 & 85.09 & 0.99 & 0.348 & 0.029 & 0.003 \\
\hline 25-May & 1500 & 81.17 & 1.04 & 0.339 & 0.030 & 0.005 \\
\hline 25-May & 1600 & 13.40 & 6.29 & 0.001 & 0.182 & 0.040 \\
\hline 25-May & 1700 & 15.00 & 5.62 & 0.002 & 0.163 & 0.042 \\
\hline 25-May & 1800 & 17.63 & 4.78 & 0.006 & 0.139 & 0.041 \\
\hline 25-May & 1900 & 19.34 & 4.36 & 0.010 & 0.126 & 0.039 \\
\hline 25-May & 2000 & 19.89 & 4.24 & 0.011 & 0.123 & 0.039 \\
\hline 25-May & 2100 & 25.95 & 3.25 & 0.037 & 0.094 & 0.031 \\
\hline 25-May & 2200 & 23.37 & 3.61 & 0.024 & 0.105 & 0.036 \\
\hline 25-May & 2300 & 21.95 & 3.84 & 0.018 & 0.111 & 0.034 \\
\hline 26-May & 0 & 25.08 & 3.36 & 0.032 & 0.097 & 0.027 \\
\hline 26-May & 100 & 22.65 & 3.72 & 0.021 & 0.108 & 0.025 \\
\hline 26-May & 200 & 22.65 & 3.72 & 0.021 & 0.108 & 0.024 \\
\hline 26-May & 300 & 24.21 & 3.48 & 0.028 & 0.101 & 0.021 \\
\hline 26-May & 400 & 24.21 & 3.48 & 0.028 & 0.101 & 0.018 \\
\hline 26-May & 500 & 24.21 & 3.48 & 0.028 & 0.101 & 0.018 \\
\hline 26-May & 600 & 25.08 & 3.36 & 0.032 & 0.097 & 0.016 \\
\hline 26-May & 700 & 21.95 & 3.84 & 0.018 & 0.111 & 0.016 \\
\hline 26-May & 800 & 103.39 & 0.82 & 0.380 & 0.024 & 0.003 \\
\hline 26-May & 900 & 103.39 & 0.82 & 0.380 & 0.024 & 0.003 \\
\hline
\end{tabular}




\begin{tabular}{|c|c|c|c|c|c|c|}
\hline \multicolumn{7}{|c|}{$\begin{array}{l}\text { Appendix G2 } \\
\text { Calculated XM99 Wave Transmission Parameters } \\
\text { XM99 Field Trials }\end{array}$} \\
\hline Date & $\begin{array}{c}\text { Time } \\
\text { (Hour) }\end{array}$ & $\begin{array}{c}L \\
(\mathbf{m})\end{array}$ & $k h$ & $\boldsymbol{T}_{F}$ & $B / L$ & $H / L$ \\
\hline 26-May & 1000 & 103.39 & 0.82 & 0.380 & 0.024 & 0.003 \\
\hline 26-May & 1100 & 70.53 & 1.19 & 0.308 & 0.035 & 0.004 \\
\hline 26-May & 1200 & 108.83 & 0.77 & 0.387 & 0.022 & 0.003 \\
\hline 26-May & 1300 & 103.39 & 0.82 & 0.380 & 0.024 & 0.003 \\
\hline 26-May & 1400 & 70.53 & 1.19 & 0.308 & 0.035 & 0.004 \\
\hline 26-May & 1500 & 108.83 & 0.77 & 0.387 & 0.022 & 0.003 \\
\hline 26-May & 1600 & 89.36 & 0.94 & 0.357 & 0.027 & 0.005 \\
\hline 26-May & 1700 & 12.42 & 6.79 & 0.001 & 0.197 & 0.043 \\
\hline 26-May & 1800 & 13.04 & 6.46 & 0.001 & 0.187 & 0.042 \\
\hline 26-May & 1900 & 14.91 & 5.65 & 0.002 & 0.164 & 0.034 \\
\hline 26-May & 2000 & 17.63 & 4.78 & 0.006 & 0.139 & 0.029 \\
\hline 26-May & 2100 & 17.63 & 4.78 & 0.006 & 0.139 & 0.028 \\
\hline 26-May & 2200 & 20.57 & 4.10 & 0.013 & 0.119 & 0.025 \\
\hline 26-May & 2300 & 19.34 & 4.36 & 0.010 & 0.126 & 0.025 \\
\hline 27-May & 0 & 21.95 & 3.84 & 0.018 & 0.111 & 0.023 \\
\hline 27-May & 100 & 24.21 & 3.48 & 0.028 & 0.101 & 0.020 \\
\hline 27-May & 200 & 25.08 & 3.36 & 0.032 & 0.097 & 0.018 \\
\hline 27-May & 300 & 25.08 & 3.36 & 0.032 & 0.097 & 0.016 \\
\hline 27-May & 400 & 25.95 & 3.25 & 0.037 & 0.094 & 0.016 \\
\hline 27-May & 500 & 25.08 & 3.36 & 0.032 & 0.097 & 0.014 \\
\hline 27-May & 600 & 25.95 & 3.25 & 0.037 & 0.094 & 0.015 \\
\hline 27-May & 700 & 25.95 & 3.25 & 0.037 & 0.094 & 0.013 \\
\hline 27-May & 800 & 24.21 & 3.48 & 0.028 & 0.101 & 0.015 \\
\hline 27-May & 1000 & 16.69 & 5.05 & 0.004 & 0.146 & 0.017 \\
\hline 27-May & 1100 & 21.95 & 3.84 & 0.018 & 0.111 & 0.013 \\
\hline 27-May & 1200 & 18.69 & 4.51 & 0.008 & 0.131 & 0.014 \\
\hline 27-May & 1300 & 103.39 & 0.82 & 0.380 & 0.024 & 0.003 \\
\hline 27-May & 1700 & 7.02 & 12.01 & 0.000 & 0.348 & 0.046 \\
\hline 27-May & 1800 & 10.07 & 8.37 & 0.000 & 0.243 & 0.033 \\
\hline 27-May & 1900 & 7.15 & 11.79 & 0.000 & 0.342 & 0.045 \\
\hline 27-May & 2000 & 81.17 & 1.04 & 0.339 & 0.030 & 0.004 \\
\hline 27-May & 2100 & 103.39 & 0.82 & 0.380 & 0.024 & 0.003 \\
\hline 27-May & 2200 & 108.83 & 0.77 & 0.387 & 0.022 & 0.002 \\
\hline
\end{tabular}




\begin{tabular}{|c|c|c|c|c|c|c|}
\hline \multicolumn{7}{|c|}{$\begin{array}{l}\text { Appendix G2 } \\
\text { Calculated XM99 Wave Transmission Parameters } \\
\text { XM99 Field Trials }\end{array}$} \\
\hline Date & $\begin{array}{c}\text { Time } \\
\text { (Hour) }\end{array}$ & $\begin{array}{c}L \\
(\mathbf{m})\end{array}$ & $k h$ & $T_{F}$ & $B / L$ & $H / L$ \\
\hline 27-May & 2300 & 108.83 & 0.77 & 0.387 & 0.022 & 0.002 \\
\hline 28-May & 1000 & 15.69 & 5.37 & 0.003 & 0.156 & 0.025 \\
\hline 28-May & 1100 & 13.40 & 6.29 & 0.001 & 0.182 & 0.028 \\
\hline 28-May & 1200 & 12.42 & 6.79 & 0.001 & 0.197 & 0.031 \\
\hline 28-May & 1400 & 12.77 & 6.60 & 0.001 & 0.191 & 0.034 \\
\hline 28-May & 1500 & 11.30 & 7.46 & 0.000 & 0.216 & 0.040 \\
\hline 28-May & 1600 & 13.77 & 6.12 & 0.001 & 0.177 & 0.035 \\
\hline 28-May & 1700 & 14.15 & 5.96 & 0.002 & 0.173 & 0.039 \\
\hline 28-May & 1900 & 20.57 & 4.10 & 0.013 & 0.119 & 0.031 \\
\hline 28-May & 2000 & 19.34 & 4.36 & 0.010 & 0.126 & 0.036 \\
\hline 28-May & 2100 & 20.57 & 4.10 & 0.013 & 0.119 & 0.030 \\
\hline 28-May & 2200 & 21.25 & 3.97 & 0.016 & 0.115 & 0.030 \\
\hline 28-May & 2300 & 21.25 & 3.97 & 0.016 & 0.115 & 0.033 \\
\hline 29-May & 0 & 21.25 & 3.97 & 0.016 & 0.115 & 0.030 \\
\hline 29-May & 100 & 25.08 & 3.36 & 0.032 & 0.097 & 0.024 \\
\hline 29-May & 200 & 21.25 & 3.97 & 0.016 & 0.115 & 0.028 \\
\hline 29-May & 300 & 73.88 & 1.14 & 0.319 & 0.033 & 0.008 \\
\hline 29-May & 400 & 25.08 & 3.36 & 0.032 & 0.097 & 0.024 \\
\hline 29-May & 500 & 23.37 & 3.61 & 0.024 & 0.105 & 0.025 \\
\hline 29-May & 600 & 30.11 & 2.80 & 0.063 & 0.081 & 0.020 \\
\hline 29-May & 700 & 32.42 & 2.60 & 0.080 & 0.075 & 0.021 \\
\hline 29-May & 800 & 25.95 & 3.25 & 0.037 & 0.094 & 0.028 \\
\hline 29-May & 900 & 35.08 & 2.40 & 0.100 & 0.070 & 0.019 \\
\hline 29-May & 1000 & 35.08 & 2.40 & 0.100 & 0.070 & 0.019 \\
\hline 29-May & 1100 & 26.97 & 3.12 & 0.043 & 0.091 & 0.024 \\
\hline 29-May & 1200 & 36.50 & 2.31 & 0.111 & 0.067 & 0.018 \\
\hline 29-May & 1300 & 41.30 & 2.04 & 0.148 & 0.059 & 0.016 \\
\hline 29-May & 1400 & 30.11 & 2.80 & 0.063 & 0.081 & 0.025 \\
\hline 29-May & 1500 & 33.67 & 2.50 & 0.089 & 0.073 & 0.032 \\
\hline 29-May & 1600 & 49.08 & 1.72 & 0.203 & 0.050 & 0.023 \\
\hline 29-May & 1700 & 53.77 & 1.57 & 0.232 & 0.045 & 0.020 \\
\hline 29-May & 1800 & 41.30 & 2.04 & 0.148 & 0.059 & 0.024 \\
\hline
\end{tabular}




\begin{tabular}{|c|c|c|c|c|c|c|}
\hline \multicolumn{7}{|c|}{$\begin{array}{l}\text { Appendix G2 } \\
\text { Calculated XM99 Wave Transmission Parameters } \\
\text { XM99 Field Trials }\end{array}$} \\
\hline Date & $\begin{array}{c}\text { Time } \\
\text { (Hour) }\end{array}$ & $\begin{array}{c}L \\
(\mathbf{m})\end{array}$ & $k h$ & $T_{F}$ & $B / L$ & $H / L$ \\
\hline 29-May & 1900 & 51.35 & 1.64 & 0.217 & 0.048 & 0.019 \\
\hline 29-May & 2000 & 49.08 & 1.72 & 0.203 & 0.050 & 0.020 \\
\hline 29-May & 2100 & 49.08 & 1.72 & 0.203 & 0.050 & 0.020 \\
\hline 29-May & 2200 & 51.35 & 1.64 & 0.217 & 0.048 & 0.019 \\
\hline \multicolumn{7}{|c|}{$\begin{array}{l}\text { Notes: } \\
\text { 1. } \mathrm{L}=\text { Wavelength, } \mathrm{m} \\
\mathrm{kh}=\text { nondimensional depth } \\
\mathrm{TF}=\text { Wiegel coefficient } \\
\mathrm{BL}=\mathrm{B} / \mathrm{L}, \text { Beam } / \text { Wavelength } \\
\mathrm{HL}=\mathrm{H} / \mathrm{L}, \text { Wave height/Wavelength } \\
\text { 2. } \mathrm{PTT}=\text { Power Transmission Theory } \\
\text { MPTT }=\text { Modified Power Transmission Theory }\end{array}$} \\
\hline
\end{tabular}


APPENDIX H

FLab DYNAMIC PRESSURES 


\begin{tabular}{|c|c|c|c|c|c|}
\hline \multicolumn{6}{|c|}{\begin{tabular}{|l|} 
Appendix H1 \\
Measured Significant $P_{1, S}$ Dynamic Wave Pressures \\
DSWG Basin Fundamental Laboratory Experiments (FLab) \\
\end{tabular}} \\
\hline \multirow{2}{*}{$\begin{array}{c}\text { Case } \\
\text { Name } \\
\end{array}$} & \multirow{2}{*}{$\begin{array}{c}\text { Run } \\
\text { No. }\end{array}$} & \multicolumn{4}{|c|}{ Gages (kPa) } \\
\hline & & Bow & Mid & Str & $\overline{\text { Avg }}$ \\
\hline \multicolumn{6}{|c|}{$45 \mathrm{deg}$ Interior Angle, $100 \%$ Penetration } \\
\hline R3_50D & 7 & 0.55 & 0.62 & 0.67 & 0.61 \\
\hline R3_52D & 7 & 0.61 & 0.63 & 0.69 & 0.65 \\
\hline R3_80D & 6 & 0.73 & 0.81 & 0.81 & 0.78 \\
\hline R3_82D & 6 & 0.77 & 0.76 & 0.76 & 0.76 \\
\hline R3120D & 6 & 0.77 & 0.89 & 0.84 & 0.84 \\
\hline $\mathrm{R} 3122 \mathrm{D}$ & 6 & 0.93 & 0.97 & 0.85 & 0.92 \\
\hline R5120D & 6 & 4.99 & 5.58 & 5.51 & 5.36 \\
\hline R5122D & 6 & 5.91 & 6.38 & 6.04 & 6.11 \\
\hline \multicolumn{6}{|c|}{$45 \mathrm{deg}$ Interior Angle, $75 \%$ Penetration } \\
\hline R3_50D & 8 & 0.57 & 0.60 & 0.58 & 0.58 \\
\hline $\mathrm{R} 3 \_52 \mathrm{D}$ & 8 & 0.59 & 0.64 & 0.71 & 0.64 \\
\hline R3_80D & 8 & 0.63 & 0.66 & 0.63 & 0.64 \\
\hline $\mathrm{R} 3 \_82 \mathrm{D}$ & 8 & 0.67 & 0.67 & 0.67 & 0.67 \\
\hline R3120D & 8 & 0.60 & 0.64 & 0.62 & 0.62 \\
\hline $\mathrm{R} 3122 \mathrm{D}$ & 8 & 0.69 & 0.69 & 0.65 & 0.68 \\
\hline R5120D & 8 & 3.85 & 4.07 & 4.12 & 4.02 \\
\hline R5122D & 8 & 4.52 & 5.03 & 5.01 & 4.85 \\
\hline \multicolumn{6}{|c|}{$45 \mathrm{deg}$ Interior Angle, $50 \%$ Penetration } \\
\hline R3_50D & 9 & 0.53 & 0.54 & 0.54 & 0.54 \\
\hline R3_52D & 9 & 0.61 & 0.61 & 0.66 & 0.63 \\
\hline R3_80D & 9 & 0.55 & 0.55 & 0.54 & 0.54 \\
\hline R3_82D & 9 & 0.58 & 0.56 & 0.58 & 0.57 \\
\hline R3120D & 9 & 0.56 & 0.55 & 0.52 & 0.54 \\
\hline $\mathrm{R} 3122 \mathrm{D}$ & 9 & 0.61 & 0.57 & 0.55 & 0.58 \\
\hline R5120D & 9 & 3.77 & 3.75 & 3.78 & 3.76 \\
\hline R5122D & 9 & 4.23 & 4.28 & 4.39 & 4.30 \\
\hline \multicolumn{6}{|c|}{45 deg Interior Angle, $25 \%$ Penetration } \\
\hline R3_50D & 10 & 0.40 & 0.40 & 0.42 & 0.41 \\
\hline R3_52D & 10 & 0.48 & 0.46 & 0.52 & 0.49 \\
\hline R3_80D & 10 & 0.46 & 0.44 & 0.43 & 0.45 \\
\hline R3 $82 \mathrm{D}$ & 10 & 0.45 & 0.43 & 0.46 & 0.45 \\
\hline R3120D & 10 & 0.53 & 0.49 & 0.47 & 0.50 \\
\hline R3122D & 10 & 0.52 & 0.49 & 0.49 & 0.50 \\
\hline R5120D & 10 & 3.35 & 3.35 & 3.42 & 3.37 \\
\hline R5122D & 10 & 3.68 & 3.71 & 3.81 & 3.73 \\
\hline
\end{tabular}




\begin{tabular}{|c|c|c|c|c|c|}
\hline \multicolumn{6}{|c|}{\begin{tabular}{|l|} 
Appendix H2 \\
Measured Maximum $P_{1, \text { Max }}$ Dynamic Wave Pressures \\
DSWG Basin Fundamental Laboratory Experiments (FLab) \\
\end{tabular}} \\
\hline \multirow{2}{*}{$\begin{array}{c}\text { Case } \\
\text { Name } \\
\end{array}$} & \multirow{2}{*}{$\begin{array}{c}\text { Run } \\
\text { No. }\end{array}$} & \multicolumn{4}{|c|}{ Gages (kPa) } \\
\hline & & Bow & Mid & Str & Avg \\
\hline \multicolumn{6}{|c|}{$45 \mathrm{deg}$ Interior Angle, $100 \%$ Penetration } \\
\hline R3 50D & 7 & 0.99 & 1.31 & 1.14 & 1.15 \\
\hline R3_52D & 7 & 0.95 & 1.45 & 1.21 & 1.20 \\
\hline R3_80D & 6 & 1.21 & 1.30 & 1.38 & 1.30 \\
\hline R3_82D & 6 & 1.53 & 1.19 & 1.15 & 1.29 \\
\hline R3120D & 6 & 1.31 & 1.57 & 1.55 & 1.48 \\
\hline $\mathrm{R} 3122 \mathrm{D}$ & 6 & 1.44 & 1.69 & 1.35 & 1.49 \\
\hline R5120D & 6 & 7.95 & 8.95 & 7.54 & 8.15 \\
\hline R5122D & 6 & 8.28 & 8.83 & 8.23 & 8.45 \\
\hline \multicolumn{6}{|c|}{$45 \mathrm{deg}$ Interior Angle, $75 \%$ Penetration } \\
\hline R3_50D & 8 & 0.96 & 1.07 & 0.88 & 0.97 \\
\hline $\mathrm{R} 3 \_52 \mathrm{D}$ & 8 & 0.89 & 1.27 & 1.35 & 1.17 \\
\hline R3_80D & 8 & 0.98 & 1.05 & 1.06 & 1.03 \\
\hline $\mathrm{R} 3 \_82 \mathrm{D}$ & 8 & 1.32 & 1.01 & 1.04 & 1.13 \\
\hline R3120D & 8 & 0.95 & 1.21 & 0.96 & 1.04 \\
\hline $\mathrm{R} 3122 \mathrm{D}$ & 8 & 1.10 & 1.21 & 1.05 & 1.12 \\
\hline R5120D & 8 & 6.46 & 6.32 & 6.09 & 6.29 \\
\hline R5122D & 8 & 6.51 & 7.56 & 7.25 & 7.11 \\
\hline \multicolumn{6}{|c|}{$45 \mathrm{deg}$ Interior Angle, $50 \%$ Penetration } \\
\hline R3_50D & 9 & 1.04 & 1.00 & 0.84 & 0.96 \\
\hline R3_52D & 9 & 0.95 & 1.28 & 1.18 & 1.14 \\
\hline R3_80D & 9 & 0.83 & 0.89 & 0.90 & 0.87 \\
\hline R3_82D & 9 & 1.12 & 0.88 & 0.86 & 0.95 \\
\hline R3120D & 9 & 0.88 & 1.03 & 0.78 & 0.89 \\
\hline $\mathrm{R} 3122 \mathrm{D}$ & 9 & 0.99 & 0.98 & 0.91 & 0.96 \\
\hline R5120D & 9 & 5.88 & 5.80 & 6.12 & 5.94 \\
\hline R5122D & 9 & 6.42 & 6.52 & 6.97 & 6.64 \\
\hline \multicolumn{6}{|c|}{45 deg Interior Angle, $25 \%$ Penetration } \\
\hline R3_50D & 10 & 0.80 & 0.78 & 0.68 & 0.76 \\
\hline R3_52D & 10 & 0.70 & 0.87 & 0.88 & 0.82 \\
\hline R3_80D & 10 & 0.70 & 0.69 & 0.72 & 0.70 \\
\hline R3 $82 \mathrm{D}$ & 10 & 0.76 & 0.67 & 0.65 & 0.70 \\
\hline R3120D & 10 & 0.92 & 0.94 & 0.74 & 0.87 \\
\hline R3122D & 10 & 0.77 & 0.87 & 0.74 & 0.79 \\
\hline R5120D & 10 & 5.69 & 5.37 & 4.75 & 5.27 \\
\hline R5122D & 10 & 5.68 & 5.47 & 5.90 & 5.68 \\
\hline
\end{tabular}




\begin{tabular}{|c|c|c|c|c|c|}
\hline \multicolumn{6}{|c|}{\begin{tabular}{|l|} 
Appendix H3 \\
Measured Average $P_{1, A v g}$ Dynamic Wave Pressures \\
DSWG Basin Fundamental Laboratory Experiments (FLab) \\
\end{tabular}} \\
\hline \multirow{2}{*}{$\begin{array}{c}\text { Case } \\
\text { Name } \\
\end{array}$} & \multirow{2}{*}{$\begin{array}{c}\text { Run } \\
\text { No. }\end{array}$} & \multicolumn{4}{|c|}{ Gages (kPa) } \\
\hline & & Bow & Mid & Str & $\overline{\text { Avg }}$ \\
\hline \multicolumn{6}{|c|}{$45 \mathrm{deg}$ Interior Angle, $100 \%$ Penetration } \\
\hline R3 50D & 7 & 0.35 & 0.40 & 0.42 & 0.39 \\
\hline R3_52D & 7 & 0.40 & 0.40 & 0.44 & 0.42 \\
\hline R3_80D & 6 & 0.46 & 0.54 & 0.52 & 0.51 \\
\hline R3_82D & 6 & 0.51 & 0.50 & 0.51 & 0.50 \\
\hline R3120D & 6 & 0.50 & 0.56 & 0.54 & 0.53 \\
\hline $\mathrm{R} 3122 \mathrm{D}$ & 6 & 0.61 & 0.63 & 0.55 & 0.59 \\
\hline R5120D & 6 & 3.24 & 3.67 & 3.69 & 3.53 \\
\hline R5122D & 6 & 3.99 & 4.27 & 4.12 & 4.13 \\
\hline \multicolumn{6}{|c|}{$45 \mathrm{deg}$ Interior Angle, $75 \%$ Penetration } \\
\hline R3_50D & 8 & 0.38 & 0.39 & 0.38 & 0.38 \\
\hline $\mathrm{R} 3 \_52 \mathrm{D}$ & 8 & 0.39 & 0.40 & 0.46 & 0.42 \\
\hline R3_80D & 8 & 0.41 & 0.44 & 0.41 & 0.42 \\
\hline R3 $82 \mathrm{D}$ & 8 & 0.44 & 0.44 & 0.44 & 0.44 \\
\hline R3120D & 8 & 0.38 & 0.40 & 0.40 & 0.40 \\
\hline $\mathrm{R} 3122 \mathrm{D}$ & 8 & 0.44 & 0.45 & 0.42 & 0.43 \\
\hline R5120D & 8 & 2.51 & 2.67 & 2.70 & 2.62 \\
\hline R5122D & 8 & 2.96 & 3.38 & 3.26 & 3.20 \\
\hline \multicolumn{6}{|c|}{$45 \mathrm{deg}$ Interior Angle, $50 \%$ Penetration } \\
\hline R3_50D & 9 & 0.35 & 0.35 & 0.36 & 0.35 \\
\hline R3_52D & 9 & 0.40 & 0.38 & 0.42 & 0.40 \\
\hline R3_80D & 9 & 0.36 & 0.36 & 0.35 & 0.36 \\
\hline R3_82D & 9 & 0.39 & 0.36 & 0.37 & 0.37 \\
\hline R3120D & 9 & 0.36 & 0.34 & 0.34 & 0.35 \\
\hline $\mathrm{R} 3122 \mathrm{D}$ & 9 & 0.39 & 0.37 & 0.35 & 0.37 \\
\hline R5120D & 9 & 2.42 & 2.41 & 2.40 & 2.41 \\
\hline R5122D & 9 & 2.76 & 2.87 & 2.86 & 2.83 \\
\hline \multicolumn{6}{|c|}{45 deg Interior Angle, $25 \%$ Penetration } \\
\hline R3_50D & 10 & 0.26 & 0.25 & 0.28 & 0.26 \\
\hline R3_52D & 10 & 0.32 & 0.29 & 0.33 & 0.31 \\
\hline R3_80D & 10 & 0.31 & 0.30 & 0.28 & 0.30 \\
\hline R3 $82 \mathrm{D}$ & 10 & 0.29 & 0.28 & 0.30 & 0.29 \\
\hline R3120D & 10 & 0.34 & 0.31 & 0.31 & 0.32 \\
\hline R3122D & 10 & 0.33 & 0.32 & 0.32 & 0.32 \\
\hline R5120D & 10 & 2.13 & 2.23 & 2.17 & 2.18 \\
\hline R5122D & 10 & 2.46 & 2.42 & 2.51 & 2.46 \\
\hline
\end{tabular}




\begin{tabular}{|c|c|c|c|c|c|}
\hline $\begin{array}{l}\text { Appendix } 1 \\
\text { Measured I } \\
\text { DSWG Bas }\end{array}$ & ized $S$ & $\begin{array}{l}\bar{p}_{I, S} \mathbf{D} \\
\text { ratory }\end{array}$ & $\begin{array}{l}\text { Nave } \\
\text { ents }(\end{array}$ & & \\
\hline Case & Run & & & & \\
\hline Name & No. & Bow & Mid & Str & Avg \\
\hline 45 deg Inter & $1 \mathrm{le}, 100$ & ation & & & \\
\hline R3 50D & 7 & 1.18 & 1.34 & 1.44 & 1.32 \\
\hline R3 52D & 7 & 1.40 & 1.45 & 1.59 & 1.48 \\
\hline R3_80D & 6 & 1.55 & 1.71 & 1.72 & 1.66 \\
\hline R3_82D & 6 & 1.64 & 1.62 & 1.61 & 1.63 \\
\hline R3120D & 6 & 1.62 & 1.88 & 1.76 & 1.75 \\
\hline R3122D & 6 & 1.94 & 2.03 & 1.76 & 1.91 \\
\hline R5120D & 6 & 4.40 & 4.93 & 4.86 & 4.73 \\
\hline R5122D & 6 & 5.07 & 5.48 & 5.19 & 5.24 \\
\hline $45 \mathrm{deg}$ Inter & le, $75^{\circ}$ & tion & & & \\
\hline R3_50D & 8 & 1.18 & 1.23 & 1.19 & 1.20 \\
\hline R3_52D & 8 & 1.29 & 1.39 & 1.54 & 1.41 \\
\hline R3_80D & 8 & 1.34 & 1.39 & 1.33 & 1.35 \\
\hline R3 82D & 8 & 1.44 & 1.44 & 1.45 & 1.44 \\
\hline R3120D & 8 & 1.22 & 1.31 & 1.27 & 1.27 \\
\hline R3122D & 8 & 1.45 & 1.44 & 1.37 & 1.42 \\
\hline R5120D & 8 & 3.36 & 3.55 & 3.59 & 3.50 \\
\hline R5122D & 8 & 3.88 & 4.32 & 4.30 & 4.17 \\
\hline $45 \mathrm{deg}$ Inter & le, $50^{\circ}$ & tion & & & \\
\hline R3_50D & 9 & 1.11 & 1.13 & 1.13 & 1.12 \\
\hline R3 52D & 9 & 1.35 & 1.34 & 1.47 & 1.38 \\
\hline R3_80D & 9 & 1.16 & 1.16 & 1.14 & 1.15 \\
\hline R3_82D & 9 & 1.27 & 1.23 & 1.26 & 1.25 \\
\hline R3120D & 9 & 1.15 & 1.13 & 1.08 & 1.12 \\
\hline R3122D & 9 & 1.29 & 1.20 & 1.17 & 1.22 \\
\hline R5120D & 9 & 3.30 & 3.28 & 3.31 & 3.30 \\
\hline R5122D & 9 & 3.67 & 3.72 & 3.82 & 3.74 \\
\hline $45 \mathrm{deg}$ Inter & le, $25^{\circ}$ & tion & & & \\
\hline R3_50D & 10 & 0.83 & 0.84 & 0.88 & 0.85 \\
\hline R3_52D & 10 & 1.08 & 1.03 & 1.18 & 1.09 \\
\hline R3_80D & 10 & 0.98 & 0.94 & 0.92 & 0.95 \\
\hline R3 82D & 10 & 0.97 & 0.94 & 1.00 & 0.97 \\
\hline R3120D & 10 & 1.10 & 1.03 & 0.97 & 1.03 \\
\hline R3122D & 10 & 1.11 & 1.06 & 1.04 & 1.07 \\
\hline R5120D & 10 & 2.94 & 2.95 & 3.01 & 2.96 \\
\hline R5122D & 10 & 3.20 & 3.22 & 3.32 & 3.25 \\
\hline
\end{tabular}




\begin{tabular}{|c|c|c|c|c|c|}
\hline \multicolumn{6}{|c|}{$\begin{array}{l}\text { Appendix H5 } \\
\text { Measured Maximum } P_{1, \text { Max }} \text { Dynamic Wave Pressures - Regular Waves } \\
\text { DSWG Basin Fundamental Laboratory Experiments (FLab) }\end{array}$} \\
\hline \multirow{2}{*}{$\begin{array}{c}\text { Case } \\
\text { Name }\end{array}$} & \multirow{2}{*}{$\begin{array}{c}\text { Run } \\
\text { No. }\end{array}$} & \multicolumn{4}{|c|}{ Gages (kPa) } \\
\hline & & Bow & Mid & Str & Avg \\
\hline \multicolumn{6}{|c|}{$45 \mathrm{deg}$ Interior Angle, $100 \%$ Penetration } \\
\hline R3_50M & 7 & 0.37 & 0.41 & 0.50 & 0.43 \\
\hline R3 52M & 7 & 0.50 & 0.35 & 0.41 & 0.42 \\
\hline R3 80M & 6 & 0.50 & 0.58 & 0.73 & 0.61 \\
\hline R3_82M & 6 & 0.64 & 0.64 & 0.70 & 0.66 \\
\hline R3120M & 6 & 0.49 & 0.56 & 0.57 & 0.54 \\
\hline R3122M & 6 & 0.55 & 0.81 & 0.81 & 0.72 \\
\hline
\end{tabular}

\begin{tabular}{|c|c|c|c|c|c|}
\hline \multicolumn{6}{|c|}{$\begin{array}{l}\text { Appendix H6 } \\
\text { Measured Average } P_{1, A v g} \text { Dynamic Wave Pressures - Regular Waves } \\
\text { DSWG Basin Fundamental Laboratory Experiments (FLab) }\end{array}$} \\
\hline \multirow{2}{*}{$\begin{array}{c}\text { Case } \\
\text { Name }\end{array}$} & \multirow{2}{*}{$\begin{array}{l}\text { Run } \\
\text { No. }\end{array}$} & \multicolumn{4}{|c|}{ Gages (kPa) } \\
\hline & & Bow & Mid & Str & Avg \\
\hline \multicolumn{6}{|c|}{45 deg Interior Angle, $100 \%$ Penetration } \\
\hline R3_50M & 7 & 0.33 & 0.38 & 0.46 & 0.39 \\
\hline R3_52M & 7 & 0.41 & 0.31 & 0.36 & 0.36 \\
\hline R3_80M & 6 & 0.44 & 0.52 & 0.65 & 0.54 \\
\hline R3_82M & 6 & 0.58 & 0.57 & 0.63 & 0.59 \\
\hline R3120M & 6 & 0.44 & 0.50 & 0.52 & 0.49 \\
\hline $\mathrm{R} 3122 \mathrm{M}$ & 6 & 0.48 & 0.76 & 0.75 & 0.67 \\
\hline
\end{tabular}




\begin{tabular}{|c|c|c|c|c|c|c|c|}
\hline \multicolumn{8}{|c|}{$\begin{array}{l}\text { Appendix } \mathrm{H} 7 \\
\text { WAMIT Predicted Normalized } \bar{p}_{W} \text { Dynamic Pressures - FL4525 } \\
\text { DSWG Basin Fundamental Laboratory Experiments (FLab) }\end{array}$} \\
\hline \multirow{2}{*}{$\begin{array}{c}X \\
(\mathbf{m})\end{array}$} & \multirow[b]{2}{*}{ Normal $X$} & \multicolumn{6}{|c|}{ Wave Case } \\
\hline & & 50 & 52 & 80 & 82 & 120 & 122 \\
\hline & & & & & & & \\
\hline 0.05 & 0.01 & -0.68 & -0.44 & -0.43 & -0.76 & 0.93 & 0.90 \\
\hline 0.17 & 0.02 & -0.65 & -0.19 & -0.60 & -0.83 & 0.92 & 0.86 \\
\hline 0.28 & 0.03 & -0.46 & 0.11 & -0.73 & -0.86 & 0.89 & 0.81 \\
\hline 0.40 & 0.04 & -0.14 & 0.42 & -0.82 & -0.86 & 0.84 & 0.75 \\
\hline 0.51 & 0.06 & 0.21 & 0.67 & -0.84 & -0.83 & 0.77 & 0.67 \\
\hline 0.63 & 0.07 & 0.50 & 0.81 & -0.82 & -0.76 & 0.68 & 0.58 \\
\hline 0.74 & 0.08 & 0.64 & 0.80 & -0.73 & -0.66 & 0.57 & 0.49 \\
\hline 0.85 & 0.09 & 0.60 & 0.64 & -0.60 & -0.53 & 0.45 & 0.38 \\
\hline 0.97 & 0.11 & 0.39 & 0.35 & -0.43 & -0.38 & 0.32 & 0.27 \\
\hline 1.08 & 0.12 & 0.05 & -0.02 & -0.23 & -0.21 & 0.17 & 0.16 \\
\hline 1.20 & 0.13 & -0.30 & -0.40 & -0.01 & -0.03 & 0.03 & 0.04 \\
\hline 1.31 & 0.14 & -0.56 & -0.70 & 0.20 & 0.15 & -0.12 & -0.08 \\
\hline 1.43 & 0.16 & -0.66 & -0.88 & 0.41 & 0.32 & -0.26 & -0.20 \\
\hline 1.54 & 0.17 & -0.57 & -0.88 & 0.58 & 0.48 & -0.40 & -0.31 \\
\hline 1.65 & 0.18 & -0.30 & -0.71 & 0.72 & 0.62 & -0.52 & -0.42 \\
\hline 1.77 & 0.19 & 0.06 & -0.39 & 0.80 & 0.73 & -0.64 & -0.52 \\
\hline 1.88 & 0.21 & 0.40 & 0.02 & 0.84 & 0.81 & -0.73 & -0.61 \\
\hline 2.00 & 0.22 & 0.63 & 0.42 & 0.81 & 0.86 & -0.81 & -0.70 \\
\hline 2.11 & 0.23 & 0.67 & 0.75 & 0.73 & 0.87 & -0.87 & -0.77 \\
\hline 2.23 & 0.24 & 0.51 & 0.93 & 0.60 & 0.84 & -0.91 & -0.83 \\
\hline 2.34 & 0.26 & 0.20 & 0.92 & 0.43 & 0.77 & -0.92 & -0.88 \\
\hline 2.45 & 0.27 & -0.18 & 0.72 & 0.23 & 0.67 & -0.91 & -0.91 \\
\hline 2.57 & 0.28 & -0.50 & 0.38 & 0.02 & 0.55 & -0.88 & -0.93 \\
\hline 2.68 & 0.29 & -0.68 & -0.04 & -0.20 & 0.39 & -0.83 & -0.94 \\
\hline 2.80 & 0.31 & -0.66 & -0.45 & -0.40 & 0.22 & -0.75 & -0.93 \\
\hline 2.91 & 0.32 & -0.44 & -0.76 & -0.58 & 0.04 & -0.66 & -0.90 \\
\hline 3.03 & 0.33 & -0.08 & -0.93 & -0.72 & -0.14 & -0.55 & -0.86 \\
\hline 3.14 & 0.34 & 0.30 & -0.90 & -0.80 & -0.32 & -0.43 & -0.81 \\
\hline 3.25 & 0.36 & 0.60 & -0.70 & -0.84 & -0.48 & -0.29 & -0.74 \\
\hline 3.37 & 0.37 & 0.71 & -0.36 & -0.81 & -0.62 & -0.15 & -0.66 \\
\hline 3.48 & 0.38 & 0.61 & 0.05 & -0.73 & -0.74 & -0.01 & -0.57 \\
\hline 3.60 & 0.39 & 0.32 & 0.44 & -0.60 & -0.82 & 0.14 & -0.47 \\
\hline 3.71 & 0.41 & -0.07 & 0.74 & -0.43 & -0.87 & 0.28 & -0.37 \\
\hline 3.83 & 0.42 & -0.44 & 0.89 & -0.23 & -0.88 & 0.41 & -0.26 \\
\hline 3.94 & 0.43 & -0.69 & 0.87 & -0.01 & -0.85 & 0.54 & -0.14 \\
\hline
\end{tabular}




\begin{tabular}{|c|c|c|c|c|c|c|c|}
\hline \multicolumn{8}{|c|}{$\begin{array}{l}\text { Appendix } \mathrm{H} 7 \\
\text { WAMIT Predicted Normalized } \bar{p}_{W} \text { Dynamic Pressures - FL4525 } \\
\text { DSWG Basin Fundamental Laboratory Experiments (FLab) }\end{array}$} \\
\hline \multirow{2}{*}{$\begin{array}{c}X \\
(\mathbf{m})\end{array}$} & \multirow[b]{2}{*}{ Normal $X$} & \multicolumn{6}{|c|}{ Wave Case } \\
\hline & & 50 & 52 & 80 & 82 & 120 & 122 \\
\hline 4.05 & 0.44 & -0.72 & 0.68 & 0.20 & -0.78 & 0.65 & -0.02 \\
\hline 4.17 & 0.46 & -0.54 & 0.36 & 0.41 & -0.68 & 0.74 & 0.10 \\
\hline 4.28 & 0.47 & -0.19 & -0.02 & 0.58 & -0.55 & 0.82 & 0.21 \\
\hline 4.40 & 0.48 & 0.22 & -0.40 & 0.72 & -0.39 & 0.88 & 0.33 \\
\hline 4.51 & 0.49 & 0.56 & -0.70 & 0.81 & -0.22 & 0.91 & 0.44 \\
\hline 4.63 & 0.51 & 0.74 & -0.86 & 0.84 & -0.04 & 0.92 & 0.54 \\
\hline 4.74 & 0.52 & 0.70 & -0.86 & 0.81 & 0.15 & 0.91 & 0.63 \\
\hline 4.85 & 0.53 & 0.44 & -0.69 & 0.73 & 0.33 & 0.87 & 0.71 \\
\hline 4.97 & 0.54 & 0.05 & -0.40 & 0.60 & 0.49 & 0.82 & 0.79 \\
\hline 5.08 & 0.56 & -0.36 & -0.02 & 0.43 & 0.63 & 0.74 & 0.84 \\
\hline 5.20 & 0.57 & -0.66 & 0.35 & 0.23 & 0.75 & 0.64 & 0.89 \\
\hline 5.31 & 0.58 & -0.76 & 0.67 & 0.01 & 0.83 & 0.53 & 0.92 \\
\hline 5.43 & 0.59 & -0.63 & 0.85 & -0.21 & 0.88 & 0.41 & 0.94 \\
\hline 5.54 & 0.61 & -0.30 & 0.88 & -0.41 & 0.88 & 0.27 & 0.94 \\
\hline 5.65 & 0.62 & 0.12 & 0.73 & -0.59 & 0.85 & 0.13 & 0.93 \\
\hline 5.77 & 0.63 & 0.51 & 0.45 & -0.72 & 0.78 & -0.02 & 0.90 \\
\hline 5.88 & 0.64 & 0.74 & 0.07 & -0.81 & 0.68 & -0.16 & 0.85 \\
\hline 6.00 & 0.66 & 0.75 & -0.32 & -0.84 & 0.54 & -0.30 & 0.80 \\
\hline 6.11 & 0.67 & 0.53 & -0.66 & -0.81 & 0.39 & -0.44 & 0.73 \\
\hline 6.23 & 0.68 & 0.15 & -0.87 & -0.73 & 0.21 & -0.56 & 0.65 \\
\hline 6.34 & 0.69 & -0.28 & -0.91 & -0.60 & 0.03 & -0.67 & 0.56 \\
\hline 6.46 & 0.71 & -0.62 & -0.77 & -0.42 & -0.16 & -0.76 & 0.46 \\
\hline 6.57 & 0.72 & -0.77 & -0.48 & -0.22 & -0.33 & -0.83 & 0.35 \\
\hline 6.68 & 0.73 & -0.69 & -0.10 & -0.00 & -0.50 & -0.88 & 0.23 \\
\hline 6.80 & 0.74 & -0.39 & 0.31 & 0.21 & -0.64 & -0.91 & 0.12 \\
\hline 6.91 & 0.76 & 0.03 & 0.66 & 0.42 & -0.75 & -0.92 & -0.00 \\
\hline 7.03 & 0.77 & 0.45 & 0.88 & 0.59 & -0.83 & -0.90 & -0.12 \\
\hline 7.14 & 0.78 & 0.73 & 0.92 & 0.73 & -0.87 & -0.87 & -0.24 \\
\hline 7.26 & 0.79 & 0.79 & 0.79 & 0.81 & -0.88 & -0.81 & -0.35 \\
\hline 7.37 & 0.81 & 0.61 & 0.50 & 0.84 & -0.85 & -0.73 & -0.46 \\
\hline 7.48 & 0.82 & 0.25 & 0.11 & 0.82 & -0.77 & -0.63 & -0.56 \\
\hline 7.60 & 0.83 & -0.19 & -0.30 & 0.73 & -0.67 & -0.51 & -0.65 \\
\hline 7.71 & 0.84 & -0.57 & -0.65 & 0.60 & -0.54 & -0.39 & -0.73 \\
\hline 7.83 & 0.86 & -0.77 & -0.87 & 0.42 & -0.38 & -0.25 & -0.80 \\
\hline 7.94 & 0.87 & -0.74 & -0.92 & 0.22 & -0.20 & -0.11 & -0.86 \\
\hline
\end{tabular}




\begin{tabular}{|c|c|c|c|c|c|c|c|}
\hline \multicolumn{8}{|c|}{$\begin{array}{l}\text { Appendix } \mathbf{H} 7 \\
\text { WAMIT Predicted Normalized } \bar{p}_{W} \text { Dynamic Pressures - FL4525 } \\
\text { DSWG Basin Fundamental Laboratory Experiments (FLab) }\end{array}$} \\
\hline \multirow{2}{*}{$\begin{array}{c}X \\
(\mathbf{m}) \\
\end{array}$} & \multirow[b]{2}{*}{ Normal $X$} & \multicolumn{6}{|c|}{ Wave Case } \\
\hline & & 50 & 52 & 80 & 82 & 120 & 122 \\
\hline & & & & & & & \\
\hline 8.06 & 0.88 & -0.48 & -0.79 & -0.00 & -0.02 & 0.04 & -0.90 \\
\hline 8.17 & 0.89 & -0.07 & -0.51 & -0.22 & 0.16 & 0.18 & -0.93 \\
\hline 8.28 & 0.91 & 0.35 & -0.12 & -0.43 & 0.34 & 0.32 & -0.94 \\
\hline 8.40 & 0.92 & 0.67 & 0.29 & -0.60 & 0.50 & 0.46 & -0.94 \\
\hline 8.51 & 0.93 & 0.78 & 0.64 & -0.73 & 0.64 & 0.58 & -0.92 \\
\hline 8.63 & 0.94 & 0.66 & 0.87 & -0.82 & 0.75 & 0.68 & -0.89 \\
\hline 8.74 & 0.96 & 0.33 & 0.94 & -0.84 & 0.82 & 0.77 & -0.84 \\
\hline 8.86 & 0.97 & -0.09 & 0.83 & -0.81 & 0.86 & 0.84 & -0.78 \\
\hline 8.97 & 0.98 & -0.47 & 0.55 & -0.72 & 0.86 & 0.89 & -0.70 \\
\hline 9.08 & 0.99 & -0.68 & 0.16 & -0.57 & 0.80 & 0.91 & -0.61 \\
\hline
\end{tabular}




\begin{tabular}{|c|c|c|c|c|c|c|c|}
\hline \multicolumn{8}{|c|}{$\begin{array}{l}\text { Appendix } \mathrm{H8} \\
\text { WAMIT Predicted Normalized } \bar{p}_{W} \text { Dynamic Pressures - FL4550 } \\
\text { DSWG Basin Fundamental Laboratory Experiments (FLab) }\end{array}$} \\
\hline \multirow{2}{*}{$\begin{array}{c}X \\
(\mathbf{m}) \\
\end{array}$} & \multirow[b]{2}{*}{ Normal $X$} & \multicolumn{6}{|c|}{ Wave Case } \\
\hline & & 50 & 52 & 80 & 82 & 120 & 122 \\
\hline & & & & & & & \\
\hline 0.05 & 0.01 & -0.19 & -0.49 & -0.39 & -0.19 & 0.95 & 0.96 \\
\hline 0.17 & 0.02 & -0.81 & -0.20 & -0.57 & -0.88 & 0.95 & 0.94 \\
\hline 0.28 & 0.03 & -0.61 & 0.21 & -0.71 & -0.95 & 0.92 & 0.90 \\
\hline 0.40 & 0.04 & -0.23 & 0.67 & -0.81 & -0.99 & 0.88 & 0.85 \\
\hline 0.51 & 0.06 & 0.25 & 1.05 & -0.87 & -0.99 & 0.81 & 0.79 \\
\hline 0.63 & 0.07 & 0.68 & 1.25 & -0.87 & -0.96 & 0.73 & 0.71 \\
\hline 0.74 & 0.08 & 0.93 & 1.20 & -0.81 & -0.89 & 0.62 & 0.62 \\
\hline 0.85 & 0.09 & 0.92 & 0.89 & -0.70 & -0.78 & 0.51 & 0.52 \\
\hline 0.97 & 0.11 & 0.64 & 0.37 & -0.54 & -0.64 & 0.38 & 0.42 \\
\hline 1.08 & 0.12 & 0.16 & -0.26 & -0.35 & -0.47 & 0.24 & 0.30 \\
\hline 1.20 & 0.13 & -0.39 & -0.86 & -0.13 & -0.28 & 0.09 & 0.18 \\
\hline 1.31 & 0.14 & -0.84 & -1.29 & 0.09 & -0.07 & -0.05 & 0.06 \\
\hline 1.43 & 0.16 & -1.05 & -1.46 & 0.31 & 0.14 & -0.20 & -0.06 \\
\hline 1.54 & 0.17 & -0.95 & -1.32 & 0.50 & 0.35 & -0.34 & -0.18 \\
\hline 1.65 & 0.18 & -0.56 & -0.89 & 0.67 & 0.54 & -0.47 & -0.30 \\
\hline 1.77 & 0.19 & 0.00 & -0.27 & 0.79 & 0.72 & -0.59 & -0.42 \\
\hline 1.88 & 0.21 & 0.58 & 0.40 & 0.85 & 0.86 & -0.69 & -0.53 \\
\hline 2.00 & 0.22 & 0.99 & 0.99 & 0.86 & 0.97 & -0.78 & -0.63 \\
\hline 2.11 & 0.23 & 1.11 & 1.36 & 0.81 & 1.04 & -0.85 & -0.72 \\
\hline 2.23 & 0.24 & 0.89 & 1.44 & 0.71 & 1.07 & -0.90 & -0.80 \\
\hline 2.34 & 0.26 & 0.39 & 1.22 & 0.56 & 1.05 & -0.92 & -0.87 \\
\hline 2.45 & 0.27 & -0.24 & 0.76 & 0.37 & 0.98 & -0.93 & -0.92 \\
\hline 2.57 & 0.28 & -0.81 & 0.15 & 0.16 & 0.87 & -0.91 & -0.96 \\
\hline 2.68 & 0.29 & -1.14 & -0.47 & -0.07 & 0.72 & -0.86 & -0.99 \\
\hline 2.80 & 0.31 & -1.12 & -0.98 & -0.29 & 0.54 & -0.80 & -0.99 \\
\hline 2.91 & 0.32 & -0.75 & -1.28 & -0.49 & 0.33 & -0.72 & -0.99 \\
\hline 3.03 & 0.33 & -0.14 & -1.33 & -0.66 & 0.11 & -0.61 & -0.97 \\
\hline 3.14 & 0.34 & 0.52 & -1.12 & -0.79 & -0.13 & -0.50 & -0.93 \\
\hline 3.25 & 0.36 & 1.03 & -0.70 & -0.86 & -0.35 & -0.37 & -0.88 \\
\hline 3.37 & 0.37 & 1.24 & -0.17 & -0.88 & -0.57 & -0.23 & -0.81 \\
\hline 3.48 & 0.38 & 1.06 & 0.39 & -0.83 & -0.76 & -0.09 & -0.73 \\
\hline 3.60 & 0.39 & 0.56 & 0.85 & -0.73 & -0.91 & 0.06 & -0.64 \\
\hline 3.71 & 0.41 & -0.12 & 1.16 & -0.58 & -1.03 & 0.20 & -0.54 \\
\hline 3.83 & 0.42 & -0.77 & 1.25 & -0.40 & -1.10 & 0.34 & -0.43 \\
\hline 3.94 & 0.43 & -1.18 & 1.11 & -0.18 & -1.13 & 0.47 & -0.31 \\
\hline
\end{tabular}




\begin{tabular}{|c|c|c|c|c|c|c|c|}
\hline \multicolumn{8}{|c|}{$\begin{array}{l}\text { Appendix } \mathrm{H8} \\
\text { WAMIT Predicted Normalized } \bar{p}_{W} \text { Dynamic Pressures - FL4550 } \\
\text { DSWG Basin Fundamental Laboratory Experiments (FLab) }\end{array}$} \\
\hline \multirow{2}{*}{$\begin{array}{c}X \\
(\mathbf{m}) \\
\end{array}$} & \multirow[b]{2}{*}{ Normal $X$} & \multicolumn{6}{|c|}{ Wave Case } \\
\hline & & 50 & 52 & 80 & 82 & 120 & 122 \\
\hline & & & & & & & \\
\hline & & $-1.2+1$ & 0.11 & & 1.10 & 0.70 & -0.17 \\
\hline 4.17 & 0.46 & -0.91 & 0.30 & 0.28 & -1.02 & 0.70 & -0.07 \\
\hline 4.28 & 0.47 & -0.30 & -0.24 & 0.48 & -0.90 & 0.78 & 0.06 \\
\hline 4.40 & 0.48 & 0.40 & -0.73 & 0.66 & -0.74 & 0.85 & 0.19 \\
\hline 4.51 & 0.49 & 0.99 & -1.10 & 0.79 & -0.54 & 0.90 & 0.31 \\
\hline 4.63 & 0.51 & 1.27 & -1.26 & 0.86 & -0.32 & 0.92 & 0.43 \\
\hline 4.74 & 0.52 & 1.15 & -1.19 & 0.88 & -0.08 & 0.92 & 0.54 \\
\hline 4.85 & 0.53 & 0.68 & -0.89 & 0.84 & 0.16 & 0.90 & 0.64 \\
\hline 4.97 & 0.54 & -0.02 & -0.41 & 0.74 & 0.40 & 0.85 & 0.73 \\
\hline 5.08 & 0.56 & -0.71 & 0.16 & 0.59 & 0.62 & 0.79 & 0.81 \\
\hline 5.20 & 0.57 & -1.19 & 0.71 & 0.40 & 0.81 & 0.70 & 0.88 \\
\hline 5.31 & 0.58 & -1.31 & 1.14 & 0.18 & 0.96 & 0.60 & 0.94 \\
\hline 5.43 & 0.59 & -1.02 & 1.35 & -0.05 & 1.07 & 0.48 & 0.98 \\
\hline 5.54 & 0.61 & -0.42 & 1.29 & -0.28 & 1.14 & 0.35 & 1.00 \\
\hline 5.65 & 0.62 & 0.31 & 0.96 & -0.49 & 1.15 & 0.21 & 1.01 \\
\hline 5.77 & 0.63 & 0.94 & 0.44 & -0.67 & 1.11 & 0.07 & 1.00 \\
\hline 5.88 & 0.64 & 1.29 & -0.19 & -0.80 & 1.02 & -0.08 & 0.97 \\
\hline 6.00 & 0.66 & 1.23 & -0.77 & -0.88 & 0.89 & -0.22 & 0.93 \\
\hline 6.11 & 0.67 & 0.80 & -1.20 & -0.90 & 0.71 & -0.36 & 0.88 \\
\hline 6.23 & 0.68 & 0.12 & -1.39 & -0.85 & 0.51 & -0.49 & 0.81 \\
\hline 6.34 & 0.69 & -0.60 & -1.29 & -0.75 & 0.28 & -0.61 & 0.73 \\
\hline 6.46 & 0.71 & -1.13 & -0.93 & -0.60 & 0.04 & -0.71 & 0.63 \\
\hline 6.57 & 0.72 & -1.32 & -0.38 & -0.40 & -0.20 & -0.80 & 0.53 \\
\hline 6.68 & 0.73 & -1.10 & 0.24 & -0.18 & -0.44 & -0.86 & 0.41 \\
\hline 6.80 & 0.74 & -0.54 & 0.81 & 0.06 & -0.65 & -0.91 & 0.30 \\
\hline 6.91 & 0.76 & 0.18 & 1.20 & 0.29 & -0.83 & -0.93 & 0.17 \\
\hline 7.03 & 0.77 & 0.83 & 1.35 & 0.50 & -0.97 & -0.93 & 0.04 \\
\hline 7.14 & 0.78 & 1.23 & 1.23 & 0.68 & -1.07 & -0.90 & -0.09 \\
\hline 7.26 & 0.79 & 1.24 & 0.87 & 0.82 & -1.13 & -0.86 & -0.21 \\
\hline 7.37 & 0.81 & 0.86 & 0.35 & 0.89 & -1.13 & -0.79 & -0.34 \\
\hline 7.48 & 0.82 & 0.23 & -0.22 & 0.91 & -1.08 & -0.70 & -0.46 \\
\hline 7.60 & 0.83 & -0.48 & -0.74 & 0.86 & -0.99 & -0.60 & -0.57 \\
\hline 7.71 & 0.84 & -1.04 & -1.10 & 0.76 & -0.85 & -0.48 & -0.67 \\
\hline 7.83 & 0.86 & -1.27 & -1.25 & 0.60 & -0.67 & -0.35 & -0.76 \\
\hline 7.94 & 0.87 & -1.11 & -1.17 & 0.40 & -0.47 & -0.21 & -0.84 \\
\hline
\end{tabular}




\begin{tabular}{|c|c|c|c|c|c|c|c|}
\hline \multicolumn{8}{|c|}{$\begin{array}{l}\text { Appendix } \mathrm{H8} \\
\text { WAMIT Predicted Normalized } \bar{p}_{W} \text { Dynamic Pressures - FL4550 } \\
\text { DSWG Basin Fundamental Laboratory Experiments (FLab) }\end{array}$} \\
\hline \multirow{2}{*}{$\begin{array}{c}X \\
(\mathbf{m}) \\
\end{array}$} & \multirow[b]{2}{*}{ Normal $X$} & \multicolumn{6}{|c|}{ Wave Case } \\
\hline & & 50 & 52 & 80 & 82 & 120 & 122 \\
\hline & & & & & & & \\
\hline 8.06 & 0.88 & -0.62 & -0.88 & 0.17 & -0.24 & -0.06 & -0.90 \\
\hline 8.17 & 0.89 & 0.06 & -0.43 & -0.07 & -0.01 & 0.08 & -0.95 \\
\hline 8.28 & 0.91 & 0.71 & 0.09 & -0.31 & 0.22 & 0.23 & -0.99 \\
\hline 8.40 & 0.92 & 1.13 & 0.59 & -0.52 & 0.43 & 0.37 & -1.00 \\
\hline 8.51 & 0.93 & 1.19 & 0.99 & -0.70 & 0.63 & 0.49 & -1.00 \\
\hline 8.63 & 0.94 & 0.88 & 1.21 & -0.83 & 0.79 & 0.61 & -0.98 \\
\hline 8.74 & 0.96 & 0.29 & 1.24 & -0.90 & 0.90 & 0.71 & -0.95 \\
\hline 8.86 & 0.97 & -0.37 & 1.06 & -0.90 & 0.97 & 0.79 & -0.89 \\
\hline 8.97 & 0.98 & -0.88 & 0.70 & -0.82 & 0.98 & 0.84 & -0.81 \\
\hline 9.08 & 0.99 & -1.04 & 0.22 & -0.66 & 0.90 & 0.88 & -0.68 \\
\hline
\end{tabular}




\begin{tabular}{|c|c|c|c|c|c|c|c|}
\hline \multicolumn{8}{|c|}{$\begin{array}{l}\text { Appendix H9 } \\
\text { WAMIT Predicted Normalized } \overline{p_{W}} \text { Dynamic Pressures - FL4575 } \\
\text { DSWG Basin Fundamental Laboratory Experiments (FLab) }\end{array}$} \\
\hline \multirow{2}{*}{$\begin{array}{c}X \\
(\mathbf{m}) \\
\end{array}$} & \multirow[b]{2}{*}{ Normal $X$} & \multicolumn{6}{|c|}{ Wave Case } \\
\hline & & 50 & 52 & 80 & 82 & 120 & 122 \\
\hline 0.05 & 0.01 & -0.77 & -0.46 & -0.44 & -0.87 & 0.98 & 1.02 \\
\hline 0.17 & 0.02 & -0.79 & -0.15 & -0.62 & -0.99 & 0.98 & 1.02 \\
\hline 0.28 & 0.03 & -0.58 & 0.28 & -0.77 & -1.08 & 0.96 & 1.00 \\
\hline 0.40 & 0.04 & -0.17 & 0.74 & -0.89 & -1.15 & 0.92 & 0.96 \\
\hline 0.51 & 0.06 & 0.32 & 1.11 & -0.96 & -1.18 & 0.86 & 0.91 \\
\hline 0.63 & 0.07 & 0.76 & 1.28 & -0.98 & -1.17 & 0.78 & 0.84 \\
\hline 0.74 & 0.08 & 0.99 & 1.17 & -0.94 & -1.12 & 0.69 & 0.76 \\
\hline 0.85 & 0.09 & 0.94 & 0.80 & -0.84 & -1.01 & 0.58 & 0.66 \\
\hline 0.97 & 0.11 & 0.61 & 0.24 & -0.69 & -0.86 & 0.45 & 0.56 \\
\hline 1.08 & 0.12 & 0.08 & -0.41 & -0.49 & -0.67 & 0.32 & 0.45 \\
\hline 1.20 & 0.13 & -0.51 & -1.00 & -0.27 & -0.44 & 0.17 & 0.32 \\
\hline 1.31 & 0.14 & -0.96 & -1.39 & -0.02 & -0.18 & 0.03 & 0.20 \\
\hline 1.43 & 0.16 & -1.14 & -1.49 & 0.23 & 0.09 & -0.12 & 0.06 \\
\hline 1.54 & 0.17 & -0.97 & -1.27 & 0.46 & 0.37 & -0.26 & -0.07 \\
\hline 1.65 & 0.18 & -0.50 & -0.76 & 0.67 & 0.64 & -0.40 & -0.20 \\
\hline 1.77 & 0.19 & 0.14 & -0.09 & 0.83 & 0.89 & -0.52 & -0.34 \\
\hline 1.88 & 0.21 & 0.75 & 0.61 & 0.95 & 1.11 & -0.64 & -0.47 \\
\hline 2.00 & 0.22 & 1.14 & 1.16 & 1.00 & 1.28 & -0.74 & -0.59 \\
\hline 2.11 & 0.23 & 1.19 & 1.45 & 0.98 & 1.40 & -0.82 & -0.70 \\
\hline 2.23 & 0.24 & 0.86 & 1.43 & 0.90 & 1.46 & -0.88 & -0.81 \\
\hline 2.34 & 0.26 & 0.26 & 1.11 & 0.76 & 1.45 & -0.92 & -0.90 \\
\hline 2.45 & 0.27 & -0.43 & 0.57 & 0.57 & 1.37 & -0.94 & -0.98 \\
\hline 2.57 & 0.28 & -1.01 & -0.06 & 0.34 & 1.24 & -0.93 & -1.05 \\
\hline 2.68 & 0.29 & -1.28 & -0.65 & 0.08 & 1.04 & -0.91 & -1.10 \\
\hline 2.80 & 0.31 & -1.16 & -1.09 & -0.19 & 0.79 & -0.86 & -1.13 \\
\hline 2.91 & 0.32 & -0.67 & -1.29 & -0.44 & 0.51 & -0.78 & -1.15 \\
\hline 3.03 & 0.33 & 0.03 & -1.24 & -0.67 & 0.19 & -0.69 & -1.15 \\
\hline 3.14 & 0.34 & 0.74 & -0.96 & -0.85 & -0.14 & -0.59 & -1.12 \\
\hline 3.25 & 0.36 & 1.23 & -0.51 & -0.99 & -0.46 & -0.46 & -1.09 \\
\hline 3.37 & 0.37 & 1.35 & 0.01 & -1.05 & -0.77 & -0.33 & -1.03 \\
\hline 3.48 & 0.38 & 1.05 & 0.51 & -1.05 & -1.04 & -0.19 & -0.96 \\
\hline 3.60 & 0.39 & 0.43 & 0.91 & -0.98 & -1.27 & -0.04 & -0.87 \\
\hline 3.71 & 0.41 & -0.34 & 1.14 & -0.84 & -1.44 & 0.10 & -0.77 \\
\hline 3.83 & 0.42 & -1.00 & 1.17 & -0.64 & -1.54 & 0.25 & -0.65 \\
\hline 3.94 & 0.43 & -1.36 & 1.00 & -0.40 & -1.58 & 0.39 & -0.53 \\
\hline
\end{tabular}




\begin{tabular}{|c|c|c|c|c|c|c|c|}
\hline \multicolumn{8}{|c|}{$\begin{array}{l}\text { Appendix H9 } \\
\text { WAMIT Predicted Normalized } \bar{p}_{W} \text { Dynamic Pressures - FL4575 } \\
\text { DSWG Basin Fundamental Laboratory Experiments (FLab) }\end{array}$} \\
\hline \multirow{2}{*}{$\begin{array}{c}X \\
(\mathbf{m}) \\
\end{array}$} & \multirow[b]{2}{*}{ Normal $X$} & \multicolumn{6}{|c|}{ Wave Case } \\
\hline & & 50 & 52 & 80 & 82 & 120 & 122 \\
\hline & & & & & & & \\
\hline & 0.44 & -1.50 & 0.07 & & 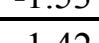 & 0.51 & \\
\hline 4.17 & 0.46 & -0.83 & 0.17 & 0.14 & -1.42 & 0.63 & -0.25 \\
\hline 4.28 & 0.47 & -0.11 & -0.35 & 0.40 & -1.24 & 0.73 & -0.10 \\
\hline 4.40 & 0.48 & 0.66 & -0.82 & 0.64 & -1.00 & 0.82 & 0.05 \\
\hline 4.51 & 0.49 & 1.22 & -1.15 & 0.84 & -0.71 & 0.88 & 0.20 \\
\hline 4.63 & 0.51 & 1.41 & -1.28 & 0.98 & -0.39 & 0.92 & 0.34 \\
\hline 4.74 & 0.52 & 1.16 & -1.17 & 1.05 & -0.05 & 0.94 & 0.49 \\
\hline 4.85 & 0.53 & 0.55 & -0.82 & 1.05 & 0.29 & 0.93 & 0.62 \\
\hline 4.97 & 0.54 & -0.24 & -0.30 & 0.98 & 0.62 & 0.91 & 0.75 \\
\hline 5.08 & 0.56 & -0.96 & 0.30 & 0.83 & 0.92 & 0.85 & 0.86 \\
\hline 5.20 & 0.57 & -1.39 & 0.86 & 0.63 & 1.17 & 0.78 & 0.96 \\
\hline 5.31 & 0.58 & -1.39 & 1.26 & 0.39 & 1.38 & 0.69 & 1.05 \\
\hline 5.43 & 0.59 & -0.96 & 1.41 & 0.11 & 1.51 & 0.58 & 1.12 \\
\hline 5.54 & 0.61 & -0.23 & 1.27 & -0.17 & 1.58 & 0.46 & 1.17 \\
\hline 5.65 & 0.62 & 0.57 & 0.87 & -0.45 & 1.57 & 0.32 & 1.20 \\
\hline 5.77 & 0.63 & 1.20 & 0.28 & -0.70 & 1.49 & 0.18 & 1.22 \\
\hline 5.88 & 0.64 & 1.45 & -0.38 & -0.90 & 1.33 & 0.03 & 1.21 \\
\hline 6.00 & 0.66 & 1.25 & -0.95 & -1.04 & 1.12 & -0.12 & 1.18 \\
\hline 6.11 & 0.67 & 0.67 & -1.33 & -1.11 & 0.86 & -0.27 & 1.14 \\
\hline 6.23 & 0.68 & -0.13 & -1.43 & -1.10 & 0.56 & -0.41 & 1.07 \\
\hline 6.34 & 0.69 & -0.89 & -1.23 & -1.02 & 0.24 & -0.54 & 0.99 \\
\hline 6.46 & 0.71 & -1.38 & -0.78 & -0.87 & -0.09 & -0.66 & 0.89 \\
\hline 6.57 & 0.72 & -1.44 & -0.18 & -0.66 & -0.42 & -0.77 & 0.78 \\
\hline 6.68 & 0.73 & -1.06 & 0.44 & -0.40 & -0.73 & -0.85 & 0.65 \\
\hline 6.80 & 0.74 & -0.35 & 0.96 & -0.11 & -0.99 & -0.92 & 0.51 \\
\hline 6.91 & 0.76 & 0.46 & 1.27 & 0.18 & -1.22 & -0.96 & 0.36 \\
\hline 7.03 & 0.77 & 1.12 & 1.32 & 0.46 & -1.38 & -0.98 & 0.21 \\
\hline 7.14 & 0.78 & 1.44 & 1.11 & 0.71 & -1.48 & -0.97 & 0.05 \\
\hline 7.26 & 0.79 & 1.30 & 0.70 & 0.91 & -1.51 & -0.95 & -0.11 \\
\hline 7.37 & 0.81 & 0.77 & 0.18 & 1.05 & -1.48 & -0.90 & -0.26 \\
\hline 7.48 & 0.82 & -0.01 & -0.35 & 1.11 & -1.37 & -0.82 & -0.42 \\
\hline 7.60 & 0.83 & -0.78 & -0.79 & 1.09 & -1.21 & -0.73 & -0.56 \\
\hline 7.71 & 0.84 & -1.30 & -1.07 & 1.00 & -0.99 & -0.62 & -0.70 \\
\hline 7.83 & 0.86 & -1.42 & -1.14 & 0.83 & -0.74 & -0.50 & -0.82 \\
\hline 7.94 & 0.87 & -1.10 & -1.02 & 0.60 & -0.45 & -0.36 & -0.94 \\
\hline
\end{tabular}




\begin{tabular}{|c|c|c|c|c|c|c|c|}
\hline \multicolumn{8}{|c|}{$\begin{array}{l}\text { Appendix H9 } \\
\text { WAMIT Predicted Normalized } \bar{p}_{W} \text { Dynamic Pressures - FL4575 } \\
\text { DSWG Basin Fundamental Laboratory Experiments (FLab) }\end{array}$} \\
\hline \multirow{2}{*}{$\begin{array}{c}X \\
(\mathbf{m})\end{array}$} & \multirow[b]{2}{*}{ Normal $X$} & \multicolumn{6}{|c|}{ Wave Case } \\
\hline & & 50 & 52 & 80 & 82 & 120 & 122 \\
\hline & & & & & & & \\
\hline 8.06 & 0.88 & -0.44 & -0.72 & 0.32 & -0.15 & -0.22 & -1.03 \\
\hline 8.17 & 0.89 & 0.35 & -0.30 & 0.02 & 0.16 & -0.07 & -1.11 \\
\hline 8.28 & 0.91 & 1.02 & 0.17 & -0.29 & 0.45 & 0.08 & -1.16 \\
\hline 8.40 & 0.92 & 1.36 & 0.62 & -0.58 & 0.71 & 0.22 & -1.20 \\
\hline 8.51 & 0.93 & 1.27 & 0.97 & -0.82 & 0.94 & 0.36 & -1.21 \\
\hline 8.63 & 0.94 & 0.79 & 1.17 & -1.01 & 1.11 & 0.48 & -1.20 \\
\hline 8.74 & 0.96 & 0.06 & 1.16 & -1.13 & 1.23 & 0.60 & -1.16 \\
\hline 8.86 & 0.97 & -0.68 & 0.96 & -1.15 & 1.26 & 0.69 & -1.09 \\
\hline 8.97 & 0.98 & -1.18 & 0.60 & -1.08 & 1.21 & 0.77 & -0.98 \\
\hline 9.08 & 0.99 & -1.23 & 0.14 & -0.87 & 1.05 & 0.82 & -0.81 \\
\hline
\end{tabular}




\begin{tabular}{|c|c|c|c|c|c|c|c|}
\hline \multicolumn{8}{|c|}{$\begin{array}{l}\text { Appendix H10 } \\
\text { WAMIT Predicted Normalized } \bar{p}_{W} \text { Dynamic Pressures - FL45100 } \\
\text { DSWG Basin Fundamental Laboratory Experiments (FLab) }\end{array}$} \\
\hline \multirow{2}{*}{$\begin{array}{c}X \\
(\mathbf{m}) \\
\end{array}$} & \multirow[b]{2}{*}{ Normal $X$} & \multicolumn{6}{|c|}{ Wave Case } \\
\hline & & 50 & 52 & 80 & 82 & 120 & 122 \\
\hline & & & & & & & \\
\hline 0.05 & 0.01 & -0.78 & -0.51 & -0.58 & -0.84 & 1.06 & 1.03 \\
\hline 0.17 & 0.02 & -0.77 & -0.18 & -0.81 & -0.98 & 1.10 & 1.06 \\
\hline 0.28 & 0.03 & -0.54 & 0.27 & -1.00 & -1.09 & 1.11 & 1.07 \\
\hline 0.40 & 0.04 & -0.12 & 0.73 & -1.14 & -1.16 & 1.09 & 1.06 \\
\hline 0.51 & 0.06 & 0.36 & 1.11 & -1.21 & -1.19 & 1.03 & 1.03 \\
\hline 0.63 & 0.07 & 0.78 & 1.28 & -1.21 & -1.17 & 0.94 & 0.98 \\
\hline 0.74 & 0.08 & 1.00 & 1.15 & -1.11 & -1.09 & 0.82 & 0.91 \\
\hline 0.85 & 0.09 & 0.93 & 0.75 & -0.94 & -0.94 & 0.66 & 0.81 \\
\hline 0.97 & 0.11 & 0.55 & 0.15 & -0.69 & -0.72 & 0.49 & 0.69 \\
\hline 1.08 & 0.12 & -0.01 & -0.50 & -0.40 & -0.45 & 0.31 & 0.54 \\
\hline 1.20 & 0.13 & -0.59 & -1.06 & -0.08 & -0.14 & 0.12 & 0.37 \\
\hline 1.31 & 0.14 & -1.01 & -1.41 & 0.26 & 0.20 & -0.07 & 0.19 \\
\hline 1.43 & 0.16 & -1.13 & -1.46 & 0.58 & 0.55 & -0.25 & -0.01 \\
\hline 1.54 & 0.17 & -0.90 & -1.18 & 0.86 & 0.89 & -0.43 & -0.21 \\
\hline 1.65 & 0.18 & -0.38 & -0.65 & 1.10 & 1.19 & -0.60 & -0.42 \\
\hline 1.77 & 0.19 & 0.26 & 0.01 & 1.26 & 1.45 & -0.76 & -0.63 \\
\hline 1.88 & 0.21 & 0.84 & 0.68 & 1.34 & 1.65 & -0.90 & -0.83 \\
\hline 2.00 & 0.22 & 1.17 & 1.18 & 1.33 & 1.77 & -1.02 & -1.03 \\
\hline 2.11 & 0.23 & 1.14 & 1.42 & 1.23 & 1.80 & -1.12 & -1.21 \\
\hline 2.23 & 0.24 & 0.76 & 1.36 & 1.05 & 1.75 & -1.19 & -1.38 \\
\hline 2.34 & 0.26 & 0.13 & 1.01 & 0.80 & 1.61 & -1.24 & -1.52 \\
\hline 2.45 & 0.27 & -0.54 & 0.47 & 0.48 & 1.38 & -1.26 & -1.64 \\
\hline 2.57 & 0.28 & -1.07 & -0.15 & 0.13 & 1.08 & -1.25 & -1.74 \\
\hline 2.68 & 0.29 & -1.27 & -0.70 & -0.24 & 0.73 & -1.22 & -1.80 \\
\hline 2.80 & 0.31 & -1.08 & -1.09 & -0.59 & 0.33 & -1.15 & -1.83 \\
\hline 2.91 & 0.32 & -0.55 & -1.24 & -0.91 & -0.09 & -1.06 & -1.82 \\
\hline 3.03 & 0.33 & 0.16 & -1.15 & -1.18 & -0.51 & -0.94 & -1.79 \\
\hline 3.14 & 0.34 & 0.83 & -0.85 & -1.37 & -0.90 & -0.80 & -1.72 \\
\hline 3.25 & 0.36 & 1.26 & -0.41 & -1.46 & -1.26 & -0.64 & -1.61 \\
\hline 3.37 & 0.37 & 1.30 & 0.08 & -1.46 & -1.55 & -0.46 & -1.47 \\
\hline 3.48 & 0.38 & 0.94 & 0.53 & -1.36 & -1.77 & -0.28 & -1.31 \\
\hline 3.60 & 0.39 & 0.30 & 0.88 & -1.17 & -1.91 & -0.08 & -1.11 \\
\hline 3.71 & 0.41 & -0.46 & 1.06 & -0.89 & -1.95 & 0.12 & -0.89 \\
\hline 3.83 & 0.42 & -1.07 & 1.07 & -0.55 & -1.90 & 0.31 & -0.66 \\
\hline 3.94 & 0.43 & -1.37 & 0.90 & -0.16 & -1.75 & 0.50 & -0.40 \\
\hline
\end{tabular}




\begin{tabular}{|c|c|c|c|c|c|c|c|}
\hline \multicolumn{8}{|c|}{$\begin{array}{l}\text { Appendix H10 } \\
\text { WAMIT Predicted Normalized } \bar{p}_{W} \text { Dynamic Pressures - FL45100 } \\
\text { DSWG Basin Fundamental Laboratory Experiments (FLab) }\end{array}$} \\
\hline \multirow{2}{*}{$\begin{array}{c}X \\
(\mathbf{m}) \\
\end{array}$} & \multirow[b]{2}{*}{ Normal $X$} & \multicolumn{6}{|c|}{ Wave Case } \\
\hline & & 50 & 52 & 80 & 82 & 120 & 122 \\
\hline & & & & & & & \\
\hline 4.05 & 0.44 & -1.24 & 0.58 & 0.23 & -1.53 & 0.68 & -0.14 \\
\hline 4.17 & 0.46 & -0.73 & 0.15 & 0.62 & -1.23 & 0.85 & 0.14 \\
\hline 4.28 & 0.47 & 0.02 & -0.32 & 0.97 & -0.88 & 0.99 & 0.41 \\
\hline 4.40 & 0.48 & 0.76 & -0.76 & 1.26 & -0.49 & 1.11 & 0.68 \\
\hline 4.51 & 0.49 & 1.27 & -1.08 & 1.46 & -0.08 & 1.21 & 0.94 \\
\hline 4.63 & 0.51 & 1.39 & -1.21 & 1.56 & 0.32 & 1.28 & 1.19 \\
\hline 4.74 & 0.52 & 1.08 & -1.11 & 1.56 & 0.71 & 1.32 & 1.41 \\
\hline 4.85 & 0.53 & 0.43 & -0.79 & 1.45 & 1.06 & 1.32 & 1.62 \\
\hline 4.97 & 0.54 & -0.36 & -0.29 & 1.24 & 1.35 & 1.30 & 1.79 \\
\hline 5.08 & 0.56 & -1.04 & 0.29 & 0.94 & 1.57 & 1.24 & 1.94 \\
\hline 5.20 & 0.57 & -1.41 & 0.85 & 0.57 & 1.72 & 1.16 & 2.05 \\
\hline 5.31 & 0.58 & -1.34 & 1.25 & 0.16 & 1.79 & 1.04 & 2.13 \\
\hline 5.43 & 0.59 & -0.85 & 1.40 & -0.26 & 1.78 & 0.90 & 2.17 \\
\hline 5.54 & 0.61 & -0.10 & 1.26 & -0.68 & 1.68 & 0.74 & 2.18 \\
\hline 5.65 & 0.62 & 0.69 & 0.84 & -1.05 & 1.51 & 0.56 & 2.14 \\
\hline 5.77 & 0.63 & 1.26 & 0.23 & -1.35 & 1.27 & 0.37 & 2.07 \\
\hline 5.88 & 0.64 & 1.45 & -0.45 & -1.56 & 0.98 & 0.17 & 1.96 \\
\hline 6.00 & 0.66 & 1.19 & -1.03 & -1.67 & 0.66 & -0.04 & 1.81 \\
\hline 6.11 & 0.67 & 0.56 & -1.40 & -1.66 & 0.31 & -0.24 & 1.63 \\
\hline 6.23 & 0.68 & -0.25 & -1.46 & -1.54 & -0.05 & -0.44 & 1.43 \\
\hline 6.34 & 0.69 & -0.98 & -1.22 & -1.32 & -0.40 & -0.63 & 1.20 \\
\hline 6.46 & 0.71 & -1.41 & -0.71 & -1.00 & -0.72 & -0.80 & 0.95 \\
\hline 6.57 & 0.72 & -1.41 & -0.07 & -0.61 & -1.01 & -0.95 & 0.68 \\
\hline 6.68 & 0.73 & -0.96 & 0.57 & -0.18 & -1.24 & -1.08 & 0.40 \\
\hline 6.80 & 0.74 & -0.23 & 1.07 & 0.27 & -1.42 & -1.18 & 0.11 \\
\hline 6.91 & 0.76 & 0.59 & 1.32 & 0.69 & -1.54 & -1.25 & -0.18 \\
\hline 7.03 & 0.77 & 1.21 & 1.30 & 1.07 & -1.58 & -1.29 & -0.46 \\
\hline 7.14 & 0.78 & 1.46 & 1.01 & 1.37 & -1.56 & -1.30 & -0.74 \\
\hline 7.26 & 0.79 & 1.26 & 0.54 & 1.58 & -1.47 & -1.27 & -1.00 \\
\hline 7.37 & 0.81 & 0.67 & -0.00 & 1.68 & -1.32 & -1.21 & -1.25 \\
\hline 7.48 & 0.82 & -0.13 & -0.51 & 1.65 & -1.12 & -1.12 & -1.47 \\
\hline 7.60 & 0.83 & $\begin{array}{l}-0.89 \\
\end{array}$ & -0.89 & 1.51 & -0.86 & -1.00 & -1.67 \\
\hline 7.71 & 0.84 & -1.37 & -1.09 & 1.26 & -0.57 & -0.85 & -1.84 \\
\hline 7.83 & 0.86 & -1.42 & -1.08 & 0.92 & -0.26 & -0.68 & -1.97 \\
\hline 7.94 & 0.87 & -1.03 & -0.90 & 0.51 & 0.06 & -0.49 & -2.07 \\
\hline
\end{tabular}




\begin{tabular}{|c|c|c|c|c|c|c|c|}
\hline \multicolumn{8}{|c|}{$\begin{array}{l}\text { Appendix H10 } \\
\text { WAMIT Predicted Normalized } \bar{p}_{W} \text { Dynamic Pressures - FL45100 } \\
\text { DSWG Basin Fundamental Laboratory Experiments (FLab) }\end{array}$} \\
\hline \multirow{2}{*}{$\begin{array}{c}X \\
(\mathbf{m})\end{array}$} & \multirow[b]{2}{*}{ Normal $X$} & \multicolumn{6}{|c|}{ Wave Case } \\
\hline & & 50 & 52 & 80 & 82 & 120 & 122 \\
\hline & & & & & & & \\
\hline 8.06 & 0.88 & -0.32 & -0.58 & 0.05 & 0.39 & -0.29 & -2.13 \\
\hline 8.17 & 0.89 & 0.49 & -0.18 & -0.41 & 0.70 & -0.08 & -2.15 \\
\hline 8.28 & 0.91 & 1.14 & 0.24 & -0.86 & 0.99 & 0.14 & -2.13 \\
\hline 8.40 & 0.92 & 1.42 & 0.61 & -1.26 & 1.25 & 0.35 & -2.07 \\
\hline 8.51 & 0.93 & 1.24 & 0.89 & -1.58 & 1.44 & 0.55 & -1.98 \\
\hline 8.63 & 0.94 & 0.68 & 1.04 & -1.80 & 1.56 & 0.74 & -1.86 \\
\hline 8.74 & 0.96 & -0.08 & 1.03 & -1.88 & 1.59 & 0.90 & -1.69 \\
\hline 8.86 & 0.97 & -0.82 & 0.85 & -1.81 & 1.53 & 1.03 & -1.47 \\
\hline 8.97 & 0.98 & -1.30 & 0.54 & -1.59 & 1.39 & 1.12 & -1.20 \\
\hline 9.08 & 0.99 & -1.28 & 0.12 & -1.20 & 1.09 & 1.16 & -0.87 \\
\hline
\end{tabular}




\begin{tabular}{|c|c|c|c|c|c|}
\hline \multicolumn{6}{|c|}{$\begin{array}{l}\text { Appendix H11 } \\
\text { WAMIT Normalized } \bar{p}_{1, W} \text { Dynamic Wave Pressures at Equivalent Gage Locations } \\
\text { DSWG Basin Fundamental Laboratory Experiments (FLab) } \\
\end{array}$} \\
\hline \multirow{2}{*}{$\begin{array}{c}\text { Case } \\
\text { Name }\end{array}$} & \multirow{2}{*}{$\begin{array}{c}\text { Run } \\
\text { No. }\end{array}$} & \multicolumn{4}{|c|}{ Gages } \\
\hline & & Bow & Mid & Str & Avg \\
\hline \multicolumn{6}{|c|}{$45 \mathrm{deg}$ Interior Angle, $100 \%$ Penetration } \\
\hline R3 50D & 7 & 1.14 & 1.38 & 1.44 & 1.32 \\
\hline $\mathrm{R} 3$ 52D & 7 & 1.44 & 1.14 & 1.20 & 1.26 \\
\hline R3_80D & 6 & 1.27 & 1.51 & 1.67 & 1.48 \\
\hline R3_82D & 6 & 1.50 & 1.87 & 1.58 & 1.65 \\
\hline R3120D & 6 & 1.18 & 1.28 & 1.31 & 1.26 \\
\hline $\mathrm{R} 3122 \mathrm{D}$ & 6 & 1.45 & 2.00 & 2.16 & 1.87 \\
\hline $\mathrm{R} 5120 \mathrm{D}$ & 6 & 1.18 & 1.28 & 1.31 & 1.26 \\
\hline $\mathrm{R} 5122 \mathrm{D}$ & 6 & 1.45 & 2.00 & 2.16 & 1.87 \\
\hline \multicolumn{6}{|c|}{$45 \mathrm{deg}$ Interior Angle, $75 \%$ Penetration } \\
\hline R3_50D & 8 & 1.16 & 1.38 & 1.43 & 1.33 \\
\hline R3_52D & 8 & 1.47 & 1.23 & 1.23 & 1.31 \\
\hline R3_80D & 8 & 0.99 & 1.05 & 1.10 & 1.05 \\
\hline R3_82D & 8 & 1.32 & 1.58 & 1.37 & 1.42 \\
\hline $\mathrm{R} 3120 \mathrm{D}$ & 8 & 0.96 & 0.94 & 0.96 & 0.95 \\
\hline $\mathrm{R} 3122 \mathrm{D}$ & 8 & 1.08 & 1.18 & 1.22 & 1.16 \\
\hline R5120D & 8 & 0.96 & 0.94 & 0.96 & 0.95 \\
\hline $\mathrm{R} 5122 \mathrm{D}$ & 8 & 1.08 & 1.18 & 1.22 & 1.16 \\
\hline \multicolumn{6}{|c|}{$45 \mathrm{deg}$ Interior Angle, $50 \%$ Penetration } \\
\hline R3_50D & 9 & 1.08 & 1.26 & 1.26 & 1.20 \\
\hline R3 52D & 9 & 1.45 & 1.26 & 1.24 & 1.32 \\
\hline R3_80D & 9 & 0.86 & 0.88 & 0.90 & 0.88 \\
\hline R3_82D & 9 & 1.02 & 1.12 & 1.14 & 1.09 \\
\hline R3120D & 9 & 0.94 & 0.92 & 0.92 & 0.93 \\
\hline $\mathrm{R} 3122 \mathrm{D}$ & 9 & 1.00 & 1.00 & 1.01 & 1.00 \\
\hline R5120D & 9 & 0.94 & 0.92 & 0.92 & 0.93 \\
\hline $\mathrm{R} 5122 \mathrm{D}$ & 9 & 1.00 & 1.00 & 1.01 & 1.00 \\
\hline \multicolumn{6}{|c|}{45 deg Interior Angle, $25 \%$ Penetration } \\
\hline R3_50D & 10 & 0.66 & 0.73 & 0.78 & 0.72 \\
\hline R3_52D & 10 & 0.90 & 0.88 & 0.92 & 0.90 \\
\hline R3_80D & 10 & 0.82 & 0.84 & 0.82 & 0.83 \\
\hline R3_82D & 10 & 0.86 & 0.88 & 0.86 & 0.87 \\
\hline R3120D & 10 & 0.92 & 0.92 & 0.92 & 0.92 \\
\hline R3122D & 10 & 0.94 & 0.94 & 0.94 & 0.94 \\
\hline R5120D & 10 & 0.92 & 0.92 & 0.92 & 0.92 \\
\hline R5122D & 10 & 0.94 & 0.94 & 0.94 & 0.94 \\
\hline
\end{tabular}




\begin{tabular}{|c|c|c|c|c|c|}
\hline $\begin{array}{l}\text { Appendix } \\
\text { Analytical } \\
\text { DSWG Bas }\end{array}$ & $\begin{array}{l}\text { alized } \overline{p_{l}} \\
\text { ndament }\end{array}$ & $\begin{array}{l}\text { aic W } \\
\text { atory }\end{array}$ & $\begin{array}{l}\text { ures } \\
\text { ents }(\end{array}$ & & \\
\hline $\begin{array}{c}\text { Case } \\
\text { Name } \\
\end{array}$ & $\operatorname{Avg} K_{T}$ & $\boldsymbol{k}_{x}$ & $\boldsymbol{k}_{v}$ & $K_{p}$ & $\bar{p}_{1, A}$ \\
\hline 45 deg Inter & igle, $100^{\circ}$ & ation & & & \\
\hline R3_50D & 0.22 & 2.01 & 4.84 & 0.65 & 1.28 \\
\hline R3_52D & 0.23 & 0.23 & 5.24 & 0.65 & 1.27 \\
\hline R3_80D & 0.28 & 0.91 & 2.19 & 0.86 & 1.68 \\
\hline R3_82D & 0.28 & 0.10 & 2.37 & 0.86 & 1.68 \\
\hline R3120D & 0.33 & 0.55 & 1.33 & 0.94 & 1.82 \\
\hline R3122D & 0.27 & 0.06 & 1.44 & 0.94 & 1.84 \\
\hline R5120D & 0.31 & 0.55 & 1.33 & 0.94 & 1.83 \\
\hline R5122D & 0.28 & 0.06 & 1.44 & 0.94 & 1.84 \\
\hline 45 deg Inter & igle, $75 \%$ & ion & & & \\
\hline R3_50D & 0.25 & 2.01 & 4.84 & 0.65 & 1.27 \\
\hline R3_52D & 0.25 & 0.23 & 5.24 & 0.65 & 1.27 \\
\hline R3_80D & 0.55 & 0.91 & 2.19 & 0.86 & 1.58 \\
\hline R3_82D & 0.49 & 0.10 & 2.37 & 0.86 & 1.61 \\
\hline R3120D & 0.71 & 0.55 & 1.33 & 0.94 & 1.59 \\
\hline R3122D & 0.71 & 0.06 & 1.44 & 0.94 & 1.60 \\
\hline R5120D & 0.68 & 0.55 & 1.33 & 0.94 & 1.62 \\
\hline R5122D & 0.61 & 0.06 & 1.44 & 0.94 & 1.68 \\
\hline 45 deg Inter & igle, $50 \%$ & ion & & & \\
\hline R3_50D & 0.37 & 2.01 & 4.84 & 0.65 & 1.25 \\
\hline R3_52D & 0.35 & 0.23 & 5.24 & 0.65 & 1.25 \\
\hline R3_80D & 0.74 & 0.91 & 2.19 & 0.86 & 1.43 \\
\hline R3_82D & 0.64 & 0.10 & 2.37 & 0.86 & 1.52 \\
\hline R3120D & 0.83 & 0.55 & 1.33 & 0.94 & 1.46 \\
\hline R3122D & 0.85 & 0.06 & 1.44 & 0.94 & 1.43 \\
\hline R5120D & 0.83 & 0.55 & 1.33 & 0.94 & 1.46 \\
\hline R5122D & 0.79 & 0.06 & 1.44 & 0.94 & 1.51 \\
\hline 45 deg Inter & igle, $25 \%$ & ion & & & \\
\hline R3_50D & 0.66 & 2.01 & 4.84 & 0.65 & 1.13 \\
\hline R3_52D & 0.64 & 0.23 & 5.24 & 0.65 & 1.14 \\
\hline R3_80D & 0.89 & 0.91 & 2.19 & 0.86 & 1.25 \\
\hline R3_82D & 0.79 & 0.10 & 2.37 & 0.86 & 1.38 \\
\hline R3120D & 0.92 & 0.55 & 1.33 & 0.94 & 1.30 \\
\hline R3122D & 0.91 & 0.06 & 1.44 & 0.94 & 1.32 \\
\hline R5120D & 0.91 & 0.55 & 1.33 & 0.94 & 1.33 \\
\hline R5122D & 0.93 & 0.06 & 1.44 & 0.94 & 1.28 \\
\hline $\begin{array}{l}\text { Notes: } \\
\text { 1. Analytic } \\
\text { FL45: } 0\end{array}$ & $67.5 \mathrm{~d}$ & $\begin{array}{l}\text { ction } \\
\Rightarrow>8\end{array}$ & & & \\
\hline
\end{tabular}




\section{APPENDIX I}

\section{XM99 ANALYTICAL DYNAMIC PRESSURES}




\begin{tabular}{|c|c|c|c|c|c|c|c|c|c|}
\hline \multicolumn{10}{|c|}{$\begin{array}{l}\text { Appendix } \mathrm{I} 1 \\
\text { Analytical } \bar{p}_{1, A} \text { Dynamic Pressures } \\
\text { XM99 Field Trials }\end{array}$} \\
\hline \multirow{2}{*}{$\begin{array}{l}\text { Code } \\
\text { Name }\end{array}$} & \multirow[b]{2}{*}{$K_{T}$} & \multicolumn{3}{|c|}{ Top Level } & \multicolumn{2}{|c|}{ Middle Level } & \multicolumn{3}{|c|}{ Bottom Level } \\
\hline & & Bow & Mid & Str & Mid & Str & Bow & Mid & Str \\
\hline 230 & 0.06 & 0.11 & 0.11 & 0.11 & 0.02 & 0.02 & 0.00 & 0.00 & 0.00 \\
\hline 260 & 0.12 & 0.11 & 0.11 & 0.11 & 0.02 & 0.02 & 0.00 & 0.00 & 0.00 \\
\hline 300 & 0.15 & 0.54 & 0.54 & 0.54 & 0.28 & 0.28 & 0.09 & 0.09 & 0.09 \\
\hline 330 & 0.16 & 0.54 & 0.54 & 0.54 & 0.28 & 0.28 & 0.09 & 0.09 & 0.09 \\
\hline 360 & 0.33 & 0.53 & 0.53 & 0.53 & 0.27 & 0.27 & 0.09 & 0.09 & 0.09 \\
\hline 400 & 0.51 & 0.90 & 0.90 & 0.90 & 0.62 & 0.62 & 0.34 & 0.34 & 0.34 \\
\hline 430 & 0.26 & 0.95 & 0.95 & 0.95 & 0.65 & 0.65 & 0.36 & 0.36 & 0.36 \\
\hline 460 & 0.30 & 0.95 & 0.95 & 0.95 & 0.65 & 0.65 & 0.36 & 0.36 & 0.36 \\
\hline 560 & 0.66 & 1.11 & 1.11 & 1.11 & 0.88 & 0.88 & 0.63 & 0.63 & 0.63 \\
\hline 660 & 0.31 & 1.44 & 1.44 & 1.44 & 1.25 & 1.25 & 1.02 & 1.02 & 1.02 \\
\hline 760 & 1.22 & 0.81 & 0.81 & 0.81 & 0.73 & 0.73 & 0.64 & 0.64 & 0.64 \\
\hline 830 & 1.24 & 0.85 & 0.85 & 0.85 & 0.79 & 0.79 & 0.72 & 0.72 & 0.72 \\
\hline 860 & 1.19 & 0.85 & 0.85 & 0.85 & 0.79 & 0.79 & 0.72 & 0.72 & 0.72 \\
\hline 930 & 1.16 & 0.88 & 0.88 & 0.88 & 0.84 & 0.84 & 0.78 & 0.78 & 0.78 \\
\hline 960 & 1.12 & 0.88 & 0.88 & 0.88 & 0.84 & 0.84 & 0.78 & 0.78 & 0.78 \\
\hline 1030 & 1.11 & 0.91 & 0.91 & 0.91 & 0.87 & 0.87 & 0.82 & 0.82 & 0.82 \\
\hline 1060 & 1.08 & 0.91 & 0.91 & 0.91 & 0.87 & 0.87 & 0.82 & 0.82 & 0.82 \\
\hline
\end{tabular}




\section{VITA}

Michael Jeffrey Briggs is a Research Hydraulic Engineer in the Coastal and Hydraulics Laboratory (CHL) at the U.S. Army Engineer Research and Development Center (ERDC) in Vicksburg, MS. His research interests include three-dimensional physical modeling, generation and analysis of directional wave spectra, wave transformation in harbors and along coasts, wave-current interaction, analytical and numerical modeling of floating breakwaters, and tsunami generation and runup. Prior to joining CHL in 1983, Mr. Briggs worked in the offshore industry for thirteen years.

He received a B.S. in petroleum engineering from The University of Texas at Austin in 1970, an M.S. in ocean engineering from the University of Southern California in 1974, and an Ocean Engineer's degree from the joint program between Massachusetts Institute of Technology and Woods Hole Oceanographic Institution in 1981.

Mr. Briggs is a Fellow in the American Society of Civil Engineers, where he has been president of the Vicksburg Branch, president of the Mississippi Section, co-chairman of the specialty conference "Civil Engineering in the Oceans V," chairman of the Ocean Engineering Technical Committee, and is currently secretary of the Waterways, Ports, Coastal, and Ocean Engineering Executive Committee. He is also a member of the International Association of Hydraulic Engineering and Research and past-chair of their Working Group on Multidirectional Waves. Mr. Briggs is a member of Sigma Xi, The Scientific Research Society, and was president of the WES Chapter. He is also a member of the American Geophysical Union, Tau Beta Pi, Pi Epsilon Tau (Petroleum Engineering Honorary Society), Who's Who Among Students in American Universities and Colleges, and Who's Who in Science and Engineering.

Mr. Briggs has authored or coauthored more than 135 reports, papers, and articles on his research, including 18 journal papers. He is a registered Professional Engineer in the state of Texas. 


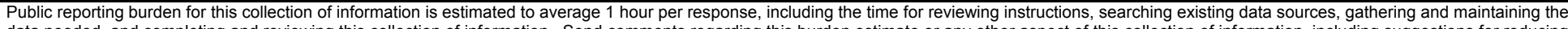

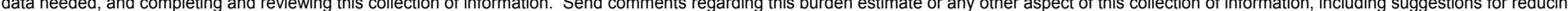

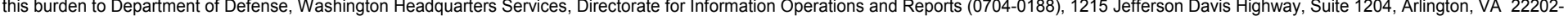

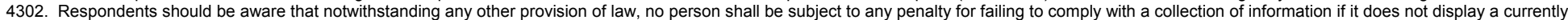
valid OMB control number. PLEASE DO NOT RETURN YOUR FORM TO THE ABOVE ADDRESS.

\begin{tabular}{l|l|l} 
1. REPORT DATE (DD-MM-YYYY) & 2. REPORT TYPE & 3. DATES COVERED (FrOm - To)
\end{tabular}

July 2001

Final Report

4. TITLE AND SUBTITLE

Performance Characteristics of a Rapidly Installed Breakwater System

5a. CONTRACT NUMBER

5b. GRANT NUMBER

5c. PROGRAM ELEMENT NUMBER

6. AUTHOR(S)

5d. PROJECT NUMBER

Michael J. Briggs

5e. TASK NUMBER

5f. WORK UNIT NUMBER

7. PERFORMING ORGANIZATION NAME(S) AND ADDRESS(ES)

8. PERFORMING ORGANIZATION REPORT NUMBER

U.S. Army Engineer Research and Development Center

Coastal and Hydraulics Laboratory

ERDC/CHL TR-01-13

3909 Halls Ferry Road

Vicksburg, MS 39180-6199

\section{SPONSORING / MONITORING AGENCY NAME(S) AND ADDRESS(ES)}

10. SPONSOR/MONITOR'S ACRONYM(S)

U.S. Army Engineer Research and Development Center

3909 Halls Ferry Road

Vicksburg, MS 39180-6199

11. SPONSOR/MONITOR'S REPORT NUMBER(S)

\section{DISTRIBUTION / AVAILABILITY STATEMENT}

Approved for public release; distribution is unlimited.

\section{SUPPLEMENTARY NOTES}

\section{ABSTRACT}

This dissertation describes an integrated study of analytical, numerical, laboratory, and field modeling efforts to quantify the performance and response of a new type of rapidly installed breakwater system (RIBS) being developed at the U.S. Army Engineer Research and Development Center's (ERDC) Coastal and Hydraulics Laboratory (CHL), located in Vicksburg, MS. RIBS is a floating breakwater with two legs in a "V" shape in plan view which provide a sheltered region from waves and currents. The performance of a floating breakwater can be quantified by the wave transmission coefficient. The structural response can be estimated using the waveinduced dynamic pressures along the structure. These pressures can be used to calculate the wave forces and moments. Both the measured wave transmission coefficient and dynamic pressure were compared to analytical and numerical predictions and several new empirical parameters have been developed.

\section{SUBJECT TERMS}

Analytical models

Breakwater performance characteristics

Breakwaters
Dynamic wave pressure

Field measurements

Floating breakwaters
Laboratory modeling

Numerical modeling

Wave transmission coefficient

\begin{tabular}{|c|c|c|c|c|c|}
\hline \multicolumn{3}{|c|}{ 16. SECURITY CLASSIFICATION OF: } & \multirow{2}{*}{$\begin{array}{l}\text { 17. LIMITATION } \\
\text { OF ABSTRACT }\end{array}$} & \multirow{2}{*}{$\begin{array}{l}\text { 18. NUMBER } \\
\text { OF PAGES }\end{array}$} & 19a. NAME OF RESPONSIBLE PERSON \\
\hline $\begin{array}{l}\text { a. REPORT } \\
\text { UNCLASSIFIED }\end{array}$ & b. ABSTRACT & $\begin{array}{l}\text { c. THIS PAGE } \\
\text { UNCLASSIFIED }\end{array}$ & & & $\begin{array}{l}\text { 19b. TELEPHONE NUMBER (include area } \\
\text { code) }\end{array}$ \\
\hline
\end{tabular}

